\title{
Anel de Witt para Semigrupos Reais, Envoltória von Neumann e B-pares
}

\author{
Hugo Rafael de Oliveira Ribeiro
}

\author{
TESE APRESENTADA \\ $\mathrm{AO}$ \\ Instituto De MATEMÁtiCA E EstatísticA \\ DA \\ UniversidAde DE SÃo PAUlo \\ PARA \\ OBTENÇÃO DO TÍTULO \\ $\mathrm{DE}$ \\ DOUTOR EM CIÊNCIAS
}

\author{
Programa: Doutorado em Matemática Pura \\ Orientador: Prof. Dr. Hugo Luiz Mariano
}

Durante o desenvolvimento deste trabalho o autor recebeu auxílio financeiro da CAPES/CNPq 


\section{Agradecimentos}

Venho através deste pequeno texto agradecer as pessoas que direta ou indiretamente contribuíram para o desenvolvimento deste trabalho.

Gostaria de agradecer primeiramente ao Prof. Hugo Luiz Mariano pela orientação e paciência ao longo dos anos em me guiar pelas teorias algébricas de formas quadráticas. Admiro a forma como você pensa a Matemática e quero ser capaz de seguir seu exemplo como pesquisador e pessoa. Também fica o meu mais sincero obrigado ao Prof. Francisco Miraglia Neto que com competência, paciência e transparência se dispôs a me ensinar. As diversas conversas por telefone tiveram um valor para além da Matemática.

Sou privilegiado em ter tido a oportunidade de contar com uma banca formada por pesquisadores cujos trabalhos formaram a base para construção do presente trabalho. Suas sugestões foram valiosas e serão úteis para muito além da tese. Obrigado Prof. Alejandro Petrovich, Prof. Max Dickmann, Prof. Peter Arndt, Prof. Hugo Luiz Mariano e Prof. Francisco Miraglia Neto.

Sou muito grato pelo companheirismo e carinho que a Priscila C. Garrido ofereceu nos momentos que eu mais precisava. Ela dormiu durante minha primeira explicação sobre o anel de Witt mas se manteve acordada enquanto eu dorbia. Agradeço também à minha mãe Sônia por ter me incentivado a aprender Matemática através de uma máquina de costurar botina e à minha avó Antônia que mesmo analfabeta soube me mostrar o valor dos estudos. Faltaram 6 dias para você, manvéia, ver o neto virar dotô, mas o seu descanso veio em hora oportuna.

Agradeço os companheiros da sala B-141: André, Dimi, Felipe, João, Rodrigo e Yuri. Também sou grato pelos irmão acadêmicos: Caio, Jean, Kaique e Luan. Todos vocês contribuíram para meu crescimento pessoal e intelectual.

Um obrigado especial aos professores que me serviram de inspiração e foram determinantes na minha escolha de carreira. Incluo o Prof. Leandro Fiorini Aurichi que me ajudou a dar os primeiros passos na pesquisa através da iniciação científica e a Prof. Rose Francia que me ensinou a ser disciplinado e determinado.

Me sinto grato também pelo ensino de qualidade, gratuito e universal recebido pela USP (ICMC e IME). Deixo um abraço aos funcionários e terceirizados da USP que fizeram parte do meu crescimento.

Por fim, agradeço à Capes e ao Cnpq pelo apoio financeiro dado com o qual pude me dedicar exclusivamente a este trabalho. Também agradeço à Univesp pela ajuda financeira e formação na área educacional dadas durante os dois últimos anos do doutorado. 


\section{Resumo}

Ribeiro, H. O. R. Anéis de Witt para Semigrupos Reais, Envoltória von Neumann e B-

pares. Tese (Doutorado) - Instituto de Matemática e Estatística, Universidade de São Paulo, 2021.

Este trabalho tem como objetivos $i$ ) generalizar o conceito de anel de Witt de grupos especiais reduzidos (RSG) para semigrupos reais (RS), ii) construir a envoltória von Neumann de um RS e iii) descrever a categoria BoolRS dos RSs Booleanos como pares $(G, \nabla)$, onde $G$ é RSG e $\nabla \subseteq G$ com propriedades de primeira ordem. Para cada RS $R$, a partir do anel de Witt $W(R)$ de $R$ é construída sua envoltória von Neumann $V(R)$, que dá origem a um adjunto à esquerda da inclusão vNRS $\hookrightarrow$ RS da categoria dos RSs von Neumann na categoria dos RSs. Além disso, existe isomorfismo canônico entre os anéis de Witt $W(R)$ e $W(V(R))$. Isso permite a análise do anel de Witt com ferramentas disponíveis para os RS von Neumann como a descrição da isometria de formas através de uma pp-fórmula. As principais aplicações de $i i$ ) em $i$ ) são o cálculo do anel graduado de Witt, a conjectura de Marshall para qualquer anel semi-real e uma axiomatização dos aneis de Witt em $\mathcal{L}_{\omega_{1}, \omega}$. O desenvolvimento de iii) permite a classificação de todos quocientes de RSs Booleanos, a construção da envoltória RS-Booleana e a existência, sob certas condições, de um adjunto à direita da inclusão vNRS $\hookrightarrow$ RS.

Palavras-chave: Anel de Witt, Semigrupo Real von Neumann, B-pares. 


\section{Abstract}

Ribeiro, H. O. R. Witt Ring for Real Semigroup, von Neumann Hull and B-pairs. Tese (Doutorado) - Instituto de Matemática e Estatística, Universidade de São Paulo, 2021.

This work purpose is threefold: $i$ ) generalize the Witt ring of reduced special groups (RSG) for real semigroups (RS), ii) build a von Neumann hull for RS and iii) describe the Boolean RS category BoolRS as pairs $(G, \nabla)$, where $G$ is a RSG and $\nabla \subseteq G$ has first-order properties. For each RS $R$, from the Witt ring $W(R)$ is built its von Neumann hull $V(R)$, which gives rise to a left adjoint of inclusion vNRS $\hookrightarrow$ RS from the von Neumann RS category in RS category. Furthermore, there is a natural ring isomorphism $W(R) \cong W(V(R))$. This allows a Witt ring analysis through von Neumann RS tools as the isometry description in first-order using a pp-formula. The main applications of $i$ ) in $i$ ) are the graded Witt ring calculation, the Marshall conjecture for any semi-real ring and an axiomatization of Witt rings in $\mathcal{L}_{\omega_{1}, \omega}$. The iii) development allows a complete classification of Boolean quotients, the construction of a RS-Boolean hull and the existence, under certain conditions, of a right adjoint to the inclusion $\mathbf{v N R S} \hookrightarrow \mathbf{R S}$.

Keywords: Witt Ring, von Neumann Real Semigroup, B-pairs. 


\section{Sumário}

Introdução 1

1 Multi-anéis $\quad 5$

1.1 Definições e exemplos básicos . . . . . . . . . . . . . . . . . . . . . . . . . . . . 5

1.2 Construções na categoria dos Multi-anéis . . . . . . . . . . . . . . . . . . . . . . . . . . 9

1.3 Pré-feixe estrutural . . . . . . . . . . . . . . . . . . . . . . . . . . . 15

1.4 Hiper-anéis von Neumann . . . . . . . . . . . . . . . . . . . . . . . . . . . . . . . . . 18

$2 \begin{aligned} & 2 \\ & 2\end{aligned}$

2.1 Preliminares . . . . . . . . . . . . . . . . . . . . . . . . 27

2.2 Semigrupos Reais como subcategoria reflexiva dos Multi-anéis . . . . . . . . . . . . . 33

2.3 RS Booleano e RS von Neumann . . . . . . . . . . . . . . . . . . . . . . . 46

2.4 Quocientes na categoria dos RS von Neumann . . . . . . . . . . . . . . . . . . . . 52

3 Anel de Witt $\quad 55$

3.1 Isometria e Witt-equivalência . . . . . . . . . . . . . . . . . . . . . . . . . 55

3.2 Qc-formas ................................ 59

3.3 Isometria em RS von Neumann . . . . . . . . . . . . . . . . . . . . . . . . 66

3.4 Anel de Witt . . . . . . . . . . . . . . . . . . . . . . . . . . . . . . . . 80

4 Envoltória von Neumann para Semigrupos Reais 89

4.1 Envoltória von Neumann . . . . . . . . . . . . . . . . . . . . . . . . . . 90

4.2 Aplicações . . . . . . . . . . . . . . . . . . . . . . . . . . . . . . . . . . . 99

4.2.1 Anel Graduado de Witt . . . . . . . . . . . . . . . . . . . . . 100

4.2.2 Quocientes em Aneis de Witt . . . . . . . . . . . . . . . . . . . . . 104

4.2.3 Anel de Witt por geradores e relações . . . . . . . . . . . . . . . . . . . . . 110

4.2.4 Axiomatização de Aneis de Witt . . . . . . . . . . . . . . . . . . . . . . 113

$\begin{array}{llr}5 & \text { B-pares } & 121\end{array}$

5.1 Categoria dos B-pares . . . . . . . . . . . . . . . . . . . . . . . . . 121

5.2 Aplicações . . . . . . . . . . . . . . . . . . . . . . . . . . . . . . . . . . . 131

5.2.1 Quocientes de RSs Booleanos . . . . . . . . . . . . . . . . . . . . 132

5.2 .2 Os inversíveis de um $\mathrm{RS}$. . . . . . . . . . . . . . . . . . . . . . 133

5.2.3 Envoltória RS-Booleana . . . . . . . . . . . . . . . . . . . . . . 139 
viii SUMÁRIO

6 Conclusões $\quad 151$

6.1 Resultados parciais . . . . . . . . . . . . . . . . . . . 151

6.2 Representações de grupos especiais e de semigrupos reais . . . . . . . . . . . . . . . 153

6.3 Outros tópicos para pesquisas futuras . . . . . . . . . . . . . . 154

$\begin{array}{ll}\text { A Quocientes em Categorias Quase-Concretas } & 157\end{array}$

$\begin{array}{lr}\text { Índice Remissivo } & 160\end{array}$

Referências Bibliográficas $\quad 163$ 


\section{Introdução}

Pode-se dizer que a teoria algébrica de formas quadráticas (TAFQ) foi fundada em 1937 por E. Witt com a introdução do conceito de anel de Witt associado a um corpo, construído a partir de suas formas quadráticas: dado um corpo $F$ de característica $\neq 2, W(F)$, o anel de Witt de $F$, classifica as formas quadráticas regulares e anisotrópicas sobre F. Questões sobre a estrutura dos anéis de Witt só puderam ser solucionadas cerca de 3 décadas após com a introdução das formas de Pfister. Desde então, a TAFQ ganhou diversas formalizações abstratas, notadamente a teoria dos grupos especiais ([DM1]) e a teoria dos semigrupos reais ([DP2]).

A teoria dos grupos especiais (SG), introduzida por M. Dickmann e F Miraglia na década de 1990, é de primeira ordem e trata simultaneamente o caso não-reduzido e reduzido. Sua axiomatização na linguagem $\mathcal{L}_{S G}$ toma como conceito primitivo a isometria de formas de dimensão $2 \mathrm{e}$ o desenvolvimento da teoria permite definir naturalmente noções da teoria algébrica de formas quadráticas como isometria entre formas de mesma dimensão finita, representantes de uma forma, equivalência de Witt entre formas, formas de Pfister, anel de Witt, etc. Com os SGs foi possível resolver dois grandes problemas em aberto: a conjectura de Marshall e a de Lam para corpos ([DM2]). Enquanto a conjectura de Lam foi formulado originalmente para corpos, a de Marshall foi formulada no contexto dual dos espaços de ordem abstratos (AOS), introduzidos por M. Marshall nos anos 1980. Um dos ingredientes importante nas resoluções, juntamente com os trabalhos de V. Voevodsky e colaboradores sobre a conjectura de Milnor, foi a envoltória Booleana de um grupo especial reduzido (RSG).

Por outro lado, a teoria dos semigrupos reais (RS) foi criada por M. Dickmann e A. Petrovich na década de 2000 como uma teoria de primeira ordem para os espectros reais de aneis ([DP2]), de forma a estender o conceito de grupo especial reduzido, mas utilizando como conceito primitivo a noção de representação (e de representação tranversal) de uma forma de dimensão 2. Anteriormente, nos anos 1990, M. Marshall havia introduzido os espectro reais abstratos (ARS) como axiomatização dos espectros reais de aneis ([Mar7]) mas esta teoria não é de primeira ordem e emprega métodos topológicos enquanto em RS são utilizados sobretudo métodos algébricos. Em [DP2] é provado que existe uma dualidade entre as categorias RS e ARS, que estende a dualidade entre RSG e AOS.

O interesse em estudar os semigrupos reais surgiu a partir dos Seminários de Teoria Algébrica de Formas Quadráticas organizados pelo Hugo Luiz Mariano e Francisco Miraglia Neto (https: //sites.google.com/site/seminarioformasquadraticas/home). Mais especificamente, o foco desta tese nos RSs Booleanos e von Neumann RSs foi por influência de algumas apresentações do Miraglia sobre o tema ocorridas o $2^{\circ}$ semestre de 2017 . De forma mais abrangente, o presente trabalho tem três objetivos principais:

- Investigar a teoria algébrica de formas quadráticas em RSs através da introdução e desenvolvimento dos conceito de isometria de formas, formas de Pfister e de anel de Witt de um RS.

- Construir a envoltória von Neumann de um RS e utilizá-la para estudar a estrutura dos aneis de Witt de RSs.

- Descrever as categorias BoolRS dos RSs Booleanos como pares $(G, \nabla), G$ RSG e $\nabla \subseteq G$ com propriedades adequadas, e com isso construir a envoltória RS-Booleana. 
O ponto de partida é a noção de multi-anel apresentada originalmente em [Mar10] que fornece um bom plano de fundo para os objetos envolvidos na presente investigação. A categoria Multi dos multi-aneis possui como subcategoria plena e fiel as categorias

- dos aneis (comutativos com unidade);

- dos semigrupos reais (em particular, a categoria dos RSGs);

- dos grupos especiais.

Iremos apresentar brevemente os funtores que fazem o trabalho acima ao longo da Seção 2.2 mas as provas completas estão em [RRM1]. Além de ser possível trabalhar dentro de uma só categoria, no primeiro capítulo são apresentadas diversas construções de álgebra comutativa para multi-aneis como quocientes por ideias, espectros primo e real e localização, bem como o quociente de Marshall, essenciais para desenvolver a teoria de formas quadráticas. Essas construções já foram definidas em [Mar10] mas aqui é dada ênfase nas propriedades universais associadas. Na seção 1.3, introduzimos o pré-feixe estrutural de um multi-anel. Quando este pré-feixe é feixe, o multi-anel é dito geométrico (o Exemplo 1.38 mostra que nem todos são geométricos). Como o pré-feixe de multi-aneis generaliza o feixe de aneis, o feixe de hiperdomínios descrito em [Jun] e de semigrupos reais (Teorema 2.15), essas três classes de multi-aneis são geométricas. Na seção seguinte, a discussão é especializada para os hiper-aneis von Neumann ( $\mathrm{HAvN})$, para os quais é obtido uma envoltória geométrica no Teorema 1.39 e seus quocientes categoriais são classificados utilizando o quociente de Marshall (Teorema 1.43).

No segundo capítulo, trabalhamos com a noção de semigrupo real que foi introduzida em [DP1], e apresentamos sua formulação em Multi chamada de multi-anel real reduzido, que possui axiomatização mais intuitiva (Definição 2.30) e será amplamente utilizada ao longo do trabalho. Na primeira seção, seguindo [DP2], encontram-se as definições e os resultados fundamentais sobre RSs. Ainda, no Teorema 1.22, é estabelecido um isomorfismo entre o pré-feixe estrutural de multianeis e o feixe canônico de RSs descrito em [DP2]. Já na segunda seção, seguindo ([Mar10]), são apresentados para conveniência do leitor alguns conceitos fundamentais de multi-álgebra real, com destaque para o funtor $Q:$ pMulti $\rightarrow \mathbf{R R M}$ da categoria dos p-multi-aneis para a categoria dos multi-aneis reais reduzidos que é adjunto à esquerda do funtor de inclusão (Teorema 2.41). A seção é finalizada com a extensão da representação de $Q$ para hipercorpos encontrada em [Mar10] para os hiper-aneis von Neumann (Teorema 2.50). Uma consequência disto é que o funtor $Q$ preserva equivalência elementar de HAvN (Teorema 2.51). Na seção 2.3, duas classes de semigrupos reais entram em foco: os Booleanos e os von Neumann. Seguindo [MR], o Teorema 2.54 fornece algumas caracterizações dos RSs Booleanos. Por outro lado, o Teorema 2.58 lista caracterizações de RSs von Neumann. Além disso, a Proposição 2.57 caracteriza a representação transversal de um RS Booleano em função do RSG formado por seus inversíveis, resultado que será essencial no desenvolvimento da Capítulo 5 (B-pares). O capítulo é finalizado com a classificação dos quocientes categoriais dos RSs von Neumann via quociente de Marshall (Teoremas 2.68 e 2.69).

No Capítulo 3, os principais objetivos são produzir uma extensão do conceito de anel de Witt dos RSGs para os RSs e dar os primeiros passos na análise desse anel, investigando como as noções de isometria $(\equiv)$ e Witt-equivalência $(\cong)$ de formas interagem para a produção das propriedades do anel. Também é destacado o bom comportamento da isometria de formas para RSs von Neumann, aspecto que será útil para analisar a estrutura de qualquer anel de Witt no próximo capítulo.

$\mathrm{Na}$ seção 1, as relações básicas entre isometria e equivalência são apresentadas. Na segunda seção, é introduzida a noção de qc-forma, tipo especial de forma onde o número de entradas é bem definido módulo isometria. No Corolário 3.10 é estabelecido que se $\varphi, \psi$ são duas formas sobre um RS $R$, então

$$
\varphi \cong \psi \Leftrightarrow \text { Existem formas } \theta, \theta^{\prime} \text { sobre } \operatorname{Id}(R) \text { tais que } \varphi \oplus \theta\langle 1,-1\rangle \equiv \psi \oplus \theta^{\prime}\langle 1,-1\rangle \text {. }
$$

A seção é finalizada com a apresentação das formas $\left\langle a^{2}, a\right\rangle, a \in R$, uma versão para RSs de formas de Pfister que será útil no decorrer do trabalho. Na seção 3, especificamos a discussão para RSs von 
Neumann, onde obtemos a representação de qualquer forma como soma de qc-formas ortogonais. Isso permite obter uma pp-fórmula que descreve a isometria de formas (Teorema 3.23) e descrever a representação transversal $D^{t}$ em função da isometria (Teorema 3.37). Além disso, a classe dos RSs para os quais vale o Teorema da Decomposição de Witt é analisada. Finalizamos a seção com uma versão do Teorema da Subforma de Pfister para RSs von Neumann (Teorema 3.43).

Na última seção do Capítulo 3, o anel de Witt é definido para qualquer RS. Descrevemos seu espectro primo (Proposição 3.48) e espectro real juntamente com suas assinaturas (Proposição 3.46). No Teorema 3.51 caracterizamos, via anel de Witt, os RSs Booleano e von Neumann. O capítulo é finalizado com a descrição do ideal fundamental $I(R)$ como aditivamente gerado pelas formas de Pfister de grau 1.

No capítulo 4, a envoltória von Neumann de um RS é introduzida e estudamos algumas aplicações para os aneis de Witt de RSs. A teoria algébrica reduzida de formas quadráticas possui algumas envoltórias que têm se mostrado frutíferas. Na teoria dos grupos especiais reduzidos (RSG), existe a envoltória Booleana ([DM1]) que foi um dos ingredientes essenciais na solução da conjectura de Marshall ([DM2]). Outra envoltória dos RSGs é a profinita, introduzida e analisada em [MM1]. Num sentido preciso, ela é um refinamento da envoltória Booleana que prova um novo princípio local-global: a reflexão de subformas. Já na teoria dos semigrupos reais, foi construída em [DP2] a envoltória de Post que compartilha algumas propriedades da envoltória Booleana de RSG como caracterização de imersões completas e comutação com quocientes arbitrários. Por outro lado, enquanto envoltória de Post de um RS tem informação apenas da topologia construtível de seu espaço de ordens, a envoltória von Neumann não apenas preserva informação do espectro real como também do espectro primo e dos RSGs construídos a partir das fibras do feixe estrutural (Teorema 4.10).

A primeira seção é dedicada à construção da envoltória von Neumann de qualquer RS a partir de seu anel de Witt e à demonstração de suas propriedades básicas. A mais importante é que dado RS $R$, o morfismo canônico para a envoltória von Neumann $v: R \rightarrow V(R)$ induz um isomorfismo natural $W(R) \cong W(V(R))$ entre os aneis de Witt, o que permitirá em seguida utilizar a isometria em RSs von Neumann para deduzir informações sobre aneis de Witt de qualquer RS.

Na segunda seção algumas aplicações da envoltória von Neumann são dadas como o cálculo do anel graduado, a axiomatização dos aneis de Witt em $\mathcal{L}_{\omega_{1}, \omega}$ na linguagem dos aneis comutativos e a classificação dos quocientes na categoria dos aneis de Witt. Os principais resultados são:

- Descrição do anel graduado de Witt de um RS em função do anel graduado de Witt do RSG formado pelos inversíveis da envoltória von Neumann (Teorema 4.30).

- O Haupsatz de Arason-Pfister vale para todo RS (Teorema 4.28).

- A conjectura de Marshall para RSs (Definição 4.31) vale para todo RS $G_{A, T}$, onde $(A, T)$ p-anel (Teorema 4.34).

- Caracterização da isometria de formas num quociente de RS von Neumann (Teorema 4.43).

- Descrição do anel de Witt de um RS por geradores e relações canônicas (Teorema 4.49).

- Na Definição 4.56 introduzimos os aneis de Witt abstratos e no Teorema 4.63 é mostrado que a categoria dos RSs von Neumann é equivalente a categoria aneis de Witt abstratos.

Na seção 2.2, a noção de RS Booleano foi estudada brevemente, sobretudo no que diz respeito a como sua estrutura geral se relaciona com o RSG formado por seus inversíveis (e.g. Proposição 2.57). O Capítulo 5 tem como um de seus objetivo tornar mais precisa a conexão entre RSs Booleanos e RSGs, a fim de serem identificados com maior clareza os resultados dos RSGs que podem ser transferidos para os RSs Booleanos.

Na primeira seção, os $B$-pares são definidos (Definição 5.5). Eles são os modelos da linguagem de primeira ordem $\mathcal{L}_{S G} \cup\{\nabla\}, \nabla$ símbolo de predicado unário, que satisfazem axiomas Horngeométricos (Proposição 5.6). O restante da seção é dedicado a estabelecer que as categorias 
BoolRS e $\mathbf{B}_{p a r}$ são equivalentes; trabalho análogo é realizado para descrever a categoria vNRS dos RSs von Neumann. Mais precisamente, os resultados que se destacam são:

- Caracterização de um morfismo $f: R \rightarrow S$ entre RSs Booleanos via propriedades das restrições $f \mid: R^{\times} \rightarrow G^{\times}$e $f \mid: \operatorname{Id}(R) \rightarrow \operatorname{Id}(G)$ (Proposição 5.10).

- Descrição do funtor $\mathfrak{R}: \mathbf{B}_{\text {par }} \rightarrow$ BoolRS que associa um a cada $B$-par um RS Booleano (Teorema 5.14).

- O funtor $\mathfrak{L}:$ BoolRS $\rightarrow \mathbf{B}_{\text {par }}$ juntamente com o funtor $\mathfrak{R}: \mathbf{B}_{p a r} \rightarrow$ BoolRS estabelecem uma equivalência de categorias (Teorema 5.16).

Na primeira subseção das aplicações, o principal resultado é o Teorema 5.21 que caracteriza os quocientes na categoria $\mathbf{B}_{\text {par }}$. Tendo em vista o equivalência de categorias BoolRS $\simeq \mathbf{B}_{\text {par }}$, tem-se uma caracterização dos quocientes em BoolRS.

Na segunda subseção das aplicações, a primeira parte consiste em introduzir as noções de RS com suficientes unidades (Definição 5.22) e de RS com suficientes qc-formas (Definição 5.26). No Teorema 5.25 é provado que num RS com suficientes qc-formas, a isometria de qc-formas é descrita por uma pp-fórmula e como corolário são obtidos axiomas Horn-Geométricos para RSs com suficientes unidades e suficientes qc-formas. Na segunda parte, é provado que se $R$ é um RS com suficientes unidades, então existe morfismo $I_{R}: V \rightarrow R, V$ RS von Neumann, final com relação a morfismos saindo de um RS von Neumann e chegando em $R$. Em particular, se todo RS tiver suficientes unidades (problema em aberto), então a inclusão vNRS $\rightarrow \mathbf{R S}$ admite um adjunto à direita. Uma recíproca parcial é obtida no Teorema 5.41: se a inclusão vNRS $\rightarrow \mathbf{R S}$ admite um adjunto à direita, então os inversíveis de todo RS formam um RSG.

Na terceira subseção, a envoltória RS-Booleana de um RS em geral é apresentada (Teoremas 5.50 e 5.52) e são dadas algumas caracterizações de monomorfismo, epimorfismo e imersão completa entre RSs utilizando as envoltórias von Neumann e RS-Booleana e aneis de Witt (Teoremas 5.63 e Teoremas 5.64).

A tese é finalizada com: (i) um capitulo curto de "conclusões", apresentando descrições de resultados parciais e de alguns temas de pesquisas futuras com os quais nos deparamos no desenvolvimento deste trabalho; (ii) um apêndice sobre morfismos quocientes, utilizados em diversas ocasiões durante o texto. 


\section{Capítulo 1}

\section{Multi-anéis}

O ponto de partida do presente trabalho é a noção de multi-anel apresentada em [Mar10]. Nosso objetivo geral é tratar a teoria algébrica de formas quadráticas através da noção de semigrupo real (RS). No Teorema 2.32 (e de forma mais detalhada em [RRM1]) é mostrado de que modo os RSs podem ser vistos como multi-aneis, facilitando o entendimento de algumas construções com semigrupos reais através do paralelo com as correspondentes noções em álgebra comutativa (como o feixe estrutural de RS no Teorema 2.15). Além disso, em [Mar10] é apresentada axiomatização mais simples dos RSs, descrita na Definição 2.30.

\subsection{Definições e exemplos básicos}

O conceito de multi-anel pode ser visto como uma generalização dos anéis comutativos adequada às formas quadráticas. Um multi-anel é um "anel"cuja soma é multivalorada.

Definição 1.1. Um multi-anel é uma tupla $(A,+,-, \cdot, 0,1)$ onde $(A, \cdot, 1)$ é um monóide comutativo, $+: A^{2} \rightarrow \mathcal{P}(A)^{*}(=\mathcal{P}(A) \backslash\{\emptyset\})$ e $-: A \rightarrow A$ são funções e $0,1 \in A$ são constantes tais que para todo $a, b, c \in R$ :

i) $a \in b+c \Rightarrow c \in-b+a$ e $b \in a+(-c)$.

ii) $a \in b+0 \Leftrightarrow a=b$.

iii) $a+b=b+a$.

iv) (Associatividade) Se $x \in g+c \operatorname{com} g \in a+b$, então existe $h \in b+c$ tal que $x \in a+h$.

v) $a .0=0$.

vi) (Distributividade fraca) Se $a \in b+c$, então $d \in A, a . d \in b . d+c . d$.

Note que, se $A$ é um multi-anel e $a \in A$, então $0=0 . a \in(1+(-1)) \cdot a \subseteq 1 \cdot a+(-1) \cdot a, \operatorname{logo}$ $(-1) \cdot a=-a$.

Fixado um multi-anel $A$ e subconjuntos $X, Y$, podemos somá-los através da regra $X+Y=\{a \in$ $A$ : existem $x \in X, y \in Y$ tais que $a \in x+y\}$. Assim, o axioma $i v$ ) assume um formato mais usual: $(a+b)+c \subseteq a+(b+c)$. Em alguns multi-anéis -como os anéis (Exemplo 1.4)-, a propriedade vi) assume uma recíproca:

$$
x \in b d+c d \Rightarrow \text { existe } a \in b+c \text { tal que } x=a d .
$$

Os multi-anéis que a satisfazem recebem o nome de hiper-anéis.

Algumas noções úteis para anéis também estão presente no contexto multi-valorado. Um multianel é dito multidomínio se para todo $a, b \in A$ com $a b=0$ tem-se $a=0$ ou $b=0$. Similarmente, 
$a \in A$ é inversível se existe $b \in A$ com $a b=1$ e é chamado de fracamente inversivel se existem $b_{1}, \ldots, b_{n} \in A$ com $1 \in a b_{1}+\cdots+a b_{n}$. Denotamos por $A^{\times}$o conjunto dos elementos inversíveis e por $A^{\times}{ }^{\times}$o conjunto dos fracamente inversíveis. Note que $A^{\times} \subseteq A^{\times}$e se $A$ é hiper-anel, então $A^{\times w}=A^{\times}$. Um multi-anel $A$ é chamado de multicorpo se todo elemento não-nulo é fracamente inversível e de hipercorpo se todo não-nulo é inversível (se $A$ é hipercorpo, então $A$ é hiperanel; de fato, dados $x \in a b+a c, a \neq 0$, então $x=a\left(a^{-1} x\right)$ e $\left.a^{-1} x \in a^{-1}\left(a b+a^{-1} a c\right) \subseteq b+c\right)$.

Comentários 1.2. - É possível definir multi-anel como um estrutura de primeira ordem interpretando a soma multivalorada como uma relação ternária: dado multi-anel $(A,+,-, \cdot, 0,1)$, a soma + pode ser vista como uma relação ternária $\pi \subseteq A^{3}$ definida como $c \in a+b$ se, e somente se, $(a, b, c) \in \pi$. Na linguagem de primeira ordem, todo axioma de multi-anel é Horn-geométrico ${ }^{1}$. Deste modo, a categoria dos multi-aneis é fechada por produtos reduzidos (em particular produtos arbitrários) e limites indutivos (para o fechamento por produtos reduzidos, confira a Proposição 6.2.2 de [CK] e para o fechamento por limites indutivos confira 1.21).

- Na definição de multi-anel, a soma multi-valorada é assumida ser não-vazia, porém isto pode ser deduzido dos axiomas. De fato, dados $a, b \in A$, como $a \in 0+a$ e $0 \in b+(-b)$, a associatividade garante a existência de $h \in a+b$ com $a \in-b+h$.

Lema 1.3. Seja $A$ um multi-anel. Dados $a, b, c \in A$ :

i) $-0=0$ e $-(-a)=a$.

ii) $a \in b+c$ se, e somente se, $-a \in-b+-c$.

Demonstração. i) $0 \in 0+0 \Rightarrow 0 \in-0+0 \Rightarrow-0=0$ e $x \in x+0 \Rightarrow 0 \in-x+x \Rightarrow x \in$ $-(-x)+0 \Rightarrow-(-x)=x$.

ii) $a \in b+c \Leftrightarrow c \in-b+a \Leftrightarrow-b \in c+-a \Leftrightarrow-a \in-b+-c$.

Exemplo 1.4. - Dado anel $(A,+,-, \cdot, 0,1)$, defina $+^{\prime}: A \times A \rightarrow \mathcal{P}(A)^{*} \operatorname{como} a+^{\prime} b=\{a+b\}$. Então $\left(A,+^{\prime},-, \cdot, 0,1\right)$ é hiper-anel.

- O hipercorpo de Krasner $\mathbb{K}=\{0,1\}$ é dado pela multiplicação usual e soma multivalorada por $0+0=\{0\}, 0+1=\{1\}, 1+1=\{0,1\}$.

- O hipercorpo de sinais $\mathbf{3}=\{1,0,-1\}$ é dado pela multiplicação usual e soma multivalorada por $0+x=\{x\} \forall x \in \mathbf{3}, 1+1=\{1\},-1+(-1)=\{-1\}, 1+(-1)=\mathbf{3}$.

Definição 1.5. Seja $f: A \rightarrow B$ um mapa entre multi-anéis. A função $f$ é um morfismo de multianéis se para todo $a, b, c \in A$ :

i) Se $a \in b+{ }_{A} c$, então $f(a) \in f(b)+_{B} f(c)$.

ii) $f\left(a \cdot \cdot_{A} b\right)=f(a) \cdot B f(b)$.

iii) $f\left(0_{A}\right)=0_{B}, f\left(1_{A}\right)=1_{B}$.

iv) $f\left(-{ }_{A} a\right)=-{ }_{B} f(a)$.

$f$ é chamado de morfismo forte se além de ser morfismo vale a seguinte recíproca do item $i)$ : se $f(a) \in f(b)+_{B} f(c)$, então existem $a^{\prime}, b^{\prime}, c^{\prime} \in A$ tais que $f\left(a^{\prime}\right)=f(a), f\left(b^{\prime}\right)=f(b), f\left(c^{\prime}\right)=f(c)$ e $a^{\prime} \in b^{\prime}+{ }_{A} c^{\prime 2}$.

\footnotetext{
${ }^{1}$ Uma fórmula $\varphi(\bar{t})$ é Horn-geométrica se é a negação de um fórmula atõmica ou é da forma $\forall \bar{v}\left(\exists \bar{w} \psi_{1}(\bar{v}, \bar{t}, \bar{w}) \rightarrow\right.$ $\left.\exists \bar{w} \psi_{2}(\bar{v}, \bar{t}, \bar{w})\right)$, onde $\psi_{1}, \psi_{2}$ são conjunções finitas de fórmulas atômicas.

${ }^{2} \mathrm{Na}$ Definição 2.22 de [DM1], é apresentada noção semelhante no contexto de grupos especiais com o nome de morfismo regular. Aqui optamos por manter a nomemclatura de [Mar10]
} 
Comentários 1.6. Seja $f: A \rightarrow B$ morfismo de multi-aneis.

- Dados $a, a_{1}, \ldots, a_{n} \in A$, se $a \in a_{1}+\cdots+a_{n}$, então $f(a) \in f\left(a_{1}\right)+\cdots+f\left(a_{n}\right)$.

- Se $B$ é um anel (para todo $a, b \in B$ vale $\operatorname{card}(a+b)=1$ ), então $f$ é morfismo forte. De fato, se $f(a) \in f(b)+f(c)$, então dado qualquer $x \in a-b-c$ vale $f(x)=0$. Fixado $x \in a-b-c$, existe $l \in-b-c$ tal que $x \in a+l$. Logo $-l \in b+c$ e $f(-l)=f(a)$.

Seja $A$ um multi-anel e $S, T \subseteq A$. Como já observamos anteriormente, podemos somar conjuntos pela fórmula $S+T=\bigcup_{s \in S, t \in T} s+t$ e multiplicá-los de maneira análoga $S \cdot T=\{s t: s \in S, t \in T\}$.

Um conjunto não vazio $\alpha \subseteq A$ é um ideal se $\alpha+\alpha \subseteq \alpha$ e $A \alpha \subseteq \alpha$; note que $0 \in \alpha$ e $-\alpha=$ $(-1) . \alpha \subseteq \alpha$. Um ideal $\alpha$ é próprio se $1 \notin \alpha$. Um ideal $\alpha$ é primo se é próprio e dados $a, b \in A$ com $a b \in \alpha$ tem-se $a \in \alpha$ ou $b \in \alpha$. O ideal $\alpha$ é maximal se é maximal com respeito a inclusão de ideais próprios. Uma forma de classificar ideias primos é como morfismos para o hipercorpo de Krasner: se $f: A \rightarrow \mathbb{K}$ é morfismo, então $f^{-1}(0)$ é ideal primo e dado $p \subseteq A$ ideal primo, a função característica $\chi_{p}: A \rightarrow \mathbb{K}$ dada por $\chi_{p}(x)=0$ se, e somente se, $x \in p$ é morfismo de multi-anéis. O conjunto de todos ideias primos do multi-anel $A$ é denotado por $\operatorname{spec}(A)$ e é chamado de espectro primo . Um conjunto $S \subseteq A$ é multiplicativo se $1 \in S$ e $S \cdot S \subseteq S$. Dado um subconjunto $X \subseteq A$, o ideal gerado por $X$ é $[X]=\bigcup\left\{t_{1} x_{1}+\cdots+t_{n} x_{n}: n \geq 1, t_{i} \in A, x_{i} \in X\right.$ for all $\left.i=1, \ldots, n\right\}$. Note que se $X=\left\{x_{1}, \ldots, x_{n}\right\}$ é finito, em geral $[X] \neq \bigcup\left\{t_{1} x_{1}+\cdots+t_{n} x_{n}: t_{i} \in A\right.$ para todo $\left.i=1, \ldots, n\right\}$ mas vale a igualdade quando $A$ é um hiper-anel.

Proposição 1.7. Seja $A$ um multi-anel.

i) Todo ideal maximal é primo.

ii) (Teorema do Ideal Primo) Sejam $I \subseteq A$ ideal e $S \subseteq A$ conjunto multiplicativo tal que $I \cap S=\emptyset$. Então existe ideal primo $p$ tal que $I \subseteq p$ e $p \cap S=\emptyset$. Em particular, $A \neq 0$ se, e somente se, $\operatorname{spec}(A) \neq \emptyset$.

iii) Dado $a \in A$, considere o conjunto $D(a)=\{p \in \operatorname{spec}(A): a \notin p\}$. Então $D(a) \cap D(b)=D(a b)$ e a família $\{D(a): a \in A\}$ é base de abertos-compactos para uma topologia espectral em $\operatorname{spec}(A)$. Além disso, dado $p \in \operatorname{spec}(A), \overline{\{p\}}=\{q \in \operatorname{spec}(A): p \subseteq q\}$.

Demonstração. i) Sejam $\mathfrak{m} \subseteq A$ um ideal maximal e $a, b \in A$ com $a b \in \mathfrak{m}$. Se $a \notin \mathfrak{m}$, considere o ideal $I$ gerado por $\mathfrak{m} \cup\{a\}$ :

$$
I=\bigcup\left\{m+t_{1} a+\cdots+t_{n} a: n \geq 1, m \in \mathfrak{m}, t_{i} \in A \text { para todo } i=1, \ldots, n\right\} .
$$

Como $\mathfrak{m}$ é ideal maximal e $a \in I \backslash m$, o ideal $I$ é impróprio. Então existem $m \in \mathfrak{m}$ e $t_{1}, \ldots, t_{n} \in A$ tais que $1 \in m+t_{1} a+\cdots+t_{n} a$. Assim $b \in b m+t_{1} a b+\cdots t_{n} a b \subseteq \mathfrak{m}$.

ii) Seja $X=\{J \subseteq A: J$ é um ideal com $I \subseteq J, J \cap S=\emptyset\}$. Com a ordem dada pela inclusão de conjuntos, $(X, \subseteq)$ é uma ordem parcial não-vazia $(I \in X)$ e é imediato verificar que a outra condição do lema de Zorn está satifeita. Logo existe $p \in X$ maximal. Se $p$ não é ideal primo, então existem $a, b \in A$ tais que $a b \in p$ e $a, b \notin p$. Considere o ideal $J=p+(a)=\bigcup\{x+$ $\left.a t_{1}+\cdots+a t_{n}: x \in p, t_{1}, \ldots, t_{n} \in A\right\}$. Como $a \in J \backslash p$ e $p \subseteq J$, pela maximalidade de $p$ temos $J \cap S \neq \emptyset$. Então existem $s_{1} \in S, x \in p$ e $t_{1}, \ldots, t_{n} \in A$ tais que $s_{1} \in x+a t_{1}+\cdots+a t_{n}$. Usando o ideal $p+(b)$, também existem $s_{2} \in S, y \in p$ e $l_{1}, \ldots, l_{k} \in A$ tais que $s_{2} \in y+b l_{1}+\cdots+b l_{k}$. Logo $s_{1} s_{2} \in x y+x b l_{1}+\cdots+x b l_{k}+y_{a} t_{1}+\cdots y a t_{n}+\sum_{i, j} a b t_{i} l_{j} \subseteq S \cap p$, um absurdo. Assim $p$ é primo.

iii) A demonstração é semelhante ao caso dos anéis e pode ser encontrada na Proposição 2.3 de [Mar10]. 
Comentário 1.8. - Se $f: A \rightarrow B$ é morfismo de multi-aneis, para cada $p \in \operatorname{spec}(B), f^{-1}(p) \in$ $\operatorname{spec}(A)$ é ideal primo. Isto induz um mapa $f^{*}=f^{-1}: \operatorname{spec}(B) \rightarrow \operatorname{spec}(A)$ tal que para cada $a \in A,\left(f^{*}\right)^{-1}\left(D_{A}(a)\right)=D_{B}(f(a))$ e $f^{*}\left(D_{B}(f(a))\right)=D_{A}(a) \cap \operatorname{Im}\left(f^{*}\right)$. Em particular, $f^{*}$ é espectral ${ }^{3}$ e se $f$ é sobrejetora, $f^{*}: \operatorname{spec}(B) \rightarrow \operatorname{Im}\left(f^{*}\right) \subseteq \operatorname{spec}(A)$ é aberta.

- Sejam $A$ multi-anel e $p \in \operatorname{spec}(A)$. Então $\overline{\{p\}}=\{q \in \operatorname{spec}(A): p \subseteq q\}$. De fato, dado $q \in$ $\operatorname{spec}(A) \operatorname{com} p \subseteq q$ e $x \in A$ com $q \in D(x)$, temos $p \in D(x)$ e assim $q \in\{p\}$. Reciprocamente, se $q \in \overline{\{p\}}$, dado $x \notin q$, isto é, $q \in D(x)$, temos $p \in D(x)$. Assim $p \subseteq q$. Em particular, $p \in \operatorname{spec}(A)$ é maximal se, e somente se, $\{p\} \subseteq \operatorname{spec}(A)$ é fechado.

Definição 1.9. Seja $f: A \rightarrow B$ morfismo de multi-aneis.

- Define-se $\operatorname{ker}(f)=\left\{(x, y) \in A^{2}: f(x)=f(y)\right\} \subseteq A^{2}$ como o núcleo de $f$.

- Define-se $\operatorname{ker}_{1}(f)=\{x \in A: f(a)=1\} \subseteq A$.

- O morfismo $f$ é dito quociente se dado morfismo $g: A \rightarrow C$ de multi-aneis com $\operatorname{ker}(f) \subseteq \operatorname{ker}(g)$, existe único morfismo $\bar{g}: B \rightarrow C$ tal que $g=\bar{g} \circ f$.

- Seja $\mathcal{C}$ uma subcategoria plena dos multi-aneis. Seja $f: A \rightarrow B$ morfismo em $\mathcal{C}$. Então $f$ é quociente em $\mathcal{C}$ se dado morfismo $g: A \rightarrow C$ em $\mathcal{C} \operatorname{com} \operatorname{ker}(f) \subseteq \operatorname{ker}(g)$, existe único morfismo $\bar{g}: B \rightarrow C$ em $\mathcal{C}$ tal que $g=\bar{g} \circ f$. Note que se $f$ é morfismo em $\mathcal{C}$ e é quociente em Multi, então é quociente em $\mathcal{C}$.

$\{$ kerSub\}

Proposição 1.10. Sejam $h: A \rightarrow B, f: B \rightarrow C, g: B \rightarrow E$ morfismos de multi-aneis. Então

i) $\operatorname{ker}(h) \subseteq \operatorname{ker}(f \circ h)$.

ii) Se $\operatorname{ker}(f) \subseteq \operatorname{ker}(g)$, então $\operatorname{ker}(f \circ h) \subseteq \operatorname{ker}(g \circ h)$.

Demonstração. i) Seja $(x, y) \in \operatorname{ker}(h)$. Então $h(x)=h(y)$ e portanto $f(h(x))=f(h(y))$. Logo $(x, y) \in \operatorname{ker}(f \circ h)$.

ii) Seja $(x, y) \in \operatorname{ker}(f \circ h)$. Então $f(h(x))=f(h(y))$ e $(h(x), h(y)) \in \operatorname{ker}(f) \subseteq \operatorname{ker}(g)$. Logo $g(h(x))=g(h(y))$ e $(x, y) \in \operatorname{ker}(g \circ h)$.

$\{r e g Q u o\}$

Proposição 1.11. Seja $f: A \rightarrow B$ morfismo de multi-aneis

i) Suponha que $f$ seja morifsmo forte e sobrejetor. Então $f$ é quociente.

ii) Suponha $f$ quociente e sobrejetor. Então o morfismo induzido

$$
f^{*}: \operatorname{spec}(B) \rightarrow\left\{p \in \operatorname{spec}(A): \operatorname{ker}(f) \subseteq \operatorname{ker}\left(\chi_{p}\right)\right\} \subseteq \operatorname{spec}(A),
$$

onde $\chi_{p}: A \rightarrow \mathbb{K}$ denota o morfismo característico de $p \subseteq A$ para o hipercorpo de Krasner, é um homeomorfismo com a imagem.

Demonstração. i) Seja $g \in A \rightarrow C$ morfismo com $\operatorname{ker}(f) \subseteq \operatorname{ker}(g)$. Deste modo, pela sobrejetividade de $f$, existe única função $\bar{g}: B \rightarrow C$ tal que $g=\bar{g} \circ f$. Vamos mostrar que $\bar{g}$ é morfismo de multi-aneis. Segue direto da definição que $\bar{g}$ preserva as constantes $0,1,-1$ e o produto. Por outro lado, sejam $x=f(a), y=f(b), z=f(c) \in B$ tais que $x \in y+z$. Como $f$ é morfismo forte, existem $a^{\prime}, b^{\prime}, c^{\prime} \in A$ tais que

- $f\left(a^{\prime}\right)=f(a), f\left(b^{\prime}\right)=f(b), f\left(c^{\prime}\right)=f(c)$;

\footnotetext{
${ }^{3}$ Dados $X, Y$ espaços topológicos espectrais, uma função $f: X \rightarrow Y$ é espectral se pré-imagem de aberto-compacto é aberto-compacto. Em particular, mapas espectrais são contínuos e mapas contínuos entre espaços topológicos Booleanos (i.e., os espaços espectrais e $T_{1}$ ) são espectrais.
} 
- $a^{\prime} \in b^{\prime}+c^{\prime}$.

Então $\bar{g}(x)=g\left(a^{\prime}\right) \in g\left(b^{\prime}\right)+g\left(c^{\prime}\right)=\bar{g}(y)+\bar{g}(z)$, como desejado.

ii) Pela propriedade universal do quociente, segue que o morfismo $f^{*}: \operatorname{spec}(B) \rightarrow\{p \in \operatorname{spec}(A): \operatorname{ker}(f) \subseteq$ $\left.\operatorname{ker}\left(\chi_{p}\right)\right\} \subseteq \operatorname{spec}(A)$ é bijetor com a imagem.

O fato de $f^{*}$ ser homeomorfismo com a imagem decorre do Comentário 1.8.

Exemplo 1.12. Seja $f: A \rightarrow B$ morfismo de multi-aneis com $B$ anel. Então $f$ é forte. Em particular, todo morfismo sobrejetor de aneis é quociente.

\subsection{Construções na categoria dos Multi-anéis}

As construções são descritas em [Mar10] e aqui adicionamos algumas proriedades como suas versões categoriais e seus efeitos no espectro primo. Em [RRM1] existe um desenvolvimento de multi-algebras comutativas (subseção 2.2). No que segue, $A$ é multi-anel, $S \subseteq A$ é conjunto multiplicativo e $I \subseteq A$ é ideal.

Quociente por ideal. Dados $a, b \in A, a$ e $b$ são equivalentes módulo $I$ se $a-b \cap I \neq \emptyset$. Seja $A / I=\{\bar{a}: a \in A\}$ o conjunto de todas as classes de equivalência. A soma multivalorada é dada por $\bar{a} \in \bar{b}+\bar{c}$ se, e somente se, existem $a^{\prime}, b^{\prime}, c^{\prime} \in A \operatorname{com} \overline{a^{\prime}}=\bar{a}, \overline{b^{\prime}}=\bar{b}$ e $\overline{c^{\prime}}=\bar{c}$ tais que $a^{\prime} \in b^{\prime}+c^{\prime}$. O produto é dado por $\bar{a} \cdot \bar{b}=\overline{a b},-\bar{a}=\overline{-a}$ e as constantes são induzidas por $A$. Então $A / I$ é multi-anel e a projeção canônica $\pi_{I}: A \rightarrow A / I$ é morfismo de multi-anéis com $\pi_{I}(I)=\{0\}$.

Proposição 1.13. Sejam $A$ multi-anel e $I$ ideal.

i) Sejam $a, b, c \in A$. São equivalentes:

a) $\pi_{I}(a) \in \pi_{I}(b)+\pi_{I}(c)$.

b) Existe $i \in I$ tal que $a \in b+c+i$.

c) Existe $a^{\prime} \in A \operatorname{com} \pi_{I}\left(a^{\prime}\right)=\pi_{I}(a)$ tal que $a^{\prime} \in b+c$.

Em particular, $\pi_{I}: A \rightarrow A / I$ é morfismo forte.

ii) Se $A$ é hiper-anel, então $A / I$ é hiper-anel.

iii) $I$ é ideal primo se, e somente se, $A / I$ é multidomínio.

iv) $I$ é maximal se, e somente se, $A / I$ é multicorpo. Em particular, se $A$ é hiper-anel, $I$ é maximal se, e somente se, $A / I$ é hipercorpo ${ }^{4}$.

v) Dado morfismo de multi-anéis $f: A \rightarrow B \operatorname{com} f(I)=\{0\}$, existe único morfismo $\bar{f}: A / I \rightarrow B$ tal que $f=\bar{f} \circ \pi_{I}$.

vi) $\pi_{I}^{*}: \operatorname{spec}(A / I) \rightarrow \operatorname{spec}(A)$ induz homeomorfismo entre $\operatorname{spec}(A / I)$ e $\{p \in \operatorname{spec}(A): I \subseteq p\}$.

vii) Sejam $f: A \rightarrow B$ morfismo de multi-anéis e $J \subseteq B$ ideal. Dado $I \subseteq f^{-1}(J)$ ideal, existe único morfismo $f_{I, J}: A / I \rightarrow B / J$ tal que

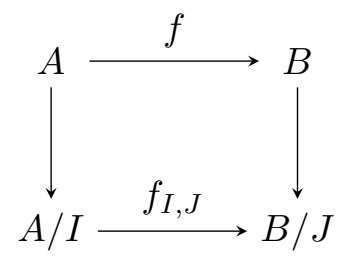

${ }^{4} \mathrm{Na}$ Proposição 1.22, os fracamente inversíveis de um multi-anel são caracterizados em termo dos ideais primos. 
é diagrama comutativo.

Demonstração. i) $a) \Rightarrow b)$ : Se $\pi_{I}(a) \in \pi_{I}(b)+\pi_{I}(c)$, existem $a^{\prime}, b^{\prime}, c^{\prime} \in A \operatorname{com} \pi_{I}\left(a^{\prime}\right)=\pi_{I}(a)$, $\pi_{I}\left(b^{\prime}\right)=\pi_{I}(b), \pi_{I}\left(c^{\prime}\right)=\pi_{I}(c)$ tais que $a^{\prime} \in b^{\prime}+c^{\prime}$. Logo existem $i, j, k \in I$ com $a \in a^{\prime}+i, b^{\prime} \in$ $b+j, c^{\prime} \in c+k$ e portanto $a \in b+c+(i+j+k)$. Logo existe $x \in i+j+k \subseteq I$ tal que $a \in b+c+x$.

$b) \Rightarrow c)$ : Seja $i \in I$ com $a \in b+c+i$. Então $-b \in c+(-a)+i$ e existe $x \in-a+i$ com $-b \in c+x$. Logo $-x \in b+c, a^{\prime}:=-x \in b+c$ e $\pi_{I}\left(a^{\prime}\right)=\pi_{I}(a)$.

$c) \Rightarrow a)$ : Direto da definição.

ii) Suponha que $A$ seja hiper-anel e sejam $a, b, c \in A$. Tome $\pi_{I}(x) \in \pi_{I}(c a)+\pi_{I}(c b)$. Então pelo item anterior existe $x^{\prime} \in A$ com $\pi_{I}\left(x^{\prime}\right)=\pi_{I}(x)$ e $x^{\prime} \in c a+c b$. Como $A$ é hiper-anel, existe $y \in a+b$ tal que $x^{\prime}=c y$. Então $\pi_{I}(y) \in \pi_{I}(a)+\pi_{I}(b)$ e $\pi_{I}(x)=\pi_{I}\left(x^{\prime}\right)=\pi_{I}(c y)$.

iii) Análogo ao caso de anéis.

iv) Basta notar que dado $m \in A$, o ideal gerado por $I \cup\{m\}$ é $\bigcup\left\{i+x_{1} m+\cdots+x_{n} m: n \geq 1, i \in\right.$ $\left.I, x_{1}, \ldots, x_{n} \in A\right\}$ e a demonstração segue como no caso de anéis.

v) Como o morfismo $\pi_{I}: A \rightarrow A / I$ é forte e sobrejetor pelo item $(i)$, segue da Proposição 1.11 que $\pi_{I}$ é quociente. Assim, para concluir o resultado é provar que dado morfismo $f: A \rightarrow B$,

$$
\operatorname{ker}\left(\pi_{I}\right) \subseteq \operatorname{ker}(f) \Leftrightarrow f(I)=\{0\} .
$$

$\mathrm{O}$ sentido $\Rightarrow$ segue direto da definição. Para a volta, suponha que $a, b \in A$ satisfazem $\pi_{I}(a)=$ $\pi_{I}(b)$. Então existe $i \in(a-b) \cap I$. Logo $0=f(i) \in f(a)-f(b)$ e $(a, b) \in \operatorname{ker}(f)$.

vi) Decorre diretamente da Proposição 1.11.(ii) e da equação $(\dagger)$ do item $(v)$.

vii) Seja $f: A \rightarrow B$ morfismo de multi-anéis, $J \subseteq B$ ideal e $I \subseteq f^{-1}(J)$. Considere o mapa $\pi_{J}: B \rightarrow B / J$. Como $\pi_{J} \circ f(I)=0$, pela propriedade universal de $\pi_{I}: A \rightarrow A / I$, existe único morfismo $f_{I, J}: A / I \rightarrow B / J$ tal que $f_{I, J} \circ \pi_{I}=\pi_{J} \circ f$.

Comentário 1.14. - Para cada multi-anel $A$, denotamos por $\operatorname{sper}(A)$ o conjunto de todos os morfismos de $A$ para 3. O conjunto $\operatorname{sper}(A)$ é chamado de espectro real de $A$ e seus elementos são as ordens de $A$. Mais adiante (seção 2.2 ) serão dadas definições equivalentes de $\operatorname{sper}(A)$.

- A função sinal sgn: $\mathbb{R} \rightarrow \mathbf{3}$ é um exemplo de morfismo que não é injetor mas satisfaz $\operatorname{sgn}(x)=$ $0 \Leftrightarrow x=0$. No entanto, se $f: A \rightarrow B$ é morfismo satisfazendo a propriedade expressa no item i) da Proposição 1.13, isto é, para $x, y, z \in A$,

$$
\text { se } f(x) \in f(y)+f(z) \text {, então existe } x^{\prime} \in A \text { tal que } f\left(x^{\prime}\right)=f(x) \text { e } x^{\prime} \in y+z \text {, }
$$

então tomando $I=f^{-1}(0)$, o morfismo induzido $\bar{f}: A / I \rightarrow B$ é um morfismo injetor forte. Em particular, se $f: A \rightarrow B$ é morfismo de multi-anéis com $B$ anel, então $A / I$ é anel e $\bar{f}: A / I \rightarrow B$ é injetor.

- Vamos denotar o mapa $f_{I, J}$ por $f_{J}$ quando $I=f^{-1}(J)$.

Localização. Os elementos de $S^{-1} A$ são da forma $a / s$ com $a \in A, s \in S$ e $a / s=b / t$ se, e somente se, existe $u \in S$ tal que $a t u=b s u$. A soma é definida por $a / s \in b / t+c / u$ se, e somente se, existe $v \in S$ tal que atuv $\in$ bsuv $+c s t v$. Define-se o oposto por $-(a / s):=(-a) / s$. O produto é $a / s \cdot b / t:=a b / s t$. A unidade é $1 / 1$ e o elemento nulo é $0 / 1$. Estas operações/constantes fazem de $S^{-1} A$ um multi-anel. O mapa canônico $\rho_{S}: A \rightarrow S^{-1} A$ dado por $\rho_{S}(a)=a / 1$ é morfismo e $\rho_{S}(S) \subseteq\left(S^{-1} A\right)^{\times}$. 
Se $A$ é multidomínio, podemos definir o hipercorpo de frações de $A$ por $f f(A):=(A \backslash 0)^{-1} A$. Além diso, dado ideal primo $p \in \operatorname{spec}(A)$, definimos $K_{A}(p):=f f(A / p)$.

Proposição 1.15. Sejam $A$ multi-anel e $S$ conjunto multiplicativo.

i) Se $A$ é hiper-anel, então $S^{-1} A$ também é.

ii) Sejam $x, a_{1}, \ldots, a_{n} \in A$. $\rho_{S}(x) \in \rho_{S}\left(a_{1}\right)+\cdots+\rho_{S}\left(a_{n}\right)$ se, e somente se, existe $s \in S$ tal que $x s \in a_{1} s+\cdots+a_{n} s$. Em particular, $\rho_{S}$ é morfismo forte.

iii) $S^{-1} A=0$ se, e somente se, $0 \in S$.

iv) Dado morfismo de multi-anéis $f: A \rightarrow B \operatorname{com} f(S) \subseteq B^{\times}$, existe único morfismo $\bar{f}: S^{-1} A \rightarrow B$ tal que $f=\bar{f} \circ \rho_{S}$. Em particular, $\rho_{S}$ é epimorfismo.

v) $\rho_{S}^{-1}: \operatorname{spec}\left(S^{-1} A\right) \rightarrow \operatorname{spec}(A)$ induz um homeomorfismo entre $\operatorname{spec}\left(S^{-1} A\right)$ e $\{P \in \operatorname{spec}(A): P \cap$ $S=\emptyset\}$.

vi) Sejam $f: A \rightarrow B$ morfismo de multi-anéis e $T \subseteq B$ conjunto multiplicativo com $S \subseteq f^{-1}(T)$. Então existe único morfismo $f_{S, T}: S^{-1} A \rightarrow T^{-1} B$ tal que

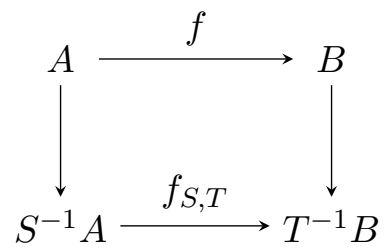

é diagrama comutativo. Em particular, dado $q \in \operatorname{spec}(B)$ e $p=f^{-1}(q)$, existe único morfismo $f_{q}: K_{A}(p) \rightarrow K_{B}(q)$ que faz o diagrama

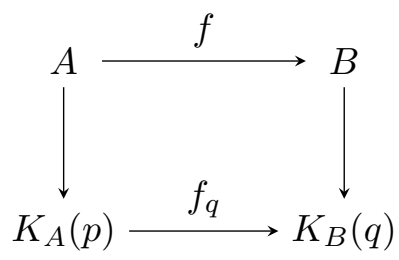

comutar.

Demonstração. i) Sejam $x, a, b, c \in A$ e $s, u, t, w \in S$ tais que $x / s \in c / w(a / u)+c / w(b / t)=$ $c a / w u+c b / w t$. Então existe $m \in S$ tal que $x m u t w^{2} \in$ camstw + cbmsuw. Como $A$ é hiperanel, existe $d \in a t+b u$ tal que $x m u t w^{2}=d(s c w m)$. Então $d / t u \in a / u+b / t$ e $x / s=d / t u \cdot c / w$. Assim $S^{-1} A$ é hiper-anel.

ii) A direção $(\Rightarrow)$ é óbvia. Para a direção $(\Leftarrow)$, vamos usar indução. O caso $n=2$ segue pela definição. Suponha $\rho_{S}(x) \in \rho_{S}\left(a_{1}\right)+\cdots+\rho_{S}\left(a_{n}\right)$. Como $S^{-1} A$ é multi-anel, existe $\rho_{S}(y) \in$ $\rho_{S}\left(a_{2}\right)+\cdots+\rho_{S}\left(a_{n}\right) \operatorname{com} \rho_{S}(x) \in \rho_{S}\left(a_{1}\right)+\rho_{S}(y)$. Por hipótese de indução, existem $s, t \in S$ tais que $y s \in a_{2} s+\cdots+a_{n} s$ tal que $x t \in a_{1} t+y t$. Então $x(s t) \in a_{1}(s t)+\cdots+a_{n}(s t)$.

iii) Se $0 \in S$, então é óbvio que $S^{-1} A=0$. Reciprocamente, se $S^{-1} A=0$, existe $s \in S$ tal que $0=0 \cdot 1 \cdot s=1 \cdot 1 \cdot s=s \in S$.

iv) Seja $f: A \rightarrow B \operatorname{com} f(S) \subseteq B^{\times}$. Note que

Afirmação. Se $a / s \in b / t+c / u$, então $f(a) f(s)^{-1} \in f(b) f(t)^{-1}+f(c) f(u)^{-1}$. Em particular, se $a / s=b / t$, então $f(a) f(s)^{-1}=f(b) f(t)^{-1}$. 
Demonstração. Seja $m \in S$ tal que amtu $\in$ bmsu + cmst. Como $f$ é morfismo de multianeis, $f(a) f(p) f(t) f(u) \in f(b) f(p) f(s) f(u)+f(c) f(p) f(s) f(t)$. Mas como por hipótese vale $f(p), f(s), f(t), f(u) \in B^{\times}, \operatorname{temos} f(a) f(s)^{-1} \in f(b) f(t)^{-1}+f(c) f(u)^{-1}$.

Assim, se definirmos $\bar{f}: S^{-1} A \rightarrow B$ por $\bar{f}(a / s)=f(a) f(s)^{-1}$, então $\bar{f}$ é morfismo de multianéis. A unicidade de $\bar{f}$ segue facilmente.

v) Pela descrição dos ideias primos por meio dos morfismos para o hipercorpo de Krasner e pela propriedade universal provada no item anterior, $\rho_{S}^{-1}: \operatorname{spec}\left(S^{-1} A\right) \rightarrow\{p \in \operatorname{spec}(A): p \cap S=\emptyset\}$ é uma bijeção.

Para finalizar, note que para todo $a \in A$ e $s \in S, \rho^{-1}(D(a / s))=\rho^{-1}(D(\rho(a)))=D(a) \cap$ $\operatorname{im}\left(\rho^{-1}\right)$ e portanto $\rho^{-1}$ é homeomorfismo com a imagem.

vi) Segue da propriedade universal do item iv) e o caso particular segue aplicando também a propriedade universal do quociente por ideias (Proposição 1.13).

Comentário 1.16. - Se $A$ é multidomínio, então o morfismo canônico $\rho: A \rightarrow f f(A)$ não é necessariamente injetivo (exemplo $2.5 \mathrm{em}[\operatorname{Mar} 10])$. Dado ideal primo $p \in \operatorname{spec}(A)$, definimos $A_{p}:=(A \backslash p)^{-1} A$. Seja $\rho: A \rightarrow A_{p}$ o morfismo canônico. Note que, pela Proposição 1.15 , o multi-anel $A_{p}$ tem único ideal maximal e ele é dado por $A_{p} \rho(p)=\left\{\frac{x}{s}: x \in p, s \in A \backslash p\right\}$ e é denotado por $p A_{p}$.

- Seja $f: A \rightarrow B$ morfismo de multi-aneis. Dado $p \in \operatorname{spec}(B)$, seja $q=f^{-1}(p) \in \operatorname{spec}(A)$. Como $A \backslash q \subseteq f^{-1}(B \backslash p)$, a Proposição 1.15 vi) nos dá morfismo natural $f_{p}: A_{q} \rightarrow B_{p}$.

- O morfismo $f_{S, T}$ é usualmente denotado por $f_{T}$ quando $S=f^{-1}(T)$.

Quociente de Marshall. Dados $a, b \in A, a, b$ são equivalentes (denotado por $a \sim_{S} b$ ) se existem $s, t \in S$ tais que $a s=b t$. O conjunto de todas as classes de equivalência é denotado por $A /{ }_{m} S$. A soma multivalorada é definida por $\bar{a} \in \bar{b}+\bar{c}$ se, e somente se, existem $a^{\prime}, b^{\prime}, c^{\prime} \in A$ tais que $a^{\prime} \sim_{S} a, b^{\prime} \sim_{S} b, c^{\prime} \sim_{S} c$ e $a^{\prime} \in b^{\prime}+c^{\prime}$. Equivalentemente, podemos definir $\bar{a} \in \bar{b}+\bar{c}$ se, e somente se, existem $s, t, u \in S$ tais que $a s \in b t+c u$. Define-se o oposto por $-\bar{a}:=\overline{-a}$. O produto é definido por $\bar{a} \cdot \bar{b}:=\overline{a b}$ e constantes são induzidas de $A$. Então $A /{ }_{m} S$ é multi-anel e o mapa natural $\pi_{S}: A \rightarrow A /{ }_{m} S$ é um morfismo sobrejetor e $\pi_{S}(S)=\{1\}$.

Definição 1.17. Sejam $A$ multi-anel e $S \subseteq A$ conjunto multiplicativo. Se para todo $x s \in S$ com $s \in S$ tivermos $x \in S$, então $S$ é chamado de cancelativo. Também definimos $\bar{S}=\{x \in A: x s \in$ $S$, para algum $s \in S\}=\left\{x \in A: x \sim_{S} 1\right\}$. Note que $S$ é cancelativo, se, e somente se, $S=\bar{S}$.

Proposição 1.18. Sejam $A$ multi-anel e $S$ conjunto multiplicativo.

i) $\bar{S} \subseteq A$ é subconjunto multiplicativo cancelativo e o mapa $S \mapsto \bar{S}$ define um operador de fecho no conjunto dos subconjuntos multilpicativos de $A$. Além disso, dado $T \subseteq A$ multiplicativo, $S, T$ induzem a mesma relação de equivalência em $A$ se, e somente se, $\bar{S}=\bar{T}$. Em particular, $A / m \bar{S}=A / m S$.

ii) Se $A$ é hiper-anel, então $A / m S$ também o é.

iii) Sejam $a, x_{1}, \ldots, x_{n} \in A, n \geq 2$. Se $\pi(a) \in \pi\left(x_{1}\right)+\cdots+\pi\left(x_{n}\right)$, então existem $s, s_{1}, \ldots, s_{n} \in S$ tais que as $\in x_{1} s_{1}+\cdots+x_{n} s_{n}$. Em particular, $\pi: A \rightarrow A / m S$ é quociente.

iv) Dado um morfismo de multi-anéis $f: A \rightarrow B$ satisfazendo $f(S)=\{1\}$, existe único morfismo $\bar{f}: A /{ }_{m} S \rightarrow B$ tal que $f=\bar{f} \circ \rho$.

v) $\pi^{*}: \operatorname{spec}(A / m S) \rightarrow \operatorname{spec}(A)$ induz homeomorfismo entre $\operatorname{spec}(A / m S)$ e $\{p \in \operatorname{spec}(A): p \cap S=$ $\emptyset\}$. 
vi) Sejam $f: A \rightarrow B$ morfismo e $T \subseteq B$ conjunto multiplicativo com $S \subseteq f^{-1}(T)$. Então existe único morfismo $f_{S, T}: A /{ }_{m} S \rightarrow B / m T$ tal que

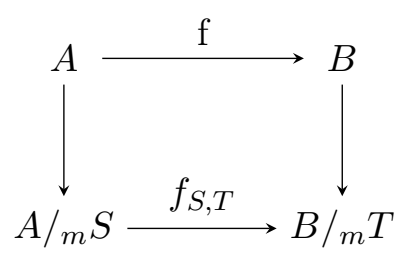

é diagrama comutativo.

Demonstração. i) Como $1 \in S$, está claro que $S \subseteq \bar{S}$. É imediato verificar que se $S \subseteq T$, então $\bar{S} \subseteq \bar{T}$.

Vamos verificar que $\bar{S}$ é multiplicativo e cancelativo.

Como $S \subseteq \bar{S}, 1 \in \bar{S}$. Dados $x, y \in \bar{S}$, existem $s, t \in S$ tais que $x s, y t \in S$. Então $x y(s t) \in S$ e assim $x y \in \bar{S}$. Também se $x s \in \bar{S}$ com $s \in \bar{S}$, então existem $s_{1}, s_{2} \in S$ tais que $x s s_{1}, s s_{2} \in S$. Logo $x\left(s s_{2}\right) s_{1} \in S$ e assim $x \in \bar{S}$. De modo análogo verificamos que $\overline{\bar{S}} \subseteq \bar{S}$.

Agora assuma que $S, T$ satisfaça $\sim_{S}=\sim_{T}$. Como $\bar{S}=\left\{x \in A: x \sim_{S} 1\right\}$, segue que $\bar{S}=\bar{T}$. Reciprocamente, se $\bar{S}=\bar{T}$, dados $x, y \in A \operatorname{com} x \sim_{S} y$, existem $s, s^{\prime} \in S$ tais que $x s=y s^{\prime}$. Logo existem $t, t^{\prime} \in T$ com $s t, s^{\prime} t^{\prime} \in T$. Então $x s\left(t t^{\prime}\right)=x s^{\prime}\left(t t^{\prime}\right)$ e $x \sim_{T} y$. Mudando os papeis de $S$ e $T$, vemos que $\sim_{S}=\sim_{T}$.

ii) Sejam $x, a, b, c \in A \operatorname{com} \pi(x) \in \pi(c a)+\pi(c b)$. Então existem $s, t, u \in S$ tais que $x s \in c a t+c b u$. Como $A$ é hiper-anel, existe $d \in a t+b u \operatorname{com} x s=c d$. Então $\pi(x)=\pi(c d)$ e $\pi(d) \in \pi(a)+\pi(b)$. Logo $A /{ }_{m} S$ é hiper-anel.

iii) A prova segue por indução em $n$. Para $n=2$ é a definição. Para $n>2$, suponha que $\pi(a) \in$ $\pi\left(x_{1}\right)+\cdots+\pi\left(x_{n}\right)$. Como $A / m S$ é multi-anel, existe $\pi(y) \in \pi\left(x_{2}\right)+\cdots+\pi\left(x_{n}\right)$ tal que $\pi(a) \in \pi\left(x_{1}\right)+\pi(y)$. Por hipótese de indução, existem $s, s_{1}, s_{2}, t, t_{2}, \ldots, t_{n} \in S$ tais que

$$
\begin{aligned}
& a s \in x_{1} s_{1}+y s_{2} \\
& y t \in x_{2} t_{2}+\cdots+x_{n} t_{n} .
\end{aligned}
$$

Portanto

$$
\begin{aligned}
\text { ast } & \in x_{1} s_{1} t+y s_{2} t \\
y t s_{2} & \in x_{2} t_{2} s_{2}+\cdots+x_{n} t_{n} s_{2}
\end{aligned}
$$

e $\operatorname{assim} a s t \in x_{1} s_{1} t+x_{2} t_{2} s_{2}+\cdots+x_{n} t_{n} s_{2}$.

iv) O morfismo $\rho: A \rightarrow A / m S$ é sobrejetor e pelo item (iii). Logo, pela Proposição 1.11, segue que $\rho$ é morfismo quociente. Para concluir a demonstração, basta notar que dado morfismo $f: A \rightarrow B$, vale que

$$
\operatorname{ker}(\rho) \subseteq \operatorname{ker}(f) \Leftrightarrow f(S)=\{1\}
$$

v) Decorre diretamente da Proposição 1.11.(ii) e da equação (†) do item (iv).

vi) Segue do item iv).

Comentário 1.19. O morfismo $f_{S, T}$ é usualmente denotado apenas por $f_{T}$ quando $S=f^{-1}(T)$.

Proposição 1.20. Sejam $A$ multi-anel. 
i) Seja $p \in \operatorname{Spec}(A)$. Existe único mapa $A_{p} / p A_{p} \rightarrow K_{A}(p)$ tal que

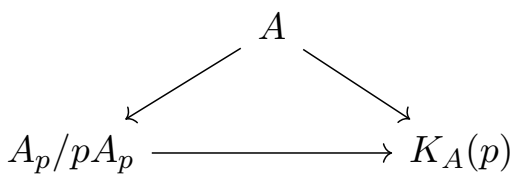

é diagrama comutativo. Além disso, tal mapa é isomorfismo.

ii) Seja $S$ um subconjunto multiplicativo de $A$ e $\pi: A \rightarrow A /{ }_{m} S$ o morfismo canônico. Dado $q \in \operatorname{spec}\left(A /{ }_{m} S\right)$, seja $p=\pi^{-1}(q) \in \operatorname{spec}(A)$. Considere o mapa canônico $g_{p}: A \rightarrow K_{A}(p)$ e seja $S_{p}=g_{p}(S)$. Então existe único morfismo $K_{A / m S}(q) \rightarrow K_{A}(p) / m S_{p}$ tal que

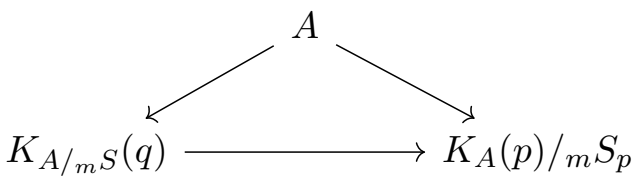

é diagrama comutativo. Além disso, tal morfismo é isomorfismo.

Demonstração. i) Considere as composições $i_{1}: A \rightarrow A / p \rightarrow K_{A}(p)$ e $i_{2}: A \rightarrow A_{p} \rightarrow A_{p} / p A_{p}$. Note que, pelas proposições 1.13 e 1.15, elas satisfazem a seguinte propriedade universal: dado mapa $f: A \rightarrow B$ tal que $f(p)=0$ e $f(A \backslash p) \subseteq B^{\times}$, então existem únicos morfismos $f_{1}: K_{A}(p) \rightarrow$ $B$ e $f_{2}: A_{p} / p A_{p} \rightarrow B$ satisfazendo $f=f_{1} \circ i_{1}=f_{2} \circ i_{2}$. Assim segue a existência do morfismo $A_{p} / p A_{p} \rightarrow K_{A}(p)$ com as propriedades desejadas.

ii) A estratégia é a mesma do item anterior: os mapas $j_{1}: A \rightarrow K_{A / m S}(q)$ e $j_{2}: A \rightarrow K_{A}(p) / m S_{p}$ satisfazem a mesma propriedade universal: dado mapa $f: A \rightarrow B$ tal que $f(S)=1, f(p)=0$ e $f(A \backslash p) \subseteq B^{\times}$, então existem únicos morfismo $f_{1}: K_{A / m}(q) \rightarrow B$ e $f_{2}: K_{A}(p) / m S_{p} \rightarrow B$ tais que $f=f_{1} \circ j_{1}=f_{2} \circ j_{2}$.

Limite indutivo. Seja $L$ uma linguagem (finitária e de $1^{a}$-ordem) e considere a categoria L-mod de todos os $L$-modelos. Dado um conjunto direcionado à direita (ou simplesmente direcionado) $\langle I, \leq\rangle$, um sistema $I$-indutivo de $L$-modelos é um funtor $\mathcal{M}:\langle I, \leq\rangle \rightarrow$ L-mod (se $i \leq j$, denotamos o morfismo $\mathcal{M}(i \leq j)$ por $\left.\mu_{i, j}\right)$. Um colimite desse sistema é denotado por $\lim \mathcal{M}$ ou $\lim _{\longrightarrow} \mathcal{M}_{i}$

Um cone dual sobre o sistema indutivo $\mathcal{M}$ é uma tupla $\left\langle A,\left\{\mu_{i}: i \in I\right\}\right\rangle$ tal que $A$ é uma $L$ estrutura e $\mu_{i}: \mathcal{M}_{i} \rightarrow A$ é um $L$-morfismo com $\mu_{i} \circ \mu_{j, i}=\mu_{j}$ para todo $j \leq i$. Para conveniência do leitor, resumimos as relações entre os conceitos apresentados em:

Proposição 1.21. Seja $\mathcal{M}=\langle I, \leq\rangle \rightarrow L-\bmod$ um sistema indutivo de $L$-estruturas. Então o colimite existe em L-mod; além disso, um cone dual sobre $\mathcal{M},\left\langle M,\left\{\mu_{i}: i \in I\right\}\right\rangle$, é isomorfo a $\lim _{\longrightarrow} \mathcal{M}$ se, e somente se,

a) $M=\bigcup_{i \in I} \mu_{i}\left(\mathcal{M}_{i}\right)$.

b) Se $\varphi\left(v_{1}, \ldots, v_{n}\right)$ é um $L$-fórmula atõmica e $s_{1}, \ldots, s_{n} \in \mathcal{M}_{i}$ satisfaz $M \vDash \varphi\left(\mu_{i}\left(s_{1}\right), \ldots, \mu_{i}\left(s_{n}\right)\right)$, então existe $k \in I$ com $k \geq i$ tal que

$$
\mathcal{M}_{k} \vDash \varphi\left(\mu_{i, k}\left(s_{1}\right), \ldots, \mu_{i, k}\left(s_{n}\right)\right) .
$$

Em particular, dados $i, j \in I$ e $a \in \mathcal{M}_{i}, b \in \mathcal{M}_{j}$, temos $\mu_{i}(a)=\mu_{j}(a)$ se, e somente se, existe $k \geq i, j$ tal que $\mu_{i, k}(a)=\mu_{j, k}(b)$. 
Além disso, seja $\varphi(\bar{v})$ disjunção de fórmulas geométricas ${ }^{5}$ e $\bar{s} \in \mathcal{M}_{i}$. Seja $S_{\varphi}=\{k \in I: k \geq$ $i$ e $\left.\mathcal{M}_{k}=\varphi\left(\mu_{i, k}(\bar{s})\right)\right\}$. Se $S_{\varphi}$ é cofinal em $I$, então $M \models \varphi\left(\mu_{i}(\bar{s})\right)$.

Demonstração. Confira seção 4 do Capítulo 17 de [Mir].

Seja $L_{\text {multi }}=\{\pi, \cdot,-, 0,1\}$ linguagem dos multianéis (aqui a soma multivalorada é vista como uma $\pi$, uma relação ternária). Seja $\mathcal{M}:\langle I, \leq\rangle \rightarrow L_{\text {multi }}$ um limite indutivo de multianéis. Então $\lim _{\longrightarrow} \mathcal{M}$ é multianel porque todos os axiomas de 1.1 são sentenças geométricas.

\subsection{Pré-feixe estrutural}

O pré-feixe estrutural de multi-aneis aqui definido generaliza o feixe estrutural de aneis, o feixe para hiperdomínios descrito em [Jun] e o feixe para semigrupos reais - estrutura considerada a partir do Capitulo 2 - apresentado em [DP2]. Assim aneis, hiperdomínios e multi-aneis reais reduzidos são geométricos, i.e, seu pré-feixe estrutural associado é um feixe. Mas existem multi-aneis não-geométricos (Comentário 1.38) e na próxima seção é dada uma caracterização em primeira ordem e finitária dos hiper-aneis von Neumann geométricos.

Sejam $A$ multi-anel, $\alpha$ ideal e $a \in A$. Definimos $\sqrt{\alpha}=\left\{x \in A\right.$ : existe $n \geq 1$ tal que $\left.x^{n} \in \alpha\right\}$ e $S_{a}=\left\{x \in A\right.$ : existe $n \geq 0$ tal que $\left.a^{n} \in(x)\right\}$ (convenção: $0^{0}=1$ ). Também definimos $A_{a}:=$ $\left\{1, a, a^{2}, \ldots\right\}^{-1} A$. Note que $S_{1}=A^{\times_{w}}$.

Proposição 1.22. Seja $A$ multi-anel. Sejam $\alpha \subseteq A$ ideal, $a \in A$ e $\rho_{a}: A \rightarrow A_{a}$ o morfismo canônico.

i) $\rho_{a}^{-1}\left(A_{a}^{\times} w\right)=S_{a}$.

ii) $\sqrt{\alpha}$ é ideal e $S_{a}$ é conjunto multiplicativo.

iii) $\sqrt{\alpha}=\bigcap_{\alpha \subseteq p \in \operatorname{spec}(A)} p$. Em particular, $\bigcap_{p \in \operatorname{spec}(A)} p$ é o conjunto dos nilpotentes.

iv) $S_{a}=\bigcap_{a \notin p \in \operatorname{spec}(A)} p^{c}$. Em particular, $\bigcap_{p \in \operatorname{spec}(A)} p^{c}=S_{1}=A^{\times_{w}}$.

v) Dados $a_{1}, \ldots, a_{n} \in A, D(a) \subseteq D\left(a_{1}\right) \cup \cdots \cup D\left(a_{n}\right)$ se, e somente se, $\sqrt{(a)} \subseteq \sqrt{\left(a_{1}\right)+\cdots+\left(a_{n}\right)}$. Além disso, para $a, b \in A$ são equivalentes:

a) $D(a) \subseteq D(b)$.

b) $\sqrt{(a)} \subseteq \sqrt{(b)}$.

c) $a \in \sqrt{(b)}$.

d) $S_{b} \subseteq S_{a}$.

vi) Se $D(a) \subseteq D(b)$, então existe único morfismo $\rho_{D(b), D(a)}: S_{b}^{-1} A \rightarrow S_{a}^{-1} A$ tal que

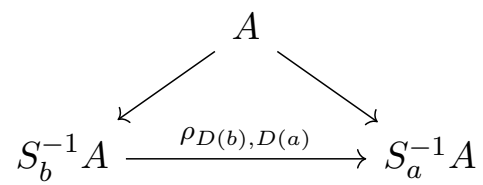

É diagrama comutativo. Além disso, se $D(a) \subseteq D(b) \subseteq D(c)$, então $\rho_{D(c), D(a)}=\rho_{D(c), D(b)} \circ$ $\rho_{D(c), D(b)}$ e $\rho_{D(a), D(a)}=I d_{S_{a}^{-1} A}{ }^{6}$.

\footnotetext{
${ }^{5}$ Uma fórmula $\varphi(\bar{t})$ é geométrica se é a negação de um fórmula atômica ou é da forma $\forall \bar{v}\left(\exists \bar{w} \psi_{1}(\bar{v}, \bar{t}, \bar{w}) \rightarrow\right.$ $\left.\exists \bar{w} \psi_{2}(\bar{v}, \bar{t}, \bar{w})\right)$, onde $\psi_{1}, \psi_{2}$ são positivas e livres de quantificadores.

${ }^{6}$ Pelo item anterior, se $D(a)=D(b)$, então $S_{a}=S_{b}$. Logo vale a identidade $S_{a}^{-1} A=S_{b}^{-1} A$
} 
vii) Existe único morfismo $A_{a} \rightarrow S_{a}^{-1} A$ tal que

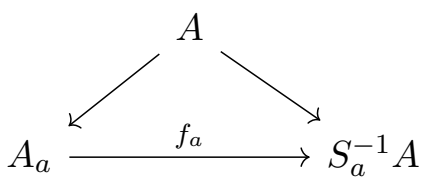

é diagrama comutativo.

Demonstração. i) $\subseteq$ : Seja $\frac{x}{1} \in A_{a}^{\times}$. Por definição, existem $\frac{t_{1}}{a^{n_{1}}}, \ldots, \frac{t_{l}}{a^{n_{l}}} \in A_{a}$ tais que $1 \in \frac{x}{1} \frac{t_{1}}{a^{n_{1}}}+$ $\cdots+\frac{x}{1} \frac{t_{1}}{a^{n_{1}}}$. Assim existem $m, n_{1}^{\prime}, \ldots, n_{l}^{\prime}$ tais que $a^{m} \in x t_{1} a^{n_{1}^{\prime}}+\cdots+x t_{l} a^{n_{l}^{\prime}}$ em $A$ e assim $x \in S_{a}$. : Se $x \in S_{a}$, então existem $t_{1}, \ldots, t_{k} \in A$ e $n \geq 1$ tais que $a^{n} \in x t_{1}+\cdots+x t_{k}$. Logo, em $A_{a}$, temos $\frac{1}{1} \in \frac{x}{1} \frac{t_{1}}{a^{n}}+\cdots+\frac{t_{k}}{a^{n}}$ e portanto $\frac{x}{1} \in A_{a}^{\times w}$.

ii) Dados $x, y \in \sqrt{\alpha}$, seja $n \geq 0$ tal que $x^{n}, y^{n} \in \alpha$. Então dado $z \in x-y$, temos $z^{2 n} \in$ $\sum_{i=0}^{2 n}\left(\begin{array}{c}2 n \\ i\end{array}\right) x^{i}(-y)^{2 n-i}$. Como para todo $i=0, \ldots, 2 n$ vale $i \geq n$ ou $2 n-i \geq n$, temos $z^{2 n} \in \alpha \mathrm{e}$ portanto $z \in \sqrt{\alpha}$. Logo $\sqrt{\alpha}$ é ideal.

Note que $1 \in S_{a}$ e dados $x, y \in S_{a}$, existem $n \geq 0$ tais que $a^{n} \in(x) \cap(y)$. Então $a^{2 n} \in(x y) \mathrm{e}$ assim $x y \in S_{a}$, provando que $S_{a}$ é multiplicativo.

iii) É fácil ver que $\sqrt{\alpha} \subseteq \bigcap_{\alpha \subseteq p} p$. Seja $x \notin \sqrt{\alpha}$ e considere o conjunto multiplicativo $S=$ $\left\{1, x, x^{2}, \ldots\right\}$. Como $\alpha \cap S=\emptyset$, pela Proposição 1.7 existe ideal primo $p$ com $\alpha \subseteq p$ e $x \notin p$. Então $x \notin \bigcap_{\alpha \subseteq p} p$.

iv) É imediato que $S_{a} \subseteq \bigcap_{a \notin p} p^{c}$. Reciprocamente, seja $x \notin S_{a}$. Então $(x) \cap\left\{1, a, a^{2}, \ldots\right\}=\emptyset$ e pela Proposição 1.7 existe ideal primo $p$ tal que $x \in p$ e $a \notin p$. Assim $x \notin \bigcap_{a \notin p} p^{c}$.

v) Seja $\alpha=\left(a_{1}\right)+\cdots+\left(a_{n}\right)$. Dado qualquer ideal primo $p$, temos $\alpha \nsubseteq p$ se, e somente se, existe $1 \leq i \leq n$ tal que $a_{i} \notin p$. Assim, pelo item $\left.i i i\right), \sqrt{\alpha}=\bigcap_{\alpha \subseteq p} p$ e $\sqrt{(a)}=\bigcap_{a \in p} p$. Além disso

$$
\begin{aligned}
D(a) \subseteq D\left(a_{1}\right) \cup \cdots \cup D\left(a_{n}\right) & \Leftrightarrow \text { para todo } p \in \operatorname{spec}(A), a \notin p \rightarrow \alpha \nsubseteq p \\
& \Leftrightarrow \sqrt{(a)} \subseteq \sqrt{\alpha} .
\end{aligned}
$$

Deste modo, fica provado também a equivalência $a) \Leftrightarrow b$ ). Pelo item $i v$ ) também temos $a) \Rightarrow d$ ). Além disso, pela definição $b) \Rightarrow c$ ) e $d) \Rightarrow c$ ).

$c) \Rightarrow b$ ): Como $a \in \sqrt{b}$, existe $n \geq 0$ tal que $a^{n} \in(b)$. Então dado $x \in \sqrt{a}$, existe $k \geq 0$ com $x^{k} \in(a)$ e portanto $x^{k+n} \in(b)$. Logo $x \in \sqrt{b}$.

vi) Se $D(a) \subseteq D(b)$, temos pelo item anterior que $S_{b} \subseteq S_{a}$ e assim a existência e unicidade de $\rho_{D(b), D(a)}$ segue por 1.15. Além disso, se $D(a) \subseteq D(b) \subseteq D(c)$, pela unicidade de $\rho_{D(c), D(a)}$ segue que $\rho_{D(b), D(a)} \circ \rho_{D(c), D(b)}=\rho_{D(c), D(a)}$.

vii) Como $\left\{1, a, a^{2}, \ldots\right\} \subseteq S_{a}$, a existência e unicidade do morfismo segue por 1.15.

Sejam $A$ um multi-anel e $a \in A$. Dizemos que $A$ tem a propriedade $a$-inversível se $A_{a}^{\times} w=A_{a}^{\times} \mathrm{e}$ $A$ tem a propriedade inversível se tem a propriedade $a$-inversível para todo $a \in A$.

$\{$ invP2\}

Proposição 1.23. Sejam $A$ multi-anel e $a \in A$. Sejam $\rho_{a}: A \rightarrow A_{a}$ e $\rho_{S_{a}}: A \rightarrow S_{a}^{-1} A$ os morfismos canônicos. Seja $\overline{\rho_{S_{a}}}: A_{a} \rightarrow S_{a}^{-1} A$ o morfismo induzido. São equivalentes:

i) $\rho_{a}\left(S_{a}\right) \subseteq A_{a}^{\times}$.

ii) $A$ satisfaz a propriedade $a$-inversível. 
iii) $S_{a}=\left\{x \in A\right.$ : existem $n \geq 1, y \in A$ tais que $\left.a^{n}=x y\right\}$.

iv) $\overline{\rho_{S_{a}}}: A_{a} \rightarrow S_{a}^{-1} A$ é um isomorfismo.

Em particular, se para todo $x \in A,(x)=A x$, então $A$ tem a propriedade inversível.

Demonstração. $i) \Rightarrow$ ii): Seja $\frac{x}{a^{k}} \in A_{a}^{\times w}$. Por definição, existem $\frac{t_{1}}{a^{n_{1}}}, \ldots, \frac{t_{l}}{a^{n_{l}}} \in A_{a}$ tais que $1 \in$ $\frac{x}{a^{k}} \frac{t_{1}}{a^{n_{1}}}+\cdots+\frac{x}{a^{k}} \frac{t_{1}}{a^{n_{1}}}$. Assim existem $m, n_{1}^{\prime}, \ldots, n_{l}^{\prime}$ tais que $a^{m} \in x t_{1} a^{n_{1}^{\prime}}+\cdots+x t_{l} a^{n_{l}^{\prime}}$ em $A$ e assim $x \in S_{a}$. Por hipótese, $\rho(x) \in A_{a}^{\times}$e existe $\frac{y}{a^{t}} \in A_{a} \operatorname{com} \frac{x}{1} \frac{y}{a^{t}}=1$. Logo $\frac{x}{a^{k}} \in A_{a}^{\times}$.

ii) $\Rightarrow$ iii): Seja $x \in S_{a}$. Então existe $n \geq 0 \operatorname{com} a^{n} \in(x)$ e assim $x \in A_{a}^{\times w}=A_{a}^{\times}$. Por definição, isto significa que existem $\frac{y}{a^{t}} \in A_{a} \operatorname{com} \frac{x}{1} \frac{y}{a^{t}}=1$. Então existe $m \geq 0$ tal que $x y a^{m}=a^{m+t}$.

iii) $\Rightarrow i v)$ : Observe que, dado $x \in S_{a}$, por hipótese existem $n \geq 0$ e $y \in A \operatorname{com} a^{n}=x y$ e assim $\rho_{a}(x) \in A_{a}^{\times}$. Assim pela propriedade universal de $\rho_{S_{a}}$, existe morfismo $\overline{\rho_{a}}: S_{a}^{-1} A \rightarrow A_{a}$ tal que $\rho_{a}=\overline{\rho_{a}} \circ \rho_{S_{a}}$. Para concluir, note que $\left(\overline{\rho_{a}} \circ \overline{\rho_{S_{a}}}\right) \circ \rho_{a}=\rho_{a}$ e $\left(\overline{\rho_{S_{a}}} \circ \overline{\rho_{a}}\right) \circ \rho_{S_{a}}=\rho_{S_{a}}$. Assim, pelas propriedades universais de $\rho_{a}$ e $\rho_{S_{a}}$, temos $\overline{\rho_{a}} \circ \overline{\rho_{S_{a}}}=I d_{A_{a}}$ e $\overline{\rho_{S_{a}}} \circ \overline{\rho_{a}}=I d_{S_{a}}$.

$i v) \Rightarrow i$ ): Seja $x \in S_{a}$. Como $\overline{\rho_{S_{a}}}\left(\rho_{a}(x)\right)=\rho_{S_{a}}(x) \in\left(S_{a}^{-1} A\right)^{\times}$e $\overline{\rho_{S_{a}}}$ é isomorfismo, temos $\rho_{a}(x) \in A_{a}^{\times}$.

Exemplo 1.24. - Se $A$ é hiperanel, então para todo $a \in A$ vale $(a)=a A$; assim $A$ tem a propriedade inversível.

- Sejam $A$ multianel real reduzido (Definição 2.30) e $a, t_{1}, \ldots, t_{n} \in A$. Seja $x \in a t_{1}+\cdots+a t_{n}$. Então dado $\sigma \in \operatorname{sper}(A)$, se $\sigma(a)=0$, temos $\sigma(x)=0$; assim $\sigma(x)=\sigma\left(a^{2} x\right)$. Como $\sigma \in$ $\operatorname{sper}(A)$ foi arbitrário, pelo Teorema 2.26, ii), $x=a^{2} x=a(a x)$. Portanto $(a)=a A$ e $A$ tem a propriedade inversível.

- Seja $A$ multianel e considere $S_{1}=\{x \in A: 1 \in(x)\}=A^{\times}$. Então $S_{1}^{-1} A$ tem a propriedade 1-inversível e o morfismo natural $A \rightarrow S_{1}^{-1} A$ é inicial entre os morfismos saindo de $A$ para multianéis com a propriedade 1-inversível.

Seja $A$ multi-anel e $\mathcal{B}_{A}:=\{D(a)\}_{a \in A}$ o conjunto dos abertos básicos de $\operatorname{spec}(A)$. Considere o funtor $\mathcal{F}_{A}: \mathcal{B}_{A} \rightarrow$ MultR dado por $\mathcal{F}_{A}(D(a))=S_{a}^{-1} A$ e se $D(a) \subseteq D(b)$, então o morfismo de restrição é dado por $\mathcal{F}_{A, D(b), D(a)}=\rho_{D(b), D(a)}$. Por 1.22 , temos que $\mathcal{F}_{A}$ é bem-definido e é de fato funtor. Se não houver risco de confusão, vamos denotar $\mathcal{F}_{A}$ apenas por $\mathcal{F}, \mathcal{F}_{A}(D(a))$ por $\mathcal{F}_{a}$ e para $D(a) \subseteq D(b)$, o mapa de restrição $\mathcal{F}_{D(b), D(a)}$ é denotado por $\mathcal{F}_{b, a}$. Além disso, dado $p \in \operatorname{spec}(A)$, denotamos por $\mathcal{F}_{p}=\underline{\lim }_{a \notin p} \mathcal{F}(D(a))$ a fibra em $p$.

Definição 1.25. Seja $A$ multi-anel. O funtor $\mathcal{F}=\mathcal{F}_{A}$ é chamado de pré-feixe estrutural associado a $A$.

i) $\mathcal{F}$ é mono-prefeixe se para toda cobertura $D(e)=\bigcup_{i \in I} D\left(e_{i}\right)$ e todos $a, b, c \in \mathcal{F}_{e}$ com

$$
\mathcal{F}_{e, e_{i}}(a) \in \mathcal{F}_{e, e_{i}}(b)+\mathcal{F}_{e, e_{i}}(c) \text { em } A_{e_{i}} \text { para todo } i \in I
$$

então $a \in b+c^{7}$.

ii) $\mathcal{F}$ é feixe se é mono-prefeixe e se dada cobertura $D(e)=\bigcup_{i \in I} D\left(e_{i}\right)$ e $x_{i} \in \mathcal{F}_{e_{i}}$ satisfazendo

$$
\mathcal{F}_{e_{i}, e_{i} e_{j}}\left(x_{i}\right)=\mathcal{F}_{e_{j}, e_{i} e_{j}}\left(x_{j}\right) \text { para todo } i, j \in I \text {, }
$$

então existe (necessariamente único) $x \in \mathcal{F}_{e}$ tal que $\mathcal{F}_{e, e_{i}}(x)=x_{i}$ para todo $i \in I$.

Definição 1.26. Seja $A$ multi-anel. Se $\mathcal{F}_{A}$ é mono-prefeixe, então $A$ é chamado de mono-multi-anel; se $\mathcal{F}_{A}$ é feixe, então $A$ é chamado de multi-anel geométrico .

\footnotetext{
${ }^{7}$ Como consequência, o morfismo natural $\mathcal{F}(D(e)) \rightarrow \prod_{i \in I} \mathcal{F}\left(D\left(e_{i}\right)\right)$ é injetor.
} 
Proposição 1.27. Seja $A$ multi-anel. Dado $p \in \operatorname{spec}(A), \mathcal{F}_{p} \cong A_{p}$ naturalmente.

Demonstração. Dado $a \notin p$, como $S_{a} \subseteq A \backslash p$, existe único mapa $i_{S_{a}}: S_{a}^{-1} A \rightarrow A_{p}$ tal que

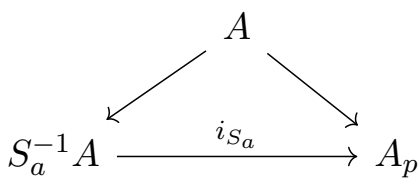

é diagrama comutativo. Assim, pela unicidade, é fácil ver que se $D(a) \subseteq D(b)$, então

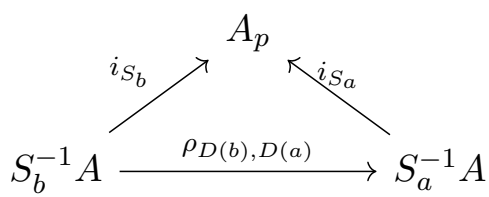

é diagrama comutativo. Assim, a família $\mathcal{I}=\left\{i_{S_{a}}: S_{a}^{-1} A \rightarrow A_{p}: a \notin p\right\}$ é cocone. Vamos mostrar em seguida que $\mathcal{I}$ é na verdade cocone limite. Considere uma família de morfismos $\left\{h_{S_{a}}: S_{a}^{-1} A \rightarrow\right.$ $B: a \notin p\}$ tal que se $D(a) \subseteq D(b)$, então $h_{S_{b}}=h_{S_{a}} \circ \rho_{D(b), D(a)}$. Vamos provar que existe único morfismo $h: A_{p} \rightarrow B$ tal que dado $a \notin p$ vale $h_{S_{a}}=h \circ i_{S_{a}}$.

Existência: Defina $h^{\prime}=h_{S_{1}} \circ \rho_{S_{1}}: A \rightarrow B$. Para usar a propriedade universal de $\rho_{p}: A \rightarrow A_{p}$, precisamos verificar que para todo $a \notin p$ tem-se $h^{\prime}(a) \in B^{\times}$. Mas note que dado $a \notin p$, como o diagrama

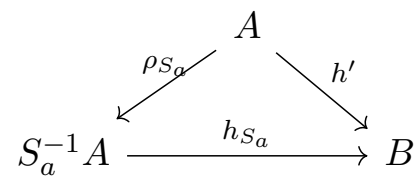

é comutativo e $\rho_{S_{a}}(a) \in S_{a}^{-1} A$ é inversível, temos $h^{\prime}(a) \in B^{\times}$. Então existem mapa $h: A_{p} \rightarrow B$ tal que

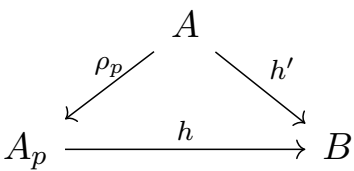

é diagrama comutativo. Então para todo $a \notin p$, como $i_{S_{a}} \circ \rho_{S_{a}}=\rho_{p}$, temos que $h \circ i_{S_{a}} \circ \rho_{S_{a}}=$ $h \circ \rho_{p}=h^{\prime}=h_{S_{a}} \circ \rho_{S_{a}}$. Mas como $\rho_{S_{a}}$ é epimorfismo, temos $h \circ i_{S_{a}}=h_{S_{a}}$.

Unicidade: Apenas note que para todo $x \in A_{p}$, existem $a \notin p$ e $y \in S_{a}^{-1} A$ tal que $i_{S_{a}}(y)=x$.

Comentário 1.28. Sejam $A$ multi-anel, $a, b, c \in A$ e $p \in \operatorname{spec}(A)$. Note que se $a \in b+c$ em $A_{p}$, então existe $x \notin p$ com $a x \in b x+c x$. Assim $a \in b+c$ em $A_{x}$, com $p \in D(x)$. Deste modo, dado $e \in A$, se $a \in b+c$ em $A_{p}$ para todo $p \in D(e)$, então pela compacidade de $D(e)$ existem $e_{1}, \ldots, e_{n}$ com $D(e)=\bigcup_{i=1}^{n} D\left(e_{i}\right)$ e $a \in b+c$ em $A_{e_{i}}$ para todo $i=1, \ldots, n$.

\subsection{Hiper-anéis von Neumann}

Nesta seção, a discussão sobre feixes de multi-anéis é especializada e um olhar cuidadoso é dado à categoria $\mathbf{H v N}$ dos hiper-anéis von Neumann. O objetivo principal é caracterizar quando um HvN é geométrico (Teorema 1.34). Como aplicação, é construída uma envoltória geométrica para $\mathbf{H v N}$ (Teorema 1.39) e é caracterizado quais quocientes de Marshall resultam em um HvN geométrico (Proposição 1.37).

$\{\mathrm{cVn}\}$

Proposição 1.29. Seja $A$ hiper-anel. As seguintes afirmações são equivalentes: 
i) $\operatorname{spec}(A)$ é um espaço topológico Booleano e $\sqrt{0}=0$.

ii) Para todo $a \in A$ existe $b \in A$ tal que $a=a^{2} b$.

Demonstração. $i) \Rightarrow$ ii) Seja $a \in A$. Como $\operatorname{spec}(A)$ é Booleano, existem $a_{1}, \ldots, a_{n} \in A$ tais que $D(a)^{c}=D\left(a_{1}\right) \cup \cdots \cup D\left(a_{n}\right)$. Em particular, $D\left(a a_{i}\right)=D(a) \cap D\left(a_{i}\right)=\emptyset=D(0)$ e assim $a a_{i} \in \sqrt{0}=$ 0 para todo $i=1, \ldots, n$. Além disso, $\operatorname{comospec}(R)=D(a) \cup D(a)^{c}=D(a) \cup D\left(a_{1}\right) \cup \cdots \cup D\left(a_{n}\right)$, pela Proposição 1.22 , devem existir $b, t_{1}, \ldots, t_{n} \in A$ tais que $1 \in a b+a_{1} t_{1}+\cdots+a_{n} t_{n}$. Assim, multiplicando esta multi-soma por $a$, temos $a=a^{2} b$.

$i i) \Rightarrow i$ ) Seja $a \in A$ e tome $b$ com $a=a^{2} b$. Então $(a b)^{2}=\left(a^{2} b\right) b=a b$. Como $A$ é hiper-anel, existe $l \in 1-a b$ tal que $a l=0$. Deste modo, dado ideal primo $p \in \operatorname{spec}(A)$, se $a \in p, a b \in p$ e então $l \notin p$ pois $l \in 1-a b$; se $a \notin p$, como $a l=0 \in p$, temos $l \in p$. Portanto $D(a)^{c}=D(l)$ e $\operatorname{spec}(A)$ é Booleano.

Definição 1.30. Seja $A$ hiper-anel. Se qualquer uma das condições equivalentes de 1.29 é válida, então $A$ é chamado hiper-anel von Neumann regular ou, simplesmente, de hiper-anel von Neumann - A classe dos hiper-anéis von Neumann com morfismos de multi-anéis é uma categoria denotada por $\mathbf{H v N}$ e a classe dos hiper-anéis von Neumann geométricos com morfismos de multi-anéis é uma categoria denotada por HvNG.

Comentários e Notações. Seja $A$ hiper-anel von Neumann.

- Como todo axioma de hiper-anel von Neumann é Horn-geométrico, segue que HvN é fechada por produtos reduzidos (Proposição 6.2.2 em [CK]) e limites indutivos (Proposição 1.21). Além disso, imagem de hiper-anel von Neumann também é HvN. Em particular, HvN é fechada por quociente por ideais e quociente de Marshall.

- Todo ideal primo $p$ de $A$ é maximal. De fato, dado $p \in \operatorname{spec}(A)$, seja $a \notin p$. Tome $b \in A$ com $a=a^{2} b$. Como $A$ é hiper-anel, existe $l \in 1-a b \operatorname{com} l a=0$. Logo $l \in p$ e $a b=1 \mathrm{em} A / p$. Pela Proposição 1.13 segue que $p$ é maximal. Além disso, dado $x \in A$, o morfismo $\rho_{x}: A \rightarrow A_{x}$ é sobrejetor. De fato, dado $\frac{a}{x^{n}} \in A$, seja $y \in A \operatorname{com} x=x^{2} y$; então $\frac{a}{x^{n}}=\frac{a y^{n}}{1}=\rho_{x}\left(a y^{n}\right)$.

- Dado $a \in A$ idempotente, como $a^{2}=a$ e $A$ é hiper-anel, existe $x \in 1-a$ com $a x=0$. Note que se temos outro $x^{\prime} \in 1-a$ com $a x^{\prime}=0$, então $x x^{\prime} \in x^{\prime}-x^{\prime} a=x^{\prime}-0=\left\{x^{\prime}\right\}$ e $x x^{\prime} \in x-x a=x-0=\{x\}$. Assim $x=x x^{\prime}=x^{\prime}$ e $x$ é unicamente determinado. Denotando $x$ por $a^{c}$, temos também $\left(a^{c}\right)^{2} \in a^{c}-a a^{c}=\left\{a^{c}\right\}$ e assim $a^{c}$ é idempotente. Também verifica-se que $D(a)^{c}=D\left(a^{c}\right)$.

- Os abertos básicos de $\operatorname{spec}(A)$ são representados por idempotentes. De fato, dado $a \in A$, seja $b \in A$ com $a=a^{2} b$; então $a b$ é idempotente e $D(a)=D(a b)$. Além disso, se existir outro $b^{\prime}$ idempotente com $D\left(b^{\prime}\right)=D(a)=D(b)$, temos $D\left(b^{\prime} b^{c}\right)=D\left(b^{\prime}\right) \cap D\left(b^{c}\right)=D\left(b^{\prime}\right) \cap D(b)^{c}=$ $\emptyset=D(0)$ e da mesma forma $D\left(b^{\prime c} b\right)=\emptyset=D(0)$. Mas como $\sqrt{0}=0$, temos $b^{\prime} b^{c}=b^{\prime c} b=0$. Como $b^{c} \in 1-b$, vale que $0=b^{\prime} b^{c} \in b^{\prime}-b b^{\prime}$ e assim $b^{\prime}=b b^{\prime}$. Da maneira análoga conclui-se que $b=b b^{\prime}$ e portanto $b=b b^{\prime}=b^{\prime}$. Assim, vamos denotar por $i(a)$ o único idempotente em $A$ tal que $D(i(a))=D(a)$. Também vamos utilizar a notação: $a^{c}=i(a)^{c}$, para elementos $a \in A$ não necessariamente idempotentes.

- Seja $f: A \rightarrow B$ morfismo de $H v N$. Dado $a \in A$, seja $b$ com $a=a^{2} b$. Como $a b$ é idempotente e $D(a b)=D(a), i(a)=a b$. Por outro lado, $f(a)=f(a)^{2} f(b)$ e pelo mesmo argumento $i(f(a))=f(a) f(b)$. Logo $f(i(a))=i(f(a))$. Morfismo também preserva o complementar: como $a^{c} \in 1-i(a)$ e $a^{c} i(a)=0, \operatorname{temos} f\left(a^{c}\right) \in 1-f(i(a))=1-i(f(a))$ e $0=f\left(a^{c}\right) f(i(a))=$ $f\left(a^{c}\right) i(f(a))$; logo pela unicidade verificada em ítem anterior, $f\left(a^{c}\right)=f(a)^{c}$.

- Sejam $a, b$ idempotentes. Se $D(a) \subseteq D(b)$, então $a \in \sqrt{b}$ e, assim, existe $l$ tal que $a=b l$. Então $a b=b b l=a$. Reversamente, se $a b=a$, entao $D(a) \subseteq D(b)$. 
Definição 1.31. Seja $A$ hiper-anel von Neumann. Um conjunto $\left\{e_{1}, \ldots, e_{n}\right\} \subseteq \operatorname{Id}(A)$ é uma partição se dados $i \neq j, e_{i} e_{j}=0$ e é uma partição da unidade se é uma partição e $1 \in e_{1}+\cdots+e_{n}$.

Lema 1.32. Sejam $A$ hiper-anel von Neumann e $B=\left\{e_{1}, \ldots, e_{n}\right\}$ uma partição.

i) Se $x \in e_{1}+\cdots+e_{n}$, então $D(x)=\bigsqcup_{i=1}^{n} D\left(e_{i}\right)$. Em particular, dado $U \subseteq \operatorname{spec}(A)$ subconjunto aberto-fechado, existe $e \in \operatorname{Id}(A)$ tal que $U=D(e)$.

ii) Seja $e \in \operatorname{Id}(R)$ tal que $D(e)=\bigsqcup_{i=1}^{n} D\left(e_{i}\right)$. Suponha que $e_{i}+e_{i}^{c}=\{1\}$ para todo $i=1, \ldots, n$. Então $e_{1}+\cdots+e_{n}=\{e\}$.

iii) Se $B=\left\{e_{1}, \ldots, e_{n}\right\}$ e $\left\{f_{1}, \ldots, f_{k}\right\}$ são partições da unidade, então $\left\{e_{1} f_{1}, \ldots, e_{1} f_{k}, \ldots, e_{n} f_{k}\right\}$ também é partição da unidade e $e_{1}+\cdots+e_{n}, f_{1}+\cdots+f_{k} \subseteq e_{1} f_{1}+\cdots+e_{1} f_{k}+e_{2} f_{1}+\cdots+e_{n} f_{k}$.

Demonstração. i) Se $p \in D(x)$, então existe $i$ com $p \in D\left(e_{i}\right)$. Reciprocamente, seja $p \in D\left(e_{i}\right)$. Para todo $j \neq i$ temos $e_{j} e_{i}=0 \in p$ e assim $e_{j} \in p$. Se valer $x \in p$, então teríamos $e_{i} \in p$, absurdo. Assim $p \in D(x)$.

Seja $U \subseteq \operatorname{spec}(A)$ aberto-fechado. Então existem $e_{1}, \ldots, e_{n} \in \operatorname{Id}(A) \operatorname{com} U=\bigcup_{i=1}^{n} D\left(e_{i}\right)$. Seja a família de idempotentes: $e_{1}^{\prime}=e_{1}, e_{i}^{\prime}=e_{i}\left(e_{1}^{c} \cdots e_{i-1}^{c}\right), i=2, \ldots, n$. Note que $D\left(e_{i}^{\prime}\right)=$ $D\left(e_{i}\right) \cap \bigcap_{k=1}^{i-1} D\left(e_{k}\right)^{c}$ e $e_{i}^{\prime} e_{j}^{\prime}=0$ se $i \neq j$. Então dado $x \in e_{1}^{\prime}+\cdots+e_{n}^{\prime}$, temos $D(x)=$ $\bigsqcup_{i=1}^{n} D\left(e_{i}^{\prime}\right)=\bigcup_{i=1}^{n} D\left(e_{i}\right)=U$. Basta tomar $e:=i(x)$ e teremos $U=D(e)$.

ii) Dados $i \neq j, e_{i} e_{j}=0$ implica que $e_{i} e_{j}^{c}=e_{i}$ porque $e_{j}^{c} \in 1-e_{j}$. Então $e_{i} e_{1}^{c} \cdots e_{i-1}^{c}=e_{i}$ para todo $i=2, \ldots, n-1$. Além disso, como $D(e)=\bigsqcup_{i=1}^{n} D\left(e_{i}\right)$, temos $e_{n}=e_{1}^{c} \cdots e_{n-1}^{c} e$ e $e_{i} e=e_{i}$ para todo $i=1, \ldots, n-1$. Assim, como $e_{i}^{c} e+e_{i} e=\{e\}$ para todo $i=1, \ldots, n$, temos

$$
\begin{aligned}
e_{1}+e_{2}+\cdots+e_{n-1}+e_{n} & =e_{1}+e_{2}+\cdots+e_{n-1} e_{1}^{c} \cdots e_{n-2}^{c}+e_{1}^{c} \cdots e_{n-1}^{c} e \\
& =e_{1}+e_{2}+\cdots+e_{1}^{c} \cdots e_{n-2}^{c}\left(e_{n-1} e+e_{n-1}^{c} e\right) \\
& =e_{1}+e_{2}+\cdots+e_{n-2} e_{1}^{c} \cdots e_{n-3}^{c}+e_{1}^{c} \cdots e_{n-2}^{c} e \\
& \vdots \\
& =e_{1} e+e_{1}^{c} e=\{e\} .
\end{aligned}
$$

iii) Se $(i, j) \neq\left(i^{\prime}, j^{\prime}\right)$, então $\left(e_{i} f_{j}\right)\left(e_{i^{\prime}} f_{j^{\prime}}\right)=0$. Além disso, a relação $1 \in e_{1}+\cdots+e_{n}$ implica que $f_{j} \in e_{1} f_{j}+\cdots+e_{n} f_{j}$ e assim $f_{1}+\cdots+f_{k} \subseteq e_{1} f_{1}+\cdots+e_{1} f_{k}+e_{2} f_{k}+\cdots+e_{n} f_{k}$. Por argumento análogo, conclui-se que $e_{1}+\cdots+e_{n} \subseteq e_{1} f_{1}+\cdots+e_{1} f_{k}+e_{2} f_{1}+\cdots+e_{n} f_{k}$. Em particular, destas inclusões concluimos que $1 \in \sum_{i, j} e_{i} f_{j}$.

Lema 1.33. Considere as seguintes afirmações sobre um hiper-anel von Neumann $A$ :

i) Dados $a, b, c \in A$ e $e_{1}, \ldots, e_{n} \in \operatorname{Id}(A)$ satisfazendo

$$
\begin{aligned}
\operatorname{spec}(A) & =\bigcup_{i=1}^{n} D\left(e_{i}\right) \\
\rho_{e_{i}}(a) \in \rho_{e_{i}}(b)+\rho_{e_{i}}(c), i & =1, \ldots, n,
\end{aligned}
$$

então $a \in b+c$.

ii) Dados $x_{1}, \ldots, x_{n} \in A$ e $e_{1}, \ldots, e_{n} \in \operatorname{Id}(A)$ satisfazendo

$$
\begin{array}{r}
\operatorname{spec}(A)=\bigcup_{i=1}^{n} D\left(e_{i}\right) \\
\rho_{e_{i} e_{j}}\left(x_{i}\right)=\rho_{e_{i} e_{j}}\left(x_{j}\right), i, j=1, \ldots, n,
\end{array}
$$


então existe $x \in A$ tal que $\rho_{e_{i}}(x)=\rho_{e_{i}}\left(x_{i}\right), i=1, \ldots, n$.

Então

a) $A$ satisfaz $i$ ) se, e somente se, $A$ é mono-hiper-anel.

b) $A$ satisfaz $i$ ) e $i i)$ se, e somente se, $A$ é geométrico.

Demonstração. a) Suponha $D(e)=\bigcup_{i \in I} D\left(e_{i}\right)$ e que para $a, b, c \in \mathcal{F}_{e}$ tenhamos

$$
\mathcal{F}_{e, e_{i}}(a) \in \mathcal{F}_{e, e_{i}}(b)+\mathcal{F}_{e, e_{i}}(c) \text { em } \mathcal{F}_{e_{i}} \text { para todo } i \in I,
$$

Queremos mostrar que $a \in b+c$ em $\mathcal{F}_{e}$. Pela compacidade de $D(e)$, é suficiente supormos $I=\{1, \ldots, n\}$ finito. Além disso, como $A$ é hiper-anel von Neumann, dado qualquer $x \in A$, $i(x) \in A$ é idempotente e $D(x)=D(i(x))$, podemos supor sem perda de generalidade que $e, e_{1}, \ldots, e_{n} \in A$ são idempotentes. Usando a Proposição 1.22 juntamente com o fato do mapa canônico $\rho_{e}: A \rightarrow A_{e}$ ser sobrejetor, podemos rescrever o problema 1.1 como

Dados $e, e_{i} \in \operatorname{Id}(A)$ com $D(e)=\cup_{i=1}^{n} D\left(e_{i}\right) e, e_{i} \in \operatorname{Id}(A)$ e $a, b, c \in A$ satisfazendo $\rho_{e_{i}}(a) \in$ $\rho_{e_{i}}(b)+\rho_{e_{i}}(c)$ em $A_{e_{i}}$, então $\rho_{e}(a) \in \rho_{e}(b)+\rho_{e}(c)$.

Trocando $a, b, c$ por $a e, b e, c e$, respectivamente, podemos supor sem perda de generalidade que $e^{c} a=e^{c} b=e^{c} c=0$. Então temos

$$
\begin{array}{r}
\operatorname{spec}(A)=D\left(e^{c}\right) \cup \bigcup_{i=1}^{n} D\left(e_{i}\right) \text { e } a, b, c \in A \text { satisfazendo } \\
\rho_{e_{i}}(a) \in \rho_{e_{i}}(b)+\rho_{e_{i}}(c) \text { em } A_{e_{i}} \text { e } \rho_{e^{c}}(a) \in \rho_{e^{c}}(b)+\rho_{e^{c}}(c) \text { em } A_{e^{c}} .
\end{array}
$$

pois $\rho_{e^{c}}(a)=\rho_{e^{c}}(a)=\rho_{e^{c}}(c)=0 \in A_{e^{c}}$. Logo, pela hipótese $\left.i\right), a \in b+c$ em $A$ e em particular $\rho_{e}(a) \in \rho_{e}(b)+\rho_{e}(c)$.

b) A demonstração segue reduções idênticas ao item $i$ ) e por isso será omitida.

Seja $A$ hiper-anel von Neumann. Pelo Lema $1.32, \mathcal{B}_{A}$ (o conjunto dos abertos básicos de $\operatorname{spec}(A)$ ) é a álgebra Booleana de abertos-fechados de $\operatorname{spec}(A)$. Seja $\mathcal{F}$ o pré-feixe estrutural de $A$. Dado $p \in \operatorname{spec}(A)$, note que $p A_{p} \subseteq A_{p}$ é o ideal zero porque dado $x \in p, x^{c} \notin p$ e $x x^{c}=0$. Então pela Proposição 1.20 e pela Proposição 1.27 a fibra $\mathcal{F}_{p} \cong A_{p}=A_{p} / p A_{p} \cong K_{A}(p)$ é hipercorpo. $\mathrm{O}$ próximo resultado da condições necessárias e suficientes para que um hiper-anel von Neumann seja geométrico e satisfaça uma forma de princípio local-global.

Teorema 1.34. Seja A hiper-anel von Neumann. São equivalentes:

i) A é mono-hiper-anel.

ii) Se $u \in A$ satisfaz $u=1$ em $K_{A}(p)$ para todo $p \in \operatorname{spec}(A)$, então $u=1$ em $A$.

iii) Para todo e $\in A$ idempotente, $e+e^{c}=\{1\}$.

Se qualquer uma das condiçôes acima é válida, $A$ é geométrico e $a \in b+c$ em $A$ se, e somente se, $a \in b+c$ em $K_{A}(p)$ para todo $p \in \operatorname{spec}(A)$. Em particular, se $a=b$ em $K_{A}(p)$ para todo $p \in \operatorname{spec}(A)$, então $a=b$ em $A$. 
Demonstração. $i) \Rightarrow i i)$ : Seja $u \in A \operatorname{com} u=1 \mathrm{em} K_{A}(p)$ para todo $p \in \operatorname{spec}(A)$. Pelo Comentário 1.28 , existem $e_{1}, \ldots, e_{n} \in A$ tais que $\operatorname{spec}(A)=\bigcup_{i=1}^{n} D\left(e_{i}\right)$ e $u=1$ em $A_{e_{i}}$. Como $\mathcal{F}_{A}$ é mono-préfeixe, segue que $u=1$.

ii) $\Rightarrow$ iii): Imediato pois todo $u \in e+e^{c}$ satisfaz $u=1$ em $K_{A}(p)$ para todo $p \in \operatorname{spec}(A)$.

iii $) \Rightarrow i$ ): Pelo Lema 1.33, basta provarmos que se $\operatorname{spec}(A)=\bigcup_{j=1}^{n} D\left(e_{j}\right), e_{j} \in I d(A)$, e $a \in b+c$ em $A_{e_{j}}$ para todo $j=1, \ldots, n$, então $a \in b+c$ em $A$. Por definição temos $a e_{i} \in b e_{i}+c e_{i}$. Trocando $e_{i}$ por $e_{i} e_{1}^{c} \cdots e_{i-1}^{c}$, podemos assumir que $D\left(e_{i}\right) \cap D\left(e_{j}\right)=\emptyset$ se $i \neq j$. Pelo Lema 1.32 temos $e_{1}+\cdots+e_{n}=\{1\}$. Deste modo, temos

$$
a \in a e_{1}+\cdots+a e_{n} \subseteq b\left(e_{1}+\cdots+e_{n}\right)+c\left(e_{1}+\cdots+e_{n}\right)=b+c .
$$

Vamos mostrar agora que se $A$ é mono-hiper-anel von Neumann, $A$ é geométrico. Novamente pelo Lema 1.33, basta mostrar que se $\operatorname{spec}(A)=\bigcup_{i=1}^{n} D\left(e_{i}\right), e_{i} \in I d(A)$ e $x_{i} \in A$ tais que $x_{i}=x_{j}$ em $A_{e_{i} e_{j}}$, então existe $x \in A$ satisfazendo $x=x_{i}$ em $A_{e_{i}}$. Por definição, como $e_{i}, e_{j} \in \operatorname{Id}(A)$, temos $x_{i} e_{i} e_{j}=x_{j} e_{i} e_{j}$. Trocando $e_{i}$ por $e_{i} e_{1}^{c} \cdots e_{i-1}^{c}$, podemos assumir que $e_{i} e_{j}=0$ se $i \neq j$. Seja $x \in e_{1} x_{1}+\cdots+e_{n} x_{n}$. Então $x e_{i}=x_{i} e_{i}$ e assim $\rho_{e_{i}}(x)=\rho_{e_{i}}\left(x_{i}\right)$ para todo $i=1, \ldots, n$.

Por último, suponha que $a \in b+c$ em $K_{A}(p)$ para todo $p \in \operatorname{spec}(A)$. Como $K_{A}(p) \cong A / p$ e $\operatorname{spec}(A)$ é compacto, existem $a_{1}, \ldots, a_{n} \in A$ tais que $\operatorname{spec}(A)=\bigcup_{i=1}^{n} D\left(a_{i}^{c}\right)$ e $a \in b+c+a_{i}$. Logo $a a_{i}^{c} \in b a_{i}^{c}+c a_{i}^{c}$ e portanto $a \in b+c$ em $A_{a_{i}^{c}}$. Como $A$ é mono-hiper-anel, $a \in b+c$.

$\{$ D_S $\}$

Definição 1.35. Sejam $A$ hiper-anel e $S \subseteq A$ conjunto multiplicativo $(1 \in S$ e $S \cdot S \subseteq S)$. Dados $a, b \in A$, considere $D_{S}(a, b):=\{x \in A$ : existem $u, v, s \in S$ tais que $x u \in a v+b s\}$.

Definição 1.36. Sejam $A$ hiper-anel von Neumann e $S \subseteq A$. O conjunto $S$ é chamado subgrupo von Neumann se

- $S$ é multiplicativo.

- Dados $a \in A$ idempotente, se $x \in D_{S}\left(a, a^{c}\right)$, então existe $s \in S$ tal que $x s \in S$.

\{quovN\}

Proposição 1.37. Seja $A$ um hiper-anel von Neumann e $S \subseteq A$ conjunto multiplicativo. Então $A / m S$ é hiper-anel von Neumann geométrico se, e somente se, $S$ é subgrupo von Neumann. Em particular, se $S$ é subgrupo von Neumann, então $\bar{S}$ também é (veja Definição 1.17 e Proposição 1.18.(i)).

Demonstração. $\Leftarrow$ : Como a categoria $\mathbf{H v N}$ é fechada por quociente de Marshall, $A /{ }_{m} S$ é hiperanel von Neumann. Assim, pelo Teorema 1.34 é suficiente provar que $x+x^{c}=\{1\}$ para todo $x \in A /{ }_{m} S$ idempotente. Considere a projeção natural $\pi: A \rightarrow A / m S$ e seja $x \in A$ tal que $\pi(x)$ seja idempotente. Como $\pi(i(x))=i(\pi(x))=\pi(x)$, podemos supor sem perda de generalidade que $x \in \operatorname{Id}(x)$. Então, dado $\pi(z) \in \pi(x)+\pi(x)^{c}=\pi(x)+\pi\left(x^{c}\right)$, existem $u, v, w \in S$ tais que $z u \in x v+x^{c} w$ e assim $z \in D_{S}\left(x, x^{c}\right)$. Como $S$ é subgrupo von Neumann, existe $o \in S$ tal que $z o \in S$ e assim $\pi(z)=1$. Logo $A /{ }_{m} S$ é hiper-anel von Neumann geométrico.

$\Rightarrow$ Sejam $a \in A$ idempotente e tome $x \in D_{S}\left(a, a^{c}\right)$. Então existem $s_{1}, s_{2}, s_{3} \in S$ com $x s_{1} \in$ $a s_{2}+a^{c} s_{3}$. Assim $\pi(x) \in \pi(a)+\pi(a)^{c}$ mas como $A /{ }_{m} S$ é geométrico, $\pi(x)=1$ e assim existe $s \in S$ tal que $x s \in S$.

Comentários 1.38. - O quociente de Marshall em geral não preserva hiper-anéis geométricos. Sejam $A=\mathbb{R} \times \mathbb{R}$ e $S=\left\{1,(2,3),\left(2^{2}, 3^{2}\right), \ldots\right\}$. Vamos verificar que embora $A$ seja hiper-anel von Neumann geométrico (todo anel é geométrico), $A / m S$ não é. Para isso vamos mostrar que $S$ não é subgrupo von Neumann. Sejam $x=\left(2^{2}, 3\right)$ e $a=(1,0)$. Note que $a^{2}=a$ é idempotente e $a^{c}=(0,1)$. Note ainda que $x=a\left(2^{2}, 3^{2}\right)+a^{c}(2,3)$ e portanto $x \in D_{S}\left(a, a^{c}\right)$. Porém dado $s=\left(2^{k}, 3^{k}\right) \in S$, temos $x s=\left(2^{2+k}, 3^{k}\right) \notin S$. Assim, $A / m S$ não é geométrico.

- A situação é um pouco melhor para alguns conjuntos multiplicativos. Se $A$ é um hiper-anel von Neumann geométrico e $S \subseteq \operatorname{Id}(A)$ é um subconjunto multiplicativo dos idempotentes (ou 
mais geralmente se $S$ é um conjunto multiplicativo tal que para todo $a, b \in S$ existe $c \in S$ tal que $a c=b c$ ), então $A /{ }_{m} S$ é geométrico também. Porém é sempre possível "corrigir" um hiper-anel von Neumann $A$, aproximando-o por um HvN-geométrico. Este é o conteúdo do próximo resultado.

Teorema 1.39. Sejam A hiper-anel von Neumann e $S=\bigcup\left\{e_{1}+\cdots+e_{n}: n \geq 1, e_{i} \in \operatorname{Id}(A), 1 \in\right.$ $e_{1}+\cdots+e_{n}, e_{i} e_{j}=0$ para $\left.i \neq j\right\}$. Então $A / m S$ é hiper-anel von Neumann geométrico e dado morfismo $f: A \rightarrow B$ com $B$ mono-hiper-anel, existe único morfismo $\bar{f}: A / m S \rightarrow B$ tal que

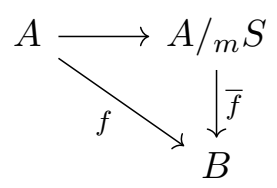

é um diagrama comutativo.

Demonstração. Primeiro note que dados $x, y \in S$, então pelo Lema 1.32 podemos assumir que existem $e_{1}, \ldots, e_{n} \in \operatorname{Id}(A)$ tais que $1 \in e_{1}+\cdots+e_{n}, e_{i} e_{j}=0$ para $i \neq j$ e $x, y \in e_{1}+\cdots+e_{n}$.

Para vermos que $A / m S$ é geométrico basta provar que $S$ é subgrupo von Neumann. Seja $a \in A$ idempotente e tome $x \in D_{S}\left(a, a^{c}\right)$. Então existem $s_{1}, s_{2}, s_{3} \in S$ tais que $x s_{1} \in a s_{2}+a^{c} s_{3}$. Pela justificativa inicial, existem $e_{1}, \ldots, e_{n} \in \operatorname{Id}(A)$ tais que $1 \in e_{1}+\cdots+e_{n}, e_{i} e_{j}=0$ para $i \neq j$ e $s_{2}, s_{3} \in e_{1}+\cdots+e_{n}$. Então $x s_{1} \in a s_{2}+a^{c} s_{3} \subseteq a e_{1}+\cdots+a e_{n}+a^{c} e_{1}+\cdots+a^{c} e_{n}$. Mas note que $1 \in a+a^{c} \subseteq a e_{1}+\cdots+a e_{n}+a^{c} e_{1}+\cdots+a^{c} e_{n}$ e se $i \neq j,\left(a e_{i}\right)\left(a e_{j}\right)=\left(a^{c} e_{i}\right)\left(a^{c} e_{j}\right)=0$ e para quaisquer $i, j\left(a e_{i}\right)\left(a^{c} e_{j}\right)=0$. Logo $x s_{1} \in S$ e assim $S$ é subgrupo von Neumann.

Para concluirmos o teorema, basta agora provar a propriedade universal. Seja $f: A \rightarrow B$ morfismo, $B$ mono-hiper-anel. Seja $x \in S$. Então existem $e_{1}, \ldots, e_{n} \in \operatorname{Id}(A)$ tais que $1 \in e_{1}+\cdots+e_{n}$, $e_{i} e_{j}=0$ para $i \neq j$ e $x \in e_{1}+\cdots+e_{n}$. Então $f(x) \in f\left(e_{1}\right)+\cdots+f\left(e_{n}\right)$ e $1 \in f\left(e_{1}\right)+\cdots+f\left(e_{n}\right)$ e $f(x) f\left(e_{i}\right)=f\left(e_{i}\right)$. Assim $f(x)=1$ em $B_{f\left(e_{i}\right)}$ para todo $i=1, \ldots, n$. Como $B$ é mono-hiper-anel, $f(x)=1$. Deste modo, como $f(S)=1$, segue da Proposição 1.18.(iv) que existe único morfismo $\bar{f}: A / m S \rightarrow B$ que faz o diagrama do teorema comutar.

Comentário 1.40. No comentário anterior, vimos que se considerarmos o anel $A=\mathbb{R} \times \mathbb{R}$ e o conjunto multiplicativo $S=\left\{(1,1),(2,3),(2,3)^{2}, \ldots\right\}$, o quociente $A / m S$ não é geométrico mas pelo teorema anterior aplicado a $A /{ }_{m} S$ deve existir um menor conjunto multiplicativo e cancelativo $S^{\prime} \supseteq S$ tal que $A / m S^{\prime}$ é geométrico. Explicitamente, temos $S^{\prime}=\left\{\left(2^{n}, 3^{k}\right) \in A: n, k \geq 0\right\}$.

Vimos na Proposição 2.69 que dado HvN, o quociente de Marshall por um subgrupo von Neumann resulta num HvN geométrico. Vamos provar um resultado complementar: todo quociente na categoria dos HvN geométricos é dado por um quociente de Marshall (Teorema 1.43). Este resultado será importante no Capítulo 2 para classificar os quocientes na categoria dos semigrupo reais von Neumann.

Lema 1.41. Seja $A$ hiper-anel von Neumann geométrico. Seja $a \in A$.

i) Se $x \in a-a^{c}$, então $x$ é inversível, $x i(a)=a$ e $x a^{c}=-a^{c}$.

ii) $\operatorname{Dados} x, y \in a-a^{c}, x=y$.

Vamos denotar por $\nabla(a)$ o único elemento em $a-a^{c}$.

iii) $x=\nabla(a)$ se, e somente se, $a=i(a) x$ e $x \in a-1+i(a)$.

iv) Se $f: A \rightarrow B$ é morfismo em HvNG, dado $x \in A$ temos $\nabla(f(x))=f(\nabla(x))$.

v) Sejam $x, y \in A$. São equivalentes:

a) $x=y$. 
b) $\nabla(x)=\nabla(y)$ e $i(x)=i(y)$.

c) $\nabla(x)=\nabla(y)$ e $\nabla(i(x))=\nabla(i(y))$.

Em particular, se $a, b \in A$ são idempotentes, $a=b$ se, e somente se, $\nabla(x)=\nabla(y)$

Demonstração. Seja $b \in A$ tal que $a=a^{2} b$. Note que $i(a)=a b$.

i) Se $x \in a-a^{c}$, então $0=x x^{c} \in a x^{c}-a^{c} x^{c}$ e $a x^{c}=a^{c} x^{c}$. Temos então $i(a) x^{c}=i(a)^{2} x^{c}=$ $i(a)\left(a b x^{c}\right)=i(a)\left(b a^{c} x^{c}\right)=0$ e $a^{c} x^{c}=\left(a^{c}\right)^{2} x^{c}=a^{c} a x^{c}=0$. Logo, como $1 \in i(a)+a^{c}$, temos $x^{c} \in i(a) x^{c}+a^{c} x^{c}=\{0\}$. Portando $0=x^{c} \in 1-i(x)$ e assim $i(x)=1$. Tomando $l \in A$ com $x=x^{2} l$, temos $1=i(x)=x l$ e deste modo $x$ é inversível.

Por último, como $x \in a-a^{c}, x a=a^{2}$ e $x a^{c}=-a^{c}$; $\operatorname{logo} x i(a)=x a b=a^{2} b=a$.

ii) Se $x, y \in a-a^{c}$, como $y$ é inversível, $x y^{-1} \in a y^{-1}-a^{c} y^{-1}$. Mas pelo item anterior yi $(a)=a \mathrm{e}$ $y a^{c}=-a^{c}$. Assim $i(a)=a y^{-1}, a^{c}=-a^{c} y^{-1}$ e $x y^{-1} \in a y^{-1}-a^{c} y^{-1}=i(a)+a^{c}=\{1\}$ pois $A$ é geométrico. Portanto $x=y$.

iii) Se $x=\nabla(a)$, pelo item $i)$, temos $a=i(a) \nabla(a)$ e $\nabla(a) \in a-a^{c}, a^{c} \in 1-i(a)$. Reciprocamente, suponha que $a=i(a) x$ e $x \in a+l, l \in-1+i(a)$. Então $x a^{c}=l a^{c}=-a^{c}$. Logo $\nabla(a) \in a-a^{c}=$ $i(a) x+a^{c} x=x\left(i(a)+a^{c}\right)=\{x\}$, pois $A$ é hiper-anel geométrico.

iv) Segue direto do item $i$ iii) pois para todo $a \in A, i(f(a))=f(i(a))$.

v) É fácil ver $a) \Rightarrow b) \Rightarrow c$ ). Vamos mostrar que $c) \Rightarrow b) \Rightarrow a)$.

$c) \Rightarrow b)$ : Basta provar que se $a, b \in \operatorname{Id}(A)$ satisfazem $\nabla(a)=\nabla(b)$, então $a=b$. Como $\nabla(b) \in b-b^{c}$, temos $b=b \nabla(b)=b \nabla(a)$ e $-b^{c}=b^{c} \nabla(b)=b^{c} \nabla(a)$. Assim, $\nabla(b) \in b-b^{c}=$ $b \nabla(a)+b^{c} \nabla(a)=\nabla(a)\left(b+b^{c}\right)=\{\nabla(a)\}$, pois $A$ é hiper-anel geométrico.

$b) \Rightarrow a)$ : Se $\nabla(x)=\nabla(y)$ e $i(x)=i(y)$ então, pelo item iii), $x=i(x) \nabla(x)=i(y) \nabla(y)=y$.

Considere o funtor esquecimento $U: \mathbf{H v N G} \rightarrow$ Sets para a categoria dos conjuntos. O pŕoximo resultado mostra, em particular, que as noções de mapa quociente em HvNG definida em 1.9 e a de $U$-quociente (Definição A.4) coincidem.

Lema 1.42. Sejam $f: A \rightarrow B$ e $g: A \rightarrow V$ morfismos em HvNG. Então $\operatorname{ker}_{1}(f) \subseteq \operatorname{ker}_{1}(g)$ se, e somente se, $\operatorname{ker}(f) \subseteq \operatorname{ker}(g)$. Em particular,

- $\operatorname{ker}_{1}(f)=\operatorname{ker}_{1}(g)$ se, e somente se, $\operatorname{ker}(f)=\operatorname{ker}(g)$.

- $f$ é injetor se, e somente se, $\operatorname{ker}_{1}(f)=1$.

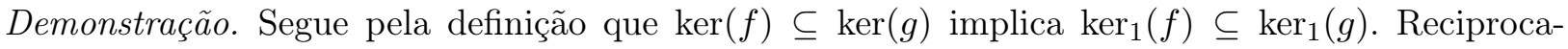
mente, seja $(a, b) \in \operatorname{ker}(f)$. Então $f(\nabla(a))=f(\nabla(b))$ e $f(\nabla(i(a)))=f(\nabla(i(b)))$. Logo

$$
f\left(\nabla(a) \nabla(b)^{-1}\right)=f\left(\nabla(i(a)) \nabla(i(b))^{-1}\right)=1
$$

. Assim, por hipótese, segue que $g\left(\nabla(a) \nabla(b)^{-1}\right)=g\left(\nabla(i(a)) \nabla(i(b))^{-1}\right)=1$ e portanto $\nabla(g(a))=$ $\nabla(g(b))$ e $\nabla(i(g(a)))=\nabla(i(g(b)))$. Pelo Lema 1.41, temos $(a, b) \in \operatorname{ker}(g)$, como desejado.

Teorema 1.43. Seja $f: A \rightarrow B$ morfismo em HvNG. Sejam $S=f^{-1}(1)$ e $\bar{f}$ o mapa induzido $A /{ }_{m} S \rightarrow B$. Então $\bar{f}$ é injetor e $S$ é subgrupo von Neumann. Além disso, as seguintes afirmações são equivalentes:

i) f é morfismo forte e sobrejetor.

ii) $f$ é morfismo quociente na categoria $\boldsymbol{H v} \boldsymbol{N G}$. 
iii) $\bar{f}: A / m S \rightarrow B$ é isomorfismo.

Demonstração. É fácil ver que $\operatorname{ker}_{1}(\bar{f})=\{1\}$ e portanto pelo Lema 1.42 temos $\bar{f}$ injetora. Para vermos que $S$ é subgrupo von Neumann, note primeiro que ele é multiplicativo. Sejam $a \in A$ idempotente e $x \in D_{S}\left(a, a^{c}\right)$. Então, tomando $s_{1}, s_{2}, s_{3} \in S$ com $x s_{1} \in a s_{2}+a^{c} s_{3}$, temos $f(x)=$ $f\left(x s_{1}\right) \in f\left(a s_{2}\right)+f\left(a^{c} s_{3}\right)=f(a)+f(a)^{c}=\{1\}$ e assim $x \in S$.

i) $\Rightarrow$ ii): Proposição 1.11 .

ii) $\Rightarrow$ iii): Vamos utilizar os conceitos de quocientes em categorias quase-concretas desenvolvidos no apêndice. Considere o funtor de esquecimento $U:$ HvNG $\rightarrow$ Set para a categoria dos conjuntos. Primeiro note que $\operatorname{ker}(f)=\operatorname{ker}_{U}(f)$ e portanto a presente noção de morfismo quociente (Definição 1.9) coincide com a de $U$-quociente apresentada em $A$.4. Vamos mostrar que $\bar{f}$ é isomorfismo utilizando o Teorema A.9. Seja $Q$ a subclasse dos morfismos de $\mathbf{H v N G}$ da forma $A \rightarrow A / m S$, onde $A$ é hiper-anel von Neumann geométrico e $S \subseteq A$ é subgrupo von Neumann. Como $\operatorname{ker}(f)=$ $\operatorname{ker}(A \rightarrow A / m S)$, segue que $Q$ é quociente-injetiva (Definição A.6). Assim, pelo Teorema A.9, $\bar{f}: A /{ }_{m} S \rightarrow B$ é isomorfismo.

iii) $\Rightarrow$ i): Como $\pi: A \rightarrow A / m S$ é sobrejetora, $f=\bar{f} \circ \pi$ também é sobrejetora. Suponha então que $f(a) \in f(b)+f(c)$, ou seja, $\bar{f}(\pi(a)) \in \bar{f}(\pi(b))+\bar{f}(\pi(c))$. Como $\bar{f}$ é isomorfismo, temos $\pi(a) \in \pi(b)+\pi(c)$. Então existem $s_{1}, s_{2}, s_{3} \in S$ tais que $a s_{1} \in b s_{2}+c s_{3}$. Observando que $\pi\left(a s_{1}\right)=$ $\pi(a), \pi\left(b s_{2}\right)=\pi(b), \pi\left(c s_{3}\right)=\pi(c)$, tem-se que $f$ é morfismo forte.

Em geral, se $A$ é um anel e $S \subseteq A$ é um subconjunto multiplicativo, então $A / m S$ não é necessariamente um anel (Exemplo do comentário 1.38). Mas vale o seguinte para aneis von Neumann.

Corolário 1.44. Seja $f: A \rightarrow B$ um homomorfismo sobrejetor entre anéis von Neumann e $S=$ $f^{-1}(1)$. Então o mapa induzido $A / m S \rightarrow B$ é isomorfismo. Em particular, $A / m S$ é anel.

Demonstração. Pelo teorema anterior, basta mostrar que $f$ é morfismo forte. Sejam $a, b, c \in A$ com $f(a)=f(b)+f(c)$. Escrevendo $x=a-b-c$ e $a^{\prime}=a-x$, temos $a^{\prime}=b+c$ e $f\left(a^{\prime}\right)=f(a)$. 


\section{Capítulo 2}

\section{Semigrupos Reais}

Neste capítulo descreveremos o ingrediente principal da tese: a noção de semigrupo real (RS). Introduzida em [DP1] como contraparte de primeira ordem da noção de espaço de ordens abstrato (encontrada em [Mar7]), a axiomatização dos RSs toma como noção primitiva a representação de formas binárias. Na primeira seção, seguindo [DP2], encontram-se as definições e os resultados fundamentais. Ainda, no Teorema 2.15, é estabelecido um isomorfismo entre o feixe estrutural de multi-aneis apresentado no primeiro capítulo e o feixe canônico de RSs encontrado em [DP2]. Já na segunda seção, seguindo o tratamento dado por Marshall ao assunto através da linguagem dos multi-aneis ([Mar10]), os multi-aneis reais reduzidos (RRM) são definidos e no Teorema 2.32 é mostrado como a noção é equivalente à RS. Ainda, para conveniência do leitor, é descrita a multiálgebra real com destaque para o funtor $Q:$ pMulti $\rightarrow \mathbf{R R M}$ da categoria dos p-multi-aneis para a categoria dos multi-aneis reais reduzidos que é adjunto à esquerda do funtor de inclusão (Teorema 2.41). A seção é finalizada com a extensão da representação de $Q$ em hipercorpos encontrada na Seção 4 de [Mar10] para os hiper-aneis von Neumann (Teorema 2.50). Uma consequência da generalização é que o funtor $Q$ preserva equivalência elementar (Teorema 2.51). Na seção 3 duas classes de semigrupos reais entram em foco: os Booleanos e os von Neumann. Seguindo [MR], o Teorema 2.54 fornece algumas caracterizações dos RSs Booleanos. Por outro lado, o Teorema 2.58 lista caracterizações de RSs von Neumann. O capítulo é finalizado com a classificação dos quocientes categoriais dos RSs von Neumann via quociente de Marshall (Teoremas 2.68 e 2.69).

\subsection{Preliminares}

Nesta seção apresentamos as definições e resultados fundamentais concernentes à teoria dos semigrupos reais. Esta noção consiste num semigrupo ternário $(R, \cdot,-1,0,1)$ munido de uma relação ternária $D$ (denotada por $a \in D(b, c)$ no lugar de $D(b, c, a)$ ). A relação $D$ é chamada representação . É útil definir uma segunda relação ternária:

$$
a \in D^{t}(b, c) \Leftrightarrow a \in D(b, c) \wedge-b \in D(-a, c) \wedge-c \in D(b,-a) .
$$

A relação $D^{t}$ é denominada representação transversal .

Definição 2.1. Um semigrupo ternário é uma estrutura $(T, \cdot,-1,0,1)$ onde $\cdot$ é operação binária sobre $T$ e $0,1,-1$ são constantes tais que

i) $(T, \cdot, 1)$ é um semigrupo comutativo com unidade.

ii) $x^{3}=x$ para todo $x \in T$.

iii) $1 \neq-1$ e $(-1) \cdot(-1)=1$.

iv) $x \cdot 0=0$ para todo $x \in T$.

v) Para todo $x \in T$, se $x=-1 \cdot x$, então $x=0$. 
Comentário 2.2. - Dado $a \in T$, utilizamos a notação $-a=-1 \cdot a$.

- Se $a \in T$ é inversível, então $a^{2}=1$ (se existe $b \in T$ com $a b=1$, então $a^{2}=a^{3} b=a b=1$ ). Denotamos por $T^{\times}$o conjunto de todos os elementos inversíveis de $T$.

- Denotamos por $\operatorname{Id}(T):=\left\{x \in T: x^{2}=x\right\}$ o conjunto dos idempotentes. Como $a^{3}=a$ em $T$, os idempotentes de $T$ coincidem com os quadrados de $T$.

Exemplo 2.3. i) O conjunto $\mathbf{3}=\{-1,0,1\}$ munido do produto usual é um semigrupo ternário. Mais geralmente, dado conjunto $X$ não-vazio, o conjunto $3^{X}$ das funções saindo de $X$ e chegando em 3 munido do produto ponto-a-ponto é semigrupo ternário.

ii) Como os axiomas de semigrupo ternário são Horn-geométricos na linguagem $\mathcal{L}_{T S}=\{\cdot,-1,0,1\}$, a classe é fechada por produtos reduzidos e limites indutivos (dirigidos para cima).

Definição 2.4. Um semigrupo real é uma tupla $(R, D, \cdot,-1,0,1)$ tal que $(R, \cdot,-1,0,1)$ é um semigrupo ternário e $D$ é uma relação ternária satisfazendo os seguintes axiomas:

RS0 $c \in D(a, b)$ se, e somente, se $c \in D(b, a)$.

RS1 $a \in D(a, b)$.

RS2 $c \in D(a, b)$ implica $c d \in D(a d, b d)$.

RS3 (Associatividade forte) Se $x \in D^{t}(a, p)$ com $p \in D^{t}(b, c)$, então existe $q \in D^{t}(a, b)$ com $x \in D^{t}(q, c)$.

RS4 $e \in D\left(c^{2} a, d^{2} b\right)$ implica $e \in D(a, b)$.

RS5 Se $a d=b d, a e=b e$ e $c \in D(d, e)$, então $a c=b c$.

RS6 $c \in D(a, b)$ implica $c \in D^{t}\left(c^{2} a, c^{2} b\right)$.

RS7 (Redução) Se $D^{t}(a,-b) \cap D^{t}(-a, b) \neq \emptyset$, então $a=b$.

RS8 $a \in D(b, c)$ implica $a^{2} \in D\left(b^{2}, c^{2}\right)$.

\{notacao D\}

Comentário 2.5. Por convenção, dado $a \in R, D(a)=a \operatorname{Id}(R)$ e $D^{t}(a)=\{a\}$.

Proposição 2.6. Seja $R$ RS. Para $a, b, c, d, e, x, y \in R$ :

i) $a \in D^{t}(b, c) \Rightarrow-b \in D^{t}(-a, c)$.

ii) $0 \in D(a, b)$.

iii) $a \in D^{t}(b, c) \Rightarrow a d \in D^{t}(b d, c d)$.

iv) $a \in D(0,1) \cup D(1,1) \Rightarrow a^{2}=a$.

v) $d \in D(c a, c b) \Rightarrow d=c^{2} d$.

vi) $a^{2} \in D(1, b)$ e $D(1,1)=\operatorname{Id}(R)$.

vii) $D^{t}(a, a)=\{a\}$.

viii) $D(0,0)=0$.

ix) $1 \in D^{t}(1, a)$.

x) $D^{t}(1,-1)=R$.

xi) $a b \in D\left(1,-a^{2}\right)$. 
xii) $0 \in D^{t}(a, b) \Leftrightarrow a=-b$.

xiii) $a \in D(b, c)$ e $b, c \in D(x, y)$, então $a \in D(x, y)$.

xiv) $a \in D(b, c) \Leftrightarrow a b \in D(1, b c) \wedge a c \in D(1, b c) \wedge a^{2} \in D\left(b^{2}, c^{2}\right)$.

xv) $D^{t}(a, b) \neq \emptyset$.

xvi) (Associatividade fraca) $x \in D(p, c) \wedge p \in D(a, b) \Rightarrow \exists y \in D(b, c): x \in D(a, y)$.

xvii) $D(a,-a)=a^{2} \cdot R$.

xviii) $a \in D^{t}(b, c) \Leftrightarrow a^{2} \in D\left(b^{2}, c^{2}\right) \wedge a b \in D^{t}\left(b^{2}, b c\right) \wedge a c \in D^{t}\left(c^{2}, b c\right)$.

xix) $a \in D(b, c) \Leftrightarrow a \in D^{t}\left(a^{2} b, a^{2} c\right)$.

Demonstração. As demonstrações podem ser encontradas em [DP1], Proposição 2.3. Em [RRM1] também existem outras provas onde se destaca o uso ou não do axioma da redução.

Exemplo 2.7. $\quad$ - O conjunto $\mathbf{3}=\{1,0,-1\}$ munido pela multiplicação usual e representação transversal dada por $D^{t}(0, x)=\{x\} \forall x \in \mathbf{3}, D^{t}(1,1)=\{1\}, D^{t}(-1,-1)=\{-1\}, D^{t}(1,-1)=$ 3 e representação por $x \in D(a, b) \Leftrightarrow x \in D^{t}\left(x^{2} a, x^{2} b\right)$ é semigrupo real ${ }^{1}$.

- Todo RSG dá origem a um RS adjuntando um elemento nulo no grupo. Mais especificamente, seja $(G, \equiv,-1)$ grupo especial ([DM1]) e considere $G^{\prime}=G \cup\{0\}$ com multiplicação induzida de $G$ e $x \cdot 0=0=0 \cdot x$ para todo $x \in G$. A definição da representação transversal é dada por:

$$
D_{G^{\prime}}^{t}(a, b)= \begin{cases}\{a\} & \text { se } b=0 \\ \{b\} & \text { se } a=0 \\ G^{\prime} & \text { se } a, b \neq 0 \text { e } a=-b \\ D_{G}(a, b) & \text { se } a, b \neq 0 \text { e } a \neq-b .\end{cases}
$$

Em [RRM1] é provado que $G^{\prime}$ satisfaz os axiomas de semigrupo real. Os semigrupos reais $R$ que são da forma $R=R^{\times} \cup\{0\} \operatorname{com} R^{\times}$um RSG são chamados de $0-\mathrm{RSG}^{2}$.

- Na próxima seção, veremos como associar a cada anel dito semi-real um semigrupo real.

Definição 2.8. Seja $f: R \rightarrow G$ um mapa entre RSs. Dizemos que $f$ é morfismo de RS para todos $a, b, c \in R$ :

- $f(a b)=f(a) f(b)$.

- $f(0)=0, f(-1)=-1$.

- Se $a \in D_{R}^{t}(b, c)$, então $f(a) \in D_{G}^{t}(f(b), f(c))$.

Pelas fórmulas que expressam a definição de $D^{t}$ em termos de $D$ e vice versa (i.e., pelo item $x i x$ ) da Proposição 2.6), segue que na definição de morfismo acima poderíamos trocar o último item por $a \in D_{R}(b, c) \Rightarrow f(a) \in D_{R}(f(b), f(c))$.

Definição 2.9. Seja $R$ RS.

- Denota-se por $X_{R}:=\{\sigma: R \rightarrow \mathbf{3}: \sigma$ é morfismo de RSs $\}$ o conjunto de todos os morfismos de $R$ para $3^{3}$. Os elementos de $X_{R}$ são chamados de ordens ou caracteres de $R$.

\footnotetext{
${ }^{1} \mathrm{~A}$ representação transversal de $\mathbf{3}$ aqui coincide com a soma multivalorada do exemplo 1.4.

${ }^{2}$ Em [DP2], os 0-RSGs são chamados de quasi-RSGs. O prefixo quasi passa a ideia de menor potência e alcance porém existe equivalência natural entre a categoria dos 0-RSGs e dos RSGs, o que justifica a adoção da nomenclatura 0 -RSG no contexto de semigrupos reais.

${ }^{3}$ Interpretando $R$ como multi-anel, a definição coincide com $\operatorname{sper}(R)$ em 1.14 .
} 
- Dados $a_{1}, \ldots, a_{n} \in R$, introduzimos as seguintes notações:

$$
\begin{aligned}
& U\left(a_{1}, \ldots, a_{n}\right)=\left\{\sigma \in X_{R}: \sigma\left(a_{1}\right)=\cdots=\sigma\left(a_{n}\right)=1\right\} \\
& Z\left(a_{1}, \ldots, a_{n}\right)=\left\{\sigma \in X_{R}: \sigma\left(a_{1}\right)=\cdots=\sigma\left(a_{n}\right)=0\right\} .
\end{aligned}
$$

- O conjunto $X_{R}$ é munido de uma topologia gerada pelos conjuntos $U\left(a_{1}, \ldots, a_{n}\right)$ para todos $a_{1}, \ldots, a_{n} \in R$.

A topologia em $X_{R}$ é espectral (Proposição 2.25) e a correspondente topologia construtível ${ }^{4}$, denotada por $\left(X_{R}\right)_{\text {const }}$, é gerada pelos conjuntos da forma $U\left(a_{1}, \ldots, a_{n}\right) \cap Z(b), a_{1}, \ldots, a_{n}, b \in R$ pois dados $b_{1}, \ldots, b_{n} \in R$ temos $Z\left(b_{1}, \ldots, b_{n}\right)=Z\left(b_{1}^{2}, \ldots, b_{n}^{2}\right)=Z(b)$ onde $b \in D^{t}\left(b_{1}^{2}, \ldots, b_{n}^{2}\right)$. A topologia de $\left(X_{R}\right)_{\text {const }}$ é Booleana (Teorema 1.3.14 de [DST]).

Abaixo enunciamos o Teorema de Separação, muito utilizado durante todo o texto. Ele também é útil para estabelecer a dualidade entre os espaços de ordem abstratos ([Mar7]) e os semigrupos reais. Mais detalhes da dualidade podem ser encontrados em [DP2].

Teorema 2.10. Seja $R$ um $R S$. Então para $a, b, c \in R$,

- $c \in D(a, b)$ se, e somente se, para todo $\sigma \in X_{R}, \sigma(c) \in D_{3}(\sigma(a), \sigma(b))$.

Em particular,

- $c \in D^{t}(a, b)$ se, e somente se, para todo $\sigma \in X_{R}, \sigma(c) \in D_{3}^{t}(\sigma(a), \sigma(b))$.

- $a=b$ se, e somente se, para todo $\sigma \in X_{R}, \sigma(a)=\sigma(b)$.

Demonstração. A demonstração pode ser encontrada em [DP1], Teorema 4.1.

Segue abaixo uma primeira aplicação do Teorema de Separação que será útil para caracterização de isometria de formas binárias (Proposição $3.4 i v$ )).

$\{123\}$

Proposição 2.11. Seja $R$ um RS. Dados $a, b, c, d \in R$ satisfazendo $a b=c d$, as seguintes afirmações são equivalentes:

i) $D(a, b)=D(c, d)$.

ii) $D^{t}(a, b)=D^{t}(c, d)$.

iii) $D^{t}(a, b) \cap D^{t}(c, d) \neq \emptyset$.

Demonstração. $i) \Rightarrow i i)$ : Sejam $x \in D^{t}(a, b)$ e $\sigma \in X_{R}$. Vamos mostrar que $\sigma(x) \in D_{3}^{t}(\sigma(c), \sigma(d))$. Como $D^{t}(a, b) \subseteq D(a, b)=D(c, d)$, já temos que $\sigma(x) \in D_{3}(\sigma(c), \sigma(d))\left(^{*}\right)$. Se $\sigma(x)=0$, então $\sigma(a)=-\sigma(b)$. Como $a b=c d$, por $i$ ) segue que $\sigma(c)=-\sigma(d)$. Logo $\sigma(x) \in D_{3}^{t}(\sigma(c), \sigma(d))$. Se $\sigma(x) \neq 0$, então por $\left(^{*}\right)$ segue que $\sigma(x) \in D_{3}^{t}(\sigma(c), \sigma(d))$. Assim, pelo Teorema 2.10, temos $x \in D^{t}(c, d)$. Simetricamente concluímos que $D^{t}(c, d) \subseteq D^{t}(a, b)$.

$i i) \Rightarrow$ iii): Imediato pois pela Proposição 2.6 vale $D^{t}(a, b) \neq \emptyset$.

iii $) \Rightarrow i$ ): Sejam $x \in D^{t}(a, b) \cap D^{t}(c, d)$ e $\sigma \in X_{R}$. Vamos mostrar que $\sigma(a) \in D_{3}(\sigma(c), \sigma(d))$ $(* *)$. Para $\sigma(a)=0$ é trivial. Se $\sigma(a) \neq 0$, temos $\sigma(b)=0$ ou $\sigma(b) \neq 0$. Caso $\sigma(b)=0$, então $\sigma(x)=\sigma(a)$ e como $a b=c d$ podemos assumir $\sigma(c)=0$. Logo $\sigma(a)=\sigma(x)=\sigma(d)$ e portanto vale $(* *)$. Caso $\sigma(b) \neq 0$, como $a b=c d$ temos $\sigma(c), \sigma(d) \neq 0$. O caso $\sigma(c)=-\sigma(d)$ é trivial e podemos assumir $\sigma(c)=\sigma(d)$. Como $a b=c d$, segue que $\sigma(a)=\sigma(b)$ e portanto $\sigma(a)=\sigma(x)=\sigma(c)=\sigma(d)$, provando $(* *)$. Pelo Teorema 2.10 temos $a \in D(c, d)$. Repetindo o mesmo argumento verifica-se que $b \in D(c, d)$ e portanto pela Proposição 2.6.(xiii) vale $D(a, b) \subseteq D(c, d)$. A outra inclusão segue analogamente.

\footnotetext{
${ }^{4}$ Mais detalhes sobre a relação entre topologia espectral e a construtível em [DST].
} 
Comentários 2.12. Seja $R$ um RS.

- Interpretando a representação transversal $D_{R}^{t}$ de $R$ como soma multivalorada e a operação unária $-: R \rightarrow R$ com a multiplicação por -1 (i.e., $-x:=(-1) \cdot x$ ), tem-se que a estrutura $\left(R, D_{R}^{t},-, \cdot, 0,1\right)$ satisfaz os axiomas de multi-anel (Definição 1.1). Assim, podemos utilizar as noções e resultados sobre multi-aneis para os semigrupos reais. Faz sentido, por exemplo, falar do espectro primo $\operatorname{spec}(R)$ de $R$. Mais detalhes sobre semigrupos reais enquanto multi-aneis são dadas na seção seguinte (Definição 2.30 e Teorema 2.32) e em [RRM1].

- Dados $x, y \in \operatorname{Id}(R)$, o conjunto $D^{t}(x, y)$ tem único elemento e este é idempotente. De fato, sejam $a, b \in D^{t}(x, y)$. Fixado $\sigma \in X_{R}$, se $\sigma(x)=0$, então $\sigma(a)=\sigma(y)=\sigma(b)$; se $\sigma(y)=0$, então $\sigma(a)=\sigma(x)=\sigma(b)$ e se $\sigma(x)=\sigma(y)=1$, então $\sigma(a)=1=\sigma(b)$. Logo, pelo Teorema da Separação, segue que $a=b$. Como $\sigma(a) \in\{0,1\}$ temos $\sigma\left(a^{2}\right)=\sigma(a)^{2}=\sigma(a)$ e, novamente pelo Teorema de Separação, concluimos que $a \in \operatorname{Id}(R)$.

- O conjunto dos idempotentes $\operatorname{Id}(R)$ admite estrutura natural de reticulado distributivo limitado. Dados $x, y \in \operatorname{Id}(R)$, diz-se que $x \leq y$ quando $x y=y$. Para todo $x \in \operatorname{Id}(R)$ vale $1 \leq x \leq 0$ e dados $x, y \in \operatorname{Id}(R), x \vee y=x y$ e $x \wedge y$ é único elemento em $D^{t}(x, y)$. Além disso, em $\operatorname{spec}(R)$ vale $D(x) \cup D(y)=D(x \wedge y)$ e $D(x) \cap D(y)=D(x \vee y)$ (veja 2.5). Por indução segue que, dados $b_{1}, \ldots, b_{n} \in \operatorname{Id}(R)$ e $x \in D^{t}\left(b_{1}, \ldots, b_{n}\right)$, vale $D(x)=D\left(b_{1}\right) \cup \ldots \cup D\left(b_{n}\right)$. Note também que, pelo Teorema de Separação 2.10, $x \leq y$ se, e somente se, para todo $\sigma \in X_{R}$ vale $\sigma(x) \leq \sigma(y)$.

Na proposição seguinte é mostrado que os RSs são fechados por localização e esta construção é mais simples do que num multi-anel em geral. A seguir um semigrupo real é visto como um multi-anel.

Proposição 2.13. Sejam $R$ RS e $S \subseteq R$ conjunto multiplicativo com $0 \notin S$. Seja $\pi: R \rightarrow S^{-1} R$ projeção canônica (morfismo de multi-anéis).

i) O morfismo $\pi$ é sobrejetor e dados $a, b \in R$, temos $\pi(a)=\pi(b)$ se, e somente se, existe $s \in S$ tal que $a s=b s$.

ii) Dados $a, b, c \in R$, temos $\pi(a) \in \pi(b)+\pi(c)$ se, e somente se, existe $s \in S$ tal que as $\in D_{R}^{t}(b s, c s)$.

iii) O multi-anel $S^{-1} R$ é RS e dado morfismo $f: R \rightarrow G$ de $R S$ satisfazendo $f(a)=f(b)$ para todos $a, b \in A$ com $\pi(a)=\pi(b)$, existe único morfismo $\bar{f}: S^{-1} R \rightarrow G$ fazendo o diagrama

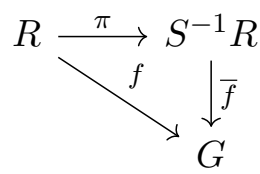

comutar. Em particular, o morfismo $\pi: R \rightarrow S^{-1} R$ é quociente na categoria dos RSs.

Demonstração. i) Dado $\frac{a}{s} \in S^{-1} R$, note que $a \cdot s^{2}=a s^{2} \cdot s^{2}$ em $R$ e portanto $\frac{a}{s}=\pi(a s)$, provando assim sobrejetividade. A equivalência em seguida sai direto da definição.

ii) Direto da definição.

iii) A propriedade universal segue da Proposição 1.15. Para vermos que $S^{-1} R$ é semigrupo real, vamos verificar os itens da Definição 2.30 .

- $\overline{1} \neq \overline{0}$ em $S^{-1} R$ pois do contrário teríamos $0 \in S$.

- Dado $a \in R$, temos $\bar{a}^{3}=\bar{a}$ em $S^{-1} R$ pois $a^{3} \cdot 1=a \cdot 1$ e $1 \in S$. 
- Dados $x, a, b \in R$, se $\bar{x} \in \bar{a}+\bar{b}^{2} \bar{a}$, pelo item $\left.i i\right)$ existe $s \in S$ com $x s \in a s+b^{2}$ as e portanto $x s^{2} \in a s^{2}+b^{2} a s^{2}=\left\{a s^{2}\right\}$. Logo $x s^{2}=a s^{2}$ e $\bar{x}=\bar{a}$ em $S^{-1} R$.

- Dados $a, b \in R$ e $\bar{x}, \bar{y} \in \bar{a}^{2}+\bar{b}^{2}$, pelo item $\left.i i\right)$ existem $s, t \in S$ tais que $x s \in a^{2} s+b^{2} s$ e $y t \in a^{2} t+b^{2} t$. Então $x s^{2} t^{2}, y s^{2} t^{2} \in a^{2} s^{2} t^{2}+b^{2} s^{2} t^{2}$ e portanto $\bar{x}=\bar{y}$ em $S^{-1} R$.

$\{$ locRS\}

Comentários 2.14. - Uma consequência imediata da proposição acima é que a definição de localização apresentada em [DP2] na Definição II.3.1 coincide com a localização de multianeis.

- Sejam $R$ RS e $a \in R$. Pelo Exemplo 1.24, $R$ tem a propriedade inversível e portanto $S_{a}=\{x \in$ $R$ : existem $n \geq 1, y \in A$ tais que $\left.a^{n}=x y\right\}=\left\{x \in R\right.$ : existe $y \in R$ tal que $\left.a^{2}=x y\right\}$. Pelas propriedades $i$ ) e $i i$ ) da proposição acima, vê-se que as relações de equivalência induzidas pela localização em $R$ por $S_{a}$ e por $\left\{1, a, a^{2}\right\}$ coincidem de modo que o isomorfismo que aparece em Proposição $1.23 \mathrm{iv}$ ) é o mapa identidade. Assim, seguindo a notação da Proposição 1.22, $\mathcal{F}_{R}(D(a))=R_{a}$. Em particular, podemos considerar $\mathcal{F}_{R}: \mathcal{B}_{R} \rightarrow \mathbf{R S}$ assumindo valores na categoria dos semigrupos reais.

O próximo resultado fornece uma descrição alternativa do feixe de semigrupos reais construído na primeira seção do capítulo 3 de [DP2]. Em particular, mostra que os semigrupos reais enquanto multi-aneis são geométricos (Definição 1.26).

$\{$ sheafrs\}

Teorema 2.15. Sejam $R R S$ e $\mathcal{B}_{R}=\{D(a)\}_{a \in R}$ o conjunto dos abertos básicos de spec $(R)$. Então $\mathcal{F}_{R}: \mathcal{B}_{R} \rightarrow \boldsymbol{R} \boldsymbol{S}$ é feixe. Assim, $R$ é multi-anel geométrico.

Demonstração. Em [DP2] é construído um feixe $\mathcal{G}_{R}$ sobre os abertos básicos de $\operatorname{spec}(R)$. Pelo Fato III.1.6 de [DP2], para cada $a \in R$ temos $\mathcal{G}_{R}(D(a))=R_{a}$. Pela Proposição $1.22, \mathcal{F}_{R}$ é funtor e pelo Comentário 2.14 temos $\mathcal{F}_{R}(D(a))=R_{a}=\mathcal{G}_{R}(D(a))$. Portanto $\mathcal{F}_{R}$ é feixe.

Comentário 2.16. A origem do feixe $\mathcal{F}_{R}$ no contexto de multi-aneis torna mais cristalina a semelhança com o feixe estrutural de anéis, já bem apontada em [DP2]. Além disso, permite a visualização mais direta de algumas informações como as fibras e os hipercorpos residuais. Dado $p \in \operatorname{spec}(R)$, pela Proposição 1.27 temos $\mathcal{F}_{p} \cong R_{p}$, onde $R_{p}=(R \backslash p)^{-1} R$ é a localização em $p$, e pela Proposição 1.20 temos $R_{p} / p R_{p} \cong K_{R}(p)$, onde $p R_{p} \subseteq R_{p}$ é o único ideal maximal de $R_{p}$. Em [DP2] é provado que $K_{R}(p)$ é um 0-RSG.

Definição 2.17. Sejam $R$ RS. Dado $\sigma \in X_{R}$, denotamos por $\operatorname{supp}(\sigma)=\sigma^{-1}(0) \in \operatorname{spec}(R)$ o suporte da ordem $\sigma$. Dado $p \in \operatorname{spec}(R)$, denotamos por $X_{p}=\left\{\sigma \in X_{R}: \operatorname{supp}(\sigma)=p\right\}$.

Note que a função supp: $X_{R} \rightarrow \operatorname{spec}(R)$ é contínua pois dado $a \in R \operatorname{temos} \operatorname{supp}^{-1}(D(a))=$ $U\left(a^{2}\right)$.

Finalizamos esta seção inicial deste segundo capítulo registrando o seguinte resultado técnico que será utilizado ao longo do texto:

Lema 2.18. Sejam $R$ RS, $p \in \operatorname{spec}(R)$ e $O \subseteq X_{R}$ um aberto na topologia construtível. Suponha que $X_{p} \subseteq O$. Então existem $a, b \in \operatorname{Id}(R)$ tais que $X_{p} \subseteq U(a) \cap Z(b) \subseteq O$.

Demonstração. Considere a função contínua supp: $X_{R} \rightarrow \operatorname{spec}(R)$. Como $O \subseteq\left(X_{R}\right)_{\text {const }}$ é aberto, $O^{c}$ é fechado e portanto compacto. $\operatorname{Logo} \operatorname{supp}\left(O^{c}\right) \subseteq(\operatorname{spec}(R))_{\text {const }}$ é compacto e $p \notin \operatorname{supp}\left(O^{c}\right)$. Deste modo $\operatorname{supp}\left(O^{c}\right)$ é fechado e existem $a, b \in \operatorname{Id}(R)$ tais que $p \in D(a) \cap D(b)^{c}$ e $\left(D(a) \cap D(b)^{c}\right) \cap$ $\operatorname{supp}\left(O^{c}\right)=\emptyset . \operatorname{Logo} X_{p} \subseteq U(a) \cap Z(b) \subseteq O$. 


\subsection{Semigrupos Reais como subcategoria reflexiva dos Multi-anéis}

Nesta seção, a multi-algebra real de um multi-anel é explorada juntamente com algumas de suas relações com os semigrupos reais -encarnados aqui como multi-aneis reais reduzidos. A principal inspiração é [Mar10] de onde alguns resultados são reproduzidos aqui para a conveniência do leitor. Os principais teoremas desta seção são:

- Em [Mar10], para cada p-multi-anel $(A, T)$ é associado o seu multi-anel real reduzido $Q_{T}(A)$. O Teorema 2.41 mostra que tal associação é um funtor adjunto à esquerda da inclusão dos multi-aneis reais reduzidos na categoria dos p-multi-aneis.

- Na seção 4 de [Mar10] é mostrado que o funtor $Q$ restrito a categoria dos p-hiper-corpos admite uma representação via quociente de Marshall. O Teorema 2.50 generaliza tal representação para p-hiper-aneis von Neumann.

- O Teorema 2.52 fornece uma forma de recíproca do Teorema 2.50 no caso de aneis. Mais especificamente, se $A$ é anel (semi-real) e $Q(A)$ é representado por algum quociente de Marshall usando aneis, então $Q(A)$ é canonicamente representado pela envoltória von Neumann regular do anel $A$.

- O Teorema 2.51 mostra que o funtor $Q$, quando restrito a categoria dos p-hiper-aneis von Neumann, preserva equivalência elementar.

Definição 2.19. Seja $A$ multi-anel. Um cone primo é um subconjunto $P \subseteq A$ tal que $A^{2} \subseteq P$, $P+P \subseteq P, P \cdot P \subseteq P, P \cup-P=A$ e $P \cap-P$ é ideal primo. Uma ordem do multi-anel $A$ é um morfismo $\sigma: A \rightarrow 3$ de multi-aneis. O conjunto de todas ordens é denotado por $\operatorname{sper}(A)$ e é chamado de espectro real. O suporte de uma ordem $\sigma$ é $\operatorname{supp}(\sigma)=\sigma^{-1}(0)$.

Estas definições são justificadas na próxima proposição:

Proposição 2.20. Seja $A$ um multi-anel. Então os seguintes conjuntos estão em correspondência bijetiva e natural:

i) $P C=\{P \subseteq A: P$ é um cone primo $\}$.

ii) $P Q=\left\{(p, P)\right.$ : onde $p \in \operatorname{spec}(A)$ e $P$ é um cone primo de $\left.K_{A}(p)\right\}$.

iii) $\operatorname{sper}(A)=\{\sigma: A \rightarrow 3: \sigma$ é um morfismo $\}$.

Demonstração. As bijeções são descritas em termos de $\operatorname{sper}(A)$. Nos limitaremos a apresentar as correpondências, pois as verificações são imediatas e serão omitidas.

i) $\leadsto$ iii): Dado cone primo $P \subseteq A$, considere o morfismo $\sigma_{P}: A \rightarrow 3$ dado por

$$
\sigma_{P}(a)= \begin{cases}1 & \text { se } a \in P \backslash-P \\ 0 & \text { se } a \in P \cap-P \\ -1 & \text { se } a \in-P \backslash P .\end{cases}
$$

Reciprocamente, se $\sigma \in \operatorname{sper}(A)$, então $P_{\sigma}:=\sigma^{-1}(\{0,1\}) \subseteq A$ é cone primo.

ii) « $\leadsto$ iii): Dado ideal primo $p \in \operatorname{spec}(A)$ e cone primo $P \subseteq K_{A}(p)$, considere o morfismo $\sigma_{(p, P)}: A \rightarrow 3$

$$
\sigma_{(p, P)}(a)= \begin{cases}1 & \text { se } a \notin p \text { e } \bar{a} \in P \\ 0 & \text { se } a \in p \\ -1 & \text { se } a \notin p \text { e }-\bar{a} \in P .\end{cases}
$$


Reciprocamente, se $\sigma: A \rightarrow 3$ é morfismo, então $p_{\sigma}:=\sigma^{-1}(0) \in \operatorname{spec}(A)$ e a imagem de $\sigma^{-1}(\{0,1\})$ pelo morfismo canônico $A \rightarrow A / p_{\sigma} \rightarrow K_{A}\left(p_{\sigma}\right)$ determina um cone primo $P_{\sigma}$ em $K_{A}\left(p_{\sigma}\right)$.

Definição 2.21. Seja $A$ um multi-anel.

- Um subconjunto $T \subseteq A$ é chamado de pré-ordem se $A^{2} \subseteq T, T \cdot T \subseteq T$ e $T+T \subseteq T$; esta pré-ordem é dita própria se $-1 \notin T$.

- Se $T$ é pré-ordem própria de $A$, o par $(A, T)$ é chamado de p-multi-anel.

Se $(A, T)$ e $(B, P)$ são p-multi-aneis, então um morfismo de multi-aneis $f: A \rightarrow B$ é morfismo de p-multi-aneis (ou simplesmente p-morfismo) se $f(T) \subseteq P$. Denotamos por pMult a categoria de todos p-multi-aneis e seus morfismos.

Comentário 2.22. A categoria pMult pode ser expressa como categoria de estruturas de primeira ordem da linguagem dos multianeis (Comentários 1.2) acrescida de um predicado unário e satisfazendo axiomas Horn-Geometricos.

$\{\mathrm{xa}\}$

Definição 2.23. Seja $A$ multi-anel e $T \subseteq A$ pré-ordem.

- Denota-se por $\operatorname{sper}_{T}(A):=\{\sigma: A \rightarrow \mathbf{3}: \sigma$ é morfismo de multi-aneis e $\sigma(T) \subseteq\{0,1\}\}$ o conjunto de todos os morfismos de $A$ para 3 que preservam $T$.

- Dados $a_{1}, \ldots, a_{n} \in A$, introduzimos as seguintes notações:

$$
\begin{aligned}
& U_{T}\left(a_{1}, \ldots, a_{n}\right)=\left\{\sigma \in \operatorname{sper}_{T}(A): \sigma\left(a_{1}\right)=\cdots=\sigma\left(a_{n}\right)=1\right\} \\
& Z_{T}\left(a_{1}, \ldots, a_{n}\right)=\left\{\sigma \in \operatorname{sper}_{T}(A): \sigma\left(a_{1}\right)=\cdots=\sigma\left(a_{n}\right)=0\right\} .
\end{aligned}
$$

Se $T=\sum A^{2}$, denotamos $U_{\sum A^{2}}$ e $Z_{\sum A^{2}}$ respectivamente por $U$ e $Z$.

- O conjunto $\operatorname{sper}_{T}(A)$ é munido de uma topologia gerada pelos conjuntos $U_{T}\left(a_{1}, \ldots, a_{n}\right)$ para todos $a_{1}, \ldots, a_{n} \in A$.

Comentários 2.24. - Pela Proposição 2.20, $\operatorname{sper}_{T}(A)$ corresponde aos cones primos $P \subseteq A$ tal que $T \subseteq P$.

- Se $T=\sum A^{2}$, então vale $\operatorname{sper}_{\sum A^{2}}(A)=\operatorname{sper}(A)($ ver Proposição 2.20).

- A topologia de $\operatorname{sper}_{T}(A)$ coincide com a topologia de subespaço induzida de $\operatorname{sper}(A)$.

- Seja $f:(A, T) \rightarrow(B, P)$ um p-morfismo. Então temos um mapa contínuo $\operatorname{sper}_{P}(B) \rightarrow$ $\operatorname{sper}_{T}(A)$ induzido pela composição com $f$ que é contínuo.

Seja $(A, T)$ p-multi-anel. Um ideal $\alpha \subseteq A$ é real se dados $a_{1}, \ldots, a_{n} \in A$ tais que $a_{1}^{2}+\cdots+a_{n}^{2} \cap \alpha \neq$ $\emptyset$, então $a_{i} \in \alpha$ para todo $i=1, \ldots, n$. O multi-anel $A$ é dito semi-real se $-1 \notin \sum A^{2}$ (ou seja, se a pré-ordem $\sum A^{2}$ é própria).

Proposição 2.25. Sejam $A$ multi-anel e $T$ pré-ordem.

i) $\operatorname{sper}_{T}(A) \neq \emptyset$ se, e somente se, $T$ é pré-ordem própria. Em particular, $\operatorname{sper}(A) \neq \emptyset$ se, e somente se, $A$ é semi-real.

ii) A topologia de $\operatorname{sper}_{T}(A)$ gerada pelos conjuntos $U_{T}\left(a_{1}, \ldots, a_{n}\right), a_{i} \in A$, espectral. Além disso, $\operatorname{sper}_{T}(A) \subseteq \operatorname{sper}(A)$ é subespaço fechado.

iii) Seja $p$ ideal primo $A$. São equivalentes: 
a) $p$ é real.

b) O hipercorpo $K_{A}(p)$ é semi-real.

c) $p$ é suporte de alguma ordem.

Demonstração. As provas estão nas proposições 5.1, 6.1 e no Corolário 5.3 de [Mar10]. forma

Seja $(A, T)$ p-multi-anel. A topologia construtível em $\operatorname{spec}_{T}(A)$ é gerada pelos conjuntos da

$$
U_{T}\left(a_{1}, \ldots, a_{n}\right) \cap Z_{T}\left(b_{1}, \ldots, b_{k}\right)
$$

com $a_{1}, \ldots, a_{n}, b_{1}, \ldots, b_{k} \in A$ e é denotada por $\left(\operatorname{sper}_{T}(A)\right)_{\text {const }}$. Este espaço topológico é Booleano (Teorema 1.3.14 de [DST]).

No caso em que $A=R$ é um semigrupo real e $T=\sum R^{2}=R^{2}=\operatorname{Id}(R)^{5}$, usamos a notação $X_{R}$ para $\operatorname{sper}(R)$ para diferenciar esse caso (notação originalmente usada em [DP1], [DP2]). Note que a Definição 2.23 e a Definição 2.9 são compatíveis.

Seja $(A, T)$ p-multi-anel e considere o mapa natural $\pi: A \rightarrow 3^{\operatorname{sper}_{T}(A)}$ dado por $\pi(a)(\sigma)=\sigma(a)$. Este mapa preserva produto e as constantes $0,1,-1$. Seja $Q_{T}(A):=\{\pi(a):$ para todo $a \in A\}$. Note que $Q_{T}(A)$ é fechado por produto em $3^{\operatorname{sper}_{T}(A)}$. Além disso, possui multi-soma dada por $\pi(a) \in \pi(b)+\pi(c)$ se, e somente se, existem $x, y, z \in A \operatorname{com} \pi(x)=\pi(a), \pi(y)=\pi(b), \pi(z)=\pi(c)$ e $x \in y+z$. Se $T=\sum A^{2}$, então $Q_{\sum A^{2}}(A)$ é denotado por $Q(A)$.

Teorema 2.26. Seja $(A, T)$ p-multi-anel.

i) $Q_{T}(A)$ é multi-anel e o mapa natural $\pi: A \rightarrow Q_{T}(A)$ é morfismo sobrejetor de multi-aneis.

ii) O mapa inclusão $Q_{T}(A) \rightarrow 3^{\text {sper }_{T}(A)}$ é imersão forte, isto é, dados a, b, $c \in A$, temos $\pi(a) \in$ $\pi(b)+\pi(c)$ se, e somente se, para todo $\sigma \in \operatorname{sper}_{T}(A)$ vale $\sigma(a) \in \sigma(b)+\sigma(c)$.

Demonstração. A demonstração pode ser encontrada em [Mar10].

Comentários 2.27. O morfismo $\pi: A \rightarrow Q_{T}(A)$ é por definição forte. Como é sobrejetor, segue pela Proposição 1.11 que é quociente na categoria dos multi-aneis.

Teorema 2.28. Seja A multi-anel semi-real. Então o mapa $\pi: A \rightarrow Q(A)$ é isomorfismo se, $e$ \{panana\} somente se, para todo $a, b \in A$

i) $a^{3}=a$.

ii) $a+b^{2} a=\{a\}$

iii) $a^{2}+b^{2}$ tem único elemento.

Demonstração. A demonstração pode ser encontrada em [Mar10].

Corolário 2.29. Seja $F$ hipercorpo semi-real. Então $\pi: F \rightarrow Q(F)$ é isomorfismo se, e somente se, para todo $a \in \dot{F}$

i) $a^{2}=1$.

ii) $1+1=\{1\}$.

\footnotetext{
${ }^{5}$ Ver comentário 2.12
} 
Demonstração. Se $\pi: F \rightarrow Q(F)$ é isomorfismo, pelo teorema anterior dado $a \in \dot{F}$ temos $a^{3}=a$ e $\operatorname{assim} a^{2}=a^{3} \cdot a^{-1}=a \cdot a^{-1}=1$ e $1+1=\{1\}$. Reciprocamente, se valem os itens $\left.\left.i\right), i i\right)$, temos $a^{3}=a$ para todo $a \in F$ e dado $x \in a+b^{2} a$, se $a=0$ ou $b=0$, temos $x=a$ e caso $a, b \neq 0$ vale $a^{2}=b^{2}=1$ e assim $x a \in a^{2}+b^{2} a^{2}=1+1=\{1\}$; logo $x=a$. Além disso, como para todo $a \in F$ temos $a^{2} \in\{0,1\}$, vale que $a^{2}+b^{2}=\{1\}$ ou $a^{2}+b^{2}=\{0\}$. Logo, pelo teorema anterior, $\pi$ é isomorfismo.

Mais adiante vamos ver que a simplificação dos axiomas no caso de hipercorpos tem como motivo mais profundo a representação de $Q(F)$ no Teorema 2.45, item iii). Além disso, será explorado até onde essa representação pode ser estendida para subcategorias maiores dos multi-aneis (Teorema 2.50 e Teorema 2.52).

Muitas vezes é útil pensar num semigrupo real como um multi-anel com alguns axiomas a mais.

Definição 2.30. Seja $A$ multi-anel.

i) $A$ é chamado de multi-anel real reduzido (RRM) se:

- $1 \neq 0$.

- $a^{3}=a$.

- $a+b^{2} a=\{a\}$.

- $a^{2}+b^{2}$ tem único elemento.

ii) $A$ é chamado de hipercorpo real reduzido (HRR) se:

- $1 \neq 0$;

- Para todo $a \neq 0, a^{2}=1$;

- $1+1=\{1\}$.

Comentários 2.31. • Em [Mar10], os hipercorpos reais reduzidos são chamados de multicorpos reais reduzidos. Optamos por uma nomenclatura mais atual haja visto que se todo elemento não-nulo é inversível nunm multi-anel ele é hiper-anel (Ver comentário logo acima de 1.2).

- Seja $F$ hipercorpo. Na definição acima, segue direto da definição $F$ é hipercorpo real reduzido se, e somente se, $F$ é multi-anel real reduzido.

- Se $(A, T)$ é um p-multi-anel, então pelo Teorema 2.26 o multi-anel $Q_{T}(A)$ é real reduzido.

- Seja $A$ multi-anel satisfazendo as condições do Teorema 2.28. Então dados $a, b \in A$ e $c \in$ $a^{2}+b^{2}$, tem-se $c^{2}=c$. De fato, como $a^{2} b^{2}+a^{2}=\left\{a^{2}\right\}$ e $a^{2} b^{2}+b^{2}=\left\{b^{2}\right\}, c^{2} \in\left(a^{2}+b^{2}\right)\left(a^{2}+b^{2}\right) \subseteq$ $a^{2}+a^{2} b^{2}+b^{2}+a^{2} b^{2}=a^{2}+b^{2}$ e assim $c^{2}=c$. Deste modo, $A$ é semi-real se, e somente se, $1 \neq 0$ (se $-1 \in \sum A^{2}$, então $-1=x^{2}$ para algum $x \in A$ e assim $0 \in 1+x^{2}=\{1\}$ ). Além disso, se $A$ é RRM1, então $\sum A^{2}=A^{2}=\operatorname{Id}(A)$, e $(A, \operatorname{Id}(A))$ é p-multi-anel.

- Se $(A, T)$ é p-multi-anel, então o morfismo natural $\pi: A \rightarrow Q_{T}(A)$ satisfaz $\pi(T) \subseteq \operatorname{Id}\left(Q_{T}(A)\right)$.

Fixado um semigrupo real $R$, podemos interpretá-lo como um multi-anel se considerarmos a representação transversal $D^{t}$ como a soma multi-valorada; com isso $R$, na verdade, é um multi-anel real reduzido. Reciprocamente, dado $A$ multi-anel real reduzido, podemos definir a seguinte relação ternária: $a \in D(b, c) \Leftrightarrow a \in a^{2} b+a^{2} c$. Então $A$ com tal relação é semigrupo real. Tais afirmações são sistematizadas no seguinte:

Teorema 2.32. Sejam A multi-anel real reduzido e $R R S$.

i) Considere a seguinte relação ternária em $A: a \in D(b, c) \Leftrightarrow a \in a^{2} b+a^{2} c$. Então $a \in D^{t}(b, c) \Leftrightarrow$ $a \in b+c$ e $(A, \cdot, D, 0,1,-1)$ é semigrupo real. 
ii) $\left(R, \cdot, D^{t}, 0,1,-1\right)$ é multi-anel real reduzido.

As associações acima determinam um isomorfismo de categorias.

Demonstração. A prova pode ser encontrada em [RRM1].

Comentário 2.33. Seja $f: R \rightarrow G$ morfismo entre semigrupos reais. Pela Proposição II.2.6 de [DP2] (p. 64), se $f$ é morfismo sobrejetor e quociente (Definição 1.9), então ker $(f)$ induz congruência (Definição II.2.1, p. 61, de [DP2]) em $R$ e $R / \operatorname{ker}(f) \cong G$.

Saindo brevemente do contexto reduzido, o Teorema 2.32 possui uma versão para grupos especiais. Ficará claro como a redução é capaz de simplificar os axiomas da versão em multi-aneis dos grupos especiais.

Definição 2.34. Seja $F$ hipercorpo. $F$ é dito hipercorpo especial se para todos $a, b, c, d \neq 0$ em $F$ vale:

- $a^{2}=1$.

- $1 \in 1+a$ e $1+a$ é fechado por multiplicação.

- Se existe $p \neq 0$ tal que

$$
\begin{aligned}
& a \in c+c p \\
& b \in p+a p \\
& d \in p+c p,
\end{aligned}
$$

então existe $l \neq 0$ satisfazendo

$$
\begin{aligned}
& a \in d+d l \\
& b \in l+a l \\
& c \in l+d l .
\end{aligned}
$$

Proposição 2.35. i) Seja $F$ hipercorpo especial. Dados $a, b, c, d \in \dot{F}$, considere a relação binária em $\dot{F}^{2}$ definida por

$$
\langle a, b\rangle \equiv_{\dot{F}}\langle c, d\rangle \Leftrightarrow a b=c d \text { e } a+b=c+d .
$$

Então $\left(\dot{F}, \cdot, 1, \equiv_{\dot{F}},-1\right)$ é grupo especial.

ii) Seja $G$ grupo especial. Então a soma multivalorada definida no Exemplo 2.7 faz de $G \cup\{0\}$ um hipercorpo especial.

iii) As associações acima determinam uma equivalência entre a categoria dos hipercorpos especiais com morfismos de multi-aneis e a categoria dos grupos especiais com seus morfismos usuais.

iv) Seja $F$ hipercorpo real reduzido. Então $F$ é hipercorpo especial e o grupo especial $\left(\dot{F}, \cdot, 1, \equiv_{\dot{F}}\right.$ $,-1)$ definido no item $i$ ) é reduzido.

Demonstração. A demonstração pode ser encontrada com todos os detalhes em [RRM1].

Comentário 2.36. A equivalência de categorias descrita no Teorema 2.32 faz corresponder os 0-RSGs -enquanto subcategoria plena dos semigrupos reais- com os hipercorpos reais reduzidos.

A seguir veremos que a associação $(A, T) \mapsto Q_{T}(A)$ define funtor da categoria pMulti para a categoria dos multi-aneis reais reduzidos. Além disso, vamos analisar algumas propriedades desse funtor como espectro primo e real, propriedade universal e sua representação como quociente de Marshall. 
Lema 2.37. Seja $A$ multi-anel real reduzido. Então todo ideal primo é real.

Demonstração. Seja $p \in \operatorname{spec}(A)$. Sejam $a_{1}, a_{2} \in A \operatorname{com}\left(a_{1}^{2}+a_{1}^{2}\right) \cap p \neq \emptyset$. Tomando $c \in\left(a_{1}^{2}+a_{2}^{2}\right) \cap p$, temos $c a_{1}^{2} \in a_{1}^{2}+a_{2}^{2} a_{1}^{2}=\left\{a_{1}^{2}\right\}$ e $c a_{2}^{2} \in a_{1}^{2} a_{2}^{2}+a_{2}^{2}=\left\{a_{2}^{2}\right\}$. Mas como $c \in p$, temos $a_{1}^{2}=c a_{1}^{2}, a_{2}^{2}=$ $c a_{2}^{2} \in p$ e, sendo $p$ ideal primo, temos $a_{1}, a_{2} \in p$. O caso $\left(a_{1}^{2}+\cdots+a_{n}^{2}\right) \cap p \neq \emptyset \Rightarrow a_{i} \in p$ segue por indução em $n$.

Definição 2.38. Seja $(A, T)$ p-multi-anel. Um ideal primo $p \in \operatorname{spec}(A)$ é chamado de $T$-convexo se dados $t_{1}, t_{2} \in T \operatorname{com}\left(t_{1}+t_{2}\right) \cap p \neq \emptyset$, então $t_{1}, t_{2} \in p$.

$\{$ tconv\}

Lema 2.39. Seja $(A, T)$ p-multi-anel. Dado $p \in \operatorname{spec}(A)$ ideal primo $T$-convexo, existe $\sigma \in \operatorname{sper}_{T}(A)$ tal que $\operatorname{supp}(\sigma)=p$.

Demonstração. Considere a pré-ordem $\bar{T}=\left\{\frac{\bar{t}}{\bar{s}^{2}} \in K_{A}(p): t \in T, s \notin p\right\}$ no hiper-anel $K_{A}(p)$. Note que $\bar{T}$ é própria pois se $-1 \in \bar{T}$, então existiriam $s, l \notin p$ e $t \in T$ tais que $\left(s^{2} l+t l\right) \cap p \neq \emptyset$. Logo $\left(s^{2} l^{2}+t l^{2}\right) \cap p \neq \emptyset$ e, como $p$ é $T$-convexo, seguiria que $s^{2} l^{2} \in p$, um absurdo. Assim, sendo $\bar{T} \subseteq$ $K_{A}(p)$ pré-ordem própria, pela Proposição 2.25 segue que existe $\bar{\sigma}: K_{A}(p) \rightarrow 3 \operatorname{com} \bar{\sigma}(\bar{T}) \subseteq\{0,1\}$.

Compondo $\bar{\sigma}$ com o morfismo natural $A \rightarrow K_{A}(p)$, obtemos o morfismo $\sigma$ desejado.

Proposição 2.40. Seja $(A, T)$ um p-multi-anel. Considere $\pi: A \rightarrow Q_{T}(A)$ o morfismo natural.

i) O mapa induzido $\operatorname{sper}\left(Q_{T}(A)\right) \rightarrow \operatorname{sper}_{T}(A)$ é homeomorfismo (com as respectivas topologias espectrais).

ii) O mapa induzido $\operatorname{spec}\left(Q_{T}(A)\right) \rightarrow\{p \in \operatorname{spec}(A): p$ é T-convexo $\} \subseteq \operatorname{spec}(A)$ é homeomorfismo com sua imagem.

Demonstração. $\quad$ i) Seja $\pi: A \rightarrow Q_{T}(A)$ o morfismo canônico e considere o mapa induzido

$$
\begin{aligned}
\tilde{\pi}: \operatorname{sper}\left(Q_{T}(A)\right) & \rightarrow \operatorname{sper}_{T}(A) \\
\sigma & \mapsto \sigma \circ \pi
\end{aligned}
$$

A injetividade de $\tilde{\pi}$ segue da sobrejetividade de $\pi$. Para a sobrejetividade de $\tilde{\pi}$ considere $\sigma^{\prime} \in \operatorname{sper}_{T}(A)$, note que dados $a, b \in A$ se $\pi(a)=\pi(b)$, então $\sigma^{\prime}(a)=\sigma^{\prime}(b)$. Defina então $\sigma: \operatorname{sper}(Q(A)) \rightarrow 3$ por $\sigma(\pi(a))=\sigma^{\prime}(a)$. É fácil ver que $\sigma(1)=1, \sigma(0)=0$ e que para todos $x, y \in Q_{T}(A)$ vale $\sigma(x y)=\sigma(x) \sigma(y)$. Suponha agora que $x, y, z \in A$ satisfaçam $\pi(x) \in$ $\pi(y)+\pi(z)$. Pelo Teorema 2.26, segue que $\sigma(x) \in \sigma(y)+\sigma(z)$. Logo $\sigma$ é morfismo de multi-anéis e portanto $\sigma \in \operatorname{sper}\left(Q_{T}(A)\right)$. Além disso, $\tilde{\pi}\left(\sigma^{\prime}\right)=\sigma$ e assim $\tilde{\pi}$ é sobrejetora. Para concluir que $\tilde{\pi}$ é homeomorfismo, basta notar que dado $\pi(a) \in Q_{T}(A)$, tem-se $\tilde{\pi}(U(\pi(a)))=U(a)$.

ii) Sejam $P \in \operatorname{spec}\left(Q_{T}(A)\right)$ e $p=\pi^{-1}(P)$. Vamos primeiro provar que $p$ é $T$-convexo. Sejam $t_{1}, t_{2} \in T \operatorname{com}\left(t_{1}+t_{2}\right) \cap p \neq \emptyset$. Logo $\left(\pi\left(t_{1}\right)+\pi\left(t_{2}\right)\right) \cap P \neq \emptyset$. Como $\pi\left(t_{i}\right)^{2}=\pi\left(t_{i}\right), i=1,2$, segue do Lema 2.37 que $\pi\left(t_{1}\right), \pi\left(t_{2}\right) \in P$ e portanto $t_{1}, t_{2} \in p$. Por outro lado, seja $p \in \operatorname{spec}(A)$ ideal primo $T$-convexo. Considere $P=\pi(p)$.

Afirmação. i) $P$ é ideal primo.

ii) $\pi^{-1}(P)=p$.

Demonstração. Pelo Lema 2.39, existe $\sigma \in \operatorname{sper}_{T}(A)$ tal que $\operatorname{supp}(\sigma)=p$.

i) Sejam $a, b \in p$ e $x \in A$. Então $\pi(x) \pi(a)=\pi(x a) \in P$ e se $\pi(x) \in \pi(a)+\pi(b)$, em particular $\sigma(x) \in \sigma(a)+\sigma(b)=\{0\}$ e portanto $x \in p$. Logo $P$ é ideal. Agora suponha que $\pi(x) \pi(y)=\pi(x y) \in P$. Então existe $c \in p$ tal que $\pi(x y)=\pi(c)$ e assim $\sigma(x y)=\sigma(c)=0$; $\operatorname{logo} \sigma(x)=0$ ou $\sigma(y)=0$, ou seja, $\pi(x) \in P$ ou $\pi(y) \in P$. 
ii) A inclusão $p \subseteq \pi^{-1}(P)$ é trivial. Seja agora $x \in \pi^{-1}(P)$. Logo existe $a \in p$ tal que $\pi(x)=\pi(a)$. Assim $\sigma(x)=\sigma(a)=0$ e $x \in p$.

Desta forma, pela afirmação acima

$$
\pi^{*}: \operatorname{spec}\left(Q_{T}(A)\right) \rightarrow\{p \in \operatorname{spec}(A): p \text { é T-conexo }\} \subseteq \operatorname{spec}(A)
$$

é sobrejetor. Por outro lado, pela sobrejetividade de $\pi, \pi^{*}$ também é injetor. Para concluirmos que $\pi^{*}$ é homeomorfismo com a imagem, basta notar que dado $a \in A$ vale $\pi^{*}(D(\pi(a)))=$ $D(a) \cap\{p \in \operatorname{spec}(A): p$ é ideal T-convexo $\}$.

Teorema 2.41. i) Seja $f:(A, T) \rightarrow(B, P)$ morfismo de p-multi-anéis. Então existe único morfismo $Q(f): Q_{T}(A) \rightarrow Q_{P}(B)$ tal que

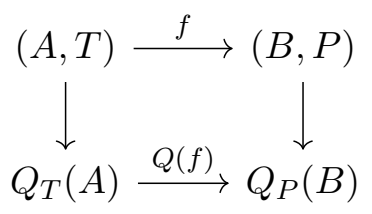

é diagrama comutativo. Além disso, Q é funtor da categoria dos p-multi-anéis para a categoria dos multi-anéis reais reduzidos.

ii) Seja $f:(A, T) \rightarrow\left(R, \sum R^{2}\right)=(R, I d(R))$ morfismo de p-multi-anéis com $R$ real reduzido. Então existe único $\bar{f}: Q_{T}(A) \rightarrow R$ tal que o diagrama

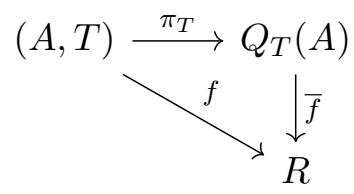

é comutativo. Em outras palavras, o funtor $Q:$ pMulti $\rightarrow \boldsymbol{R} \boldsymbol{R} \boldsymbol{M} 1$ é adjunto à esquerda do funtor de inclusão $i: \mathbf{R} \boldsymbol{R} \mathbf{M} \mathbf{1} \rightarrow \boldsymbol{p M u l t i}$ dado por $i(R)=\left(R, \sum R^{2}\right)$.

Demonstração. $\quad$ i) Sejam $\pi_{T}: A \rightarrow Q_{T}(A)$ e $\pi_{P}: B \rightarrow Q_{P}(B)$ as projeções canônicas. Considere o mapa $Q(f): Q_{T}(A) \rightarrow Q_{P}(B)$ dado por $Q(f)\left(\pi_{T}(a)\right)=\pi_{P}(f(a))$ (note que se $\pi_{T}(a)=\pi_{T}(b)$, então em particular para todo $\sigma \in \operatorname{sper}_{P}(B)$ temos $\sigma \circ f \in \operatorname{sper}_{T}(A)$ e $\sigma(f(a))=\sigma(f(b)) ; \log 0$ $\left.\pi_{P}(f(a))=\pi_{P}(f(b))\right)$. É claro que $Q(f)$ preserva $0,1,-1$. Sejam $a, b, x \in A$

- Note que $Q(f)\left(\pi_{T}(a) \pi_{T}(b)\right)=Q(f)\left(\pi_{T}(a b)\right)=\pi_{P}(f(a b))=\pi_{P}(f(a)) \pi_{P}(f(b))$ $=Q(f)\left(\pi_{T}(a)\right) Q(f)\left(\pi_{T}(b)\right)$.

- Se $\pi_{T}(x) \in \pi_{T}(a)+\pi_{T}(b)$, então, em particular, para todo $\sigma \in \operatorname{sper}_{P}(B)$ temos $\sigma \circ f \in$ $\operatorname{sper}_{T}(A)$ e assim $\sigma \circ f(x) \in \sigma \circ f(a)+\sigma \circ f(b)$. Portanto, pelo Teorema 2.26, $\pi_{P}(f(x)) \in$ $\pi_{P}(f(a))+\pi_{P}(f(b))$, isto é, $Q(f)\left(\pi_{T}(x)\right) \in Q(f)\left(\pi_{T}(a)\right)+Q(f)\left(\pi_{T}(b)\right)$,

Logo $Q(f): Q_{T}(A) \rightarrow Q_{P}(B)$ é morfismo de multianéis que faz o diagrama em questão comutar e, pela sobrejetividade de $\pi_{T}$, este é o único com tais propriedades. 
ii) Pelo Teorema 2.28, o morfismo natural $\pi_{R}: R \rightarrow Q(R)$ é isomorfimo. Defina $\bar{f}=\pi_{R}^{-1}$ 。 $Q(f): Q_{T}(A) \rightarrow R$. Então $f=\bar{f} \circ \pi_{T}$ e da sobrejetividade de $\pi_{T}$ segue a unicidade de $\bar{f}$.

Corolário 2.42. Seja $f:(A, T) \rightarrow(B, P)$ morfismo de p-multi-aneis. Considere o morfismo induzido $Q(f): Q_{T}(A) \rightarrow Q_{P}(B)$.

i) Se $f: A \rightarrow B$ for morfismo quociente sobrejetor, então $Q(f): Q_{T}(A) \rightarrow Q_{P}(B)$ é quociente sobrejetor em Multi. Em particular, se $f: A \rightarrow B$ for morfismo sobrejetor de aneis, então $Q(f): Q_{T}(A) \rightarrow Q_{P}(B)$ é quociente.

ii) Se $f: A \rightarrow B$ for morfismo forte e sobrejetor, então $Q(f): Q_{T}(A) \rightarrow Q_{P}(B)$ é morfismo forte e sobrejetor. Em particular, o morfismo $Q(A) \rightarrow Q_{T}(A)$ é morfismo forte.

Demonstração. Considere os morfismo $\pi_{T}: A \rightarrow Q_{T}(A)$ e $\pi_{P}: B \rightarrow Q_{P}(B)$. Pelo Teorema 2.41, segue que $Q(f) \circ \pi_{T}=\pi_{P} \circ f$. Assim, como $\pi_{P}$ é sobrejetor, se $f$ também for, o mesmo vale para $Q(f)$.

i) Para mostrar que $Q(f)$ é quociente, seja $g: Q_{T}(A) \rightarrow R, R$ multi-anel, morfismo satisfazendo $\operatorname{ker}(Q(f)) \subseteq \operatorname{ker}(g)$.

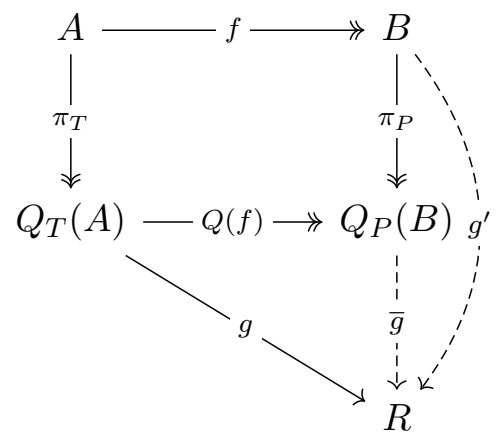

Então, pela Proposição 1.10, segue que

$$
\operatorname{ker}(f) \subseteq \operatorname{ker}\left(\pi_{P} \circ f\right)=\operatorname{ker}\left(Q(f) \circ \pi_{T}\right) \subseteq \operatorname{ker}\left(g \circ \pi_{T}\right) .
$$

Como $f$ é quociente, existe $g^{\prime}: B \rightarrow R$ tal que $g \circ \pi_{T}=g^{\prime} \circ f$. Assim, temos $\operatorname{ker}\left(\pi_{P}\right) \subseteq$ $\operatorname{ker}\left(g^{\prime}\right)$. De fato, dado $(x, y) \in \operatorname{ker}\left(\pi_{P}\right)$, pela sobrejetividade de $f$ existe $(a, b) \in A^{2}$ tal que $(f(a), f(b))=(x, y)$ e portanto

$$
\begin{aligned}
(a, b) \in \operatorname{ker}\left(\pi_{P} \circ f\right)=\operatorname{ker}\left(Q(f) \circ \pi_{T}\right) & \Rightarrow\left(\pi_{P}(a), \pi_{P}(b)\right) \in \operatorname{ker}(Q(f)) \subseteq \operatorname{ker}(g) \\
& \Rightarrow(a, b) \in \operatorname{ker}\left(g \circ \pi_{P}\right)=\operatorname{ker}\left(g^{\prime} \circ f\right) \\
& \Rightarrow(x, y)=(f(a), f(b)) \in \operatorname{ker}\left(g^{\prime}\right) .
\end{aligned}
$$

Deste modo, como o morfismo $\pi_{P}$ é quociente (Comentário 2.27), segue que existe $\bar{g}: Q_{P}(B) \rightarrow$ $R$ tal que $g^{\prime}=\bar{g} \circ \pi_{P}$. Assim, temos que

$$
\bar{g} \circ Q(f) \circ \pi_{T}=\bar{g} \circ \pi_{P} \circ f=g^{\prime} \circ f=g \circ \pi_{T} .
$$

Como $\pi_{T}$ é sobrejetora, segue que $g=\bar{g} \circ Q(f)$. Por outro lado, como $f$ é sobrejetor, segue que $Q(f)$ também é e portanto temos a unicidade de $\bar{g}$.

ii) Sejam $a, b, c \in A$ tal que $Q(f)\left(\pi_{T}(a)\right) \in Q(f)\left(\pi_{T}(b)\right)+Q(f)\left(\pi_{T}(c)\right)$. Então $\pi_{P}(f(a)) \in$ $\pi_{P}(f(b))+\pi_{P}(f(c))$. Como $f: A \rightarrow B$ é morfismo sobrejetor e $\pi_{P}: B \rightarrow Q_{P}(B)$ é morfismo forte (Comentário 2.27), existem $\left(f\left(a^{\prime}\right), f(a)\right),\left(f\left(b^{\prime}\right), f(b)\right),\left(f\left(c^{\prime}\right), f(c)\right) \in \operatorname{ker}\left(\pi_{P}\right)$ tais que $f\left(a^{\prime}\right) \in$ 
$f\left(b^{\prime}\right)+f\left(c^{\prime}\right)$. Por outro lado, como $f: A \rightarrow B$ é morfismo forte, existem $\left(a^{\prime \prime}, a^{\prime}\right),\left(b^{\prime \prime}, b^{\prime}\right),\left(c^{\prime \prime}, c^{\prime}\right) \in$ $\operatorname{ker}(f)$ tais que $a^{\prime \prime} \in b^{\prime \prime}+c^{\prime \prime}$. Logo $\pi_{T}\left(a^{\prime \prime}\right) \in \pi_{T}\left(b^{\prime \prime}\right)+\pi_{T}\left(c^{\prime \prime}\right)$ e $Q(f)\left(\pi_{T}\left(a^{\prime \prime}\right)\right)=\pi_{P}\left(f\left(a^{\prime \prime}\right)\right)=$ $\pi_{P}\left(f\left(a^{\prime}\right)\right)=\pi_{P}(f(a))=Q(f)\left(\pi_{T}(a)\right), Q(f)\left(\pi_{T}\left(b^{\prime \prime}\right)\right)=Q(f)\left(\pi_{T}(b)\right), Q(f)\left(\pi_{T}\left(c^{\prime \prime}\right)\right)=Q(f)\left(\pi_{T}(c)\right)$. Assim, $Q(f)$ é morfismo forte.

Proposição 2.43. Sejam $A$ multi-anel, $S \subseteq A$ multiplicativo e $I \subseteq A$ ideal próprio. Então

i) Seja $\pi: A \rightarrow A / I$ projeção canônica. Então $\pi$ induz homeomorfismo $\operatorname{sper}(A / I) \cong\{\sigma \in$ $\operatorname{sper}(A): \sigma(I)=0\}$.

ii) Seja $\rho: A \rightarrow S^{-1} A$ a projeção canônica. Então $\rho$ induz homeomorfismo $\operatorname{sper}\left(S^{-1} A\right) \cong\{\sigma \in$ $\left.\operatorname{sper}(A): \sigma\left(S^{2}\right)=1\right\}$.

iii) Seja $\pi: A \rightarrow A /{ }_{m} S$ projeção canônica. Então $\pi$ induz homeomorfismo $\operatorname{sper}(A / m S) \cong\{\sigma \in$ $\operatorname{sper}(A): \sigma(S)=1\}$.

Demonstração. Todos os itens seguem, respectivamente, pelas propriedades universais do quociente por ideal e localização e quociente de Marshall por conjunto multiplicativo que podem ser encontradas na seção 1.2.

Lema 2.44. Seja $f: A \rightarrow B$ morfismo sobrejetor de multi-aneis com $A, B$ RRMs. Se o mapa induzido $f^{*}: \operatorname{sper}(B) \rightarrow \operatorname{sper}(A)$ é sobrejetor, então $f$ é isomorfismo. Em particular, dado morfismo $g:(A, T) \rightarrow(B, S)$ de p-multi-aneis, o morfismo induzido $Q(g): Q_{T}(A) \rightarrow Q_{S}(B)$ é isomorfismo quando:

- $Q(g): Q_{T}(A) \rightarrow Q_{S}(B)$ é sobrejetor e $g^{*}: \operatorname{sper}_{S}(B) \rightarrow \operatorname{sper}_{T}(A)$ é sobrejetor.

Demonstração. Primeiro vamos provar que $f$ é injetor. Sejam $x, y \in A$ com $f(x)=f(y)$. Dado $\sigma \in \operatorname{sper}(A)$, pela sobrejetividade de $\operatorname{sper}(f)$ existe $\tau \in \operatorname{sper}(B)$ tal que $\sigma=\tau \circ f$. Logo $\sigma(x)=$ $\tau(f(x))=\tau(f(y))=\sigma(y)$. Como $\sigma \in \operatorname{sper}(A)$ foi arbitrário, temos $x=y$. Para que $f$ seja isomorfismo, resta mostrar que se $f(x) \in f(y)+f(z)$ para $x, y, z \in A$, então $x \in y+z$. Mas, usando a mesma argumentação para injetividade acima, temos que $\sigma(x) \in \sigma(y)+\sigma(z)$ para todo $\sigma \in \operatorname{sper}(A)$. Logo $x \in y+z$.

O que segue em particular no enunciado decorre do isomorfismo na Proposição 2.40.(i).

Teorema 2.45. Sejam $(A, T)$ p-multi-anel. Considere os morfismos canônicos de multi-aneis $\pi: A \rightarrow$ $Q_{T}(A)$ e $\rho: A \rightarrow A / m(1+T)$.

i) Seja $\tilde{T}=\rho(T)$. Então $(A / m(1+T), \tilde{T})$ é p-multi-anel e o morfismo $\rho$ induz isomorfismo $Q_{T}(A) \cong Q_{\tilde{T}}(A / m(1+T))$. Em particular, se $B$ é multi-anel semi-real, então $Q(B) \cong Q(B / m(1+$ $\left.\left.\sum B^{2}\right)\right)$.

ii) Sejam $B=(1+T)^{-1} A$ e $S=\left\{\frac{t}{x^{2}} \in B: t \in T\right.$ e $\left.x \in 1+T\right\}$. Então

- $(B, S)$ é p-anel.

- $1+S \subseteq B^{\times}$.

- O morfismo canônico $(A, T) \rightarrow(B, S)$ induz isomorfismo $Q_{T}(A) \cong Q_{S}(B)$.

iii) Suponha que A seja hipercorpo. Então $Q_{T}(A) \cong A / m(1+T) \cong A / m \dot{T}$.

Demonstração. i) Primeiro vamos provar que $\tilde{T} \subseteq A / m(1+T)$ é pré-ordem própria. Segue direto da definição que $\tilde{T} \cdot \tilde{T} \subseteq \tilde{T}$ e que, como $\rho$ é sobrejetor, $(A / m(1+T))^{2} \subseteq \tilde{T}$. Por outro lado, dados $a, b \in T$ e $x \in \rho(a)+\rho(b)$, novamente pela sobrejetividade de $\rho$, existe $c \in A$ tal que 
$x=\rho(c) \in \rho(a)+\rho(b)$. Assim, pela definição, existem $t, s, v \in T$ e $t^{\prime} \in 1+t, s^{\prime} \in 1+s, v^{\prime} \in 1+v$ tais que

$$
c t^{\prime} \in a s^{\prime}+b v^{\prime} \subseteq T .
$$

$\operatorname{Logo} \rho(c) \in \tilde{T}$. Por fim, se tivessemos $-1 \in \tilde{T}$, então existiriam $t \in T$ e $s, v \in 1+T$ tais que $-s=t v$. Então valeria $-t v \in 1+T$ e portanto $0 \in 1+T$, o que contraria o fato de $T$ ser própria.

Agora, aplicando o funtor $Q$ no morfismo de p-multi-aneis $\rho:(A, T) \rightarrow\left(A /{ }_{m}(1+T), \tilde{T}\right)$, temos o seguinte diagrama

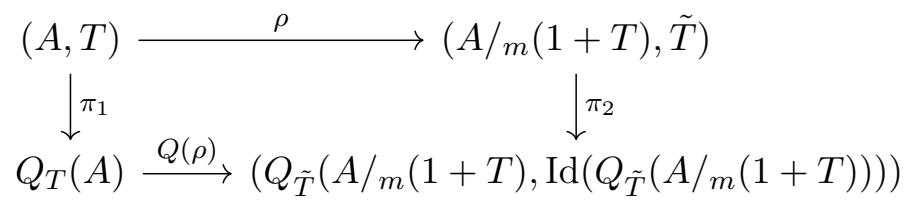

Como os morfismos $\rho: A \rightarrow A /{ }_{m}(1+T), \pi_{1}: A \rightarrow Q_{T}(A), \pi_{2}: A /{ }_{m}(1+T) \rightarrow Q_{\tilde{T}}(A / m(1+T))$ são sobrejetores, a comutação do diagrama acima permite concluir que $Q(\rho)$ é sobrejetor. Para concluir que $Q(\rho)$ é isomorfismo, pelo Lema 2.44 resta mostrar que $Q(\rho)^{*}: \operatorname{sper}\left(Q_{\tilde{T}}(A / m(1+\right.$ $T))) \rightarrow \operatorname{sper}\left(Q_{T}(A)\right)$ é sobrejetor. Note que dado $\sigma \in \operatorname{sper}\left(Q_{T}(A)\right)$, o morfismo $\sigma_{1}:=\sigma \circ$ $\pi_{1} \in \operatorname{sper}_{T}(A)$. Logo $\sigma_{1}(1+T)=\{1\}$ e portanto existe $\tilde{\sigma_{1}}: A / m(1+T) \rightarrow 3$ tal que $\sigma_{1}=$ $\tilde{\sigma_{1}} \circ \rho$. Deste modo, $\tilde{\sigma_{1}}(\tilde{T}) \subseteq\{0,1\}$ e portanto $\left.\tilde{\sigma_{1}} \in \operatorname{sper}_{\tilde{T}}(A / m(1+T))\right)$. Pela Proposição 2.40.(i), o mapa $\pi_{2}^{*}: \operatorname{sper}\left(Q_{\tilde{T}}(A / m(1+T))\right) \rightarrow \operatorname{sper}_{\tilde{T}}(A / m(1+T))$ é sobrejetor. Logo existe $\tilde{\sigma} \in \operatorname{sper}\left(Q_{\tilde{T}}(A / m(1+T))\right)$ tal que $\tilde{\sigma_{1}}=\tilde{\sigma} \circ \pi_{2}$. Como

$$
\pi_{1}^{*} \circ Q(\rho)^{*}=\left(Q(\rho) \circ \pi_{1}\right)^{*}=\left(\pi_{2} \circ \rho\right)^{*}=\rho^{*} \circ \pi_{2}^{*},
$$

temos $\pi_{1}^{*}\left(Q(\rho)^{*}(\tilde{\sigma})\right)=\sigma_{1}=\pi_{1}^{*}(\sigma)$. Como pela Proposição 2.40.(i) o mapa $\pi_{1}^{*}$ é injetor, segue que $Q(\rho)^{*}(\tilde{\sigma})=\sigma$.

ii) Primeiro, segue direto da definição que $S \subseteq B$ é pré-ordem. Se tivéssemos $-1 \in S$, então existiriam $t \in T$ e $x, a \in 1+T$ tais que $-x^{2} a=t a$ em $A$. Mas como $x^{2} a \in 1+T$ e $t a \in T$, teríamos $0 \in x^{2} a+t a \subseteq 1+T$, o que seria um absurdo. Logo $S \subseteq B$ é próprio.

Para provarmos que $1+S \subseteq B^{\times}$, sejam $\frac{t}{x^{2}} \in S(t \in T, x \in 1+T)$ e $\frac{a}{y} \in 1+\frac{t}{x^{2}}(a \in A$, $y \in 1+T)$. Então existe $z \in 1+T$ tal que

$$
a\left(x^{2} z\right) \in x^{2} y z+t y z \subseteq 1+T
$$

pois $x^{2} y z \in 1+T$ e $t y z \in T$. Logo existe $w \in 1+T$ tal que $a\left(x^{2} z\right)=w$ e assim $\frac{a}{y}=\frac{w}{y x^{2} z} \in B^{\times}$. Resta provar que o morfismo natural $\rho:(A, T) \rightarrow(B, S)$ induz isomorfismo $Q_{T}(A) \rightarrow Q_{S}(B)$. Para isso, vamos utilizar o Lema 2.44 e assim precisamos provar que $\rho^{*}: \operatorname{sper}_{S}(B) \rightarrow \operatorname{sper}_{T}(A)$ e $Q(\rho): G_{A, T} \rightarrow G_{B, S}$ são sobrejetores. Seja $\sigma \in \operatorname{sper}_{T}(A)$. Então $\sigma(1+T)=\{1\}$ e portanto existe $\bar{\sigma}: B \rightarrow 3$ tal que $\sigma=\bar{\sigma} \circ \rho$. Como $\bar{\sigma}(S) \subseteq\{0,1\}$, segue que $\bar{\sigma} \in \operatorname{sper}_{S}(B)$. $\operatorname{Logo} \rho^{*}(\bar{\sigma})=\sigma$ e portanto $\rho^{*}$ é sobrejetor. Por outro lado, seja $m=\frac{a}{x} \in B$. Note que toda ordem em $\operatorname{sper}_{S}(B)$ leva $\frac{x}{1}$ em 1 . Logo, pela definição de $G_{B, S}, \bar{m}=Q(\rho)(a)$. Portanto $Q(\rho)$ é sobrejetor.

iii) Considere os morfismos canônicos $\pi: A \rightarrow Q_{T}(A)$ e $\rho: A \rightarrow A / m(1+T)$. Primeiro note que

i) Como $(A, T)$ é p-hipercorpo, vale que $1 \neq 0$ em $A / m(1+T)$.

ii) Se $x \neq 0$ em $A$, então $x^{2}\left(1+x^{-2}\right)=\left(1+x^{2}\right)$ e portanto $x^{2}=1 \mathrm{em} A / m(1+A)$.

iii) Se $y \in 1+1 \mathrm{em} A / m(1+T)$, então existem $s_{1}, s_{2}, s_{3} \in 1+T$ tais que $y s_{1} \in s_{2}+s_{3}$. Como $1+T$ é fechado por soma, temos $y s_{1} \in 1+T$ e portanto $y=1 \mathrm{em} A / m(1+T)$. 
Então pelo Corolário 2.29 o hipercorpo $A / m(1+T)$ é real reduzido. Deste modo, a propriedade universal de $\pi: A \rightarrow Q_{T}(A)$ (Teorema 2.41.(ii)) garante a existência de único morfismo $\bar{\rho}: Q_{T}(A) \rightarrow A / m(1+T)$ tal que $\rho=\bar{\rho} \circ \pi$. Por outro lado, como $\pi(1+T)=\{1\}$, a propriedade universal de $\rho$ garante a existência de único morfismo $\bar{\pi}: A / m(1+T) \rightarrow Q_{T}(A)$ tal que $\pi=\bar{\pi} \circ \rho$. Assim, pelas unicidades das propriedades universais de $\pi$ e $\rho$, segue que $\bar{\pi} \circ \bar{\rho}=I d_{Q_{T}(A)}$ e $\bar{\rho} \circ \bar{\pi}=I d_{A / m(1+T)}$.

Por outro lado, como $1+T \subseteq \dot{T}$, temos um morfismo sobrejetor canônico $\alpha: A / m(1+T) \rightarrow$ $A /{ }_{m} \dot{T}$.

Afirmação. Sejam $x, y, z \in A$ tais que $x \in y+z \mathrm{em} A /{ }_{m} \dot{T}$, então $x \in y+z \mathrm{em} A / m(1+T)$.

Demonstração. Por hipótese, existem $t_{1}, t_{2}, t_{3} \in \dot{T}$ tais que $x t_{1} \in y t_{2}+z t_{3}(*)$. Como $t_{i}(1+$ $\left.t_{i}^{-1}\right)=1+t_{i}$, existe $s_{i} \in 1+t_{i}^{-1} \subseteq 1+T$ tal que $t_{i} s_{i} \in 1+T$. Multiplicando $(*)$ por $\left(s_{1} s_{2} s_{3}\right)$, temos

$$
x\left(t_{1} s_{1}\right)\left(s_{2} s_{3}\right) \in y\left(t_{2} s_{2}\right)\left(s_{1} s_{3}\right)+z\left(t_{3} s_{3}\right)\left(s_{1} s_{2}\right)
$$

Logo $x \in y+z$ em $A / m(1+T)$.

Pela afirmação acima temos que o morfismo $\alpha$ reflete soma. Para que $\alpha$ seja isomorfismo basta que seja injetora mas se $\alpha(x)=\alpha(y)$, então $x \in y+0 \mathrm{em} A /{ }_{m} \dot{T}$ e pela Afirmação acima $x \in y+0$ em $A / m(1+T)$, ou seja, $x=y$ e portanto $\alpha$ é injetora.

Deste modo $Q_{T}(A) \cong A / m(1+T) \cong A /{ }_{m} \dot{T}$.

Comentário 2.46. Seguindo a notação do Teorema 2.45.(ii), o p-multi-anel $(B, S)$ satisfaz $1+$ $S \subseteq B^{\times}$, que é chamada de propriedade da inversão limitada (em inglês é bounded inversion property ou simplesmente BIP). Assim, pelo Teorema 2.45.(ii), ao considerarmos propriedades de semigrupos reais da forma $Q_{T}(A)$ para p-aneis $(A, T)$, podemos supor sem perda de generalizadade que $(A, T)$ satisfaz BIP. Essa simplicação tem consequências interessantes para teoria dos aneis fielmente quadráticos (veja o capítulo 7 de [DM10]).

Teorema 2.47. Seja A multi-anel semi-real. Considere o morfismo canônico $\pi: A \rightarrow Q(A)$. Sejam $p \in \operatorname{spec}(Q(A))$ e $q=\pi^{-1}(p)$. Então o mapa canônico $A \rightarrow A_{q}$ induz isomorfismo $Q(A)_{p} \cong Q\left(A_{q}\right)$. Em particular, temos que

- $K_{Q(A)}(p) \cong Q\left(K_{A}(q)\right)$ e $K_{Q(A)}(p)$ é hipercorpo real reduzido.

- O morfismo canônico $A \rightarrow K_{A}(p)$ induz bijeção $\operatorname{sper}\left(K_{A}(p)\right) \cong\left\{\sigma \in \operatorname{sper}(A): \sigma^{-1}(0)=p\right\}$.

Demonstração. Pela Proposição 2.13, $Q(A)_{p}$ é RS e pela Proposição $\left.2.40 i i\right), q \subseteq A$ é ideal primo real. Logo $A_{q}$ é multi-anel semi-real (Proposição 2.25). Além disso, pelo Comentário 1.16, existe morfismo natural $\pi_{p}: A_{q} \rightarrow Q(A)_{p}$ que faz o diagrama

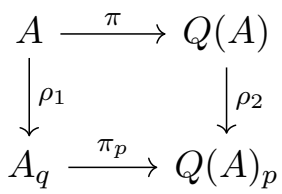

comutar.

Vamos mostrar que o morfismo $\pi_{p}$ satisfaz a mesma propriedade universal que $A_{q} \rightarrow Q\left(A_{q}\right)$. Primeiro note que, como $\rho_{1}, \rho_{2}$ e $\pi$ são sobrejetores, o mesmo vale para $\pi_{p}$. Seja $f: A_{q} \rightarrow R$ morfismo para multi-anel real reduzido. Então $f \circ \rho_{1}: A \rightarrow R$ é morfismo para RRM e pela propriedade universal de $\pi$ existe um único morfismo $f^{\prime}: Q(A) \rightarrow R$ tal que $f \circ \rho_{1}=f^{\prime} \circ \pi$. Por isso, $f^{\prime}(Q(A) \backslash p) \subseteq$ 
$R^{\times}$. Logo, pela Proposição $\left.2.13 v i\right)$, existe um único morfismo $\bar{f}: Q(A)_{p} \rightarrow R$ tal que $f^{\prime}=\bar{f} \circ \rho_{2}$. Assim, $f \circ \rho_{1}=f^{\prime} \circ \pi=\bar{f} \circ \rho_{2} \circ \pi=\bar{f} \circ \pi_{p} \circ \rho_{1}$. Como $\rho_{1}$ é sobrejetor, segue que $f=\bar{f} \circ \pi_{p}$. A unicidade de $\bar{f}$ segue da sobrejetividade de $\pi_{p}$.

Portanto, como $\pi_{p}$ satisfaz a mesma propriedade universal de $A_{q} \rightarrow Q\left(A_{q}\right)$, segue que $Q(A)_{p} \cong$ $Q\left(A_{q}\right)(*)$ naturalmente.

Seja $p Q(A)_{p} \in \operatorname{spec}\left(Q(A)_{p}\right)$ o único ideal maximal (Comentário 1.16) e $\bar{q} \in \operatorname{spec}\left(Q\left(A_{q}\right)\right)$ também único ideal maximal (a bijeção em 2.40 preserva a inclusão de ideias primos). Como o isomorfismo (*) leva $\bar{p}$ em $\bar{q}$, temos pela Proposição 1.20 i)

$$
K_{Q(A)}(p) \cong Q(A)_{p} / p Q(A)_{p} \cong Q\left(A_{q}\right) / \bar{q} \cong K_{Q\left(A_{q}\right)}(\bar{q}) .
$$

Por outro lado, considere $f: A_{q} \rightarrow K_{A}(q)$ o morfismo canônico sobrejetor. Então o morfismo induzido $Q(f): Q\left(A_{q}\right) \rightarrow Q\left(K_{A}(q)\right)$ satisfaz $Q(f)(\bar{q})=\{0\}$ e é sobrejetor. Assim, pela propriedade universal de quocientes por ideais (Proposição 1.13), temos que existe $\overline{Q(f)}: Q\left(A_{q}\right) / \bar{q} \rightarrow Q\left(K_{A}(q)\right.$ ) tal que

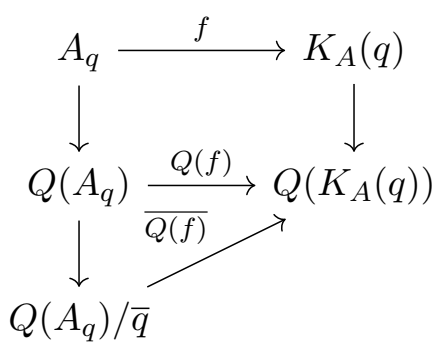

é diagrama comutativo. Por outro lado, pelo Teorema 2.26 e pelas proposições $2.43,2.40$ i), temos

$$
\begin{aligned}
\operatorname{sper}\left(Q\left(A_{q}\right) / \bar{q}\right) & \cong\left\{\sigma \in \operatorname{sper}\left(Q\left(A_{q}\right)\right): \sigma(\bar{q})=0\right\} \\
& \cong\left\{\sigma \in \operatorname{sper}\left(A_{q}\right): \sigma\left(q A_{q}\right)=0\right\} \\
& \cong\{\sigma \in \operatorname{sper}(A): \sigma(q)=0 \text { e } \sigma(A \backslash q) \in\{1,-1\}\} \\
& \cong \operatorname{sper}\left(K_{A}(q)\right) \\
& \cong \operatorname{sper}\left(Q\left(K_{A}(q)\right)\right) .
\end{aligned}
$$

Logo pelo Lema 2.44 temos que $\overline{Q(f)}: Q\left(A_{q}\right) / \bar{q} \rightarrow Q\left(K_{A}(q)\right)$ é isomorfismo e portanto vale $K_{Q(A)}(p) \cong Q\left(K_{A}(q)\right)$.

Para concluir, pela Proposição 2.43 temos

$$
\operatorname{sper}\left(K_{A}(p)\right) \cong\{\sigma \in \operatorname{sper}(A): \sigma(p)=\{0\} \text { e } \sigma(A \backslash p) \in\{1,-1\}\}=\left\{\sigma \in \operatorname{sper}(A): \sigma^{-1}(0)=p\right\} .
$$

Proposição 2.48. Seja $A$ multi-anel. São equivalentes:

i) $A$ é multi-anel real reduzido e para cada $a \in A$, existe $x \in A$ tal que $a x=0$ e $x \in 1-a^{2}$.

ii) $A$ é hiper-anel real reduzido.

iii) $A$ é hiper-anel von Neumann geométrico e para todo $a \in A$ vale $1+a^{2}=\{1\}$.

Demonstração. $i) \Rightarrow i i)$ : Seja $d \in b a+c a$. Precisamos provar que existe $f \in b+c$ tal que $d=f a$. Por hipótese, existe $x \in 1-a^{2}$ satisfazendo $a x=0$. Seja $l \in b+c$ e considere $f \in l x+d a$.

Pelo Teorema 2.28, é suficiente provar que para todo $\sigma \in \operatorname{sper}(A), \sigma(f) \in \sigma(b)+\sigma(c)$ e $\sigma(d)=$ $\sigma(f) \sigma(a)$. Fixe $\sigma \in \operatorname{sper}(A)$. Note que se $\sigma(a)=0$, então $\sigma(x) \in 1-\sigma(a)^{2}=\{1\}$ e se $\sigma(a) \neq 0$, $0=\sigma(x) \sigma(a)=\sigma(x) \sigma(a)^{2}=\sigma(x)$ e $\sigma(a)^{2}=1$. 
- $\sigma(f) \in \sigma(b)+\sigma(c)$.

Se $\sigma(a)=0$, então $\sigma(x)=1$ e $\sigma(f) \in \sigma(l x)+\sigma(d a)=\{\sigma(l)\} \subseteq \sigma(b)+\sigma(c)$. Se $\sigma(a) \neq 0$, então $\sigma(x)=0, \sigma(a)^{2}=1$ e assim $\sigma(f) \in \sigma(l x)+\sigma(d a)=\{\sigma(d a)\} \in \sigma(b) \sigma(a)^{2}+\sigma(c) \sigma(a)^{2}=$ $\sigma(b)+\sigma(c)$.

- $\sigma(d)=\sigma(f a)$.

Se $\sigma(a)=0$, então $\sigma(d) \in \sigma(b a)+\sigma(c a)=\{0\}$ e assim $\sigma(d)=0=\sigma(f a)$. Se $\sigma(a) \neq 0$, então $\sigma(x)=0, \sigma(a)^{2}=1$ e $\sigma(f a) \in \sigma(l x a)+\sigma\left(d a^{2}\right)=\{\sigma(d)\}$.

$i i) \Rightarrow$ iii): Como $A$ é multi-anel real reduzido, pelo Teorema $2.15 A$ é geométrico. Por outro lado, como vale $x=x^{3}$ para todo $x \in A$, pela Proposição 1.29 temos que $A$ é hiper-anel von Neumann.

iii $) \Rightarrow i$ ): Primeiro vamos provar que $A$ é multi-anel real reduzido.

- $\forall a \in A, a^{3}=a$.

Por hipótese, temos $1+\nabla(a)^{-2}=\{1\}$. Assim

$$
\left\{\nabla(a)^{2}\right\}=\nabla(a)^{2}\left(1+\nabla(a)^{-2}\right)=1+\nabla(a)^{2}=\{1\} .
$$

Assim $a^{3}=(i(a) \nabla(a))^{3}=i(a) \nabla(a)=a$.

- $a+a b^{2}=\{a\}$.

Como $A$ é hiper-anel, por hipótese $a+a b^{2}=a\left(1+b^{2}\right)=a \cdot\{1\}=\{a\}$.

- Se $x, y \in a^{2}+b^{2}$, então $x=y$.

Primeiro note que, como $a^{2}, b^{2} \in \operatorname{Id}(A)$, temos $i(a)=a^{2}$. Note também que

$$
\begin{aligned}
& x a^{2}, y a^{2} \in a^{2}+b^{2} a^{2}=\left\{a^{2}\right\} \\
& x a^{c}, y a^{c} \in 0+b^{2} a^{c}=\left\{b^{2} a^{c}\right\} .
\end{aligned}
$$

Assim $x a^{2}=y a^{2}, x a^{c}=y a^{c}$. Como $a^{2}+a^{c}=\{1\}$,

$$
x \in x\left(a^{2}+a^{c}\right)=x a^{2}+x a^{c}=y a^{2}+y a^{c}=y\left(a^{2}+a^{c}\right)=\{y\} .
$$

Para completar a prova, note que dado $a \in A$, o elemento $a^{c}$ satisfaz $a a^{c}=0$ e $a^{c} \in 1-i(a)=$ $1-a^{2}$.

Lema 2.49. Sejam $A$ hiper-anel von Neumann e $T \subseteq A$ uma pré-ordem própria. Então $1+T \subseteq A$ é subgrupo von Neumann.

Demonstração. Sejam $a \in A$ idempotente e $w \in a+a^{c}$. Primeiro note, por definição de $a^{c}, w$ não pode pertencer a nenhum ideal primo de $A$; assim, pelo Proposição $1.22 \mathrm{iv}$ ) segue $w$ é inversível (num hiper-anel, todo fracamente inversível é inversível). Observe que $w \in \sum A^{2} \subseteq T$ e $w^{-1}=w^{-2} w \in T$. Logo a expressão $\left(1+w^{-1}\right) w=1+w$ garante a existência de $y \in 1+T$ tal que $w y \in 1+T$.

Seja $x \in D_{1+T}\left(a, a^{c}\right)$. Então existem $s, u, v \in 1+T$ tais que $x s \in a u+a^{c} v$. Tomando $u^{\prime}, v^{\prime} \in T$ com $u \in 1+u^{\prime}, v \in 1+v^{\prime}$, temos

$$
x s \in a u+a^{c} v \subseteq\left(a+a u^{\prime}\right)+\left(a^{c}+a^{c} v^{\prime}\right)=\left(a+a^{c}\right)+\left(a u^{\prime}+a^{c} v^{\prime}\right) .
$$

Seja $w \in a+a^{c}$ tal que $x s \in w+\left(a u^{\prime}+a^{c} v^{\prime}\right)$. Tomando $y \in 1+T$ com $w y \in 1+T$, como $a, a^{c}$ são quadrados temos $x s y \in w y+a u^{\prime} y+a^{c} v^{\prime} y \in 1+T$. Assim $1+T \subseteq A$ é subgrupo von Neumann.

Teorema 2.50. Seja A hiper-anel von Neumann e $T \subseteq A$ uma pré-ordem própria. Então a projeção natural $\pi: A \rightarrow Q_{T}(A)$ induz isomorfismo $Q_{T}(A) \cong A / m(1+T)$. 
Demonstração. Seja $\rho: A \rightarrow A / m(1+T)$ a projeção natural. Pelo lema anterior, $1+T \subseteq A$ é subgrupo von Neumann e assim pela Proposição $1.37 A / m(1+T)$ é hiper-anel von Neumann geométrico. Por outro lado, dados $x, a \in A$ tais que $\rho(x) \in 1+\rho(a)^{2}$, existem $t_{1}, t_{2}, t_{3} \in 1+T$ com $x t_{1} \in t_{2}+a^{2} t_{3} \subseteq 1+T$ e assim $\rho(x)=1$. Deste modo, pela Proposição 2.48, o hiperanel $A / m(1+T)$ é hiper-anel real reduzido. Por outro lado, o morfismo $\rho: A \rightarrow A / m(1+T)$ satisfaz a mesma propriedade universal de $\pi: A \rightarrow Q_{T}(A)$ (Teorema 2.41). De fato, seja $f: A \rightarrow R$ morfismo de multi-aneis com $R$ multi-anel real reduzido tal que $f(T) \subseteq \sum R^{2}=R^{2}=I d(R)$. Então $f(1+T) \subseteq 1+R^{2}=\{1\}$ e pela Proposição 1.18 existe único morfismo $\bar{f}: A / m(1+T) \rightarrow R$ tal que $f=\bar{f} \circ \rho$. Portanto, como os mapas $\rho: A \rightarrow A / m(1+T)$ e $\pi: A \rightarrow Q_{T}(A)$ satisfazem a mesma propriedade universal, segue que $Q_{T}(A) \cong A / m(1+T)$ naturalmente.

O próximo resultado mostra como o funtor $Q$ preserva equivalência elementar quando restrito a subcategoria dos p-hiper-naies von Neumann. Ele é essencialmente um corolário do teorema precedente e foi inicialmente vislumbrado pelo Francisco Miraglia em conversa privada.

\{elemEqui\}

Teorema 2.51. Sejam $(A, T),(B, P)$ p-hiper-aneis von Neumann. Se $(A, T) \equiv(B, P)$ (elementarmente equivalentes) na linguagem $\mathcal{L}_{\text {multi }} \cup\{T\}$ de p-multi-anéis, então $Q_{T}(A) \equiv Q_{P}(B)$ na linguagem $\mathcal{L}_{\text {multi }}$ (ou equivalentemente na linguagem dos semigrupos reais).

Demonstração. A demonstração detalhada pode ser encontrada no Teorema 3.16 de [RM3]. A ideia é, usando o Teorema de Keisler-Shelah, provar que o funtor $Q$ preserva colimites indutivos e produtos arbitrários. É possível provar que o funtor $Q:$ pMult $\rightarrow$ RRM1 preserva colimites dirigidos mas em geral não preserva produtos arbitrários. Então o cerne da demonstração é usar o Teorema anterior para provar que o funtor que $Q$ restrito a subcategoria dos p-hiper-aneis von Neumann preserva produtos arbitrários.

Teorema 2.52. Seja $A$ um anel semi-real. Assuma que exista anel $B$ e subconjunto multiplicativo $S \subseteq B$ tal que $Q(A) \cong B / m S$. Então $Q(A) \cong V(A) / m\left(1+\sum V(A)^{2}\right)$ é representado pela envoltória von Neumann regular $V(A)$.

Demonstração. A demonstração está no Corolário 4.13 de [RM3]. Nela é usado o funtor envoltória von Neumann de multi-anel em geral e que ele comuta com o funtor $Q$.

\subsection{RS Booleano e RS von Neumann}

A presente seção se concentra em cobrir resultados básicos sobre os RSs Booleanos e von Neumann. Os Teoremas 2.54 e 2.58 fornecem, respectivamente, caracterizações de RSs Booleanos e von Neumann. Neste último é provado que um RS é von Neumann se, e somente se, é um hiper-anel. Além disso, a Proposição 2.57 fornece uma caracterização da representação transversal de um RS Booleano em função do RSG dos inversíveis, resultado que será útil no desenvolvimento da Capítulo 5 (B-pares). As principais referências são [DP2] e [MR].

Definição 2.53. Seja $R$ RS. $R$ é dito Booleano se $X_{R}$ é espaço topológico Booleano e $R$ é dito von Neumann se $\operatorname{spec}(R)$ é espaço topológico Booleano. ${ }^{6}$

Teorema 2.54. Seja R RS. São equivalentes:

i) Para todo $a \in R$, existe $u \in R^{\times}$tal que $a=u a^{2}$ e $u \in D^{t}(-1, a)$.

ii) Para todo $a \in I d(R)$, existe $u \in R^{\times}$tal que $a=u a$ e $u \in D^{t}(-1, a)$.

iii) $R$ é $R S$ Booleano.

\footnotetext{
${ }^{6}$ Os semigrupos reais correspondem, na linguagem dos AOS, aos espaços de ordem abstratos zero-dimensionais definidos na Seção 7.6 de [Mar7].
} 
iv) $R^{\times}$, com a representação induzida de $R$, é $R S G$ e o morfismo induzido $X_{R} \rightarrow X_{R^{\times}}$é homemomorfismo.

Demonstração. As equivalências entre $i$ ), iii) e $i v$ ) estão no Teorema 3.6 de [MR]. É imediato que $i) \Rightarrow i i)$. Vamos provar $i i) \Rightarrow i i i)$. Precisamos mostrar que para todo $a \in R,[[a \leq 0]] \subseteq X_{R}$ é aberto. Por $i i)$, existe $u \in R^{\times}$tal que $a^{2}=u a^{2}$ e $u \in D^{t}\left(-1, a^{2}\right)$. Assim $[[a=0]]=\left[\left[a^{2}=0\right]\right]=[[u=-1]]$ e portanto $[[a \leq 0]]=[[a=-1]] \cup\left[\left[a^{2}=0\right]\right] \subseteq X_{R}$ é aberto.

Seja $R$ RS Booleano. Pelo Teorema da Separação (2.10), o elemento $u$ no item $i$ ) do Teorema 2.54 é único. Dado $a \in R$, denota-se por $\nabla(a) \in R^{\times}$o único elemento satisfazendo $a=\nabla(a) a^{2}$ e $\nabla(a) \in D^{t}(-1, a)$ e $\Delta(a):=-\nabla(-a)$.

Proposição 2.55. Seja $R$ RS Booleano. Dados $a, b, c \in R$

i) $a=\Delta(a) a^{2}$ e $\Delta(a) \in D^{t}(1, a)$.

ii) Se $f: R \rightarrow G$ é morfismo de RS Booleanos, então dado $a \in R$ vale $f(\nabla(a))=\nabla(f(a))$ e $f(\Delta(a))=\Delta(f(a))$.

iii) $[[a=1]]=[[\nabla(a)=1]],[[a=-1]]=[[\Delta(a)=-1]]$ e $[[a=0]]=\left[\left[a^{2}=0\right]\right]=\left[\left[\nabla\left(a^{2}\right)=-1\right]\right]$. Em particular, dado $\sigma \in X_{R}$, se $\sigma(a) \neq 0$, então $\sigma(a)=\sigma(\nabla(a))=\sigma(\Delta(a))$.

iv) São equivalentes:
a) $a=b$.
b) $\nabla(a)=\nabla(b)$ e $\Delta(a)=\Delta(b)$.
c) $\nabla(a)=\nabla(b)$ e $\nabla\left(a^{2}\right)=\nabla\left(b^{2}\right)$.

Em particular, se $a, b \in I d(R)$, então $a=b$ se, e somente se, $\nabla(a)=\nabla(b)$.

v) Sejam $f, g: R \rightarrow G$ morfismo com $G$ RS qualquer. Se $\left.f\right|_{R^{\times}}=\left.g\right|_{R^{\times}}$, então $f=g$.

vi) $\nabla(a) \Delta(a)=\nabla\left(a^{2}\right)$.

vii) Se $a=x y \operatorname{com} x \in R^{\times}$e $y \in I d(R)$, então $y=a^{2}$ e $\Delta(a) \leq x \leq \nabla(a)$.

viii) Se $a \in D^{t}(b, c)$, então $\nabla(a) \in D^{t}(\nabla(b), \nabla(c))$.

Demonstração. i) Por definição, $-a=\nabla(-a) a^{2}$ e $\nabla(-a) \in D^{t}(-1,-a)$. Logo $a=\Delta(a) a^{2}$ e $\Delta(a) \in D^{t}(1, a)$.

ii) Imediato da unicidade das definições de $\nabla$ e $\Delta$.

iii) Primeiro vamos mostrar que $[[a=1]]=[[\nabla(a)=1]]$. Dado $\sigma \in X_{R}$, se $\sigma(a)=1$, então $\sigma(\nabla(a))=\sigma\left(\nabla(a) a^{2}\right)=\sigma(a)=1$. Reciprocamente, se $\sigma(\nabla(a))=1$, então $1=\sigma(\nabla(a)) \in$ $D^{t}(-1, \sigma(a)) ; \log \sigma(a)=1$. Agora note que $[[a=-1]]=[[-a=1]]=[[\nabla(-a)=1]]=$ $[[\Delta(a)=-1]]$ e $[[a=0]]=\left[\left[a^{2}=0\right]\right]=\left[\left[a^{2}=1\right]\right]^{c}=\left[\left[\nabla\left(a^{2}\right)=1\right]\right]^{c}=\left[\left[\nabla\left(a^{2}\right)=-1\right]\right]$.

iv) $a) \Rightarrow b$ ): Imediato.

$b) \Rightarrow c)$ : Seja $\sigma \in X_{R}$. Se $\sigma\left(\nabla\left(a^{2}\right)\right)=1$, então $\sigma(a) \in\{1,-1\}$. Se $\sigma(a)=1$, então $1=$ $\sigma(\nabla(a))=\sigma(\nabla(b))$; logo $\sigma(b)=1$ e portanto $\sigma\left(\nabla\left(a^{2}\right)\right)=\sigma\left(\nabla\left(b^{2}\right)\right)$. O caso $\sigma(a)=-1$ segue de forma análoga. Como $\sigma$ é arbitrário, segue que $\nabla\left(a^{2}\right)=\nabla\left(b^{2}\right)$.

$c) \Rightarrow a)$ : Vamos mostrar que $a^{2}=b^{2}$. Seja $\sigma \in X_{R}$. Se $\sigma(a)=0$, então $-1=\sigma\left(\nabla\left(a^{2}\right)\right)=$ $\sigma\left(\nabla\left(b^{2}\right)\right)$ e portanto $\sigma(b)=0$. Analogamente, se $\sigma(b)=0$, então $\sigma(a)=0$. Logo, pelo Teorema da Separação, $a^{2}=b^{2}$. Deste modo, $a=\nabla(a) a^{2}=\nabla(b) b^{2}=b$. 
v) Pelo Teorema 2.10, podemos supor sem perda de generalidade que $G=\mathbf{3}$. Assim, dado $x \in R$, temos $\nabla(f(x))=f(\nabla(x))=g(\nabla(x))=\nabla(g(x))$ e $\Delta(f(x))=f(\Delta(x))=g(\Delta(x))=\Delta(g(x))$. Pelo item anterior, segue que $f=g$.

vi) Seja $\sigma \in X_{R}$. Se $\sigma(a)=0$, então pelo item iii) segue que $\sigma(\nabla(a))=-1, \sigma(\Delta(a))=1 \mathrm{e}$ portanto $\sigma(\nabla(a) \Delta(a))=-1=\sigma\left(\nabla\left(a^{2}\right)\right)$. Se $\sigma(a) \neq 0$, então $\sigma(\nabla(a))=\sigma(\Delta(a))=\sigma(a)$ e $\sigma(\nabla(a) \Delta(a))=1=\sigma\left(\nabla\left(a^{2}\right)\right)$.

vii) Sejam $x \in R^{\times}$e $y \in \operatorname{Id}(R) \operatorname{com} a=x y$. Se $\sigma(a)=0$, então $\sigma(\Delta(a))=1 \leq \sigma(x) \leq-1=$ $\sigma(\nabla(a))$. Se $\sigma(a)=1$, então $\sigma(x)=1$ e $\sigma(\Delta(a))=1=\sigma(x)=\sigma(\nabla(a))$. Se $\sigma(a)=-1$, então $\sigma(x)=-1$ e $\sigma(\Delta(a))=-1=\sigma(x)=\sigma(\nabla(a))$. Como $\sigma \in X_{R}$ é arbitrário, temos $\Delta(a) \leq x \leq \nabla(a)$.

viii) Sejam $a, b, c \in R$ com $a \in D^{t}(b, c)$. Seja $\sigma \in X_{R}$. Se $\sigma(b)=0$, então $\sigma(a)=\sigma(c) ; \log \sigma(\nabla(a))=$ $\sigma(\nabla(c))$ e portanto $\sigma(\nabla(a)) \in D^{t}(\sigma(\nabla(b)), \sigma(\nabla(c)))(*)$. De maneira análoga concluímos $(*)$ se $\sigma(c)=0$. Caso $\sigma(a)=0$, então $\sigma(b)=-\sigma(c) ; \operatorname{logo} \sigma(\nabla(b))=-1$ ou $\sigma(\nabla(c))=-1$ e portanto vale $(*)$. No caso $\sigma(a), \sigma(b), \sigma(c) \neq 0$, temos $\sigma(\nabla(a))=\sigma(a), \sigma(\nabla(b))=\sigma(b), \sigma(\nabla(c))=\sigma(c)$ e portanto vale $(*)$. Como $\sigma \in X_{R}$ é arbitrário, temos $\nabla(a) \in D^{t}(\nabla(b), \nabla(c))$.

Para o próximo resultado, antecipamos parte de Definição 3.1. Com a noção de Witt-equivalência 7 podemos caracterizar a representação transversal de um RS Booleano.

Definição 2.56. Seja $R$ RS.

- Uma forma sobre $R$ é uma tupla $\left\langle a_{1}, \ldots, a_{n}\right\rangle$, onde $n \geq 1$.

- Dada forma $\varphi=\left\langle a_{1}, \ldots, a_{n}\right\rangle$ e $\sigma \in X_{R}$, definimos $\operatorname{sgn}_{\sigma}(\varphi)=\sigma\left(a_{1}\right)+\cdots+\sigma\left(a_{n}\right)$ (soma em $\mathbb{Z})$.

- Dadas duas formas $\varphi, \psi$, dizemos que elas são Witt-equivalentes quando para todo $\sigma \in X_{R}$ vale $\operatorname{sgn}_{\sigma}(\varphi)=\operatorname{sgn}_{\sigma}(\psi)$. (Notação $\varphi \cong_{R} \psi$ ).

Note que, se $\varphi=\left\langle a_{1}, \ldots, a_{n}\right\rangle$ é forma sobre $3=\{1,0,-1\}$, então $\operatorname{sgn}(\varphi)=a_{1}+\cdots+a_{n}$ (soma em $\mathbb{Z}$ ). Além disso, pelo Teorema da Separação (2.10), dados $x, y \in R$ vale $\left\langle x \cong_{R}\langle y\rangle\right.$ se, e somente se, $x=y$.

Proposição 2.57. Seja $R$ um RS Booleano. Dados $a, b, c \in R$, os seguintes itens são equivalentes:

i) $a \in D^{t}(b, c)$.

ii) $\nabla(a) \in D^{t}(\nabla(b), \nabla(c)) \mathrm{e}$

- $b\left\langle 1,-a^{2}\right\rangle \cong_{R}-c\left\langle 1,-a^{2}\right\rangle$.

- $a\left\langle 1,-b^{2}\right\rangle \cong_{R} c\left\langle 1,-b^{2}\right\rangle$.

- $a\left\langle 1,-c^{2}\right\rangle \cong_{R} b\left\langle 1,-c^{2}\right\rangle$.

Além disso, dados $x, y \in R$ e $a \in \operatorname{Id}(R)$, temos

$$
\begin{aligned}
x\langle 1,-a\rangle \cong_{R} y\langle 1,-a\rangle \Leftrightarrow & \nabla(x)\langle 1,-\nabla(a)\rangle \cong_{R} \nabla(y)\langle 1,-\nabla(a)\rangle \\
& \nabla(-x)\langle 1,-\nabla(a)\rangle \cong_{R} \nabla(-y)\langle 1,-\nabla(a)\rangle
\end{aligned}
$$

\footnotetext{
${ }^{7}$ Foi originalmente definida em [DP2]
} 
Demonstração. Pelo Teorema de Separação 2.10, é suficiente supor que $R=3$.

$i) \Rightarrow i i)$ : Suponha $a \in D_{3}^{t}(b, c)$. Pela Proposição 2.55 , temos $\nabla(a) \in D_{3}^{t}(\nabla(b), \nabla(c))$. Por outro lado, se $a=0$, então $b=-c$ e portanto $b\left\langle 1,-a^{2}\right\rangle \cong_{3}-c\left\langle 1,-a^{2}\right\rangle(*)$; se $a \neq 0$, então (*) vale trivialmente pois $\left\langle 1,-a^{2}\right\rangle \cong_{3}\langle 0\rangle$. Como $a \in D^{t}(b, c) \Leftrightarrow b \in D^{t}(a,-c) \Leftrightarrow c \in D^{t}(a,-b)$, as outras duas Witt-equivalências decorrem do caso que acabamos de provar por mera substituição.

$i i) \Rightarrow i)$ : Se $a, b, c \neq 0$, então $\nabla(a)=a, \nabla(b)=b, \nabla(c)=c$ e portanto vale $a \in D_{3}^{t}(b, c)$. Caso $a=0$, temos por $b\left\langle 1,-a^{2}\right\rangle \cong_{3}-c\left\langle 1,-a^{2}\right\rangle$ que $b=-c$ e portanto $a \in D_{3}^{t}(b, c)$. Os casos $b=0$ e $c=0$ seguem de maneira análoga e permitem concluir que $a \in D_{3}^{t}(b, c)$.

Por fim, para provar a última equivalência, pela definição de equivalência de formas também podemos supor $R=3$. $\Rightarrow$ : Se $a=0$, por hipótese temos $x=y$. Logo, como $\nabla(a)=-1$, temos

$$
\begin{aligned}
\operatorname{sgn}(\nabla(x)\langle 1,-\nabla(a)\rangle) & =2 \nabla(x)=2 \nabla(y)=\operatorname{sgn}(\nabla(y)\langle 1,-\nabla(a)\rangle) \\
\operatorname{sgn}(\nabla(-x)\langle 1,-\nabla(a)\rangle) & =2 \nabla(-x)=2 \nabla(-y)=\operatorname{sgn}(\nabla(-y)\langle 1,-\nabla(a)\rangle) .
\end{aligned}
$$

Se $a=1$, então $\nabla(a)=1 \mathrm{e}$

$$
\begin{aligned}
\operatorname{sgn}(\nabla(x)\langle 1,-\nabla(a)\rangle) & =0=\operatorname{sgn}(\nabla(y)\langle 1,-\nabla(a)\rangle) \\
\operatorname{sgn}(\nabla(-x)\langle 1,-\nabla(a)\rangle) & =0=\operatorname{sgn}(\nabla(-y)\langle 1,-\nabla(a)\rangle) .
\end{aligned}
$$

$\Leftarrow$ : Se $a=0$, então $\nabla(a)=-1$. Por hipótese, temos $2 \nabla(x)=2 \nabla(y)$ e $2 \nabla(-x)=2 \nabla(-y)$. Logo $\nabla(x)=\nabla(y)$ e $\Delta(x)=-\nabla(-x)=-\nabla(-y)=\Delta(y)$ e portanto pela Proposição $2.55 i v)$ temos $x=y$. Deste modo, $\operatorname{sgn}(x\langle 1,-a\rangle)=2 x=2 y=\operatorname{sgn}(y\langle 1,-a\rangle)$. Se $a=1$, então $\operatorname{sgn}(x\langle 1,-a\rangle)=0=$ $\operatorname{sgn}(y\langle 1,-a\rangle)$.

Teorema 2.58. Seja R RS. São equivalentes:

i) Todo ideal primo é maximal.

ii) $R$ é um $R S$ von Neumann.

iii) Para todo $a \in R$, existe $x \in D^{t}\left(1,-a^{2}\right)$ tal que ax $=0$.

iv) $R$ é um hiper-anel.

v) $\operatorname{Id}(R)$ é uma álgebra Booleana.

Além disso, se $R$ é von Neumann, então $R$ é Booleano e $R^{\times}$é $R S G$.

Demonstração. $i) \Rightarrow$ ii): Como $\operatorname{spec}(R)$ é espaço topológico espectral, é suficiente provar que $\operatorname{spec}(R)$ é Hausdorff. Sejam $p, q \in \operatorname{spec}(R)$ ideais primos diferentes. Considere o conjunto

$$
\mathcal{C}=\{D(a) \cap D(b): p \in D(a), q \in D(b), a, b \in R\} .
$$

Suponha que $\emptyset \notin \mathcal{C}$. Como $\mathcal{C}$ é fechado por interseç̧ão finita e seus elementos são fechados em $(\operatorname{spec}(R))_{\text {cons }}$, existe ideal primo $r \in \bigcap \mathcal{C}$. Assim $r \subseteq p$ e $r \subseteq q$, o que implica que $r$ não é maximal, um absurdo.

$i i) \Rightarrow$ iii): Seja $a \in R$. Como $\operatorname{spec}(R)$ é Booleano, $D(a)^{c}$ é aberto-fechado. Deste modo, existem $b_{1}, \ldots, b_{n} \in R$ tal que $D(a)^{c}=D\left(b_{1}\right) \cup \cdots \cup D\left(b_{n}\right)$. Tome então $x \in D^{t}\left(b_{1}^{2}, \cdots, b_{n}^{2}\right)$. Logo, pelos comentários em 2.12, segue que $D(x)=D\left(b_{1}^{2}\right) \cup \cdots \cup D\left(b_{n}^{2}\right)=D\left(b_{1}\right) \cup \cdots \cup D\left(b_{n}\right)=D(a)^{c}(*)$. Vamos mostrar que $x \in D^{t}\left(1,-a^{2}\right)$ e $a x=0$ comparando as assinaturas. Seja $\sigma \in X_{R}$. Se $\sigma(a)=0$, então por $(*)$ vale $\sigma(x)=1$ e portanto $\sigma(x) \in D_{3}^{t}\left(1,-\sigma(a)^{2}\right), \sigma(a x)=0(* *)$. Se $\sigma(a) \neq 0$, então por $(*)$ temos $\sigma(x)=0$ e assim vale $(* *)$. Logo, pelo Teorema da Separação $(2.10), x \in D^{t}\left(1,-a^{2}\right)$ e $a x=0$.

iii $) \Rightarrow i v$ ): É a implicação $i) \Rightarrow i i)$ da Proposição 2.48 . 
$i v) \Rightarrow v)$ : Seja $a \in I d(R)$. Como $0 \in D^{t}\left(a,-a^{2}\right)$, existe $b \in D^{t}(1,-a)$ com $a b=0$. Então $a \vee b=1$ e $a \wedge b=a b=0^{8}$; portanto $a$ admite complementar em $\operatorname{Id}(R)$. Logo $\operatorname{Id}(R)$ é álgebra Booleana.

$v) \Rightarrow i)$ : Sejam $p \in \operatorname{spec}(R)$ ideal primo e $I$ ideal com $p \subseteq I$. Suponha que exista $x \in I \backslash p$. Como $p$ é primo, podemos supor $x \in I d(R)$. Tome $x^{c} \in I d(R)$ tal que $x \vee x^{c}=1$ e $x \wedge x^{c}=0$, ou seja, $1 \in D^{t}\left(x, x^{c}\right)$ e $x x^{c}=0$. Como $0=x x^{c} \in p$ e $x \notin p, x^{c} \in p \subseteq I$. Logo $1 \in D^{t}\left(x, x^{c}\right) \subseteq I$. Deste modo, o ideal $p$ é maximal.

Por último, seja $R$ RS von Neumann. Vamos mostrar que $R$ é Booleano. Dado $a \in R$, seja $x \in D^{t}\left(1,-a^{2}\right)$ com $a x=0$. Considere $u \in D^{t}(a,-x)$. Comparando as assinaturas, vê-se que $u \in R^{\times}, u \in D^{t}(1,-a)$ e $a=u a^{2}$. Logo, pelo Teorema 2.54, temos que $R$ é Booleano.

Seja $R$ RS von Neumann. Dado $a \in R$, pelo Teorema 2.58 existe $x \in D^{t}\left(1,-a^{2}\right), x a=0$. Comparando as assinaturas, pelo Teorema da Separação (2.10) tal $x$ é único. Vamos denominá-lo por $a^{c}$. Note que $a^{c} \in \operatorname{Id}(R)$ e dado $\sigma \in X_{R}$, temos $\sigma(a)=0$ se, e somente se, $\sigma\left(a^{c}\right)=1$. Além disso, $a^{c}=\left(a^{2}\right)^{c}$.

\{idvn\}

Proposição 2.59. Seja $R$ RS von Neumann. Sejam $x \in R$ e $e \in \operatorname{Id}(R)$.

i) $(x e)^{c} e=x^{c} e$.

ii) $x^{c}(x e)^{c}=x^{c}$.

iii) $D^{t}\left(x,-x^{c}\right)=\{\nabla(x)\}$.

iv) $\left\langle x^{c}\right\rangle \cong_{R}\left\langle 1,-x^{2}\right\rangle$

Demonstração. i) Note que $(x e)^{c}=\left(x^{2} e\right)^{c}$. Usando a estrutura de reticulado de $\operatorname{Id}(R)$, temos $\left(x^{2} e\right)^{c} e=\left(x^{c} \wedge e^{c}\right) \vee e=x^{c} \vee e=x^{c} e$.

ii) É a lei de absorção de reticulados.

iii) Sejam $y \in D^{t}\left(x,-x^{c}\right)$ e $\sigma \in X_{R}$. Se $\sigma(x)=0$, então $\sigma(y) \in D^{t}(0,-1)=\{-1\}$. Se $\sigma(x) \neq 0$, então $\sigma(y) \in D^{t}(\sigma(x), 0)=\{\sigma(x)\}$. Em qualquer caso, $\sigma(y)=\sigma(\nabla(x))$ e portanto $y=\nabla(x)$.

iv) Seja $\sigma \in X_{R}$. Se $\sigma(x)=0$, então $\operatorname{sgn}_{\sigma}\left(\left\langle x^{c}\right\rangle\right)=\sigma\left(x^{c}\right)=1=\operatorname{sgn}_{\sigma}\left(\left\langle 1,-x^{2}\right\rangle\right)$. Se $\sigma(x) \neq 0$, então $\operatorname{sgn}_{\sigma}\left(\left\langle x^{c}\right\rangle\right)=\sigma\left(x^{c}\right)=0=\operatorname{sgn}_{\sigma}\left(\left\langle 1,-x^{2}\right\rangle\right)$.

\{sumVN2\}

Proposição 2.60. Seja $R$ RS von Neumann e $a, b, c \in R$. Então são equivalentes:

i) $a \in D^{t}(b, c)$.

ii) $\nabla(a) \in D^{t}(\nabla(b), \nabla(c))$ e $b a^{c}=-c a^{c}, a b^{c}=c b^{c}, a c^{c}=b c^{c}$.

Demonstração. Como todo RS von Neumann é Booleano, o resultado segue da Proposição 2.57 usando a Proposição $2.59 \mathrm{iv}$ ).

O próximo resultado descreve melhor quem são as fibras de RS von Neumann em termos do quociente dos RSGs formado pelos inversíveis. Para mais detalhes sobre quociente na categoria dos RSGs, confira o capítulo 2 de [DM1].

\{fiberq\}

Proposição 2.61. Seja $R$ RS von Neumann. Sejam $p \in \operatorname{spec}(R)$ e $\pi: R \rightarrow R_{p}=(R \backslash p)^{-1} R$. Então

i) $R_{p} \cong K_{R}(p)$ é hipercorpo real reduzido.

\footnotetext{
${ }^{8} \mathrm{~A}$ estrutura de reticulado de $\operatorname{Id}(R)$ foi definida em 2.12
} 
ii) $\pi_{R^{\times}}: R^{\times} \rightarrow R_{p}^{\times}$é RSG-morfismo sobrejetor e

$$
\Delta=\pi_{\mid R^{\times}}^{-1}(1)=\left\{a \in R^{\times} \text {: existe } i \notin p \text { tal que } a i=i\right\}
$$

é subgrupo saturado de RSG.

iii) O mapa induzido $R^{\times} / \Delta \cong R_{p}^{\times}$é isomorfismo de RSGs.

Demonstração. i) Como $R$ é von Neumann, pelo Teorema 2.58 o ideal primo $p \in \operatorname{spec}(R)$ é maximal e $R$ é hiper-anel. Então pela Proposição 1.13 o quociente $R / p$ é hipercorpo; logo $K_{R}(p) \cong R / p$. Por outro lado, dado $x \in p$, temos $x^{c} \notin p$ e $x x^{c}=0 ; \log \bar{x}=0$ em $R_{p}$. Deste modo, $p R_{p}$ é o ideal nulo e pela Proposição 1.20 temos que $R_{p}=R_{p} / p R_{p} \cong K_{R}(p)$ é hipercorpo real reduzido.

ii) Primeiro note que, sendo $R$ e $R_{p}$ RSs von Neumann, eles o são Booleanos. Assim, dado $x \in R_{p}^{\times}$, temos $\pi(\nabla(x))=\nabla(\pi(x))=\pi(x)$ e portanto o mapa $\pi_{R^{\times}}$é sobrejetor. Dados $a, b \in S$, sejam $i, j \notin p$ tais que $a i=i$ e $b j=j$. Assim, $a b(i j)=(i j)$ e $a b \in S$. Além disso, se $x \in R^{\times}$satisfaz $x \in D^{t}(1, a)$, então $x i \in D^{t}(i, a i)=D^{t}(i, i)=\{i\} ; \operatorname{logo} x \in S$. Portanto $S \subseteq R^{\times}$é subgrupo saturado.

iii) O mapa induzido $R^{\times} / \Delta \rightarrow R_{p}^{\times}(*)$ é um morfismo bijetor de RSGs. Sejam $a, b \in R^{\times}$e suponha que $\pi(a) \in D_{R_{p}^{\times}}(1, \pi(b))$. Assim, pela definição da representação em $R_{p}$, segue que existem $i \notin p$ tal que ai $\in D_{R}^{t}(i, b i)$. Assim o mapa (*) é isomorfismo.

Teorema 2.62. Seja $R R S$ von Neumann. Seja $\theta\left(v_{1}, \ldots, v_{n}\right)$ uma $\mathcal{L}_{R S}$-fórmula positiva e existencialmente fechada e $\bar{a} \in R^{n}$.

i) Seja $p \in \operatorname{spec}(R)$ com $R_{p}=\theta\left(\pi_{p}(\bar{a})\right)$. Então existe $x \in I d(R)$ onde $p \in D(x)$ e tal que para todo $q \in D(x), R_{q} \models \theta\left(\pi_{q}(\bar{a})\right)$.

ii) Sejam $x \in R$ e $\rho_{x}: R \rightarrow R_{x}$ a projeção canônica, onde $R_{x}=\left\{1, x, x^{2}\right\}^{-1} R$. Então são equivalentes:

- $R_{x}=\theta\left(\rho_{x}(\bar{a})\right)$.

- Para todo $q \in D(x), R_{q}=\theta\left(\pi_{q}(\bar{a})\right)$.

Demonstração. Pelo Teorema 2.15, $R$ é multi-anel geométrico, ou seja, o pré-feixe estrutural é feixe. Deste modo, o resultado segue de um fato bem mais geral de Teoria dos Modelos e pode ser encontrada na Proposição 3.2 de [DM9].

Definição 2.63. Seja $R$ RS. Uma forma sobre $R$ é simplesmente um elemento de $\bigcup_{n \geq 1} R^{n}$, geralmente denotada por $\varphi=\left\langle a_{1}, \ldots, a_{n}\right\rangle$. Além disso, dado $p \in \operatorname{spec}(R)$, considerando a projeção $\pi: R \rightarrow K_{R}(p)$, denotamos por $\varphi_{p}$ a forma $\left\langle\pi\left(a_{1}\right), \ldots, \pi\left(a_{n}\right)\right\rangle$ sem as entradas elementos nulas. Se todas forem nulas, $\varphi_{p}$ é simplesmente $\langle 0\rangle$.

Corolário 2.64. Seja $R$ RS von Neumann e $\varphi$ uma forma. Então $a \in D_{R}^{t}(\varphi)$ se, e somente se, $\{d t L o c a l\}$ $a_{p} \in D_{R_{p}}^{t}\left(\varphi_{p}\right)$ para todo $p \in \operatorname{spec}(R)$.

Demonstração. Basta notar que a fórmula $v \in D^{t}\left(v_{1}, \ldots, v_{n}\right)$ é positivamente existencialmente fechada.

Comentário 2.65. O corolário anterior vale para qualquer semigrupo real mas a prova é mais trabalhosa. O chamado princípio local-global fraco de Brocker está provado no Teorema 6.7.1 de [Mar7]. Note que, pelo Teorema 2.47, temos que $X_{K_{R}(p)} \cong\left\{\sigma \in X_{R}: \operatorname{supp}(\sigma)=0\right\}$ (comparar com o espaço residual definido na página 126 de [Mar7]). 


\subsection{Quocientes na categoria dos RS von Neumann}

Na seção anterior, apresentamos duas classes de semigrupos reais: os Booleanos e os von Neumann ([DP2], [MR]). Nesta seção, vamos explorar o quociente de Marshall em RSs von Neumann. Primeiro é introduzida a noção de subgrupo von Neumann, que da origem a um quociente na categoria dos RSs von Neumann (RSvN). Posteriormente é mostrado que todo quociente em RSvN vem de um subgrupo von Neumann. Como consequência, é mostrado no Corolário 2.71 que todo morfismo quociente em RSvN é transversalmente 2-regular. No Teorema 5.21 é classificado os quocientes na categoria dos RSs Booleanos utilizando a noção de B-pares (a ser introduzida). Até a finalização deste trabalho, não está claro para o autor se existe alguma noção de subgrupo para RSs Booleanos que permita classificar todos os quocientes em RSBool.

Sejam $R$ RS e $S \subseteq R$ subconjuntos saturado. Relembremos a Definição 1.35: dados $a, b \in R$, considere $D_{S}(a, b)=\left\{x \in R\right.$ : existem $s, t, u \in S$ tais que $\left.x s \in D^{t}(a t, b u)\right\}$.

Definição 2.66. Seja $R$ RS von Neumann. Um subconjunto $S \subseteq R$ é chamado de subgrupo saturado se $S$ é subgrupo von Neumann e para todo $a \in R$ e $x \in D_{S}\left(1, a^{2}\right)$ existe $s \in S$ tal que $x s \in S$.

Quando $R$ é um 0-RSG, a noção de subgrupo saturado coincide com àquela já existente para RSG. O que é proposto aqui é uma extensão desta noção para RSs von Neumann.

Lema 2.67. Seja $R$ RS von Neumann e $S \subseteq R$ subgrupo saturado.

i) Seja $\bar{S}=\{x \in R$ : existe $s \in S$ tal que $x s \in S\}$. Então $\bar{S}$ é subgrupo saturado cancelativo e $R / m \bar{S} \cong R / m S$.

ii) Para todo $a \in R, D_{S}(1, a)$ é conjunto multiplicativo.

Demonstração. i) Pelo Teorema 2.58, $R$ é hiper-anel von Neumann. Logo, pela Proposição 1.37, temos que $\bar{S}$ é subgrupo cancelativo von Neumann e $R / m \bar{S} \cong R /{ }_{m} S$. Resta provar que $\bar{S}$ é saturado. Seja $a \in R$ e tome $x \in D_{\bar{S}}\left(1, a^{2}\right)$. Então existem $u, v, w \in \bar{S}$ tais que $x u \in D^{t}\left(v, a^{2} w\right)$. Seja $s \in S$ tal que $u s, v s, w s \in S$. Então $x u s \in D^{t}\left(v s, a^{2} w s\right)$ implica $x \in D_{S}\left(1, a^{2}\right)$. Como $S$ é saturado, existe $s^{\prime} \in S$ tal que $x s^{\prime} \in S$. Logo $x \in \bar{S}$ e $\bar{S}$ é saturado.

ii) Seja $a \in R$ e tome $x, y \in D_{S}(1, a)$. Então existem $t_{1}, t_{2}, t_{3}, s_{1}, s_{2}, s_{3} \in S$ tais que

$$
\begin{aligned}
& x t_{1} \in D^{t}\left(t_{2}, a t_{3}\right) \\
& y s_{1} \in D^{t}\left(s_{2}, a s_{3}\right) .
\end{aligned}
$$

Logo $x y t_{1} s_{1} \in D^{t}\left(t_{2} s_{2}, a^{2} t_{3} s_{3}, a t_{3} s_{2}, a t_{2} s_{3}\right)$. Como $R$ é hiper-anel, existe $m \in D^{t}\left(t_{2} s_{2}, a^{2} t_{3} s_{3}\right)$ e $n \in D^{t}\left(t_{3} s_{2}, t_{2} s_{3}\right)=D^{t}\left(t_{3} s_{2}, 1^{2} t_{2} s_{3}\right)$ tais que $x y t_{1} s_{1} \in D^{t}(m, a n)$. Mas como $S$ é subgrupo saturado, existem $s, t \in S$ tais que $m s, n t \in S$. Assim

$$
x y t_{1} s_{1} s t \in D^{t}(m s t, \text { ants })
$$

e portanto $x y \in D_{S}(1, a)$.

\{satu\}

Teorema 2.68. Sejam $R R S$ von Neumann e $S \subseteq R$ subgrupo saturado. Então $R / m S$ é $R S$ von Neumann.

Demonstração. Como $R$ é hiper-anel von Neumann por 2.58, pela Proposição 1.37 segue que $G=$ $R /{ }_{m} S$ é hiper-anel von Neumann geométrico. Além disso, como $S$ é subgrupo saturado, $G$ satisfaz $1+a^{2}=\{1\}$ para todo $a \in G$. Assim, pela Proposição 2.48 segue que $G=R / m S$ é RS von Neumann. 
Teorema 2.69. Seja $f: R \rightarrow G$ morfismo de RSs von Neumann. Seja $S=f^{-1}(1)$. Então $S \subseteq R$ é subgrupo saturado e o mapa induzido $\bar{f}: R / m S \rightarrow G$ é injetor. Além disso, são equivalentes:

i) $f$ é morfismo forte e sobrejetor.

ii) $f$ é quociente em $\boldsymbol{R} \boldsymbol{S} \boldsymbol{v} \boldsymbol{N}$.

iii) $\bar{f}: R / m S \rightarrow G$ é isomorfismo.

Em particular, se $I \subseteq R$ é ideal próprio, então, considerando a projeção natural $\pi: R \rightarrow R / I$, temos $S=\pi^{-1}(1) \subseteq R$ é subgrupo saturado e $R /{ }_{m} S \cong R / I$.

Demonstração. Pelo Teorema 1.43, segue que $S$ é subgrupo von Neumann. Além disso, dado $a \in R$ e $x \in D_{S}\left(1, a^{2}\right)$, existem $s_{1}, s_{2}, s_{3} \in S$ tais que $x s_{1} \in D^{t}\left(s_{2}, a^{2} s_{3}\right)$ e portanto $f(x)=f\left(x s_{1}\right) \in$ $D^{t}\left(s_{2}, f(a)^{2} s_{3}\right)=D^{t}\left(1, f(a)^{2}\right)=\{1\}$. Logo $x \in S$ e portanto $S$ é subgrupo saturado. A injetividade de $\bar{f}$ segue do Teorema 1.43 .

$i) \Rightarrow i i)$ : Teorema 1.43 .

ii) $\Rightarrow$ iii): Vamos utilizar o apêndice sobre quocientes em categorias quase-concretas. Considere o funtor de esquecimento $U: \mathbf{R S v N} \rightarrow$ Sets da categoria dos RSs von Neumann para a categoria dos conjuntos. Note que $\operatorname{ker}(f)=\operatorname{ker}_{U}(f)$ e portanto a presente noção de morfismo quociente (Definição 1.9) coincide com a de $U$-quociente apresentada em A.4. Vamos mostrar que $\bar{f}$ é isomorfismo utilizando o Teorema $A$.9. Considere a subclasse $Q$ dos morfismos da forma $R \rightarrow R /{ }_{m} S$ onde $R$ é RS von Neumann e $S \subseteq R$ é subgrupo von Neumann. Como $\operatorname{ker}(f)=\operatorname{ker}(R \rightarrow R / m S)$, segue que $Q$ é quociente-injetiva (Definição A.6). Logo, pelo Teorema A.9, segue que $\bar{f}$ é isomorfismo.

iii) $\Rightarrow$ i): Teorema 1.43 .

Definição 2.70 (Definição III.3.1 de [DP2]). Seja $f: R \rightarrow G$ morfismo de RS. O morfismo $f$ é dito transversalmente 2-regular se para todos $a, b, c, d \in R$,

$$
\begin{aligned}
D_{G}^{t}(f(a), f(b)) \cap D_{G}^{t}(f(c), f(d)) \neq \emptyset \Rightarrow & \text { Existem }\left(a, a^{\prime}\right), \ldots,\left(d, d^{\prime}\right) \in \operatorname{ker}(f) \text { tais que } \\
& D_{R}^{t}\left(a^{\prime}, b^{\prime}\right) \cap D_{R}^{t}\left(c^{\prime}, d^{\prime}\right) \neq \emptyset .
\end{aligned}
$$

Corolário 2.71. Seja $f: R \rightarrow G$ morfismo de RSs von Neumann quociente em RSvN. Então $f$ é transversalmente 2-regular.

Demonstração. Seja $S=f^{-1}(1)$.Pelo Teorema 2.69, o morfismo induzido $\bar{f}: R / m S \rightarrow G$ é isomorfismo. Considere $a, b, c, d \in R$ tais que $D_{G}^{t}(f(a), f(b)) \cap D_{G}^{t}(f(c), f(d))$. Como $f$ é sobrejetor, existe $f(x) \in D_{G}^{t}(f(a), f(b)) \cap D_{G}^{t}(f(c), f(d))$. Sendo $\bar{f}$ isomorfismo, existem $s_{i}, t_{i} \in S, i=1,2,3$, tais que $x s_{1} \in D_{R}^{t}\left(a s_{2}, b s_{3}\right)$ e $x t_{1} \in D_{R}^{t}\left(c t_{2}, d t_{3}\right)$. Portanto

$$
x s_{1} t_{1} \in D_{R}^{t}\left(a s_{2} t_{1}, b s_{3} t_{1}\right) \cap D_{R}^{t}\left(c t_{2} s_{1}, d t_{3} s_{1}\right)
$$

e $\left(a, a s_{2} t_{1}\right),\left(b, b s_{3} t_{1}\right),\left(c, c t_{2} s_{1}\right),\left(d, d t_{3} s_{1}\right) \in \operatorname{ker}(f)$. Logo $f: R \rightarrow G$ é transversalmente 2-regular.

Comentários 2.72. Segue direto da definição que se $f$ é transversalmente 2-regular, então $f$ é morfismo forte. Por outro lado, a recíproca não vale em geral. No Exemplo III.3.5 de [DP2] é exibido um anel $A$ e um pré-ordem própria $T \subseteq A$ tal que $\iota: Q(A) \rightarrow Q_{T}(A)$ não é transversalmente 2-regular mas pelo Corolário 2.42 o morfismo $\iota$ é forte. 


\section{Capítulo 3}

\section{Anel de Witt}

Neste capítulo, os principais objetivos são produzir uma extensão do conceito de anel de Witt dos RSGs para os RSs e dar os primeiros passos na análise desse anel, investigando como as noções de isometria $(\equiv)$ e Witt-equivalência $(\cong)^{1}$ de formas interagem para a produção das propriedades do anel. Também vamos destacar o bom comportamento da isometria de formas para RSs von Neumann, fato que será útil para analisar a estrutura de qualquer anel de Witt no próximo capítulo.

$\mathrm{Na}$ seção 1, vamos apresentar as relações básicas entre isometria e equivalência, válidas em qualquer RS. Na segunda seção, é introduzida a noção de qc-formas, uma extensão útil de forma de dimensão $k \geq 1$ no contexto dos RSs. Ainda é estabelecido em 3.10 que se $\varphi, \psi$ são duas formas sobre um RS $R$, então

$$
\varphi \cong \psi \Leftrightarrow \text { Existem formas } \theta, \theta^{\prime} \text { sobre } \operatorname{Id}(R) \text { tais que } \varphi \oplus \theta\langle 1,-1\rangle \equiv \psi \oplus \theta^{\prime}\langle 1,-1\rangle \text {. }
$$

A seção é finalizada com a apresentação das formas $\left\langle a^{2}, a\right\rangle, a \in R$, uma versão para RSs de formas de Pfister que será útil no decorrer do trabalho. Na seção 3, especificamos a discussão para RSs von Neumann, onde obtemos a representação de qualquer forma como soma de qc-formas ortogonais. Isso permite obter uma pp-fórmula que descreve a isometria de formas (Teorema 3.23) e a representação transversal $D^{t}$ em função da isometria (Teorema 3.37). Além disso, analisamos alguns casos onde toda forma admite uma parte anisotrópica. Finalizamos a seção com uma versão do Teorema da Subforma de Pfister para RSs von Neumann (Teorema 3.43).

Por último, na seção 4, é definido o anel de Witt de qualquer RS. Descrevemos seu espectro primo (Proposição 3.48) e espectro real juntamente com as assinaturas (Proposição 3.46). No Teorema 3.51 caracterizamos, via anel de Witt, os RSs Booleano e von Neumann. O capítulo é finalizado com a descrição do ideal fundamental $I(R)$ como aditivamente gerado pelas formas de Pfister de grau 1.

\subsection{Isometria e Witt-equivalência}

A motivação central da tese foi desenvolver uma noção de isometria que favorecesse um comportamento parecido com o que acontece nos RGSs -como indica a Proposição 3.4 e o Corolário 3.10. Esta seção é dedicada a explorar as propriedades básicas da isometria e suas relações com a Witt-equivalência.

Definição 3.1. Sejam $R$ um RS.

i) Seja $\varphi=\left\langle a_{1}, \ldots, a_{n}\right\rangle$ forma sobre $R$, i.e., $\varphi \in \bigcup_{n \in \mathbb{N}} R^{n}$.

\footnotetext{
${ }^{1} \mathrm{~A}$ Witt-equivalência já foi introduzida em [DP2] mas, até onde o autor e orientador conhecem, a noção de isometria para RS é nova.
} 
- Para cada $\sigma \in X_{R}$, definimos as seguintes assinaturas :

$$
\begin{aligned}
\operatorname{sgn}_{\sigma}(\varphi) & =\sigma\left(a_{1}\right)+\cdots+\sigma\left(a_{n}\right)(\text { soma em } \mathbb{Z}) \\
\operatorname{sgn}_{\sigma}^{+}(\varphi) & =\left|\left\{i: \sigma\left(a_{i}\right)=1\right\}\right| \\
\operatorname{sgn}_{\sigma}^{-}(\varphi) & =\left|\left\{i: \sigma\left(a_{i}\right)=-1\right\}\right|
\end{aligned}
$$

- Dimensão local de $\varphi$ : é a função $\operatorname{dim}(\varphi): p \in \operatorname{spec}(R) \mapsto \operatorname{dim}_{p}(\varphi) \in \mathbb{Z}$, dada por $\operatorname{dim}_{p}(\varphi)=$ $\left|\left\{i: a_{i} \notin p\right\}\right|$.

- Vamos também usar a função $\operatorname{dim}(\varphi)$ com domínio $X_{R}$ : dada $\sigma \in X_{R}$, definimos $\operatorname{dim}_{\sigma}(\varphi)=$ $\operatorname{dim}_{\operatorname{supp}(\sigma)}(\varphi)$

- Dadas formas $\varphi, \psi$, dizemos que $\operatorname{dim}(\varphi) \leq \operatorname{dim}(\psi)$ quando para todo $p \in \operatorname{spec}(R)$ vale $\operatorname{dim}_{p}(\varphi) \leq \operatorname{dim}_{p}(\psi)$

ii) Sejam formas $\varphi \in R^{n}$ e $\psi \in R^{m}$ formas sobre $R$ (possivelmente com $n \neq m$ ).

- $\varphi$ e $\psi$ são ditas Witt-equivalentes ou simplesmente equivalentes (notação: $\varphi \cong \psi$ ) se para todo $\sigma \in X_{R}, \operatorname{sgn}_{\sigma}(\varphi)=\operatorname{sgn}_{\sigma}(\psi)$.

- $\varphi$ e $\psi$ são ditas isométricas (notação: $\varphi \equiv \psi$ ) se para todo $\sigma \in X_{R}, \operatorname{sgn}_{\sigma}^{+}(\varphi)=\operatorname{sgn}_{\sigma}^{+}(\psi)$ e $\operatorname{sgn}_{\sigma}^{-}(\varphi)=\operatorname{sgn}_{\sigma}^{-}(\psi)$.

iii) Sejam $\varphi=\left\langle a_{1}, \ldots, a_{n}\right\rangle$ e $\psi=\left\langle b_{1}, \ldots, b_{k}\right\rangle$ duas formas sobre $R$. As seguintes operações sobre formas são definidas:

- Soma de formas: $\varphi \oplus \psi=\left\langle a_{1}, \ldots, a_{n}, b_{1}, \ldots, b_{k}\right\rangle$.

- Produto de formas: $\varphi \otimes \psi=\left\langle a_{1} b_{1}, \ldots, a_{1} b_{k}, a_{2} b_{1}, \ldots, a_{2} b_{k}, \ldots, a_{n} b_{k}\right\rangle$.

Dado $a \in R$, denotamos $\langle a\rangle \otimes \varphi$ por $a \varphi$. Além dissso, dado $n \geq 1$, denotamos a forma $\bigoplus_{1 \leq i \leq n} \varphi$ por $n \varphi$.

Exemplo 3.2. Sejam $\left(G, \equiv_{G},-1\right)$ RSG e considere o 0-RSG $R=G \cup\{0\}$ (Exemplo 2.7). Considere, respectivamente, as relações $\equiv \mathrm{e} \cong$ de isometria e Witt-equivalência para $R$ definidas acima. Dadas formas $\varphi=\left\langle a_{1}, \ldots, a_{n}\right\rangle, \psi=\left\langle b_{1}, \ldots, b_{n}\right\rangle$ com $a_{i}, b_{i} \in R^{\times}=G$, pelo Princípio local-global de Pfister vale

- $\varphi \equiv_{G} \psi \Leftrightarrow \varphi \equiv \psi$.

- Existem $n, k \geq 1 \operatorname{com} \varphi \otimes n\langle 1,-1\rangle \equiv_{G} \psi \otimes k\langle 1,-1\rangle \Leftrightarrow \varphi \cong \psi$.

Comentários 3.3. Sejam $R$ RS e $\varphi=\left\langle a_{1}, \ldots, a_{n}\right\rangle$.

- Para todo $\sigma \in X_{R}$, vale $\operatorname{dim}_{\sigma}(\varphi)=\operatorname{sgn}_{\sigma}\left(\left\langle a_{1}^{2}, \ldots, a_{n}^{2}\right\rangle\right)$.

- $\varphi \equiv\langle 0\rangle$ se, e somente se, para todo $p \in \operatorname{spec}(R)$ vale $\operatorname{dim}_{p}(\varphi)=0$. Assim, se $\varphi \equiv\langle 0\rangle$, então $a_{1}=\cdots=a_{n}=0$.

- Para todo $n, k \geq 1$, temos

$$
\begin{aligned}
& \text {. } n\langle 1,-1\rangle \cong\langle 0\rangle . \\
& \text {. } n\langle 0\rangle \equiv k\langle 0\rangle . \\
& \text {. } \varphi \oplus\langle 0\rangle \equiv \varphi .
\end{aligned}
$$

- Se $\varphi$ é uma forma sobre $\operatorname{Id}(R)$, então para todo $\sigma \in X_{R}, \operatorname{sgn}_{\sigma}(\varphi)=\operatorname{dim}_{\sigma}(\varphi)$. Reciprocamente, se $\varphi=\left\langle a_{1}, \ldots, a_{n}\right\rangle$ satisfaz $\operatorname{sgn}_{\sigma}(\varphi)=\operatorname{dim}_{\sigma}(\varphi)$ para todo $\sigma \in X_{R}$, então $\left\langle a_{1}^{2},-a_{1}\right\rangle \oplus \cdots \oplus$ $\left\langle a_{n}^{2},-a_{n}\right\rangle \cong 0$. Mas a forma $\left\langle a^{2},-a\right\rangle$ tem sempre assinatura positiva e portanto $\left\langle a_{i}^{2},-a_{i}\right\rangle \cong 0$ para todo $i=1, \ldots, n$. Logo para todo $\sigma \in X_{R}$ vale $\sigma\left(a_{i}\right)^{2}=\sigma\left(a_{i}\right)$. Deste modo, $a_{i}^{2}=a_{i} \in$ $\operatorname{Id}(R)$. 
- Pela Lema 2.37, o mapa supp: $X_{R} \rightarrow \operatorname{spec}(R)$ é sobrejetor. Assim, dada forma $\psi$, temos $\operatorname{dim}_{\sigma}(\varphi)=\operatorname{dim}_{\sigma}(\psi)$ para todo $\sigma \in X_{R}$ se, e somente se, $\operatorname{dim}_{p}(\varphi)=\operatorname{dim}_{p}(\psi)$ para todo $p \in \operatorname{spec}(R)$.

Proposição 3.4. Seja $R$ RS. Sejam $\varphi, \psi, \theta$ formas e $a, b, c, d \in R$. Então:

i) $\operatorname{Dados} \sigma \in X_{R}$ e $p \in \operatorname{spec}(R)$,
a) $\operatorname{sgn}_{\sigma}(\varphi \oplus \psi)=\operatorname{sgn}_{\sigma}(\varphi)+\operatorname{sgn}_{\sigma}(\psi)$
b) $\operatorname{sgn}_{\sigma}(\varphi \otimes \psi)=\operatorname{sgn}_{\sigma}(\varphi) \cdot \operatorname{sgn}_{\sigma}(\psi)$
c) $\operatorname{dim}_{p}(\varphi \oplus \psi)=\operatorname{dim}_{p}(\varphi)+\operatorname{dim}_{p}(\psi)$
d) $\operatorname{dim}_{p}(\varphi \otimes \psi)=\operatorname{dim}_{p}(\varphi) \cdot \operatorname{dim}_{p}(\psi)$
e) $\operatorname{dim}_{\sigma}(\varphi) \equiv_{2} \operatorname{sgn}_{\sigma}(\varphi)$. Em particular, se $\varphi \cong \psi, \operatorname{dim}_{p}(\varphi) \equiv \operatorname{dim}_{p}(\psi)(\bmod 2)$.
f) As funções $\operatorname{sgn}(\varphi), \operatorname{sgn}^{+}(\varphi), \operatorname{sgn}^{-}(\varphi):\left(X_{R}\right)_{\text {const }} \rightarrow \mathbb{Z}$ são contínuas.
g) $\operatorname{dim}(\varphi):(\operatorname{spec}(R))_{\text {const }} \rightarrow \mathbb{Z}$ é contínua.

ii) Escreva $\varphi=\left\langle a_{1}, \ldots, a_{n}\right\rangle, \psi=\left\langle b_{1}, \ldots, b_{k}\right\rangle$. Então $\varphi \equiv \psi \Leftrightarrow \varphi \cong \psi \mathrm{e} \operatorname{dim}(\varphi)=\operatorname{dim}(\psi) \Leftrightarrow \varphi \cong$ $\psi$ e $\left\langle a_{1}^{2}, \ldots, a_{n}^{2}\right\rangle \cong\left\langle b_{1}^{2}, \ldots, b_{k}^{2}\right\rangle$.

iii) $\varphi \cong \psi \Rightarrow \varphi \oplus \theta \cong \psi \oplus \theta$ e $\varphi \otimes \theta \cong \psi \otimes \theta$. (O mesmo vale para isometria no lugar de Witt-equivalência)

iv) As seguintes afirmações são equivalentes:
a) $\langle a, b\rangle \equiv\langle c, d\rangle$.
b) $\langle a, b\rangle \cong\langle c, d\rangle$ e $a b=c d$.
c) $D(a, b)=D(c, d)$ e $a b=c d$.
d) $D^{t}(a, b)=D^{t}(c, d)$ e $a b=c d$.
e) $D^{t}(a, b) \cap D^{t}(c, d) \neq \emptyset$ e $a b=c d$.

v) $a \in D^{t}(b, c) \Leftrightarrow\langle a, a b c\rangle \cong\langle b, c\rangle$.

vi) As seguintes afirmações são equivalentes:
a) $a \in D(b, c)$.
b) $a \in D^{t}\left(a^{2} b, a^{2} c\right)$.
c) $\langle a, a b c\rangle \cong\left\langle a^{2} b, a^{2} c\right\rangle$.
d) $D(a, a b c)=D\left(a^{2} b, a^{2} c\right)$.
e) $D^{t}(a, a b c)=D^{t}\left(a^{2} b, a^{2} c\right)$.
f) $D^{t}(a, a b c) \cap D^{t}\left(a^{2} b, a^{2} c\right) \neq \emptyset$.

Demonstração. i) Sejam $\varphi=\left\langle a_{1}, \ldots, a_{n}\right\rangle$ e $\psi=\left\langle b_{1}, \ldots, b_{k}\right\rangle$ formas, $\sigma \in X_{R}$ e $p \in \operatorname{spec}(R)$. Então a)

$$
\begin{aligned}
\operatorname{sgn}_{\sigma}(\varphi \oplus \psi) & =\operatorname{sgn}_{\sigma}\left(\left\langle a_{1}, \ldots, a_{n}, b_{1}, \ldots, b_{k}\right\rangle\right) \\
& =\sigma\left(a_{1}\right)+\cdots+\sigma\left(a_{n}\right)+\sigma\left(b_{1}\right)+\cdots+\sigma\left(b_{k}\right) \\
& =\operatorname{sgn}_{\sigma}(\varphi)+\operatorname{sgn}_{\sigma}(\psi) .
\end{aligned}
$$


b)

$$
\begin{aligned}
\operatorname{sgn}_{\sigma}(\varphi \otimes \psi) & =\operatorname{sgn}_{\sigma}\left(\left\langle a_{1} b_{1}, a_{1} b_{2}, \ldots, a_{2} b_{1}, \ldots, a_{n} b_{k}\right\rangle\right) \\
& =\sigma\left(a_{1} b_{1}\right)+\sigma\left(a_{1} b_{2}\right)+\cdots+\sigma\left(a_{2} b_{1}\right)+\cdots+\sigma\left(a_{n} b_{k}\right) \\
& =\sigma\left(a_{1}\right) \cdot\left[\sigma\left(b_{1}\right)+\cdots+\sigma\left(b_{k}\right)\right]+\cdots+\sigma\left(a_{n}\right) \cdot\left[\sigma\left(b_{1}\right)+\cdots+\sigma\left(b_{k}\right)\right] \\
& =\operatorname{sgn}_{\sigma}(\varphi) \cdot \operatorname{sgn}_{\sigma}(\psi) .
\end{aligned}
$$

c)

$$
\begin{aligned}
\operatorname{dim}_{p}(\varphi \oplus \psi) & =\mid\left(\left\{i: a_{i} \notin p\right\}|+|\left\{j: b_{j} \notin p\right\} \mid\right. \\
& =\operatorname{dim}_{p}(\varphi)+\operatorname{dim}_{p}(\psi)
\end{aligned}
$$

d)

$$
\begin{aligned}
\operatorname{dim}_{p}(\varphi \otimes \psi) & =\operatorname{dim}_{p}\left(\left\langle a_{1} b_{1}, a_{1} b_{2}, \ldots, a_{2} b_{1}, \ldots, a_{n} b_{k}\right\rangle\right) \\
& =\left|\left\{(i, j): a_{i} b_{j} \notin p\right\}\right| \\
& =\mid\left\{(i, j): a_{i} \notin p \text { e } b_{j} \notin p\right\} \mid \\
& =\operatorname{dim}_{p}(\varphi) \cdot \operatorname{dim}_{p}(\psi)
\end{aligned}
$$

e) Primeiro note que $\operatorname{dim}_{\sigma}(\varphi)=\operatorname{sgn}_{\sigma}^{+}(\varphi)+\operatorname{sgn}_{\sigma}^{-}(\varphi)$ e $\operatorname{sgn}_{\sigma}(\varphi)=\operatorname{sgn}_{\sigma}^{+}(\varphi)-\operatorname{sgn}_{\sigma}^{-}(\varphi) . \operatorname{Logo}$ $\operatorname{dim}_{\sigma}(\varphi) \equiv_{2} \operatorname{dim}_{\sigma}(\varphi)-2 \operatorname{sgn}_{\sigma}^{-}(\varphi)=\operatorname{sgn}_{\sigma}(\varphi)$.

f) Vamos mostrar que a função $\operatorname{sgn}(\varphi):\left(X_{R}\right)_{\text {const }} \rightarrow \mathbb{Z}$ é localmente constante. Escreva $\varphi=$ $\left\langle a_{1}, \ldots, a_{n}\right\rangle$ e tome $\sigma \in X_{R}$ com $\operatorname{sgn}_{\sigma}(\varphi)=k$. Considere o conjunto $I_{t}=\left\{i: \sigma\left(a_{i}\right)=t\right\}$ para $t=-1,0,1$. Considere também o aberto-fechado $U=\bigcap_{i \in I_{-1}} U\left(-a_{i}\right) \cap \bigcap_{i \in I_{1}} U\left(a_{i}\right) \cap$ $\bigcap_{i \in I_{0}} Z\left(a_{i}\right)$ na topologia construtível. Então para todo $\sigma^{\prime} \in U$, temos $\operatorname{sgn}_{\sigma^{\prime}}(\varphi)=\operatorname{sgn}_{\sigma}(\varphi)=$ $k$. A continuidade das funções $\operatorname{sgn}^{+}(\varphi), \operatorname{sgn}^{-}(\varphi):\left(X_{R}\right)_{\text {const }} \rightarrow \mathbb{Z}$ seguem de forma análoga.

g) Seja $\varphi=\left\langle a_{1}, \ldots, a_{n}\right\rangle$ e tome $p \in \operatorname{spec}(R)$. Sejam $I, J \subseteq\{1, \ldots, n\}$ tais que se $i \in I$, $a_{i} \in p$ e se $i \in J, a_{i} \notin p$. Assim, dado qualquer $q \in \bigcap_{i \in I} D\left(a_{i}\right)^{c} \cap \bigcap_{i \in J} D\left(a_{i}\right)$ temos $\operatorname{dim}_{q}(\varphi)=\operatorname{dim}_{p}(\varphi)$, provando que $\operatorname{dim}(\varphi)$ é localmente constante.

ii) Seja $\sigma \in X_{R}$. Note que

$$
\begin{aligned}
& \operatorname{sgn}_{\sigma}^{+}(\varphi)=\operatorname{sgn}_{\sigma}^{+}(\psi) \\
& \operatorname{sgn}_{\sigma}^{-}(\varphi)=\operatorname{sgn}_{\sigma}^{-}(\psi)
\end{aligned} \Leftrightarrow \begin{aligned}
& \operatorname{sgn}_{\sigma}^{+}(\varphi)-\operatorname{sgn}_{\sigma}^{-}(\varphi)=\operatorname{sgn}_{\sigma}^{+}(\psi)-\operatorname{sgn}_{\sigma}^{-}(\psi) \\
& \operatorname{sgn}_{\sigma}^{+}(\varphi)+\operatorname{sgn}_{\sigma}^{-}(\varphi)=\operatorname{sgn}_{\sigma}^{+}(\psi)+\operatorname{sgn}_{\sigma}^{-}(\psi) .
\end{aligned}
$$

Como $\operatorname{sgn}_{\sigma}(\varphi)=\operatorname{sgn}_{\sigma}^{+}(\varphi)-\operatorname{sgn}_{\sigma}^{-}(\varphi)$ e $\operatorname{dim}_{\sigma}(\varphi)=\operatorname{sgn}_{\sigma}^{+}(\varphi)+\operatorname{sgn}_{\sigma}^{-}(\varphi)$, temos $\varphi \equiv \psi \Leftrightarrow \varphi \cong \psi$ e $\operatorname{dim}(\varphi)=\operatorname{dim}(\psi)$. A outra equivalência segue do Comentário 3.3.

iii) Assuma que $\varphi \cong \psi$. Então, pelo item $i$ ), dado $\sigma \in X_{R}$

$$
\begin{gathered}
\operatorname{sgn}_{\sigma}(\varphi \oplus \theta)=\operatorname{sgn}_{\sigma}(\varphi)+\operatorname{sgn}_{\sigma}(\theta)=\operatorname{sgn}_{\sigma}(\psi)+\operatorname{sgn}_{\sigma}(\theta)=\operatorname{sgn}_{\sigma}(\psi \oplus \theta) \\
\operatorname{sgn}_{\sigma}(\varphi \otimes \theta)=\operatorname{sgn}_{\sigma}(\varphi) \cdot \operatorname{sgn}_{\sigma}(\theta)=\operatorname{sgn}_{\sigma}(\psi) \cdot \operatorname{sgn}_{\sigma}(\theta)=\operatorname{sgn}_{\sigma}(\psi \otimes \theta) .
\end{gathered}
$$

Então $\varphi \oplus \theta \cong \psi \oplus \theta$ e $\varphi \otimes \theta \cong \psi \otimes \theta$.

iv) Pela Proposição 2.11, é suficiente provar que $a) \Rightarrow b) \Rightarrow d) \Rightarrow a$ ).

$a) \Rightarrow b)$ : É suficiente provar que se $\langle a, b\rangle \equiv\langle c, d\rangle$ então $a b=c d$. Seja $\sigma \in X_{R}$. Vamo proceder em alguns casos. Se $\sigma(a)=0$, então pela isometria $\sigma(c)$ ou $\sigma(d)$ é zero e assim $\sigma(a b)=\sigma(c d)$. De maneira análoga, concluímos que $\sigma(a b)=\sigma(c d)$ sempre que $\sigma(a b c d)=0$. Suponha então que $\sigma(a), \sigma(b), \sigma(c), \sigma(d) \neq 0$. Se $\sigma(a)=\sigma(b)$, então pela isometria $\sigma(c)=\sigma(d)=\sigma(a)=\sigma(b)$ 
e assim $\sigma(a b)=1=\sigma(c d)$. Se $\sigma(a)=-\sigma(b)$, então $\sigma(c)=-\sigma(d)$ e assim $\sigma(a b)=-1=\sigma(c d)$. Portanto $a b=c d$.

$b) \Rightarrow d)$ : Precisamos mostrar que se $\langle a, b\rangle \cong\langle c, d\rangle$ e $a b=c d$, então $D^{t}(a, b)=D^{t}(c, d)$. Pela simetria da Witt-equivalência, basta provar que a hipótese implica $D^{t}(a, b) \subseteq D^{t}(c, d)$. Sejam $x \in D^{t}(a, b)$ e $\sigma \in X_{R}$. Se $\sigma(a)=0$, por $a b=c d$ podemos assumir que $\sigma(c)=0$. Então, pela hipótese, $\sigma(b)=\sigma(d)$; $\log \sigma \sigma(x)=\sigma(b)=\sigma(d) \in D_{3}^{t}(\sigma(c), \sigma(d))$. De maneira similar, podemos concluir que $\sigma(x) \in D_{3}^{t}(\sigma(c), \sigma(d))$ sempre que $\sigma(a b c d)=0$. Assuma que $\sigma(a), \sigma(b), \sigma(c), \sigma(d) \neq 0$. Se $\sigma(a)=\sigma(b)$, pela equivalência temos $\sigma(c)=\sigma(d)=\sigma(a)=\sigma(b)$. Então $\sigma(x)=\sigma(a)=\sigma(c) \in D_{3}^{t}(\sigma(c), \sigma(d))$. Se $\sigma(a)=-\sigma(b)$, pela equivalência $\sigma(c)=-\sigma(d)$ e então $\sigma(x) \in D_{3}^{t}(\sigma(c), \sigma(d))$. Portanto $x \in D^{t}(c, d)$.

$d) \Rightarrow a$ ): Sejam $x \in D^{t}(a, b)=D^{t}(c, d)$ e $\sigma \in X_{R}$. Se $\sigma(a)=0$, como $a b=c d$, podemos assumir $\sigma(c)=0$. Como $D^{t}(a, b)=D^{t}(c, d), \sigma(b)=\sigma(x)=\sigma(d)$ e assim $\sigma(a)+\sigma(b)=$ $\sigma(c)+\sigma(d), \operatorname{dim}_{\sigma}(\langle a, b\rangle)=\operatorname{dim}_{\sigma}(\langle c, d\rangle)(*)$. De maneira análoga podemos concluir que valem as duas afirmações de $(*)$ sempre que $\sigma(a b c d)=0$. Assuma que $\sigma(a), \sigma(b), \sigma(c), \sigma(d) \neq 0$. Então naturalmente vale $\operatorname{dim}_{\sigma}(\langle a, b\rangle)=2=\operatorname{dim}_{\sigma}(\langle c, d\rangle)$. Se $\sigma(a)=\sigma(b)$, então por $a b=c d$ temos $\sigma(c)=\sigma(d) ; \operatorname{logo} \sigma(a)+\sigma(b)=\sigma(c)+\sigma(d)$. Se $\sigma(a)=-\sigma(b)$, por $a b=c d$ temos $\sigma(c)=-\sigma(d)$ e assim $\sigma(a)+\sigma(b)=\sigma(c)+\sigma(d)$. Portanto $\langle a, b\rangle \cong\langle c, d\rangle$ e $\operatorname{dim}(\langle a, b\rangle)=\operatorname{dim}(\langle c, d\rangle)$. Pelo item ii), segue que $\langle a, b\rangle \equiv\langle c, d\rangle$.

$\mathrm{v}) \Rightarrow$ : Seja $\sigma \in X_{R}$. Se $\sigma(a)=0$, como $a \in D^{t}(b, c), \sigma(b)=-\sigma(c)$ e $\sigma(a)+\sigma(a b c)=0=\sigma(b)+\sigma(c)$. Se $\sigma(b)=0$, então $\sigma(a)=\sigma(c)$ e $\sigma(a)+\sigma(a b c)=\sigma(a)=\sigma(b)+\sigma(c)$. Argumento similar resolve o caso $\sigma(c)=$ e assim podemos assumir $\sigma(a), \sigma(b), \sigma(c) \neq 0$. Se $\sigma(b)=\sigma(c)$, então $\sigma(a)=\sigma(b)$ e $\sigma(a)+\sigma(a b c)=2 \sigma(a)=\sigma(b)+\sigma(c)$. Se $\sigma(b)=-\sigma(c), \sigma(a)+\sigma(a b c)=\sigma(a)-\sigma(a)=0=$ $\sigma(b)+\sigma(c)$. Deste modo $\langle a, a b c\rangle \cong\langle b, c\rangle$.

$\Leftarrow$ : Seja $\sigma \in X_{R}$. Se $\sigma(a)=0$, como $\langle a, a b c\rangle \cong\langle b, c\rangle$, então temos $\sigma(b)=-\sigma(c)$ e $\sigma(a) \in$ $D_{3}^{t}(\sigma(b), \sigma(c))$. Se $\sigma(b)=0$, então $\sigma(a)=\sigma(c)$ e $\sigma(a) \in D_{3}^{t}(\sigma(b), \sigma(c))$. Argumento semelhante resolve o caso $\sigma(c)=0$ e assim podemos assumir $\sigma(a), \sigma(b), \sigma(c) \neq 0$. Se $\sigma(b)=\sigma(c)$, então $\sigma(a)\left(1+\sigma(b)^{2}\right)=2 \sigma(b)$ e, como $\sigma(b)^{2}=1$, temos $\sigma(a)=\sigma(b)$. Então $\sigma(a) \in D_{3}^{t}(\sigma(b), \sigma(c))$. Se $\sigma(b)=-\sigma(c)$, então $\sigma(a) \in D_{3}^{t}(\sigma(b), \sigma(c))$ por definição. Logo $a \in D^{t}(b, c)$.

vi) A equivalência entre $a$ ) e $b$ ) segue da Proposição 2.6, item xix. O restante da equivalência é uma combinação de $i i)$ e $v$ ).

\subsection{Qc-formas}

Uma forma é qc-forma (qc de quadrado-constante) se existe $k \geq 1$ cuja dimensão local é sempre $k$ ou 0 e atinge $k$ em pelo menos um ideal primo. Essas formas são mais bem comportadas: é possível escrevê-las com exatas $k$-entradas (Corolário 3.9). Num 0-RSG, toda forma não-nula é uma qc-forma e a maneira de escrevê-la apenas com $k$ entradas é retirar todas as entradas nulas excedentes. Por outro lado, num RS geral, o processo é diferente. As qc-formas serão úteis sobretudo para descrever as formas gerais num RS von Neumann.

Definição 3.5. Seja $R$ RS. Sejam $\varphi$ e $\psi$ formas sobre $R$.

- As formas $\varphi$ e $\psi$ são ditas ortogonais quando $\varphi \otimes \psi \equiv\langle 0\rangle$.

- O suporte de uma forma $\varphi$ é $\operatorname{supp}(\varphi)=\left\{p \in \operatorname{spec}(R): \operatorname{dim}_{p}(\varphi) \neq 0\right\}$.

- Dado $n \geq 0$, definimos $\operatorname{Od}_{n}(\varphi)=\left\{p \in \operatorname{spec}(R): \operatorname{dim}_{p}(\varphi)=n\right\}$.

- Definimos a dimensão máxima de $\varphi$ como $\operatorname{mdim}(\varphi)=\max \left\{n \geq 0: \operatorname{Od}_{n}(\varphi) \neq \emptyset\right\}$.

- A forma $\varphi$ é dita qc-forma se existe $k \geq 1$ tal que $\operatorname{supp}(\varphi)=\operatorname{Od}_{k}(\varphi) \neq \emptyset$. Nesse caso, $\varphi$ também é chamada de $k$-forma. 
- Um conjunto ordenado de formas $\left(\varphi_{1}, \ldots, \varphi_{n}\right)$ é um sistema ortogonal se $\varphi_{i}$ é uma $d_{i}$-forma, $d_{1}<\cdots<d_{n}$ e para $i \neq j, \varphi_{i}$ é ortogonal a $\varphi_{j}$.

$\{3344\}$

Comentário 3.6. - Se $\varphi$ e $\psi$ são formas $\operatorname{com} \varphi \equiv \psi$, então $\operatorname{Od}_{n}(\varphi)=\operatorname{Od}_{n}(\psi)$ para todo $n \geq 0$.

- Para uma forma $\varphi$ temos $\operatorname{supp}(\varphi)=\bigsqcup_{n \geq 1} \operatorname{Od}_{n}(\varphi)$.

- Duas formas $\varphi, \psi$ são ortogonais se, e somente se, $\operatorname{dim}_{p}(\varphi \otimes \psi)=0$ para todo $p \in \operatorname{spec}(R)$.

- Dado $k \geq 1, \varphi$ é uma $k$-forma se para todo $p \in \operatorname{spec}(R)$ vale $\operatorname{dim}_{p}(\varphi) \in\{0, k\}$ e existe $p \in \operatorname{spec}(R)$ tal que $\operatorname{dim}_{p}(\varphi)=k$.

- Dado $k \geq 1, \varphi=\left\langle a_{1}, \ldots, a_{k}\right\rangle$ é $k$-forma se, e somente se, $a_{i}^{2}=a_{j}^{2} \neq 0$ para todo $1 \leq i, j \leq k$. De fato, suponha $\varphi k$-forma. Então existe $\tau \in X_{R}$ tal que $\tau\left(a_{i}\right) \neq 0$ e assim $a_{i}^{2} \neq 0, i=1, \ldots, k$. Seja $\sigma \in X_{R}$. Se $\sigma\left(a_{1}\right)=0$, então $\operatorname{dim}_{\sigma}(\varphi)<k$. Como $\varphi$ é $k$-forma, segue que $\operatorname{dim}_{\sigma}(\varphi)=0$ e portanto $\sigma\left(a_{i}^{2}\right)=0$ para todo $i=1, \ldots, k$. Se $\sigma\left(a_{1}\right) \neq 0$, então $\operatorname{dim}_{\sigma}(\varphi) \geq 1$. Como $\varphi$ é $k$-forma, temos $\operatorname{dim}_{\sigma}(\varphi)=k$ e portanto $\sigma\left(a_{i}^{2}\right)=1$ para todo $i=1, \ldots, k$. Como $\sigma \in X_{R}$ foi arbitrário, segue pelo Teorema da Separação que $a_{i}^{2}=a_{j}^{2} \neq 0$ para todos $1 \leq i, j \leq k$. A volta é imediata.

- Seja $R$ um 0-RSG. Então toda forma não-nula é uma $k$-forma para algum $k \geq 1$.

Proposição 3.7. Seja $R$ RS. Sejam $\varphi, \psi$ formas sobre $R$.

i) Duas formas $\varphi$ e $\psi$ são ortogonais se, e somente se, $\operatorname{supp}(\varphi) \cap \operatorname{supp}(\psi)=\emptyset$. Como consequência, fixado $k \geq 1$, uma soma de $k$-formas mutualmente ortogonais é uma $k$-forma.

ii) Se $\varphi \equiv \varphi_{1} \oplus \cdots \oplus \varphi_{k}$, então para $n \geq 0$

$$
\operatorname{Od}_{n}(\varphi)=\bigsqcup_{\substack{\left(i_{1}, \ldots, i_{k}\right) \in \mathbb{N}^{k} \\ i_{1}+\cdots+i_{k}=n}} \operatorname{Od}_{i_{1}}\left(\varphi_{1}\right) \cap \cdots \cap \operatorname{Od}_{i_{k}}\left(\varphi_{k}\right) .
$$

iii) Se $\varphi \equiv \varphi_{1} \otimes \cdots \otimes \varphi_{k}$, então para $n \geq 0$

$$
\operatorname{Od}_{n}(\varphi)=\bigsqcup_{\substack{\left(i_{1}, \ldots, i_{k}\right) \in \mathbb{N}^{k} \\ i_{1} \cdots i_{k}=n}} \operatorname{Od}_{i_{1}}\left(\varphi_{1}\right) \cap \cdots \cap \operatorname{Od}_{i_{k}}\left(\varphi_{k}\right) .
$$

iv) Se $\left(\varphi_{1}, \ldots, \varphi_{n}\right)$ é sistema ortogonal com $\varphi_{i}$ uma $d_{i}$-forma tal que $\varphi \equiv \varphi_{1} \oplus \cdots \oplus \varphi_{n}$, então para cada $i=1, \ldots, n$ :

$$
\operatorname{Od}_{d_{i}}(\varphi)=\operatorname{supp}\left(\varphi_{i}\right)
$$

Além disso, $\operatorname{Od}_{0}(\varphi)=\bigcap_{i=1}^{n} \operatorname{Odd}_{0}\left(\varphi_{i}\right)$ e $\operatorname{Od}_{k}(\varphi)=\emptyset$ para $k \notin\left\{d_{1}, \ldots, d_{n}\right\} \cup\{0\}$.

v) Se $\left(\varphi_{1}, \ldots, \varphi_{n}\right)$ e $\left(\psi_{1}, \ldots, \psi_{k}\right)$ são sistema ortogonais e $\varphi_{1} \oplus \cdots \oplus \varphi_{n} \equiv \psi_{1} \oplus \cdots \oplus \psi_{k}$, então $n=k$ e $\varphi_{i} \equiv \psi_{i}$ para $i=1, \ldots, n$.

Demonstração. i) $\Rightarrow: \operatorname{Seja} p \in \operatorname{supp}(\varphi)$. Como $\varphi \otimes \psi \equiv\langle 0\rangle$, temos $0=\operatorname{dim}_{p}(\varphi \otimes \psi)=\operatorname{dim}_{p}(\varphi)$. $\operatorname{dim}_{p}(\psi)$ e então $\operatorname{dim}_{p}(\psi)=0 . \operatorname{Logo} p \notin \operatorname{supp}(\psi)$.

$\Leftarrow$ : Pelo Comentário 3.6, precisamos provar que para todo $p \in \operatorname{spec}(R), \operatorname{dim}_{p}(\varphi \otimes \psi)=$ $\operatorname{dim}_{p}(\varphi) \operatorname{dim}_{p}(\psi)=0$. Dado $p \in \operatorname{spec}(R)$, assuma que $\operatorname{dim}_{p}(\varphi) \neq 0 . \operatorname{Assim} p \in \operatorname{supp}(\varphi)$ e portanto por hipótese $p \notin \operatorname{supp}(\psi)$. Deste modo $\operatorname{dim}_{p}(\psi)=0$ e $\operatorname{assim} \operatorname{dim}_{p}(\varphi \otimes \psi)=0$.

Assim se $\left\{\varphi_{i}\right\}_{i=1}^{n}$ é uma família de $k$-formas mutualmente ortogonais, considere a forma $\varphi=$ $\bigoplus_{1 \leq i \leq n} \varphi_{i}$. Dado $p \in \operatorname{spec}(R)$, se $p \notin \operatorname{Od}_{k}\left(\varphi_{i}\right)$ para todo $1 \leq i \leq n$, então $\operatorname{dim}_{p}(\varphi)=$ $\sum_{1 \leq i \leq n} \operatorname{dim}_{p}\left(\varphi_{i}\right)=0$. Se existe algum $i_{0}$ tal que $p \in \operatorname{Od}_{k}\left(\varphi_{i_{0}}\right)$, como $\varphi_{i_{0}}$ é ortogonal a $\varphi_{i}$ para todo $i \neq i_{0}$, então $\operatorname{dim}_{p}(\varphi)=\sum_{1 \leq i \leq n} \operatorname{dim}_{p}\left(\varphi_{i}\right)=\operatorname{dim}_{p}\left(\varphi_{i_{0}}\right)=k$. 
ii), iii) Basta observarmos que se $\varphi=\psi \oplus \theta$ e $\tau=\psi \otimes \theta$, dado $p \in \operatorname{spec}(R)$, então $\operatorname{dim}_{p}(\varphi)=$ $\operatorname{dim}_{p}(\psi)+\operatorname{dim}_{p}(\theta)$ e $\operatorname{dim}_{p}(\tau)=\operatorname{dim}_{p}(\psi) \cdot \operatorname{dim}_{p}(\theta)$.

iv) Seja $\varphi_{i}$ a $d_{i}$-forma. Como $\left(\varphi_{1}, \ldots, \varphi_{n}\right)$ é ortogonal, pelos itens $\left.\left.i\right), i i\right)$ temos

$$
\operatorname{Od}_{d_{i}}(\varphi)=\operatorname{Od}_{d_{i}}\left(\varphi_{i}\right) \cap \bigcap_{j=1, j \neq i}^{n} \operatorname{Od}_{0}\left(\varphi_{j}\right)=\operatorname{Od}_{d_{i}}\left(\varphi_{i}\right)=\operatorname{supp}\left(\varphi_{i}\right) .
$$

Ainda pelo item $i$ ), vemos que $\operatorname{Od}_{0}(\varphi)=\bigcap_{i=1}^{n} \operatorname{Od}_{0}\left(\varphi_{i}\right)$.

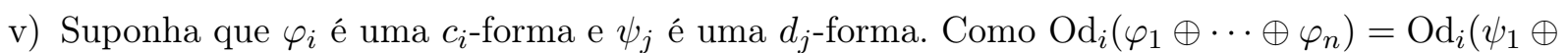
$\cdots \oplus \psi_{k}$ ), para todo $i \geq 1$, pelo item $i v$ )

$$
\begin{aligned}
& n=\left|\left\{i: i \geq 1 \mathrm{e} \operatorname{Od}_{i}\left(\varphi_{1} \oplus \cdots \oplus \varphi_{n}\right) \neq \emptyset\right\}\right| \\
& =\left|\left\{i: i \geq 1 \operatorname{e~Od}_{i}\left(\psi_{1} \oplus \cdots \oplus \psi_{k}\right) \neq \emptyset\right\}\right|=k .
\end{aligned}
$$

Agora vamos provar por indução que $c_{i}=d_{i}$ e $\varphi_{i} \equiv \psi_{i}$ para todo $i=1, \ldots, k$. Primeiro note que $\left.c_{1}=\min \left\{\operatorname{dim}_{p}\left(\varphi_{1} \oplus \cdots \oplus \varphi_{k}\right)\right): p \in \operatorname{spec}(R)\right\}=\min \left\{\operatorname{dim}_{p}\left(\psi_{1} \oplus \cdots \oplus \psi_{k}\right)\right): p \in$

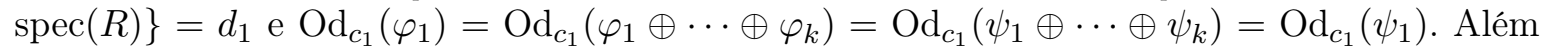
disso, dado $\sigma \in X_{R}$, se $\operatorname{supp}(\sigma) \in \operatorname{Od}_{c_{1}}\left(\varphi_{1}\right)$ temos

$$
\begin{aligned}
\operatorname{sgn}_{\sigma}^{ \pm}\left(\varphi_{1}\right) & =\operatorname{sgn}_{\sigma}^{ \pm}\left(\varphi_{1} \oplus \cdots \oplus \varphi_{n}\right) \\
& =\operatorname{sgn}_{\sigma}^{ \pm}\left(\psi_{1} \oplus \cdots \oplus \psi_{n}\right) \\
& =\operatorname{sgn}_{\sigma}^{ \pm}\left(\psi_{1}\right) .
\end{aligned}
$$

Se $\sigma \in X_{R}$ satisfaz $\operatorname{supp}(\sigma) \notin \operatorname{Od}_{c_{1}}\left(\varphi_{1}\right)$, então $\operatorname{sgn}_{\sigma}^{ \pm}\left(\varphi_{1}\right)=0=\operatorname{sgn}_{\sigma}^{ \pm}\left(\psi_{1}\right)$, o que prova o caso $i=1$. Isso implica que $\varphi_{2} \oplus \cdots \oplus \varphi_{k} \equiv \psi_{2} \oplus \cdots \oplus \psi_{k}$ e a demonstração do caso geral segue por indução.

O próximo resultado permite separar uma forma em suas partes com dimensão fixa. Esse ingrediente será básico para descrevermos as qc-formas (Corolário 3.9) e para caracterizar a equivalência de Witt em função da isometria (Corolário 3.10).

Notação. • Dados $1 \leq k \leq n$ inteiros, denotamos por

$$
S_{n, k}=\{f:\{1, \ldots, k\} \rightarrow\{1, \ldots, n\}: f \text { é estritamente crescente }\}
$$

o conjunto de todas as sub-uplas de $(1, \ldots, n)$ de comprimento $k$.

- Dada forma $\varphi=\left\langle a_{1}, \ldots, a_{n}\right\rangle, 1 \leq k \leq n$ e $f \in S_{n, k}$, definimos $\pi_{\varphi, f}=\Pi_{1 \leq i \leq k} a_{f_{i}}^{2}$ e $\Sigma_{\varphi, f}$ o único idempotente (quadrado) em $D^{t}\left(\left\langle a_{j}^{2}\right\rangle_{j \notin \operatorname{Im}(f)}\right)$ (se $k=n$, defina $\left.\Sigma_{\varphi, f}=0\right)$. Note que para $f \in S_{n, k}, \bigcap_{1 \leq i \leq k} D\left(a_{f_{i}}\right)=D\left(\pi_{\varphi, f}\right)$ e $\bigcap_{j \notin \operatorname{Im}(f)} D\left(a_{j}\right)^{c}=D\left(\Sigma_{\varphi, f}\right)^{c}$. Quando $\varphi$ é clara pelo contexto, vamos denotar $\pi_{\varphi, f}$ e $\Sigma_{\varphi, f}$ apenas por $\pi_{f}$ e $\Sigma_{f}$, respectivamente.

Teorema 3.8. Sejam $R R S$ e $\varphi=\left\langle a_{1}, \ldots, a_{n}\right\rangle$ uma forma. Então

i) Seja $e \in D^{t}\left(a_{1}^{2}, \ldots, a_{n}^{2}\right)$. Então $\operatorname{supp}(\varphi)=D(e)$.

ii) Para $1 \leq k \leq n$,

$$
\operatorname{Od}_{k}(\varphi)=\bigsqcup_{f \in S_{n, k}} D\left(\pi_{f}\right) \cap D\left(\Sigma_{f}\right)^{c}
$$

iii) $\varphi \cong \bigoplus_{1 \leq k \leq n} \bigoplus_{f \in S_{n, k}} \pi_{f}\left\langle 1,-\Sigma_{f}\right\rangle\left\langle a_{f_{1}}, \ldots, a_{f_{k}}\right\rangle$. 
iv) Seja $\operatorname{mdim}(\varphi)=k$. Então existe $b \in I d(R)$ tal que $\operatorname{Od}_{k}(\varphi)=D(b)$. Se $\varphi$ é não nula, existem $b_{1}, \ldots, b_{k} \in R$ com $b_{i}^{2}=b$ para todo $i=1, \ldots, k$ tais que

$$
b \varphi \equiv\left\langle b_{1}, \ldots, b_{k}\right\rangle .
$$

Demonstração. i) Note que, por 2.12, $\operatorname{supp}(\varphi)=D\left(a_{1}^{2}\right) \cup \cdots \cup D\left(a_{n}^{2}\right)=D(e)$

ii) Seja $1 \leq k \leq n$. Então

$$
\begin{aligned}
\operatorname{Od}_{k}(\varphi) & =\bigcup_{f \in S_{n, k}}\left[D\left(a_{f_{1}}\right) \cap \cdots \cap D\left(a_{f_{k}}\right) \cap \bigcap_{j \notin i m(f)} D\left(a_{j}\right)^{c}\right] \\
& =\bigsqcup_{f \in S_{n, k}}\left[D\left(a_{f_{1}}^{2}\right) \cap \cdots \cap D\left(a_{f_{k}}^{2}\right) \cap \bigcap_{j \notin i m(f)} D\left(a_{j}^{2}\right)^{c}\right] \\
& =\bigsqcup_{f \in S_{n, k}} D\left(\pi_{f}\right) \cap D\left(\Sigma_{f}\right)^{c} .
\end{aligned}
$$

iii) Pelo item anterior, podemos escrever $\operatorname{spec}(R)$ como

$$
\operatorname{spec}(R)=\bigsqcup_{0 \leq k \leq n} \operatorname{Od}_{k}(\varphi)=\operatorname{Od}_{0}(\varphi) \sqcup \bigsqcup_{1 \leq k \leq n} \bigsqcup_{f \in S_{n, k}} D\left(\pi_{f}\right) \cap D\left(\Sigma_{f}\right)^{c} .
$$

Seja $\psi=\bigoplus_{1 \leq k \leq n} \bigoplus_{f \in S_{n, k}} \pi_{f}\left\langle 1,-\Sigma_{f}\right\rangle\left\langle a_{f_{1}}, \ldots, a_{f_{k}}\right\rangle$. Tome $\sigma \in X_{R}$ e assuma que $p=\operatorname{supp}(\sigma) \in$ $D\left(\pi_{g}\right) \cap D\left(\Sigma_{g}\right)^{c}$ para algum $g \in S_{n, l}$ e $1 \leq l \leq n$. Note que $\sigma\left(\pi_{g}\right)=1$ e $\sigma\left(\Sigma_{g}\right)=0$. Como a união acima é disjunta, dado $1 \leq m \leq n$ e $h \in S_{n, m} \operatorname{com}(l, g) \neq(m, h)$, temos $p \notin D\left(\pi_{h}\right) \cap D\left(\Sigma_{h}\right)^{c}$ e assim $\operatorname{sgn}_{\sigma}\left(\pi_{h}\left\langle 1,-\Sigma_{h}\right\rangle\right)=0$. Logo

$$
\begin{aligned}
\operatorname{sgn}_{\sigma}(\psi) & =\sum_{1 \leq k \leq n} \sum_{f \in S_{n, k}} \operatorname{sgn}_{\sigma}\left(\pi_{f}\left\langle 1,-\Sigma_{f}\right\rangle\left\langle a_{f_{1}}, \ldots, a_{f_{k}}\right\rangle\right) \\
& =\operatorname{sgn}_{\sigma}\left(\pi_{g}\left\langle 1,-\Sigma_{g}\right\rangle\left\langle a_{g_{1}}, \ldots, a_{g_{k}}\right\rangle\right) \\
& =\sigma\left(a_{g_{1}}\right)+\cdots+\sigma\left(a_{g_{k}}\right) .
\end{aligned}
$$

Como $\sigma\left(\Sigma_{g}\right)=0, \sigma\left(a_{j}\right)=0$ para todo $j \notin \operatorname{im}(g)$. Então $\operatorname{sgn}_{\sigma}(\psi)=\sigma\left(a_{g_{1}}\right)+\cdots+\sigma\left(a_{g_{k}}\right)=$ $\operatorname{sgn}_{\sigma}(\phi)$. Se $\sigma \in \operatorname{Od}_{0}(\varphi)$, então $\operatorname{sgn}_{\sigma}(\varphi)=0=\operatorname{sgn}_{\sigma}(\psi)$. Logo $\varphi \cong \psi$.

iv) Seja $k=\operatorname{mdim}(\varphi)$. Se $k=0$, então $\varphi \equiv\langle 0\rangle$ e daí basta tomar $b=1$. Assuma $k \geq 1$. Fixado $f \in S_{n, k}$, pela maximalidade de $k$ temos $D\left(\pi_{f}\right) \subseteq D\left(a_{j}^{2}\right)^{c}$ para todo $j \notin \operatorname{im}(f)$ e portanto $D\left(\pi_{f}\right) \subseteq D\left(\Sigma_{f}\right)^{c}$. Logo, pelo item anterior, $\operatorname{Od}_{k}(\varphi)=\bigsqcup_{f \in S_{n, k}} D\left(\pi_{f}\right)$. Então basta tomar $b \in D^{t}\left(\left\langle\pi_{f}\right\rangle_{f \in S_{n, k}}\right)$ para termos para $\operatorname{Od}_{k}(\varphi)=\bigsqcup_{f \in S_{n, k}} D\left(\pi_{f}\right)=D(b)(*)$. Por outro lado, também pelo item anterior, temos

$$
b \varphi \cong \bigoplus_{f \in S_{n, k}} \pi_{f}\left\langle a_{f_{1}}, \ldots, a_{f_{k}}\right\rangle .
$$

Seja $\varphi_{i}=\bigoplus_{f \in S_{n, k}}\left\langle\pi_{f} a_{f_{i}}\right\rangle, i=1, \ldots, k$. Pela união disjunta em $(*), \varphi_{i}$ é 1-forma com suporte $D(b)$. Assim, tomando $b_{i} \in D^{t}\left(\varphi_{i}\right)$, temos $\varphi_{i} \equiv\left\langle b_{i}\right\rangle$ e $b_{i}^{2}=b$. Deste modo, $b \varphi \cong\left\langle b_{1}, \ldots, b_{k}\right\rangle$. Mas como as dimensões coincidem, pela Proposição 3.4 iii), temos $b \varphi \equiv\left\langle b_{1}, \ldots, b_{k}\right\rangle$.

Corolário 3.9. Sejam $R$ RS e $\varphi=\left\langle a_{1}, \ldots, a_{n}\right\rangle$ uma $k$-forma, $k \geq 1$. Então existem $b \in \operatorname{Id}(R)$, 
$b_{1}, \ldots, b_{k} \in R \operatorname{com} b_{i}^{2}=b$ para $1 \leq i, j \leq k$ tais que $\operatorname{Od}_{k}(\varphi)=D(b)$ e

$$
\begin{aligned}
\varphi & \equiv\left\langle b_{1}, \ldots, b_{k}\right\rangle \\
\left\langle a_{1}^{2}, \ldots, a_{n}^{2}\right\rangle & \cong k\langle b\rangle
\end{aligned}
$$

Demonstração. Imediato pelo teorema anterior. Note que a demonstração do teorema acima fornece um algoritmo para obtenção de $b_{1}, \ldots, b_{k}$ em termos das entradas de $\varphi$.

Corolário 3.10. Sejam $R$ RS e $\varphi, \psi$ formas. Então

$\varphi \cong \psi \Leftrightarrow$ existem formas $\theta, \theta^{\prime}$ sobre $\operatorname{Id}(R)$ tais que $\varphi \oplus \theta\langle 1,-1\rangle \equiv \psi \oplus \theta^{\prime}\langle 1,-1\rangle$.

Além disso, se $R$ é von Neumann, $\varphi \cong \psi$ e $\operatorname{dim}(\varphi) \geq \operatorname{dim}(\psi)$, então existe formas $\theta$ sobre $\operatorname{Id}(R)$ tal que $\varphi \equiv \theta\langle 1,-1\rangle \oplus \psi$.

Demonstração. Sejam $\varphi=\left\langle a_{1}, \ldots, a_{n}\right\rangle$ e $\psi=\left\langle b_{1}, \ldots, b_{k}\right\rangle$. Suponha que $\varphi \cong \psi$. Por $\left.3.4 i\right)$, $\operatorname{dim}_{p}(\varphi) \equiv \operatorname{dim}_{p}(\psi)(\bmod 2)$ para todo $p \in \operatorname{spec}(R)$. Defina $z_{i}=a_{i}^{2}$ para $i=1, \ldots, n, z_{j+n}=-b_{i}^{2}$ para $j=1, \ldots, k$ e $\tau=\left\langle z_{1}, \ldots, z_{q}\right\rangle, q=k+n$. Então para todo $\sigma \in X_{R}, \operatorname{sgn}_{\sigma}(\tau)=\operatorname{dim}_{\sigma}(\varphi)-$ $\operatorname{dim}_{\sigma}(\psi) \equiv 0(\bmod 2)$.

Pelo Teorema $3.8 \mathrm{iii}$ ), podemos escrever

$$
\tau \cong \bigoplus_{1 \leq k \leq q} \bigoplus_{f \in S_{q, k}} \pi_{f}\left\langle 1,-\Sigma_{f}\right\rangle\left\langle z_{f_{1}}, \ldots, z_{f_{k}}\right\rangle
$$

Mas como $\tau$ tem sempre assinatura par, para todo $1 \leq k \leq q$ e $f \in S_{q, k}$ existe $n_{f} \in \mathbb{Z}$ par tal que

$$
\pi_{f}\left\langle 1,-\Sigma_{f}\right\rangle\left\langle z_{f_{1}}, \ldots, z_{f_{k}}\right\rangle \cong n_{f} \pi_{f}\left\langle 1,-\Sigma_{f}\right\rangle .
$$

Defina $I_{+}=\left\{(k, f): 1 \leq k \leq q\right.$ e $f \in S_{q, k}$ tais que $\left.n_{f} \geq 0\right\}$ e $I_{-}=\{(k, f): 1 \leq k \leq q$ e $f \in$ $S_{q, k}$ tais que $\left.n_{f}<0\right\}$. Então defina

$$
\begin{aligned}
\theta & =\bigoplus_{(k, f) \in I_{+}} \frac{n_{f}}{2} \pi_{f} \Sigma_{f}-\bigoplus_{(k, f) \in I_{-}} \frac{n_{f}}{2} \pi_{f} \\
\theta^{\prime} & =\bigoplus_{(k, f) \in I_{+}} \frac{n_{f}}{2} \pi_{f}-\bigoplus_{(k, f) \in I_{-}} \frac{n_{f}}{2} \pi_{f} \Sigma_{f}
\end{aligned}
$$

Note que $\theta$ e $\theta^{\prime}$ são formas sobre $\operatorname{Id}(R)$ e

$$
\theta^{\prime}-\theta=\bigoplus_{(k, f) \in I_{+}} \frac{n_{f}}{2} \pi_{f}\left\langle 1,-\Sigma_{f}\right\rangle+\bigoplus_{(k, f) \in I_{-}} \frac{n_{f}}{2} \pi_{f}\left\langle 1,-\Sigma_{f}\right\rangle .
$$

Logo

$$
\begin{aligned}
2\left(\theta^{\prime}-\theta\right) & =\bigoplus_{(k, f) \in I_{+}} n_{f} \pi_{f}\left\langle 1,-\Sigma_{f}\right\rangle+\bigoplus_{(k, f) \in I_{-}} n_{f} \pi_{f}\left\langle 1,-\Sigma_{f}\right\rangle \\
& \cong \bigoplus_{(k, f) \in I_{+}} \pi_{f}\left\langle 1,-\Sigma_{f}\right\rangle\left\langle z_{f_{1}}, \ldots, z_{f_{k}}\right\rangle+\bigoplus_{(k, f) \in I_{-}} \pi_{f}\left\langle 1,-\Sigma_{f}\right\rangle\left\langle z_{f_{1}}, \ldots, z_{f_{k}}\right\rangle \\
& =\tau .
\end{aligned}
$$


Como $\theta$ e $\theta^{\prime}$ são formas sobre $\operatorname{Id}(R)$, dado $\sigma \in X_{R}$

$$
\begin{aligned}
\operatorname{dim}_{\sigma}(\varphi \oplus \theta\langle 1,-1\rangle) & =\operatorname{dim}_{\sigma}(\varphi)+2 \operatorname{dim}_{\sigma}(\theta) \\
& =\operatorname{dim}_{\sigma}(\varphi)+2 \operatorname{sgn}_{\sigma}(\theta) \\
& =\operatorname{dim}_{\sigma}(\varphi)+2 \operatorname{sgn}_{\sigma}\left(\theta^{\prime}\right)-\operatorname{sgn}_{\sigma}(\tau) \\
& =\operatorname{dim}_{\sigma}(\varphi)+2 \operatorname{sgn}_{\sigma}\left(\theta^{\prime}\right)-\operatorname{dim}_{\sigma}(\varphi)+\operatorname{dim}_{\sigma}(\psi) \\
& =\operatorname{dim}_{\sigma}(\psi)+2 \operatorname{dim}_{\sigma}\left(\theta^{\prime}\right)=\operatorname{dim}_{\sigma}\left(\psi \oplus \theta^{\prime}\langle 1,-1\rangle\right) .
\end{aligned}
$$

Deste modo, pela Proposição 3.4 iii), concluímos que $\varphi \oplus \theta\langle 1,-1\rangle \equiv \psi \oplus \theta^{\prime}\langle 1,-1\rangle$.

Assuma que $R$ é von Neumann e $\operatorname{dim}_{p}(\varphi) \geq \operatorname{dim}_{p}(\psi)$ para todo $p \in \operatorname{spec}(R)$. A prova acima será adaptada e usaremos a mesma notação. Defina $\tau$ exatamente da mesma forma. Assim $\operatorname{sgn}_{\sigma}(\tau) \geq 0$ para todo $\sigma \in X_{R}$. Como $R$ é von Neumann, $\left\langle 1,-x^{2}\right\rangle \cong\left\langle x^{c}\right\rangle$ para todo $x \in R$ e então a equivalência 3.2 pode ser reescrita como

$$
\tau \cong \bigoplus_{1 \leq k \leq q} \bigoplus_{f \in S_{q, k}} \pi_{f} \Sigma_{f}^{c}\left\langle z_{f_{1}}, \ldots, z_{f_{k}}\right\rangle
$$

Como fizemos anteriormente, dado $1 \leq k \leq q$ e $f \in S_{q, k}$, obtemos um inteiro positivo par $n_{f}$ tal que

$$
\pi_{f} \Sigma_{f}^{c}\left\langle z_{f_{1}}, \ldots, z_{f_{k}}\right\rangle \cong n_{f} \pi_{f} \Sigma_{f}^{c} .
$$

Defina então $\theta=\bigoplus_{1 \leq k \leq q} \bigoplus_{f \in S_{q, k}} \frac{n_{f}}{2} \pi_{f} \Sigma_{f}^{c}$. Pelo mesmo argumento dado acima, concluímos que $\{\operatorname{com} 1\}$ $2 \theta \cong \tau$ e $\operatorname{assim} \varphi \equiv \theta\langle 1,-1\rangle \oplus \psi$.

Comentário 3.11. Uma bela consequência do resultado acima é que se $R$ é von Neumann e $\varphi$ uma forma $\operatorname{com} \varphi \cong\langle 0\rangle$, então existe forma $\theta$ sobre $\operatorname{Id}(R)$ tal que $\varphi \equiv \theta\langle 1,-1\rangle$.

Vamos finalizar a seção apresentando uma generalização de forma de Pfister de grupos especiais para o contexto de semigrupos reais. Seja $R$ RS. Em [DP2], uma forma de Pfister é definida como $\bigotimes_{i=1}^{n}\left\langle 1, a_{i}\right\rangle$ com $a_{i} \in R$. Resultados importantes são provados com essa definição como a Proposição IV.5.6 e o Teorema IV.5.7 que, usando a envoltória de Post, sedimentam a conexão entre formas de Pfister e o espectro real de um RS. Ao longo da tese, vamos utilizar a versão $\left\langle a^{2}, a\right\rangle, a \in R$, como forma de Pfister ${ }^{2}$ por sua maior versatilidade em provar resultados como o Teorema que classifica as formas multiplicativas (6.10), uma demonstração mais simples de um resultado de densidade no produto de RSs (Proposição 3.32) provada incialmente em [DMP] e a descrição da isometria num quociente de RS (Teorema 4.43). Além disso, como veremos adiante, num RS von Neumann toda forma $\bigotimes_{i=1}^{n}\left\langle 1, a_{i}\right\rangle$ é isométrica à soma de formas do tipo $\bigotimes_{i=1}^{k}\left\langle b_{i}^{2}, b_{i}\right\rangle$ mas o contrário não vale.

Definição 3.12. Seja $R$ RS. Uma forma $\varphi$ é dita de Pfister de grau $n \geq 1$ se existem $a_{1}, \ldots, a_{n} \in R$ tais que $\varphi=\bigotimes_{i=1}^{n}\left\langle a_{i}^{2}, a_{i}\right\rangle$. Por convenção, a forma $\varphi$ é de Pfister de grau 0 se $\varphi=\langle e\rangle$ para algum $e \in \operatorname{Id}(R)$.

Comentários 3.13. Seja $R \operatorname{RS}$ e $\varphi=\bigotimes_{i=1}^{n}\left\langle a_{i}^{2}, a_{i}\right\rangle$ forma de Pfister de grau $n \geq 1$.

- O produto de formas de Pfister é uma forma de Pfister. Em particular, dado $e \in \operatorname{Id}(R)$, e $\varphi$ continua sendo forma de Pfister.

- Seja $R$ é von Neumann. Dado $a \in R$, a forma $\langle 1, a\rangle$ é isométrica a soma ortogonal $\left\langle a^{2}, a\right\rangle \oplus\left\langle a^{c}\right\rangle$ de formas de Pfister. Portanto, dados $a_{1}, \ldots, a_{n} \in R$, a forma $\bigotimes_{i=1}^{n}\left\langle 1, a_{i}\right\rangle$ é isométrica a uma soma de formas de Pfister. No entando, a recíproca não vale: enquanto a forma $\left\langle a^{2}, a\right\rangle$ tem suporte $D(a) \subseteq \operatorname{spec}(R)$, qualquer forma construída a partir das formas $\langle 1, b\rangle$ usando produto e soma tem suporte $D(1)=\operatorname{spec}(R)$.

\footnotetext{
${ }^{2}$ Ficamos sabendo durante a defesa que o Prof. Alejandro Petrovich considerou há alguns anos que seria interessante a análise das formas de Pfister nesta acepção.
} 
- Considere $\left\langle a^{2}, a\right\rangle$ forma de Pfister. Dado $\sigma \in X_{R}$, temos $\operatorname{sgn}_{\sigma}\left(\left\langle a^{2}, a\right\rangle\right)=0$ quando $\sigma(a) \in$ $\{0,-1\}$ e $\operatorname{sgn}_{\sigma}\left(\left\langle a^{2}, a\right\rangle\right)=2$ quando $\sigma(a)=1$. Mais geralmente, temos para todo $\sigma \in X_{R}$ que $\operatorname{sgn}_{\sigma}(\varphi) \in\left\{0,2^{n}\right\} \operatorname{com} \operatorname{sgn}_{\sigma}(\varphi)=2^{n}$ apenas quando $\sigma \in \bigcap_{i=1}^{n}\left[\left[a_{i}=1\right]\right]$.

- Dado $x \in R$, $\left[\left[a_{1}=1\right]\right] \cap \cdots \cap\left[\left[a_{n}=1\right]\right] \cap[[x=0]]=\emptyset$ se, e somente se, $x^{2} \varphi \cong \varphi$. De fato, se existe $\sigma \in\left[\left[a_{1}=1\right]\right] \cap \cdots \cap\left[\left[a_{n}=1\right]\right] \cap[[x=0]]$, então $\operatorname{sgn}_{\sigma}\left(x^{2} \varphi\right)=0 \neq 2^{n}=\operatorname{sgn}_{\sigma}(\varphi)$. Por outro lado, se $x^{2} \varphi \neq \varphi$, existe $\sigma \in X_{R}$ tal que $\sigma(x)=0$ e $\operatorname{sgn}_{\sigma}(\varphi) \neq 0$; logo, pelo comentário acima, $\sigma \in\left[\left[a_{1}=1\right]\right] \cap \cdots \cap\left[\left[a_{n}=1\right]\right]$ e portanto $\sigma \in\left[\left[a_{1}=1\right]\right] \cap \cdots \cap\left[\left[a_{n}=1\right]\right] \cap[[x=0]]$.

- Seja $a=\prod_{i=1}^{n} a_{i}^{2}$. Note que $a \varphi=\varphi$. Além disso, $\operatorname{Od}_{2^{n}}(\varphi)=D(a)$ e se $a \neq 0$ então $\varphi$ é $2^{n}$-forma.

Proposição 3.14. Sejam $R$ RS, $\varphi=\bigotimes_{i=1}^{n}\left\langle a_{i}^{2}, a_{i}\right\rangle$ forma de Pfister e $x \in R$. Seja $a=\prod_{i=1}^{n} a_{i}^{2}$. Então

$$
x \in D^{t}(\varphi) \Leftrightarrow x=a x \text { e } x \varphi \cong \varphi .
$$

Demonstração. Pelo Teorema IV.5.7 (página 155) de [DP2], considerando $3=\{1<0<-1\}$, temos

$$
x \in D^{t}(\varphi) \Leftrightarrow x=a x \text { e para todo } \sigma \in X_{R}, \sigma(x a) \leq \sigma(a) \vee \bigvee_{i=1}^{n} \Delta\left(\sigma\left(a_{i}\right)\right),
$$

onde $\Delta(0)=\Delta(1)=1$ e $\Delta(-1)=-1$. Agora vamos provar 3.4:

$\Rightarrow$ : Seja $\sigma \in X_{R}$. Se $\sigma(x)=0$, então por 3.5 temos que $\sigma(a)=0$ ou existe $1 \leq i \leq n$ com $\sigma\left(a_{i}\right)=-1$; em ambos os casos, $\operatorname{sgn}_{\sigma}(\varphi)=0$ e portanto $\operatorname{sgn}_{\sigma}(x \varphi)=\operatorname{sgn}_{\sigma}(\varphi)(*)$. Se $\sigma(a)=0$, verifica-se facilmente $(*)$. Caso $\sigma(x)=-1$ e $\sigma(a)=1$, por 3.5 existe $1 \leq i \leq n$ com $\sigma\left(a_{i}\right)=-1$ e portanto vale $(*)$. O caso $\sigma(x)=1$ é trivial.

$\Leftarrow$ : Seja $\sigma \in X_{R}$. Vamos provar que $\sigma(x a) \leq \sigma(a) \vee \bigvee_{i=1}^{n} \Delta\left(\sigma\left(a_{i}\right)\right)(* *)$. Se $\sigma(x) \in\{0,-1\}$, então $\operatorname{sgn}_{\sigma}(\varphi)=0$ e portanto existe $1 \leq i \leq n$ tal que $\sigma\left(a_{i}\right) \in\{0,-1\}$; logo vale $(* *)$. Se $\sigma(x)=1$, então $(* *)$ vale trivialmente.

Corolário 3.15. Sejam $R$ RS, $\varphi=\bigotimes_{i=1}^{n}\left\langle a_{i}^{2}, a_{i}\right\rangle$ forma de Pfister e $x \in R$. Seja $a=\prod_{i=1}^{n} a_{i}^{2}$.

i) $a \in D^{t}(\varphi)$ e $D^{t}(\varphi)$ é fechado por multiplicação.

ii) $D^{t}(\varphi) \cap R^{\times} \neq \emptyset$ se, e somente se, $a_{1}, \ldots, a_{n} \in R^{\times}$e $\varphi=\bigotimes_{i=1}^{n}\left\langle 1, a_{n}\right\rangle$.

iii) $x \in D^{t}(\varphi) \Leftrightarrow x \varphi \oplus a\langle 1,-1\rangle^{n} \equiv \varphi \oplus x^{2}\langle 1,-1\rangle^{n}$. Em particular, supondo $x^{2}=a$ temos que $x \in D^{t}(\varphi)$ se, e somente se, $x \varphi \equiv \varphi$.

iv) $x a \in D^{t}(\varphi)$ se, e somente se, $x \varphi \cong \varphi$. Em particular, $\varphi \cong 0$ apenas quando $0 \in D^{t}(\varphi)$ e neste caso $\varphi \equiv n\langle-a, a\rangle$.

Demonstração. i) Como $a \varphi=\varphi$, pela Proposição 3.14 temos $a \in D^{t}(\varphi)$. Além disso, se $x, y \in$ $D^{t}(\varphi)$, então $x=a x, y=a y$ e $x \varphi \cong \varphi \cong y \varphi$. $\operatorname{Logo} x y=a(x y)$ e $(x y) \varphi \cong x \varphi \cong \varphi$. Novamente pela Proposição 3.14 temos $x y \in D^{t}(\varphi)$.

ii) Pela proposição anterior, se existe $x \in D^{t}(\varphi) \cap R^{\times}$, então $x=b x$ e assim $b=1$.

iii) Pela Proposição 3.14, precisamos mostrar que

$$
x=a x \text { e } x \varphi \cong \varphi \Leftrightarrow x \varphi \oplus a\langle 1,-1\rangle^{n} \equiv \varphi \oplus x^{2}\langle 1,-1\rangle^{n} .
$$

$(\Rightarrow)$ : Seja $\sigma \in X_{R}$. Vamos provar que $\operatorname{sgn}_{\sigma}^{ \pm}\left(x \varphi \oplus a\langle 1,-1\rangle^{n}\right)=\operatorname{sgn}_{\sigma}^{ \pm}\left(\varphi \oplus x^{2}\langle 1,-1\rangle^{n}\right)(*)$. Suponha $\sigma(x)=0$. Se $\sigma(a)=0$, então $\operatorname{sgn}^{ \pm}(\varphi)=0$ e portanto vale $(*)$. Caso $\sigma(a)=1$, 
então a equivalência $x \varphi \cong \varphi$ implica que existe $i \in\{1,2, \ldots, n\}$ tal que $\sigma\left(a_{i}\right)=-1$. Logo $\operatorname{sgn}_{\sigma}^{ \pm}(\varphi)=2^{n-1}$ e vale $(*)$.

Agora suponha $\sigma\left(x^{2}\right)=1$. Então $\sigma(a)=1$. Deste modo, da congruência $x \varphi \cong \varphi$ segue que vale $(*)$.

$(\Leftarrow)$ : De imediato já temos $x \varphi \cong x \varphi \oplus a\langle 1,-1\rangle^{n} \equiv \varphi \oplus x^{2}\langle 1,-1\rangle^{n} \cong \varphi$ e resta mostrar que $x=a x$. Se $\sigma(a)=0$, então por hipótese $0=\operatorname{sgn}_{\sigma}^{+}\left(x \varphi \oplus a\langle 1,-1\rangle^{n}\right)=\operatorname{sgn}_{\sigma}^{+}\left(\varphi \oplus x^{2}\langle 1,-1\rangle\right)=$ $\operatorname{sgn}_{\sigma}^{+}\left(x^{2}\langle 1,-1\rangle\right)$. $\log \sigma(x)=0$. Como $\sigma \in X_{R}$ foi arbitrário, segue que $x=a x$.

iv) Consequência imediata da Proposição 3.14.

\subsection{Isometria em RS von Neumann}

Nesta seção, veremos como a isometria e a representação de formas num RS von Neumann possuem propriedades parecidas com as mesmas noções em RSGs. Mais especificamente:

- A isometria de formas é de primeira ordem e é descrita por uma pp-fórmula (Teorema 3.23);

- Caracterização da representação de formas $D^{t}$ em função da isometria (Teorema 3.37).

Além disso, no Teorema 3.29 caracterizamos quando vale o Teorema da Decomposição de Witt para uma forma em função da continuidade do índice de Witt. Já no Teorema 3.34 damos uma caracterização topológica dos elementos da classe $W I(R S)$, composta por RSs von Neumann onde vale o Teorema da Decomposição de Witt. Também provamos que $W I(R S)$ é uma classe elementar fechada por produtos arbitrários.

\{idvn1\}

Lema 3.16. Seja $R$ RS. Dados $e, e_{1}, \ldots, e_{n} \in \operatorname{Id}(R) \operatorname{com} e_{i} e_{j}=0$ para $i \neq j$, são equivalentes:

i) $e \in D^{t}\left(e_{1}, \ldots, e_{n}\right)$.

ii) $\langle e\rangle \equiv\left\langle e_{1}, \ldots, e_{n}\right\rangle$.

iii) $D(e)=\bigsqcup_{i=1}^{n} D\left(e_{i}\right)$.

Além disso, dados $x, x_{1}, \ldots, x_{n} \in R$ satisfazendo $x e_{i}=x_{i} e_{i}$ para todo $i=1, \ldots, n$, então $x e \in D_{R}^{t}\left(x_{1} e_{1}, \ldots, x_{n} e_{n}\right)$, onde $e \in D^{t}\left(e_{1}, \ldots, e_{n}\right)$.

Demonstração. $i) \Rightarrow i i)$ : Segue por indução em $n$. Para $n=1$ é imediato. Se $n=2$, então $e \in$ $D^{t}\left(e_{1}, e_{2}\right)$ implica, pela Proposição $\left.3.4 v\right)$, que

$$
\langle e\rangle \equiv\left\langle e, e e_{1} e_{2}\right\rangle \cong\left\langle e_{1}, e_{2}\right\rangle
$$

Mas como a dimensão de ambas as formas acima são iguais, novamente pela Proposição 3.4 iii) temos $\langle e\rangle \equiv\left\langle e_{1}, e_{2}\right\rangle$. Para $n \geq 3$, seja $f \in D^{t}\left(e_{2}, \ldots, e_{n}\right)$ tal que $e \in D^{t}\left(e_{1}, f\right)$. Por hipótese de indução, temos $\langle f\rangle \equiv\left\langle e_{2}, \ldots, e_{n}\right\rangle$ e, como $e_{1} f=0,\langle e\rangle \equiv\left\langle e_{1}, f\right\rangle$. Logo

$$
\langle e\rangle \equiv\left\langle e_{1}, f\right\rangle \equiv\left\langle e_{1}, e_{2}, \ldots, e_{n}\right\rangle .
$$

ii $) \Rightarrow$ iii): Dado $p \in D(e)$, temos $1=\operatorname{dim}_{p}(\langle e\rangle)=\sum_{i=1}^{n} \operatorname{dim}_{p}\left(\left\langle e_{i}\right\rangle\right)$ e portanto existe $i$ tal que $p \in D\left(e_{i}\right)$. Reciprocamente, dado $p \in D\left(e_{j}\right)$, temos $1 \leq \sum_{i=1}^{n} \operatorname{dim}_{p}\left(\left\langle e_{i}\right\rangle\right)=\operatorname{dim}_{p}(\langle e\rangle)$ e assim $p \in D(e)$.

iii) $\Rightarrow$ i): Comentário 2.12 .

A última afirmação segue por indução em $n$. Para $n=1$, é trivial. Supondo $n \geq 2$, seja $f \in D^{t}\left(e_{2}, \ldots, e_{n}\right)$. Note que $e \in D^{t}\left(e_{1}, f\right)$. Então por hipótese de indução segue que $x f \in$ $D^{t}\left(x_{2} e_{2}, \ldots, x_{n} e_{n}\right)$. Como $x e \in D^{t}\left(x e_{1}, x f\right)=D^{t}\left(x_{1} e_{1}, x f\right)$, temos que $x e \in D^{t}\left(x_{1} e_{1}, \ldots, x_{n} e_{n}\right)$. 
Comentário 3.17. Sejam $R$ RS e $e_{1}, \ldots, e_{n} \in \operatorname{Id}(R)$ idempotentes satisfazendo $e_{i} e_{j}=0$ se $i \neq j$. Sejam $x, x_{1}, \ldots, x_{n} \in R$ tais que $x e_{i}=x_{i} e_{i}$ para todo $i=1 \ldots, n$. Então $x e \in D^{t}\left(x_{1} e_{1}, \ldots, x_{n} e_{n}\right)$, onde $e \in D^{t}\left(e_{1}, \ldots, e_{n}\right)$.

Teorema 3.18. Sejam $R R S$ e $\varphi$ forma não-nula com $\operatorname{supp}(\varphi)=D(e)$. Então existe sistema ortogonal $\left(\varphi_{1}, \cdots, \varphi_{k}\right)$ tal que $\varphi \equiv \varphi_{1} \oplus \cdots \oplus \varphi_{k}$ se, e somente se, para todo $i \geq 1, \operatorname{Od}_{i}(\varphi) \subseteq \operatorname{spec}(R)$ é aberto (na topologia espectral). Neste caso, existem $e_{1}, \ldots, e_{k} \in \operatorname{Id}(R)$ tais que

i) $e_{i} e_{j}=0$ para $i \neq j$.

ii) $\langle e\rangle \equiv\left\langle e_{1}, \ldots, e_{k}\right\rangle$.

iii) $e \varphi \equiv \varphi$ e $e_{i} \varphi \equiv \varphi_{i}$.

Demonstração. Assuma que $\varphi \equiv \varphi_{1} \oplus \cdots \oplus \varphi_{k}$ para algum sistema ortogonal $\left(\varphi_{1}, \ldots, \varphi_{k}\right), \varphi_{j}$ $d_{j}$-forma. Pela Proposição $\left.3.8 i v\right)$, para cada $1 \leq j \leq k$ existe $e_{j} \in \operatorname{Id}(R)$ tal que $\operatorname{Od}_{d_{j}}\left(\varphi_{j}\right)=$ $D\left(e_{j}\right)$. Então pela Proposição $\left.3.7 i\right), \operatorname{Od}_{d_{j}}(\varphi)=\operatorname{Od}_{d_{j}}\left(\varphi_{j}\right)=D\left(e_{j}\right)$ é aberto e se $i \notin\left\{d_{1}, \ldots, d_{k}\right\}$, $\operatorname{Od}_{i}(\varphi)=\emptyset$. Neste caso, como $D(e)=\operatorname{supp}(\varphi)=\bigsqcup_{i=1}^{k} \operatorname{Od}_{d_{i}}(\varphi)=\bigsqcup_{i=1}^{k} D\left(e_{i}\right)$, pelo lema anterior temos $\langle e\rangle \equiv\left\langle e_{1}, \ldots, e_{n}\right\rangle$. Além disso, é fácil ver que

$$
\begin{aligned}
& \text {. } e_{i} e_{j}=0 \text { para } i \neq j . \\
& \text {. e } \varphi \equiv \varphi \text { e } e_{i} \varphi \equiv e_{i} \varphi_{i} \equiv \varphi_{i} .
\end{aligned}
$$

Reciprocamente, assuma que para todo $i \geq 1, \operatorname{Od}_{i}(\varphi)$ é aberto. Seja $\left\{d_{1}, \ldots, d_{k}\right\}=\{i: i \geq$ 1 e $\left.\operatorname{Od}_{i}(\varphi) \neq \emptyset\right\}$ com $i \leq j$ implicando $d_{i}<d_{j}$. Como para cada $j=1, \ldots, k$ o conjunto $\operatorname{Od}_{d_{j}}(\varphi) \subseteq$ $(\operatorname{spec}(R))_{\text {const }}$ é aberto-fechado na topologia espectral, existem $a_{(j, 1)}, \ldots, a_{\left(j, t_{j}\right)} \in \operatorname{Id}(R)$ tais que $\operatorname{Od}_{d_{j}}(\varphi)=\cup_{l=1}^{t_{j}} D\left(a_{(j, l)}\right)$. Tomando $e_{j} \in D^{t}\left(\left\langle a_{(j, l)}\right\rangle_{l \leq t_{j}}\right)$, temos que $\operatorname{Od}_{d_{j}}(\varphi)=D\left(e_{j}\right)$. Assim $e_{j} \varphi$ é $d_{j}$-forma e se $i \neq j, e_{i} e_{j}=0$. Deste modo $\left(e_{1} \varphi, \ldots, e_{k} \varphi\right)$ é sistema ortogonal e $\varphi \equiv e_{1} \varphi \oplus \cdots \oplus e_{k} \varphi$.

Corolário 3.19. Seja $R$ RS. São equivalentes:

i) $R$ é von Neumann.

ii) Para toda forma $\varphi \not \equiv 0$, existe único sistema ortogonal $\left(\varphi_{1}, \ldots, \varphi_{k}\right) \operatorname{com} \varphi \equiv \varphi_{1} \oplus \cdots \oplus \varphi_{k}$.

Demonstração. Se $R$ é von Neumann, então $\operatorname{spec}(R)$ é espaço Booleano e portanto $\operatorname{Od}_{i}(\varphi)$ é aberto para todo $i \geq 1$. Então pelo Teorema 3.18, existe sistema ortogonal para $\varphi$. A unicidade segue pela Proposição 3.7. Reciprocamente, seja $\varphi=\left\langle 1,-x^{2}\right\rangle$. Como $\varphi$ admite sistema ortogonal, pelo Teorema 3.18, o conjunto $D(x)^{c}=\operatorname{Od}_{1}(\varphi)$ é aberto. Então $\operatorname{spec}(R)$ é Booleano. Logo $R$ é von Neumann.

Comentário 3.20. Sejam $\varphi=\left\langle a_{1}, \ldots, a_{n}\right\rangle$ forma e $\left(\varphi_{1}, \ldots, \varphi_{k}\right)$ sistema ortogonal com $\varphi \equiv \varphi_{1} \oplus$ $\cdots \oplus \varphi_{k}$. Então como as dimensões das qc-formas $\varphi_{i}$ são diferentes, vale a desigualdade

$$
k \leq \operatorname{mim}(\varphi) \leq n
$$

Em particular $\operatorname{Od}_{t}(\varphi)=\emptyset$ para todo $t>n$

Lema 3.21. Seja $R$ RS Booleano. Sejam $\varphi=\left\langle a_{1}, \ldots, a_{k}\right\rangle, \psi=\left\langle b_{1}, \ldots, b_{k}\right\rangle k$-formas, $k>2$. Então $\varphi \equiv \psi$ se, e somente se, existem $\alpha, \beta \in R$ e uma $k-2$-forma $\theta$ tais que

$$
\begin{aligned}
\left\langle a_{1}, \alpha\right\rangle & \equiv\left\langle b_{1}, \beta\right\rangle \\
\left\langle a_{2}, \ldots, a_{k}\right\rangle & \equiv\langle\alpha\rangle \oplus \theta \\
\left\langle b_{2}, \ldots, b_{k}\right\rangle & \equiv\langle\beta\rangle \oplus \theta .
\end{aligned}
$$


Demonstração. Assuma que $\varphi \equiv \psi$. Seja então $a \in \operatorname{Id}(R) \operatorname{com} \operatorname{Od}_{k}(\varphi)=\operatorname{Od}_{k}(\psi)=D(a) . \operatorname{Em} R_{a}$, as formas $\bar{\varphi}$ e $\bar{\psi}$ tem coeficientes invertíveis e são isométricas no RSG $R_{a}^{\times}$. Então como a relação de isometria em RSG é indutiva e $x \in R$ é invertível em $R_{a}$ somente quando $x^{2}=a$, existem $\alpha, \beta \in R$ e $c_{2}, \cdots, c_{k} \in R \operatorname{com} c_{i}^{2}=a, i=2, \cdots, n, \alpha^{2}=\beta^{2}=a$ tais que a forma $\theta=\left\langle c_{2}, \ldots, c_{k}\right\rangle$ satisfaz

$$
\begin{aligned}
\left\langle\overline{a_{1}}, \bar{\alpha}\right\rangle & \equiv_{R_{a}^{\times}}\left\langle\overline{b_{1}}, \bar{\beta}\right\rangle \\
\left\langle\overline{a_{2}}, \ldots, \overline{a_{k}}\right\rangle & \equiv_{R_{a}^{\times}}\langle\bar{\alpha}\rangle \oplus \bar{\theta} \\
\left\langle\overline{b_{2}}, \ldots, \overline{b_{k}}\right\rangle & \equiv_{R_{a}^{\times}}\langle\bar{\beta}\rangle \oplus \bar{\theta} .
\end{aligned}
$$

Note que, pela Proposição 1.15, $X_{R_{a}^{\times}} \cong X_{R_{a}} \cong\left\{\sigma \in X_{R}: \sigma(a)=1\right\}$. Assim, como todos os coeficientes das formas acima têm valores nulos para $\sigma \in X_{R}$ com $\sigma(a)=0$, temos

$$
\begin{aligned}
\left\langle a_{1}, \alpha\right\rangle & \equiv\left\langle b_{1}, \beta\right\rangle \\
\left\langle a_{2}, \ldots, a_{k}\right\rangle & \equiv\langle\alpha\rangle \oplus \theta \\
\left\langle b_{2}, \ldots, b_{k}\right\rangle & \equiv\langle\beta\rangle \oplus \theta .
\end{aligned}
$$

Reciprocamente, se as equações acima valem, temos

$$
\begin{aligned}
\varphi=\left\langle a_{1}, \ldots, a_{k}\right\rangle & \equiv\left\langle a_{1}\right\rangle \oplus\langle\alpha\rangle \oplus \theta \\
& \equiv\left\langle a_{1}, \alpha\right\rangle \oplus \theta \\
& \equiv\left\langle b_{1}, \beta\right\rangle \oplus \theta \\
& \equiv\left\langle b_{1}, \ldots, b_{k}\right\rangle=\psi
\end{aligned}
$$

Comentário 3.22. Se $\varphi=\left\langle a_{1}, \ldots, a_{k}\right\rangle, \psi=\left\langle b_{1}, \ldots, b_{k}\right\rangle$ são $k$-formas, $k>2$, e existem $\alpha, \beta \in R$ e $k-2$-forma $\theta=\left\langle z_{3}, \ldots, z_{k}\right\rangle$ satisfazendo

$$
\begin{aligned}
\left\langle a_{1}, \alpha\right\rangle & \equiv\left\langle b_{1}, \beta\right\rangle \\
\left\langle a_{2}, \ldots, a_{k}\right\rangle & \equiv\langle\alpha\rangle \oplus \theta \\
\left\langle b_{2}, \ldots, b_{k}\right\rangle & \equiv\langle\beta\rangle \oplus \theta,
\end{aligned}
$$

então temos $a_{1}^{2}=b_{1}^{2}=\alpha^{2}=\beta^{2}=z_{3}^{2}$. De fato, sejam $a, b \in \operatorname{Id}(R)$ tais que $\operatorname{Od}_{k}(\varphi)=D(a)$ e $\operatorname{Od}_{k}(\psi)=D(b)$. Como $\varphi \equiv \psi$, temos $a_{1}^{2}=a=b=b_{1}^{2}$ e assim pela primeira isometria acima vale $\alpha^{2}=\beta^{2}$. A segunda isometria nos diz que $(k-1)\langle a\rangle \cong\left\langle\alpha^{2}\right\rangle \oplus(k-2)\left\langle z_{3}^{2}\right\rangle$. Comparando as assinaturas, vemos que $a=\alpha^{2}=z_{3}^{2}$ e portanto $a_{1}^{2}=b_{1}^{2}=\alpha^{2}=\beta^{2}=z_{3}^{2}$.

Teorema 3.23. Sejam $R R S$ von Neumann e considere a linguagem $\mathcal{L}_{R S}=\left\{\cdot,-, D^{t}, 0,1\right\}$ dos $R S s$. Dados $n, k \geq 1$, existe pp-fórmula $\theta_{n, k}\left(x_{1}, \ldots, x_{n}, y_{1}, \ldots, y_{k}\right)$ tal que dadas formas $\varphi=\left\langle a_{1}, \ldots, a_{n}\right\rangle$ $e \psi=\left\langle b_{1}, \ldots, b_{k}\right\rangle$,

$$
\varphi \equiv \psi \text { se, e somente se, } R \models \theta_{n, k}\left(a_{1}, \ldots, a_{n}, b_{1}, \ldots, b_{k}\right)
$$

Demonstração. Notação: Somente para esta demonstração, dado $k \geq 1$ vamos chamar uma forma $\varphi$ de $k$-forma* se $\varphi \equiv\langle 0\rangle$ ou $\varphi$ é $k$-forma.

A prova seguirá depois da construção de algumas fórmulas intermediárias. Note que a fórmula $v \in D^{t}\left(v_{1}, \ldots, v_{n}\right)$ é pp.

Afirmação. Sejam $n \geq k \geq 1$. Então existe pp-fórmula $\theta_{n, k}^{1}\left(x_{1}, \ldots, x_{n}\right)$ tal que dada forma $\varphi=$ $\left\langle a_{1}, \ldots, a_{n}\right\rangle$,

$$
\varphi \text { é k-forma* se, e somente se, } R \models \theta_{k, n}^{1}\left(a_{1}, \ldots, a_{n}\right) \text {. }
$$


Demonstração. Defina

$$
\begin{aligned}
\theta_{n, k}^{1}\left(x_{1}, \ldots, x_{n}\right) & =\exists b\left(b^{2}=b\right) \wedge\left(\bigwedge_{i=1}^{n} b x_{i}^{2}=x_{i}^{2}\right) \\
& \wedge\left(b \in D^{t}\left(\left\langle x_{f_{1}}^{2} \cdot \ldots \cdot x_{f_{k}}^{2}\right\rangle_{f \in S_{n, k}}\right)\right) \\
& \wedge\left(\bigwedge_{f \in S_{n, k+1}} x_{f_{1}}^{2} \cdot \ldots \cdot x_{f_{k+1}}^{2}=0\right) .
\end{aligned}
$$

Se $\varphi \equiv\langle 0\rangle$, então $a_{1}=\cdots=a_{n}=0$ e basta tomar $b=0$ para termos $R \models \theta_{n, k}^{1}\left(a_{1}, \ldots, a_{n}\right)$. Se $\varphi$ é uma $k$-forma, tome $b \in D^{t}\left(\left\langle a_{f_{1}}^{2} \cdot \ldots \cdot a_{f_{k}}^{2}\right\rangle_{f \in S_{n, k}}\right)$. Então $b \in \operatorname{Id}(R)$ e pela demonstração do Teorema $3.8 \mathrm{iv}$ ) temos $\operatorname{Od}_{k}(\varphi)=D(b)$. Assim, como $\operatorname{dim}_{p}(\varphi)=0$ para todo $p \notin \operatorname{Od}_{k}(\varphi)$, temos $D\left(a_{i}\right) \subseteq D(b)$ e portanto $a_{i}^{2} b=a_{i}^{2}$. Por outro lado, se existisse $f \in S_{n, k+1} \operatorname{com} a_{f_{1}}^{2} \cdot \ldots \cdot a_{f_{k+1}}^{2} \neq 0$, existiria $p \in \operatorname{spec}(R)$ tal que $a_{f_{i}}^{2} \notin p$ para $i=1, \ldots, k+1$ e portanto $\operatorname{dim}_{p}(\varphi) \geq k+1$, o que contradiz o fato de $\varphi$ ser $k$-forma. Logo $R \models \theta_{n, k}^{1}\left(a_{1}, \ldots, a_{n}\right)$.

Reciprocamente, suponha que $R \models \theta_{n, k}^{1}\left(a_{1}, \ldots, a_{n}\right)$ e seja $b \in \operatorname{Id}(R)$ a testemunha. Se $b=0$, temos $\varphi \equiv\langle 0\rangle$. Assuma $b \neq 0$. Como $\bigwedge_{f \in S_{n, k+1}} a_{f_{1}}^{2} \cdot \ldots \cdot a_{f_{k+1}}^{2}=0$ temos $\operatorname{Od}_{t}(\varphi)=\emptyset$ para todo $t \geq k+1$ pois se existisse $p \in \operatorname{Od}_{t}(\varphi)$, existiria $f \in S_{n, t}$ com $a_{f_{i}}^{2} \notin p$ para todo $i=1, \ldots, t$ e portanto $a_{f_{1}}^{2} \cdot \ldots \cdot a_{f_{t}}^{2} \neq 0$ em $R$, um absurdo. Por outro lado, a relação $b \in D^{t}\left(\left\langle a_{f_{1}}^{2} \cdot \ldots \cdot a_{f_{k}}^{2}\right\rangle_{f \in S_{n, k}}\right)$ implica que

$$
D(b)=\bigcup_{f \in S_{n, k}} D\left(a_{f_{1}}^{2} \cdot \ldots \cdot a_{f_{k}}^{2}\right) .
$$

Fixe $p \in \operatorname{spec}(R) \operatorname{com} \operatorname{dim}_{p}(\varphi) \neq 0$. Já vimos que $\operatorname{dim}_{p}(\varphi) \leq k$. Por outro lado, como existe $i$ com $p \in D\left(a_{i}^{2}\right)$, a igualdade $b a_{i}^{2}=a_{i}^{2}$ implica que $p \in D(b)$. Assim deve existir $f \in S_{n, k}$ tal que $p \in D\left(a_{f_{1}}^{2} \cdot \ldots \cdot a_{f_{k}}^{2}\right)$ e portanto $\operatorname{dim}_{p}(\varphi) \geq k . \operatorname{Logo} \operatorname{dim}_{p}(\varphi)=k$. Logo $\varphi$ é $k$-forma.

Afirmação. Seja $k \geq 1$. Então existe pp-fórmula $\theta_{k}^{2}\left(x_{1}, \ldots, x_{k}, y_{1}, \ldots, y_{k}\right)$ tal que para todas $k$ formas* $\varphi=\left\langle a_{1}, \ldots, a_{k}\right\rangle, \psi=\left\langle b_{1}, \ldots, b_{k}\right\rangle$

$$
\varphi \equiv \psi \text { se, e somente se, } R \models \theta_{k}^{2}\left(a_{1}, \ldots, a_{k}, b_{1}, \ldots, b_{k}\right) .
$$

Demonstração. A definição da fórmula segue por indução em $k$. Para $k=1$, defina $\theta_{1}^{2}\left(x_{1}, y_{1}\right)=$ $\left(x_{1}=y_{1}\right)$ e para $k=2$, usando 3.4 , defina

$$
\theta_{2}^{2}\left(x_{1}, x_{2}, y_{1}, y_{2}\right)=\left(D^{t}\left(x_{1}, x_{2}\right) \cap D^{t}\left(y_{1}, y_{2}\right) \neq \emptyset\right) \wedge\left(x_{1} x_{2}=y_{1} y_{2}\right) .
$$

Para $k \geq 3$, definimos $\theta_{k}^{2}$ como

$$
\begin{aligned}
\theta_{k}^{2}\left(x_{1}, \ldots, x_{k}, y_{1}, \ldots, y_{k}\right) & =\exists \alpha, \beta, z_{3}, \ldots, z_{k}\left(\alpha^{2}=\beta^{2}=x_{1}^{2}=y_{1}^{2}\right) \\
& \wedge\left(\bigwedge_{i=3}^{k} z_{i}^{2}=\alpha^{2}\right) \\
& \wedge \theta_{2}^{2}\left(x_{1}, \alpha, y_{1}, \beta\right) \\
& \wedge \theta_{k-1}^{2}\left(x_{2}, \ldots, x_{k}, \alpha, z_{3}, \ldots, z_{k}\right) \\
& \wedge \theta_{k-1}^{2}\left(y_{2}, \ldots, y_{k}, \beta, z_{3}, \ldots, z_{k}\right) .
\end{aligned}
$$

Vamos mostrar por indução em $k$ a implicação $\Rightarrow$ de 3.6. Se $\varphi$ é identicamente nula, é fácil ver que $R \models \theta_{k}^{2}\left(a_{1}, \ldots, a_{k}, b_{1}, \ldots, b_{k}\right)$. Além disso, o caso $k=2$ decorre da Proposição $3.4(k=1$ é trivial). Suponha então que ambas são $k$-formas, $k \geq 3$. Pelo Lema 3.21, existem $\alpha, \beta \in R$ e 
$k$ - 2-forma $\theta$ tais que

$$
\begin{aligned}
\left\langle a_{1}, \alpha\right\rangle & \equiv\left\langle b_{1}, \beta\right\rangle \\
\left\langle a_{2}, \ldots, a_{k}\right\rangle & \equiv\langle\alpha\rangle \oplus \theta \\
\left\langle b_{2}, \ldots, b_{k}\right\rangle & \equiv\langle\beta\rangle \oplus \theta .
\end{aligned}
$$

Pelo Corolário 3.9 podemos assumir que $\theta=\left\langle z_{3}, \ldots, z_{k}\right\rangle$ e $z_{i}^{2}=z_{j}^{2}$ para todo $3 \leq i, j \leq k$. Também sabemos pelo comentário 3.22 que $\alpha^{2}=\beta^{2}=a_{1}^{2}=b_{1}^{2}=z_{i}^{2}, i=3, \ldots, k$. Assim, pela hipótese de indução, temos $R \models \theta_{k}^{2}\left(a_{1}, \ldots, a_{k}, b_{1}, \ldots, b_{k}\right)$.

Reciprocamente, o sentido $\Leftarrow$ de 3.6 também é provado por indução em $k$. Para $k=2$ segue da Proposição 3.4. Assuma que $R=\theta_{k}^{2}\left(a_{1}, \ldots, a_{k}, b_{1}, \ldots, b_{k}\right), k \geq 3$. Por hipótese de indução, vale que

$$
\begin{aligned}
\left\langle a_{1}, \alpha\right\rangle & \equiv\left\langle b_{1}, \beta\right\rangle \\
\left\langle a_{2}, \ldots, a_{k}\right\rangle & \equiv\langle\alpha\rangle \oplus\left\langle z_{3}, \ldots, z_{k}\right\rangle \\
\left\langle b_{2}, \ldots, b_{k}\right\rangle & \equiv\langle\beta\rangle \oplus\left\langle z_{3}, \ldots, z_{k}\right\rangle .
\end{aligned}
$$

$\operatorname{Logo} \varphi \equiv \psi$

Afirmação. Sejam $n_{1}, n_{2} \geq k \geq 1$. Então existe pp-fórmula $\theta_{n_{1}, n_{2}, k}^{3}\left(x_{1}, \ldots, x_{n_{1}}, y_{1}, \ldots, y_{n_{2}}\right)$ tal que para todas $k$-formas ${ }^{*} \varphi=\left\langle a_{1}, \ldots, a_{n_{1}}\right\rangle$ e $\psi=\left\langle b_{1}, \ldots, b_{n_{2}}\right\rangle$

$$
\varphi \equiv \psi \text { se, e somente se, } R \models \theta_{n_{1}, n_{2}, k}^{3}\left(a_{1}, \ldots, a_{n_{1}}, b_{1}, \ldots, b_{n_{2}}\right) \text {. }
$$

Demonstração. Usando a prova do Teorema 3.8, podemos definir

$$
\begin{aligned}
\theta_{n_{1}, n_{2}, k}^{3}\left(x_{1}, \ldots, x_{n}, y_{1}, \ldots, y_{n}\right) & =\exists v_{1}, \ldots, v_{k}, w_{1}, \ldots, w_{k}\left(\bigwedge_{i=1}^{k} v_{i} \in D^{t}\left(\left\langle x_{f_{1}}^{2} \cdot \ldots \cdot x_{f_{k}}^{2} x_{f_{i}}\right\rangle_{f \in S_{n_{1}, k}}\right)\right) \\
& \wedge\left(\bigwedge_{i=1}^{k} w_{i} \in D^{t}\left(\left\langle y_{f_{1}}^{2} \cdot \ldots \cdot y_{f_{k}}^{2} y_{f_{i}}\right\rangle_{f \in S_{n_{2}, k}}\right)\right) \\
& \wedge \theta_{k}^{2}\left(v_{1}, \ldots, v_{k}, w_{1}, \ldots, w_{k}\right) .
\end{aligned}
$$

Agora estamos prontos para provar o teorema. Sem perda de generalidade suponha $k=n$. Defina $\theta_{n, n}$ como

$$
\begin{aligned}
\theta_{n, n}\left(x_{1}, \ldots, x_{n}, y_{1}, \ldots, y_{n}\right) & =\exists e_{0}, e_{1}, \ldots, e_{n}\left(\bigwedge_{i=0}^{n} e_{i}^{2}=e_{i}\right) \wedge\left(\bigwedge_{i, j=0, i \neq j}^{n} e_{i} e_{j}=0\right) \\
& \wedge\left(1 \in D^{t}\left(e_{0}, \ldots, e_{n}\right)\right) \\
& \wedge\left(\bigwedge_{i=1}^{n}\left(\theta_{i}^{1}\left(e_{i} x_{1}, \ldots, e_{i} x_{n}\right) \wedge \theta_{i}^{1}\left(e_{i} y_{1}, \ldots, e_{i} y_{n}\right)\right)\right. \\
& \wedge\left(\bigwedge_{i=1}^{n} \theta_{n, n, i}^{3}\left(e_{i} x_{1}, \ldots, e_{i} x_{n}, e_{i} y_{1}, \ldots, e_{i} y_{n}\right)\right) .
\end{aligned}
$$

Sejam $\varphi=\left\langle a_{1}, \ldots, a_{n}\right\rangle, \psi=\left\langle b_{1}, \ldots, b_{n}\right\rangle$ formas $\operatorname{com} \varphi \equiv \psi$. Sejam $e_{i} \in \operatorname{Id}(R), i=0, \ldots, n$, tais que $\operatorname{Od}_{i}(\varphi)=\operatorname{Od}_{i}(\psi)=D\left(e_{i}\right)$. Note que para $i \neq j$ temos $e_{i} e_{j}=0$ e $1 \in D^{t}\left(e_{0}, \ldots, e_{n}\right)$. Além disso $e_{i} \varphi$ e $e_{i} \psi$ são $i$-formas* e $e_{i} \varphi \equiv e_{i} \psi$. Logo $R \models \theta_{n, n}(\varphi, \psi)$. Reciprocamente, suponha $\theta_{n, n}(\varphi, \psi)$ válida. Então existem $e_{0}, \ldots, e_{n}$ tais que $1 \in D^{t}\left(e_{0}, \ldots, e_{n}\right), e_{i} e_{j}=0$ para $i \neq j$ e, pelas afirmações 
anteriores, $e_{i} \varphi \equiv e_{i} \psi$. Deste modo $\langle 1\rangle \equiv\left\langle e_{0}, \ldots, e_{n}\right\rangle$ e

$$
\begin{aligned}
\varphi & \equiv\left\langle e_{0}, \ldots, e_{n}\right\rangle \otimes \varphi \\
& \equiv e_{0} \varphi \oplus \cdots \oplus e_{n} \varphi \\
& \equiv e_{0} \psi \oplus \cdots \oplus e_{n} \psi \\
& \equiv\left\langle e_{0}, \ldots, e_{n}\right\rangle \otimes \psi \equiv \psi
\end{aligned}
$$

Comentário 3.24. Sejam $R$ RS Booleano e formas $\varphi=\left\langle a_{1}, \ldots, a_{n}\right\rangle, \psi=\left\langle b_{1}, \ldots, b_{k}\right\rangle$ com $\operatorname{Od}_{i}(\varphi)$ aberto para todo $i \geq 1$. Então a mesma demonstração acima mostra que

$$
\varphi \equiv \psi \text { se, e somente se, } R=\theta_{n, k}\left(a_{1}, \ldots, a_{n}, b_{1}, \ldots, b_{k}\right) \text {. }
$$

Definição 3.25. Sejam $R$ RS e $\varphi=\left\langle a_{1}, \ldots, a_{n}\right\rangle$ forma sobre $R$. $\varphi$ é dita isotrópica se existe $x \in \operatorname{Id}(R)$ não-nulo e forma $\theta$ tal que $\varphi \equiv x\langle 1,-1\rangle \oplus \theta$ e anisotrópica caso contrário. $\varphi$ é l-isotrópica se existe $p \in \operatorname{spec}(R)$ tal que $\varphi_{p}$ é isotrópica em $R_{p}$ e é dita l-anisotrópica caso contrário.

Comentários 3.26. - Note que a forma nula $\langle 0\rangle$ é anisotrópica e todas as formas isotrópicas são l-isotrópicas.

- Seja $R$ RS von Neumann e $\varphi$ uma forma sobre $R^{\times}$. Se $\varphi$ é isotrópica no RSG $R^{\times}, \varphi$ é isotrópica em $R$ mas a recíproca não é verdade em geral. De fato, se $R$ não é 0-RSG, existe $x \in \operatorname{Id}(R)$ tal que $x \neq 0,1$. Como $1 \in D^{t}\left(x, x^{c}\right)$ e $\nabla(x) \in D^{t}\left(x,-x^{c}\right)$ (Proposição 2.59), temos $\langle 1\rangle \equiv\left\langle x, x^{c}\right\rangle$ $\mathrm{e}\langle\nabla(x)\rangle \equiv\left\langle x,-x^{c}\right\rangle$. Assim $\langle 1,-\nabla(x)\rangle \equiv\left\langle x, x^{c}\right\rangle \oplus\left\langle-x, x^{c}\right\rangle \equiv x\langle 1,-1\rangle \oplus x^{c}\langle 1,1\rangle$. Por outro lado $\langle 1,-\nabla(x)\rangle$ é anisotrópica em $R^{\times}$pois do contrário teríamos $\nabla(x)=1$, contrariando $x \neq 1$.

Definição 3.27. Sejam $R$ RS e $\varphi$ forma. Para cada $p \in \operatorname{spec}(R)$, seja $n_{p} \geq 0$ inteiro tal que exista forma $\theta$ sobre $R_{p}$ e anisotrópica em $R_{p} \operatorname{com~} \varphi_{p} \equiv_{p} n_{p}\langle 1,-1\rangle \oplus \theta$. Vamos denotar por $\operatorname{ind}_{W}(\varphi): \operatorname{spec}(R) \rightarrow \mathbb{N}$ a função $\operatorname{ind}_{W}(\varphi)_{p}=n_{p}$; esta função é chamada de indice de Witt de $\varphi$.

Lema 3.28. Sejam $R$ RS von Neumann e $\varphi, \psi$ formas. Seja $p \in \operatorname{spec}(R)$.

i) Se $\varphi_{p} \cong_{p} \psi_{p}$, então existe $x \in \operatorname{Id}(R)$ com $p \in D(x)$ tal que $x \varphi \cong x \psi$.

ii) Se $\varphi_{p} \equiv_{p} \psi_{p}$, então existe $x \in \operatorname{Id}(R)$ com $p \in D(x)$ tal que $x \varphi \equiv x \psi$.

iii) Para todo $x \in \operatorname{Id}(R)$, vale $\varphi \equiv x \varphi \oplus x^{c} \varphi$.

Demonstração. Note que $i i) \Rightarrow i$ ). De fato, se $\varphi_{p} \cong{ }_{p} \psi_{p}$, existem formas $\varphi^{\prime}, \psi^{\prime}$ tais que $\varphi^{\prime} \cong \varphi, \psi^{\prime} \cong$ $\psi$ e $\varphi_{p}^{\prime} \equiv \psi_{p}^{\prime}$. Então, por $\left.i i\right)$, existe $x \in \operatorname{Id}(R) \operatorname{com} x \varphi^{\prime} \equiv x \psi^{\prime}$. Assim

$$
\begin{aligned}
x \varphi & \cong x \varphi^{\prime} \\
& \equiv x \psi^{\prime} \cong x \psi .
\end{aligned}
$$

Suponha $\varphi_{p} \equiv \psi_{p}$. Considere o conjunto $O=\left\{\sigma \in X_{R}: \operatorname{sgn}_{\sigma}^{ \pm}(\varphi)=\operatorname{sgn}_{\sigma}^{ \pm}(\psi)\right\}$. Como a assinatura de uma forma é função contínua, $O \subseteq X_{R}$ é aberto. Logo, como $X_{p} \subseteq O$, pelo Lema 2.18 existe $x \in \operatorname{Id}(R)$ tal que $p \in D(x)$ e $U(x) \subseteq O$. Logo $x \varphi \equiv x \psi$.

Para iii), basta notar que $\langle 1\rangle \equiv\left\langle x, x^{c}\right\rangle$.

Teorema 3.29. Seja $R$ RS von Neumann.

i) Dada forma $\varphi$ e $p \in \operatorname{spec}(R)$ tal que $\varphi_{p}$ é isotrópica em $R_{p}$, existe $x \operatorname{com} p \in D(x)$ e forma $\theta$ tal que $\varphi \equiv x\langle 1,-1\rangle \oplus \theta$. Em particular, uma forma é l-isotrópica se, e somente se, é isotrópica. 
ii) Assuma que $\operatorname{spec}(R)=\bigcup_{i=1}^{n} D\left(x_{i}\right), x_{i} \in I d(R)$, e para cada $i=1, \ldots, n$ seja $\theta_{i}$ uma forma. Se $x_{i} x_{j} \theta_{i} \equiv x_{i} x_{j} \theta_{j}$ para todo $1 \leq i, j \leq n$, então existe forma $\theta$ tal que $x_{i} \theta \equiv x_{i} \theta_{i}$ para todo $i$. Além disso $\theta$ é unicamente determinada módulo isometria.

iii) (Teorema da decomposição de Witt) Dada forma $\varphi$, existe forma anisotrópica $\psi$ com $\varphi \cong \psi$ se, e somente se, $\operatorname{ind}_{W}(\varphi)$ é função contínua. Neste caso, existe forma $\theta$ sobre $\operatorname{Id}(R)$ tal que $\varphi \equiv \theta\langle 1,-1\rangle \oplus \psi$.

Demonstração. i) Seja $\varphi$ forma com $\varphi_{p}$ isotrópica em $R_{p}$. Então existe forma $\theta$ tal que $\varphi_{p} \equiv_{p}$ $\langle 1,-1\rangle \oplus \theta_{p}$. Então pelo Lema 3.28, existe $x \in \operatorname{Id}(R) \operatorname{com} p \in D(x)$ tal que $x \varphi \equiv x\langle 1,-1\rangle \oplus x \theta$. Então

$$
\varphi \equiv x \varphi \oplus x^{c} \varphi \equiv x\langle 1,-1\rangle \oplus\left(x \theta \oplus x^{c} \varphi\right) .
$$

ii) Para $2 \leq i \leq n$, defina $y_{i}=x_{i} \cdot x_{1}^{c} \cdot x_{2}^{c} \cdot \ldots \cdot x_{i-1}^{c}$ e $y_{1}=x_{1}$. Note que $D\left(y_{i}\right)=D\left(x_{i}\right) \backslash \bigcup_{j=1}^{i-1} D\left(x_{j}\right)$ e portanto $D\left(x_{i}\right)=D\left(x_{i} y_{1}\right) \sqcup \cdots \sqcup D\left(x_{i} y_{i}\right)$. Logo $\left\langle x_{i}\right\rangle \equiv\left\langle x_{i} y_{1}, \ldots, x_{i} y_{i}\right\rangle$.

Defina $\theta=y_{1} \theta_{1} \oplus y_{2} \theta_{2} \oplus \ldots \oplus y_{n} \theta_{n}$. Como $D\left(y_{i}\right) \cap D\left(y_{j}\right)=\emptyset$ para $i \neq j$, temos $y_{i} \theta \equiv y_{i} \theta_{i}$. Então dado $1 \leq i \leq n$, como $x_{i} x_{j} \theta_{j} \equiv x_{i} x_{j} \theta_{i}$, temos

$$
\begin{aligned}
x_{i} \theta & \equiv x_{i} y_{1} \theta \oplus \cdots \oplus x_{i} y_{i} \theta \\
& \equiv x_{i} y_{1} \theta_{1} \oplus \cdots \oplus x_{i} y_{i} \theta_{i} \\
& \equiv x_{i} y_{1} \theta_{i} \oplus \cdots \oplus x_{i} y_{i} \theta_{i} \\
& \equiv\left\langle x_{i} y_{1}, \ldots, x_{i} y_{i}\right\rangle \otimes \theta_{i} \\
& \equiv x_{i} \theta_{i} .
\end{aligned}
$$

A unicidade de $\theta$ segue facilmente da hipótese $\operatorname{spec}(R)=\bigcup_{i=1}^{n} D\left(x_{i}\right)$.

iii) $\Rightarrow$ : Assuma que $\varphi \cong \psi$ com $\psi$ anisotrópica (l-anisotrópica). Então para todo $p \in \operatorname{spec}(R)$, $\operatorname{dim}_{p}(\varphi) \geq \operatorname{dim}_{p}(\psi)$. Pelo Corolário 3.10, existe forma $\theta$ sobre $\operatorname{Id}(R)$ tal que $\varphi \equiv \theta\langle 1,-1\rangle \oplus \psi$. Então para $n \geq 0$ temos $\operatorname{ind}_{W}(\varphi)^{-1}(n)=\operatorname{Od}_{n}(\theta)$ é conjunto aberto.

$\Leftarrow$ : Primeiro vamos provar que, em algum sentido, a anisotropia local pode ser levantada para um aberto de $\operatorname{spec}(R)$.

Afirmação. Sejam $p \in \operatorname{spec}(R)$ e $n=\operatorname{ind}_{W}(\varphi)_{p}$. Então existe $x \in \operatorname{Id}(R) \operatorname{com} p \in D(x)$ e uma forma $\theta$ tal que $\varphi \equiv n x\langle 1,-1\rangle \oplus \theta$ e $\theta_{q}$ é anisotrópica em $R_{q}$ para todo $q \in D(x)$.

Demonstração. Como $\operatorname{ind}_{W}(\varphi)$ é contínua, existe $e \in \operatorname{Id}(R)$ com $p \in D(e)$ tal que para todo $q \in D(e), \operatorname{ind}_{W}(\varphi)_{q}=n$. Usando a prova do primeiro item, podemos obter $x \in \operatorname{Id}(R)$ com $p \in D(x) \subseteq D(e)$ e forma $\psi$ com $\psi_{p}$ anisotrópica tal que

$$
\varphi \equiv n x\langle 1,-1\rangle \oplus x \psi \oplus x^{c} \varphi
$$

Como $\operatorname{ind}_{W}(\varphi)$ é constante em $D(x), \psi_{q}$ é anisotrópica para todo $q \in D(x)$. Então dado $q \in D(x)$, temos $\left(x \psi \oplus x^{c} \varphi\right)_{q} \equiv \psi_{q}$ anisotrópica em $R_{q}$.

Usando a compacidade de $\operatorname{spec}(R)$, pela afirmação acima existem $x_{1}, \ldots, x_{n} \in \operatorname{Id}(R)$ e formas $\theta_{1}, \ldots, \theta_{n}$ tais que

a) $\operatorname{spec}(R)=\bigcup_{i=1}^{n} D\left(x_{i}\right)$.

b) $\theta_{i}$ é anisotrópica em $D\left(x_{i}\right)$ para $i=1, \ldots, n$.

c) Para cada $i$, existe $n_{i}$ tal que $\varphi \equiv n_{i} x_{i}\langle 1,-1\rangle \oplus \theta_{i}$. 
Para usarmos $i i$, precisamos provar que as formas $\theta_{i}, \theta_{j}$ concordem em $D\left(x_{i} x_{j}\right)$. Se $x_{i} x_{j}=0$, então $x_{i} x_{j} \theta_{i} \equiv x_{i} x_{j} \theta_{j}$. Se não, seja $p \in D\left(x_{i} x_{j}\right)$. Como $\theta_{i, p}$ e $\theta_{j, p}$ são anisotrópicas, por $\left.c\right)$, $n_{i}=n_{j}$. Então escrevendo $\left.k=n_{i}, c\right)$ implica

$$
n x_{i} x_{j}\langle 1,-1\rangle \oplus x_{i} x_{j} \theta_{i} \equiv n x_{i} x_{j}\langle 1,-1\rangle \oplus x_{i} x_{j} \theta_{j}
$$

Cancelando de ambos os lados, temos $x_{i} x_{j} \theta_{i} \equiv x_{i} x_{j} \theta_{j}$. Assim, usando $i i$, é possível obter forma $\theta$ tal que $x_{i} \theta \equiv x_{i} \theta_{i}$. Então $\theta$ é anisotrópica por $b$ ) e $c$ ) implica $\varphi \cong \theta$.

Por fim, a existência da forma $\theta$ segue pelo Corolário 3.10.

Comentário 3.30. É possível provar que algumas das propriedades acima são suficientes para concluir que $R$ é von Neumann. Mais precisamente, analisando a forma $\left\langle 1,-x^{2}\right\rangle$ é possível provar que as seguintes afirmações são equivalentes:

i) $R$ é von Neumann;

ii) Para cada $\varphi$ tal que $\operatorname{ind}_{W}(\varphi):(\operatorname{spec}(R))_{\text {cons }} \rightarrow \mathbb{N}$ é contínua, existe forma $\psi$ l-anisotrópica tal que $\varphi \cong \psi$.

iii) a) Dado forma $\varphi$ e $p \in \operatorname{spec}(R)$ tal que $\varphi_{p}$ é isotrópica em $R_{p}$, existem $x \in \operatorname{Id}(R)$ e forma $\theta$ com $p \in D(x)$ tal que $\varphi \equiv x\langle 1,-1\rangle \oplus \theta$

b) Assuma que $\operatorname{spec}(R)=\bigcup_{i=1}^{n} D\left(x_{i}\right), x_{i} \in \operatorname{Id}(R)$, e para cada $i=1, \ldots, n$ seja $\theta_{i}$ forma. Se $x_{i} x_{j} \theta_{i} \equiv x_{i} x_{j} \theta_{j}$ para todo $1 \leq i, j \leq n$, então existe forma $\theta$ tal que $x_{i} \theta \equiv x_{i} \theta_{i}$ para todo $i$.

Seja WI(RS) a classe de todos os semigrupos reais $R$ tal que $\operatorname{ind}_{W}(\varphi)(\varphi): \operatorname{spec}(R) \rightarrow \mathbb{N}$ é uma função contínua para toda forma $\varphi$. Note que todo RS nessa classe é von Neumann pois se $\operatorname{ind}_{W}\left(\left\langle 1,-x^{2}\right\rangle\right)$ é contínua, então $\operatorname{ind}_{W}\left(\left\langle 1,-x^{2}\right\rangle\right)^{-1}(0)=D(x)^{c} \subseteq \operatorname{spec}(R)$ é aberto. Não sabemos se a recíproca é verdadeira mas podemos descrever alguns RS nesta classe bem como o fechamento dela por algumas construções (Teorema 3.34).

Num RS von Neumann toda forma pode ser escrita com uma quantidade mínima de entradas. Este é o conteúdo do próximo resultado que será útil para provarmos que a classe $W I(R S)$ é elementar (Teorema 3.34).

Proposição 3.31. Sejam $R$ RS von Neumann e $\varphi$ forma não-nula. Seja $n \geq \operatorname{mdim}(\varphi)$. Então existem $y_{1}, \ldots, y_{n} \in R$ tais que $\varphi \equiv\left\langle y_{1}, \ldots, y_{n}\right\rangle$.

Demonstração. Sem perda de generalidade podemos supor $n=\operatorname{mdim}(\varphi)$ pois podemos completar as entradas com $0(\varphi \equiv \varphi \oplus\langle 0\rangle)$. Seja $\left(\varphi_{1}, \ldots, \varphi_{k}\right)$ sistema ortogonal para $\varphi$. Vamos provar o desejado por indução em $k$.

$\varphi_{i}$ é uma $c_{i}$-forma com $\operatorname{Od}_{c_{i}}\left(\varphi_{i}\right)=D\left(e_{i}\right)$ para algum $e_{i} \in \operatorname{Id}(R)$ e $c_{1}<\cdots<c_{k}$. Note que $c_{k}=n$.

Se $k=1$, então $\varphi$ é $c_{1}$-forma. Então pelo Corolário 3.9 existem $a_{1}, \ldots, a_{c_{1}}$ tal que $\varphi \equiv$ $\left\langle a_{1}, \ldots, a_{c_{1}}\right\rangle$. Suponha então $k>1$. Por hipótese de indução, existem $a_{1}, \ldots, a_{c_{k-1}} \in R$ tais que $\varphi_{1} \oplus \cdots \oplus \varphi_{k-1} \equiv\left\langle a_{1}, \ldots, a_{c_{k-1}}\right\rangle$. Por outro lado, pelo Corolário 3.9, exitem $x_{1}, \ldots, x_{c_{k}} \in R$ com $x_{i}^{2}=e_{k}$ para todo $i=1, \ldots, c_{k}$ tais que $\varphi_{k} \equiv\left\langle x_{1}, \ldots, x_{c_{k}}\right\rangle$. Como $e_{k} e_{j}=0$ para todo $j=1, \ldots, k-1$, temos para todo $i=1, \ldots, c_{k}$

$$
x_{i}\left\langle a_{1}, \ldots, a_{c_{k-1}}\right\rangle \equiv x_{i}\left(\varphi_{1} \oplus \cdots \oplus \varphi_{k-1}\right) \equiv\langle 0\rangle
$$


e portanto $x_{i} a_{l}=0$ para todo $l=1, \ldots, c_{k-1}$. Para $l=1, \ldots, c_{k-1}$, seja $y_{l} \in D^{t}\left(a_{l}, x_{l}\right)$ e para $l=c_{k-1}+1, \ldots, c_{k}$ defina $y_{l}=x_{l}$. Como $x_{l} a_{l}=0$, fica fácil ver que $\left\langle y_{l}\right\rangle \equiv\left\langle a_{l}, x_{l}\right\rangle$. Deste modo

$$
\begin{aligned}
\left\langle y_{1}, \ldots, y_{c_{k}}\right\rangle & \equiv\left\langle y_{1}, \ldots, y_{c_{k-1}}\right\rangle \oplus\left\langle y_{c_{k-1}+1}, \ldots, y_{c_{k}}\right\rangle \\
& \equiv \bigoplus_{l=1}^{c_{k-1}}\left\langle a_{l}, x_{l}\right\rangle \oplus\left\langle x_{c_{k-1}+1}, \ldots, x_{c_{k}}\right\rangle \\
& \equiv \varphi_{1} \oplus \cdots \oplus \varphi_{k-1} \oplus\left\langle x_{1}, \ldots, x_{c_{k}}\right\rangle \\
& \equiv \varphi_{1} \oplus \cdots \oplus \varphi_{k} \equiv \varphi .
\end{aligned}
$$

Na próxima proposição, caracterizamos a isometria e a equivalência de formas num produto arbitrário de RSs. O ingrediente principal é o item $i v$ ) que foi provado originalmente em [DMP] e aqui demonstração mais simples é dada utilizando formas de Pfister.

Proposição 3.32. Sejam $\left\{R_{i}\right\}_{i \in I}$ família de RS e $R=\prod_{i \in I} R_{i}$. Considere também $\pi_{i}: R \rightarrow R_{i}$ projeção canônica e $\varphi, \psi$ formas.

i) O subconjunto $\bigsqcup_{i \in I} \operatorname{spec}\left(R_{i}\right):=\left\{\pi_{i}^{-1}(p) \in \operatorname{spec}(R): i \in I, p \in \operatorname{spec}\left(R_{i}\right)\right\} \subseteq \operatorname{spec}(R)$ é denso na topologia construtível.

ii) $\varphi$ é anisotrópica se, e somente se, $\pi_{i}(\varphi)$ é anisotrópica em $R_{i}$ para todo $i \in I$.

iii) $D_{R}^{t}(\varphi)=\prod_{i \in I} D_{R_{i}}^{t}\left(\pi_{i}(\varphi)\right)$.

iv) O subconjunto $\bigsqcup_{i \in I} X_{R_{i}}:=\left\{\sigma \circ \pi_{i}: i \in I, \sigma \in X_{R_{i}}\right\} \subseteq X_{R}$ é denso na topologia construtível.

v) $\varphi \equiv \psi$ se, e somente se, $\pi_{i}(\varphi) \equiv \pi_{i}(\psi)$.

vi) $\varphi \cong \psi$ se, e somente se, $\pi_{i}(\varphi) \cong \pi_{i}(\psi)$.

Demonstração. i) Seja $a, b \in \operatorname{Id}(R)$ com $D(a) \cap D(b)^{c} \neq \emptyset$. Vamos mostrar que $D(a) \cap D(b)^{c} \cap$ $\bigsqcup_{i \in I} \operatorname{spec}\left(R_{i}\right) \neq \emptyset$. Podemos escrever $a=\left(a_{i}\right)_{i \in I}, b=\left(b_{i}\right)_{i \in I}$ com $a_{i}, b_{i} \in \operatorname{Id}\left(R_{i}\right)$ para todo $i \in I$. Como $D(a) \cap D(b)^{c} \neq \emptyset$, temos $a \neq a b$ e portanto existe $i \in I$ tal que $a_{i} \neq a_{i} b_{i}$ em $R_{i}$. Logo deve existir $p \in \operatorname{spec}\left(R_{i}\right)$ tal que $\pi_{i}^{-1}(p) \in D(a) \cap D(b)^{c}$.

ii) $\Rightarrow$ : Suponha que $\varphi$ seja anisotrópica em $R$ e fixe $i \in I$. Dado $p \in \operatorname{spec}\left(R_{i}\right)$, note que $R_{\pi_{i}^{-1}(p)} \cong$ $R_{i, p}$; deste modo, como $\varphi_{\pi_{i}^{-1}(p)}$ é anisotrópica em $R_{\pi_{i}^{-1}(p)}$, temos $\pi_{i}(\varphi)_{p}$ anisotrópica em $R_{i, p}$. Como $p \in \operatorname{spec}\left(R_{i}\right)$ foi arbitrário, temos $\pi_{i}(\varphi)$ anisotrópica em $R_{i}$.

$\Leftarrow$ : Vamos provar pela contra-positiva. Se $\varphi$ é isotrópica em $R$, então existe $x \in \operatorname{Id}(R)$ não-nulo e forma $\psi$ tais que $\varphi \equiv x\langle 1,-1\rangle \oplus \psi$. Como $D(x) \neq \emptyset$, pelo item $i)$ existe $i \in I$ e $p \in \operatorname{spec}\left(R_{i}\right)$ tal que $q=\pi_{i}^{-1}(p) \in D(x)$. Assim $p \in D\left(\pi_{i}(x)\right)$ e portanto $\pi_{i}(\varphi) \equiv \pi_{i}(x)\langle 1,-1\rangle \oplus \pi_{i}(\theta)$ é isotrópica em $R_{i}$.

iii) Segue por indução no número de entradas de $\varphi$.

iv) Seja $\left[\left[a_{1}=1\right]\right] \cap \cdots \cap\left[\left[a_{n}=1\right]\right] \cap[[x=0]] \neq \emptyset$ aberto-básico não-vazio da topologia construtível. Sejam $\varphi=\bigotimes_{i=1}^{n}\left\langle a_{i}^{2}, a_{i}\right\rangle$ e $a=\prod_{i=1}^{n} a_{i}^{2}$. Pelo Comentário 3.13, temos $x^{2} \varphi \neq_{R} \varphi$. Assim, pelo Corolário $3.15 i v), x^{2} a \notin D^{t}(\varphi)$. Logo, pelo item iii) acima, existe $i \in I$ tal que $\pi_{i}\left(x^{2} a\right) \notin$ $D^{t}\left(\pi_{i}(\varphi)\right)$. Novamente pelo Corolário $\left.3.15 i v\right)$, temos que $\pi_{i}\left(x^{2}\right) \pi_{i}(\varphi) \approx_{R_{i}} \pi_{i}(\varphi)$. Portanto pelo Comentário 3.13 existe $\sigma \in\left[\left[\pi_{i}\left(a_{1}\right)=1\right]\right] \cap \cdots \cap\left[\left[\pi_{i}\left(a_{n}\right)=1\right]\right] \cap\left[\left[\pi_{i}(x)=0\right]\right]$, isto é, $\sigma \circ \pi_{i} \in\left[\left[a_{1}=1\right]\right] \cap \cdots \cap\left[\left[a_{n}=1\right]\right] \cap[[x=0]]$. 
v) A direção $\Rightarrow$ é imediata pois $\pi_{i}$ é morfismo de RS. Para a direção oposta, pelo item $\left.i v\right)$, é suficiente provarmos que para quaisquer formas $\varphi, \psi$, os conjuntos $\left\{\sigma \in X_{R}: \operatorname{sgn}_{\sigma}^{+}(\varphi)=\operatorname{sgn}_{\sigma}^{+}(\psi)\right\}$ e $\left\{\sigma \in X_{R}: \operatorname{sgn}_{\sigma}^{-}(\varphi)=\operatorname{sgn}_{\sigma}^{-}(\psi)\right\}$ são fechados na topologia construtível. Mas isto segue da continuidade das funções $\operatorname{sgn}_{+}(\varphi), \operatorname{sgn}_{-}(\varphi), \operatorname{sgn}_{+}(\psi), \operatorname{sgn}_{-}(\psi):\left(X_{R}\right)_{\text {cons }} \rightarrow \mathbb{Z}$ (Proposição $3.4 i)-f)$ ).

vi) O resultado segue pela argumentação análoga a utilizada no item iii).

Definição 3.33. Sejam $R$ RS e forma $\varphi$. Definimos os seguintes subconjuntos

$$
\begin{aligned}
\operatorname{Ani}(\varphi) & :=\left\{p \in \operatorname{spec}(R): \varphi_{p} \text { é anisotrópica em } R_{p}\right\} \\
\operatorname{Iso}(\varphi) & :=\left\{p \in \operatorname{spec}(R): \varphi_{p} \text { é isotrópica em } R_{p}\right\}
\end{aligned}
$$

Pelo Teorema 3.29, $\operatorname{Iso}(\varphi) \subseteq \operatorname{spec}(R)$ é aberto e $\operatorname{Ani}(\varphi)=\operatorname{Iso}(\varphi)^{c}$ é fechado.

Teorema 3.34. i) Seja $R R S$ von Neumann. Então $R \in W I(R S)$ se, e somente se, para toda forma $\varphi$, o subconjunto $\operatorname{Ani}(\varphi) \subseteq \operatorname{spec}(R)$ é aberto.

ii) WI(RS) é uma classe elementar. Em particular, WI(RS) é fechada por ultraprodutos.

iii) $W I(R S)$ é fechado por produtos arbitrários. Em particular, se $\left\{R_{i}\right\}_{i \in I}$ é família de 0-RSG, então $\Pi_{i \in I} R_{i} \in W I(R S)$.

Demonstração. i) $\Rightarrow$ : Suponha que $R \in \mathrm{WI}(\mathrm{RS})$ e seja $\varphi$ forma. Pelo teorema anterior existe forma $\psi$ anisotrópica tal que $\varphi \cong \psi$. Então, $\operatorname{como} \operatorname{dim}_{p}(\varphi) \geq \operatorname{dim}_{p}(\psi)$ para todo $p \in \operatorname{spec}(R)$, pelo Corolário 3.10 existe forma $\theta$ sobre $\operatorname{Id}(R)$ tal que $\varphi \equiv \theta\langle 1,-1\rangle \oplus \psi$. Portanto $\operatorname{Ani}(\varphi)=$ $\operatorname{Od}_{0}(\theta)$ é aberto.

$\Leftarrow$ : Seja $\varphi$ forma. Sejam $p \in \operatorname{spec}(R)$ e $n \in \mathbb{N}$ com $\operatorname{ind}_{W}(\varphi)_{p}=n$. Vamos provar que existe $e \in \operatorname{Id}(R)$ com $p \in D(e)$ tal que para todo $q \in D(e) \operatorname{temos} \operatorname{ind}_{W}(\varphi)_{q}=n$.

Pela prova do Teorema $3.29 i$ ), existe $x \in \operatorname{Id}(R)$ com $p \in D(x)$ e forma $\theta$ tal que

$$
\varphi \equiv n x\langle 1,-1\rangle \oplus x \theta \oplus x^{c} \varphi
$$

onde $\theta_{p}$ é anisotrópica. Como $\operatorname{Ani}(\theta)$ é aberto, existe $e \in \operatorname{Id}(R)$ com $p \in D(e) \subseteq D(x)$ tal que $\theta_{q}$ é anisotrópica para todo $q \in D(e)$. Então para todo $q \in D(e)$, temos em $R_{q}$

$$
\varphi_{q} \equiv n\langle 1,-1\rangle \oplus \theta_{q}
$$

$\mathrm{e} \operatorname{assim}_{\operatorname{ind}}(\varphi)_{q}=n$

ii) Dado $n \geq 1$, considere a fórmula $\theta_{n}^{1}\left(x_{1}, \ldots, x_{n}\right)$ dada por

$$
\exists x \exists y_{1}, \ldots, y_{n}:\left\langle x_{1}, \ldots, x_{n}\right\rangle \equiv x\langle 1,-1\rangle \oplus\left\langle y_{1}, \ldots, y_{n}\right\rangle \Rightarrow x=0 .
$$

Pelo Teorema 3.23 a fórmula $\theta_{n}^{1}$ é de primeira ordem na linguagem $\mathcal{L}_{R S}$.

Afirmação. Seja $\varphi=\left\langle a_{1}, \ldots, a_{n}\right\rangle$ forma. Então

$$
\varphi \text { é anisotrópica se, e somente se, } R \models \theta_{n}^{1}(\varphi) \text {. }
$$

Demonstração. Se $R \not \models \theta_{n}^{1}(\varphi)$, é fácil ver que $\varphi$ é isotrópica. Reciprocamente, se $\varphi$ é isotrópica, existe $x \in \operatorname{Id}(R)$ com $x \neq 0$ e forma $\psi$ tais que $\varphi \equiv x\langle 1,-1\rangle \oplus \theta$. Como para todo $p \in$ $\operatorname{spec}(R)$ temos $n \geq \operatorname{dim}_{p}(\varphi) \geq \operatorname{dim}_{p}(\theta)$, pela Proposição 3.31 existem $y_{1}, \ldots, y_{n}$ tais que $\theta \equiv\left\langle y_{1}, \ldots, y_{n}\right\rangle$. Logo $R \not \models \theta_{n}^{1}(\varphi)$. 
Fixado $n \geq 1$, considere a sentença $\theta_{n}$ dada por

$$
\begin{aligned}
\forall a_{1}, \ldots, a_{n} \exists b_{1}, \ldots, b_{n}, c_{1}, \ldots, c_{n}: & \left\langle a_{1}, \ldots, a_{n}\right\rangle \equiv\left\langle b_{1}, \ldots, b_{n}\right\rangle\langle 1,-1\rangle \oplus\left\langle c_{1}, \ldots, c_{n}\right\rangle \\
& \wedge \theta_{n}^{1}\left(c_{1}, \ldots, c_{n}\right) .
\end{aligned}
$$

Vamos mostrar $R \in \mathrm{WI}(\mathrm{RS})$ se, e somente se, para todo $n \geq 1$ vale $R \models \theta_{n}$.

$\Rightarrow$ : Seja $n \geq 1$ e considere uma forma $\varphi=\left\langle a_{1}, \ldots, a_{n}\right\rangle$. Pelo Teorema 3.29 iii $)$ e Corolário 3.10 , existem forma $\theta$ sobre $\operatorname{Id}(R)$ e forma $\psi$ anisotrópica tais que $\varphi \equiv \theta\langle 1,-1\rangle \oplus \psi$. Como $n \geq \operatorname{dim}_{p}(\varphi) \geq \operatorname{dim}_{p}(\theta)$ e $n \geq \operatorname{dim}_{p}(\psi)$ para todo $p \in \operatorname{spec}(R)$, pela Proposição 3.31 existem $b_{1}, \ldots, b_{n}, c_{1}, \ldots, c_{n} \in R$ tais que

$$
\begin{aligned}
\theta & \equiv\left\langle b_{1}, \ldots, b_{n}\right\rangle \\
\psi & \equiv\left\langle c_{1}, \ldots, c_{n}\right\rangle .
\end{aligned}
$$

Logo, pela afirmação anterior $R \models \theta_{n}$.

$\Leftarrow$ : Imediato pelo Teorema 3.29 iii).

iii) Sejam $\left\{R_{i}\right\}_{i \in I}$ família de RS em WI(RS) e $R=\prod_{i \in I} R_{i}$. Seja $\pi_{i}: R \rightarrow R_{i}$ a projeção canônica.

Afirmação. Para cada $i \in I$, seja $\varphi_{i}$ forma sobre $R_{i}$. Suponha que exista $n \geq 1$ tal que para todo $p \in \operatorname{spec}\left(R_{i}\right)$ vale $\operatorname{dim}_{p}\left(\varphi_{i}\right) \leq n$ para todo $i \in I$. Então existe forma $\varphi$ sobre $R$ tal que $\pi_{i}(\varphi) \equiv \varphi_{i}$ em $R_{i}$ para todo $i \in I$.

Demonstração. Dado $i \in I$, pela Proposição 3.31 podemos assumir $\varphi_{i}=\left\langle a_{1}^{i}, \ldots, a_{n}^{i}\right\rangle$ para $a_{1}^{i}, \ldots, a_{n}^{i} \in R_{i}$. Para $j=1, \ldots, n$, seja $a_{j}=\left(a_{j}^{i}\right)_{i \in I} \in R$ e defina $\theta=\left\langle a_{1}, \ldots, a_{n}\right\rangle$.

Vamos mostrar que $R \in \mathrm{WI}(\mathrm{RS})$. Seja $\varphi=\left\langle a_{1}, \ldots, a_{n}\right\rangle$ forma sobre $R \operatorname{com} a_{j}=\left(a_{j}^{i}\right)_{i \in I}$. Considere as formas $\varphi_{i}=\left\langle a_{1}^{i}, \ldots, a_{n}^{i}\right\rangle$ sobre $R_{i}$. Como $R_{i} \in \mathrm{WI}(\mathrm{RS})$, existe forma $\psi_{i}$ anisotrópica tal que $\varphi_{i} \cong \psi_{i}$. Pela afirmação acima, existe forma $\psi$ sobre $R$ tal que $\pi_{i}(\psi) \equiv \psi_{i}$. Logo, pela Proposição $3.32, \psi$ é anisotrópica e $\varphi \cong \psi$.

No que segue é apresentada uma caracterização da representação transversal $D^{t}$ em termos da isometria (Teorema 3.37) que generaliza a representação na teoria de formas quadráticas sobre corpos ou, mais geralmente, nos grupos especiais. Começamos com dois lemas.

Lema 3.35. Sejam $R$ RS von Neumann e $\varphi, \psi$ formas. Se $\varphi \equiv \psi$, então $D^{t}(\varphi)=D^{t}(\psi)$. Em particular, dado $a \in R$, temos que $a \in D^{t}(\varphi)$ se, e somente se, $0 \in D^{t}\left(a^{c} \varphi\right)$ e $a \in D^{t}\left(a^{2} \varphi\right)$.

Demonstração. Assumindo que $\varphi \equiv \psi$, pelo Corolário 2.64 temos

$$
\begin{aligned}
a \in D^{t}(\varphi) & \Leftrightarrow a_{p} \in D^{t}\left(\varphi_{p}\right) \text { para todo } p \in \operatorname{spec}(R) \\
& \Leftrightarrow a_{p} \in D^{t}\left(\psi_{p}\right) \text { para todo } p \in \operatorname{spec}(R) \\
& \Leftrightarrow a \in D^{t}(\psi) .
\end{aligned}
$$

Logo $D^{t}(\varphi)=D^{t}(\psi)$

Assim se $a \in D^{t}(\varphi)$, então $0=a a^{c} \in D^{t}\left(a^{c} \varphi\right)$ e $a=a a^{2} \in D^{t}\left(a^{2} \varphi\right)$. Reciprocamente, suponha que $0 \in D^{t}\left(a^{c} \varphi\right)$ e $a \in D^{t}(a \varphi)$. Como $\langle 1\rangle \equiv\left\langle a^{2}, a^{c}\right\rangle$, temos $\varphi \equiv a^{2} \varphi \oplus a^{c} \varphi$. Logo $a \in D^{t}(a, 0) \subseteq$ $D^{t}\left(a^{2} \varphi \oplus a^{c} \varphi\right)=D^{t}(\varphi)$.

Lema 3.36. Sejam $R$ RS von Neumann e $\varphi$ uma qc-forma $\operatorname{com} \operatorname{supp}(\varphi)=D(e), e \in \operatorname{Id}(R)$. Seja $a \in R$.

i) $0 \in D^{t}(\varphi)$ se, e somente se, existe forma $\theta$ tal que $e\langle 1,-1\rangle \oplus e \theta \equiv \varphi$. 
ii) Se $a^{2}=e$, então $a \in D^{t}(\varphi)$ se, e somente se, existe forma $\psi$ tal que $\langle a\rangle \oplus e \psi \equiv \varphi$.

iii) $a \in D^{t}(\varphi)$ se, e somente se, existem forma $\theta, \psi$ tais que

$$
\varphi \equiv\left(\langle a\rangle \oplus a^{2} \psi\right) \oplus\left(a^{c} e\langle 1,-1\rangle \oplus a^{c} \theta\right) .
$$

Demonstração. Pelo Lema 3.35 podemos assumir que $\varphi=\left\langle a_{1}, \ldots, a_{k}\right\rangle$ com $a_{i}^{2}=e$ para algum $k \geq 1$.

i) $\Rightarrow: \operatorname{Em} R_{e}$, temos $0 \in D_{R_{e}}^{t}(\bar{\varphi})$. Logo $-\overline{a_{1}} \in D_{R_{e}}^{t}\left(\overline{a_{2}}, \ldots, \overline{a_{k}}\right)$. Como $R_{e}^{\times}$é RSG e $\overline{a_{i}} \in R_{e}^{\times}$para todo $i=1, \ldots, k$, existem $\overline{b_{3}}, \ldots, \overline{b_{k}} \in R_{e}^{\times}$tais que

$$
\left\langle-\overline{a_{1}}, \overline{b_{3}}, \ldots, \overline{b_{k}}\right\rangle \equiv_{R_{e}}\left\langle\overline{a_{2}}, \ldots, \overline{a_{k}}\right\rangle .
$$

Portanto $\bar{\varphi} \equiv_{R_{e}}\left\langle\overline{a_{1}},-\overline{a_{1}}, \overline{b_{3}}, \ldots, \overline{b_{k}}\right\rangle \equiv_{R_{e}}\langle 1,-1\rangle \oplus\left\langle\overline{b_{3}}, \ldots, \overline{b_{k}}\right\rangle$. Deste modo, em $R$ vale

$$
\varphi \equiv e \varphi \equiv e\langle 1,-1\rangle \oplus e\left\langle b_{3}, \ldots, b_{k}\right\rangle .
$$

$\Leftarrow$ : Observe que $1 \in D^{t}(\langle 1\rangle \oplus \theta)$ e assim $e \in D^{t}(\langle e\rangle \oplus e \theta)$. Como $0 \in D^{t}(-e, e)$, temos $0 \in D^{t}(\langle-e\rangle \oplus(\langle e\rangle \oplus e \theta))=D^{t}(e\langle 1,-1\rangle \oplus e \theta)$. Pelo Lema 3.35 temos $0 \in D^{t}(\varphi)$.

ii) $\Rightarrow$ : Em $R_{e}$, temos $\bar{a} \in D_{R_{e}}^{t}(\bar{\varphi})$ com $\bar{a}$ inversível assim como os coeficientes de $\bar{\varphi}$. Como $R_{e}^{\times}$ é RSG, existem $\overline{b_{2}}, \ldots, \overline{b_{k}} \in R_{e}^{\times}$tais que $\bar{\varphi} \equiv_{R_{e}}\left\langle\bar{a}, \overline{b_{2}}, \ldots, \overline{b_{k}}\right\rangle$. Assim em $R$ temos $\varphi=e \varphi \equiv$ $\left\langle a e, e b_{2}, \ldots, e b_{k}\right\rangle \equiv\left\langle a, e b_{2}, \ldots, e b_{k}\right\rangle$.

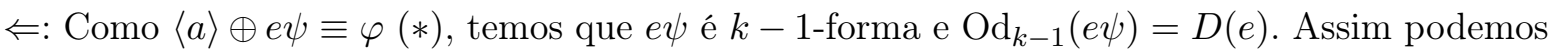
supor que $e \psi=\left\langle b_{1}, \ldots, b_{k-1}\right\rangle$ com $b_{i}^{2}=e$. Deste modo a isometria $(*)$ em $R_{e}$ implica que $\bar{a} \in D_{R_{e}}^{t}(\bar{\varphi})$. Logo, pela Proposição 1.15, em $R$ vale $a=a e \in D^{t}(e \varphi)=D^{t}(\varphi)$.

iii) Primeiro note que qualquer uma das condições da equivalência implicam $a^{2} e=a^{2}$. Com isso, pelo lema anterior e pelos itens $i$ ), $i i$ ) temos

$$
\begin{aligned}
a \in D^{t}(\varphi) & \Leftrightarrow 0 \in D^{t}\left(a^{c} \varphi\right) \text { e } a \in D^{t}\left(a^{2} \varphi\right) \\
& \Leftrightarrow \text { existem formas } \theta, \psi \text { tais que } a^{c} e\langle 1,-1\rangle \oplus a^{c} e \theta \equiv a^{c} \varphi \text { e }\langle a\rangle \oplus a^{2} e \psi \equiv a^{2} \varphi .
\end{aligned}
$$

Mas a última condição é equivalente a $\varphi \equiv a^{2} \varphi \oplus a^{c} \varphi \equiv\left(\langle a\rangle \oplus a^{2} \psi\right) \oplus\left(a^{c} e\langle 1,-1\rangle \oplus a^{c} \theta\right)$.

Teorema 3.37. Seja $R R S$ von Neumann. Sejam $\varphi$ forma com $\operatorname{supp}(\varphi)=D(f), f \in I d(R) e$ $a \in R$. Então

$$
a \in D^{t}(\varphi) \Leftrightarrow \text { existem formas } \psi, \theta \text { tais que } \varphi \equiv\left(\langle a\rangle \oplus a^{2} \psi\right) \oplus\left(a^{c} f\langle 1,-1\rangle \oplus a^{c} \theta\right) .
$$

Demonstração. A equivalência é trivial quando $\varphi \equiv\langle 0\rangle$ pois nesse caso $D^{t}(\varphi)=\{0\}$. Supondo $\varphi \not \equiv\langle 0\rangle$, seja $\left(\varphi_{1}, \ldots, \varphi_{k}\right)$ sistema ortogonal para $\varphi \operatorname{com} \varphi_{i}$ uma $c_{i}$-forma $\operatorname{Od}_{c_{i}}=D\left(e_{i}\right), e_{i} \in \operatorname{Id}(R)$, para todo $i=1, \ldots, k$. Note que $e_{i} \varphi \equiv \varphi_{i}$ para todo $i=1, \ldots, k$ e que $\langle 1\rangle \equiv\left\langle f^{c}, e_{1}, \ldots, e_{k}\right\rangle(*)$.

$\Rightarrow$ : Se $a \in D^{t}(\varphi)$, então $a_{i}:=a e_{i} \in D^{t}\left(\varphi_{i}\right)$ para $i=1, \ldots, k$ e $a f^{c}=0$. Pelo Lema 3.36, para cada $i=1, \ldots, k$ existem formas $\psi_{i}, \theta_{i}$ tais que

$$
\varphi_{i} \equiv\left(\left\langle a_{i}\right\rangle \oplus a_{i}^{2} \psi_{i}\right) \oplus\left(a_{i}^{c} e_{i}\langle 1,-1\rangle \oplus a_{i}^{c} \theta_{i}\right) .
$$


Deste modo temos

$$
\begin{aligned}
\varphi & \equiv \varphi_{1} \oplus \cdots \oplus \varphi_{k} \\
& \equiv \bigoplus_{i=1}^{k}\left(\left\langle a_{i}\right\rangle \oplus a_{i}^{2} \psi_{i}\right) \oplus\left(a_{i}^{c} e_{i}\langle 1,-1\rangle \oplus a_{i}^{c} \theta_{i}\right) \\
& \equiv\left(\left\langle a_{1}, \ldots, a_{k}\right\rangle \oplus \bigoplus_{i=1}^{k} a_{i}^{2} \psi_{i}\right) \oplus\left(\left\langle a_{1}^{c} e_{1}, \ldots a_{k}^{c} e_{k}\right\rangle\langle 1,-1\rangle \oplus \bigoplus_{i=1}^{k} a_{i}^{c} \theta_{i}\right) .
\end{aligned}
$$

Observe que, pela isometria $(*)$

$\{$ soki1\}

$$
\begin{aligned}
\left\langle a_{1}, \ldots, a_{k}\right\rangle & \equiv a\left\langle e_{1}, \ldots, e_{k}\right\rangle \\
& \equiv a\left\langle f^{c}, e_{1}, \ldots, e_{k}\right\rangle \\
& \equiv\langle a\rangle .
\end{aligned}
$$

Além disso, temos $\langle f\rangle \equiv\left\langle e_{1}, \ldots, e_{k}\right\rangle$ pois $f^{c} e_{i}=0$ para todo $i=1, \ldots, k$. Pela Proposição 2.59 vale $a_{i}^{c} e_{i}=a^{c} e_{i}$ e portanto

\{soki2\}

$$
\begin{aligned}
\left\langle a_{1}^{c} e_{1}, \ldots, a_{k}^{c} e_{k}\right\rangle & =\left\langle a^{c} e_{1}, \ldots, a^{c} e_{k}\right\rangle \\
& \equiv a^{c}\left\langle e_{1}, \ldots, e_{k}\right\rangle \\
& \equiv\left\langle a^{c} f\right\rangle .
\end{aligned}
$$

Substituindo 3.9 e 3.10 em 3.8 temos

$$
\varphi \equiv\left(\langle a\rangle \oplus \bigoplus_{i=1}^{k} a_{i}^{2} \psi_{i}\right) \oplus\left(a^{c} f\langle 1,-1\rangle \oplus \bigoplus_{i=1}^{k} a_{i}^{c} \theta_{i}\right) .
$$

Sejam $\psi=\bigoplus_{i=1}^{k} a_{i}^{2} \psi_{i}$ e $\theta=\bigoplus_{i=1}^{k} a_{i}^{c} \theta_{i}$. É fácil ver que $a^{2} \psi=\psi$. Por outro lado, por 3.7 temos $\langle 0\rangle \equiv e_{i}^{c} \varphi \equiv e_{i}^{c} a_{i}^{c} \theta_{i}$. Logo $a_{i}^{c} e_{i} \theta_{i} \equiv a_{i}^{c} \theta_{i}$. Assim como $a_{i}^{c} e_{i}=a^{c} e_{i}$ temos

$$
\begin{aligned}
a^{c} \theta & =\bigoplus_{i=1}^{k} a^{c} a_{i}^{c} \theta_{i} \equiv \bigoplus_{i=1}^{k} a^{c} a_{i}^{c} e_{i} \theta_{i} \\
& =\bigoplus_{i=1}^{k} a_{i}^{c} e_{i} \theta_{i} \equiv \bigoplus_{i=1}^{k} a_{i}^{c} \theta_{i}=\theta .
\end{aligned}
$$

Portanto vale $\varphi \equiv\left(\langle a\rangle \oplus a^{2} \psi\right) \oplus\left(a^{c} f\langle 1,-1\rangle \oplus a^{c} \theta\right)$.

$\Leftarrow$ : Suponha que existam formas $\psi, \theta$ satifazendo $\varphi \equiv\left(\langle a\rangle \oplus a^{2} \psi\right) \oplus\left(a^{c} f\langle 1,-1\rangle \oplus a^{c} \theta\right)$. Em particular como $f^{c} \varphi \equiv\langle 0\rangle$, temos $a f^{c}=0$ e para todo $i$ vale que $a_{i}:=a e_{i}$ satisfaz

$$
\varphi_{i} \equiv e_{i} \varphi \equiv\left(\left\langle a_{i}\right\rangle \oplus a_{i}^{2} \psi\right) \oplus\left(a_{i}^{c} e_{i}\langle 1,-1\rangle \oplus a_{i}^{c} e_{i} \theta\right)
$$

pois $a_{i}^{c} e_{i}=a^{c} e_{i}$ e $a^{c} e^{c} e_{i}=a^{c} e_{i}=a_{i}^{c} e_{i}$. Logo, pelo Lema 3.36, $a_{i} \in D^{t}\left(\varphi_{i}\right)$. Assim, como $1 \in D^{t}\left(f^{c}, e_{1}, \ldots, e_{k}\right)$, temos $a \in D^{t}\left(a f^{c}, a_{1}, \ldots, a_{k}\right)=D^{t}\left(a_{1}, \ldots, a_{k}\right)$. Logo $a \in D^{t}\left(\varphi_{1} \oplus \cdots \oplus \varphi_{k}\right)=$ $D^{t}(\varphi)$.

$\{$ aniHG\}

Corolário 3.38. Sejam $R$ RS von Neumann e $\varphi$ uma forma. Seja $e \in \operatorname{Id}(R) \operatorname{com} \operatorname{supp}(\varphi)=D(e)$. São equivalentes:

i) $\varphi$ é anisotrópica.

ii) Para todo $x \in D^{t}(\varphi), x^{2}=e$. 
Demonstração. $i) \Rightarrow i i)$ : Seja $x \in D^{t}(\varphi)$. Pelo Teorema 3.37, temos $x^{c} e=0$. Por outro lado, $x e^{c} \in D^{t}\left(e^{c} \varphi\right)=\{0\}$. Logo $x^{2}=e$.

$i i) \Rightarrow i)$ : Suponha que $\varphi$ é isotrópica. Vamos mostrar que existe $z \in D^{t}(\varphi)$ tal que $z^{c} e \neq 0$.

Afirmação. Seja $\psi$ uma qc-forma $\operatorname{com} \operatorname{supp}(\psi)=D(f), \theta$ forma e $x \in \operatorname{Id}(R)$ não-nulo. Se $\psi \equiv$ $x\langle 1,-1\rangle \oplus \theta(*)$, então existe $y \in D^{t}(\psi)$ tal que $y^{c} f=x$.

Demonstração. Note que $x^{c} \theta \equiv x^{c} \psi$ é forma nula ou qc-forma com suporte $D\left(x^{c} f\right)$. Em qualquer caso, existe $y \in D^{t}\left(x^{c} \theta\right)$ tal que $y^{2}=x^{c} f(* *)$. Primeiro note que $0 \in D^{t}(x\langle 1,-1\rangle \oplus x \theta)$ pois dado qualquer $l \in D^{t}(\theta)$, temos $-x l \in D^{t}(x,-x), x l \in D^{t}(x \theta)$ e $0 \in D^{t}(-x l, x l)$. Deste modo, como $\psi \equiv x \psi \oplus x^{c} \psi \equiv x\langle 1,-1\rangle \oplus x \theta \oplus x^{c} \theta$, temos que $y \in D^{t}(\psi)$. Assim a relação $(* *)$ implica que $y^{c} \in D^{t}\left(f^{c}, x\right)$; mas como por $(*)$ temos $x f=x$ vale $y^{c} f \in D^{t}(0, f x)=\{x\}$.

Seja $\left(\varphi_{1}, \ldots, \varphi_{k}\right)$ sistema ortogonal para $\varphi$ onde $\varphi_{i}$ é uma $c_{i}$-forma com $\operatorname{Od}_{c_{i}}\left(\varphi_{i}\right)=D\left(e_{i}\right)$. Assumindo que $\varphi$ é isotrópica, seja $x \in \operatorname{Id}(R)$ não-nulo e $\theta$ forma tais que $\varphi \equiv x\langle 1,-1\rangle \oplus \theta$. Como $x \neq 0$, existe $i=1, \ldots, k$ tal que $x e_{i} \neq 0$. Então $\varphi_{i} \equiv e_{i} \varphi \equiv x e_{i}\langle 1,-1\rangle \oplus e_{i} \theta$. Pela afirmação, existe $y \in D^{t}\left(\varphi_{i}\right)$ tal que $y^{c} e_{i}=x e_{i}$. Para cada $j=1, \ldots, k, j \neq i$, escolha algum $y_{j} \in D^{t}\left(\varphi_{j}\right)$. Seja $z \in D^{t}\left(y_{1} e_{1}, \ldots, y_{i-1} e_{i-1}, y e_{i}, y_{i+1} e_{i+1}, \ldots, y_{k} e_{k}\right)$. Note que $z e^{c}=0$ e $z e_{j} \in D^{t}\left(\varphi_{j}\right)$ para todo $j=1, \ldots, k$. Então $z \in D^{t}(\varphi)$. Como $x e_{i} \neq 0$, tomando $\sigma \in X_{R}$ com $\sigma\left(x e_{i}\right)=1$, vemos que $\sigma(e)=1$ e $\sigma\left(z^{c}\right)=\sigma\left(y^{c}\right)=1$. Assim $z^{c} e \neq 0$.

Como último resultado dessa seção, vamos provar o Teorema da Subforma de Pfister para RSs von Neumann, o que generaliza o resultado homônio para RSG em [AS]. Diferentemente do que acontece em RSG, num RS se $\psi \equiv \varphi \oplus \theta$ não temos necessariamente que $D^{t}(\varphi) \subseteq D^{t}(\psi)$ : $\langle 1,1\rangle \equiv\langle 0,0\rangle \oplus\langle 1,1\rangle$ mas $D^{t}(\langle 0,0\rangle) \nsubseteq D^{t}(\langle 1,1\rangle)$. No entanto, como preliminar do resultado principal, vale o seguinte:

Proposição 3.39. Sejam $R \operatorname{RS}$ e $\varphi, \psi$ formas com $\operatorname{Ani}(\psi) \cap \operatorname{supp}(\psi) \subseteq \operatorname{supp}(\varphi)$. Se existe forma $\theta$ com $\psi \equiv \varphi \oplus \theta$, então $D^{t}(\varphi) \subseteq D^{t}(\psi)$.

Demonstração. Seja $x \in D^{t}(\varphi)$. Considere $p \in \operatorname{spec}(R)$ ideal primo e $\pi: R \rightarrow K_{R}(p)$ a projeção canônica. Então $\pi(x) \in D_{K_{R}(p)}^{t}(\pi \star \varphi)$ e $\pi \star \psi \equiv_{p} \pi \star \varphi \oplus \pi \star \theta$. Se $\pi \star \varphi \equiv_{p}\langle 0\rangle$, ou seja, $\operatorname{dim}_{p}(\varphi)=0$, então $\pi(x)=0$ e por hipótese vale $\operatorname{dim}_{p}(\psi)=0$ ou $\pi \star \psi$ isotrópica. Em qualquer um dos casos, segue que $\pi(x) \in D_{K_{R}(p)}^{t}(\pi \star \psi)$. Se $\operatorname{dim}_{p}(\varphi) \neq 0$, então $\operatorname{dim}_{p}(\psi) \neq 0$ e portanto temos $\pi(x) \in$ $D_{K_{R}(p)}^{t}(\pi \star \psi)$. Assim, pelo Princípio local-global fraco de Brocker (Teorema 6.7.1 de [Mar7]) vale $x \in D^{t}(\psi)$.

Comentário 3.40. Não é possível enfraquecer a hipótese da Proposição 3.39 porque se temos $D^{t}(\varphi) \subseteq D^{t}(\psi)$, então $\operatorname{Ani}(\psi) \cap \operatorname{supp}(\psi) \subseteq \operatorname{supp}(\varphi)$.

Definição 3.41. Sejam $R$ RS e $\varphi, \psi$ formas. $\varphi$ é dita subforma de $\psi$ se existe forma $\theta$ tal que $\psi \equiv \varphi \oplus \theta$.

Comentários 3.42. • A forma nula é uma subforma de toda forma.

- Suponha $R$ é von Neumann e $\varphi, \psi$ formas. Se $\varphi \cong \psi$ e $\operatorname{dim}(\psi) \geq \operatorname{dim}(\varphi)$, então pelo Corolário 3.10 temos que $\varphi$ é subforma de $\psi$.

Dado $R$ RS, pelo Teorema $2.32 R$ é multi-anel com a multi-soma dada por $D_{R}^{t}$.

Teorema 3.43 (Teorema de subforma de Pfister). Sejam $R$ RS von Neumann e $\varphi, \psi=\left\langle a_{1}, \ldots, a_{n}\right\rangle$ \{subform\} formas. Suponha que $\psi$ seja anisotrópica e que para todo morfismo de multi-aneis $f: R \rightarrow G$ com $G$ hipercorpo especial valha $D_{G}^{t}(f \star \varphi) \subseteq f\left(a_{1}\right)+\cdots+f\left(a_{n}\right)$. Então existe forma $\theta$ sobre $R$ tal que $\psi \equiv_{R} \varphi \oplus \theta$. 
Demonstração. Sejam $p \in \operatorname{spec}(R)$ ideal primo de $R$ e $\pi_{p}: R \rightarrow R_{p}$ a projeção natural. Então $\pi_{p} \star \psi \equiv \psi_{p}$ é anisotrópica sobre $R_{p}$ e dado $\bar{f}: R_{p} \rightarrow L$ morfismo com $L$ hipercorpo especial temos $D_{L}^{t}\left(\bar{f} \star \varphi_{p}\right)=D_{L}^{t}\left(\left(\bar{f} \circ \pi_{p}\right) \star \varphi\right) \subseteq \bar{f}\left(\pi_{p}\left(a_{1}\right)\right)+\cdots+\bar{f}\left(\pi_{p}\left(a_{n}\right)\right)$. Supondo $\varphi_{p}, \psi_{p}$ formas não-nulas, pelo Teorema de subforma de Pfister para RSG (Teorema 3 de [AS]), o 0-RSG $R_{p}$ satisfaz a seguinte fórmula:

$$
\exists a_{1}, \ldots, a_{n}: \pi_{p} \star \varphi \oplus\left\langle a_{1}, \ldots, a_{n}\right\rangle \equiv \pi_{p} \star \psi,
$$

onde $n \geq 1$ é a quantidade de entradas de $\psi$. Note que esta é uma pp-fórmula para RSs von Neumann na linguagem dos RSs pelo Teorema 3.23. Se $\psi_{p} \equiv_{p}\langle 0\rangle$, então $D_{R_{p}}^{t}\left(\varphi_{p}\right)=\{0\}$ e assim $\varphi_{p} \equiv_{p}\langle 0\rangle$. Neste caso, $R_{p}$ também satisfaz a fórmula acima. Se $\varphi_{p} \equiv_{p}\langle 0\rangle$, então a fórmula acima é trivialmente satisfeita por $R_{p}$. Assim, pelo Teorema 2.62, existe forma $\theta$ sobre $R$ tal que $\varphi \oplus \theta \equiv \psi$.

Comentário 3.44. $\quad$ - O Teorema acima só vale para RSs von Neumann. De fato, seja $R$ RS. Dado $x \in \operatorname{Id}(R), x \neq 1$, considere as formas anisotrópicas $\psi=\langle 1,1, x\rangle$ e $\varphi=\langle 1, x, x\rangle$. Dado morfismo $f: R \rightarrow G$ com $G$ hipercorpo especial, temos dois casos: se $f(x)=0$, então $D_{G}^{t}(f \star \varphi)=\{1\} \subseteq 1+1=f(1)+f(1)+f(x)$; se $f(x)=1$, então $D_{R}^{t}(f \star \varphi)=\{1\} \subseteq$ $1+1+1=f(1)+f(1)+f(x)$. Assim, se o Teorema 3.43 valer para $R$, existe forma $\theta$ sobre $R$ tal que $\langle 1,1, x\rangle \equiv\langle 1, x, x\rangle \oplus \theta$. Então $\theta$ é uma 1-forma com $\operatorname{Od}_{1}(\theta)=D(x)^{c}$. Pelo Teorema $3.8 \mathrm{iv})$ temos que $D(x)^{c}=\operatorname{Od}_{1}(\theta) \subseteq \operatorname{spec}(R)$ é aberto. Logo $R$ é von Neumann.

- A noção de forma anisotrópica para semigrupos reais aqui adotada pode ser muito restritiva e na conclusão da tese mostramos uma alternativa. No entanto, para qualquer enfraquecimento, o argumento do comentário acima continua valendo. Seria interessante pensar em generalizações do Teorema da subforma de Pfister que sejam válidas em classes de RSs mais amplas como a dos Booleanos. Um palpite é que, em virtude do Teorema 4.12 ii), alguma condição além da continência de representação transversal precisa ser acrescentada.

\subsection{Anel de Witt}

Seja $R$ RS. O anel de Witt de $R$ é definido como o conjunto $W(R)$ de todas as formas sobre $R$ módulo a equivalência de Witt munido da noção usual de soma e produto de formas. Pela Proposição 3.4 as operações estão bem definidas. O elemento nulo do anel é $\langle 0\rangle$, a unidade é $\langle 1\rangle$ e dada forma $\varphi=\left\langle a_{1}, \ldots a_{n}\right\rangle$, o oposto no anel é $-\varphi=\left\langle-a_{1}, \ldots-a_{n}\right\rangle$. Quando não haver risco de confusão, a forma $\varphi$ e sua classe de equivalência em $W(R)$ serão denotadas pelo mesmo símbolo.

Nesta seção, vamos mostrar alguns resultados estruturais sobre $W(R)$. Note que quando $R$ é 0-RSG, pelo Exemplo 3.2 a presente noção de anel de Witt coincide com a noção clássica. Além disso, dado $f: R \rightarrow G$ morfismo de RSs, então mapa $\mathrm{W}(f): \mathrm{W}(R) \rightarrow \mathrm{W}(G)$ dado por $\mathrm{W}(f)\left(\left\langle a_{1}, \ldots, a_{n}\right\rangle\right)=\left\langle f\left(a_{1}\right), \ldots, f\left(a_{n}\right)\right\rangle$ é morfismo de anéis (denotamos $\left\langle f\left(a_{1}\right), \ldots, f\left(a_{n}\right)\right\rangle$ por $f \star \varphi)$.

Lema 3.45. Sejam $A$ multi-anel, $B$ anel e $f: A \rightarrow B$ mapa. Suponha que o mapa $f$ satisfaça as seguintes propriedades para $a, b, c \in A$ :

i) $f(0)=0, f(1)=1$ e $f(-a)=-f(a)$.

ii) $f(a b)=f(a) f(b)$.

iii) Se $a \in b+c$, então $f(a)+f(a b c)=f(b)+f(c)$.

Então $\sigma \circ f: A \rightarrow 3 \in \operatorname{sper}(A)$ para todo $\sigma \in \operatorname{sper}(B)$.

Demonstração. Pelas propriedades $i), i i)$ de $f$, é suficiente provar que se $a \in b+c$, então $\sigma(f(a)) \in$ $\sigma(f(b))+\sigma(f(c))(*)$. Pela propriedade $i i i)$, temos $f(a)+f(a b c)=f(b)+f(c)$. 
Caso 1: $\sigma(f(b))=0$ ou $\sigma(f(c))=0$.

Se $\sigma(f(b))=0$, então $\sigma(f(b)+f(c)) \in \sigma(f(b))+\sigma(f(c))=\{\sigma(f(c))\}$. Por outro lado $\sigma(f(a)+$ $f(a b c)) \in \sigma(f(a))+\sigma(f(a b c))=\{f(a)\}$. Logo $\sigma(f(a))=\sigma(f(c))$ e a relação $(*)$ se verifica. O caso $\sigma(f(c))=0$ é análogo.

Caso 2: $\sigma(f(b))=\sigma(f(c)) \neq 0$.

Neste caso temos $\sigma(f(b)+f(c)) \in \sigma(f(b))+\sigma(f(c))=\{\sigma(f(b))\}$ e como $\sigma(f(b c))=1$ também temos $\sigma(f(a)+\sigma(f(a b c))) \in \sigma(f(a))+\sigma(f(a b c))=\sigma(f(a))+\sigma(f(a))=\{\sigma(f(a))\}$. Logo $\sigma(f(a))=\sigma(f(b))$ e a relação $(*)$ se verifica.

Caso 3: $\sigma(f(b))=-\sigma(f(c)) \neq 0$.

Aqui vale trivialmente $\sigma(f(a)) \in \sigma(f(b))+\sigma(f(c))$.

Deste modo, pelos 3 casos acima, temos $\sigma \circ f$ morfismo de multi-anéis e portanto $\sigma \circ f \in$ $\operatorname{sper}(A)$.

Seja $R$ RS e considere o mapa $i: R \rightarrow \mathrm{W}(R)$ dado por $i(x)=\langle x\rangle$ para todo $x \in R$. Pela Proposição $3.4 v)$, dados $a, b, c \in R$, se $a \in D^{t}(b, c)$, então $i(a)+i(a b c)=i(b)+i(c)$. Assim, pelo lema anterior, dado $\tau \in \operatorname{sper}(W(R))$, temos $\tau \circ i \in X_{R}$. Para o próximo resultado, denota-se por sgn: $\mathbb{Z} \rightarrow 3$ a única ordem de $\mathbb{Z}$

Proposição 3.46. Seja $R$ RS. Considere os seguintes mapas:

$$
\begin{aligned}
& f:\left(X_{R}\right)_{\text {const }} \rightarrow \operatorname{sper}(\mathrm{W}(R)) \\
& \sigma \mapsto f_{\sigma}: \mathrm{W}(R) \rightarrow 3 \\
& \varphi \quad \mapsto \operatorname{sgn}\left(\operatorname{sgn}_{\sigma}(\varphi)\right) \\
& g: X_{R} \rightarrow \operatorname{Hom}(\mathrm{W}(R), \mathbb{Z}) \\
& \sigma \mapsto g_{\sigma}: \mathrm{W}(R) \quad \rightarrow \quad \mathbb{Z} \\
& \varphi \quad \mapsto \operatorname{sgn}_{\sigma}(\varphi) \\
& h: \operatorname{sper}(\mathrm{W}(R)) \rightarrow \operatorname{Hom}(\mathrm{W}(R), \mathbb{Z}) \\
& \tau \mapsto h_{\tau}: \mathrm{W}(R) \quad \rightarrow \quad \mathbb{Z} \\
& \varphi \quad \mapsto \operatorname{sgn}_{\tau \circ i}(\varphi)
\end{aligned}
$$

Então todos esses mapas são bijetores e $f$ é homeomorfismo. Além disso o diagrama abaixo comuta.

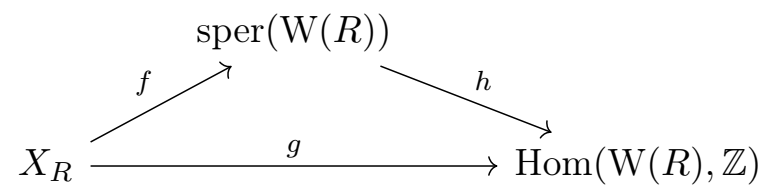

Demonstração. $\quad f:$ Dado $\sigma \in X_{R}$ temos que $f_{\sigma}=\operatorname{sgn} \circ \operatorname{sgn}_{\sigma}$ onde $\operatorname{sgn}_{\sigma}: W(R) \rightarrow \mathbb{Z}$ é morfismo de anéis pela Proposição 3.4 e sgn: $\mathbb{Z} \rightarrow 3$ é a única ordem de $\mathbb{Z}$. Assim $f_{\sigma} \in \operatorname{sper}(W(R))$. Note agora que o mapa $f$ é injetor pois dados $\sigma \neq \tau$ em $X_{R}$, existe $a \in R$ tal que $\sigma(a) \neq \tau(a)$ e portanto $f_{\sigma}(\langle a\rangle)=\sigma(a) \neq \tau(a)=f_{\tau}(\langle a\rangle)$. Para a sobrejetividade, dado $\Sigma \in \operatorname{sper}(W(R))$, defina $\sigma=\Sigma \circ i$, onde $i: R \rightarrow W(R)$ é o mapa de inclusão. Pelo lema anterior, $\sigma \in X_{R}$. Para vermos que $\Sigma=f_{\sigma}$ é suficiente provarmos que dados $a_{1}, \ldots, a_{n} \in R, \Sigma\left(\left\langle a_{1}, \cdots, a_{n}\right\rangle\right)=$ $\operatorname{sgn}\left(\sigma\left(a_{1}\right)+\cdots+\sigma\left(a_{n}\right)\right)$. Seja $P=\operatorname{supp}(\Sigma)$ ideal primo de $\mathrm{W}(R)$. Dado $a \in R$, como $a^{3}=a$, existe $t \in\{1,0,-1\}$ tal que $\langle a,-t\rangle \in P$. Assim existem $t_{1}, \ldots, t_{n} \in\{-1,0,1\}$ tal que $\left\langle a_{i},-t_{i}\right\rangle \in P$. Logo $\Sigma(\varphi)=\Sigma\left(\left\langle t_{1}, \ldots, t_{n}\right\rangle\right)$. Além disso, como $\Sigma\left(\left\langle a_{i}\right\rangle\right)=\sigma\left(t_{i}\right)=t_{i}$ e $\Sigma\left(\left\langle t_{1}, \ldots, t_{n}\right\rangle\right)=\operatorname{sgn}\left(t_{1}+\cdots+t_{n}\right), \operatorname{temos} \Sigma(\varphi)=\operatorname{sgn}\left(t_{1}+\cdots+t_{n}\right)=\operatorname{sgn}\left(\sigma\left(a_{1}\right)+\cdots+\sigma\left(a_{n}\right)\right)$. 
Para concluir que $f$ é homeomorfismo, note que dada forma $\varphi$,

$$
f^{-1}\left(U_{W(R)}(\varphi)\right)=\left\{\sigma \in X_{R}: \operatorname{sgn}_{\sigma}(\varphi) \geq 1\right\}=\operatorname{sgn}(\varphi)^{-1}(\{n: n \geq 1\})
$$

é aberto pela Proposição 3.4. Por outro lado, dado $a \in R$, temos

$$
\begin{aligned}
& f\left(U_{R}(a)\right)=\{\tau \in \operatorname{sper}(W(R)): \tau \circ i(a)=1\}=U_{W(R}(\langle a\rangle) \\
& f\left(Z_{R}(a)\right)=\{\tau \in \operatorname{sper}(W(R)): \tau \circ i(a)=0\}=U_{W(R)}\left(\left\langle 1,-a^{2}\right\rangle\right)
\end{aligned}
$$

e portanto $f$ é homeomorfismo.

$g:$ Note que dado $\sigma \in X_{R}$, temos que $g_{\sigma}=\operatorname{sgn}_{\sigma}: W(R) \rightarrow \mathbb{Z}$ é morfismo de anéis por 3.4. A injetividade de $g$ segue pelo mesmo argumento da injetividade de $f$. Para a sobrejetividade, dado $\alpha: \mathrm{W}(R) \rightarrow \mathbb{Z}$ morfismo de anéis, defina $\Sigma=\operatorname{sgn} \circ \alpha \in \operatorname{sper}(W(R))$ e considere $\sigma=$ $\Sigma \circ i \in X_{R}$, onde $i: R \rightarrow \mathrm{W}(R)$ denota o morfismo de inclusão. Então dados $a_{1}, \ldots, a_{n} \in R$ temos que $\alpha\left(\left\langle a_{1}, \ldots, a_{n}\right\rangle\right)=\alpha\left(i\left(a_{1}\right)\right)+\cdots+\alpha\left(i\left(a_{n}\right)\right)=\sigma\left(a_{1}\right)+\cdots+\sigma\left(a_{n}\right)=g_{\sigma}\left(\left\langle a_{1}, \ldots, a_{n}\right\rangle\right)$ para todo . Logo $g_{\sigma}=\alpha$ e $g$ é sobrejetora.

$h$ : Dado $\tau \in \operatorname{sper}(W(R))$, pelo demonstração da sobrejetividade de $f$ vale $\tau \circ i=f^{-1}(\tau)$. Então para toda forma $\varphi$ em $W(R)$ temos $h_{\tau}(\varphi)=\operatorname{sgn}_{f^{-1}(\tau)}(\varphi)=g_{f^{-1}(\tau)}(\varphi)$. Logo $h=g f^{-1}$ e $h$ é bijetora.

Seja $R$ RS. Para cada $\sigma \in X_{R}$ e $p \in \mathbb{Z}$ número primo, sejam $P_{\sigma, p}:=\left\{\varphi \in \mathrm{W}(R): \operatorname{sgn}_{\sigma}(\varphi) \in(p)\right\}$ e $P_{\sigma}:=\left\{\varphi \in \mathrm{W}(R): \operatorname{sgn}_{\sigma}(\varphi)=0\right\}$. Como $\operatorname{sgn}_{\sigma}: W(R) \rightarrow \mathbb{Z}$ é morfismo de anéis (sobrejetor), $P_{\sigma, p}$ e $P_{\sigma}$ são ideais primos e temos os isomorfismos $W(R) / P_{\sigma} \cong \mathbb{Z}$ e ${ }^{W(R)} / P_{\sigma, p} \cong \mathbb{Z}_{p}$. Note que $P_{\sigma} \subseteq P_{\sigma, p}$, para todo inteiro $n \in \mathbb{Z}$ não-nulo vale $n\langle 1\rangle \notin P_{\sigma}$ e $p\langle 1\rangle \in P_{\sigma, p}$.

Proposição 3.47. Sejam $R$ RS e $\sigma, \tau \in X_{R}$. Então $P_{\sigma, 2}=P_{\tau, 2}$ se, e somente se, $\operatorname{supp}(\sigma)=$ $\operatorname{supp}(\tau) \in \operatorname{spec}(R)$.

Demonstração. Basta notar que $P_{\sigma, 2} \cap R=\operatorname{supp}(\sigma)$ e que pela proposição 3.4 para uma forma $\varphi$ temos $\varphi \in P_{\sigma, 2}$ se, e somente se, $\operatorname{dim}_{\operatorname{supp}(\sigma)}(\varphi)$ é par.

Note que, diferentemente do que acontece com RSG, o anel de Witt de um semigrupo real pode ter mais de um ideal primo $P$ com $2 \in P$. De fato, $\mathrm{W}(R)$ tem apenas um ideal primo contendo o número 2 se, e somente se, $R$ é 0-RSG (se $R$ é RS com apenas um ideal primo, então $R$ é 0-RSG).

Proposição 3.48. Seja $R$ RS. Então $\operatorname{spec}(\mathrm{W}(R))=\left\{P_{\sigma, p}: \sigma \in X_{R}\right.$ e $p \in \mathbb{Z}$ número primo $\} \cup$ $\left\{P_{\sigma}: \sigma \in X_{R}\right\}$.

Demonstração. Seja $P$ ideal primo de $\mathrm{W}(R)$. Dado $a \in R$, como $a^{3}=a$ e $P \subseteq \mathrm{W}(R)$ é primo, existe $t \in\{0,1,-1\}$ tal que $\langle a,-t\rangle \in P$. Por outro lado, como $R$ gera $\mathrm{W}(R)$ aditivamente, a inclusão canônica $i_{P}: \mathbb{Z} \rightarrow \mathrm{W}(R) / P$ é sobrejetora. Se este mapa é injetor, pela Proposição 3.46 existe $\sigma \in X_{R}$ tal que $i_{P}^{-1} \circ \pi=\operatorname{sgn}_{\sigma}: W(R) \rightarrow \mathbb{Z}$ onde $\pi: W(R) \rightarrow W(R) / P$ é a projeção canônica. Então $P=\pi^{-1}(0)=\left(i_{p}^{-1} \circ \pi\right)^{-1}(0)=\operatorname{sgn}_{\sigma}^{-1}(0)=P_{\sigma}$.

Se o mapa $i_{p}$ não é injetor, então existe $p \in \mathbb{Z}$ número primo tal que o morfismo $i_{P}$ induz $\mathbb{Z}_{p} \cong \mathrm{W}(R) / P$. Assim $P$ é maximal e $p\langle 1\rangle \in P$. Nessas condições deve existir ideal primo $Q$ tal que $Q \subsetneq P$. De fato, caso contrário, o ideal primo $P$ seria maximal e minimal ao mesmo tempo e isso implicaria que a localização $W(R)_{P}$ do anel $\mathrm{W}(R)$ no ideal primo $P$ teria único ideal primo $P W(R)_{P}$. Mas como a intersecção de todos os ideais primos são os nilpotentes, dada forma $\varphi \in P$, existiria $n \geq 1$ e forma $\psi \notin P$ tais que $\varphi^{n} \psi=0$. Aplicando isso para $\varphi=p\langle 1\rangle \in P$, vemos que deveria existir forma $\psi$ não-nula tal que $\psi \oplus \cdots \oplus \psi=0$ em $W(R)$, o que é um absurdo. Considere então $Q$ ideal primo propriamente contido em $P$.

Se $i_{Q}$ não fosse injetivo, então $Q$ também seria maximal e assim $Q=P$, uma contradição. Então $i_{Q}$ é injetivo. Vimos que, nesse caso, deve existir $\sigma \in X_{R}$ tal que $Q=P_{\sigma}$. Mas como $\mathbb{Z} \cong \mathrm{W}(R) / Q$ temos que $P$ é o único ideal primo $I$ tal que $Q \subseteq I$ e $p\langle 1\rangle \in I$. Logo $P=P_{\sigma, p}$. 
Corolário 3.49. Seja $R$ RS. Então $Q(W(R))$ é a álgebra de Post de $R$.

Demonstração. Primeiro vamos provar que $Q(W(R))$ é álgebra de Post usando a Proposição IV.7.1 de [DP2] (nessa referência, para um anel semi-real $A, Q(A)$ é denotado por $G_{A}$ ). Pela Proposição 3.48 , os ideias primos reais de $\mathrm{W}(R)$ são da forma $P_{\sigma}, \sigma \in X_{R}$, e ${ }^{W(R)} / P_{\sigma} \cong \mathbb{Z}$. Deste modo, ${ }^{W(R)} / P_{\sigma}$ admite única ordem. Para verificarmos que os ideias primos reais são dois a dois incomparáveis, sejam $\sigma, \tau \in X_{R}$ diferentes. Seja $a \in R \operatorname{com} \sigma(a) \neq \tau(a)$. Se $\sigma(a)=0$ ou $\tau(a)=0$, então $\langle a\rangle \in P_{\sigma} \triangle P_{\tau}$ (diferença simétrica); se $\sigma(a)=-\tau(a) \neq 0$, então $\langle 1, a\rangle \in P_{\sigma} \triangle P_{\tau}$ e portanto $P_{\sigma}$ e $P_{\tau}$ são incomparáveis.

Para concluir que $Q(W(R))$ é a álgebra de Post de $R$, é suficiente mostrar que $X_{Q(W(R))} \cong$ $\left(X_{R}\right)_{\text {const }}$. Pela Proposição 2.40 , temos $X_{Q(W(R))} \cong \operatorname{sper}(W(R))$ e pela Proposição 3.46 vale $\operatorname{sper}(W(R)) \cong\left(X_{R}\right)_{\text {const }}$. Logo $X_{Q(W(R))} \cong\left(X_{R}\right)_{\text {const }}$.

Note que se $\varphi \in \mathrm{W}(R)$ é inversível, $\operatorname{sgn}_{\sigma}(\varphi) \in\{1,-1\}$ para todo $\sigma \in X_{R}$ e assim $\varphi^{2}=1 \mathrm{em}$ $\mathrm{W}(R)$. Em geral é possível que existam formas $\varphi \operatorname{com} \varphi^{2}=1$ sem que $\varphi$ seja representável em $R$, ou seja, sem que exista $r \in R$ tal que $\varphi \cong\langle r\rangle$. O fenômeno acontece quando $R$ não é Booleano. De fato, dado $a \in R$, considere a forma $\varphi_{a}=\left\langle a, a^{2},-1\right\rangle$. É fácil ver que $\varphi_{a}^{2}=1 \mathrm{em} W(R)$ mas $\varphi_{a}$ só é representável precisamente quando $\nabla(a) \in R$ e neste caso $\langle\nabla(a)\rangle \cong \varphi_{a}$. O curioso é que, em certo sentido, as formas $\varphi_{a}$ são os únicos exemplos desse fenômeno. Este é o conteúdo do próximo resultado.

Definição 3.50. Sejam $R$ RS. Uma forma $\varphi$ é dita regular se $\operatorname{dim}_{p}(\varphi) \equiv \operatorname{dim}_{q}(\varphi) \bmod 2$ para todos $p, q \in \operatorname{spec}(R)$.

Teorema 3.51. Seja $R R S$.

i) São equivalentes:

a) $R$ é Booleano.

b) Se $\varphi \in W(R)$ é inversivel, então existe $r \in R^{\times}$tal que $\varphi \cong\langle r\rangle$.

c) Se $\varphi$ é uma forma regular, então existem $b_{1}, \ldots, b_{k} \in R^{\times}$tais que $\varphi \cong\left\langle b_{1}, \ldots, b_{k}\right\rangle$, onde $k=\operatorname{mdim}(\varphi)$.

ii) São equivalentes:

a) $R$ é von Neumann.

b) Se $\varphi$ é forma satisfazendo $\varphi^{3}=\varphi$ em $W(R)$, então existe $a \in R$ tal que $\varphi \cong\langle a\rangle$.

c) Se $\varphi$ é forma satisfazendo $\varphi^{2}=\varphi$, então existe $a \in \operatorname{Id}(R)$ tal que $\varphi \cong\langle a\rangle$.

d) Suponha que $\varphi$ é forma com $\operatorname{sgn}_{\sigma}(\varphi) \geq 0$ para todo $\sigma \in X_{R}$ e se $\sigma, \tau \in X_{R}$ são ordens com mesmo suporte, então $\operatorname{sgn}_{\sigma}(\varphi)=\operatorname{sgn}_{\tau}(\varphi)$. Então existem $a_{1}, \ldots, a_{n} \in I d(R)$ tais que $\varphi \cong\left\langle a_{1}, \ldots, a_{n}\right\rangle$.

Demonstração. i) As equivalências vão ser provadas na sequência $a) \Rightarrow b) \Rightarrow c) \Rightarrow a$ ).

$a) \Rightarrow b)$ : Seja $\varphi=\left\langle a_{1}, \ldots, a_{n}\right\rangle$ forma satisfazendo $\operatorname{sgn}_{\sigma}(\varphi) \in\{1,-1\}$ para todo $\sigma \in X_{R}$.

Afirmação. Existem $e_{1}, \ldots, e_{d} \in R^{\times}$e $n_{1}, \ldots, n_{d} \in\{0,1\}$ tais que $X_{R}=\bigsqcup_{i=1}^{d} U\left(e_{i}\right)$ e para todo $\sigma \in U\left(e_{i}\right),(-1)^{\left.\operatorname{dim}_{\sigma}(\varphi)\left(\operatorname{dim}_{\sigma}(\varphi)-1\right)\right) / 2}=(-1)^{n_{i}}$.

Demonstração. Pelo Teorema 3.8 existem $a_{i}, b_{i} \in \operatorname{Id}(R)$ e $d_{i} \geq 0, i=1, \ldots, d$, tais que $\operatorname{spec}(R)=\bigsqcup_{i=1}^{d} D\left(a_{i}\right) \cap D\left(b_{i}\right)^{c}$ e para todo $p \in D\left(a_{i}\right) \cap D\left(b_{i}\right)^{c}$ temos $\operatorname{dim}_{p}(\varphi)=d_{i}$. Deste modo $X_{R}=\bigsqcup_{i=1}^{d} U\left(a_{i}\right) \cap Z\left(b_{i}\right)$ e para todo $\sigma \in U\left(a_{i}\right) \cap Z\left(b_{i}\right) \operatorname{temos}(-1)^{\left.\operatorname{dim}_{\sigma}(\varphi)\left(\operatorname{dim}_{\sigma}(\varphi)-1\right)\right) / 2}=(-1)^{n_{i}}$ onde $n_{i} \in\{0,1\}$ satisfaz $n_{i} \equiv \frac{d_{i}\left(d_{i}-1\right)}{2} \bmod 2$. Para finalizarmos basta notar que $U\left(a_{i}\right) \cap Z\left(b_{i}\right)=$ $U\left(\nabla\left(a_{i} \cdot-\nabla\left(b_{i}\right)\right)\right)$ : se $\sigma \in U\left(a_{i}\right) \cap Z\left(b_{i}\right)$, então $\sigma\left(-\nabla\left(b_{i}\right)\right)=1$ e $\nabla\left(a_{i} \cdot-\nabla\left(b_{i}\right)\right)=1$; reciprocamente, se $\sigma\left(\nabla\left(a_{i} \cdot-\nabla\left(b_{i}\right)\right)\right)=1$, então $\sigma\left(a_{i}\right)=1$ e $\sigma\left(b_{i}\right)=0$ pois $a_{i}, b_{i} \in \operatorname{Id}(R)$. 
Afirmação. Existe $x \in R^{\times}$tal que para todo $\sigma \in X_{R}$ temos $\sigma(x)=(-1)^{\left.\operatorname{dim}_{\sigma}(\varphi)\left(\operatorname{dim}_{\sigma}(\varphi)-1\right)\right) / 2}$.

Demonstração. Usando a afirmação anterior, defina $x=\left(-e_{1}\right)^{n_{1}} \cdot \ldots \cdot\left(-e_{d}\right)^{n_{d}}$. Assim, dado $\sigma \in X_{R}$, existe único $i$ com $\sigma \in U\left(e_{i}\right)$ e portanto $\sigma(x)=\left(-\sigma\left(e_{1}\right)\right)^{n_{1}} \cdot \ldots \cdot\left(-\sigma\left(e_{d}\right)\right)^{n_{d}}=(-1)^{n_{i}}=$ $(-1)^{\left.\operatorname{dim}_{\sigma}(\varphi)\left(\operatorname{dim}_{\sigma}(\varphi)-1\right)\right) / 2}$.

Agora podemos provar o desejado. Seja $x \in R^{\times}$o elemento da afirmação anterior e defina $r=x \Delta\left(a_{1}\right) \cdot \ldots \cdot \Delta\left(a_{n}\right)$. Vamos provar que para todo $\sigma \in X_{R}$ vale $\sigma(r)=\operatorname{sgn}_{\sigma}(\varphi)$. Fixe $\sigma \in X_{R}$. Sejam $k=\operatorname{dim}_{\sigma}(\varphi), P=\operatorname{sgn}_{\sigma}^{+}(\varphi)$ e $N=\operatorname{sgn}_{\sigma}^{-}(\varphi) . \operatorname{Comosgn}_{\sigma}(\varphi) \in\{1,-1\}$, considere também $e \in\{0,1\}$ tal que $\operatorname{sgn}_{\sigma}(\varphi)=(-1)^{e}=1-2 e$. Notando que $\sigma(r)=(-1)^{k(k-1) / 2}$. $(-1)^{N}$, precisamos mostrar que $k(k-1) / 2+N \equiv e \bmod 2$. Primeiro observe que as constantes satisfazem $P+N=k$ e $P-N=(-1)^{e}=1-2 e$. Deste modo $k=2 P-(-1)^{e}=2(P+e)-1$ é impar e $k(k-1) / 2=k(P+e-1) \equiv P+e-1 \bmod 2$. Portanto

$$
k(k-1) / 2+N \equiv N+P+e-1=(k-1)+e \equiv e \bmod 2 .
$$

Portanto temos $\sigma(r)=\operatorname{sgn}_{\sigma}(\varphi)$.

$b) \Rightarrow c)$ : Seja $\varphi=\left\langle a_{1}, \ldots, a_{n}\right\rangle$ forma regular. Considere o conjunto $\left\{k_{1}<\ldots<k_{t}\right\}=\{k \geq$ 1: $\left.\operatorname{Od}_{k}(\varphi) \neq \emptyset\right\}$. Note que $k_{t}=\operatorname{mdim}(\varphi)$ e pela regularidade de $\varphi$ temos $k_{i} \equiv k_{t} \bmod 2$ para todo $i=1, \ldots, t$. Suponha inicialmente que $\operatorname{supp}(\varphi)=\operatorname{spec}(R)$.

Pelo Teorema 3.8, podemos escrever $\varphi$ como

$$
\begin{aligned}
\varphi & \cong \bigoplus_{1 \leq i \leq t} \bigoplus_{f \in S_{n, k_{i}}} \pi_{f}\left\langle 1,-\Sigma_{f}\right\rangle\left\langle a_{f_{1}}, \ldots, a_{f_{k_{i}}}\right\rangle \\
& \cong \bigoplus_{1 \leq i \leq t} \bigoplus_{f \in S_{n, k_{i}}} \pi_{f}\left\langle 1,-\Sigma_{f}\right\rangle\left[\left\langle a_{f_{1}}, \ldots, a_{f_{k_{i}}}\right\rangle \oplus \frac{k_{t}-k_{i}}{2}\langle 1,-1\rangle\right] .
\end{aligned}
$$

Fixe $1 \leq i \leq t$ e $f \in S_{n, k_{i}}$. Para cada $j=1, \ldots, k_{t}$, defina

$$
x_{i, f, j}= \begin{cases}a_{f_{j}} & \text { if } j \leq k_{i} \\ (-1)^{j} & \text { if } j>k_{i} .\end{cases}
$$

Então podemos reescrever $\varphi$ como

$$
\begin{aligned}
\varphi & \cong \bigoplus_{1 \leq i \leq t} \bigoplus_{f \in S_{n, k_{i}}} \pi_{f}\left\langle 1,-\Sigma_{f}\right\rangle\left\langle x_{i, f, 1}, \ldots, x_{i, f, k_{t}}\right\rangle \\
& \cong \bigoplus_{1 \leq j \leq k_{t}} \bigoplus_{1 \leq i \leq t} \bigoplus_{f \in S_{n, k_{i}}} \pi_{f}\left\langle 1,-\Sigma_{f}\right\rangle\left\langle x_{i, f, j}\right\rangle .
\end{aligned}
$$

Deste modo, para cada $j=1, \ldots, k_{t}$, defina $\varphi_{j}=\bigoplus_{1 \leq i \leq t} \bigoplus_{f \in S_{n, k_{i}}} \pi_{f}\left\langle 1,-\Sigma_{f}\right\rangle\left\langle x_{i, f, j}\right\rangle$. Para podermos usar a hipótese vamos verificar que $\varphi_{j}^{2}=1 \mathrm{em} W(R)$.

Note que, $\operatorname{como} \operatorname{supp}(\varphi)=\operatorname{spec}(R)$, temos pelo Teorema 3.8

$$
\begin{aligned}
\operatorname{spec}(R) & =\bigsqcup_{i=1}^{t} \operatorname{Od}_{k_{i}}(\varphi) \\
& =\bigsqcup_{i=1}^{t} \bigsqcup_{f \in S_{n, k_{i}}} D\left(\pi_{f}\right) \cap D\left(\Sigma_{f}\right)^{c} .
\end{aligned}
$$


Seja $\sigma \in X_{R}$. Seja $1 \leq l \leq t$ e $g \in S_{n, k_{i}}$ tais que $\operatorname{supp}(\sigma) \in D\left(\pi_{g}\right) \cap D\left(\Sigma_{g}\right)^{c}$. Como a união em 3.12 é disjunta, temos

$$
\begin{aligned}
\operatorname{sgn}_{\sigma}\left(\varphi_{j}\right) & =\operatorname{sgn}_{\sigma}\left(\pi_{g}\left\langle 1,-\Sigma_{g}\right\rangle\left\langle x_{l, g, j}\right\rangle\right) \\
& =\sigma\left(x_{l, g, j}\right) \in\{1,-1\} .
\end{aligned}
$$

Assim, por hipótese, deve existir $b_{j} \in R^{\times}$tal que $\varphi_{j} \cong\left\langle b_{j}\right\rangle$ e portanto por 3.11 temos $\varphi \cong$ $\left\langle b_{1}, \ldots, b_{k_{t}}\right\rangle$.

Suponha agora que $\operatorname{supp}(\varphi)=D(b) \operatorname{com} b \in \operatorname{Id}(R)$ satisfazendo $b \neq 1$. Isto significa que $\operatorname{Od}_{0}(\varphi)=D(b)^{c} \neq \emptyset$ e portanto pela regularidade temos $k_{i} \equiv 0 \bmod 2$ para todo $i=1, \ldots, t$. Neste caso podemos adaptar a demonstração acima para obtermos o mesmo resultado. Seguindo a mesma notação acima, para cada $j=1, \ldots, k_{t}$ defina

$$
\varphi_{j}=(-1)^{j}\langle 1,-b\rangle \oplus \bigoplus_{1 \leq i \leq t} \bigoplus_{f \in S_{n, k_{i}}} \pi_{f}\left\langle 1,-\Sigma_{f}\right\rangle\left\langle x_{i, f, j}\right\rangle .
$$

Como $k_{t}$ é par, temos pela equação 3.11

$$
\bigoplus_{j=1}^{k_{t}} \varphi_{j} \cong \frac{k_{t}}{2}\langle 1,-1\rangle\langle 1,-b\rangle \oplus \varphi \cong \varphi .
$$

Por outro lado, pelo Teorema 3.8 podemos escrever

$$
\begin{aligned}
\operatorname{spec}(R) & =\bigsqcup_{k \geq 0} \operatorname{Od}_{k}(\varphi) \\
& =D(b)^{c} \sqcup \bigsqcup_{i=1}^{t} \bigsqcup_{f \in S_{n, k_{i}}} D\left(\pi_{f}\right) \cap D\left(\Sigma_{f}\right)^{c}
\end{aligned}
$$

Pela mesma argumentação do primeiro caso é possível provar que $\varphi_{j}^{2}=1 \mathrm{em} W(R)$ para todo $j$. Logo, por hipótese, devem existir $b_{1}, \ldots, b_{k_{t}} \in R^{\times}$tais que $\varphi \cong\left\langle b_{1}, \ldots, b_{k_{t}}\right\rangle$.

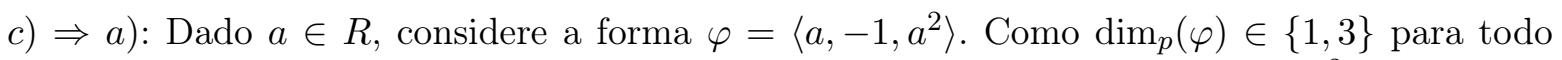
$p \in \operatorname{spec}(R), \varphi$ é regular e por $c)$ devem existir $x, y, z \in R^{\times}$tais que $\left\langle a,-1, a^{2}\right\rangle \cong\langle x, y, z\rangle$. Então note que $\nabla(a)=-x y z \in R^{\times}$. De fato, seja $\sigma \in X_{R}$. Se $\sigma(a) \in\{0,-1\}$, então $\sigma(x)+$ $\sigma(y)+\sigma(z)=\operatorname{sgn}(\varphi)=-1 ; \operatorname{logo} \sigma(x y z)=1$ e portanto $\sigma(\nabla(a))=-1=\sigma(-x y z)$. Se $\sigma(a)=1$, então $\sigma(x)+\sigma(y)+\sigma(z)=\operatorname{sgn}_{\sigma}(\varphi)=1$. Logo $\sigma(x y z)=-1$ e portanto $\sigma(\nabla(a))=1=\sigma(-x y z)$.

ii) A prova segue a sequência $a) \Rightarrow b) \Rightarrow c) \Rightarrow a), a) \Rightarrow d) \Rightarrow a$ ).

$a) \Rightarrow b$ ): Vamos começar provando o resultado em um caso particular.

Afirmação. Seja $\varphi$ qc-forma $\operatorname{com} \varphi^{3}=\varphi$. Então existe $r \in R$ tal que $\varphi \cong\langle r\rangle$.

Demonstração. Pelo Corolário 3.9 podemos assumir que $\varphi=\left\langle a_{1}, \ldots, a_{k}\right\rangle$ para algum $k \geq 1 \mathrm{e}$ que existe $b \in \operatorname{Id}(R)$ tal que $a_{i}^{2}=b$ para todo $i=1, \ldots, k$. Notando que para todo $\sigma \in X_{R}$ vale $\operatorname{sgn}_{\sigma}(\varphi) \in\{1,0,-1\}$, pela Proposição $\left.3.4 i\right) e$ ) temos dois casos: ou $k$ é par e para todo $\sigma \in X_{R}$ vale $\operatorname{sgn}_{\sigma}(\varphi)=0$ ou $k$ é ímpar e para todo $\sigma \in \operatorname{supp}(\varphi)=D(b)$ vale $\operatorname{sgn}_{\sigma}(\varphi) \in\{1,-1\}$. Se $k$ é par, basta tomar $r=0 \in R$. Supondo $k$ ímpar, seja $\bar{\varphi}$ a imagem de $\varphi$ em $R_{b}$. Como $R_{b}$ é von Neumann, pelo Teorema $2.58 R_{b}$ é Booleano. Além disso, $\bar{\varphi}^{2}=1 \mathrm{em} W\left(R_{b}\right)$. Logo, pelo item anterior existe, $r \in R$ tal que $\bar{\varphi} \cong\langle\bar{r}\rangle$. $\operatorname{Logo} \varphi \equiv b \varphi \cong\langle b r\rangle$.

Assuma agora que $\varphi$ é uma forma qualquer satisfazendo $\varphi^{3}=\varphi$ e suponha sem perda de generalidade que $\varphi \not \equiv\langle 0\rangle$. Como $R$ é von Neumann, pelo Corolário 3.19 existe sistema ortogonal $\left(\varphi_{1}, \cdots, \varphi_{k}\right)$ tal que $\varphi \equiv \varphi_{1} \oplus \ldots \oplus \varphi_{k}$. Como $\operatorname{sgn}_{\sigma}(\varphi) \in\{1,0,-1\}$ para todo $\sigma \in X_{R}$, temos que 
o mesmo vale para cada $\varphi_{i}$ e portanto pela afirmação acima existe $r_{i} \in R$ tal que $\varphi_{i} \cong\left\langle r_{i}\right\rangle(*)$. Tome então $r \in D^{t}\left(r_{1}, \ldots, r_{k}\right)$. Vamos mostrar que $\varphi \cong\langle r\rangle$. Seja $\sigma \in X_{R}$. Se $\operatorname{supp}(\sigma) \in$ $\operatorname{supp}(\varphi)$, então existe único $i$ tal que $\operatorname{supp}(\sigma) \in \operatorname{supp}\left(\varphi_{i}\right)$. Logo, por $(*)$, temos $\sigma\left(r_{j}\right)=0$ para todo $j \neq i$ e portanto $\sigma(r)=\sigma\left(r_{i}\right)$. Deste modo $\operatorname{sgn}_{\sigma}(\varphi)=\operatorname{sgn}_{\sigma}\left(\varphi_{i}\right)=\sigma\left(r_{i}\right)=\sigma(r)$. Se $\operatorname{supp}(\sigma) \notin \operatorname{supp}(\varphi)$, então $0=\operatorname{sgn}_{\sigma}\left(\varphi_{i}\right)=\sigma\left(r_{i}\right)$ e portanto $\operatorname{sgn}_{\sigma}(\varphi)=0=\sigma(r)$.

b) $\Rightarrow c)$ : Imediato.

c) $\Rightarrow a$ ): Dado $a \in \operatorname{Id}(R)$, considere a forma $\varphi=\left\langle 1,-a^{2}\right\rangle$. Como $\varphi^{2}=\varphi$ em $\mathrm{W}(R)$, por hipótese existe $x \in \operatorname{Id}(R)$ tal que $\varphi \cong\langle x\rangle$. Comparando as assinaturas fica fácil ver que $x=a^{c}$.

$a) \Rightarrow d)$ : Seja $\varphi$ forma satisfazendo as hipóteses de $d$ ). Pelo Lema 2.18, para cada $p \in \operatorname{spec}(R)$ existe $x_{p} \in \operatorname{Id}(R)$ e $n_{p} \in \mathbb{N}$ tais que $p \in D\left(x_{p}\right)$ e $x_{p} \varphi \cong x_{p} n_{p}\langle 1\rangle$. Então pela compacidade de $\operatorname{spec}(R)$ existem $x_{1}, \ldots, x_{k} \in \operatorname{Id}(R)$ e $n_{1}, \ldots, n_{k} \in \mathbb{N}$ tais que $\operatorname{spec}(R)=\bigcup_{i=1}^{n} D\left(x_{i}\right)$ e para todo $i=1, \ldots, k$ vale $x_{i} \varphi \cong x_{i} n_{i}\langle 1\rangle$. Então definindo $y_{1}=x_{1}$ e $y_{i}=x_{i} \cdot x_{1}^{c} \cdot \ldots \cdot x_{i-1}^{c}$ para $i=2, \ldots, k$, temos $\operatorname{spec}(R)=\bigsqcup_{i=1}^{n} D\left(y_{i}\right)$ e $y_{i} \varphi \cong y_{i} n_{i}\langle 1\rangle$. Logo $\varphi \cong y_{1} n_{1}\langle 1\rangle \oplus \cdots \oplus y_{k} n_{k}\langle 1\rangle$.

$d) \Rightarrow a)$ : Dado $a \in R$, seja $\varphi=\left\langle 1,-a^{2}\right\rangle$. Note que $\varphi$ satisfaz a hipótese de $d$ ) e assim existe forma $\theta$ sobre $\operatorname{Id}(R)$ tal que $\varphi \cong \theta$. Mas como $\operatorname{dim}_{\sigma}(\theta)=\operatorname{sgn}_{\sigma}(\theta) \in\{0,1\}$ para todo $\sigma \in X_{R}$, temos que $\theta$ é uma 1-forma. Então pelo Corolário 3.9 existe $x \in R$ tal que $\left\langle 1,-a^{2}\right\rangle \cong \theta \cong\langle x\rangle$. Comparando as assinaturas fica fácil ver que $x=a^{c}$.

Comentários 3.52. Alguns dos resultados provados no Teorema 3.51 acima já foram apresentados em [Mar7] na linguagem dos ARSs. Aqui, porém, as demonstrações são diferentes.

No item $i$ ) do Teorema 3.51:

- O Teorema 8.3.5 de [Mar7] é uma versão mais geral da implicação $a) \Rightarrow b$ ). A demonstração aqui apresentada é construtível, permitindo fabricar $r$ a partir das entradas de $\varphi$.

- Uma versão levemente mais fraca do item $c$ ) é apresentada no Teorema 7.6.6 de [Mar7]. A diferença está na limitação da quantidade de entradas inversíveis suficientes para construir forma equivalente a $\varphi$ e, novamente, a demonstração aqui apresentada é construtível.

No item $i$ ) do Teorema 3.51:

- O Corolário 8.3.6 de [Mar7] prova versões mais gerais que os itens $b$ ) e $c$ ) (note que num RSG se uma forma é inversível, então ela é representável).

Corolário 3.53. Sejam $R$ RS von Neumann e $\varphi$ forma. Então existe único $a \in \operatorname{Id}(R)$ e formas $\theta, \varphi_{0}, \varphi_{1}$ satisfazendo as seguintes propriedades:

i) $\theta$ é forma sobre $\operatorname{Id}(R)$.

ii) $\varphi_{0}$ e $\varphi_{1}$ são formas sobre $R^{\times}$.

iii) A dimensão de $\varphi_{0}$ é $\max \left\{\operatorname{dim}_{p}(\varphi): p \in \operatorname{spec}(R)\right.$ e $\operatorname{dim}_{p}(\varphi)$ é $\left.\operatorname{par}\right\}$.

iv) A dimensão de $\varphi_{1}$ é $\max \left\{\operatorname{dim}_{p}(\varphi): p \in \operatorname{spec}(R)\right.$ e $\operatorname{dim}_{p}(\varphi)$ é ímpar $\}$.

v) $\varphi \oplus \theta\langle 1,-1\rangle \equiv a \varphi_{0} \oplus a^{c} \varphi_{1}$.

Além disso $\theta$ é unicamente determinada módulo isometria e se $\varphi_{0}^{\prime}$ e $\varphi_{1}^{\prime}$ são outras formas $R^{\times}$ satisfazendo as propriedades acima, então $a \varphi_{0} \equiv a \varphi_{0}^{\prime}$ e $a^{c} \varphi_{1} \equiv a^{c} \varphi_{1}^{\prime}$.

Demonstração. Considere os números

$$
\begin{aligned}
& l_{0}=\max \left\{\operatorname{dim}_{p}(\varphi): p \in \operatorname{spec}(R) \text { e } \operatorname{dim}_{p}(\varphi) \text { é } \operatorname{par}\right\} \\
& l_{1}=\max \left\{\operatorname{dim}_{p}(\varphi): p \in \operatorname{spec}(R) \text { e } \operatorname{dim}_{p}(\varphi) \text { é ímpar }\right\}
\end{aligned}
$$


Como $R$ é von Neumann e o mapa de dimensão é contínuo, existe $a \in \operatorname{Id}(R)$ tal que $\{p \in$ $\operatorname{spec}(R): \operatorname{dim}_{p}(\varphi)$ é par $\}=D(a)$. Então como a imagem de $\varphi$ em $R_{a}$ e $R_{a^{c}}$ é regular, podemos aplicar o Teorema $3.51 c$ ) (note que todo von Neumann é Booleano) para obter formas $\varphi_{0}$ e $\varphi_{1}$ sobre $R^{\times}$com dimensões $l_{0}$ e $l_{1}$ respectivamente tais que $\varphi \cong a \varphi_{0} \oplus a^{c} \varphi_{1}$. Mas como temos $\operatorname{dim}_{p}(\varphi) \leq$ $\operatorname{dim}_{p}\left(a \varphi_{0} \oplus a^{c} \varphi_{1}\right)$ para todo $p \in \operatorname{spec}(R)$, pelo Corolário 3.10 existe forma $\theta$ sobre $\operatorname{Id}(R)$ tal que $\varphi \oplus \theta\langle 1,-1\rangle \equiv a \varphi_{0} \oplus a^{c} \varphi_{1}$.

Assuma que $b \in \operatorname{Id}(R)$ e $\theta^{\prime}, \varphi_{0}^{\prime}, \varphi_{1}^{\prime}$ sejam formas satisfazendo $\left.i\right)-v$ ). Então pela isometria $v$ ) temos $D(b)=\left\{p \in \operatorname{spec}(R): \operatorname{dim}_{p}(\varphi)\right.$ é par $\}=D(a)$ e assim $a=b$. Isto também implica que $\varphi \oplus \theta\langle 1,-1\rangle$ e $\varphi \oplus \theta^{\prime}\langle 1,-1\rangle$ têm sempre a mesma dimensão e portando $\varphi \oplus \theta\langle 1,-1\rangle \equiv \varphi \oplus \theta^{\prime}\langle 1,-1\rangle$. Assim $\theta\langle 1,-1\rangle \equiv \theta^{\prime}\langle 1,-1\rangle$. Como $\theta, \theta^{\prime}$ são formas sobre $\operatorname{Id}(R)$, isto implica que $\theta \equiv \theta^{\prime}$. Para terminarmos, notando que $a \varphi_{0} \oplus a^{c} \varphi_{1} \equiv a \varphi_{0}^{\prime} \oplus a^{c} \varphi_{1}^{\prime}$, temos $a \varphi_{0} \equiv a\left(a \varphi_{0} \oplus a^{c} \varphi_{1}\right) \equiv a\left(a \varphi_{0}^{\prime} \oplus a^{c} \varphi_{1}^{\prime}\right) a \varphi_{0}^{\prime}$ e da mesma maneira $a^{c} \varphi_{1}^{\prime} \equiv a^{c} \varphi_{1}^{\prime}$.

Definição 3.54. Seja $R$ RS. Considere $\mathrm{I}(R)=\left\{\varphi \in \mathrm{W}(R): \operatorname{dim}_{p}(\varphi) \equiv 0 \bmod 2\right.$ para todo $p \in$ $\operatorname{spec}(R)\}$ o ideal das formas de dimensão sempre par. $\mathrm{I}(R)$ é chamado de ideal fundamental de $\mathrm{W}(R)$.

Lema 3.55. Seja $R$ RS. Sejam $a_{i}, b_{i} \in \operatorname{Id}(R), i=1, \ldots, n$. Então existem $c_{j}, d_{j} \in \operatorname{Id}(R), j=$ $1, \ldots, k$, tais que $\left\{D\left(c_{j}\right) \cap D\left(d_{j}\right)^{c}: j=1, \ldots, k\right\}$ é um refinamento disjunto de $\left\{D\left(a_{i}\right) \cap D\left(b_{i}\right)^{c}: i=\right.$ $1, \ldots, n\}$, ou seja,

i) $\left\{D\left(c_{j}\right) \cap D\left(d_{j}\right)^{c}: j=1, \ldots, k\right\}$ é família disjunta.

ii) Dado $j \in\{1, \ldots, k\}$, existe $i \in\{1, \ldots, n\}$ tal que $D\left(c_{j}\right) \cap D\left(d_{j}\right)^{c} \subseteq D\left(a_{i}\right) \cap D\left(b_{i}\right)^{c}$.

iii) $\bigsqcup_{j=1}^{k} D\left(c_{j}\right) \cap D\left(d_{j}\right)^{c}=\bigcup_{i=1}^{n} D\left(a_{i}\right) \cap D\left(b_{i}\right)^{c}$.

Demonstração. Considere a família $\mathcal{F}=\{(J, K): J, K \subseteq\{1, \ldots, n\}$ tais que $J \cap K \neq \emptyset\}$. Para cada $(J, K) \in \mathcal{F}$, sejam

- $a_{J, K}:=\Pi_{j \in J} a_{j} \Pi_{k \notin K} b_{k}$ (convenção: se $K=\{1, \ldots, n\}$, então $\Pi_{k \notin K} b_{k}=1$ ).

- $b_{J, K}$ é o único idempontente em $D^{t}\left(c_{J, K}, d_{J, K}\right)$, onde $c_{J, K} \in D^{t}\left(\left\langle a_{j}\right\rangle_{j \notin J}\right)$ e $d_{J, K} \in D^{t}\left(\left\langle b_{k}\right\rangle_{k \in K}\right)$ (convenção: se $J=\{1, \ldots, n\}$, então $D^{t}\left(\left\langle a_{j}\right\rangle_{j \notin J}\right)=\{0\}$ ).

Deste modo, no espaço $\operatorname{spec}(R)$ valem as seguintes igualdades

$$
\begin{aligned}
D\left(a_{J, K}\right) & =\left(\bigcap_{j \in J} D\left(a_{j}\right)\right) \cap\left(\bigcap_{k \notin K} D\left(b_{k}\right)\right) \\
D\left(b_{J, K}\right) & =D\left(c_{J, K}\right) \cup D\left(d_{J, K}\right) \\
& =\left(\bigcup_{j \notin J} D\left(a_{j}\right)\right) \cup\left(\bigcup_{k \in K} D\left(b_{k}\right)\right) .
\end{aligned}
$$

Vamos mostrar que a família $\left\{D\left(a_{J, K}\right) \cap D\left(b_{J, K}\right)^{c}:(J, K) \in \mathcal{F}\right\}$ é o refinamento disjunto do enunciado começando pelas propriedades $i i)$ e iii). Fixados $(J, K) \in \mathcal{F}$, note que dado $i \in J \cap K$, vale $D\left(a_{J, K}\right) \subseteq D\left(a_{i}\right)$ e $D\left(b_{i}\right) \subseteq D\left(b_{J, K}\right)$. Logo $D\left(a_{J, K}\right) \cap D\left(b_{J, K}\right)^{c} \subseteq D\left(a_{i}\right) \cap D\left(b_{i}\right)^{c}$. Por outro lado, fixado $i \in\{1, \ldots, n\}$ e $p \in D\left(a_{i}\right) \cap D\left(b_{i}\right)^{c}$, considere os conjuntos $J=\left\{j \in\{1, \ldots, n\}: p \in D\left(a_{j}\right)\right\}$ e $K=\left\{k \in\{1, \ldots, n\}: p \notin D\left(b_{k}\right)\right\}$. Então $i \in J \cap K \mathrm{e}$

$$
p \in D\left(a_{J, K}\right) \cap D\left(b_{J, K}\right)^{c} .
$$

Para completar a prova, vamos provar que a família em questão é disjunta. Sejam $(J, K),\left(J^{\prime}, K^{\prime}\right) \in$ $\mathcal{F}$ pares distintos. Se existe $j \in J \backslash J^{\prime}$, então $D\left(a_{J, K}\right) \cap D\left(b_{J, K}\right)^{c} \subseteq D\left(a_{J, K}\right) \subseteq D\left(a_{j}\right)$ e $D\left(a_{J^{\prime}, K^{\prime}}\right) \cap$ $D\left(b_{J^{\prime}, K^{\prime}}\right)^{c} \subseteq D\left(b_{J^{\prime}, K^{\prime}}\right)^{c} \subseteq D\left(a_{j}\right)^{c}$. Por outro lado, se existe $k \in K \backslash K^{\prime}$, então $D\left(a_{J, K}\right) \cap D\left(b_{J, K}\right)^{c} \subseteq$ $D\left(b_{J, K}\right)^{c} \subseteq D\left(b_{k}\right)^{c}$ e $D\left(a_{J^{\prime}, K^{\prime}}\right) \cap D\left(b_{J^{\prime}, K^{\prime}}\right)^{c} \subseteq D\left(a_{J^{\prime}, K^{\prime}}\right) \subseteq D\left(b_{k}\right)$. Em ambos os casos, $D\left(a_{J, K}\right) \cap$ $D\left(b_{J, K}\right)^{c}$ e $D\left(a_{J^{\prime}, K^{\prime}}\right) \cap D\left(b_{J^{\prime}, K^{\prime}}\right)^{c}$ são disjuntos. 
Teorema 3.56. Seja R RS. Então

i) $W(R) /_{I(R)} \cong C\left((\operatorname{spec}(R))_{\text {cons }}, \mathbb{Z}_{2}\right)$.

ii) $I(R)$ é ideal primo se, e somente se, $R$ é 0-RSG;

iii) $I(R)$ é aditivamente gerado por formas de Pfister de grau 1.

Demonstração. i) Considere o mapa $\operatorname{dim}_{2}: \mathrm{W}(R) \rightarrow C\left(\operatorname{spec}(R)_{\text {cons }}, \mathbb{Z}_{2}\right)$ definido como $\operatorname{dim}_{2}(\varphi)=$ $\pi \circ \operatorname{dim}(\varphi)$ onde $\operatorname{dim}(\varphi): \operatorname{spec}(R)_{\text {cons }} \rightarrow \mathbb{Z}$ é o mapa usual de dimensão e $\pi: \mathbb{Z} \rightarrow \mathbb{Z}_{2}$ é o único morfismo de anéis que satisfaz $\pi(x) \equiv x \bmod 2$ para todo $x \in \mathbb{Z}$. Pela Proposição $3.4 i$ ), $\operatorname{dim}_{2}$ é morfismo de anéis. Além disso $\operatorname{dim}_{2}^{-1}(0)=I(R)$; assim, pelo teorema do homomorfismo para anéis, para concluir $i)$ é suficiente provar que $\operatorname{dim}_{2}$ é sobrejetor. Seja $H \in C\left((\operatorname{spec}(R))_{\text {con }}, \mathbb{Z}_{2}\right)$. Como $H^{-1}(1)$ é aberto-fechado em $(\operatorname{spec}(R))_{c o n s}$, existem famílias $\left(a_{i}\right)_{i=1, \ldots, n},\left(b_{i}\right)_{i=1, \ldots, n}$ de idempotentes tais que $H^{-1}(1)=\bigcup_{i=1, \ldots, n} D\left(a_{i}\right) \cap D\left(b_{i}\right)^{c}(*)$. Pelo Lema 3.55, podemos supor que a união em $(*)$ é disjunta. Defina $\varphi=\bigoplus_{i=1}^{n} a_{i}\left\langle 1,-b_{i}\right\rangle$. Notando que dados $a, b \in \operatorname{Id}(R)$ temos $\operatorname{dim}_{p}(a\langle 1,-b\rangle) \equiv 1 \bmod 2$ se, e somente se, $p \in D(a) \cap D(b)^{c}$, dado $p \in \operatorname{spec}(R)$

$$
\begin{aligned}
\operatorname{dim}_{p}(\varphi) & =\sum_{i=1}^{n} \operatorname{dim}_{p}\left(a_{i}\left\langle 1,-b_{i}\right\rangle\right) \\
& \equiv H(p) \bmod 2 .
\end{aligned}
$$

Logo $\operatorname{dim}_{2}(\varphi)=H$ e dim 2 é sobrejetora

ii) É imediato do item anterior pois $\operatorname{se} \operatorname{spec}(R)$ tem mais de um elemento, existe aberto-fechado $A \subseteq(\operatorname{spec}(R))_{\text {cons }}$ tal que $A, A^{c} \neq \emptyset$. Assim, tomando as funções características $f$ e $g$ em $C\left((\operatorname{spec}(R))_{\text {con }}, \mathbb{Z}_{2}\right)$ de $A$ e $A^{c}$, respectivamente, temos $f, g \neq 0$ mas $f g=0$.

iii) Seja $\varphi=\left\langle a_{1}, \ldots, a_{n}\right\rangle$ forma com $\varphi \in \mathrm{I}(R)$. Considere a forma $\varphi_{2}=\left\langle a_{1}^{2}, \ldots, a_{n}^{2}\right\rangle$. É fácil ver que $\varphi_{2} \in \mathrm{I}(R)$. Pela prova do Teorema 3.8, é possível obter formas $\theta=\left\langle x_{1}^{2}, \ldots, x_{l}^{2}\right\rangle, \theta^{\prime}=\left\langle y_{1}^{2}, \ldots, y_{k}^{2}\right\rangle$ tais que $\varphi_{2}=2\left(\theta-\theta^{\prime}\right)$ em $\mathrm{W}(R)$. Então podemos escrever $\varphi$ em $\mathrm{W}(R)$ como

$$
\begin{aligned}
\varphi & =\sum_{i=1}^{n}\left\langle a_{i}, a_{i}^{2}\right\rangle-\varphi_{2} \\
& =\sum_{i=1}^{n}\left\langle a_{i}, a_{i}^{2}\right\rangle-2\left(\theta-\theta^{\prime}\right) \\
& =\sum_{i=1}^{n}\left\langle a_{i}, a_{i}^{2}\right\rangle-\sum_{i=1}^{l}\left\langle x_{i}^{2}, x_{i}^{2}\right\rangle+\sum_{i=1}^{k}\left\langle y_{i}^{2}, y_{i}^{2}\right\rangle .
\end{aligned}
$$




\section{Capítulo 4}

\section{Envoltória von Neumann para Semigrupos Reais}

$\mathrm{Na}$ teoria algébrica reduzida de formas quadráticas algumas envoltórias têm se mostrado frutíferas. Na Teoria dos Grupos Especiais Reduzidos (RSG), existe a envoltória Booleana introduzida e estudada em [DM1] que foi um dos ingredientes essenciais na solução da conjectura de Marshall ([DM2]). Outra envoltória dos RSGs é a profinita, introduzida e analisada em [MM1]. Num sentido preciso, ela é um refinamento da envoltória Booleana que prova um novo princípio local-global: a reflexão de subformas.

Já na teoria dos Semigrupos Reais, a envoltória de Post foi construída em [DP2] e compartilha boas propriedades da envoltória Booleana de RSG como caracterização de imersões completas e comutação com quocientes arbitrários. Por outro lado, enquanto envoltória de Post de um RS tem informação apenas da topologia construtível de seu espaço de ordens, a envoltória von Neumann de um RS (Definição 4.15) não apenas preserva informação do espectro real como também do espectro primo e dos RSGs associados às fibras do feixe estrutural (Teorema 4.10).

A primeira seção é dedicada a construção da envoltória von Neumann de qualquer RS a partir do anel de Witt. Muitas das propriedades algébricas desta envoltória são parecidas com o caso da envoltória von Neumann regular de aneis ([AM]), paralelo que ficará claro ao longo da exposição. Logo abaixo é elencado os principais resultados. Sejam $R \mathrm{RS}$ e $v: R \rightarrow V(R)$ o morfismo natural para a envoltória von Neumann.

- A associação $R \mapsto V(R)$ define um funtor adjunto à esquerda da inclusão $\mathbf{R S v N} \hookrightarrow \mathbf{R S}$ da categoria dos RSs von Neumann na categoria dos RSs (Teorema 4.14).

- O morfismo $v: R \rightarrow V(R)$ reflete a representação transversal de formas arbitrárias (Teorema 4.12).

- O morfismo induzido $W(v): W(R) \rightarrow W(V(R))$ entre os aneis de Witt é isomorfismo. Essa e outras propriedades algébricas da envoltória são provadas no Teorema 4.10.

Na segunda seção algumas aplicações da envoltória von Neumann são dadas como o cálculo do anel graduado, axiomatização dos aneis de Witt em $\mathcal{L}_{\omega_{1}, \omega}$ na linguagem dos aneis comutativos e a classificação dos quocientes na categoria dos aneis de Witt. Os principais resultados são:

- Descrição do anel graduado de Witt de um RS em função do anel graduado de Witt do RSG formado pelos inversíveis da envoltória von Neumann (Teorema 4.30).

- A conjectura de Marshall (Definição 4.31) vale para todo RS da forma $G_{A, T}$, onde $(A, T)$ é um p-anel (Teorema 4.34).

- Descrição da isometria de formas num quociente de RS von Neumann (Teorema 4.43).

- Descrição do anel de Witt de um RS por geradores e relações canônicas (Teorema 4.49). 
- Na Definição 4.56 introduzimos os aneis de Witt abstratos e no Teorema 4.63 é mostrado que a categoria dos RSs von Neumann é equivalente a categoria dos aneis de Witt abstratos.

\subsection{Envoltória von Neumann}

Seja $R$ RS. Considere $\mathrm{V}(R):=\left\{\varphi \in \mathrm{W}(R): \varphi^{3}=\varphi\right\}$ com o produto induzido pelo anel de Witt $\mathrm{W}(R)$ e representação transversal dada por

$\{37\}$

$$
\varphi \in \mathrm{D}_{V(R)}^{t}(\psi, \theta) \Leftrightarrow \varphi+\varphi \cdot \psi \cdot \theta=\psi+\theta \mathrm{em} \mathrm{W}(R) .
$$

Note que para todo $\varphi \in V(R)$ e $\sigma \in X_{R}$ vale $\operatorname{sgn}_{\sigma}(\varphi) \in\{1,0,-1\}$ e $\varphi \in \mathrm{D}_{V(R)}^{t}(\psi, \theta)$ se, e somente se, para todo $\sigma \in X_{R}$ vale $\operatorname{sgn}_{\sigma}(\varphi) \in D_{3}^{t}\left(\operatorname{sgn}_{\sigma}(\psi), \operatorname{sgn}_{\sigma}(\theta)\right)(*)$ (Proposição $\left.3.4 v\right)$ aplicado a $3=\{1,0,-1\})$. Considere também $v: R \rightarrow V(R)$ a inclusão natural dada por $v(a)=\langle a\rangle$. Note que, exceto a associatividade, todos os outros axiomas de semigrupo real são universais (Definição $2.30)$. Deste modo, a caracterização $(*)$ permite concluir que $\left(V(R), \cdot, D_{V(R)}^{t},-, 0,1\right)$ verifica todos os axiomas de RS menos, possivelmente, a associatividade.

Definição 4.1. Seja $R$ RS. Dados $a, b \in R$, vamos denotar por $R_{(a, b)}=R_{a} /(\bar{b})$ onde $(\bar{b}) \subseteq R_{a}$ é o ideal gerado por $\bar{b}$.

Lema 4.2. Sejam $R$ RS e $a, b \in \operatorname{Id}(R)$. São equivalentes:

i) $1 \neq 0$ em $R_{(a, b)}$.

ii) $a b \neq a$.

iii) $D(a) \cap D(b)^{c} \neq \emptyset$.

iv) $a\langle 1,-b\rangle \not \equiv\langle 0\rangle$.

Além disso, se $1 \neq 0$ em $R_{(a, b)}$, então $R_{(a, b)}$ é RS e o mapa canônico $R \rightarrow R_{(a, b)}$ induz homeomorfismo $X_{R_{(a, b)}} \cong\left\{\sigma \in X_{R}: \sigma(a)=1\right.$ e $\left.\sigma(b)=0\right\}$.

Demonstração. $i) \Rightarrow i i):$ Se $a b=a$, então $1 \in(\bar{b})$ e portanto $1=0$ em $R_{(a, b)}$.

$i i) \Rightarrow i$ ): Se $1=0$ em $R_{(a, b)}$, então $1 \in(\bar{b})$. Logo existem $x_{1}, \ldots, x_{n} \in R$ tais que

$$
a \in D^{t}\left(b x_{1} a, \ldots, b x_{n} a\right)
$$

. Assim, dado $\sigma \in X_{R}$, se $\sigma(b)=0$, entao $\sigma(a)=0$. Portanto $a=a b$.

$i i) \Leftrightarrow i$ ): Imediato.

$i i) \Leftrightarrow i i i)$ : Teorema da Separação 2.10.

$\{$ lem1\}

Lema 4.3. Sejam $R$ be a $\mathrm{RS}$ e $\varphi_{1}, \ldots, \varphi_{l} \in \mathrm{V}(R)$. Então existem $a_{i}, b_{i} \in \operatorname{Id}(R)$ e $x_{1, i}, \ldots, x_{l, i} \in R$, $i=1, \ldots, n$, tais que

- $\operatorname{spec}(R)=\bigsqcup_{i=1}^{n} D\left(a_{i}\right) \cap D\left(b_{i}\right)^{c}$ e $D\left(a_{i}\right) \cap D\left(b_{i}\right)^{c} \neq \emptyset$ para todo $i=1, \ldots, n$.

- $\varphi_{j} \cong_{R} \bigoplus_{i=1}^{n} x_{j, i} a_{i}\left\langle 1,-b_{i}\right\rangle$.

Demonstração. Vamos provar o resultado por indução em $l$. Se $l=1$ e $\varphi=\left\langle a_{1}, \ldots, a_{n}\right\rangle$ é forma em $\mathrm{V}(R)$, então pelo Teorema 3.8 podemos escrever

$$
\varphi \cong \bigoplus_{1 \leq k \leq n} \bigoplus_{f \in S_{n, k}} \pi_{f}\left\langle 1,-\Sigma_{f}\right\rangle\left\langle a_{f_{1}}, \ldots, a_{f_{k}}\right\rangle
$$


Seja $b \in \operatorname{Id}(R)$ tal que $\operatorname{supp}(\varphi)=D(b)$. Novamente pelo Teorema 3.8 temos

$$
\begin{aligned}
\operatorname{spec}(R) & =\bigsqcup_{k \geq 0} \operatorname{Od}_{k}(\varphi) \\
& =D(b)^{c} \sqcup \bigsqcup_{k=1}^{n} \bigsqcup_{f \in S_{n, k}} D\left(\pi_{f}\right) \cap D\left(\Sigma_{f}\right) .
\end{aligned}
$$

Fixe $1 \leq k \leq n$ e $f \in S_{n, k}$. Então temos

Afirmação. Existe $x_{f} \in R$ tal que

$$
\pi_{f}\left\langle 1,-\Sigma_{f}\right\rangle\left\langle a_{f_{1}}, \ldots, a_{f_{k}}\right\rangle \cong x_{f} \pi_{f}\left\langle 1,-\Sigma_{f}\right\rangle .
$$

Demonstração. Vamos dividir a prova em dois casos.

Caso 1: $k$ é par.

Neste vamos provar que $x_{f}=0$ satisfaz 4.4. Seja $\sigma \in X_{R}$. Se $\operatorname{supp}(\sigma) \notin D\left(\pi_{f}\right) \cap D\left(\Sigma_{f}\right)^{c}$, então $\operatorname{sgn}_{\sigma}\left(\pi_{f}\left\langle 1,-\Sigma_{f}\right\rangle\right)=0$ e a relação 4.4 é verificada. Se $\operatorname{supp}(\sigma) \in D\left(\pi_{f}\right) \cap D\left(\Sigma_{f}\right)^{c}$, então pela Proposição $3.4 e)$ temos $0 \equiv k=\operatorname{dim}_{\sigma}(\varphi) \equiv \operatorname{sgn}_{\sigma}(\varphi) \bmod 2$. Mas como $\operatorname{sgn}_{\sigma}(\varphi) \in\{1,0,-1\}$, temos $0=\operatorname{sgn}_{\sigma}(\varphi)=\operatorname{sgn}_{\sigma}\left(\left\langle a_{f_{1}}, \ldots, a_{f_{k}}\right\rangle\right)$ e portanto temos 4.4 válida para $x_{f}=0$.

Caso 2: $k$ é ímpar.

Neste caso vamos provar que $x_{f}=(-1)^{k(k-1) / 2} a_{f_{1}} \cdot \ldots \cdot a_{f_{k}}$ satisfaz 4.4. Seja $\sigma \in X_{R}$. Se $\operatorname{supp}(\sigma) \notin D\left(\pi_{f}\right) \cap D\left(\Sigma_{f}\right)^{c}$, então $\operatorname{sgn}_{\sigma}\left(\pi_{f}\left\langle 1,-\Sigma_{f}\right\rangle\right)=0$ e portanto 4.4 é verificada. Se $\operatorname{supp}(\sigma) \in$ $D\left(\pi_{f}\right) \cap D\left(\Sigma_{f}\right)^{c}$, então $\operatorname{sgn}_{\sigma}\left(\pi_{f}\left\langle 1,-\Sigma_{f}\right\rangle\right)=1$ e $\sigma\left(a_{f_{i}}\right) \neq 0$ para todo $i=1, \ldots, k$. Sejam $P=$ $\operatorname{card}\left(\left\{i: \sigma\left(a_{f_{i}}\right)=1\right\}\right)$ e $N=\operatorname{card}\left(\left\{i: \sigma\left(a_{f_{i}}\right)=-1\right\}\right)$. Como $k$ é impar, temos

$$
1 \equiv k=\operatorname{dim}_{\sigma}(\varphi) \equiv \operatorname{sgn}_{\sigma}(\varphi) \bmod 2 .
$$

Mas por hipótese $\operatorname{sgn}_{\sigma}(\varphi) \in\{1,0,-1\}$; logo podemos assumir que $\operatorname{sgn}_{\sigma}(\varphi)=(-1)^{e}$ para algum $e \in\{0,1\}$. Note então que $P+N=k$ e $P-N=\operatorname{sgn}_{\sigma}\left(\left\langle a_{f_{1}}, \ldots, a_{f_{k}}\right\rangle\right)=\operatorname{sgn}_{\sigma}(\varphi)=(-1)^{e}$. Pelo $x_{f}$ escolhido, temos $\sigma\left(x_{f}\right)=(-1)^{k(k-1) / 2} \cdot(-1)^{N}$ e assim precisamos mostrar que $k(k-1) / 2+$ $N \equiv e \bmod 2$. Como $(-1)^{e}=1-2 e$, temos que $k=2 P-(-1)^{e}=2(P+e)-1$ é impar e $k(k-1) / 2=k(P+e-1) \equiv P+e-1 \bmod 2 . \log \mathrm{O}$

$$
k(k-1) / 2+N \equiv N+P+e-1=(k-1)+e \equiv e \bmod 2 .
$$

Portanto temos $\sigma\left(x_{f}\right)=\operatorname{sgn}_{\sigma}\left(\left\langle a_{f_{1}}, \ldots, a_{f_{k}}\right\rangle\right)$.

Assim, pela afirmação e por 4.2,4.3, temos o resultado provado. Note que podemos assumir $D\left(\pi_{f}\right) \cap D\left(\Sigma_{f}\right)^{c} \neq \emptyset$ pois do contrário $\pi_{f}\left\langle 1,-\Sigma_{f}\right\rangle=0$ e daí podemos eliminá-lo da representação de $\varphi$.

Assuma o resultado válido para $l \geq 1$ e sejam $\varphi_{1}, \ldots, \varphi_{l+1} \in \mathrm{V}(R)$. Aplicando a hipótese de indução para $\varphi_{1}, \ldots, \varphi_{l}$ e para $\varphi_{l+1}$, obtemos famílias $\left(a_{i}\right)_{i=1}^{n},\left(b_{i}\right)_{i=1}^{n},\left(e_{j}\right)_{j=1}^{m},\left(f_{j}\right)_{j=1}^{m}$ de idempotentes e $x_{p, i}, y_{j} \in R, p=1, \ldots, l$, tais que

- $\operatorname{spec}(R)=\bigsqcup_{i=1}^{n} D\left(a_{i}\right) \cap D\left(b_{i}\right)^{c}=\bigsqcup_{j=1}^{m} D\left(e_{j}\right) \cap D\left(f_{j}\right)^{c}$.

- $\varphi_{p} \cong_{R} \bigoplus_{i=1}^{n} x_{p, i} a_{i}\left\langle 1,-b_{i}\right\rangle$ para $p=1, \ldots, l$.

- $\varphi_{l+1} \cong_{R} \bigoplus_{j=1}^{m} y_{j} e_{j}\left\langle 1,-f_{j}\right\rangle$. 
Note então que

$$
\operatorname{spec}(R)=\bigsqcup_{1 \leq i \leq n, 1 \leq j \leq m}\left(D\left(a_{i}\right) \cap D\left(b_{i}\right)^{c}\right) \cap\left(D\left(e_{j}\right) \cap D\left(f_{j}\right)^{c}\right)=\bigsqcup_{1 \leq i \leq n, 1 \leq j \leq m} D\left(c_{i, j}\right) \cap D\left(d_{i, j}\right)^{c},
$$

onde $c_{i, j}=a_{i} e_{j}$ e $d_{i, j} \in D^{t}\left(b_{i}, f_{j}\right)$. Deste modo para $p=1, \ldots, l$ podemos verificar pela comparação das assinaturas que

- $\varphi_{p} \cong_{R} \bigoplus_{i \leq n, j \leq m} x_{p, i} c_{i, j}\left\langle 1,-d_{i, j}\right\rangle$.

- $\varphi_{l+1} \cong_{R} \bigoplus_{i \leq n, j \leq m} y_{j} c_{i, j}\left\langle 1,-d_{i, j}\right\rangle$.

Retirando as formas $c_{i, j}\left\langle 1,-d_{i, j}\right\rangle$ com $D\left(c_{i, j}\right) \cap D\left(d_{i, j}\right)=\emptyset$ temos o desejado.

Comentário 4.4. Seja $R$ RS. Dados $a, b \in \operatorname{Id}(R)$, a forma $\varphi=a\langle 1,-b\rangle=\langle a,-a b\rangle$ satisfaz $\varphi^{2}=\langle a,-a b,-a b, a b\rangle \cong\langle a,-a b\rangle=\varphi$. O Lema 4.3 prova, em particular, que se $\varphi$ é uma forma que satisfaz $\varphi^{2} \cong \varphi(*)$, então existem $a_{i}, b_{i} \in \operatorname{Id}(R), i=1, \ldots, n$ de modo que os conjuntos $\left\{D\left(a_{i}\right) \cap D\left(b_{i}\right)^{c}\right\}_{i=1}^{n}$ são disjuntos e $\varphi \cong \bigoplus_{i=1}^{n} a_{i}\left\langle 1,-b_{i}\right\rangle$. Por outro lado, supondo $R$ é von Neumann, se $\varphi$ é uma forma satisfazendo (*), então pelo Teorema 3.51 existe $a \in \operatorname{Id}(R)$ tal que $\varphi \cong\langle a\rangle$.

Lema 4.5. Seja $R$ RS. Sejam $a_{i}, b_{i} \in \operatorname{Id}(R) \operatorname{com} D\left(a_{i}\right) \cap D\left(b_{i}\right)^{c} \neq \emptyset, i=1, \ldots, n$ tais que $D\left(a_{i}\right) \cap$ $D\left(b_{i}\right)^{c} \cap D\left(a_{j}\right) \cap D\left(b_{j}\right)^{c}=\emptyset$ para $i \neq j$. Para cada $i=1, \ldots, n$ seja $\theta_{i}=a_{i}\left\langle 1,-b_{i}\right\rangle \in V(R)$ e considere uma forma $\varphi=\bigoplus_{i=1}^{n} x_{i} \theta_{i} \in V(R)$, com $x_{i} \in R$. Dadas formas $\psi=\bigoplus_{i=1}^{n} y_{i} \theta_{i}, \tau=$ $\bigoplus_{i=1}^{n} z_{i} \theta_{i} \in V(R)$, vale que

$$
\varphi \in D_{V(R)}^{t}(\psi, \tau) \Leftrightarrow \overline{x_{i}} \in D_{R_{\left(a_{i}, b_{i}\right)}^{t}}^{t}\left(\overline{y_{i}}, \overline{z_{i}}\right) \text { em } R_{\left(a_{i}, b_{i}\right)} \text { para todo } i=1, \ldots, n \text {. }
$$

Além disso, $\varphi \cdot \theta_{i}=x_{i} \theta_{i}$ em $V(R)$.

Demonstração. Primeiro note que dados $i \neq j$, como $D\left(a_{i}\right) \cap D\left(b_{i}\right)^{c} \cap D\left(a_{j}\right) \cap D\left(b_{j}\right)^{c}=\emptyset$, então $\theta_{i} \cdot \theta_{j}=0$ em $W(R)$. Deste modo $\varphi \cdot \theta_{i} \cong \bigoplus_{j=1}^{n} x_{j} \theta_{j} \theta_{i} \cong x_{i} \theta_{i}^{2}=x_{i} \theta_{i}$. Se $\varphi \in D_{V(R)}^{t}(\psi, \tau)$, então dado $i$ temos $x_{i} \theta_{i} \in D_{V(R)}^{t}\left(y_{i} \theta_{i}, z_{i} \theta_{i}\right)$. Logo para todo $\sigma \in X_{R}$ com $\sigma\left(a_{i}\right)=1$ e $\sigma\left(b_{i}\right)=0$ vale $\sigma\left(x_{i}\right)+\sigma\left(x_{i} y_{i} z_{i}\right)=\sigma\left(y_{i}\right)+\sigma\left(z_{i}\right)$. Pelo Lema 4.2 temos $\overline{x_{i}} \in D_{R_{\left(a_{i}, b_{i}\right)}^{t}}\left(\overline{y_{i}}, \overline{z_{i}}\right)(*)$. Reciprocamente, se vale $(*)$ para todo $i=1, \ldots, n$, então novamento pelo Lema 4.2 temos para todo $\sigma \in X_{R}$ que $\operatorname{sgn}_{\sigma}\left(\theta_{i}\right)\left(\sigma\left(x_{i}\right)+\sigma\left(x_{i} y_{i} z_{i}\right)\right)=\operatorname{sgn}_{\sigma}\left(\theta_{i}\right)\left(\sigma\left(y_{i}\right)+\sigma\left(z_{i}\right)\right)$ e portanto $x_{i} \theta_{i}+x_{i} y_{i} z_{i} \theta_{i}=y_{i} \theta_{i}+z_{i} \theta_{i}$ em $\mathrm{W}(R)$. Logo

$$
\varphi+\varphi \psi \tau=\sum_{i=1}^{n} x_{i} \theta_{i}+x_{i} y_{i} z_{i} \theta_{i}=\sum_{i=1}^{n} y_{i} \theta_{i}+z_{i} \theta_{i}=\psi+\tau
$$

$\mathrm{e} \operatorname{assim} \varphi \in D_{V(R)}^{t}(\psi, \tau)$

Teorema 4.6. Seja $R R S$. Então $V(R)$ é $R S$ von Neumann.

Demonstração. Para vermos que $V(R)$ é semigrupo real é suficiente provarmos que $V(R)$ satisfaz a propriedade associativa (4.1). Sejam $\varphi, \psi, \theta, \Gamma, \phi \in \mathrm{V}(R)$ tais que

$$
\Gamma \in D_{V(R)}^{t}(\varphi, \phi) \text { e } \phi \in D_{V(R)}^{t}(\psi, \theta) .
$$

Precisamos provar que existe $\phi^{\prime} \in \mathrm{V}(R)$ tal que $\Gamma \in D_{V(R)}^{t}\left(\phi^{\prime}, \theta\right) \operatorname{com} \phi^{\prime} \in D_{V(R)}^{t}(\varphi, \psi)$.

Pelo Lema 4.3, existem $a_{i}, b_{i} \in \operatorname{Id}(R)$ e $v_{i}, w_{i}, x_{i}, y_{i}, z_{i} \in R, i=1, \ldots, n$, tais que

- $\operatorname{spec}(R)=\bigsqcup_{i=1}^{n} D\left(a_{i}\right) \cap D\left(b_{i}\right)^{c}, D\left(a_{i}\right) \cap D\left(b_{i}\right)^{c} \neq \emptyset$.

- $\Gamma \cong \bigoplus_{i=1}^{n} v_{i} a_{i}\left\langle 1,-b_{i}\right\rangle$. 
- $\varphi \cong \bigoplus_{i=1}^{n} w_{i} a_{i}\left\langle 1,-b_{i}\right\rangle$.

- $\phi \cong \bigoplus_{i=1}^{n} x_{i} a_{i}\left\langle 1,-b_{i}\right\rangle$.

- $\psi \cong \bigoplus_{i=1}^{n} y_{i} a_{i}\left\langle 1,-b_{i}\right\rangle$.

- $\theta \cong \bigoplus_{i=1}^{n} z_{i} a_{i}\left\langle 1,-b_{i}\right\rangle$.

Por outro lado, pelo Lema 4.5, as relações acima implicam que $\overline{v_{i}} \in D_{R_{\left(a_{i}, b_{i}\right)}^{t}}\left(\overline{w_{i}}, \overline{x_{i}}\right)$ e $\overline{x_{i}} \in$ $D_{R_{\left(a_{i}, b_{i}\right)}^{t}}\left(\overline{y_{i}}, \overline{z_{i}}\right)$. Como $R_{\left(a_{i}, b_{i}\right)}$ é RS, para cada $i$ existe $x_{i}^{\prime} \in R$ tal que $\overline{v_{i}} \in D_{R_{\left(a_{i}, b_{i}\right)}^{t}}\left(\overline{x_{i}^{\prime}}, \overline{z_{i}}\right)$ com $\overline{x_{i}^{\prime}} \in D_{R_{\left(a_{i}, b_{i}\right)}^{t}}\left(\overline{w_{i}}, \overline{y_{i}}\right)$. Defina $\psi^{\prime}=\bigoplus_{i=1}^{n} x_{i}^{\prime} a_{i}\left\langle 1,-b_{i}\right\rangle$. Então $\phi^{\prime} \in \mathrm{V}(R)$ e pelo Lema 4.5 temos $\Gamma \in D_{V(R)}^{t}\left(\phi^{\prime}, \theta\right) \operatorname{com} \phi^{\prime} \in D_{V(R)}^{t}(\varphi, \psi)$.

Para finalizarmos a prova, precisamos mostrar que $\mathrm{V}(R)$ é von Neumann. Vamos utilizar a caracterização do Teorema 2.58. Dada forma $\varphi \in \mathrm{V}(R)$, defina $\varphi^{c}=\langle 1\rangle-(\varphi \oplus \varphi)$. Então $\varphi \varphi^{c}=$ $\varphi-\varphi^{3} \cong 0$ e $\varphi^{c}-\varphi^{c} \varphi^{2} \cong \varphi^{c}=1-\varphi^{2}$. Logo $\varphi \cdot \varphi^{c}=0, \varphi^{c} \in D_{V(R)}^{t}\left(1,-\varphi^{2}\right)$ e portanto $\mathrm{V}(R)$ é von Neumann.

Lema 4.7. Seja $R$ RS. Sejam $a_{i}, b_{i} \in \operatorname{Id}(R) \operatorname{com} D\left(a_{i}\right) \cap D\left(b_{i}\right)^{c} \neq \emptyset, i=1, \ldots, n$ tais que $D\left(a_{i}\right) \cap$ $\{$ lem3\} $D\left(b_{i}\right)^{c} \cap D\left(a_{j}\right) \cap D\left(b_{j}\right)^{c}=\emptyset$ para $i \neq j$. Para cada $i=1, \ldots, n$ seja $\theta_{i}=a_{i}\left\langle 1,-b_{i}\right\rangle \in V(R)$ e considere uma forma $\varphi=\bigoplus_{i=1}^{n} x_{i} \theta_{i} \in V(R)$, com $x_{i} \in R$. Então

$$
\varphi \in D_{V(R)}^{t}\left(v\left(x_{1}\right) \theta_{1}, \ldots, v\left(x_{n}\right) \theta_{n}\right) .
$$

Demonstração. A demonstração segue por indução em $n \geq 1$. Para $n=1$ é trivial. Supondo $n \geq 2$, seja $\varphi^{\prime}=\bigoplus_{i=2}^{n} x_{i} \theta_{i}$. Por hipótese de indução, temos $\varphi^{\prime} \in D_{V(R)}^{t}\left(v\left(x_{2}\right) \theta_{2}, \ldots, v\left(x_{n}\right) \theta_{n}\right)$. Por outro lado, pelo Lema 4.5 temos que $\varphi \in D^{t}\left(v\left(x_{1}\right) \theta_{1}, \varphi^{\prime}\right)$. Portando $\varphi \in D_{V(R)}^{t}\left(v\left(x_{1}\right) \theta_{1}, \ldots, v\left(x_{n}\right) \theta_{n}\right)$.

Definição 4.8. Um morfismo $f: R \rightarrow G$ de RSs é chamado de imersão completa quando dados $\{$ DefimerCom $a_{1}, \ldots, a_{n}, b_{1}, \ldots, b_{k} \in R$,

$$
\left\langle f\left(a_{1}\right), \ldots, f\left(a_{n}\right)\right\rangle \cong_{G}\left\langle f\left(b_{1}\right), \ldots, f\left(b_{k}\right)\right\rangle \Rightarrow\left\langle a_{1}, \ldots, a_{n}\right\rangle \cong_{R}\left\langle b_{1}, \ldots, b_{k}\right\rangle .
$$

Comentários 4.9. Seja $f: R \rightarrow G$ morfismo de RSs.

- Se $f^{*}: X_{G} \rightarrow X_{R}$ é sobrejetor, então $f$ é imersão completa. No Teorema 5.64 a recíproca é provada.

- Suponha que $f$ seja imersão completa. Então pela Proposição 3.4.(v) vale que dados $a, b, c \in R$,

$$
a \in D_{R}^{t}(b, c) \Leftrightarrow f(a) \in D_{G}^{t}(f(b), f(c)) .
$$

Em particular, $f$ é injetora.

- No Teorema 5.64 várias caracterizações de imersão completa são provadas. Uma delas assegura que é possível trocar a equivalência de formas na Definição 4.8 pela isometria.

Teorema 4.10. Seja $R R S$. Considere o morfismo natural $v: R \rightarrow V(R)$. Então

i) Os mapas espectrais induzidos por $v, s(v): \operatorname{spec}(V(R)) \rightarrow(\operatorname{spec}(R))_{\text {cons }}$ e $\operatorname{rs}(v): X_{V(R)} \rightarrow$ $\left(X_{R}\right)_{\text {cons }}$, são homeomorfismos. Em particular, $v$ é imersão completa. 
ii) Para cada $p^{\prime} \in \operatorname{spec}(V(R))$, seja $p=s(v)\left(p^{\prime}\right)$. Então o morfismo $v_{p^{\prime}}: K_{p}(R) \rightarrow K_{p^{\prime}}(V(R))$ induzido faz o diagrama

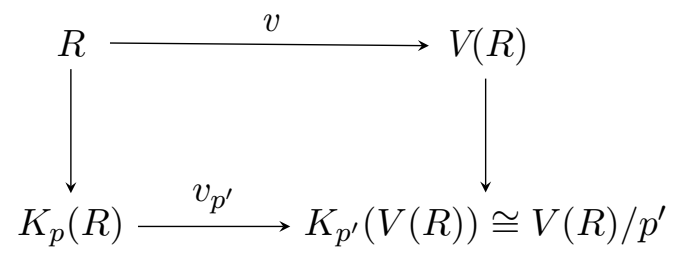

comutar e é isomorfismo.

iii) O mapa induzido $W(v): W(R) \rightarrow W(V(R))$ é isomorfismo de anéis que preserva os ideias fundamentais.

Demonstração. $\quad$ i) Basta mostrarmos que $s(v)$ e $r s(v)$ são bijetores para concluirmos que as funções são homeomorfismo pois seus domínios e a imagens são espaços Booleanos.

- $s(v): \operatorname{spec}(\mathrm{V}(R)) \rightarrow(\operatorname{spec}(R))_{\text {cons }}$ é injetor.

Sejam $P, Q \in \operatorname{spec}(\mathrm{V}(R))$ e assuma que $v^{-1}(P)=v^{-1}(Q)$. Seja $\varphi \in P$. Pelo Lema 4.3, existem $\theta_{1}, \ldots, \theta_{n} \in \mathrm{V}(R)$ e $x_{i} \in R$ tais que

$$
\begin{aligned}
\theta_{i} & =a_{i}\left\langle 1,-b_{i}\right\rangle, a_{i}, b_{i} \in \operatorname{Id}(R) \\
\operatorname{spec}(R) & =\bigsqcup_{i=1}^{n} D\left(a_{i}\right) \cap D\left(b_{i}\right)^{c} \\
\varphi \cdot \theta_{i} & =v\left(x_{i}\right) \theta_{i} .
\end{aligned}
$$

Seja $j$ com $v^{-1}(P)=v^{-1}(Q) \in D\left(a_{j}\right) \cap D\left(b_{j}\right)^{c}$. Notando que $v\left(b_{j}\right)^{c}=\left\langle 1,-b_{j}\right\rangle$, temos $v\left(a_{j}\right) \notin P$ e $\left\langle 1,-b_{j}\right\rangle \notin P$. Como $P$ é ideal primo, segue que $\theta_{j}=a_{j}\left\langle 1,-b_{j}\right\rangle \notin P$ e de forma análoga $\theta_{j} \notin Q$. Então, como $\varphi \in P$, pela última identidade de 4.5 temos $v\left(x_{j}\right) \in P$ e assim $x_{j} \in v^{-1}(P)=v^{-1}(Q)$. Logo $\varphi \cdot \theta_{j}=v\left(x_{j}\right) \theta_{j} \in Q$ implica $\varphi \in Q$ e assim $P \subseteq Q$. De forma semelhante verifica-se que $Q \subseteq P$.

- $s(v)$ é sobrejetor.

Seja $p \in \operatorname{spec}(R)$. Defina $P=\left\{\varphi \in \mathrm{V}(R): \operatorname{dim}_{p}(\varphi)=0\right\}$. É fácil verificar que $P$ é ideal primo de $V(R)$. Para vermos que $v^{-1}(P)=p$, note que dado $x \in v^{-1}(P)$, temos que para todo $\sigma \in X_{p}$ vale $\sigma(x)=0$ e assim $x \in p$. Reciprocamente se $x \in p$, então $\operatorname{dim}_{p}(\langle x\rangle)=0$ e $\operatorname{assim} v(x) \in P$.

- $r s(v): X_{V(R)} \rightarrow\left(X_{R}\right)_{\text {cons }}$ é injetor.

Dado $\Sigma \in X_{V(R)}$, seja $\sigma=r s(v)(\Sigma)=\Sigma \circ v \in X_{R}$. Dado $\varphi \in V(R)$, pelo lema 4.3 existem $a_{i}, b_{i} \in \operatorname{Id}(R)$ e $x_{i} \in R, i=1, \ldots, n$, tais que $\operatorname{spec}(R)=\bigsqcup_{i=1}^{n} D\left(a_{i}\right) \cap D\left(b_{i}\right)^{c}$ e $\varphi \cong \bigoplus_{i=1}^{n} x_{i} a_{i}\left\langle 1,-b_{i}\right\rangle$.

Afirmação. Se $v^{-1}(\operatorname{supp}(\Sigma)) \in D\left(a_{j}\right) \cap D\left(b_{j}\right)^{c}$, então $\Sigma(\varphi)=\sigma\left(x_{j}\right)$.

Demonstração. Para cada $i$ seja $\theta_{i}=a_{i}\left\langle 1,-b_{i}\right\rangle \in V(R)$. Como $p=v^{-1}(\operatorname{supp}(\Sigma)) \notin$ $D\left(a_{i}\right) \cap D\left(b_{i}\right)^{c}$ para $i \neq j$, temos $\Sigma\left(\theta_{i}\right)=0$. De fato, se $p \notin D\left(a_{i}\right)$, ou seja, $a_{i} \in p$, então $\Sigma\left(v\left(a_{i}\right)\right)=0$ e segue $\Sigma\left(\theta_{i}\right)=0$. Se $p \notin D\left(b_{i}\right)^{c}$, ou seja, $b_{i} \notin p$, temos $\Sigma\left(v\left(b_{i}\right)\right)=1$ pois $v\left(b_{i}\right) \in V(R)$ é idempotente; logo $0=\Sigma\left(v\left(b_{i}\right)^{c}\right)=\Sigma\left(\left\langle 1,-b_{i}\right\rangle\right)$ e portanto $\Sigma\left(\theta_{i}\right)=0$. Por argumentos semelhantes verifica-se que a hipótese da afirmação implica $\Sigma\left(\theta_{j}\right)=1$.

Deste modo, pelo Lema 4.7 temos $\varphi \in D_{V(R)}^{t}\left(v\left(x_{1}\right) \theta_{1}, \ldots, v\left(x_{n}\right) \theta_{n}\right)$. Deste modo $\Sigma(\varphi) \in$ $D_{V(R)}^{t}\left(\sigma\left(x_{1}\right) \Sigma\left(\theta_{1}\right), \ldots, \sigma\left(x_{n}\right) \Sigma\left(\theta_{n}\right)\right)=\left\{\sigma\left(x_{j}\right)\right\}$.

Em particular, a afirmação mostra que $\Sigma$ é determinado por $\sigma$ e portanto $r s(v)$ é injetor. 
- $r s(v)$ é sobrejetora.

Seja $\sigma \in X_{R}$. Defina $\Sigma: V(R) \rightarrow 3$ por: se $\varphi=\left\langle a_{1}, \ldots, a_{n}\right\rangle \in V(R)$, então $\Sigma(\varphi)=$ $\operatorname{sgn}_{\sigma}(\varphi)$. Note que pela Proposição $\left.3.4 i\right)$ vale $\Sigma(\varphi \cdot \psi)=\Sigma(\varphi) \cdot \Sigma(\psi)$. Por outro lado, se $\varphi \in D_{V(R)}^{t}(\psi, \tau)$, então em particular vale $\operatorname{sgn}_{\sigma}(\varphi) \in D_{3}^{t}\left(\operatorname{sgn}_{\sigma}(\psi), \operatorname{sgn}_{\sigma}(\theta)\right)(4.1)$ e portanto $\Sigma(\varphi) \in D_{3}^{t}(\Sigma(\psi), \Sigma(\tau))$. Assim $\Sigma \in X_{V(R)}$ e $r s(v)(\Sigma)=\sigma$.

ii) Pela Proposição 1.15, $v_{p^{\prime}}$ existe e é unico fazendo o diagrama em questão comutar. Pelo Lema 2.44 para provarmos que $v_{p^{\prime}}$ é isomorfismo é suficiente que $v_{p^{\prime}}$ é sobrejetora pois pelo item i) acima, o mapa induzido entre os espectros reais é bijetor. Seja $\varphi \in V(R)$ e escreva $\varphi \cong$ $\bigoplus_{i=1}^{n} x_{i} a_{i}\langle 1,-b\rangle$ com $a_{i}, b_{i} \in \operatorname{Id}(R)$ e $\operatorname{spec}(R)=\bigsqcup_{i=1}^{n} D\left(a_{i}\right) \cap D\left(b_{i}\right)^{c}$. Seja $j$ tal que $p \in$ $D\left(a_{j}\right) \cap D\left(b_{j}\right)^{c}$. Seja $\theta=a_{j}\left\langle 1,-b_{j}\right\rangle$. Então $\theta \notin p^{\prime}$. Como $\varphi \cdot \theta=v\left(x_{j}\right) \cdot \theta$ e $\mathrm{V}(R)$ é hiper-anel por 2.58 , existe $\psi \in D_{V(R)}^{t}\left(\varphi,-v\left(x_{j}\right)\right)$ tal que $\psi \theta=0$. Deste modo, como $\theta \notin p^{\prime}, \psi \in p^{\prime}$ e em $K_{p^{\prime}}(V(R)) \cong V(R) / p^{\prime}$ temos $\bar{\varphi}=\overline{v\left(x_{i}\right)}$. Logo $v_{p}\left(\overline{x_{i}}\right)=\bar{\varphi}$.

iii) Como $v$ é imersão completa pelo item $i)$, temos que $\mathrm{W}(v): \mathrm{W}(R) \rightarrow \mathrm{W}(\mathrm{V}(R))$ é injetor. Vamos mostrar a sobrejetividade.

Afirmação. Sejam $\varphi=\left\langle c_{1}, \ldots, c_{n}\right\rangle \in \mathrm{V}(R), \Sigma \in X_{V(R)}$ e $\sigma=\Sigma \circ v \in X_{R}$. Então $\Sigma(\varphi)=$ $\sigma\left(c_{1}\right)+\cdots+\sigma\left(c_{n}\right)$.

Demonstração. Pelo Lema 4.3, podemos escrever $\varphi \cong \bigoplus_{i=1}^{n} x_{i} a_{i}\left\langle 1,-b_{i}\right\rangle$ onde $a_{i}, b_{i} \in \operatorname{Id}(R)$ e $\operatorname{spec}(R)=\bigsqcup_{i=1}^{n} D\left(a_{i}\right) \cap D\left(b_{i}\right)^{c}$. Deste modo, se $v^{-1}(\operatorname{supp}(\Sigma)) \in D\left(a_{i}\right) \cap D\left(b_{j}\right)^{c}$, então pela afirmação localizada na demonstração da injetividade de $r s(v)$ no item $i)$ acima vale que $\Sigma(\varphi)=$ $\sigma\left(x_{i}\right)=\operatorname{sgn}_{\sigma}(\varphi)=\sigma\left(c_{1}\right)+\cdots+\sigma\left(c_{n}\right)$.

Assim dada forma $\varphi=\left\langle a_{1}, \ldots, a_{n}\right\rangle \in \mathrm{V}(R)$ vale que $\mathrm{W}(v)\left(\left\langle a_{1}, \ldots, a_{n}\right\rangle\right)=\langle\varphi\rangle \in W(V(R))$. Mas como $W(V(R))$ é gerado aditivamente por $V(R)$ segue que $\mathrm{W}(v)$ é sobrejetor. A preservação dos ideias fundamentais segue pelo Comentário 4.11.

Comentários 4.11. - Sejam $f: R \rightarrow G$ morfismo de RSs e $\varphi$ forma sobre $R$. Dado $q \in \operatorname{spec}(G)$, seja $p=f^{-1}(q) \in \operatorname{spec}(R)$. Então $\operatorname{dim}_{p}(\varphi)=\operatorname{dim}_{q}(f \star \varphi)$. Em particular, se $s(f): \operatorname{spec}(G) \rightarrow$ $\operatorname{spec}(R)$ é sobrejetor e $\psi$ é outra forma:

$-\operatorname{dim}(\varphi) \leq \operatorname{dim}(\psi)$ se, e somente se, $\operatorname{dim}(f \star \varphi) \leq \operatorname{dim}(f \star \psi)$.

$-\operatorname{dim}(\varphi) \leq n$ se, e somente se, $\operatorname{dim}(f \star \varphi) \leq n$.

$-\varphi \in I(R) \subseteq W(R)$ se, e somente se, $f \star \varphi \in I(G) \subseteq W(G)$.

- Sejam $R$ RS e $\varphi$ forma sobre $V(R)$. O item $i i i)$ do Teorema 4.10 implica que existe forma $\psi$ sobre $R$ tal que $\varphi \cong_{V(R)} v \star \psi$. Porém não vale em geral que exista forma $\psi$ sobre $R$ tal que $\varphi \equiv_{V(R)} v \star \psi$. De fato, suponha que vale e fixe $a \in R$. Considerando a forma $\varphi:=\left\langle v(a)^{c}\right\rangle$, existiria $\psi$ sobre $R$ tal que $\varphi \equiv_{V(R)} v \star \psi$. Logo teríamos que $\psi$ é 1-forma e pelo Corolário 3.9 existiria $b \in R \operatorname{com} \psi \equiv\langle b\rangle$. Assim $b=a^{c} \in R$. Como $a \in R$ foi arbitrário, teríamos que $R$ já seria von Neumann.

Teorema 4.12. Sejam $R R S$ e $\varphi, \psi$ formas. Considere $v: R \rightarrow V(R)$.

i) Dado $a \in R, a \in D_{R}^{t}(\varphi)$ se, e somente se, $v(a) \in D_{V(R)}^{t}(v \star \varphi)$.

ii) $D_{R}^{t}(\varphi) \subseteq D_{R}^{t}(\psi)$ se, e somente se, $D_{V(R)}^{t}(v \star \varphi) \subseteq D_{V(R)}^{t}(v \star \psi)$. 
Demonstração. i) Pelo Teorema 4.10, o mapa $\operatorname{sp}(v): \operatorname{spec}(V(R)) \rightarrow \operatorname{spec}(R)$ é bijetor e para cada $p \in \operatorname{spec}(V(R))$, o mapa induzido $v_{p}: K_{R}(q) \rightarrow K_{V(R)}(p), q=s p(v)(p)$, é isomorfismo. Deste modo, pelo Teorema 6.7.1 de [Mar7] temos

$$
\begin{aligned}
a \in D_{R}^{t}(\varphi) & \Leftrightarrow \text { Para todo } p \in \operatorname{spec}(R) \text { vale } \bar{a} \in D_{K_{R}(p)}^{t}(\bar{\varphi}) \\
& \Leftrightarrow \text { Para todo } q \in \operatorname{spec}(V(R)) \text { vale } \overline{v(a)} \in D_{K_{V(R)}(q)}^{t}(\overline{v \star \varphi}) \Leftrightarrow v(a) \in D_{V(A)}^{t}(v \star \varphi) .
\end{aligned}
$$

ii) O item $i$ ) acima prova a direção $\Leftarrow$. Para a direção oposta, suponha $D_{R}^{t}(\varphi) \subseteq D_{R}^{t}(\psi)$. Então para todo $p \in \operatorname{spec}(R)$ vale $D_{K_{R}(p)}^{t}(\bar{\varphi}) \subseteq D_{K_{R}(p)}^{t}(\psi)$. Assim, pelo Teorema 4.10, temos que para todo $p \in \operatorname{spec}(V(R))$ vale $D_{K_{V(R)}(p)}^{t}(\overline{v \star \varphi}) \subseteq D_{K_{V(R)}(p)}^{t}(\overline{v \star \psi})$. Pelo Corolário 2.64, segue que $D_{V(R)}^{t}(v \star \varphi) \subseteq D_{V(R)}^{t}(v \star \psi)$.

\{isovon\}

Lema 4.13. Seja $R$ RS von Neumann. Então o mapa $v: R \rightarrow V(R)$ é isomorfismo.

Demonstração. Pelo Teorema $4.10 i)$, rs $(v): X_{V(R)} \rightarrow X_{R}$ é bijetor. Assim, para concluir que $v$ é isomorfismo, pelo Lema 2.44, basta provar que $v$ é sobrejetor mas isto segue pelo Teorema $3.51 i i)-b)$.

\{univeVon\}

Teorema 4.14. Seja R RS. Então

i) A associação $R \mapsto V(R)$ define funtor da categoria dos semigrupos reais para a categoria dos von Neumann.

ii) Dado morfismo $f: R \rightarrow G$, onde $G$ é $R S$ von Neumann, existe unico morfismo $\bar{f}: V(R) \rightarrow V$ tal que

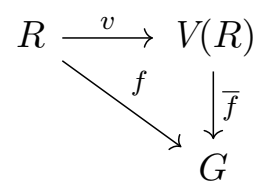

é diagrama comutativo. Em particular $V(V(R))$ é canonicamente isomorfo a $V(R)$.

iii) Com a notação do item acima, se f é imersão completa, então $\bar{f}$ também é.

Demonstração. i) Seja $f: R \rightarrow S$ morfismo de RSs. Dado $\varphi=\left\langle a_{1}, \ldots, a_{n}\right\rangle \in V(R)$, defina $v(f)(\varphi)=\left\langle f\left(a_{1}\right), \ldots, f\left(a_{n}\right)\right\rangle$. Segue da definição que $v(f): V(R) \rightarrow V(S)$ é a restrição do mapa $W(f): \mathrm{W}(R) \rightarrow \mathrm{W}(S)$ e portanto é morfismo de RS pois o produto e a representação transversal de $V(R)$ são definiveis pelas operações de aneis de $W(R)$. Se $g: S \rightarrow G$ é outro morfismo de RS temos $v(g \circ f)=v(g) \circ v(f)$ e portanto $v: \mathbf{R S} \rightarrow \mathbf{v N R S}$ é funtor.

ii) Seja $f: R \rightarrow G$ morfismo de RSs com $G$ von Neumann. Note que o morfismo $W(f): W(R) \rightarrow$ $W(G)$ preserva elementos ternários e pelo Lema 4.13 o mapa $v_{G}: G \rightarrow V(G) \subseteq W(G)$ é isomorfismo. Defina então $\bar{f}=v_{G}^{-1} \circ W(f)_{V(R)}$. Então $\bar{f}$ é morfismo de RS porque o produto e a representação transversal de $V(R)$ são definidas pela estrutura de anel de $W(R)$. Além disso $f=\bar{f} \circ v$. Se $g: V(R) \rightarrow V$ é outro mapa com $f=g \circ v$, então $W(g) \circ W(v)=W(\bar{f}) \circ W(v)$; mas pelo Teorema 4.10 o mapa $W(v)$ é isomorfismo e portanto $W(g)=W(\bar{f})$. Logo $g=\bar{f}$.

iii) Como $f$ é imersão completa, então pelo Teorema 5.64.(iii) o mapa $f^{*}: X_{G} \rightarrow X_{R}$ é sobrejetor. Além disso, pelo Teorema 4.10.(i) o morfismo $v^{*}: X_{V(R)} \rightarrow X_{R}$ é injetor. Por outro lado, como vale $f^{*}=v^{*} \circ \bar{f}^{*}$, segue que $\bar{f}^{*}: X_{G} \rightarrow X_{V(R)}$ é sobrejetor. Novamente pelo Teorema 5.64 temos que $\bar{f}: V(R) \rightarrow G$ é imersão completa. 
Definição 4.15. Seja $R$ RS. O semigrupo real $\mathrm{V}(R)$ é chamado de envoltória von Neumann de $R$.

Comentário 4.16. O Teorema 4.10 descreve algumas propriedades algébricas da envoltória von Neumann. Por outro lado, elas são suficientes para caracterizar a envoltória. Mais precisamente, fixado $R$ RS e $f: R \rightarrow V$ morfismo para um RS von Neumann, são equivalentes:

- $f: R \rightarrow V$ é inicial entre morfismos saindo de $R$ e chegando num RS von Neumann (em outras palavras, $V \cong V(R))$;

- $f$ satisfaz os itens $i$ ) e $i$ i) do Teorema 4.10;

- $W(f): W(R) \rightarrow W(V)$ é isomorfismo de aneis (item $i i i$ ) do Teorema 4.10).

Teorema 4.17. Seja $A$ anel semi-real. Seja $V(A)$ a envoltória von Neumann regular do anel $A$. Então o morfismo natural $A \rightarrow V(A)$ induz isomorfismo $V\left(G_{A}\right) \cong G_{V(A)}$.

Demonstração. Consequência imediata do Teorema 4.12 de [RM3].

Comentários 4.18. - Sejam $A$ e $V(A)$ como no Teorema 4.17. Este resultado mostra que a envoltória von Neumann preserva a categoria dos semigrupos reais representados por aneis semi-reais. Além disso, mostra também que o morfismo natural de multi-aneis $V(A) \rightarrow V\left(G_{A}\right)$ satisfaz a propriedade universal do morfismo $V(A) \rightarrow G_{V(A)}$ (Teorema 2.41).

- O uso da mesma notação para envoltória von Neumann de um anel e de um semigrupo real no Teorema 4.17 não é ao acaso. O resultado pode ser largamente estendido para a categoria dos multi-aneis. É possível definir nesta categoria uma noção de envoltória von Neumann regular que generaliza simultaneamente as envoltórias von Neumann de aneis e semigrupos reais. Para mais detalhes, veja [RM3].

Seja $R$ RS. Considere o morfismo $\varepsilon: R \rightarrow P_{R}=C\left(\left(X_{R}\right)_{c o n s t}, 3\right)$ para a envoltória de Post de $R$ (para mais detalhes, confira o início da Seção 5.2.3 e em especial a Proposição 5.47). Pela Proposição 5.46, o RS $P_{R}$ é von Neumann. Logo, pelo Teorema 4.14, existe único morfismo $\bar{\varepsilon}: V(R) \rightarrow P_{R}$ satisfazendo $\varepsilon=\bar{\varepsilon} \circ v$.

Proposição 4.19. Seguindo a notação acima, vale:

i) $\bar{\varepsilon}: V(R) \rightarrow P_{R}$ é imersão completa. Em particular, $\bar{\varepsilon}$ é injetora e dados $a, b, c \in V(R)$ temos que

$$
a \in D_{V(R)}^{t}(b, c) \Leftrightarrow \bar{\varepsilon}(a) \in D_{P_{R}}^{t}(\bar{\varepsilon}(b), \bar{\varepsilon}(c)) .
$$

ii) Dada forma $\varphi \in V(R)$ e $\sigma \in X_{R}$, vale que

$$
\bar{\varepsilon}(\varphi)(\sigma)=\operatorname{sgn}_{\sigma}(\varphi) .
$$

iii) $\operatorname{Im}(\bar{\varepsilon})=\left\{f \in P_{R}: \forall p \in \operatorname{spec}(R), \exists x \in R\right.$ tal que $\left.\left.f\right|_{X_{p}}=\left.\varepsilon(x)\right|_{X_{p}}\right\}$.

Demonstração. i) Pela Proposição 5.47, o morfismo $\varepsilon: R \rightarrow P_{R}$ é imersão completa. Logo pelo Teorema 4.14.(iii) o morfismo $\bar{\varepsilon}: V(R) \rightarrow P_{R}$ também é imersão completa.

ii) Seja $\varrho: V(R) \rightarrow P_{R}$ dada por $\left(\varphi \in V(R), \sigma \in X_{R}\right)$ :

$$
\begin{aligned}
\varrho(\varphi): X_{R} & \rightarrow 3 \\
\sigma & \mapsto \operatorname{sgn}_{\sigma}(\varphi) .
\end{aligned}
$$

Pela Proposição 3.4. $(i)$, @ preserva produtos e pelos itens $(i),(v)$ da Proposição 3.4 segue que $\varrho$ também preserva a representação transversal. Assim, $\varrho$ é morfismo de RSs satisfazendo $\varepsilon=\varrho \circ v$. Pela unicidade da propriedade universal $v: R \rightarrow V(R)$ (Teorema 4.14) segue que $\bar{\varepsilon}=\varrho$. 
iii) Seja $\varphi \in V(R)$. Pelo Lema 4.3, existem $a_{i}, b_{i} \in \operatorname{Id}(R)$ e $x_{i} \in R, i=1, \ldots, n$, tais que

- $\operatorname{spec}(R)=\bigsqcup_{i=1}^{n} D\left(a_{i}\right) \cap D\left(b_{i}\right)^{c}$.

- $\varphi \cong_{R} \bigoplus_{i=1}^{c} x_{i} a_{i}\left\langle 1,-b_{i}\right\rangle$.

Seja $p \in \operatorname{spec}(R)$. Então existe $i \in\{1, \ldots, n\}$ tal que $p \in D\left(a_{i}\right) \cap D\left(b_{i}\right)^{c}$. Fixe $\sigma \in X_{p}$. Assim $\operatorname{sgn}_{\sigma}(\varphi)=\sigma\left(x_{i}\right)$ e pelo item $\left.i i\right)$

$$
\begin{aligned}
\bar{\varepsilon}(\varphi)(\sigma) & =\operatorname{sgn}_{\sigma}(\varphi) \\
& =\sigma\left(x_{i}\right)=\varepsilon\left(x_{i}\right)(\sigma),
\end{aligned}
$$

o que prova a inclusão $\subseteq$. Reciprocamente, seja $f \in P_{R}$ tal que para todo $p \in \operatorname{spec}(R)$, existe $x \in R$ tal que $\left.f\right|_{X_{p}}=\left.\varepsilon(x)\right|_{X_{p}}(\star)$. Fixado $p \in \operatorname{spec}(R)$, seja $x \in R$ uma testemunha da igualdade $(\star)$. Considere o conjunto

$$
U=\left\{\sigma \in X_{R}: f(\sigma)=\sigma(x)\right\} .
$$

Como $f:\left(X_{R}\right)_{\text {const }} \rightarrow 3$ é contínua, $U \subseteq\left(X_{R}\right)_{\text {const }}$ é aberto-fechado e por hipótese $X_{p} \subseteq U$. Pelo Lema 2.18 segue que existem $a, b \in \operatorname{Id}(R)$ tais que $X_{p} \subseteq U(a) \cap Z(b) \subseteq U$. Deste modo,

- $p \in D(a) \cap D(b)^{c}$.

- Para todo $\sigma \in X_{R}$ com $\operatorname{supp}(\sigma) \in D(a) \cap D(b)^{c}$ vale $f(\sigma)=\sigma(x)$.

Pela compacidade de $(\operatorname{spec}(R))_{\text {const }}$ e pelo Lema 3.55, existem $c_{i}, d_{i} \in \operatorname{Id}(R)$ e $x_{i} \in R, i=$ $1, \ldots, k$, tais que

- $\operatorname{spec}(R)=\bigsqcup_{i=1}^{k} D\left(c_{i}\right) \cap D\left(d_{i}\right)^{c}$.

- Para todo $\sigma \in X_{R}$ com $\operatorname{supp}(\sigma) \in D\left(c_{i}\right) \cap D\left(d_{i}\right)^{c}$ vale $f(\sigma)=\sigma\left(x_{i}\right)$.

Seja $\varphi=\bigoplus_{i=1}^{k} x_{i} c_{i}\left\langle 1,-d_{i}\right\rangle$. Então dado $\sigma \in X_{R} \operatorname{com} \operatorname{supp}(\sigma) \in D\left(c_{i}\right) \cap D\left(d_{i}\right)^{c}$ vale

$$
\operatorname{sgn}_{\sigma}(\varphi)=\sigma\left(x_{i}\right)=f(\sigma) .
$$

Assim pelo item $i i$ ) vale que $\bar{\varepsilon}(\varphi)=f$.

Exemplo 4.20. - Seja $R$ semigrupo real espectral (confira Definição V.1.8, pag. 173, de [DP2] ou caracterização de primeira ordem no Teorema V.7.4, pág. 194, da mesma referência). Então $V(R) \cong P_{R}$. Para justificar isso, vamos utilizar a Proposição 4.19.(iii) e mostrar que

$$
\left\{f \in P_{R}: \forall p \in \operatorname{spec}(R), \exists x \in R \text { tal que }\left.f\right|_{X_{p}}=\left.\varepsilon(x)\right|_{X_{p}}\right\}=P_{R}
$$

Pelo Teorema V.9.1 de [DP2] (pág. 199), o mapa espectral supp: $X_{R} \rightarrow \operatorname{spec}(R)$, que associa para cada ordem seu suporte, é homeomorfismo. Logo $X_{p}$ é unitário para todo $p \in \operatorname{spec}(R)$, de onde segue facilmente a igualdade $(\star)$.

- Seja $R$ um RS-fan (Definição VI.2.6 de [DP2]). Pela Proposição VI.1.2 de [DP2] (pág. 207), os ideias primos de $R$ são linearmente ordenados. Como os ideias primos de $V(R)$ são dois a dois incomparáveis, $V(R)$ é RS-fan apenas quando $R$ é 0-RSG.

Comentários 4.21. - A Proposição 4.19 fornece uma forma equivalente de definir a envoltória von Neumann como o subconjunto $\left\{f \in P_{R}: \forall p \in \operatorname{spec}(R), \exists x \in R\right.$ tal que $\left.f\right|_{X_{p}}=$ $\left.\left.\varepsilon(x)\right|_{X_{p}}\right\} \subseteq P_{R}$ com as noções de produto e de representação transveral induzidas da envoltória de Post $P_{R}$. 
- No artigo [RM3], é apresentada outra maneira de construir a envoltória von Neumann de um semigrupo real utilizando o pré-feixe estrutural (Definido logo acima da Definição 1.25). Neste paper é construído o funtor $V:$ Multi $\rightarrow$ GvNH que associa a cada multi-anel um hiper-anel von Neumann geométrico. No Teorema 4.8 de [RM3] é provado que o funtor $V$ é adjunto à esquerda do funtor de inclusão GvNH $\hookrightarrow$ Multi. Em seguida, no Corolário 4.13 de [RM3] é provado que a restrição desse funtor na categoria dos multi-aneis reais reduzidos (versão dos RSs na linguagem dos multianeis - Teorema 2.32) fornece um adjunto à esquerda da inclusão dos hiper-aneis reais reduzidos na categoria dos multi-aneis reais reduzidos. Mas pelo Teorema 2.58, os RSs que são hiper-aneis dão exatamente os RSs von Neumann e portanto o isomorfismo de categorias do Teorema 2.32 se restringe a um isomorfismo entre a categoria dos RSs von Neumann e dos hiper-aneis reais reduzidos. Deste modo, o Corolário 4.13 de [RM3] é uma versão na linguagem dos multi-aneis da propriedade universal do Teorema 4.14.(ii).

Para finalizar a seção, é mostrado que a envoltória von Neumann de RSs preserva quocientes. Mais do que isso, a envoltória von Neumann leva morfismos quocientes para morfismos transversalmente 2-regulares.

Proposição 4.22. Seja $f: R \rightarrow G$ morfismo quociente de RSs. Então $V(f): V(R) \rightarrow V(G)$ é morfismo transversalmente-2 regular e sobrejetor. Em particular, $V(f)$ morfismo forte e quociente.

Demonstração. Primeiro vamos provar que $V(f): V(R) \rightarrow V(G)$ é quociente na categoria dos RSs von Neumann, isto é, satisfaz a propriedade universal da Definição 1.9. Seja $g: V(R) \rightarrow V$ morfismo para RS von Neumann $V$ satisfazendo $\operatorname{ker}(V(f)) \subseteq \operatorname{ker}(g)$. Então pela Proposição 1.10

$$
\operatorname{ker}(f) \subseteq \operatorname{ker}\left(v_{G} \circ f\right)=\operatorname{ker}\left(V(f) \circ v_{R}\right) \subseteq \operatorname{ker}\left(g \circ v_{R}\right)
$$

Assim, como $f$ é quociente, existe $\widetilde{g}: G \rightarrow V$ tal que $g \circ v_{R}=\widetilde{g} \circ f$.

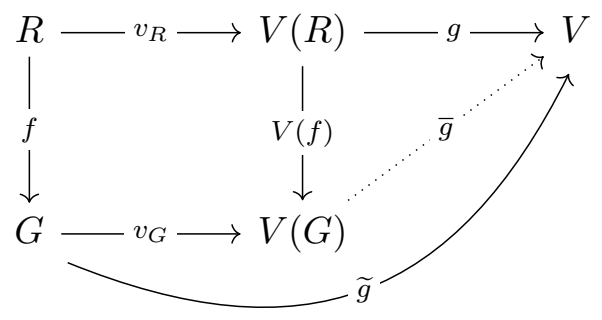

Por outro lado, pela propriedade universal da envoltória von Neumann $v_{G}$, existe $\bar{g}: V(G) \rightarrow V$ tal que $\widetilde{g}=\bar{g} \circ v_{G}$. Deste modo,

$$
g \circ v_{R}=\bar{g} \circ v_{G} \circ f=\bar{g} \circ V(f) \circ v_{R} .
$$

Pela unicidade da propriedade universal da envoltória von Neumann $v_{R}$, segue que $g=\bar{g} \circ V(f)$. A unicidade de $\bar{g}$ segue pela unicidade das propriedades universais de $f$ e $v_{G}$. Assim, segue do Teorema 2.69 que $V(f): V(R) \rightarrow V(G)$ é morfismo forte e sobrejetor. Além disso, pelo Corolário 2.71, $V(f)$ é transversalmente 2-regular.

\subsection{Aplicações}

Nesta seção, algumas aplicações da envoltória von Neumann são apresentadas como o cálculo do anel graduado, axiomatização dos aneis de Witt em $\mathcal{L}_{\omega_{1}, \omega}$ na linguagem dos aneis comutativos e a classificação dos quocientes na categoria dos aneis de Witt. Nos parágrafos seguintes são dadas descrições mais precisas das subseções.

Na subseção 4.2.1, primeiro é provado que se $R$ é RS, então $W_{g}(R)$, seu anel graduado de Witt, é isomorfo a $W_{g}(V(R))$. Em seguida, é mostrado como reduzir o cálculo de $W_{g}(V), V$ RS 
von Neumann, para o cálculo do anel graduado de Witt clássico do RSG $V^{\times}$. Essas simplificações permitem provar o Teorema Haupsatz de Arason-Pfister para todo RS. Além disso, utilizando uma versão natural da conjectura de Marshall ([MC]) para RS, provamos no Teorema 4.32 que dado qualquer p-anel $(A, T)$, o RS $G_{A, T}$ satisfaz [MC].

Na subseção 4.2.2, os esforços são concentrados em descrever a isometria e equivalência de Witt de um quociente de RS von Neumann. Pelo Teorema 2.69, basta considerar os quocientes de Marshall por um subgrupo von Neumann. O principal resultado é o Teorema 4.43 que generaliza a Proposição 2.21 de [DM1].

Na subseção 4.2.3, é descrito anel de semigrupo ternário para o caso dos inteiros e com isso o anel de Witt de um RS é descrito por geradores e relações canônicas.

Por fim, na subção 4.2.4, os aneis de Witt de um RS são axiomatizados em $L_{\omega_{1}, \omega}$ e é provado que a categoria dos aneis de Witt abstratos é equivalente a categoria dos RSs von Neumann.

\subsubsection{Anel Graduado de Witt}

Seja $R$ RS. Para cada $n \geq 1$, considere o quociente $\bar{I}^{n}(R):=I(R)^{n} / I(R)^{n+1}$ como grupo abeliano e $\bar{I}^{0}(R)=W(R) / I(R)$ (para simplificar a exposição, adotaremos a convenção $I(R)^{0}=$ $W(R))$. Note que $\bar{I}^{n}(R)$ é grupo de expoente 2 pois dada forma $\varphi \in I(R)^{n}$ tem-se $\varphi \oplus \varphi=$ $2 \otimes \varphi \in I(R)^{n+1}$. Para cada $n \geq 0$, considere o elemento distinguido $\top_{n}=2^{n} \in \bar{I}^{n}(R)$ e o mapa $h_{n}: \bar{I}^{n}(R) \rightarrow \bar{I}^{n+1}(R)$ dado por $h_{n}(\bar{\varphi})=\overline{2 \otimes \varphi}$. Note que $h_{n}$ é morfismo de grupos abelianos e $h_{n}\left(\top_{n}\right)=\top_{n+1}$.

Dados $n, m \geq 0$, considere a multiplicação $*: \bar{I}^{n}(R) \times \bar{I}^{m}(R) \rightarrow \bar{I}^{n+m}(R)$ induzida pela multiplicação de formas dada por $\bar{\varphi} * \bar{\psi}=\overline{\varphi \otimes \psi}$.

Como a notação apresentada acima, denotamos por $W_{g}(R)=\left(W(R) / I(R) \stackrel{h_{0}}{\longrightarrow} \bar{I}(R) \stackrel{h_{1}}{\longrightarrow}\right.$ $\left.\bar{I}^{2}(R) \stackrel{h_{2}}{\longrightarrow} \ldots\right)$ o anel graduado de Witt. $W_{g}(R)$ é um anel graduado indutivo de expoente 2 .

Proposição 4.23. Seja $R$ RS. Então existe isomorfismo canônico $W_{g}(R) \cong W_{g}(V(R))$ de anéis graduados.

Demonstração. Pelo Teorema 4.10, o morfismo de de RS $v: R \rightarrow V(R)$ induz isomorfismo de aneis de Witt $\mathrm{W}(R) \cong \mathrm{W}(V(R))$ que preserva os ideias fundamentais. Portanto induz isomorfismo $\mathrm{W}_{g}(G) \cong \mathrm{W}_{g}(V(R))$ de anéis graduados indutivos.

Definição 4.24. Seja $R \mathrm{RS}$. Uma forma $\varphi$ é regular se para todo $p, q \in \operatorname{spec}(R)$ tem-se $\operatorname{dim}_{p}(\varphi) \equiv$ $\operatorname{dim}_{q}(\varphi) \bmod 2$.

Proposição 4.25. Seja $R$ RS von Neumann. Sejam $\varphi, \psi$ formas regulares.

i) Se $\tau$ é uma forma qualquer e $\tau \cong \varphi$, então $\tau$ é regular.

ii) Se $a_{1}, \ldots, a_{n} \in R^{\times}$, então $\left\langle a_{1}, \ldots, a_{n}\right\rangle$ é regular.

iii) $\varphi \oplus \psi, \varphi \otimes \psi$ e $-\varphi$ são regulares.

Demonstração. i) Dado $\sigma \in X_{R}$, temos por 3.4 que

$$
\begin{aligned}
\operatorname{dim}_{\sigma}(\tau) & \equiv \operatorname{sgn}_{\sigma}(\tau)=\operatorname{sgn}_{\sigma}(\varphi) \\
& \equiv \operatorname{dim}_{\sigma}(\varphi) \bmod 2
\end{aligned}
$$

Dados $p, q \in \operatorname{spec}(R)$, temos $\operatorname{dim}_{p}(\tau) \equiv \operatorname{dim}_{p}(\varphi) \equiv \operatorname{dim}_{q}(\varphi) \equiv \operatorname{dim}_{q}(\tau) \bmod 2$. Logo $\tau$ é regular.

ii) Basta notar que para todo $p \in \operatorname{spec}(R)$ vale $\operatorname{dim}_{p}\left(\left\langle a_{1}, \ldots, a_{n}\right\rangle\right)=n$.

iii) O resultado segue da Proposição 3.4 pois para qualquer $p \in \operatorname{spec}(R)$ temos 
- $\operatorname{dim}_{p}(\varphi \oplus \psi)=\operatorname{dim}_{p}(\varphi)+\operatorname{dim}_{p}(\psi)$.

- $\operatorname{dim}_{p}(\varphi \otimes \psi)=\operatorname{dim}_{p}(\varphi) \cdot \operatorname{dim}_{p}(\psi)$.

- $\operatorname{dim}_{p}(-\varphi)=\operatorname{dim}_{p}(\varphi)$.

Definição 4.26. Seja $R$ RS von Neumann. O anel de Witt regular é definido por $W_{2}(R):=\{\varphi \in$ $W(R): \varphi$ é regular\}. Note que $I(R) \subseteq W_{2}(R)$.

Seja $R$ RS von Neumann. Pelo Teorema 2.58, o conjunto dos inversíveis $R^{\times}$é RSG. O próximo resultado relaciona o anel de Witt de $R^{\times} \operatorname{com} W_{2}(R)$.

Proposição 4.27. Seja $R$ RS von Neumann. Então o morfismo $\rho: W\left(R^{\times}\right) \rightarrow W_{2}(R)$ dado por $\rho\left(\left\langle a_{1}, \ldots, a_{n}\right\rangle\right)=\left\langle a_{1}, \ldots, a_{n}\right\rangle$ para todos $a_{1}, \ldots, a_{n} \in R^{\times}$é isomorfismo e preserva os ideais fundamentais.

Demonstração. $\quad-\rho$ está bem-definida e é morfismo de anéis.

Sejam $\varphi=\left\langle a_{1}, \ldots, a_{n}\right\rangle$ e $\psi=\left\langle b_{1}, \ldots, b_{k}\right\rangle$ duas formas sobre $R^{\times}$tais que $\varphi \cong_{R^{\times}} \psi$, ou seja, $\varphi=\psi$ em $W\left(R^{\times}\right)$. Como $R$ é Booleano também, pelo Teorema 2.54 temos $X_{R^{\times}} \cong X_{R}(*)$. Logo pelo princípio local-global de Pfister temos $\varphi \cong_{R} \psi$. Além disso, como a soma e produto é feito coordenada a coordenada tanto em $W\left(R^{\times}\right)$quanto em $W_{2}(R), \rho$ é morfismo de anéis.

- $\rho$ é injetora.

Se $\rho(\varphi)=0$, então por (*) segue que $\varphi$ é forma hiperbólica sobre $R^{\times}$. Logo $\varphi=0$ em $W\left(R^{\times}\right)$.

- $\rho$ é sobrejetora.

Seja $\varphi \in W_{2}(R)$ forma regular. Como $R$ é também Booleano, pelo Teorema $\left.3.51 i\right)-c$ ) existem $b_{1}, \ldots, b_{k} \in R^{\times}$tais que $\varphi \cong\left\langle b_{1}, \ldots, b_{k}\right\rangle$. Segue pela bijeção $(*)$ que $\rho\left(\left\langle b_{1}, \ldots, b_{k}\right\rangle\right)=\varphi$.

Considere agora $I\left(R^{\times}\right)=\left\{\left\langle a_{1}, \ldots, a_{n}\right\rangle \in W\left(R^{\times}\right): n\right.$ é par $\}$. É fácil ver que $\rho\left(I\left(R^{\times}\right)\right) \subseteq I(R)$. Reciprocamente, dada $\varphi \in I(R)$, temos que $k=\operatorname{mdim}(\varphi)$ é par e novamente pelo Teorema 3.51 existem $b_{1}, \ldots, b_{k} \in R^{\times}$tais que $\varphi \cong\left\langle b_{1}, \ldots, b_{k}\right\rangle$. Deste modo $\left\langle b_{1}, \ldots, b_{k}\right\rangle \in I\left(R^{\times}\right)$e portanto $\rho\left(I\left(R^{\times}\right)\right)=I(R)$.

Teorema 4.28 (Arason-Pfister Haupsatz). Sejam $R R S$ e $\varphi$ forma. Se $\varphi \in I(R)^{n}$ para algum $n \geq 1$ e $\operatorname{dim}(\varphi)<2^{n}$, então $\varphi \cong 0$.

Demonstração. Pelo Teorema $4.10 \mathrm{iii)}$ e Comentário 4.11, podemos assumir que $R$ é von Neumann. Além disso, como $\varphi$ é regular, pelo Teorema $3.51 i)-c$ ), existem $b_{1}, \ldots, b_{k} \in R^{\times}$tais que $\rho\left(\left\langle b_{1}, \ldots, b_{k}\right\rangle\right)=\varphi$ e $k<2^{n}$. Pelo Teorema 4.27 segue que $\left\langle b_{1}, \ldots, b_{k}\right\rangle \in I\left(R^{\times}\right)^{n}$ e portanto pelo Teorema Arason-Pfister Haupsatz aplicado ao RSG $R^{\times}$(Teorema 7.31 de [DM1]) segue que $\left\langle b_{1}, \ldots, b_{k}\right\rangle \cong_{R^{\times}} 0 . \operatorname{Logo} \varphi \cong 0$.

Corolário 4.29. Seja $R$ RS. Então $\bigcap_{n \geq 1} I(R)^{n}=\{0\}$.

Demonstração. Imediato pelo teorema anterior.

Teorema 4.30. Sejam $R R S$ von Neumann e $I=I(R) \subseteq W(R)$ ideal fundamental. Então

- o morfismo natural de aneis $f_{0}: W(R) / I(R) \rightarrow C\left(\operatorname{spec}(R), \mathbb{Z}_{2}\right)$ é isomorfismo.

- Para $i \geq 1$, o morfismo natural de grupos abelianos $f_{i}: I\left(R^{\times}\right)^{i} / I\left(R^{\times}\right)^{i+1} \rightarrow I(R)^{i} / I(R)^{i+1} \dot{e}$ isomorfismo.

Demonstração. O isomorfismo de anéis $f_{0}$ segue do Teorema 3.56 e os morfismos $f_{i}$ seguem do isomorfismo $\rho$ descrito na Proposição 4.27. 
Pelo teorema acima e pela Proposição 4.23, se $R$ é um RS qualquer e $V(R)$ é sua envoltória von Neumann, então

$$
W_{g}(R) \cong\left(W(R) / I(R) \cong C\left((\operatorname{spec}(R))_{\text {const }}, \mathbb{Z}_{2}\right) \rightarrow I\left(V(R)^{\times}\right) / I\left(V(R)^{\times}\right)^{2} \rightarrow \cdots\right)
$$

Seja $R$ RS. Note que dada forma $\varphi \in I(R)^{n}$, pelo Teorema 3.56 tem-se que $\operatorname{sgn}_{\sigma}(\varphi) \equiv 0 \bmod 2$ para todo $\sigma \in X_{R}$.

$\{\mathrm{mcDef}\}$

Definição 4.31. Seja $R$ RS. Dizemos que $R$ satisfaz [MC] (conjectura de Marshall) quando vale a seguinte afirmação:

Se $\varphi$ é uma forma satisfazendo $\operatorname{sgn}_{\sigma}(\varphi) \equiv 0 \bmod 2^{n}$ para todo $\sigma \in X_{R}$, então $\varphi \in I(R)^{n}$.

$\{\mathrm{mc}\}$

Proposição 4.32. Seja $R$ RS. São equivalentes:

i) $R$ satisfaz $[\mathrm{MC}]$;

ii) $V(R)$ satisfaz $[\mathrm{MC}]$;

iii) O RSG $V(R)^{\times}$satisfaz $[\mathrm{MC}]$;

Demonstração. Vamos provar $i i) \Rightarrow i) \Rightarrow i i i) \Rightarrow i i)$.

ii) $\Rightarrow i$ ): Seja $\varphi$ forma sobre $R$ satisfazendo $\operatorname{sgn}_{\sigma}(\varphi) \equiv 0 \bmod 2^{n}$ para todo $\sigma \in X_{R}$. Assim, para todo $\tau \in X_{V(R)}$ vale que $\operatorname{sgn}_{\tau}(v \star \varphi) \equiv 0 \bmod 2^{n}$. Como $V(R)$ satisfaz [MC], segue que $v \star \varphi \in I(V(R))^{n}$. Pelo Teorema $\left.4.10 \mathrm{iii}\right)$ tem-se $\varphi \in I(R)^{n}$.

i) $\Rightarrow$ iii): Seja $\varphi$ forma sobre $V(R)^{\times}$satisfazendo $\operatorname{sgn}_{\tau}(\varphi) \equiv 0 \bmod 2^{n}$ para todo $\tau \in X_{V(R)^{\times}}$. Pelo Teorema $4.10 \mathrm{iii}$ ) segue que existe forma $\varphi^{\prime}$ sobre $R$ tal que $v \star \varphi^{\prime}=\varphi$ em $W(V(R))$. Assim, novamente pelo Teorema $4.10 \mathrm{iii})$ e por 2.54 segue que $\operatorname{sgn}_{\sigma}\left(\varphi^{\prime}\right) \equiv 0 \bmod 2^{n}$ para todo $\sigma \in X_{R}$. Logo, por hipótese temos $\varphi^{\prime} \in I(R)^{n}$ e portanto $\varphi \in I(V(R))^{n}$. Pela Proposição 4.27 segue que $\varphi \in I\left(V(R)^{\times}\right)^{n}$.

iii $) \Rightarrow i$ : Seja $\varphi$ forma sobre $V(R)$ satisfazendo $\operatorname{sgn}_{\tau}(\varphi) \equiv 0 \bmod 2^{n}$ para todo $\tau \in X_{V(R)}$. Assim $\varphi$ é regular e pelo Teorema 3.51 existem $b_{1}, \ldots, b_{k} \in V(R)^{\times}$tal que $\varphi \cong\left\langle b_{1}, \ldots, b_{k}\right\rangle$. Novamente pelo Teorema 2.54 segue que $\operatorname{sgn}_{\sigma}\left(\left\langle b_{1}, \ldots, b_{k}\right\rangle\right) \equiv 0 \bmod 2^{n}$ para todo $\sigma \in X_{V(R)^{\times}}$. Por \{qvcomm\} hipótese segue que $\left\langle b_{1}, \ldots, b_{k}\right\rangle \in I\left(V(R)^{\times}\right)^{n}$ e a Proposição 4.27 implica que $\varphi \in I(V(R))^{n}$.

Teorema 4.33. Seja $(A, T)$ p-anel. Então existe $\tilde{T} \subseteq V(A)$ pré-ordem própria na envoltória von Neumann regular do anel $A$ tal que o morfismo canônico $(A, T) \rightarrow(V(A), \tilde{T})$ induz isomorfismo $V\left(G_{A, T}\right) \cong G_{V(A), \tilde{T}}$, onde $V\left(G_{A, T}\right)$ denota a envoltória von Neumann do $R S G_{A, T}$.

Demonstração. Consequência imediata do Teorema 4.12 de [RM3].

Teorema 4.34. Seja $(A, T)$ p-anel. Então $G_{A, T}$ satisfaz [MC].

Demonstração. Pela Proposição 4.32 e pelo Teorema 4.33, podemos supor $A$ anel von Neumann regular. Por outro lado, considerando o anel $B=(1+T)^{-1} A$ e o subconjunto $S=\left\{\frac{t}{x^{2}} \in B: t \in\right.$ $T, x \in 1+T\}$, pelo Teorema 2.45.(ii) temos que

- $(B, S)$ é p-anel.

- $1+S \subseteq B^{\times}$.

- O morfismo canônico $(A, T) \rightarrow(B, S)$ induz isomorfismo $G_{A, T} \cong G_{B, S}$.

Assim, como $B$ é von Neumann regular, podemos supor sem perda de generalidade que $A$ é von Neumann regular e $1+T \subseteq A^{\times}$. 
Note que $2 \in 1+T \subseteq A^{\times}$. No artigo [DM9] é construido o RSG $G_{T}(A)$ a partir dos inversíveis do anel von Neumann regular $A$. Além disso, pelo Teorema 7.2 de [DM9] o RSG $G_{T}(A)$ satisfaz [MC]. Por outro lado, como $(A, T)$ tem BIP, pela Proposição 7.8, pag. 73 de [DM10], segue que $G_{T}(A)$ é canonicamente isomorfo a $\left(G_{A, T}\right)^{\times}$na linguagem dos grupos especiais. Portanto, $\left(G_{A, T}\right)^{\times}$ satisfaz [MC] e pela Proposição 4.32 segue que $G_{A, T}$ também satisfaz [MC].

Comentário 4.35. Sejam $A$ anel semi-real e $\varphi$ forma sobre $G_{A}$. Fixe $n \geq 1$. O Teorema 4.34 mostra que

$$
\text { se para todo } \sigma \in X_{G_{A}} \cong \operatorname{sper}(A) \text { valer } \operatorname{sgn}_{\sigma}(\varphi) \equiv 0 \bmod 2^{n},(\star)
$$

então existem formas de Pfister $\varphi_{1}, \ldots, \varphi_{t}$ de grau $n$ sobre o semigrupo real $G_{A}$ tais que $\varphi \cong_{G_{A}}$ $\pm \varphi_{1} \pm \cdots \pm \varphi_{t}$. No entando, o Teorema 4.34 não prova que se a forma inicial $\varphi$ tiver coeficientes em $\left(G_{A}\right)^{\times}$, então as formas de Pfister $\varphi_{1}, \ldots, \varphi_{t}$ podem ser assumidas com coeficientes em $\left(G_{A}\right)^{\times}$.

Sendo assim, contínua em aberto o seguinte problema:

Seja $(A, T)$ p-anel com bounded inversion property $\left(1+T \subseteq A^{\times}\right)$tal que $A$ é $T$-fielmente quadrático $^{1}{ }^{2}$. Será que o RSG $G_{T}(A)$, construído como quociente de $A^{\times}$por $T^{\times 3}$, satisfaz a conjectura de Marshall?

O próximo resulta fornece outra caracterização dos primeiros níveis do anel de Witt graduado usando a já conhecida bijeção entre um RSG e o quociente do ideal fundamental por seu quadrado. Dado $a \in R$, vamos usar no próximo resultado que $\langle\nabla(a)\rangle \cong\left\langle a, a^{2},-1\right\rangle$.

Teorema 4.36. Seja $R R S$ von Neumann. Considere as funções $\alpha: \operatorname{Id}(R) \rightarrow W(R) / I(R) e$ \{iniGradWR\} $\beta: R^{\times} \rightarrow I(R) / I(R)^{2}$ dadas por

$$
\alpha(a)=\langle 1,-a\rangle, \beta(g)=\langle 1,-g\rangle .
$$

Então $\alpha$ e $\beta$ são bijetoras satisfazendo as seguintes propriedades:

- Dados $a, b \in I d(R)$, temos $\alpha(a \wedge b)=\alpha(a) \otimes \alpha(b)$ e $\alpha(a \vee b)=\alpha(a) \oplus \alpha(b) \oplus \alpha(a) \alpha(b)$. Além disso, $\alpha(0)=1$ e $\alpha(1)=0$.

- $\beta$ é morfismo de grupos abelianos ( $R^{\times}$multiplicativo e $\bar{I}(R)$ aditivo) que preserva elemento distinguido.

Além disso, o diagrama

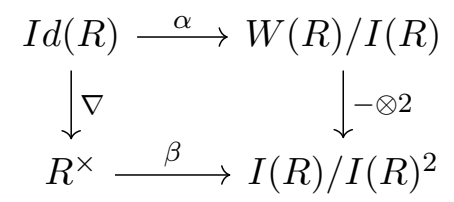

é comutativo.

Demonstração. $\quad$ - $\alpha$ é injetora.

Sejam $a, b \in I d(R)$ tais que $\alpha(a)=\alpha(b)$, ou seja, $\langle 1,-a\rangle-\langle 1,-b\rangle=\langle-a, b\rangle \in I(R)$. Deste modo, para todo $p \in \operatorname{spec}(R)$ temos $\operatorname{dim}_{p}(\langle-a, b\rangle) \in\{0,2\}$. Logo, dado $p \in \operatorname{spec}(R)$ temos que $a \in p$ se, e somente se, $b \in p$. Como $a, b$ são idempotentes, temos $a=b$.

- $\alpha$ é sobrejetora.

Seja $\varphi \in W(R)$ forma. Como $R$ é von Neumann, pelo Teorema 3.8 existem $e, f \in \operatorname{Id}(R)$ tais

\footnotetext{
${ }^{1}$ Com a bounded inversion property, garante-se que $G_{T}(A) \cong\left(G_{A, T}\right)^{\times}$na linguagem dos grupos especiais. Ver Proposição 7.8, pag. 73, de [DM10]

${ }^{2}$ Para consultar a noção de $T$-fielmente quadrático, veja a Definição 3.1, pag. 27, de [DM10]

${ }^{3}$ Para mais detalhes, consulte a seção 2 do segundo capítulo de [DM10]
} 
que

$$
\begin{aligned}
& D(e)=\left\{p \in \operatorname{spec}(R): \operatorname{dim}_{p}(\varphi) \text { é } \operatorname{par}\right\} \\
& D(f)=\left\{p \in \operatorname{spec}(R): \operatorname{dim}_{p}(\varphi) \text { é ímpar }\right\}
\end{aligned}
$$

Note que $\operatorname{spec}(R)=D(e) \sqcup D(f)$ e portanto

$$
\begin{aligned}
\varphi & \equiv e \varphi \oplus f \varphi \cong e \varphi \oplus f \varphi \oplus\langle-f, f\rangle \\
& \equiv e \varphi \oplus(f \varphi \oplus\langle-f\rangle) \oplus\langle f\rangle .
\end{aligned}
$$

Mas note que, por definição de $e, f$, temos $e \varphi, f \varphi \oplus\langle-f\rangle \in I(R)$. Logo, como $e^{c}=f$, em $W(R) / I(R)$ vale que $\varphi=\langle f\rangle=\langle 1 .-e\rangle=\alpha(e)$.

- $\beta$ é injetora.

Sejam $g, h \in R^{\times}$tais que $\beta(g)=\beta(h)$, ou seja, $\langle-a, b\rangle=\langle 1,-a\rangle-\langle 1,-b\rangle \in I(R)^{2}$. Como $\operatorname{dim}(\langle-a, b\rangle)=2<2^{2}$, pelo Teorema 4.28 segue que $\langle-a, b\rangle \cong 0$. Logo $a=b$.

- $\beta$ é morfismo de grupos que preserva elementos distinguido e é sobrejetora.

Dados $g, h \in R^{\times}$, é fácil verificar que $\langle 1,-g h\rangle \oplus\langle 1,-g\rangle \otimes\langle 1,-h\rangle \cong\langle 1,-g\rangle \oplus\langle 1,-h\rangle$. Dessa equivalência segue que $\beta(g h)=\beta(g)+\beta(h)$. Além disso, $\beta(-1)=\langle 1,1\rangle=2$.

Pelo Teorema $3.56 \mathrm{iii}), I(R)$ é gerado aditivamente por formas de Pfister de grau 1. Logo, como $\beta$ é morfismo, para provar sobrejetividade de $\beta$ é suficiente provarmos que dado $a \in R$, existe $g \in R^{\times}$tal que $\beta(g)=\left\langle a, a^{2}\right\rangle$.Mas pelo comentário que precede o presente teorema, temos que $\langle 1, \nabla(a)\rangle=\left\langle 1,-1, a, a^{2}\right\rangle \cong\left\langle a, a^{2}\right\rangle$ e portanto $\beta(-\nabla(a))=\left\langle a, a^{2}\right\rangle$.

- Dados $a, b \in I d(R)$, vale $\alpha(a \wedge b)=\alpha(a) \otimes \alpha(b)$ e $\alpha(a \vee b)=\alpha(a) \oplus \alpha(b) \oplus \alpha(a) \alpha(b)$.

Primeiro note que $\langle a \wedge b\rangle \cong\langle a, b,-a b\rangle(*)$. Fixado $\sigma \in X_{R}$, se $\sigma(a)=0$, então $\sigma(\langle a \wedge b\rangle)=\sigma(b)$ e portanto $\operatorname{sgn}_{\sigma}(a \wedge b)=\sigma(a \wedge b)=\sigma(b)=\operatorname{sgn}_{\sigma}(\langle a, b,-a b\rangle)$. De forma análoga verifica-se $(*)$ caso $\sigma(b)=0$. Se $\sigma(a)=\sigma(b)=1$, então $\sigma(a \wedge b)=1$ e $\operatorname{sgn}_{\sigma}(\langle a \wedge b\rangle)=1=\operatorname{sgn}_{\sigma}(\langle a, b,-a b\rangle)$.

Deste modo, temos em $W(R) / I(R)$

$$
\begin{aligned}
\alpha(a) \otimes \alpha(b) & =\langle 1,-a\rangle \otimes\langle 1,-b\rangle=\langle 1,-a,-b, a b\rangle=\langle 1,-a \wedge b\rangle=\alpha(a \wedge b) . \\
\alpha(a) \oplus \alpha(b) \oplus \alpha(a) \alpha(b) & =\langle 1,-a\rangle \oplus\langle 1,-b\rangle \oplus\langle 1,-a,-b, a b\rangle \\
& =\langle 1,1\rangle \oplus\langle-a,-a\rangle \oplus\langle-b,-b\rangle \oplus\langle 1, a b\rangle \\
& =\langle 1, a b\rangle=\langle 1,-a b\rangle=\alpha(a \vee b) .
\end{aligned}
$$

Para finalizar a demonstração, precisamos verificar que o diagrama comuta. Mas note que, pelo comentário que precede o presente teorema, dado $a \in I d(R)$ temos em $I(R) / I(R)^{2}$ que $\beta(\nabla(a))=$ $\langle 1,-\nabla(a)\rangle=2\langle 1,-a\rangle=2 \otimes \alpha(a)$.

\subsubsection{Quocientes em Aneis de Witt}

Definição 4.37. Sejam $R$ RS von Neumann e $\varphi, \psi$ formas. Considere também $S \subseteq R$ subgrupo saturado e $I \subseteq R$ ideal próprio. Sejam $\pi_{1}: R \rightarrow R /{ }_{m} S$ e $\pi_{2}: R \rightarrow R / I$ as projeções canônicas.

i) Denota-se por $\varphi \equiv_{S} \psi$ a fórmula $\pi_{1} \star \varphi \equiv_{R / m S} \pi_{1} \star \psi$.

ii) Denota-se por $\varphi \equiv_{I} \psi$ a fórmula $\pi_{2} \star \varphi \equiv_{R / I} \pi_{2} \star \psi$.

iii) Dizemos que $\varphi \equiv_{S}^{*} \psi$ quando existir forma de Pfister $\theta$ sobre $S$ (ou seja, existem $s_{1}, \ldots, s_{n} \in S$ tais que $\left.\theta=\bigotimes_{i=1}^{n}\left\langle s_{i}^{2}, s_{i}\right\rangle\right)$ tal que $\varphi \otimes \theta \equiv \psi \otimes \theta$.

iv) Dizemos que $\varphi \equiv_{I}^{+} \psi$ se existem formas $\theta$, $\theta^{\prime}$ sobre $I$ tais que $\varphi \oplus \theta \equiv \psi \oplus \theta^{\prime}$. 
Comentário 4.38. Com a notação da definição acima, pela Proposição 2.43 vale que $X_{R / m} \cong$ $\left\{\sigma \in X_{R}: \sigma(S)=\{1\}\right\}$ e $X_{R / I} \cong\left\{\sigma \in X_{R}: \sigma(I)=\{0\}\right\}$. Logo

$$
\begin{aligned}
& \varphi \equiv_{S} \psi \Leftrightarrow \text { para todo } \sigma \in X_{R} \operatorname{com} \sigma(S)=\{1\} \text { vale } \operatorname{sgn}_{\sigma}^{ \pm}(\varphi)=\operatorname{sgn}_{\sigma}^{ \pm}(\psi) \\
& \varphi \equiv_{I} \psi \Leftrightarrow \text { para todo } \sigma \in X_{R} \operatorname{com} \sigma(I)=\{0\} \text { vale } \operatorname{sgn}_{\sigma}^{ \pm}(\varphi)=\operatorname{sgn}_{\sigma}^{ \pm}(\psi) .
\end{aligned}
$$

Proposição 4.39. Sejam $R$ RS von Neumann e $S \subseteq R$ subgrupo saturado. Sejam $\varphi, \psi, \tau$ formas.

i) $\equiv_{S}^{*}$ é relação de equivalência.

ii) Se $\varphi \equiv_{S}^{*} \psi$, então $\varphi \equiv_{S} \psi$.

iii) Se $\varphi \equiv_{S}^{*} \psi$, então $\varphi \oplus \tau \equiv_{S}^{*} \psi \oplus \tau$ e $\varphi \otimes \tau \equiv_{S}^{*} \psi \otimes \tau$.

iv) Se $\varphi \equiv_{S}\langle 0\rangle$, então existe $s \in S$ tal que $s \varphi \equiv\langle 0\rangle$.

v) Se $\varphi=\left\langle a_{1}, \ldots, a_{n}\right\rangle$ e $s_{1}, \ldots, s_{n} \in S$, então $\left\langle a_{1} s_{1}, \ldots, a_{n} s_{n}\right\rangle \equiv_{S}^{*} \varphi$.

vi) Se $\varphi$ é $k$-forma em $R / m S$, então existe forma $\theta$ tal que $\theta$ é $k$-forma em $R$ e $\varphi \equiv_{S}^{*} \theta$.

vii) Seja $e \in \operatorname{Id}(R)$. Se $e \varphi \equiv_{S} \varphi$, então $e \varphi \equiv_{S}^{*} \varphi$.

Demonstração. i) É fácil ver que a relação $\equiv_{S}^{*}$ é reflexiva e simétrica, restando mostrar que é transitiva. Suponha que $\varphi \equiv_{S}^{*} \psi$ e $\psi \equiv_{S}^{*} \tau$. Então existem $\theta, \theta^{\prime}$ formas de Pfister sobre $S$ tais que $\varphi \otimes \theta \equiv \psi \otimes \theta$ e $\psi \otimes \theta^{\prime} \equiv \tau \otimes \theta^{\prime}$. Deste modo $\varphi \otimes \theta \otimes \theta^{\prime} \equiv \psi \otimes \theta \otimes \theta^{\prime} \equiv \tau \otimes \theta \otimes \theta^{\prime}$. Como $\theta \otimes \theta^{\prime}$ é forma de Pfister sobre $S$, temos $\varphi \equiv_{S}^{*} \tau$.

ii) Seja $\theta=\bigotimes_{i=1}^{n}\left\langle s_{i}^{2}, s_{i}\right\rangle, s_{i} \in S$, forma de Pfister sobre $S$ tal que $\varphi \otimes \theta \equiv \psi \otimes \theta$. Então dado $\sigma \in X_{R} \operatorname{com} \sigma(S)=\{1\}$ temos

$$
2^{n} \operatorname{sgn}_{\sigma}^{ \pm}(\varphi)=\operatorname{sgn}_{\sigma}^{ \pm}(\varphi \otimes \theta)=\operatorname{sgn}_{\sigma}^{ \pm}(\psi \otimes \theta)=2^{n} \operatorname{sgn}_{\sigma}^{ \pm}(\psi)
$$

e assim $\operatorname{sgn}_{\sigma}^{ \pm}(\varphi)=\operatorname{sgn}_{\sigma}^{ \pm}(\psi)$. Como o $\sigma \in X_{R}$ escolhido foi arbitrário, temos $\varphi \equiv_{S} \psi$.

iii) Seja $\theta$ forma de Pfister sobre $S$ tal que $\varphi \otimes \theta \equiv \psi \otimes \theta$. Então

$$
\begin{aligned}
(\varphi \oplus \tau) \otimes \theta \equiv \varphi \otimes \theta \oplus \tau \otimes \theta & \equiv \psi \otimes \theta \oplus \tau \otimes \theta \equiv(\psi \oplus \tau) \otimes \theta \\
\varphi \otimes \tau \otimes \theta & \equiv \psi \otimes \tau \otimes \theta .
\end{aligned}
$$

$\operatorname{Logo} \varphi \oplus \tau \equiv_{S}^{*} \psi \oplus \tau$ e $\varphi \otimes \tau \equiv_{S}^{*} \psi \otimes \tau$.

iv) Seja $\varphi=\left\langle a_{1}, \ldots, a_{n}\right\rangle$. Se $\varphi \equiv_{S}\langle 0\rangle$, então $a_{i}=0 \mathrm{em} R /{ }_{m} S$ para todo $i=1, \ldots, n$. Existe então $s_{i} \in S$ tal que $a_{i} s_{i}=0$ em $R$. Tomando $s=s_{1} \cdot \ldots \cdot s_{n} \in S$, temos $s \varphi \equiv\langle 0\rangle$.

v) Considere a forma $\theta=\bigotimes_{i=1}^{n}\left\langle s_{i}^{2}, s_{i}\right\rangle$. Como $s_{i}\left\langle s_{i}^{2}, s_{i}\right\rangle=\left\langle s_{i}^{2}, s_{i}\right\rangle$, temos

$$
\begin{aligned}
\left\langle a_{1} s_{1}, \ldots, a_{n} s_{n}\right\rangle \otimes \theta & \equiv \bigoplus_{i=1}^{n} a_{i} s_{i} \theta \\
& \equiv \bigoplus_{i=1}^{n} a_{i} \theta \equiv \varphi \otimes \theta
\end{aligned}
$$

$\mathrm{e} \operatorname{assim}\left\langle a_{1} s_{1}, \ldots, a_{n} s_{n}\right\rangle \equiv_{S}^{*} \varphi$. 
vi) Seja $\left(\varphi_{1}, \ldots, \varphi_{p}\right)$ sistema ortogonal para $\varphi$ em $R \operatorname{com} \varphi_{i}$ uma $c_{i}$-forma. Como $\varphi$ é $k$-forma em $R / m S$ e vale $\varphi \equiv_{S} \varphi_{1} \oplus \cdots \oplus \varphi_{p}$, existe $1 \leq j \leq p$ tal que $c_{j}=k$ e $\varphi_{i} \equiv_{S}\langle 0\rangle$ para $i \neq j$. Assim, para cada $i \neq j$, existe $s_{i} \in S$ tal que $s_{i} \varphi_{i} \equiv\langle 0\rangle$. Portanto, pelo item anterior desta proposição,

$$
\begin{aligned}
\varphi & \equiv \varphi_{1} \oplus \cdots \oplus \varphi_{j} \oplus \cdots \oplus \varphi_{p} \\
& \equiv{ }_{S}^{*} s_{1} \varphi_{1} \oplus \cdots \oplus \varphi_{j} \oplus \cdots \oplus s_{p} \varphi_{p} \\
& \equiv \varphi_{j} .
\end{aligned}
$$

vii) Escreva $\varphi=\left\langle a_{1}, \ldots, a_{n}\right\rangle$. Se $e \varphi \equiv_{S} \varphi$, então $a_{i} e=a_{i}$ em $R /{ }_{m} S$ para $i=1, \ldots, n$. Logo, pelo item $i v)$ desta proposição, $e \varphi \equiv_{S}^{*} \varphi$.

Proposição 4.40. Sejam $R$ RS von Neumann e $I \subseteq R$ ideal próprio. Sejam $\varphi, \psi, \tau$ formas.

i) $\equiv_{I}^{+}$é relação de equivalência.

ii) Se $\varphi \equiv_{I}^{+} \psi$, então $\varphi \equiv_{I} \psi$.

iii) Se $\varphi \equiv_{I}^{+} \psi$, então $\varphi \oplus \tau \equiv_{I}^{+} \psi \oplus \tau$ e $\varphi \otimes \tau \equiv_{I}^{+} \psi \otimes \tau$.

iv) Se $2 \varphi \equiv_{I}^{+} 2 \psi$, então $\varphi \equiv_{I}^{+} \psi$.

Demonstração. i) É fácil ver que $\equiv_{I}^{+}$é reflexiva e simétrica. Para a transitividade, suponha que $\varphi \equiv_{I}^{+} \psi$ e $\psi \equiv_{I}^{+} \tau$. Então existem formas $\theta_{i}$ sobre $I, i=1,2,3,4$ tais que $\varphi \oplus \theta_{1} \equiv \psi \oplus \theta_{2}$ e $\psi \oplus \theta_{3} \equiv \tau \oplus \theta_{4}$. Logo $\varphi \oplus \theta_{1} \oplus \theta_{3} \equiv \psi \oplus \theta_{2} \oplus \theta_{3} \equiv \tau \oplus \theta_{4} \oplus \theta_{2}$ e assim $\varphi \equiv_{I}^{+} \tau$.

ii) Sejam $\theta, \theta^{\prime}$ formas sobre $I$ tais que $\varphi \oplus \theta \equiv \psi \oplus \theta^{\prime}$. Assim dado $\sigma \in X_{R} \operatorname{com} \sigma(I)=\{0\}$, temos

$$
\begin{aligned}
\operatorname{sgn}_{\sigma}^{ \pm}(\varphi) & =\operatorname{sgn}_{\sigma}^{ \pm}(\varphi \oplus \theta) \\
& =\operatorname{sgn}_{\sigma}^{ \pm}\left(\psi \oplus \theta^{\prime}\right) \\
& =\operatorname{sgn}_{\sigma}^{ \pm}(\psi) .
\end{aligned}
$$

Deste modo $\varphi \equiv_{I} \psi$.

iii) Se $\varphi \equiv_{I}^{+} \psi$, então existem $\theta, \theta^{\prime}$ formas sobre $I$ tais que $\varphi \oplus \theta \equiv \psi \oplus \theta^{\prime}$. Logo $(\varphi \oplus \tau) \oplus \theta \equiv$ $(\psi \oplus \tau) \oplus \theta^{\prime}$ e $(\varphi \otimes \tau) \oplus \theta \otimes \tau \equiv(\psi \otimes \tau) \oplus \theta^{\prime} \otimes \tau$. Então $\varphi \oplus \tau \equiv_{I}^{+} \psi \oplus \tau$ e $\varphi \otimes \tau \equiv_{I}^{+} \psi \otimes \tau$ pois sendo $I$ ideal, as formas $\theta \otimes \tau, \theta^{\prime} \otimes \tau$ tem entradas em $I$.

iv) Se $2 \varphi \equiv_{I}^{+} 2 \psi$, então existem formas $\theta, \theta^{\prime}$ sobre $I$ tais que $2 \varphi \oplus \theta \equiv 2 \psi \oplus \theta^{\prime}$. Sejam $e, e^{\prime} \in \operatorname{Id}(R)$ com $\operatorname{supp}(\theta)=D(e)$ e $\operatorname{supp}\left(\theta^{\prime}\right)=D\left(e^{\prime}\right)$. Pelo Teorema 3.8 temos $e, e^{\prime} \in I$. Seja $f \in D^{t}\left(e, e^{\prime}\right) \subseteq$ I. Como $f^{c}=e^{c} e^{c}$, vale

$$
\begin{aligned}
2 f^{c} \varphi & \equiv f^{c}(2 \varphi \oplus \theta) \\
& \equiv f^{c}\left(2 \psi \oplus \theta^{\prime}\right) \\
& \equiv 2 f^{c} \psi
\end{aligned}
$$

Deste modo $f^{c} \varphi \equiv f^{c} \psi$. Logo

$$
\begin{aligned}
\varphi \oplus f \psi & \equiv\left(f \varphi \oplus f^{c} \varphi\right) \oplus f \psi \\
& \equiv f \varphi \oplus\left(f^{c} \psi \oplus f \psi\right) \equiv \psi \oplus f \varphi .
\end{aligned}
$$

Como $f \in I$, temos $\varphi \equiv_{I}^{+} \psi$.

Lema 4.41. Seja $R$ RS von Neumann. Então 
i) $i \in D^{t}(y,-z)$ se, e somente se, $\langle y\rangle \oplus\langle-i, i y z\rangle \equiv\langle z\rangle \oplus i^{2} y^{2}\langle 1,-1\rangle$.

ii) $a \in D^{t}(b, c)$ se, e somente se, $\langle a, a b c\rangle \oplus a^{c}(b c)^{2}\langle 1,-1\rangle \equiv\langle b, c\rangle$.

Demonstração. i) Pela Proposição $3.4 v$ ), temos

$$
\begin{aligned}
i \in D^{t}(y,-z) & \Leftrightarrow\langle i,-i y z\rangle \cong\langle y,-z\rangle \\
& \Leftrightarrow\langle y\rangle \oplus\langle-i, i y z\rangle \cong\langle z\rangle \\
& \Leftrightarrow\langle y\rangle \oplus\langle-i, i y z\rangle \cong\langle z\rangle \oplus i^{2} y^{2}\langle 1,-1\rangle .
\end{aligned}
$$

Assim para provarmos $i$ ), pela Proposição 3.4 iii), é suficiente provarmos que se $i \in D^{t}(y,-z)$, então para todo $p \in \operatorname{spec}(R)$ vale $\operatorname{dim}_{p}(\langle y\rangle \oplus\langle-i, i y z\rangle)=\operatorname{dim}_{p}\left(\langle z\rangle \oplus i^{2} y^{2}\langle 1,-1\rangle\right)$. Fixe $p \in$ $\operatorname{spec}(R)$. Sejam $\varphi=\langle y\rangle \oplus\langle-i, i y z\rangle$ e $\psi=\langle z\rangle \oplus i^{2} y^{2}\langle 1,-1\rangle$.

Caso 1: $y, z \in p$.

Então $i \in p$ e $\operatorname{dim}_{p}(\varphi)=0=\operatorname{dim}_{p}(\psi)$.

Caso 2: $y \in p$ e $z \notin p$.

Como $i \in D^{t}(y,-z)$, temos $i \notin p$. Logo $\operatorname{dim}_{p}(\varphi)=1=\operatorname{dim}_{p}(\psi)$.

Caso 3: $y \notin p$ e $z \in p$.

Então $i \notin p$. $\operatorname{Logo} \operatorname{dim}_{p}(\varphi)=2=\operatorname{dim}_{p}(\psi)$.

Caso 4: $y, z \notin p$.

Se $i \in p$, então $\operatorname{dim}_{p}(\varphi)=1=\operatorname{dim}_{p}(\psi)$. Se $i \notin p$, então $\operatorname{dim}_{p}(\varphi)=3=\operatorname{dim}_{p}(\psi)$.

ii) Pela Proposição $3.4 v$ ) vale

$$
\begin{aligned}
a \in D^{t}(b, c) & \Leftrightarrow\langle a, a b c\rangle \cong\langle b, c\rangle \\
& \Leftrightarrow\langle a, a b c\rangle \oplus a^{c}(b c)^{2}\langle 1,-1\rangle \cong\langle b, c\rangle .
\end{aligned}
$$

Seguindo o mesma linha de argumento usada no item anterior, é suficiente provarmos que se $a \in D^{t}(b, c)$, então para todo $p \in \operatorname{spec}(R)$ vale $\operatorname{dim}_{p}\left(\langle a, a b c\rangle \oplus a^{c}(b c)^{2}\langle 1,-1\rangle\right)=\operatorname{dim}_{p}(\langle b, c\rangle)$. $\operatorname{Sejam} \varphi=\langle a, a b c\rangle \oplus a^{c}(b c)^{2}\langle 1,-1\rangle$ e $\psi=\langle b, c\rangle$. Fixe $p \in \operatorname{spec}(R)$. Vamos analisar alguns casos:

Caso 1: $b, c \in p$.

Então $a \in p$ e $\operatorname{dim}_{p}(\varphi)=0=\operatorname{dim}_{p}(\psi)$.

Caso 2: $b \in p$ e $c \notin p$.

Como $a \in D^{t}(b, c)$, temos $a \notin p . \operatorname{Logo} \operatorname{dim}_{p}(\varphi)=1=\operatorname{dim}_{p}(\psi)$.

Caso 3: $b \notin p$ e $c \in p$.

Então $a \notin p . \operatorname{Logo} \operatorname{dim}_{p}(\varphi)=1=\operatorname{dim}_{p}(\psi)$.

Caso 4: $b, c \notin p$.

Se $a \in p$, então $a^{c} \notin p$ e $\operatorname{dim}_{p}(\varphi)=2=\operatorname{dim}_{p}(\psi)$. Se $a \notin p$, então $a^{c} \in p$ e $\operatorname{dim}_{p}(\varphi)=2=$ $\operatorname{dim}_{p}(\psi)$.

Lema 4.42. Seja $R$ RS von Neumann e $S \subseteq R$ subgrupo saturado. Sejam $a, b, c, d \in R$. São equivalentes:

i) $\langle a, b\rangle \equiv_{S}\langle c, d\rangle$.

ii) $\langle a, b\rangle \equiv_{S}^{*}\langle c, d\rangle$.

Demonstração. A direção $i i) \Rightarrow i$ ) segue do lema anterior. Suponha então que $\langle a, b\rangle \equiv_{S}\langle c, d\rangle$. Então pela Proposição $3.4 i v)$ temos $a b=c d$ em $R /{ }_{m} S$ e existe $z \in D_{S}(a, b) \cap D_{S}(c, d)$. Logo existem 
$t, t^{\prime}, t_{1}, t_{2}, t_{3}, s_{1}, s_{2}, s_{3} \in S$ tais que

$$
\begin{aligned}
& a b t=c d t^{\prime} \\
& z t_{1} \in D^{t}\left(a t_{2}, b t_{3}\right) \\
& z s_{1} \in D^{t}\left(c s_{2}, d s_{3}\right) .
\end{aligned}
$$

Assim, pelo Lema 4.41, temos

$$
\begin{aligned}
\left\langle z t_{1}, z a b t_{1} t_{2} t_{3}\right\rangle \oplus\left(z t_{1}\right)^{c}\left(a b t_{2} t_{3}\right)^{2}\langle 1,-1\rangle & \equiv\left\langle a t_{2}, b t_{3}\right\rangle \\
\left\langle z s_{1}, z c d s_{1} s_{2} s_{3}\right\rangle \oplus\left(z s_{1}\right)^{c}\left(c d s_{2} s_{3}\right)^{2}\langle 1,-1\rangle & \equiv\left\langle c s_{2}, d s_{3}\right\rangle .
\end{aligned}
$$

Por outro lado, pelo Lema 2.59, vale $\left(z t_{1}\right)^{c} t_{1}^{2}=\left(z t_{1}^{2}\right)^{c} t_{1}^{2}=z^{c} t_{1}^{2}$ e $\left(z s_{1}\right)^{c} s_{1}^{2}=z^{c} s_{1}^{2}$. Logo, pelo Proposição $4.39 v$ ), temos

$$
\begin{aligned}
\left\langle a b t_{2} t_{3}\right\rangle \equiv_{S}^{*}\langle a b t\rangle & =\left\langle c d t^{\prime}\right\rangle \equiv_{S}^{*}\left\langle c d s_{2} s_{3}\right\rangle \\
\left\langle a b t_{1} t_{2} t_{3}\right\rangle & \equiv_{S}^{*}\left\langle c d s_{1} s_{2} s_{3}\right\rangle \\
\left\langle\left(z t_{1}\right)^{c}\right\rangle & \equiv_{S}^{*}\left\langle\left(z s_{1}\right)^{c}\right\rangle .
\end{aligned}
$$

Logo, novamente pela Proposição 4.39,

$$
\begin{aligned}
\langle a, b\rangle \equiv_{S}^{*}\left\langle a t_{2}, b t_{3}\right\rangle & \equiv\left\langle z t_{1}, z a b t_{1} t_{2} t_{3}\right\rangle \oplus\left(z t_{1}\right)^{c}\left(a b t_{2} t_{3}\right)^{2}\langle 1,-1\rangle \\
& \equiv_{S}^{*}\left\langle z s_{1}, z c d s_{1} s_{2} s_{3}\right\rangle \oplus\left(z s_{1}\right)^{c}\left(c d s_{2} s_{3}\right)^{2}\langle 1,-1\rangle \\
& \equiv\left\langle c s_{2}, d s_{3}\right\rangle \equiv_{S}^{*}\langle c, d\rangle .
\end{aligned}
$$

O próximo resultado, central nessa subseção, é uma generalização da Proposição 2.21 de [DM1] que caracteriza a isometria num quociente de grupo de especial por subgrupo de Pfister.

Teorema 4.43. Sejam $R R S$ von Neumann $R S$ e $S \subseteq R$ subgrupo saturado. Sejam $\varphi, \psi$ formas sobre R. São equivalentes:

i) $\varphi \equiv_{S} \psi$.

ii) Existe forma de Pfister $\theta$ sobre $S$ tal que $\varphi \otimes \theta \equiv \psi \otimes \theta$.

Em particular,

- $\varphi \cong_{S} 0$ se, e somente se, existe forma de Pfister $\theta$ sobre $S$ tal que $\varphi \otimes \theta \cong 0$.

- O conjunto $I_{S}:=\{\varphi \in W(S)$ : Existe forma de Pfister $\theta$ sobre $S$ tal que $\varphi \otimes \theta=0\} \subseteq W(R)$ é ideal e $W\left(R /{ }_{m} S\right) \cong W(R) / I_{S}$ de forma natural.

Demonstração. Pela Proposição 4.39 é suficiente provarmos a direção $i) \Rightarrow i i)$. Suponha inicialmente que $\varphi \equiv_{S}\langle 0\rangle \equiv_{S} \psi(*)$. Pela Proposição 4.39 , existem $s, t \in S$ tais que $s \varphi \equiv\langle 0\rangle \equiv t \psi$. Logo, pela mesma proposição, temos $\varphi \equiv_{S}^{*}\langle 0\rangle \equiv_{S}^{*} \psi$. A partir de agora vamos supor $\varphi, \psi \not_{S}\langle 0\rangle$.

Afirmação. Suponha que $\varphi, \psi$ sejam $k$-formas sobre $R$. Se $\varphi \equiv_{S} \psi$, então $\varphi \equiv_{S}^{*} \psi$.

Demonstração. Pelo Corolário 3.9 podemos assumir que $\varphi=\left\langle a_{1}, \ldots, a_{k}\right\rangle, \psi=\left\langle b_{1}, \ldots, b_{k}\right\rangle$. Como $\varphi, \psi \not \equiv_{S}\langle 0\rangle$, temos que $\varphi, \psi$ são $k$-formas em $R /{ }_{m} S$. A prova da afirmação segue por indução em $k$. Para $k=1$ a prova segue por definiçao e para $k=2$ segue do Lema 4.42. Para $k>2$, pelo Lema 3.21 aplicado a $R /{ }_{m} S$, existem $\alpha, \beta \in R$ e forma $\theta$ tais que $\theta$ é $k-2$-forma sobre $R /{ }_{m} S$ e

$$
\begin{aligned}
\left\langle a_{1}, \alpha\right\rangle & \equiv_{S}\left\langle b_{1}, \beta\right\rangle \\
\left\langle a_{2}, \ldots, a_{k}\right\rangle & \equiv_{S}\langle\alpha\rangle \oplus \theta \\
\left\langle b_{2}, \ldots, b_{k}\right\rangle & \equiv_{S}\langle\beta\rangle \oplus \theta .
\end{aligned}
$$


Pelo Lema 4.42 temos $\left\langle a_{1}, \alpha\right\rangle \equiv_{S}^{*}\left\langle b_{1}, \beta\right\rangle$. Por outro lado, pela Proposição $4.39 v i$ ), existem formas $\theta_{1}, \theta_{2}$ tais que

- $\theta_{1}, \theta_{2}$ são $k-1$-formas sobre $R$

- $\theta_{1} \equiv_{S}^{*}\langle\alpha\rangle \oplus \theta$

- $\theta_{2} \equiv{ }_{S}^{*}\langle\beta\rangle \oplus \theta$.

Assim, por hipótese de indução, temos

$$
\begin{aligned}
\left\langle a_{2}, \ldots, a_{k}\right\rangle & \equiv_{S}^{*} \theta_{1} \\
\left\langle b_{2}, \ldots, b_{k}\right\rangle & \equiv{ }_{S}^{*} \theta_{2} .
\end{aligned}
$$

Deste modo

$$
\begin{aligned}
\varphi & =\left\langle a_{1}, \ldots, a_{k}\right\rangle \equiv_{S}^{*}\left\langle a_{1}\right\rangle \oplus \theta_{1} \\
& \equiv_{S}^{*}\left\langle a_{1}, \alpha\right\rangle \oplus \theta \equiv_{S}^{*}\left\langle b_{1}, \beta\right\rangle \oplus \theta \\
& \equiv_{S}^{*}\left\langle b_{1}\right\rangle \oplus \theta_{2} \equiv_{S}^{*}\left\langle b_{1}, b_{2}, \ldots, b_{k}\right\rangle=\psi .
\end{aligned}
$$

Note que, na afirmação acima, se trocarmos a hipótese por $\varphi, \psi k$-formas em $R /{ }_{m} S$ ela continua valendo pela Proposição 4.39 vi). Assuma agora que $\varphi \equiv_{S} \psi$. Como $\varphi, \psi \not_{S}\langle 0\rangle$, pelo Teorema 3.18 existem $e, e_{1}, \ldots, e_{k} \in \operatorname{Id}(R)$ e inteiros $1 \leq n_{1}<\cdots<n_{k}$ tais que

- $\operatorname{supp}_{R / m} S(\varphi)=D(e)=\operatorname{supp}_{R / m}(\psi)$. Em particular, $e \varphi \equiv_{S} \varphi$ e $e \psi \equiv_{S} \psi$.

- $e_{i} e_{j}=0$ em $R / m S$ para $i \neq j$ e $\langle e\rangle \equiv_{S}\left\langle e_{1}, \ldots, e_{n}\right\rangle$.

- $e_{i} \varphi \equiv_{S} e_{i} \psi$ são $n_{i}$-formas em $R /{ }_{m} S$.

Como $\langle e\rangle,\left\langle e_{1}, \ldots, e_{k}\right\rangle$ são 1 -formas em $R /{ }_{m} S$ e $e_{i} \varphi, e_{i} \psi n_{i}$-formas, pela afirmação acima vale $\langle e\rangle \equiv_{S}^{*}\left\langle e_{1}, \ldots, e_{n}\right\rangle$ e $e_{i} \varphi \equiv_{S}^{*} e_{i} \psi$. Portanto, pela Proposição 4.39, temos

$$
\begin{aligned}
\varphi \equiv_{S}^{*} e \varphi & \equiv_{S}^{*}\left\langle e_{1}, \ldots e_{k}\right\rangle \otimes \varphi \equiv_{S}^{*} \bigoplus_{i=1}^{k} e_{i} \varphi \\
& \equiv_{S}^{*} \bigoplus_{i=1}^{k} e_{i} \psi \equiv_{S}^{*} e \psi \equiv_{S}^{*} \psi .
\end{aligned}
$$

Corolário 4.44. Sejam $R$ RS von Neumann e $I \subseteq R$ ideal próprio. Sejam $\varphi, \psi$ formas sobre $R$. São equivalentes:

i) $\varphi \equiv_{I} \psi$.

ii) Existem formas $\theta, \theta^{\prime}$ sobre $I$ tais que $\varphi \oplus \theta \equiv \psi \oplus \theta^{\prime}$ em $R$.

Em particular, $\varphi \cong_{I} 0$ se, e somente se, existe forma $\theta$ sobre $I$ tal que $\varphi \cong \theta$.

Demonstração. A direção $\Leftarrow$ segue da Proposição 4.40. Considere $\pi: R \rightarrow R / I$ a projeção canônica e $S=\pi^{-1}(1)$. Pelo Teorema 2.69 o morfismo $\pi$ induz isomorfismo $R / m S \cong R / I$. Deste modo, se $\varphi \equiv_{I} \psi$, então $\varphi \equiv_{S} \psi$. Pelo Teorema 4.43, existe forma de Pfister $\theta=\bigotimes_{i=1}^{n}\left\langle s_{i}, s_{i}^{2}\right\rangle$ com $s_{i} \in S$ tal 
que $\varphi \otimes \theta \equiv \psi \otimes \theta(*)$. Por outro lado, como $s_{i}=1$ em $R / I$, existe $x_{i} \in D^{t}\left(s_{i},-1\right) \cap I$. Logo pelo Lema 4.41 temos

$$
\left\langle s_{i}\right\rangle \oplus\left\langle-x_{i}, x_{i} s_{i}\right\rangle \equiv\langle 1\rangle \oplus x_{i}^{2} s_{i}^{2}\langle 1,-1\rangle
$$

e portanto $\left\langle s_{i}\right\rangle \equiv_{I}^{+}\langle 1\rangle$. Deste modo, pela Proposição 4.40 , temos $\left\langle s_{i}^{2}, s_{i}\right\rangle \equiv_{I}^{+}\langle 1,1\rangle$ e assim $\theta \equiv_{I}^{+}$ $2^{n}$. Então a isometria $(*)$ implica que $2^{n} \varphi \equiv_{I}^{+} 2^{n} \psi$. Novamente pela Proposição 4.40 concluímos que $\varphi \equiv_{I}^{+} \psi$.

Comentários 4.45. • Na categoria dos 0-RSGs, a noção de quocientes por ideias é trivial (o único ideal próprio é o nulo).

- Sejam $R$ RS e $I \subseteq R$ ideal próprio.

- Utilizando a Proposição $1.13-i$ ), prova-se que o multi-anel $R / I$ é multi-anel real reduzido. Tendo em mente que o conceito de ideal aqui utilizado corresponde a ideal saturado em [DM2], como semigrupo real o quociente $R / I$ é isomorfo ao quociente por ideal saturado definido em [DP2].

- É possível provar que o morfismo para envoltória von Neumann $v: R \rightarrow V(R)$ preserva quocientes por ideias e, com isso, concluir que dada forma $\varphi$ sobre $R$,

$$
\varphi=0 \text { em } W(R / I) \Leftrightarrow \text { Existe forma } \theta \text { sobre } I \text { tal que } \varphi \cong \theta \text {. }
$$

\subsubsection{Anel de Witt por geradores e relações}

Nesta subseção, vamos generalizar a descrição de anel de Witt clássico por geradores e relações. Para aneis de Witt abstratos (versão do Marshall), o resultado é provado no Teorema 4.3 de [Mar6].

Definição 4.46. Seja $T$ semigrupo ternário. Definimos

$$
\mathbb{Z}[T]:=\{f: T \rightarrow \mathbb{Z}: \text { existe } I \subseteq T \text { finito tal que } f(T \backslash I)=\{0\} \text { e } f(0)=0\} .
$$

Dados $f, g \in \mathbb{Z}[T]$, definimos as seguintes operações:

- $s=f+g$ é a função definida por $s(t)=f(t)+g(t)$ para todo $t \in T$.

- $p=f \cdot g$ é a função definida por $p(0)=0$ e para $t \neq 0$

$$
p(t)=\sum_{x, y \in T, x y=t} f(x) g(y)
$$

Além disso, seja $[0] \in \mathbb{Z}[T]$ a função constante igual a $0 \in \mathbb{Z}$ e dado $t \in T$ não-nulo, considere $[t] \in \mathbb{Z}[T]$ dada por $[t](x)=0$ para todo $x \neq t$ e $[t](t)=1$. Dado $f \in \mathbb{Z}[T]$, definimos $\operatorname{supp}(f)=$ $\{t \in R: f(t) \neq 0\}$.

Proposição 4.47. Seja $T$ semigrupo ternário. Então $\mathbb{Z}[T]$ é anel comutativo com unidade. Além disso, dados $x, y \in T$, temos $[x] \cdot[y]=[x y]$.

Demonstração. Dado $f \in \mathbb{Z}[T]$, defina $(-f)(t)=-f(t)$ para todo $t \in T$. Segue direto da definição que $(\mathbb{Z}[T],+,-,[0])$ é grupo abeliano comutativo e que o produto em $\mathbb{Z}[T]$ também é comutativo. Vamos mostrar que $i)[1] \in \mathbb{Z}[T]$ é unidade e $f \cdot[0]=[0]$ para todo $f \in \mathbb{Z}[R]$, ii) o produto é associativo e iii) o produto é distributivo. 
i) Seja $f \in \mathbb{Z}[T]$. Precisamos mostrar que $f \cdot[1]=f$ e que $f \cdot[0]=[0]$. Dado $t \in T$, se $t=0$, então por definição $f \cdot[1](0)=0=f(0)$ e $f \cdot[0](0)=0=[0](0)$; se $t \neq 0$, temos

$$
\begin{aligned}
& f \cdot[1](t)=\sum_{x y=t} f(x)[1](y)=f(t)[1](1)=f(t) \\
& f \cdot[0](t)=\sum_{x y=t} f(x)[0](y)=0=[0](t) .
\end{aligned}
$$

ii) Sejam $f, g, h \in \mathbb{Z}[T]$. Vamos mostrar que $(f \cdot g) \cdot h=f \cdot(g \cdot h)$. Dado $t \in T, t \neq 0$, temos

$$
\begin{aligned}
(f \cdot g) \cdot h(t) & =\sum_{x y=t}(f \cdot g)(x) h(y)=\sum_{x y=t} \sum_{a b=x} f(a) g(b) h(y) \\
& =\sum_{(a b) y=t} f(a) g(b) h(y)=\sum_{a(b y)=t} f(a) g(b) h(y) \\
& =\sum_{a x=t} \sum_{b y=x} f(a) g(b) h(y)=\sum_{a x=t} f(a)(g \cdot h)(x)=f \cdot(g \cdot h)(t) .
\end{aligned}
$$

iii) Sejam $f, g, h \in \mathbb{Z}[T]$. Vamos mostrar que $f \cdot(g+h)=f \cdot g+f \cdot h$. Dado $t \in T, t \neq 0$, temos

$$
\begin{aligned}
f \cdot(g+h)(t) & =\sum_{x y=t} f(x)(g(y)+h(y))=\sum_{x y=t} f(x) g(y)+\sum_{x y=t} f(x) h(y) \\
& =f \cdot g(t)+f \cdot h(t) .
\end{aligned}
$$

Por último, sejam $x, y \in T$. Vamos mostrar que $[x] \cdot[y]=[x y]$, ou seja, que para todo $t \in T$ não-nulo vale $[x] \cdot[y](t)=[x y](t)$.

Caso 1: $x=0$ ou $y=0$.

Neste caso, temos $[x] \cdot[y]=0_{\mathbb{Z}[R]}=[0]=[x y]$.

Caso 2: $x, y \neq 0$ e $x y=0$.

Seja $t \in R$ não-nulo. Então, como $t \neq 0=x y$,

$$
[x] \cdot[y](t)=\sum_{p m=t}[x](p)[y][m]=0=[0](t)=[x y](t) .
$$

Caso 3: $x y \neq 0$.

Seja $t \in T$ não-nulo. Se $t \neq x y$, então pela mesma conta do caso anterior temos $[x] \cdot[y](t)=0=$ $[x y](t)$. Se $t=x y$, então

$$
[x] \cdot[y](t)=\sum_{p m=t}[x](p)[y][m]=1=[x y](t) .
$$

Comentário 4.48. Seja $G$ RSG e considere o semigrupo ternário $T=G \cup\{0\}$. Então o anel $Z[T]$ é isomorfo ao anel de grupo $Z[G]$.

Teorema 4.49. Seja $R R S$ von Neumann e considere o morfismo $J: \mathbb{Z}[R] \rightarrow W(R)$ dado por: $J(f)=\bigoplus_{r \in R} f(r)\langle r\rangle$. Então o ideal kef $(J(f))$ é gerado por $[a]+[a b c]-[b]-[c]$ tal que a $\in D^{t}(b, c)$.

Demonstração. Seja $I$ o ideal gerado por $\left\{[a]+[a b c]-[b]-[c] \in \mathbb{Z}[R]: a \in D^{t}(b, c)\right\}$. Então, pela Proposição $3.4 v$ ), é fácil ver que $I \subseteq \operatorname{ker}(f)$. Vamos mostrar a inclusão reversa através de algumas afirmações preliminares. 
Afirmação. Sejam $e_{1}, \ldots, e_{n} \in \operatorname{Id}(R)$ tais que $e_{i} e_{j}=0$ para $i \neq j$. Então dado $e \in D^{t}\left(e_{1}, \ldots, e_{n}\right)$,

$$
[e] \equiv\left[e_{1}\right]+\cdots+\left[e_{n}\right] \bmod I \text {. }
$$

Em particular, dados $x_{1}, \ldots, x_{k} \in R$ e $x \in D^{t}\left(x_{1} e_{1}, \ldots, x_{n} e_{n}\right)$, então

$$
[x] \equiv\left[x_{1} e_{1}\right]+\cdots+\left[x_{n} e_{n}\right] \bmod I .
$$

Demonstração. Para $n=1,2$ o resultado é imediato. Para $n \geq 3$, seja $t \in D^{t}\left(e_{2}, \ldots, e_{n}\right)$,e $\in$ $D^{t}\left(e_{1}, t\right)$. Então $t \in \operatorname{Id}(R)$ e $t e_{1} \in D^{t}\left(e_{2} e_{1}, \ldots, e_{n} e_{1}\right)=\{0\}$. Logo, por hipótese de indução temos

$$
\begin{aligned}
{[e] } & \equiv\left[e_{1}\right]+[t] \bmod I \\
{[t] } & \equiv\left[e_{2}\right]+\cdots+\left[e_{n}\right] \bmod I .
\end{aligned}
$$

Assim $[e] \equiv\left[e_{1}\right]+\cdots+\left[e_{n}\right] \bmod I$.

Agora suponha que $x \in D^{t}\left(x_{1} e_{1}, \ldots, x_{n} e_{n}\right)$. Então $x e_{i}=x_{i} e_{i}$ e tomando $e \in D^{t}\left(e_{1}, \ldots, e_{n}\right)$ temos $x e=x$. Deste modo, como $[e] \equiv\left[e_{1}\right]+\cdots+\left[e_{n}\right] \bmod I$,

$$
\begin{aligned}
{[x]=[x e] } & \equiv\left[x e_{1}\right]+\cdots+\left[x e_{n}\right] \bmod I \\
& \equiv\left[x_{1} e_{1}\right]+\cdots+\left[x_{n} e_{n}\right] \bmod I .
\end{aligned}
$$

Afirmação. Sejam $\varphi=\left\langle a_{1}, \ldots, a_{m}\right\rangle, \psi=\left\langle b_{1}, \ldots, b_{n}\right\rangle k$-formas tais que $\varphi \equiv \psi$. Então

$$
\left[a_{1}\right]+\cdots+\left[a_{m}\right] \equiv\left[b_{1}\right]+\cdots+\left[b_{n}\right] \bmod I .
$$

Demonstração. Para cada $1 \leq i \leq k$, tome

$$
x_{i} \in D^{t}\left(\left\langle\pi_{f} a_{f_{i}}\right\rangle_{f \in S_{m, k}}\right) .
$$

Pelo Teorema 3.9, sabemos que $\operatorname{Od}_{k}(\varphi)=\bigsqcup_{f \in S_{m, k}} D\left(\pi_{f}\right)=D(e)$, onde $e \in D^{t}\left(\left\langle\pi_{f}\right\rangle_{f \in S_{m, k}}\right)$, e que $\varphi \equiv\left\langle x_{1}, \ldots, x_{k}\right\rangle$. Com isso, temos alguns fatos:

- $a_{i} e=a_{i} \operatorname{pois} \operatorname{supp}(\varphi)=\operatorname{Od}_{k}(\varphi)=D(e)$.

- Dado $f \in S_{m, k}$ e $i \notin \operatorname{Im}(f), \pi_{f} a_{i}=0$. Em particular, em $\mathbb{Z}[R]$ vale $\left[\pi_{f}\right]\left(\left[a_{f_{1}}\right]+\cdots+\left[a_{f_{k}}\right]\right)=$ $\left[\pi_{f}\right]\left(\left[a_{1}\right]+\cdots+\left[a_{m}\right]\right)$.

Deste modo, por 4.6 e pela afirmação anterior, temos

$$
\begin{aligned}
{\left[x_{1}\right]+\cdots+\left[x_{k}\right] } & \equiv \sum_{i=1}^{k} \sum_{f \in S_{m, k}}\left[\pi_{f}\right]\left[a_{f_{i}}\right] \bmod I \\
& =\sum_{f \in S_{m, k}}\left[\pi_{f}\right]\left(\left[a_{f_{1}}\right]+\cdots+\left[a_{f_{k}}\right]\right) \bmod I \\
& =\sum_{f \in S_{m, k}}\left[\pi_{f}\right]\left(\left[a_{1}\right]+\cdots+\left[a_{m}\right]\right) \bmod I \\
& =\left(\left[a_{1}\right]+\cdots+\left[a_{m}\right]\right) \sum_{f \in S_{m, k}}\left[\pi_{f}\right] \bmod I \\
& \equiv\left(\left[a_{1}\right]+\cdots+\left[a_{m}\right]\right)[e] \bmod I \\
& =\left[a_{1}\right]+\cdots+\left[a_{m}\right] \bmod I .
\end{aligned}
$$

Por causa disso, para provar a afirmação podemos assumir que $m=n=k$. Agora podemos provar a afirmação por indução em $k$. Para $k=1$ é trivial. Para $k=2$, como $\varphi \equiv \psi$, pela Proposição 
3.4 , existe $z \in D^{t}\left(a_{1}, a_{2}\right) \cap D^{t}\left(b_{1}, b_{2}\right)$ e $a_{1} a_{2}=b_{1} b_{2}$. Logo

$$
\begin{aligned}
{\left[a_{1}\right]+\left[a_{2}\right] } & \equiv[z]+\left[z a_{1} a_{2}\right] \bmod I \\
& \equiv[z]+\left[z b_{1} b_{2}\right] \bmod I \\
& \equiv\left[b_{1}\right]+\left[b_{2}\right] \bmod I .
\end{aligned}
$$

Para $k \geq 3$, pelo Lema 3.21, existem $\alpha, \beta \in R$ e $k-2$-forma $\theta=\left\langle x_{2}, \ldots, x_{k}\right\rangle$ tais que

$$
\begin{aligned}
\left\langle a_{1}, \alpha\right\rangle & \equiv\left\langle b_{1}, \beta\right\rangle \\
\left\langle a_{2}, \ldots, a_{k}\right\rangle & \equiv\langle\alpha\rangle \oplus \theta \\
\left\langle b_{2}, \ldots, b_{k}\right\rangle & \equiv\langle\beta\rangle \oplus \theta .
\end{aligned}
$$

Deste modo, por hipótese de indução, temos

$$
\begin{aligned}
{\left[a_{1}\right]+\cdots+\left[a_{k}\right] } & \equiv\left[a_{1}\right]+\left([\alpha]+\left[x_{2}\right]+\cdots+\left[x_{k}\right]\right) \bmod I \\
& \equiv\left(\left[a_{1}\right]+[\alpha]\right)+\left[x_{2}\right]+\cdots+\left[x_{k}\right] \bmod I \\
& \equiv\left(\left[b_{1}\right]+[\beta]\right)+\left[x_{2}\right]+\cdots+\left[x_{k}\right] \bmod I \\
& \equiv\left[b_{1}\right]+\left[b_{2}\right]+\cdots+\left[b_{k}\right] \bmod I .
\end{aligned}
$$

Juntanto as afirmações acima, podemos provar que para duas formas quaisquer $\varphi=\left\langle a_{1}, \ldots, a_{n}\right\rangle$ e $\psi=\left\langle b_{1}, \ldots, b_{k}\right\rangle$, se $\varphi \equiv \psi$, então $\left[a_{1}\right]+\cdots+\left[a_{n}\right] \equiv\left[b_{1}\right]+\cdots+\left[b_{k}\right] \bmod I$. De fato, suponha sem perda de generalidade que $\varphi, \psi \neq \equiv\langle 0\rangle$. Pelo Teorema 3.18, existem $e, e_{1}, \ldots, e_{n} \in \operatorname{Id}(R)$ tais que

$. \operatorname{supp}(\varphi)=\operatorname{supp}(\psi)=D(e)$.

. $e_{i} e_{j}=0$ para $i \neq j$ e $e \in D^{t}\left(e_{1}, \ldots, e_{n}\right)$.

. $e_{i} \varphi \equiv e_{i} \psi$ são $n_{i}$-formas.

Pela primeira afirmação temos $[e] \equiv\left[e_{1}\right]+\cdots+\left[e_{n}\right] \bmod I$ e pela segunda afirmação $\left[e_{i} a_{1}\right]+$ $\cdots+\left[e_{i} a_{n}\right] \equiv\left[e_{i} b_{1}\right]+\cdots+\left[e_{i} b_{k}\right] \bmod I$. Assim

$$
\begin{aligned}
{\left[a_{1}\right]+\cdots+\left[a_{n}\right] } & =\left(\left[a_{1}\right]+\cdots+\left[a_{n}\right]\right) \cdot[e] \equiv \sum_{i=1}^{n}\left[e_{i} a_{1}\right]+\cdots+\left[e_{i} a_{n}\right] \bmod I \\
& \equiv \sum_{i=1}^{n}\left[e_{i} b_{1}\right]+\cdots+\left[e_{i} b_{k}\right]=\left(\left[b_{1}\right]+\cdots+\left[b_{k}\right]\right) \cdot[e]=\left[b_{1}\right]+\cdots+\left[b_{k}\right] \bmod I
\end{aligned}
$$

Para provar que $\operatorname{ker}(J) \subseteq I$, seja $f \in \mathbb{Z}[R] \operatorname{com} J(f)=0$. Seja $\operatorname{supp}(f)=\left\{a_{1}, \ldots, a_{n}\right\}$ e considere a forma $\varphi=J(f)=\bigoplus_{i=1}^{n} f\left(a_{i}\right)\left\langle a_{i}\right\rangle$. Como $\varphi=0 \mathrm{em} \mathrm{W}(R)$, pelo Comentário 3.11, existe forma $\theta=\left\langle b_{1}, \ldots, b_{k}\right\rangle$ tal que $\varphi \equiv \theta\langle 1,-1\rangle$. Logo

$$
\sum_{i=1}^{n} f\left(a_{i}\right)\left[a_{i}\right] \equiv \sum_{j=1}^{k}\left[b_{j}\right]+\left[-b_{j}\right] \bmod I .
$$

Mas observe que, como $0 \in D^{t}\left(b_{j},-b_{j}\right)$, temos $\left[-b_{j}\right] \equiv-\left[b_{j}\right] \bmod I$. Deste modo, a equivalência 4.7 implica que $\sum_{i=1}^{n} f\left(a_{i}\right)\left[a_{i}\right] \in I$.

\subsubsection{Axiomatização de Aneis de Witt}

No que segue, $A$ é um anel comutativo reduzido $(\sqrt{0}=0)$ e $2 \in A$ é não-divisor de zero. Seja $T_{A}=\left\{x \in A: x^{3}=x\right\}$ o semigrupo dos ternários associados a $A$. Também consideramos a relação 
ternária $D^{t}$ em $T_{A}$ dada por $a \in D^{t}(b, c)$ se, e somente se, $a+a b c=b+c$ em $A$. Além disso, dado $\{$ fact 2$\}$ $a \in T_{A}$, definimos $a^{c}=1-a^{2}$ e $\nabla(a)=a-a^{c}=a-1+a^{2}$.

Fato 4.50. Dados $a, b, c \in T_{A}$, as seguintes propriedades são verdadeiras:

i) $\left(a^{c}\right)^{2}=a^{c}$ e $\nabla(a)^{2}=1$. Em particular, vale $a^{c}, \Delta(a) \in T_{A}$.

ii) $a a^{c}=0$ e $a^{c} \in D^{t}\left(1,-a^{2}\right)$. Em particular, $0^{c}=1$ e se $a^{2}=1, a^{c}=0$.

iii) $a=a^{2} \nabla(a)$ e $\nabla(a) \in D^{t}(-1, a)$. Em particular, $\nabla(0)=-1$ e se $a^{2}=1, \nabla(a)=a$.

iv) $a \in D^{t}(b, c)$ se, e somente se, $\nabla(a) \in D^{t}(\nabla(b), \nabla(c)), b a^{c}=-c a^{c}, a b^{c}=c b^{c}$ e $a c^{c}=b c^{c}$.

v) Dados $a, b \in T_{A}$ idempotentes, se $a b^{c}=a^{c} b=0$, então $a=b$.

Demonstração. i) $\left(a^{c}\right)^{2}=\left(1-a^{2}\right)^{2}=1-2 a^{2}+a^{2}=1-a^{2}=a^{c}$ e $\nabla(a)^{2}=\left(a-a^{c}\right)^{2}=$ $a^{2}-2 a a^{c}+\left(a^{c}\right)^{2}=a^{2}-2 a\left(1-a^{2}\right)+a^{c}=a^{2}-2(a-a)+a^{c}=a^{2}+1-a^{2}=1$.

ii) $a a^{c}=a\left(1-a^{2}\right)=(a-a)=0$ e $a^{c}-a^{c} a^{2}=a^{c}=1-a^{2} ; \log 0 a^{c} \in D^{t}\left(1,-a^{2}\right)$. Assim, $0^{c} \in D^{t}(1,0)=\{1\}$ e se $a^{2}=1$, temos $a^{c}=a^{2} a^{c}=a\left(a a^{c}\right)=0$.

iii) $a^{2} \nabla(a)=a^{2}\left(a-a^{c}\right)=a$ e $\nabla(a)-\nabla(a) a=a-a^{c}-\left(a-a^{c}\right) a=a-1+a^{2}-a^{2}=-1+a$; logo $\nabla(a) \in D^{t}(-1, a)$. Assim, $\nabla(0) \in D^{t}(-1,0)=\{-1\}$ e se $a^{2}=1, a=a^{2} \nabla(a)=\nabla(a)$.

iv) Como a fórmula desse item é livre de quantificadores e $\sqrt{0}=0$, se provarmos a equivalência em $K_{A}(p)=\mathrm{ff}(A / p)$ para todo $p \in \operatorname{spec}(A)$, ela é válida em $A$. Então podemos assumir que $A$ é corpo (mas não necessariamente $2 \neq 0$ ). Como $A$ é corpo, dado $x \in T_{A}$, temos $x=0$ ou $x^{2}=1$. Precisamos mostrar que

$$
\begin{aligned}
a+a b c=b+c \Leftrightarrow & \left(a-a^{c}\right)+\left(a-a^{c}\right)\left(b-b^{c}\right)\left(c-c^{c}\right)=\left(b-b^{c}\right)+\left(c-c^{c}\right) \\
& b a^{c}=-c a^{c}, a b^{c}=c b^{c}, a c^{c}=b c^{c} .
\end{aligned}
$$

Temos alguns casos:

Caso 1: $a=0$.

Então precisamos mostrar que $b=-c$ se, e somente se, $-1-\nabla(b) \nabla(c)=\nabla(b)+\nabla(c)$ e $b=-c, 0=c b^{c}, 0=b c^{c}$. Como $b=-c$ implica $c b^{c}=c\left(1-b^{2}\right)=c\left(1-c^{2}\right)=0=b\left(1-b^{2}\right)=$ $b\left(1-c^{2}\right)=b c^{c}$, basta mostrarmos que $b=-c$ implica $-1-\nabla(b) \nabla(c)=\nabla(b)+\nabla(c)$. Mas note que, como $b^{c}=1-b^{2}=1-c^{2}=c^{c}$, temos

$$
\begin{aligned}
-1-\nabla(b) \nabla(c) & =-1-\left(b-b^{c}\right)\left(c-c^{c}\right)=-1-\left(b-b^{c}\right)\left(-b-b^{c}\right) \\
& =-1+\left(b-b^{c}\right)\left(b+b^{c}\right)=-1+\left(b^{2}-b^{c}\right) \\
& =-2 b^{c}=\left(b-b^{c}\right)+\left(-b-b^{c}\right) \\
& =\left(b-b^{c}\right)+\left(c-c^{c}\right)=\nabla(b)+\nabla(c) .
\end{aligned}
$$

Logo, $-1 \in D^{t}(\nabla(b), \nabla(c))$.

Caso 2: $b=0$.

Então precisamos mostrar que $a=c$ se, e somente se, $\nabla(a)-\nabla(a) \nabla(c)=-1+\nabla(c)$ e $0=c a^{c}, a=c, a c^{c}=0$. Novamente, como $a=c$ implica $0=c a^{c}=a c^{c}$, é suficiente provar que $a=c$ implica $\nabla(a)-\nabla(a) \nabla(c)=-1+\nabla(c)$. Note que

$$
\begin{aligned}
\nabla(a)-\nabla(a) \nabla(c) & =\nabla(c)-\nabla(c) \nabla(c) \\
& =-1+\nabla(c) .
\end{aligned}
$$

Assim, $\nabla(a) \in D^{t}(-1, \nabla(c))$. 
Caso 3: $c=0$.

Segue por argumento simétrico ao caso 2 .

Caso 3: $a, b, c \neq 0$.

Neste caso, temos $\nabla(a)=a, \nabla(b)=b, \nabla(c)=c$ e $a^{c}=b^{c}=c^{c}=0$ e assim a equivalência 4.8 segue sem dificuldades.

v) Se $a b^{c}=a^{c} b=0$, então $0=a(1-b)=a-a b$ e $0=(1-a) b=b-a b$. Logo $a=a b=b$.

Fato 4.51. Dados $a, b \in T_{A}$,

i) $T_{A}$ é semigrupo ternário e $\left(T_{A}, D^{t}, \cdot, 0,1\right)$ satisfaz todos os axiomas de multi-anéis menos, possivelmente, a associatividade.

ii) $D^{t}\left(a, b^{2} a\right)=\{a\}$.

iii) $D^{t}\left(a^{2}, b^{2}\right)$ tem um único elemento.

iv) Seja $T_{A}^{\times}=\left\{x \in A: x^{2}=1\right\}$. Então $\left(T_{A}^{\times} \cup\{0\}, D^{t}, \cdot, 1,-1\right)$ satisfaz todos os axiomas de hipercorpo real reduzido menos, possivelmente, a associatividade.

Demonstração. i) É óbvio que $T_{A}$ é fechado por produtos e $1,-1,0 \in T_{A}$. Além disso, dado $x \in T_{A}$ com $x=-x$, então $2 x=0$. Logo, como 2 é não-divisor de zero, temos $x=0$. Assim $T_{A}$ é semigrupo ternário. Além disso os axiomas de multi-anéis menos a associatividade decorrem facilmente do fato de $A$ ser anel comutativo.

ii) Note que, por definição, temos $a \in D^{t}\left(a, b^{2} a\right)$. Por outro lado, seja $x \in T_{A}$ satisfazendo $x \in$ $D^{t}\left(a, b^{2} a\right)$, ou seja, $x+x a^{2} b^{2}=a+a b^{2}$. Então dado $p \in \operatorname{spec}(A)$, vale $2(x-a)=0 \mathrm{em} K_{A}(p)$. De fato, se $a \in p$, temos $x=0=a$ em $K_{A}(p)$; se $b \in p$, temos $x=a$ em $K_{A}(p)$ e se $a, b \notin p$, $a^{2}=b^{2}=1$ em $K_{A}(p)$ e portanto $x+x=a+a$, ou seja, $2(x-a)=0$. Como $\sqrt{0}=0$, temos $2(x-a)=0$ em $A$ e portanto $x=a$ já que 2 não é divisor de zero.

iii) Seja $c=a^{2} b^{2}+a^{2} b^{c}+a^{c} b^{2}$. Então, como $b b^{c}=c c^{c}=0$, temos $c^{2}=\left(a^{2} b^{2}+a^{2} b^{c}+a^{c} b^{2}\right)^{2}=$ $a^{2} b^{2}+a^{2} b^{c}+a^{c} b^{2}=c \in T$. Além disso, $c+c a^{2} b^{2}=a^{2} b^{2}+a^{2} b^{c}+a^{c} b^{2}+a^{2} b^{2}=a^{2}\left(b^{2}+\right.$ $\left.b^{c}\right)+b^{2}\left(a^{2}+a^{c}\right)=a^{2}+b^{2}$. Logo, $c \in D^{t}\left(a^{2}, b^{2}\right)$. Agora suponha que $x, y \in D^{t}\left(a^{2}, b^{2}\right)$, ou seja, $x+x a^{2} b^{2}=y+y a^{2} b^{2}=a^{2}+b^{2}$. Vamos motrar que $2(x-y)=0$ em $K_{A}(p)$ para todo $p \in \operatorname{spec}(A)$ e disso seguirá que $x=y$. Se $a \in p$ ou $b \in p$, então $a^{2} b^{2}=0$ em $K_{A}(p)$ e portanto $x=y$; se $a, b \notin p$, então $a^{2}=b^{2}=1$ em $K_{A}(p)$ e portanto $2(x-y)=0$.

iv) Consequência dos itens $i$ ), $i i$ ) e $i i i)$.

Assim, pelos fatos anteriores, se provarmos que $\left(T_{A}, D^{t}, \cdot,-, 0,1\right)$ satisfaz a propriedade associativa, $T_{A}$ é um semigrupo real von Neumann. O próximo teorema da condições suficientes (e, a posteriori, necessárias) para que isso aconteça. Note antes que, dado $a \in T_{A}$, temos em $\operatorname{spec}(R)$ que $D(a)^{c}=D\left(a^{c}\right)$.

Teorema 4.52. Seja A anel comutativo tal que $\sqrt{0}=0$ e $2 \in A$ é não-divisor de zero. Considere o conjunto $T_{A}^{\times}=\left\{x \in A: x^{2}=1\right\}$. Se $\left(T_{A}^{\times}, \cdot, D^{t},-1\right)$ é $R S G$, então $\left(T_{A}, D^{t}, \cdot,-, 0,1\right)$ é semigrupo real von Neumann.

Demonstração. Pelos fatos 4.51 e 4.50, é suficiente provarmos a associatividade de $\left(T_{A}, D^{t}, \cdot,-, 0,1\right)$. Sejam $a, b, c, l, x \in T_{A}$ tais que $x \in D^{t}(l, c)$ e $l \in D^{t}(a, b)\left(^{*}\right)$. Então pelo Fato 4.50, $\nabla(x) \in$ $D^{t}(\nabla(l), \nabla(c))$ e $\nabla(l) \in D^{t}(\nabla(a), \nabla(b))$. Como $T_{A}^{\times}$é RSG, existe $m \in T_{A}^{\times}$tal que $\nabla(x) \in$ $D^{t}(\nabla(a), m), m \in D^{t}(\nabla(b), \nabla(c))(* *)$. Defina

$$
y=a^{c} x+a^{2} b^{c} c+a^{2} b^{2} c^{c} b+a^{2} b^{2} c^{2} m .
$$


Observe que $\operatorname{spec}(A)=D\left(a^{c}\right) \sqcup D\left(a^{2} b^{c}\right) \sqcup D\left(a^{2} b^{2} c^{c}\right) \sqcup D\left(a^{2} b^{2} c^{2}\right)$. Para provar que $y \in T_{A}$, note que

i) Se $p \in D\left(a^{c}\right), y=x$ em $K_{A}(p)$.

ii) Se $p \in D\left(a^{2} b^{c}\right), y=c$ em $K_{A}(p)$.

iii) Se $p \in D\left(a^{2} b^{2} c^{c}\right), y=b$ em $K_{A}(p)$.

iv) Se $p \in D\left(a^{2} b^{2} c^{2}\right), y=m$ em $K_{A}(p)$.

Logo $y^{3}=y$ em $K_{A}(p)$ para todo $p \in \operatorname{spec}(A)$ e assim $y \in T_{A}$.

Afirmação. $x \in D^{t}(a, y)$ e $y \in D^{t}(b, c)$.

Demonstração. i) Se $p \in D\left(a^{c}\right)$, então $y=x, a=0$ em $K_{A}(p)$. Assim, por $\left(^{*}\right)$, temos $l=b$ e portanto $x+x b c=b+c$. Então $x+x a y=a+y$ e $y+y b c=b+c$ em $K_{A}(p)$.

ii) Se $p \in D\left(a^{2} b^{c}\right)$, então $y=c, b=0$ em $K_{A}(p)$. Assim, por $\left(^{*}\right)$, temos $l=a$ e portanto $x+x a c=a+c$. Então $x+x a y=a+y$ e $y+y b c=b+c$ em $K_{A}(p)$.

iii) Se $p \in D\left(a^{2} b^{2} c^{c}\right)$, então $y=b, c=0$ em $K_{A}(p)$. Assim, por $\left(^{*}\right), x=l$ e portanto $x+x a b=a+b$. Então $x+x a y=a+y$ e $y+y b c=b+c$ em $K_{A}(p)$.

iv) Se $p \in D\left(a^{2} b^{2} c^{2}\right)$, então $y=m$ e $a, b, c \neq 0$ em $K_{A}(p)$. Como $\nabla(a)=a, \nabla(b)=b, \nabla(c)=c$ em $K_{A}(p)$, por $\left({ }^{* *}\right)$ temos $x+x a m=a+m$ e $m+m b c=b+c$. Logo $x+x a y=a+y$ e $y+y b c=b+c$ são válidas em $K_{A}(p)$.

Então como $x+x a y=a+y$ e $y+y b c=b+c$ são válidas em $K_{A}(p)$ para todo $p \in \operatorname{spec}(A)$, temos $x \in D^{t}(a, y), y \in D^{t}(b, c)$.

Definição 4.53. Seja $A$ anel comutativo. $A$ é dito real se para todo $a \in A$, se existir $m \geq 1$ tal que $-a^{2 m} \in \sum A^{2}$, então $a=0$.

Lema 4.54. Seja $A$ anel real.

i) A intersecção de todos ideias primos reais é nula.

ii) Todo inteiro $k=1+\cdots+1 \in A$ é não divisor de zero. Em particular, $A$ tem característica nula.

iii) Suponha que $A=\sum T_{A}$. Então dado $p \in \operatorname{spec}(A)$ ideal primo real, existe $f \in \operatorname{Hom}(A, \mathbb{Z})$ tal que $f^{-1}(0)=p$. Em particular, se $x, y \in A$ satisfazem $f(x)=f(y)$ para toda $f \in \operatorname{Hom}(A, \mathbb{Z})$, então $x=y$.

Demonstração. i) Segue da aplicação direta do Teorema Positivstellensatz (Teorema 5.4 .2 de $[\mathrm{Mar} 7])$.

ii) Seja $x \in A$ tal que $k x=x+\cdots+x=0$. Então $x^{2}+\cdots+x^{2}=0$ e, como $A$ é real, segue que $x=0$. Logo $k \in A$ é não divisor de zero.

iii) Seja $p \in \operatorname{spec}(A)$ ideal primo real. Seja $x=a_{1}+\cdots+a_{n} \in A$ com $a_{i} \in T_{A}$ para todo $i=1, \ldots, n$. Como $a_{i}^{3}=a_{i}$, temos $a_{i} \cdot\left(a_{i}+1\right) \cdot\left(a_{i}-1\right)=0 \in p$ e portanto existe $t_{i} \in\{1,0,-1\}$ tal que $a_{i}-t_{i} \in p$. Logo $x-\left(t_{1}+\cdots+t_{n}\right) \in p$. Defina $f_{p}: A \rightarrow \mathbb{Z}$ por $f_{p}(x)=t_{1}+\cdots+t_{n}$.

- Primeiro note que se existem $l_{j} \in\{1,0,-1\}, j=1, \ldots, k$, com $x-\left(l_{1}+\cdots+l_{k}\right) \in p$, então $\left(t_{1}+\cdots+t_{n}\right)-\left(l_{1}+\cdots+l_{k}\right)=\left[x-\left(t_{1}+\cdots+t_{n}\right)\right]-\left[x-\left(l_{1}+\cdots+l_{k}\right)\right] \in p$. Como $p$ é real, segue que $t_{1}+\cdots+t_{n}=l_{1}+\cdots+l_{k}$ em $A$. Mas como a característica de $A$ é 0 , segue que $t_{1}+\cdots+t_{n}=l_{1}+\cdots+l_{k}$ em $\mathbb{Z}$ 
- Segue direto da definição que $f(1)=1, f(0)=0, f(-1)=-1$ e $f_{p}^{-1}(0)=p$. Sejam $x, y \in A$ $\operatorname{com} x-\left(t_{1}+\cdots+t_{n}\right), y-\left(l_{1}+\cdots+l_{k}\right) \in p$. Então $(x+y)-\left(t_{1}+\cdots+t_{n}+l_{1}+\cdots+l_{k}\right) \in p$. Além disso, escrevendo $x+t=t_{1}+\cdots+t_{n}, y+l=l_{1}+\cdots+l_{k}, t, l \in p$, temos $x y-\sum_{i, j} t_{i} l_{j}=$ $x y-\sum_{i} t_{i} \sum_{j} l_{j}=x y-\sum_{i} t_{i}(y+l)=x y-(x+t)(y+l)=-t y-l x-t l \in p$. Logo $f_{p}(x+y)=f_{p}(x)+f_{p}(y)$ e $f_{p}(x y)=f_{p}(x) f_{p}(y)$. Logo $f_{p}$ é morfismo.

Provado a existência do morfismo $f$, o restante segue de imediato do item $i$ ).

Definição 4.55. Seja $(G, \cdot)$ um semigrupo (a operação · é binária e associativa). Uma coleção de elementos $a_{1}, \ldots, a_{n} \in G$ é dita quadrado-constante se $a_{1}^{2}=\cdots=a_{n}^{2}$.

Definição 4.56. Seja $A$ anel. $A$ é anel de Witt abstrato se é real, $A=\sum T_{A}$ e a seguinte propriedade é válida:

Sejam $a_{1}, \ldots, a_{k}, b_{1}, \ldots, b_{k} \in T_{A}$ coleção quadrado-constante, $k \geq 3$, tais que $a_{1}+\cdots+a_{k}=$ $b_{1}+\cdots+b_{k}$. Então existem $\alpha, \beta, z_{3}, \ldots, z_{k} \in T_{A}$ quadrado-constantes tais que $\alpha^{2}=a_{1}^{2} \mathrm{e}$

$$
\begin{aligned}
a_{1}+\alpha & =b_{1}+\beta \\
a_{2}+\cdots+a_{k} & =\alpha+z_{3}+\cdots+z_{k} \\
b_{2}+\cdots+b_{k} & =\beta+z_{3}+\cdots+z_{k} .
\end{aligned}
$$

A categoria dos aneis de Witt abstratos com morfimos de aneis é denotada por $\mathbf{A b s}_{W i t t}$.

Note que o conceito de anel de Witt abstrato pode ser descrito por sentenças na linguagem $L_{\omega_{1}, \omega}$, onde $L$ é a linguagem usual para descrever os aneis com unidade.

Proposição 4.57. i) Seja $R$ RS von Neumann. Então o morfismo natural $R \rightarrow T_{W(R)}$ na linguagem $L_{R S}$ é isomorfismo. Em particular, $T_{W(R)}$ é RS von Neumann.

ii) Seja $R$ RS. Então $W(R)$ é anel de Witt abstrato. Assim, $W: \mathbf{R S} \rightarrow \mathbf{A b s}_{W i t t}$ é funtor.

Demonstração. i) Considere o mapa natural $f: R \rightarrow T_{W(R)}$ dado por $f(x)=\langle x\rangle$. Note que $f$ é injetor e é morfismo de semigrupos ternários. Pelo Teorema $3.51 f$ é sobrejetor. Por fim, a Proposição $3.4, v)$ mostra que $f$ preserva e reflete a representação transversal. Logo, $f$ é isomorfismo na linguagem $L_{R S}$.

ii) Pelo Teorema 4.10, podemos supor $R$ von Neumann. Primeiro vamos mostrar que $W(R)$ é real. Seja $\varphi$ forma sobre $R$ tal que $-\varphi^{2 m} \in \sum W(R)^{2}$. Então para toda ordem $\tau \in \operatorname{sper}(W(R))$ vale $\tau\left(\varphi^{2 m}\right) \in\{0,-1\}$. Pela Proposição 3.46, temos que para todo $\sigma \in X_{R}$ vale $\operatorname{sgn}_{\sigma}\left(\varphi^{2 m}\right)=$ $\operatorname{sgn}_{\sigma}(\varphi)^{2 m} \in\{-1,0\}$. Logo $\operatorname{sgn}_{\sigma}(\varphi)=0 . \operatorname{Assim} \varphi=0$ em $W(R)$.

A fim de verificar o axioma de indutividade do anel abstrato de Witt, pelo isomorfismo do item $i$ ) podemos identificar $T_{W(R)}$ e $R$. Deste modo, dados $a_{1}, \ldots, a_{k}, b_{1}, \ldots, b_{k} \in R$ quadradoconstantes, se $\left\langle a_{1}, \ldots, a_{k}\right\rangle \cong_{R}\left\langle b_{1}, \ldots, b_{k}\right\rangle$, então $\left\langle a_{1}, \ldots, a_{k}\right\rangle \equiv_{R}\left\langle b_{1}, \ldots, b_{k}\right\rangle$. Assim, pelo Lema 3.21 e Corolário 3.9, existem $\alpha, \beta, z_{3}, \ldots, z_{k} \in R$ quadrado-iguais tais que $\alpha^{2}=a_{1}^{2}$ e vale 4.10 .

O funtor $W: \mathbf{R S} \rightarrow$ Rings é definido no início da seção 3.4. Pelo provado no parágrafo anterior, a imagem $W$ cai na subcategoria plena $\mathbf{A b s}_{W i t t}$ dos anéis comutativos com unidade.

Lema 4.58. Seja $A$ anel de Witt abstrato. Sejam $a, b, c, d \in T_{A}$ quadrado-constantes. Se $a+b=c+d$, então $a \in D^{t}(c, d)$.

Demonstração. Precisamos mostrar que se $a+b=c+d$, então $b=a c d$. Seja $f \in \operatorname{Hom}(A, \mathbb{Z})$. Vamos mostrar que $f(b)=f(a c d)(*)$ e o resultado seguirá do Lema 4.54. Note que, para todo $x \in T_{A}$, $f(x) \in\{1,0,-1\}$. 
Caso 1: $f(c)=f(d)=1$.

Logo $f(a)+f(b)=2$. Como $a, b, c, d$ são quadrado-constantes, temos $f(a)=f(b)=1$, o que prova $(*)$.

Caso 2: $f(c)=f(d)=-1$.

Logo $f(a)+f(b)=0$. Como $a, b, c, d$ são quadrado-constantes, temos $f(a)=-f(b)$, o que prova $(*)$.

Caso 3: $f(c)=-f(d)$.

Análogo ao caso anterior.

Caso 4: $f(c)=f(d)=0$.

Novamente pelo fato de $a, b, c, d$ serem quadrado-constantes, temos $f(a)=f(b)=0$ e portanto vale $(*)$.

$\{$ RSG1\}

Proposição 4.59. Seja $A$ anel de Witt abstrato. Então $\left(T_{A}^{\times} \cup\{0\}, D^{t}, \cdot, 1,-1\right)$ é hipercorpo real reduzido. Além disso, se $f: A \rightarrow B$ é morfismo de aneis de Witt abstratos, então $f \mid: T_{A}^{\times} \cup\{0\} \rightarrow$ $T_{B}^{\times} \cup\{0\}$ é morfismo de multi-aneis.

Demonstração. Pelo Fato 4.51, é suficiente provar que $D^{t}$ é associativo. Sejam $x, a, b, c, l \in T_{A}^{\times} \cup\{0\}$ tais que $x \in D^{t}(l, c), l \in D^{t}(a, b)$. Vamos mostrar que existe $m \in D^{t}(b, c)$ com $x \in D^{t}(a, m)$. Os casos onde alguma entrada $x, a, b, c, l$ é nula são triviais e portanto vamos assumir $x, a, b, c, l \in T_{A}^{\times}$. Por definição, $x+x l c=l+c$ e $l+l a b=a+b$. Assim $a+b+c=x+x l c+l a b$. Mas como $A$ é anel de Witt abstrato, existem $m, n, z \in T_{A}^{\times}$tais que

$\{478\}$

$$
\begin{aligned}
a+m & =x+n \\
b+c & =m+z \\
x l c+l a b & =n+z .
\end{aligned}
$$

Assim, pelo Lema 4.58, temos $x \in D^{t}(a, m)$ e $m \in D^{t}(b, c)$. Logo $D^{t}$ é associativa em $T_{A}^{\times}$.

Por último, se $f: A \rightarrow B$ é morfismo de aneis de Witt abstratos, então $f \mid: T_{A}^{\times} \cup\{0\} \rightarrow T_{B}^{\times} \cup\{0\}$ é mapa que preserva produto e dados $a, b, c \in T_{A}^{\times} \cup\{0\}$, se $a \in D^{t}(b, c)$, então $a+a b c=b+c$. Logo $f(a)+f(a) f(b) f(c)=f(b)+f(c)$ e portanto $f(a) \in D^{t}(f(b), f(c))$, o que completa a prova.

Corolário 4.60. Seja $A$ anel de Witt abstrato. Então $\left(T_{A}, D^{t}, \cdot, 0,1\right)$ é semigrupo real von Neumann. Além disso, se $f: A \rightarrow B$ é morfismo de aneis de Witt abstratos, então $f \mid: T_{A} \rightarrow T_{B}$ é morfismo de RS von Neumann.

Demonstração. Segue diretamente da proposição anterior combinada com o Teorema 4.52. Além disso, se $f: A \rightarrow B$ é morfismo de aneis de Witt abstratos, então $f \mid: T_{A} \rightarrow T_{B}$ é morfismo de RS von Neumann por argumento parecido ao dado no final da demonstração da proposição anterior.

Pelo corolário anterior, a associação $T: A \in \mathbf{A b s}_{W i t t} \mapsto T_{A} \in \mathbf{v N R S}$ é funtorial.

Lema 4.61. Seja $A$ anel de Witt abstrato. Sejam $f \in \operatorname{Hom}(A, \mathbb{Z})$ e $a_{1}, \ldots, a_{k}, b_{1}, \ldots, b_{k} \in T_{A}$ quadrado-constantes.

i) O mapa

$$
\begin{aligned}
\tau_{f}: T_{A} & \rightarrow 3 \\
a & \mapsto f(a) .
\end{aligned}
$$

está bem-definido e $\tau_{f} \in X_{T_{A}}$. Em particular, $\varphi=\left\langle a_{1}, \ldots, a_{n}\right\rangle, \psi=\left\langle b_{1}, \ldots, b_{k}\right\rangle$ são formas sobre $T_{A} \operatorname{com} \varphi \cong_{T_{A}} \psi$, então $a_{1}+\cdots+a_{n}=b_{1}+\cdots+b_{k}$ em $A$.

ii) Se $a_{1}+\cdots+a_{k}=b_{1}+\cdots+b_{k}$, então $\left\langle a_{1}, \ldots, a_{k}\right\rangle \cong_{T_{A}}\left\langle b_{1}, \ldots, b_{k}\right\rangle$.

Demonstração. i) Considere o mapa de inclusão $i: T_{A} \rightarrow A$. Note que para todo $x \in T_{A}$, vale $f(x) \in\{1,0,-1\}$ e portanto podemos escrever $\tau_{f}=\operatorname{sgn} \circ f \circ i$, onde sgn: $\mathbb{Z} \rightarrow 3$ é a única ordem de $\mathbb{Z}$. Pelo Lema 3.45 , temos que $\tau_{f}=(\operatorname{sgn} \circ f) \circ i \in X_{T_{A}}$. 
ii) Vamos mostrar por indução em $k \geq 1$. Para $k=1$ é trivial e para $k=2$ segue do Lema 4.58. Supondo $k \geq 3$, pela definição de anel de Witt abstrato, existem $\alpha, \beta, z_{3}, \ldots, z_{k} \in T_{A}$ quadrado-constantes tais que $\alpha^{2}=a_{1}^{2}$ e

$$
\begin{aligned}
a_{1}+\alpha & =b_{1}+\beta \\
a_{2}+\cdots+a_{k} & =\alpha+z_{3}+\cdots+z_{k} \\
b_{2}+\cdots+b_{k} & =\beta+z_{3}+\cdots+z_{k} .
\end{aligned}
$$

Logo, por hipótese de indução temos

$$
\begin{aligned}
\left\langle a_{1}, \alpha\right\rangle & \cong_{T_{A}}\left\langle b_{1}, \beta\right\rangle \\
\left\langle a_{2}, \ldots, a_{k}\right\rangle & \cong_{T_{A}}\left\langle\alpha, z_{3}, \ldots, z_{k}\right\rangle \\
\left\langle b_{2}, \ldots, b_{k}\right\rangle & \cong_{T_{A}}\left\langle\beta, z_{3}, \ldots, z_{k}\right\rangle .
\end{aligned}
$$

e portanto $\left\langle a_{1}, \ldots, a_{k}\right\rangle \cong_{T_{A}}\left\langle a_{1}, \alpha, z_{3}, \ldots, z_{k}\right\rangle \cong_{T_{A}}\left\langle b_{1}, \beta, z_{3}, \ldots, z_{k}\right\rangle \cong_{T_{A}}\left\langle b_{1}, \ldots, b_{k}\right\rangle$.

Teorema 4.62. Seja A anel de Witt abstrato. Então $\left(T_{A}, D^{t}, \cdot, 0,1\right)$ é $R S$ von Neumann e o morfismo natural $\zeta: W\left(T_{A}\right) \rightarrow A$ é isomorfismo.

Demonstração. Pelo Lema 4.61, o mapa natural $\zeta: \mathrm{W}\left(T_{A}\right) \rightarrow A$ dado por $\zeta\left(\left\langle a_{1}, \ldots, a_{n}\right\rangle\right)=a_{1}+$ $\cdots+a_{n}$ está bem definido. Note que $\zeta$ é morfismo de aneis e como $A=\sum T_{A}$, segue que $\zeta$ é sobrejetor. Resta provar que $\zeta$ é injetor. Sejam $a_{1}, \ldots, a_{n} \in T_{A}$ tais que $a_{1}+\cdots+a_{n}=0$ em $A$. Considere a forma $\varphi=\left\langle a_{1}, \ldots, a_{n}\right\rangle$. Supondo $\varphi \not_{T_{A}}\langle 0\rangle$, pelo Teorema 3.18 e Corolário 3.9, existem $e_{1}, \ldots, e_{n} \in \operatorname{Id}\left(T_{A}\right)$ não-nulos e para cada $i=1, \ldots, n$, existe coleção $\left\{a_{i, 1}, \ldots, a_{i, k_{i}}\right\} \subseteq T_{A}\left(k_{i} \geq 1\right)$ quadrado-constante tais que

- $e_{i} e_{j}=0$ para $i \neq j$;

- $\varphi \equiv_{T_{A}} e_{1} \varphi \oplus \cdots \oplus e_{n} \varphi$.

- Para cada $i=1, \ldots, n$, vale $e_{i} \varphi \equiv_{T_{A}}\left\langle a_{i, 1}, \ldots, a_{i, k_{i}}\right\rangle$ e $a_{i, 1}^{2}=e_{i}$.

Pelo Lema 4.61, segue que para cada $i=1, \ldots, n$ vale $a_{i, 1}+\cdots+a_{i, k_{i}}=0$. Como $a_{i, 1}^{2}=e_{i} \neq 0$, segue que $k_{i}$ é par e portanto $a_{i, 1}+\cdots+a_{i, k_{i}}=0=\frac{k_{i}}{2}\left(e_{i}-e_{i}\right)$. Pelo Lema 4.61, segue que $\left\langle a_{i, 1}, \ldots, a_{i, k_{i}}\right\rangle \cong_{T_{A}}\langle 0\rangle$. Logo $\varphi=\left\langle a_{1}, \ldots, a_{n}\right\rangle \cong_{T_{A}}\langle 0\rangle$.

Teorema 4.63. Os funtores $W: \boldsymbol{v N R S} \rightarrow \boldsymbol{A b s}_{W i t t}$ e $T: \boldsymbol{A b s _ { W i t t }} \rightarrow \boldsymbol{v N R S}$ descrevem uma equivalência de categorias.

Demonstração. Pelo Teorema 4.62, dado $A$ anel de Witt abstrato, temos $W(T(A)) \cong A$ naturalmente e pela Proposição 4.57 dado RS von Neumann $R, T(W(R)) \cong R$ naturalmente. 


\section{Capítulo 5}

\section{B-pares}

Na seção 2.3, a noção de RS Booleano foi estudada brevemente, sobretudo no que diz respeito a como sua estrutura geral se relaciona com o RSG formado pelos inversíveis (e.g. Proposição 2.57). O presente capítulo tem por objetivo tornar mais precisa a conexão entre RSs Booleanos e RSGs, a fim de serem identificados com maior clareza os resultados da categoria dos RSGs que podem ser transferidos para a categoria dos RSs Booleanos.

Na primeira seção, é definida a categoria $\mathbf{B}_{\text {par }}$ dos $B$-pares $(G, \nabla)$, onde $G$ é um RSG e $\nabla \subseteq G$ subconjunto com propriedades adequadas (Definição 5.5). Na Proposição 5.6 é provado os $B$-pares são Horn-geométricos axiomatizáveis na linguagem $\mathcal{L}_{S G} \cup\{\nabla\}$, onde $\nabla$ denota um símbolo de predicado unário. O restante da seção é dedicado em estabelecer que as categorias BoolRS e $\mathbf{B}_{\text {par }}$ são equivalentes.

Na primeira subseção das aplicações, o principal resultado é o Teorema 5.21 que caracteriza os quocientes na categoria $\mathbf{B}_{\text {par }}$. Tendo em vista o equivalência de categorias BoolRS $\cong \mathbf{B}_{\text {par }}$, tem-se uma caracterização dos quocientes em BoolRS. Os principais resultados da primeira seção e desta primeira subseção foram feitos na linguagem dos semigrupos reais no artigo [MR]. A principal diferença entre as abordagens é a axiomatização Horn-Geométrica dos $B$-pares e uma noção de morfismo mais simples.

Na segunda subseção das aplicações, a primeira parte consiste em introduzir as noções de RS com suficientes unidades (Definição 5.22) e de RS com suficientes qc-formas (Definição 5.26). No Teorema 5.25 é provado que num RS com suficientes qc-formas, a isometria de qc-formas é de primeira ordem (na verdade, descrita por uma pp-fórmula) e como corolário são obtidos axiomas Horn-Geométricos para RSs com suficientes unidades e suficientes qc-formas. Na segunda parte, é provado que se $R$ é um RS com suficientes unidades, então existe morfismo $I_{R}: V \rightarrow R, V$ RS von Neumann, final com relação a morfismos saindo de um RS von Neumann e chegando em $R$. Em particular, se todo RS tiver suficientes unidades (problema em aberto), então a inclusão $\mathbf{v N R S} \rightarrow \mathbf{R S}$ admite um adjunto à direita. Uma recíproca parcial é obtida no Teorema 5.41: se a inclusão vNRS $\rightarrow$ RS admite um adjunto à direita, então os inversíveis de todo RS formam um RSG.

Na terceira subseção, é apresentada a envoltória RS-Booleana de um RS em geral (Teoremas 5.50 e 5.52) e são dadas algumas caracterizações de monomorfismo, epimorfismo e imersão completa entre RSs utilizando as envoltórias von Neumann e RS-Booleana e aneis de Witt.

\subsection{Categoria dos B-pares}

Nesta seção, a categoria $\mathbf{B}_{\text {par }}$ dos $B$-pares é definida e é construído o funtor $\mathfrak{R}: \mathbf{B}_{\text {par }} \rightarrow$ BoolRS que associa a cada $B$-par um RS Booleano. Os principais resultados da seção são:

- Caracterização de morfismo $f: R \rightarrow G$ entre RSs Booleanos via propriedades das restrições $f \mid: R^{\times} \rightarrow G^{\times}$e $f \mid: \operatorname{Id}(R) \rightarrow \operatorname{Id}(G)$ (Proposição 5.10). Este resultado aparece no artigo [MR] 
com um prova diferente. No Comentário 5.11, é apresentada uma generalização onde $G$ pode ser um RS arbitrário.

- Descrição do funtor $\mathfrak{R}: \mathbf{B}_{\text {par }} \rightarrow$ BoolRS que associa um a cada $B$-par um RS Booleano (Teorema 5.14). A primeira aparição desta construção (na linguagem dos Espaço de Ordens Abstratas) ocorre no Teorema 8.9.3 de [Mar7] que difere em demonstração e não trata de como levantar morfismos dos $B$-pares.

- O funtor $\mathfrak{L}:$ BoolRS $\rightarrow \mathbf{B}_{\text {par }}$ juntamente com o funtor $\mathfrak{R}: \mathbf{B}_{p a r} \rightarrow$ BoolRS estabelecem uma equivalência de categorias (Teorema 5.16).

Ao longo do texto, é assumido o conhecimento de propriedades básicas de grupos especiais reduzidos e sua envoltória Booleana (que podem ser encontrados em [DM1]). Para conveniência do leitor, vamos relembrar alguns fatos essenciais para o que segue e fixar notação.

Sejam $G$ RSG e $X_{G}=\left\{\sigma: G \rightarrow \mathbb{Z}_{2}: \sigma\right.$ é morfismo de RSG $\}$ seu espaço de ordens. Para cada $a \in G$, considere o subconjunto $[[a=-1]]=\left\{\sigma \in X_{G}: \sigma(a)=-1\right\} \subseteq X_{G}$. A família $\{[[a=-1]]: a \in G\}$ forma uma sub-base de uma topologia Booleana em $X_{G}$.

\section{Envoltória Booleana}

A álgebra Booleana (BA) $B_{G}=\mathbf{C}\left(X_{G}, \mathbb{Z}_{2}\right)=\left\{f: X_{G} \rightarrow \mathbb{Z}_{2}: f\right.$ é contínua $\}$ dos abertos-fechados de $X_{G}$ é chamada de envoltória Booleana de $G$. Ela tem estrutura natural de RSG induzida pontoa-ponto de $\mathbb{Z}_{2}=\{1,-1\}(1 \leq-1)$-que também é uma BA. Mais precisamente, dados $f, g, h \in B_{G}$

- Para todo $\sigma \in X_{G},(f \cdot g)(\sigma)=f(\sigma) g(\sigma)$.

- $f \in D_{B_{G}}(g, h)$ se, e somente se, para todo $\sigma \in X_{G}$ vale $f(\sigma) \in D_{\mathbb{Z}_{2}}(g(\sigma), h(\sigma))$.

Equivalentemente, considerando as operações Booleanas de $B_{G}$, dados $f, g, h \in B_{G}$, temos (f $\triangle g=(-f \wedge g) \vee(f \wedge-g)$ denota a diferença simétrica $)$

- $f \cdot g=f \triangle g$.

- $f \in D_{B_{G}}(g, h)$ se, e somente se, $f g \leq h g$.

O mapa $\varepsilon_{G}: G \rightarrow B_{G}$ dado por $\varepsilon_{G}(g)(\sigma)=\sigma(g)$ para $g \in G$ e $\sigma \in X_{G}$ é uma imersão completa de RSG. Além disso, a associação $G \mapsto B_{G}$ define um funtor da categoria dos RSG para a das álgebras Booleanas que é adjunto a esquerda do funtor de inclusão (daí o nome envoltória Booleana). Dados $x, y, z \in G$, dizemos que $x \vee y=z$ se $\varepsilon_{G}(x) \vee \varepsilon_{G}(y)=\varepsilon_{G}(z)$ (analogamente definimos $x \wedge y=z$ ). Note que, como $\varepsilon_{G}$ é injetora, tal $z$ é único e $x \vee y=z$ se, e somente se, para todo $\sigma \in X_{G}$ vale $\sigma(x) \vee \sigma(y)=\sigma(z)$ em $\mathbb{Z}_{2}$. Assim $\vee, \wedge$ são operações parciais em $G$. Além disso, se $f: G \rightarrow H$ é um morfismo de RSG e $x \vee y=z$ (ou $x \wedge y=z)$ em $G$, então $f(x) \vee f(y)=f(z)$ $(f(x) \wedge f(y)=f(z))$.

Um subconjunto $\nabla \subseteq G$ é chamado de reticulado se $1,-1 \in \nabla$ e dados $x, y \in \nabla$, tem-se $x \wedge y, x \vee y \in \nabla$.

\section{Quociente de RSG}

Sejam $G$ RSG e $\Delta \subseteq G$ subgrupo. Dizemos que $\Delta$ é saturado se para todo $a \in G$ valer $D_{G}(1, a) \subseteq \Delta$. Então o subgrupo quociente $G / \Delta$ admite estrutura de RSG dada por

$$
\langle\bar{a}, \bar{b}\rangle \equiv_{G / \Delta}\langle\bar{c}, \bar{d}\rangle \Leftrightarrow \text { Existem } s, t, u, v \in \Delta \text { tais que }\langle a s, b t\rangle \equiv_{G}\langle c u, d v\rangle .
$$

Assim, tem-se $\bar{a} \in D_{G / \Delta}(\bar{b}, \bar{c})$ se, e somente se, existem $s, t, u \in \Delta$ tais que as $\in D_{G}(b t, c u)$. Além disso, temos $\bar{a}=\bar{b}$ em $G / \Delta$ se, e somente se, para todo $\sigma \in X_{G} \operatorname{com} \sigma(\Delta)=\{1\}$ vale $\sigma(a)=\sigma(b)$. 
Definição 5.1. Seja $G$ RSG. Dizemos que $a \in G$ é elemento particionador se dado qualquer $g \in G$, $a \wedge g, a \vee g \in G$.

O seguinte lema fornece uma útil caracterização de elementos particionadores.

Lema 5.2. Sejam $G$ RSG e $a \in G$. São equivalentes:

i) $a$ é particionador.

ii) Para todo $g \in G, g \vee a \in G$.

iii) Para todo $g \in G, g \wedge a \in G$.

Demonstração. $i) \Rightarrow i i)$ : Imediato.

ii) $\Rightarrow$ iii): Seja $g \in G$. Note que

$$
\begin{aligned}
\left(\varepsilon_{G}(-g) \vee \varepsilon_{G}(a)\right) \triangle \varepsilon_{G}(-g) & =\left[\left(\varepsilon_{G}(-g) \vee \varepsilon_{G}(a)\right) \wedge \varepsilon_{G}(g)\right] \vee\left[\left(\varepsilon_{G}(g) \wedge \varepsilon_{G}(-a)\right) \wedge \varepsilon_{G}(-g)\right] \\
& =\left[\left(\varepsilon_{G}(-g) \vee \varepsilon_{G}(a)\right) \wedge \varepsilon_{G}(g)\right]=\varepsilon_{G}(g) \wedge \varepsilon_{G}(a) .
\end{aligned}
$$

Portanto, como $-g \vee a \in G$ por hipótese, temos $g \wedge a=(-g \vee a) \cdot(-g) \in G$. G.

$i i i) \Rightarrow i)$ : Seja $g \in G$. De forma análoga à implicação $i i) \Rightarrow i i i)$, prova-se que $g \vee a=(-g \wedge a) \cdot g \in$

Note que se $a \in G$ é particionador, $-a \in G$ tambem o é. A nomenclatura é justificada no resultado seguinte $([\mathrm{MR}])$.

Proposição 5.3. Seja $G$ RSG.

i) Dado $a \in G$ particionador, o mapa natural $\rho: G \rightarrow G / D(1, a) \times G / D(1,-a)$ é isomorfismo.

ii) Sejam $S, S^{\perp} \subseteq G$ subgrupos saturados tais que o mapa $G \rightarrow G / S \times G / S^{\perp}$ é isomorfismo. Então existe $a \in G$ particionador tal que $S=D(1, a)$ e $S^{\perp}=D(1,-a)$.

Demonstração. i) A injetividade segue do fato $D(1, a) \cap D(1,-a)=\{1\}$ para qualquer $a \in G$. Para a sobrejetividade, sejam $x, y \in G$ e considere

$$
z=-(x \vee a) \cdot(y \wedge(-a)) \in G .
$$

Note que dado $\sigma \in X_{G}$, se $\sigma(a)=1$, então $\sigma(z)=\sigma(x)$ e se $\sigma(a)=-1$, então $\sigma(z)=\sigma(y)$. Logo $\rho(z)=(\bar{z}, \bar{z})=(\bar{x}, \bar{y})$ e portanto $\rho$ é sobrejetor. Para concluir, precisamos provar que $\rho$ é imersão. Sejam $x, y \in G$ tais que $\bar{x} \in D_{G / D(1, a)}(\overline{1}, \bar{y})(*)$ e $\bar{x} \in D_{G / D(1,-a)}(\overline{1}, \bar{y})(* *)$. Assim, dado $\sigma \in X_{G}$, se $\sigma(a)=1$ por $(*)$ temos $\sigma(x) \in D_{\mathbb{Z}_{2}}(1, \sigma(y))$; se $\sigma(a)=-1$, então por (**) temos $\sigma(x) \in D_{\mathbb{Z}_{2}}(1, \sigma(y))$. Logo $x \in D_{G}(1, y)$.

ii) Seja $\varphi: G \rightarrow G / S \times G / S^{\perp}$ o morfismo induzido das projeções. Como $\varphi$ é sobrejetor, existe $a \in G$ tal que $\varphi(a)=(\overline{1},-\overline{1})$.

Primeiro vamos provar que $S=D(1, a)\left(S^{\perp}=D(1,-a)\right.$ seguirá de forma análoga). Dado $x \in D(1, a)$, temos $\varphi(x) \in D((\overline{1}, \overline{1}), \varphi(a))=D((\overline{1}, \overline{1}),(\overline{1},-\overline{1}))$. Logo $\varphi(x)=(\overline{1}, \bar{x})$ e $x \in S$. Reciprocamente, dado $y \in S$, temos $\varphi(y)=(\overline{1}, \bar{y}) \in D((\overline{1}, \overline{1}),(\overline{1},-\overline{1}))=D(\overline{1}, \varphi(a))$. Como $\varphi$ é isomorfismo, segue que $y \in D(1, a)$.

Por último, vamos verificar que $a$ é particionador. Seja $g \in G$. Considere $x \in G$ tal que $\varphi(x)=(\overline{1}, \bar{g})$. Note que $x \in S=D(1, a)$ e $x g \in S^{\perp}=D(1,-a)(*)$. Comparando as assinaturas, vemos que $a \wedge g=x$. De fato, dado $\sigma \in X_{G}$, se $\sigma(a)=1$, então por (*) temos $\sigma(x)=1$; se $\sigma(a)=-1$, então novamente por $(*)$ temos $\sigma(x)=\sigma(g)$. Segue pelo Lema 5.2 que $a \in G$ é particionador. 
Proposição 5.4. Seja $G$ RSG.

i) Se $a, b \in G$ são particionadores, então $a \wedge b, a \vee b \in G$ também são. Em particular, se $a_{1}, \ldots, a_{n} \in$ $G$ são particionadores, então $a_{1} \vee \cdots \vee a_{n} \in G$.

ii) Se $a_{1}, \ldots, a_{n} \in G$ são particionadores e $\left\{x_{p} \in G: p \in\{1,-1\}^{n}\right\}$ é família de elementos de $G$, então existe $x \in G$ tal que fixado $p \in\{1,-1\}^{n}$, para todo $\sigma \in\left[\left[\bigvee_{i=1}^{n} p(i) a_{i}=1\right]\right]$ vale $\sigma(x)=\sigma\left(x_{p}\right)$. Em outras palavras, o mapa $G \rightarrow \prod_{p \in E} G / D\left(1, \bigvee_{i=1}^{n} p(i) a_{i}\right)$ é isomorfismo, onde $E=\left\{p \in\{1,-1\}^{n}: \bigvee_{i=1}^{n} p(i) a_{i} \neq-1\right\}$.

Demonstração. i) Seja $g \in G$. Note que $(a \vee b) \vee g=a \vee(b \vee g) \in G$ e $(a \wedge b) \wedge g=a \wedge(b \wedge g) \in G$. Pelo Lema 5.2, $a \vee b, a \wedge b \in G$ são particionadores.

ii) Dado $p \in\{1,-1\}^{n}$, pelo item anterior $e_{p}:=\bigvee_{i=1}^{n} p(i) a_{i} \in G$ é particionador. Considere então o elemento

$$
x=\Pi_{p}\left(-e_{p} \wedge x_{p}\right) \in G .
$$

Fixado $p \in\{1,-1\}^{n}$, seja $\sigma \in\left[\left[e_{p}=1\right]\right]$. Isto significa que para todo $1 \leq i \leq n$ temos $\sigma\left(a_{i}\right)=p(i)$ e dado $q \in\{1,-1\}^{n}$ diferente de $p$ vale $\sigma\left(e_{q}\right)=-1$. Logo $\sigma(x)=\sigma\left(x_{p}\right)$.

$\{$ defBpar\}

Definição 5.5. Seja $G$ um RSG. Um subconjunto $\nabla \subseteq G$ é chamado de $B$-conjunto se

- Todo elemento de $\nabla$ é particionador;

- $\nabla$ é um reticulado.

O par $(G, \nabla)$ é chamada de $B$-par. Se além disso temos $-\nabla \subseteq \nabla, \nabla$ é chamado de $v N$-conjunto e $(G, \nabla) v N$-par. Um morfismo de B-pares $(G, \nabla) \rightarrow\left(G^{\prime}, \nabla^{\prime}\right)$ consiste num morfismo $f: G \rightarrow G^{\prime}$ de RSG satisfazendo $f(\nabla) \subseteq \nabla^{\prime}$. Vamos denotar pelo mesmo símbolo o morfismo de B-pares e o morfismo de RSG subjacente.

Denotamos por $\mathbf{B}_{\text {pares }}$ a categoria de todos os B-pares com a noção de morfismo acima. Note que se $(G, \nabla)$ é um vN-par, então $\nabla$ é um subgrupo pois dados $x, y \in \nabla$, temos $x \cdot y=(x \wedge-y) \vee(-x \wedge y) \in$ \{bparHorn\} $\nabla$.

Proposição 5.6. Considere a linguagem de primeira ordem $\mathcal{L}_{B p a r}=\mathcal{L}_{S G} \cup\{\nabla\}=\{\cdot, 1,-1, \equiv, \nabla\}$ dos $B$-pares constituída da linguagem dos SGs junto com um símbolo de predicado unário $\nabla$. Então os $B$-pares são axiomatizáveis em $\mathcal{L}_{\text {Bpar }}$ por sentenças Horn-Geométricas.

Demonstração. Considere a pp-fórmula $\varphi(x, y, z)$ na linguagem $\mathcal{L}_{S G}$ dada por

$$
\langle 1, x\rangle \otimes\langle 1, y\rangle \equiv\langle 1,1\rangle \otimes\langle 1, z\rangle .
$$

Pelo Corolário 7.20, p. 155, [DM1], segue que dado grupo especial reduzido $G$ e $a, b, c \in G$, temos $G=\varphi(a, b, c)$ se, e somente se, $c=a \vee b$. Como consequência, segue que $c=a \wedge b$ se, e somente se, $G \models \varphi(-a,-b,-c)$

Considere agora as seguintes sentenças Horn-Geométricas na linguagem $\mathcal{L}_{\text {Bpar }}$ :

- $\nabla(1), \nabla(-1)$.

- $\forall a, b(\nabla(a) \wedge \nabla(b) \Rightarrow \exists c, d \nabla(c) \wedge \nabla(d) \wedge \varphi(a, b, c) \wedge \varphi(-a,-b,-d))$.

- $\forall a, g(\nabla(a) \Rightarrow \exists c \varphi(a, b, c))$. 
Seja $\mathcal{T}$ o conjunto formado pelas sentenças acima juntamente com os axiomas de grupo especial reduzido. Naturalmente, $\mathcal{T}$ possui apenas sentenças Horn-geométricas. Seja $(G, \nabla) \models \mathcal{T}$. As sentenças ( $\dagger$ ) e $(\dagger \dagger)$ afirmam que $\nabla \subseteq G$ é reticulado enquanto pelo Lema 5.2 a sentença ( $\dagger \dagger \dagger)$ afirma que todo elemento de $\nabla$ é particionador. $\operatorname{Logo}(G, \nabla)$ é $B$-par.

Lema 5.7. Seja $f: G \rightarrow H$ morfismo de RSGs.

i) Seja $a, b \in G$ com $a \vee b \in G$. Então $f(a) \vee f(b) \in H$ e $f(a) \vee f(b)=f(a \vee b)$. (Vale enunciado análogo para $\wedge$ ).

ii) Se $f: G \rightarrow H$ é sobrejetor, então $f$ preserva elementos particionadores.

iii) Se $f: G \rightarrow H$ é sobrejetor e $\nabla \subseteq G$ é B-conjunto, então $f(\nabla) \subseteq H$ é B-conjunto.

Demonstração. i) Seja $c \in G \operatorname{com} a \vee b=c$. Vamos mostrar que $f(a) \vee f(b)=f(c)$. Basta notar que, dado $\sigma \in X_{H}, \sigma \circ f \in X_{G}$ e portanto $\sigma(f(a)) \vee \sigma(f(b))=\sigma(f(c))$.

ii) Seja $a \in G$ particionador. Dado $h=f(g) \in H$, temos $f(g) \vee f(a)=f(g \vee a) \in H$. Logo, pela sobrejetividade de $f, f(a) \in H$ é particionador.

iii) Imediato pelos itens anteriores.

Seja $R$ RS Booleano. A envoltória de Post de $R$ (Proposição 5.47) induz uma ordem parcial em $R$ (dados $a, b \in R, a \leq b \Leftrightarrow$ para todo $\sigma \in X_{R}$ vale $\sigma(a) \leq \sigma(b)$ em $\left.3=\{1,0,-1\}\right)$. O RSG $R^{\times}$ dado pelos inversíveis de $R$ também possui ordem parcial induzida pela envoltória Booleana. Como o mapa de restrição $X_{R} \rightarrow X_{R^{\times}}$é homeomorfismo, a restrição da ordem parcial de $R$ a $R^{\times}$coincide com aquela inerente do RSG $R^{\times}$. Portanto, podemos utilizar operações de sup e inf em $R^{\times}$sem fazer distinção de qual ordem estamos considerando.

Lema 5.8. Seja $R$ RS Booleano. Sejam $e, f \in \operatorname{Id}(R), e^{\prime} \in D^{t}(e, f)$ e $g \in R^{\times}$. Então

i) $\nabla(e) \vee \nabla(f)=\nabla(e \cdot f)$.

ii) $\nabla(e) \wedge \nabla(f)=\nabla\left(e^{\prime}\right)$.

iii) $\nabla(e) \vee g=\nabla(e g)$.

iv) $\nabla(e) \wedge g=\nabla(e) \cdot g \cdot \nabla(e g)$.

Demonstração. Vamos provar o item $i$ ) comparando assinaturas. Os demais seguem de maneira análoga. Dado $\sigma \in X_{R}$, vamos verificar que $\sigma(\nabla(e) \vee \nabla(f))=\sigma(\nabla(e f))$. Considere os seguintes casos:

Caso 1: $\sigma(e)=0$ ou $\sigma(f)=0$.

Supondo $\sigma(e)=0$, temos $\sigma(\nabla(e))=-1$ e $\sigma(\nabla(e f))=-1$. Logo $\sigma(\nabla(e) \vee \nabla(f))=-1=\sigma(\nabla(e f))$.

Caso 2: $\sigma(e)=\sigma(f)=1$.

Assim $\sigma(\nabla(e))=\sigma(\nabla(f))=1=\sigma(\nabla(e f))$ e portanto $\sigma(\nabla(e) \vee \nabla(f))=1=\sigma(\nabla(e f))$.

Como $\sigma \in X_{R}$ foi arbitrário, temos $\nabla(e) \vee \nabla(f)=\nabla(e \cdot f) \in \nabla_{R}$.

Proposição 5.9. Seja $R$ um RS Booleano e considere o conjunto $\nabla_{R}:=\nabla(\operatorname{Id}(R)) \subseteq R^{\times}$. Então $\left(R^{\times}, \nabla_{R}\right)$ é um B-par e $\nabla: I d(R) \rightarrow \nabla_{R}$ é isomorfismo de reticulados. Além disso, $\left(R^{\times}, \nabla_{R}\right)$ é vN-par se, e somente se, $R$ é von Neumann.

Demonstração. Note que $\nabla(1)=1, \nabla(0)=-1 \in \nabla_{R}$. Tome $e, f \in I d(R)$ e $g \in R^{\times}$. Pelo lema anterior, temos 
i) $\nabla(e) \vee \nabla(f) \in \nabla_{R}$ e $\nabla(e) \wedge \nabla(f) \in \nabla_{R}$.

ii) $\nabla(e) \vee g \in R^{\times}$e $\nabla(e) \wedge g \in R^{\times}$.

Logo, $\left(R^{\times}, \nabla_{R}\right)$ é B-par. Os itens $\left.\left.i\right), i i\right)$ do lema anterior também provam que $\nabla: \operatorname{Id}(R) \rightarrow \nabla_{R}$ é morfismo de reticulados (note que $\nabla(0)=-1$ ). Resta mostrar que $\nabla: I d(R) \rightarrow \nabla_{R}$ é injetivo. Sejam $e, f \in R$ idempontentes com $\nabla(e)=\nabla(f)$. Dado $\sigma \in X_{R}$, se $\sigma(e)=0$, então $\nabla(\sigma(f))=\sigma(\nabla(f))=$ $\nabla(\sigma(e))=-1$ e portanto $\sigma(f)=0$; se $\sigma(e)=1$, então $\nabla(\sigma(f))=\sigma(\nabla(f))=\nabla(\sigma(e))=1$ e portanto $\sigma(f)=1$. Logo $e=f$.

Se $\left(R^{\times}, \nabla_{R}\right)$ é vN-par, então dado $e \in \operatorname{Id}(R)$ existe $f \in \operatorname{Id}(R)$ tal que $-\nabla(e)=\nabla(f)$. Assim, dado $\sigma \in X_{R}$, se $\sigma(e)=0$, então $\sigma(\nabla(f))=-\sigma(\nabla(e))=1$ e portanto $\sigma(f)=1$. Se $\sigma(e)=1$, então $\sigma(\nabla(f))=-\sigma(\nabla(e))=-1$ e portanto $\sigma(f)=0$. Logo $f=e^{c}$ e deste modo $R$ é von Neumann. Reciprocamente, se $R$ é von Neumann, então dado $e \in \operatorname{Id}(R)$, temos $-\nabla(e)=\nabla\left(e^{c}\right) \in \nabla(R)$. Assim $\left(R^{\times}, \nabla_{R}\right)$ é vN-par.

A associação $\mathfrak{L}:$ BoolRS $\rightarrow \mathbf{B}_{\text {par }}$ definida por $\mathfrak{L}(R)=\left(R^{\times}, \nabla_{R}\right)$ é um funtor: dado $f: R \rightarrow G$ morfismo de RS Booleanos, a restrição $\left.f\right|_{R^{\times}}: R^{\times} \rightarrow G^{\times}$é morfismo de RSG e satisfaz $f\left(\nabla_{R}\right) \subseteq \nabla_{G}$.

Proposição 5.10. Seja $f: R \rightarrow G$ um mapa entre RS Booleanos. Suponha que

i) $f\left(R^{\times}\right) \subseteq G^{\times}$e $f(\operatorname{Id}(R)) \subseteq \operatorname{Id}(G)$.

ii) $\left.f\right|_{R^{\times}}: R^{\times} \rightarrow G^{\times}$é um morfismo de RSG.

iii) Para todo $a \in R$, tem-se $f(a)=f\left(a^{2}\right) \cdot f(\nabla(a))$.

iv) Para todo $a \in R$ idempotente, vale $f(\nabla(a))=\nabla(f(a))$.

Então $f$ é um morfismo de RS.

Demonstração. Usando 2.10, temos que se para todo $\sigma \in X_{G}, \sigma \circ f$ é um morfismo de RS, então $f$ é um morfismo de RS. Assim, podemos supor sem perda de generalidade que $G=3$.

Afirmação. Sejam $a, b \in \operatorname{Id}(R)$. Então $f(a b)=f(a) f(b)$.

Demonstração. Note que, pela Lema 5.8, temos $\nabla(f(a b))=f(\nabla(a b))=f(\nabla(a) \vee \nabla(b))=f(\nabla(a)) \vee$ $f(\nabla(b))=\nabla(f(a)) \vee \nabla(f(b))=\nabla(f(a) f(b))$. Pela Proposição 2.55, $f(a b)=f(a) f(b)$.

Afirmação. Se $x \in R^{\times}$e $a \in \operatorname{Id}(R)$, então $f(x a)=f(x) f(a)$. Como consequência, temos que para todos $a, b \in R, f(a b)=f(a) f(b)$ e $f(-a)=-f(a)$.

Demonstração. Escreva $y=x a$. Note que $y^{2}=a$ e assim $f(y)=f(a) f(\nabla(y))$. Como $f(a) \in\{0,1\}$, temos dois casos. Se $f(a)=0$, então $f(y)=0=f(x) f(a)$. Suponha $f(a)=1$. Pela Proposição 2.55, temos $\triangle(y) \leq x \leq \nabla(y)$. Como $f\left(R^{\times}\right) \subseteq G^{\times}$é um morfismo de RSG, temos $f(\triangle(y)) \leq f(x) \leq$ $f(\nabla(y))(*)$. Mas pela novamente pela Proposição 2.55 temos $\triangle(y) \nabla(y)=\nabla\left(y^{2}\right)=\nabla(a)$ e assim $f(\triangle(y)) f(\nabla(y))=f(\nabla(a))=\nabla(f(a))=1$. Então $f(\nabla(y))=f(\triangle(y))$ e portanto a desigualdade (*) implica $f(\nabla(y))=f(x)$. Logo $f(y)=f(a) f(\nabla(y))=f(a) f(x)$. Deste modo, dados $a, b \in R$, como $a b=a^{2} \nabla(a) b^{2} \nabla(b)$, temos pela conclusão precedente que $f(a b)=f\left(a^{2} b^{2}\right) f(\nabla(a) \nabla(b))$. Mas pela afirmação anterior vale $f\left(a^{2} b^{2}\right)=f\left(a^{2}\right) f\left(b^{2}\right)$ e por hipótese $f(\nabla(a) \nabla(b))=f(\nabla(a)) f(\nabla(b))$. Logo $f(a b)=f\left(a^{2}\right) f(\nabla(a)) f\left(b^{2}\right) f(\nabla(b))=f(a) f(b)$. Por último, como $f(-1)=-1$ (propriedade ii)), temos para todo $a \in R$ que $f(-a)=-f(a)$.

Afirmação. Para todo $x \in R, f(\nabla(x))=\nabla(f(x))$.

Demonstração. Note que se $f(x) \neq 0$, então $f\left(x^{2}\right)=f(x)^{2}=1$ e $f(x)=f\left(x^{2}\right) f(\nabla(x))=$ $f(\nabla(x)) \neq 0$. Logo $\nabla(f(x))=f(x)=f(\nabla(x))$. Se $f(x)=0$, como $\nabla(x) \triangle(x)=\nabla\left(x^{2}\right)$, temos por $i v)$ que $f(\nabla(x)) f(\triangle(x))=f\left(\nabla\left(x^{2}\right)\right)=-1$. Assim $f(\nabla(x))$ e $f(\triangle(x))$ tem sinais não-nulos opostos e como $\triangle(x) \leq \nabla(x)$ vale $f(\triangle(x)) \leq f(\nabla(x))$. Logo $f(\nabla(x))=-1=\nabla(f(x))$. 
Afirmação. Sejam $a \in \operatorname{Id}(R)$ e $x, y \in R$ tais que $x\langle 1,-a\rangle \cong_{R} y\langle 1,-a\rangle$. Se $f(a)=0$, então $f(x)=f(y)$.

Demonstração. Pela Proposição 2.57, temos $\nabla(x)\langle 1,-\nabla(a)\rangle \equiv_{R^{\times}} \nabla(y)\langle 1,-\nabla(a)\rangle$. Então, como $\left.f\right|_{R^{\times}}: R^{\times} \rightarrow G^{\times}$é morfismo de RSG, se $f(a)=0, f(\nabla(a))=\nabla(f(a))=-1$ e assim

$$
f(\nabla(x))\langle 1,1\rangle \equiv_{3} f(\nabla(y))\langle 1,1\rangle
$$

. Logo, $\nabla(f(x))=f(\nabla(x))=f(\nabla(y))=\nabla(f(y))$. Repetindo o mesmo argumento para a Wittequivalência $-x\langle 1,-a\rangle \cong_{R}-y\langle 1,-a\rangle$, concluímos que $\nabla(-f(x))=\nabla(-f(y))$. Logo $f(x)=f(y)$.

Agora estamos prontos para provar que se $a \in D_{R}^{t}(b, c)$, então $f(a) \in D_{3}^{t}(f(b), f(c))$. Pela Proposição 2.57 , se $a \in D_{R}^{t}(b, c)$, então $\nabla(a) \in D_{R}^{t}(\nabla(b), \nabla(c))$ e $b\left\langle 1,-a^{2}\right\rangle \cong-c\left\langle 1,-a^{2}\right\rangle, a\left\langle 1,-b^{2}\right\rangle \cong$ $c\left\langle 1,-b^{2}\right\rangle, a\left\langle 1,-c^{2}\right\rangle \cong b\left\langle 1,-c^{2}\right\rangle$. Como $\left.f\right|_{R^{\times}}: R^{\times} \rightarrow\{1,-1\}$ é um morfismo de RSG, $\nabla(f(a)) \in$ $D_{3}^{t}(\nabla(f(b)), \nabla(f(c)))$. Se $f(a), f(b), f(c) \neq 0$, temos $f(a) \in D_{3}^{t}(f(b), f(c))$. Se $f(a)=0$, pela última afirmação temos $f(b)=-f(c)$ e assim $f(a) \in D_{3}^{t}(f(b), f(c))$. Os outros casos seguem por argumento idêntico e assim $f(a) \in D_{3}^{t}(f(b), f(c))$.

Comentário 5.11. É possível provar o resultado anterior sem supor $G$ Booleano. Fixado $R$ RS qualquer, dado $a \in R$ dizemos que $\nabla(a) \in R^{\times}$se existir $g \in R^{\times}$tal que $a=g a^{2}, g \in D^{t}(-1, a)$; tal $g$ é único e o chamamos de $\nabla(a)$. Seja $I d_{\nabla}(R)=\{a \in I d(R): \nabla(a) \in R\}$.

Assim dado morfismo $f: R \rightarrow G$ entre quasiquer RS, pela definição segue que $f\left(I_{\nabla}(R)\right) \subseteq I d_{\nabla}(G)$. A mesma demonstração da proposição acima funciona para provar a seguinte

Proposição 5.12. Sejam $R, G$ RS, $R$ Booleano e $f: R \rightarrow G$ um mapa. Suponha que

i) $f\left(R^{\times}\right) \subseteq G^{\times}$e $f(I d(R)) \subseteq I d_{\nabla}(G)$.

ii) $\left.f\right|_{R^{\times}}: R^{\times} \rightarrow G^{\times}$é um morfismo de pré-RSG.

iii) Para todo $a \in R$, tem-se $f(a)=f\left(a^{2}\right) \cdot f(\nabla(a))$.

iv) Para todo $a \in R$ idempotente, vale $f(\nabla(a))=\nabla(f(a))$.

Então $f$ é um morfismo de RS.

Seja $(G, \nabla)$ um B-par. Dados $g \in G, a \in \nabla$, considere a função contínua $\gamma(g, a): X_{G} \rightarrow 3$ dada por

$$
\gamma(g, a)(\sigma)=\operatorname{sgn}_{\sigma}(\langle g, g a\rangle) / 2= \begin{cases}\sigma(g) & \text { se } \sigma(a)=1 \\ 0 & \text { se } \sigma(a)=-1 .\end{cases}
$$

Com a estrutura de álgebra de Post de $C\left(X_{G}, 3\right)$ (Exemplo 5.44), é possível multiplicar e fazer operações da álgebra com as funções acima.

Lema 5.13. Seja $(G, \nabla)$ um B-par. Sejam $g, h \in G$ e $a, b \in \nabla$. Então

i) Se $\gamma(g, a)(\sigma) \in\{1,-1\}$ para todo $\sigma \in X_{G}$, então $\gamma(g, a)=\gamma(g, 1)$.

Se $\gamma(g, a)(\sigma) \in\{1,0\}$ para todo $\sigma \in X_{G}$, então $\gamma(g, a)=\gamma(1, a)$.

ii) $\gamma(g, 1)=\gamma(h, 1)$ se, e somente se, $g=h$.

$\gamma(1, a)=\gamma(1, b)$ se, e somente se, $a=b$.

iii) $\gamma(1, a) \cdot \gamma(1, b)=\gamma(1, a) \vee \gamma(1, b)=\gamma(1, a \vee b)$.

$\gamma(1, a) \wedge \gamma(1, b)=\gamma(1, a \wedge b)$.

iv) $-\gamma(g, 1)=\gamma(-g, 1)$.

$\gamma(g, 1) \cdot \gamma(h, 1)=\gamma(g h, 1)$. 
v) $\gamma(g, a)^{2}=\gamma(1, a)$.

vi) $\nabla(\gamma(g, a))=\gamma(g \vee a, 1)$. Em particular, $\nabla(\gamma(1, a))=\gamma(a, 1)$.

vii) $\gamma(g, a)=\gamma(h, b)$ se, e somente se, $a=b$ e $g \vee a=h \vee a$.

viii) $\gamma(g, a)=\gamma(g, 1) \cdot \gamma(1, a)$.

ix) $\gamma(g, a) \cdot \gamma(h, b)=\gamma(g h, a \vee b)$.

Demonstração. i) Se $\gamma(g, a)(\sigma) \neq 0, \sigma \in X_{G}$, então $\sigma(a)=1$. Logo $a=1$ e $\gamma(g, a)=\gamma(g, 1)$. Se $\gamma(g, a)(\sigma) \in\{0,1\}$, então $\sigma(a)=\sigma(g)=1$ ou $\sigma(a)=-1$. Em ambos os casos, $\gamma(g, a)(\sigma)=$ $\gamma(1, a)(\sigma)$ e portanto $\gamma(g, a)=\gamma(1, a)$.

ii) Basta notar que para todo $\sigma \in X_{G}$ vale $\gamma(g, 1)(\sigma)=\sigma(g)$ e $\gamma(1, a)(\sigma)=(\sigma(a)+1) / 2$.

iii) Seja $\sigma \in X_{G}$. Vamos mostrar que 1) $\gamma(1, a) \vee \gamma(1, b)(\sigma)=\gamma(1, a \vee b)(\sigma)$ e 2) $\gamma(1, a) \wedge \gamma(1, b)(\sigma)=$ $\gamma(1, a \wedge b)(\sigma)$. Vamos dividir em alguns casos:

Caso 1: $\sigma(a)=\sigma(b)$.

Neste caso, $\gamma(1, a)(\sigma)=\gamma(1, b)(\sigma)=\gamma(1, a \vee b)(\sigma)=\gamma(1, a \wedge b)(\sigma)$ e portanto vale 1$), 2)$.

Caso 2: $\sigma(a)=1=-\sigma(b)$.

Neste caso, $\gamma(1, a)(\sigma)=\gamma(1, a \wedge b)(\sigma)=1$ e $\gamma(1, b)(\sigma)=\gamma(1, a \vee b)(\sigma)=0$. Logo $\gamma(1, a) \vee$ $\gamma(1, b)(\sigma)=0=\gamma(1, a \vee b)(\sigma)$ e $\gamma(1, a) \wedge \gamma(1, b)(\sigma)=1=\gamma(1, a \wedge b)(\sigma)$.

Caso 3: $\sigma(a)=-1=-\sigma(b)$

Segue por argumento simétrico ao dado no caso anterior.

iv) Imediato.

v) Seja $\sigma \in X_{G}$. Vamos mostrar que $\gamma(g, a)^{2}(\sigma)=\gamma(1, a)(\sigma)$. Se $\sigma(a)=1$, então $\gamma(g, a)^{2}(\sigma)=$ $\sigma(g)^{2}=1=\gamma(1, a)(\sigma)$. Se $\sigma(a)=-1$, então $\gamma(g, a)^{2}(\sigma)=0=\gamma(1, a)(\sigma)$.

vi) Seja $\sigma \in X_{G}$. Vamos mostrar que $\nabla(\gamma(g, a))(\sigma)=\gamma(g \vee a, 1)(\sigma)$. Se $\sigma(a)=1$, então $\gamma(g, a)(\sigma)=$ $\sigma(g)=\sigma(g \vee a)$ e portanto $\nabla(\gamma(g, a))(\sigma)=\sigma(g)=\gamma(g \vee a, 1)(\sigma)$. Se $\sigma(a)=-1$, então $\gamma(g, a)(\sigma)=0$ e $\sigma(g \vee a)=-1 . \operatorname{Logo} \nabla(\gamma(g, a))(\sigma)=-1=\gamma(g \vee a, 1)(\sigma)$.

vii) Se $\gamma(g, a)=\gamma(h, b)$, então $\gamma(1, a)=\gamma(g, a)^{2}=\gamma(h, b)^{2}=\gamma(1, b)$ e $\gamma(g \vee a, 1)=\nabla(\gamma(g, a))=$ $\nabla(\gamma(h, b))=\gamma(h \vee b, 1)$. Logo pelo item $i i)$ temos $a=b$ e $g \vee a=h \vee b$. Reciprocamente, se $a=b$ e $g \vee a=h \vee b$, então $\gamma(g, a)^{2}=\gamma(h, b)^{2}$ e $\nabla(\gamma(g, a))=\nabla(\gamma(h, b))$. Logo $\gamma(g, a)=$ $\gamma(g, a)^{2} \nabla(\gamma(g, a))=\gamma(h, b)^{2} \nabla(\gamma(h, b))=\gamma(h, b)$.

viii) $\gamma(g, a) \cdot \gamma(h, b)=\gamma(g, 1) \cdot \gamma(1, a) \cdot \gamma(h, 1) \cdot \gamma(1, b)=\gamma(g h, 1) \cdot \gamma(1, a \vee b]=\gamma(g h, a \vee b)$.

Dado B-par $L=(G, \nabla)$, considere o conjunto $\mathfrak{R}(L)=\left\{f \in \mathbf{C}\left(X_{G}, 3\right)\right.$ : existem $g \in G, a \in$ $\nabla$ such that $f=\gamma(g, a)\}$.

Teorema 5.14. Seja $L=(G, \nabla)$ um B-par. Considere o conjunto $\mathfrak{R}(L)$ munido da multiplicação e soma multi-valorada induzida por $C\left(X_{G}, 3\right)$.

i) Seja Iv $v_{L}: G \rightarrow \mathfrak{R}(L)$ mapa dado por $(g \in G)$

$$
I v_{L}(g)=\gamma(g, 1)
$$

\section{Então}

a) $\mathfrak{R}(L)$ é um $R S$ Booleano. 
b) $I v_{L}(G)=\mathfrak{R}(L)^{\times}$e $I v_{L}(\nabla)=\nabla_{\mathfrak{R}(L)}$.

c) $I v_{L}:(G, \nabla) \rightarrow\left(\mathfrak{R}(L)^{\times}, \nabla_{\mathfrak{R}(L)}\right)$ é um isomorfismo natural de B-pares.

ii) Sejam $R R S$ Booleano e $i: L=(G, \nabla) \rightarrow\left(R^{\times}, \nabla_{R}\right)$ morfismo de B-pares. Então existe único morfismo $h: \mathfrak{R}(L) \rightarrow R$ de modo que o diagrama

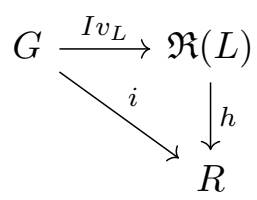

seja comutativo.

iii) Seja $f: L=(G, \nabla) \rightarrow L^{\prime}=\left(G^{\prime}, \nabla^{\prime}\right)$ um morfismo de B-pares. Então existe único morfismo $\mathfrak{R}(f): \mathfrak{R}(L) \rightarrow \mathfrak{R}\left(L^{\prime}\right)$ tal que o diagrama

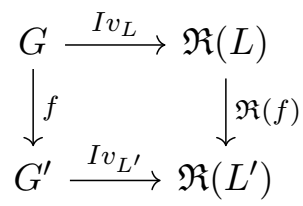

é comutativo. Além disso, tal associação define um funtor $\mathfrak{R}: \boldsymbol{B}_{\text {pares }} \rightarrow \boldsymbol{B o o l R} \boldsymbol{S}$.

Demonstração. i) a) Como $\mathbf{C}\left(X_{G}, 3\right)$ é $\mathrm{RS}$ e $\mathfrak{R}(L) \subseteq \mathbf{C}\left(X_{G}, 3\right)$ induz suas operações, $\mathfrak{R}(L)$ satisfaz todos axiomas de RS menos possivelmente a associatividade forte (pois são axiomas universais). Sejam $f, g, h, x, y \in G, a, b, c, d, e \in \nabla$ e suponha que

$$
\gamma(x, d) \in D^{t}(\gamma(f, a), \gamma(y, e)), \gamma(y, e) \in D^{t}(\gamma(g, b), \gamma(h, c)) .
$$

Precisamos provar que existe $z \in G$ e $v \in \nabla$ tais que $\gamma(x, d) \in D^{t}(\gamma(z, v), \gamma(h, c)), \gamma(z, v) \in$ $D^{t}(\gamma(f, a), \gamma(g, b))(*)$.

Para isso, será usado a Proposição 5.4, item $i i)$, com $a_{1}=a, a_{2}=b, a_{3}=c, a_{4}=d, a_{5}=e$. Para cada $p \in\{1,-1\}^{5}$, vamos obter $z_{p} \in G$ cuja colagem constitui um $z \in G$ que satisfaz $(*)$. Como $\{1,-1\}^{5}$ tem $2^{5}=32$ permutações, vamos usar as restrições que 5.1 impõe para reduzir os casos a serem analisados para 13 .

Note que dado $\sigma \in X_{G}$, os conjuntos $(\sigma(d), \sigma(a), \sigma(e))$ e $(\sigma(e), \sigma(b), \sigma(c))$ não podem ter exatamente dois -1 : se, por exemplo, $\sigma(d)=\sigma(a)=-1$ e $\sigma(e)=1$, então teríamos pela primeira soma multivalorada de 5.1 que $0 \in D_{3}^{t}(0, \sigma(y))$, o que é um absurdo pois $\sigma(y) \in$ $\{1,-1\}$. Em outras palavras, se $p \in\{1,-1\}^{5}$ é tal que $(p(4), p(1), p(5))$ ou $(p(5), p(2), p(3))$ tem exatamente duas entradas com -1 , então $\bigvee_{i=1}^{5} p(i) a_{i}=1\left(\left[\left[\bigvee_{i=1}^{5} p(i) a_{i}=1\right]\right]=\emptyset\right)$. Com esse tipo de restrição atingimos os 13 casos.

Na tabela abaixo, cada caso é esquematizado. Por exemplo, na primeira linha é definida a permutação $p_{1}$ por $p_{1}(i)=1$ para qualquer $i=1, \ldots, 5$. Usamos a notação $e_{p_{j}}=$ $\bigvee_{i=1}^{5} p_{j}(i) a_{i}, j=1, \ldots, 13$, e a coluna das restrições representa as relações válidas em $G / D\left(1, e_{p_{j}}\right)$ derivadas de 5.1. Na última coluna estão as definições de cada $z_{j}$ : na primeira linha, como $\bar{x} \in D(\bar{f}, \bar{y}), \bar{y} \in D(\bar{g}, \bar{h})$ em $G / D\left(1, e_{p_{1}}\right)$, existe $z_{1} \in G$ tal que $\bar{x} \in$ $D\left(\bar{z}_{1}, \bar{h}\right), \bar{z}_{1} \in D(\bar{f}, \bar{g})$. 


\begin{tabular}{|l|r|r|r|r|r|l|l|}
\hline & $\mathrm{a}$ & $\mathrm{b}$ & $\mathrm{c}$ & $\mathrm{d}$ & $\mathrm{e}$ & Restrição válida em $G / D\left(1, e_{p_{i}}\right)$ & \\
\hline$p_{1}$ & 1 & 1 & 1 & 1 & 1 & $\bar{x} \in D(\bar{f}, \bar{y}), \bar{y} \in D(\bar{g}, \bar{h})$ & $\bar{x} \in D(\bar{z} 1, \bar{h}), \bar{z}_{1} \in D(\bar{f}, \bar{g})$ \\
\hline$p_{2}$ & 1 & 1 & 1 & 1 & -1 & $\bar{x}=\bar{f}, \bar{g}=-\bar{h}$ & $\bar{z}_{2}=\bar{f}$ \\
\hline$p_{3}$ & 1 & 1 & 1 & -1 & 1 & $-\bar{f} \in D(\bar{g}, \bar{h})$ & $\bar{z}_{3}=-\bar{h}$ \\
\hline$p_{4}$ & 1 & 1 & -1 & 1 & 1 & $\bar{x} \in D(\bar{f}, \bar{g})$ & $\bar{z}_{4}=\bar{x}$ \\
\hline$p_{5}$ & 1 & 1 & -1 & -1 & 1 & $\bar{f}=-\bar{g}$ & $\bar{z}_{5}=\overline{1}$ \\
\hline$p_{6}$ & 1 & -1 & 1 & 1 & 1 & $\bar{x} \in D(\bar{f}, \bar{h})$ & $\bar{z}_{6}=\bar{f}$ \\
\hline$p_{7}$ & 1 & -1 & 1 & -1 & 1 & $\bar{f}=-\bar{h}$ & $\bar{z}_{7}=-\bar{h}$ \\
\hline$p_{8}$ & 1 & -1 & -1 & 1 & -1 & $\bar{x}=\bar{f}$ & $\bar{z}_{8}=\bar{f}$ \\
\hline$p_{9}$ & -1 & 1 & 1 & 1 & 1 & $\bar{x} \in D(\bar{g}, \bar{h})$ & $\bar{z}_{9}=\bar{g}$ \\
\hline$p_{10}$ & -1 & 1 & 1 & -1 & -1 & $\bar{g}=-\bar{h}$ & $\bar{z}_{10}=\bar{g}$ \\
\hline$p_{11}$ & -1 & 1 & -1 & 1 & 1 & $\bar{x}=\bar{g}$ & $\bar{z}_{11}=\bar{x}$ \\
\hline$p_{12}$ & -1 & -1 & 1 & 1 & 1 & $\bar{x}=\bar{h}$ & $\bar{z}_{12}=\overline{1}$ \\
\hline$p_{13}$ & -1 & -1 & -1 & -1 & -1 & & $\bar{z}_{13}=\overline{1}$ \\
\hline
\end{tabular}

Pela Proposição 5.4, existe $z \in G$ tal que dado $\sigma \in\left[\left[e_{p_{j}}=1\right]\right]$, tem-se $\sigma(z)=\sigma\left(z_{j}\right)$. Seja $v=(a \wedge b) \vee(c \wedge d) \in \nabla$.

Assim, dado $\sigma \in X_{G}$, existe algum $j=1, \ldots, 13$ tal que $\sigma \in\left[\left[e_{p_{j}}=1\right]\right]$ e, pela relação correspodente na última coluna da tabela, $\gamma(x, d)(\sigma) \in D^{t}(\gamma(z, v)(\sigma), \gamma(h, c))(\sigma), \gamma(z, v)(\sigma) \in$ $D^{t}(\gamma(f, a)(\sigma), \gamma(g, b)(\sigma))$. Logo $\gamma(x, d) \in D^{t}(\gamma(z, v), \gamma(h, c)), \gamma(z, v) \in D^{t}(\gamma(f, a), \gamma(g, b))$ e $\mathfrak{R}(L)$ satisfaz a associatividade forte. Por último, pelo Lema 5.13, dado $x \in \mathfrak{R}(L)$ temos que existe $y=\nabla(x) \in \mathfrak{R}(L)$ satisfazendo $x=y x^{2}, y \in D_{\mathbf{C}\left(X_{G}, 3\right)}^{t}(-1, x)$. Logo $\mathfrak{R}(L)$ é RS Booleano.

b) Note que $I v_{L}(G) \subseteq \mathfrak{R}(L)^{\times}$. Note também que dado $\gamma(g, a) \in \mathfrak{R}(L)$ inversível, temos $\gamma(g, a)(\sigma) \in\{1,-1\}$ para todo $\sigma \in X_{G}$ e portanto pelo Lema 5.13 vale $\gamma(g, a)=\gamma(g, 1)=$ $I v_{L}(g)$. Por outro lado, dado $a \in \nabla$, temos pelo Lema 5.13 que $I v_{L}(a)=\gamma(a, 1)=$ $\nabla(\gamma(1, a)) \in \nabla_{\mathfrak{R}(L)}$. Além disso, dado $x \in \nabla_{\mathfrak{R}(L)}$, pelo Lema 5.13, existe $a \in \nabla$ tal que $x=\nabla(\gamma(1, a))=\gamma(a, 1)$ e $\operatorname{assim} I v_{L}(a)=x$.

c) Pelo item anterior, é suficiente provar que $I v_{L}: G \rightarrow \mathfrak{R}(L)^{\times}$é imersão. Pelo Lema 5.13, Iv $v_{L}$ é um morfismo de grupos injetor que preserva a constante -1 . Além disso, pelo teorema de Pfister para RSG, dado $g, h \in G, \gamma(g, 1) \in D^{t}(\gamma(1,1), \gamma(h, 1)) \Leftrightarrow g \in D_{G}(1, h)$ e portanto $I v_{L}: G \rightarrow \mathfrak{R}(L)^{\times}$é imersão.

ii) Como $i(\nabla) \subseteq \nabla_{R}$, pelo lema anterior podemos definir a função $h: \mathfrak{R}(L) \rightarrow R$ por $h(\gamma(g, a))=$ $i(g \vee a) \nabla_{R}^{-1}(i(a))$, onde $\nabla_{R}: \operatorname{Id}(R) \rightarrow \nabla_{R}$ é isomorfismo de reticulados da Proposição 5.9. Para provar que $h$ é um morfismo de RS, vamos utilizar a Proposição 5.10. Sejam $g \in G$ e $a \in \nabla$.

(a) $h(\gamma(g, 1))=i(g \vee 1) \nabla_{R}^{-1}(i(1))=i(g)$. Logo $h\left(\mathfrak{R}(L)^{\times}\right) \subseteq R^{\times}$e $h: \mathfrak{R}(L)^{\times} \rightarrow R^{\times}$é morfismo de RSG pois $\left.h\right|_{\mathfrak{R}(L) \times}=i \circ I v_{L}^{-1}$.

(b) $h(\gamma(1, a))=i(1 \vee a) \nabla_{R}^{-1}(i(a))=i(a) \nabla_{R}^{-1}(i(a))$. Mas como vale $y \nabla(y)=y$ para todo $y \in \operatorname{Id}(R)$, temos $i(a) \nabla_{R}^{-1}(i(a))=\nabla_{R}^{-1}(i(a))$ e $\operatorname{assim} h(\operatorname{Id}(\mathfrak{R}(L))) \subseteq \operatorname{Id}(R)$.

(c) Pelo lema anterior, temos que $\gamma(g, a)^{2}=\gamma(1, a)$ e $\nabla(\gamma(g, a))=\gamma(g \vee a, 1)$. Assim, $h\left(\gamma(g, a)^{2}\right) h(\nabla(\gamma(g, a)))=h(\gamma(1, a)) h(\gamma(g \vee a, 1))=\nabla_{R}^{-1}(i(a)) i(g \vee a)=h(\gamma(g, a))$.

(d) Também pelo lema anterior, temos $\nabla(h(\gamma(1, a)))=\nabla\left(\nabla_{R}^{-1}(i(a))\right)=i(a)=h(\gamma(a, 1))=$ $h(\nabla(\gamma(1, a)))$.

Deste modo, pela Proposição 5.10, $h$ é um morfismo de RS e pelos itens $a$ ) e $b$ ) acima $h$ faz o diagrama em questão comutar. Se $h^{\prime}$ é outro morfismo que faz o diagrama comutar, temos pelo lema anterior $h^{\prime}(\gamma(g, a))=h^{\prime}(\gamma(g \vee a, 1)) h^{\prime}(\gamma(1, a))=h^{\prime}\left(\operatorname{Iv}_{L}(g \vee a)\right) h^{\prime}\left(\nabla^{-1}(\gamma(a, 1))\right)=$ $h^{\prime}\left(\operatorname{Iv}_{L}(g \vee a)\right) h^{\prime}\left(\nabla^{-1}\left(I v_{L}(a)\right)\right)=i(g \vee a) \nabla_{R}^{-1}(i(a))=h(\gamma(g, a))$, provando assim a unicidade de $h$. 
iii) Considere o mapa $f_{v}=I v_{L^{\prime}} \circ f$. Note que $f_{v}: G \rightarrow \mathfrak{R}\left(L^{\prime}\right)^{\times}$é um morfismo de RSG e $f_{v}(\nabla) \subseteq$ $\nabla_{\mathfrak{R}\left(L^{\prime}\right)}$. Assim, pelo item anterior, existe único morfismo $h: \mathfrak{R}(L) \rightarrow \mathfrak{R}\left(L^{\prime}\right)$ que faz o diagrama em questão comutar. Pela demonstração acima, temos $h(\gamma(g, a))=\gamma(f(g), f(a))$ e dessa expressão fica fácil deduzirmos que se $g:\left(G^{\prime}, \nabla^{\prime}\right) \rightarrow\left(G^{\prime \prime}, \nabla^{\prime \prime}\right)$ é outro morfismo de B-pares, então $\mathfrak{R}(g \circ f)=\mathfrak{R}(g) \circ \mathfrak{R}(f)$. Daí como $\mathfrak{R}\left(I d_{L}\right)=I d_{\mathfrak{R}(L)}$, a associação $\mathfrak{R}: \mathbf{B}_{\text {pares }} \rightarrow$ BoolRS é funtorial.

Lema 5.15. Seja $f: R \rightarrow G$ um morfismo de RS Booleano. Então $f$ é isomorfismo de RS se, e somente se, $\left.f\right|_{R^{\times}}: R^{\times} \rightarrow G^{\times}$é isomorfismo de RSG e $\left.f\right|_{I d(R)}: I d(R) \rightarrow I d(G)$ é sobrejetora. Em particular, dado RS Booleano $R$, temos $\mathfrak{R}\left(R^{\times}, \nabla_{R}\right) \cong R$ naturalmente.

Demonstração. Se $f$ é isomorfismo, é fácil ver que as restrições aos invertíveis e aos idempotentes também são isomorfismos. Para a volta, primeiro vamos provar que $f$ é bijetora. Dado $g \in G$, pela sobrejetividade das restrições, existem $x \in R^{\times}$e $y \in I d(R)$ tais que $f(x)=\nabla(g)$ e $f(y)=g^{2}$. Assim $f(x y)=\nabla(g) g^{2}=g$ e $f$ é sobrejetora. Dados $a, b \in R \operatorname{com} f(a)=f(b)$, temos $f(\nabla(a))=\nabla(f(a))=$ $\nabla(f(b))=f(\nabla(b))$ e $f\left(\nabla\left(a^{2}\right)\right)=\nabla\left(f\left(a^{2}\right)\right)=\nabla\left(f\left(b^{2}\right)\right)=f\left(\nabla\left(b^{2}\right)\right)$. Logo pela injetividade da restrição, $\nabla(a)=\nabla(b)$ e $\nabla\left(a^{2}\right)=\nabla\left(b^{2}\right)$ e portanto $a=b$. Para concluirmos que $f$ é isomorfismo, note que, como $\left.f\right|_{R^{\times}}: R^{\times} \rightarrow G^{\times}$, pelo Teorema 2.54, segue que o mapa induzido $f^{*}: X_{G} \rightarrow X_{R}$ é homemomorfismo. Assim, pelo Lema 2.44, segue que $f: R \rightarrow G$ é isomorfismo. Em particular, pelo teorema anterior, existe mapa canônico $\mathfrak{R}\left(R^{\times}, \nabla_{R}\right) \rightarrow R$ cujas restrições a $\mathfrak{R}\left(R^{\times}, \nabla(R)\right)^{\times}$e $\operatorname{Id}\left(\mathfrak{R}\left(R^{\times}, \nabla_{R}\right)\right)$ são isomorfismos e portanto pelo que foi provado $\mathfrak{R}\left(R^{\times}, \nabla_{R}\right) \cong R$.

Teorema 5.16. Os funtores $\mathfrak{R}: \boldsymbol{B}_{\text {par }} \rightarrow$ BoolRS e $\mathfrak{L}:$ BoolR $\boldsymbol{S} \rightarrow \boldsymbol{B}_{\text {par }}$ definem uma equivalência de categorias.

Demonstração. Pelo Teorema 5.14,i)-c), dado $B$-par $L=(G, \nabla)$, vale $\mathfrak{L}(\Re(L)) \cong L$ naturalmente. Por outro lado, pelo Lema 5.15 dado RS Booleano $R$, temos $\mathfrak{R}(\mathfrak{L}(R)) \cong R$.

Proposição 5.17. Sejam $L=(G, \nabla)$ um B-par e $R=\mathfrak{R}(L)$. Considere $\nabla^{\prime}$ a subalgebra Booleana gerada por $\nabla$. Então $\nabla^{\prime} \subseteq G$ é vN-conjunto e existe isomorfismo canônico $V(R) \cong \Re\left(G, \nabla^{\prime}\right)$.

Demonstração. Pela Proposição 5.4, $\nabla^{\prime} \subseteq G$ é vN-conjunto. Para concluirmos que $\mathfrak{R}\left(G, \nabla^{\prime}\right) \cong$ $V(R)$, pelo Teorema 5.16, é suficiente provarmos que o mapa de inclusão $i:(G, \nabla) \rightarrow\left(G, \nabla^{\prime}\right)$ satisfaz a propriedade universal do Teorema 4.14 na categoria $\mathbf{B}_{\text {par }}$, ou seja, dado morfismo $h:(G, \nabla) \rightarrow$ $\left(H, \nabla^{\prime \prime}\right)$ de B-pares com $\left(H, \nabla^{\prime \prime}\right)$ vN-par, existe único morfismo $\bar{h}:\left(G, \nabla^{\prime}\right) \rightarrow\left(H, \nabla^{\prime \prime}\right)$ fazendo o diagrama

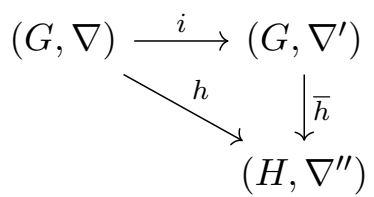

comutar. Mas como $\nabla^{\prime \prime} \subseteq H$ é vN-conjunto, podemos provar por indução na complexidade de $x \in \nabla^{\prime}$ que $h(x) \in \nabla^{\prime \prime}$, ou seja, $h\left(\nabla^{\prime}\right) \subseteq \nabla^{\prime \prime}$ o que garante a existência de $\bar{h}$. A unicidade é imediata.

\subsection{Aplicações}

Nesta seção de aplicações, vamos utilizar os $B$-pares para uma desenvolver uma série de resultados interessantes. Mais notadamente:

- Classificar todos os quocientes de RSs Booleanos (subseção 5.2.1); 
- Estudar a classe dos RSs com suficientes qc-formas -que estende substancialmente a classe dos RSs Booleano e ainda possui uma boa teoria de formas quadráticas (??)- e mostrar que, sob certas condições, a inclusão de categorias vNRS $\rightarrow$ RS possui adjunto à esquerda (subseção $5.2 .2)$

- Definir a envoltória RS-Booleana e caracterizar monomorfismos, epimorfismos e imerções completas de RSs gerais em termos das envoltórias von Neumann e RS-Booleana e dos aneis de Witt (subseção 5.2.3).

\subsubsection{Quocientes de RSs Booleanos}

Definição 5.18. Seja $f: G \rightarrow G^{\prime}$ morfismo de RSG. Define-se seu núcleo como $\operatorname{ker}_{1}=\{x \in$ $G: f(x)=1\}^{1}$.

\{quoDefbool\}

Definição 5.19. Seja $f:(G, \nabla) \rightarrow\left(G^{\prime}, \nabla^{\prime}\right)$ morfismo de $B_{\text {pares. }}$. Dizemos que $f$ é mapa quociente se dado morfismo $g:(G, \nabla) \rightarrow\left(G^{\prime \prime}, \nabla^{\prime \prime}\right)$ de $B_{\text {pares }}$ satisfazendo $\operatorname{ker}_{1}(f) \subseteq \operatorname{ker}_{1}(g)$, então existe único $\bar{g}:\left(G^{\prime}, \nabla^{\prime}\right) \rightarrow\left(G^{\prime \prime}, \nabla^{\prime \prime}\right)$ satisfazendo $g=\bar{g} \circ f$.

$\{$ kervn\}

Lema 5.20. Sejam $f: R \rightarrow G$ e $g: R \rightarrow H$ morfismos em BoolRS. São equivalentes:

i) $\operatorname{ker}_{1}(f) \subseteq \operatorname{ker}_{1}(g)$;

ii) $\operatorname{ker}_{1}(f) \cap R^{\times} \subseteq \operatorname{ker}_{1}(g) \cap R^{\times}$;

iii) $\operatorname{ker}(f) \subseteq \operatorname{ker}(g)$;

Em particular,

- $\operatorname{ker}_{1}(f)=\operatorname{ker}_{1}(g)$ se, e somente se, $\operatorname{ker}_{U}(f)=\operatorname{ker}_{U}(g)$.

- $f$ é injetor se, e somente se, $\operatorname{ker}_{1}(f)=1$.

- Sejam $\mathfrak{L}(f):\left(R^{\times}, \nabla_{R}\right) \rightarrow\left(G^{\times}, \nabla_{G}\right)$ e $\mathfrak{L}(g):\left(R^{\times}, \nabla_{R}\right) \rightarrow\left(H^{\times}, \nabla_{H}\right)$. Então $\operatorname{ker}(f) \subseteq \operatorname{ker}(g)$ se, e somente se, $\operatorname{ker}_{1}(\mathfrak{L}(f)) \subseteq \operatorname{ker}_{1}(\mathfrak{L}(g))$.

Demonstração. Os sentidos $i) \Rightarrow i i)$ e $i i i) \Rightarrow i$ ) são imediatos. Para $i i) \Rightarrow i i i)$, sejam $(x, y) \in$ $\operatorname{ker}(f)$. Como $f(x)=f(y)$, temos $f\left(\nabla(x) \nabla(y)^{-1}\right)=f\left(\nabla\left(x^{2}\right) \nabla\left(y^{2}\right)^{-1}\right)=1$. Por hipótese segue que $g\left(\nabla(x) \nabla(y)^{-1}\right)=g\left(\nabla\left(x^{2}\right) \nabla\left(y^{2}\right)^{-1}\right)=1$, ou seja, $\nabla(g(x))=\nabla(g(y))$ e $\nabla\left(g\left(x^{2}\right)\right)=\nabla\left(g(y)^{2}\right)$. Logo pela Proposição 2.55 vale $g(x)=g(y)$. Deste modo, $\operatorname{ker}(f) \subseteq \operatorname{ker}(g)$.

Pelo Lema anterior, o funtor $\mathfrak{L}: \mathbf{B}_{\text {par }} \rightarrow$ BoolRS preserva morfismos quocientes (Definição 5.19). Como $\mathfrak{L}$ é parte de uma equivalência de categorias (Teorema 5.16), podemos nos concentrar nos quocientes em $\mathbf{B}_{\text {par }}$ a fim de caracterizar os quocientes em BoolRS.

Sejam $(G, \nabla)$ B-par, $S \subseteq G$ subgrupo saturado e $\pi: G \rightarrow G / S$ morfismo quociente. Pelo Lema 5.7, $(G / S, \pi(\nabla))$ é B-par e é fácil verificar que $\pi:(G, \nabla) \rightarrow(G / S, \pi(\nabla))$ é morfismo quociente de B-pares.

Teorema 5.21. Seja $f:(G, \nabla) \rightarrow\left(G^{\prime}, \nabla^{\prime}\right)$ morfismo quociente. Seja $S=k_{\text {er }}(f)$. Então o morfismo induzido $\bar{f}:(G / S, \pi(\nabla)) \rightarrow\left(G^{\prime}, \nabla^{\prime}\right)$ é isomorfismo. Em particular, o morfismo $f: G \rightarrow G^{\prime}$ é regular ${ }^{2}$ e sobrejetor.

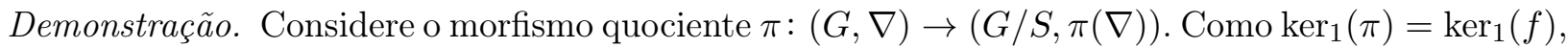
existe único $\bar{f}:(G / S, \pi(\nabla)) \rightarrow\left(G^{\prime}, \nabla^{\prime}\right)$ tal que $f=\bar{f} \circ \pi$. Além disso, pela propriedade universal de $f$, existe $g:\left(G^{\prime}, \nabla^{\prime}\right) \rightarrow(G / S, \pi(\nabla))$ tal que $g \circ f=\pi$.

\footnotetext{
${ }^{1}$ Utilizamos a mesma notação para pré-imagem do 1 em multi-aneis (Definição 1.9)

${ }^{2}$ Definição 2.22, p. 43, de [DM1]
} 


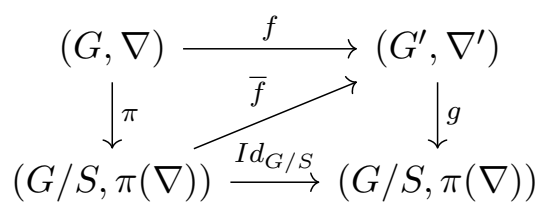

Assim, como $g \circ \bar{f} \circ \pi=g \circ f=I d_{G / S} \circ \pi$ e $\pi$ é sobrejetora, segue que $g \circ \bar{f}=I d_{G / S}$. Por outro lado, $\bar{f} \circ g \circ f=\bar{f} \circ g \circ \bar{f} \circ \pi=\bar{f} \circ \pi=f=I d_{G^{\prime}} \circ f$. Como $f$ é epimorfismo de B-pares, segue que $\bar{f} \circ g=I d_{G^{\prime}}$.

\subsubsection{Os inversíveis de um RS}

Nesta subseção, é introduzida a classe dos RSs com suficientes qc-formas e mostrado que, sob certas condições, a inclusão vNRS $\rightarrow$ RS possui adjunto à esquerda (subseção 5.2.2). Os principais resultados desta subseção são:

- Na Definição 5.26, é apresentada a noção de RSs com suficientes qc-formas. A nomenclatura é justificada no Teorema de caracterização 5.25, que mostra como a isometria de qc-formas é de primeira ordem. Além disso, tem como corolário que a teoria dos RSs com suficientes qc-formas é Horn-Geométrica axiomatizável (Teorema 5.27).

- Se $R$ tem suficientes unidades, então existe morfismo $I_{R}: V \rightarrow R$ de RS com $V$ von Neumann tal que $I_{R}$ é final com relação a morfismos saindo de um von Neumann e chegando em $R$ ( Teorema 5.36). Em particular, se todo RS tiver suficientes unidades (problema em aberto), então a subcategoria vNRS de RS é birreflexiva (inclusão tem adjuntos à esquerda e à direita).

- Vale uma reciproca partical do Teorema 5.36. O Teorema 5.41 prova que se a inclusão vNRS $\hookrightarrow \mathbf{R S}$ tiver adjunto à direita, então os inversíveis de todo RS é RSG.

Definição 5.22. Seja $R$ RS. Considere o pré-RSG $\left(R^{\times}, D_{R^{\times}},-1, \cdot\right)$, onde $D_{R^{\times}}$é dado por:

$$
a \in D_{R^{\times}}(b, c) \Leftrightarrow a \in D_{R}^{t}(b, c), \text { onde } a, b, c \in R^{\times} .
$$

Dizemos que $R$ tem suficientes unidades se $\left(R^{\times}, D_{R^{\times}},-1, \cdot\right)$ é RSG e o mapa de restrição $X_{R} \rightarrow X_{R^{\times}}$ é sobrejetor.

Proposição 5.23. Seja $R$ RS com $R^{\times}$RSG. São equivalentes:

i) O mapa $X_{R} \rightarrow X_{R^{\times}}$é sobrejetor.

ii) Para toda forma de Pfister $\varphi$ sobre $R^{\times}$, vale $D_{R^{\times}}(\varphi)=D_{R}^{t}(\varphi) \cap R^{\times}$.

iii) Para toda forma de Pfister $\varphi=\left\langle 1, a_{1}\right\rangle \otimes \cdots \otimes\left\langle 1, a_{n}\right\rangle$, com $a_{i} \in R^{\times}$e $n \geq 2$, se $a \in D_{R}^{t}(\varphi) \cap R^{\times}$, então existem $x, y \in R^{\times}$tais que $a \in D_{R}^{t}\left(x, a_{1} y\right), x, y \in D_{R}^{t}\left(\left\langle 1, a_{2}\right\rangle \otimes \cdots \otimes\left\langle 1, a_{n}\right\rangle\right)$.

iv) Para toda forma de Pfister $\varphi$ sobre $R^{\times}$, se $-1 \in D_{R}^{t}(\varphi)$, então $-1 \in D_{R^{\times}}(\varphi)$.

Demonstração. $i) \Rightarrow$ ii): Seja $x \in D_{R}^{t}(\varphi) \cap R^{\times}$. Então pelo Corolário 3.15 iii), segue que $x \varphi \equiv_{R} \varphi$. Como o mapa de restrição $X_{R} \rightarrow X_{R^{\times}}$é sobrejetor, temos que $x \varphi \equiv_{R^{\times}} \varphi$. Assim, novamente pelo Corolário 3.15 aplicado ao 0 -RSG $R^{\times} \cup\{0\}$, vale que $x \in D_{R^{\times}}(\varphi)$.

ii $) \Rightarrow$ iii): Seja $a \in D^{t}(\varphi) \cap R^{\times}$. Então $a \in D_{R^{\times}}(\varphi)=D_{R^{\times}}\left(\varphi^{\prime} \oplus a_{1} \varphi^{\prime}\right)$, onde $\varphi^{\prime}=\left\langle 1, a_{2}\right\rangle \otimes \ldots \otimes\left\langle 1, a_{n}\right\rangle$. Logo existem $x, y \in R^{\times}$tais que $a \in D_{R^{\times}}\left(x, a_{1} y\right) \subseteq D_{R}^{t}\left(x, a_{1} y\right)$ e $x, y \in D_{R^{\times}}\left(\varphi^{\prime}\right) \subseteq D_{R}^{t}\left(\varphi^{\prime}\right)$.

iii) $\Rightarrow$ ii): Seja $\varphi=\left\langle 1, a_{1}\right\rangle \otimes \cdots \otimes\left\langle 1, a_{n}\right\rangle$. Vamos provar por indução em $n \geq 1$ que $D_{R}^{t}(\varphi) \cap R^{\times} \subseteq$ $D_{R^{\times}}(\varphi)$. Para $n=1$ é imediato. Supondo $n \geq 2$, seja $a \in D_{R}^{t}(\varphi) \cap R^{\times}$. Então pela hipótese 
existem $x, y \in D_{R}^{t}\left(\left\langle 1, a_{2}\right\rangle \otimes \cdots \otimes\left\langle 1, a_{n}\right\rangle\right)$ tais que $a \in D_{R}^{t}\left(x, a_{1} y\right)$. Assim, por hipótese de indução, $x, y \in D_{R^{\times}}\left(\left\langle 1, a_{2}\right\rangle \otimes \cdots \otimes\left\langle 1, a_{n}\right\rangle\right)$ e $a \in D_{R^{\times}}\left(x, a_{1} y\right)$. Logo $a \in D_{R^{\times}}(\varphi)$.

ii) $\Rightarrow$ iv): Imediato.

$i v) \Rightarrow i$ ): Vamos mostrar que a imagem da função $X_{R} \rightarrow X_{R^{\times}}$é densa. Sendo $X_{R}$ compacto e $X_{R^{\times}}$Hausdorff, concluíremos que $X_{R} \rightarrow X_{R^{\times}}$é sobrejetora. Sejam $a_{1}, \ldots, a_{n} \in R^{\times}$tais que $\bigcap_{i=1}^{n}\left[\left[a_{i}=1\right]\right]_{R^{\times}} \neq \emptyset$. Por conta análoga ao Comentário 3.13, segue que $\varphi \neq_{R^{\times}} 0$. Pelo Corolário 3.15 iii), segue que $-1 \notin D_{R^{\times}}(\varphi)$. Deste modo, por hipótese, temos $-1 \notin D_{R}^{t}(\varphi)$. Pelo mesmo corolário temos $\varphi \approx_{R} 0$ e portanto existe $\sigma \in \bigcap_{i=1}^{n}\left[\left[a_{i}=1\right]\right]_{R} \subseteq X_{R}$.

Teorema 5.24. Seja $R$ semigrupo real. Então $R$ tem suficientes unidades se, e somente se, para todos $a_{1}, \ldots, a_{n}, b_{1}, \ldots, b_{n} \in R^{\times}$tais que $\left\langle a_{1}, \ldots, a_{n}\right\rangle \equiv_{R}\left\langle b_{1}, \ldots, b_{n}\right\rangle$, existem $a, b, z_{3}, \ldots, z_{n} \in R^{\times}$ satisfazendo

$$
\begin{aligned}
\left\langle a_{1}, a\right\rangle & \equiv_{R}\left\langle b_{1}, b\right\rangle \\
\left\langle a_{2}, \ldots, a_{n}\right\rangle & \equiv_{R}\left\langle a, z_{3}, \ldots, z_{n}\right\rangle \\
\left\langle b_{2}, \ldots, b_{n}\right\rangle & \equiv_{R}\left\langle b, z_{3}, \ldots, z_{n}\right\rangle .
\end{aligned}
$$

Demonstração. $(\Rightarrow)$ : Sejam $a_{1}, \ldots, a_{n}, b_{1}, \ldots, b_{n} \in R^{\times}$tais que $\left\langle a_{1}, \ldots, a_{n}\right\rangle \equiv_{R}\left\langle b_{1}, \ldots, b_{n}\right\rangle$. Como o mapa de restrição $X_{R} \rightarrow X_{R^{\times}}$é sobrejetor, temos $\left\langle a_{1}, \ldots, a_{n}\right\rangle \equiv_{R^{\times}}\left\langle b_{1}, \ldots, b_{n}\right\rangle$. Assim, existem $a, b, z_{3}, \ldots, z_{n} \in R^{\times}$tais que

$$
\begin{aligned}
\left\langle a_{1}, a\right\rangle & \equiv_{R_{\times}}\left\langle b_{1}, b\right\rangle \\
\left\langle a_{2}, \ldots, a_{n}\right\rangle & \equiv_{R^{\times}}\left\langle a, z_{3}, \ldots, z_{n}\right\rangle \\
\left\langle b_{2}, \ldots, b_{n}\right\rangle & \equiv_{R^{\times}}\left\langle b, z_{3}, \ldots, z_{n}\right\rangle .
\end{aligned}
$$

Deste modo, vale

$$
\begin{aligned}
\left\langle a_{1}, a\right\rangle & \equiv_{R}\left\langle b_{1}, b\right\rangle \\
\left\langle a_{2}, \ldots, a_{n}\right\rangle & \equiv_{R}\left\langle a, z_{3}, \ldots, z_{n}\right\rangle \\
\left\langle b_{2}, \ldots, b_{n}\right\rangle & \equiv_{R}\left\langle b, z_{3}, \ldots, z_{n}\right\rangle .
\end{aligned}
$$

$(\Leftarrow)$ : Primeiro vamos mostrar que $R^{\times}$é RSG. Sejam $a, b, c, x, l \in R^{\times}$tais que $x \in D_{R}^{t}(a, l), l \in$ $D_{R}^{t}(b, c)$. Então pela Proposição $\left.3.4 v\right)$ temos $\langle x, a l x\rangle \equiv_{R}\langle a, l\rangle$ e $\langle l, l b c\rangle \equiv_{R}\langle b, c\rangle$. Portanto $\langle x, a l x, l b c\rangle \equiv_{R}\langle a, b, c\rangle \equiv_{R}\langle c, a, b\rangle$. Deste modo, pela hipótese, existem $\alpha, \beta, z \in R^{\times}$tais que

$$
\begin{aligned}
\langle x, \alpha\rangle & \equiv_{R}\langle c, \beta\rangle \\
\langle a l x, l b c\rangle & \equiv_{R}\langle\alpha, z\rangle \\
\langle a, b\rangle & \equiv_{R}\langle\beta, z\rangle .
\end{aligned}
$$

Pela Proposição $3.4 i v)$ segue que $x \in D_{R}^{t}(\beta, c)$ com $\beta \in D_{R}^{t}(a, b)$. Logo $R^{\times}$é RSG.

Resta provar que o mapa de restrição $X_{R} \rightarrow X_{R^{\times}}$é sobrejetor. Primeiro, vamos provar por indução em $n \geq 2$ que dados duas formas $\varphi, \psi$ sobre $R^{\times}$de dimensão $n$, se $\varphi \equiv_{R} \psi$, então $\varphi \equiv_{R^{\times}} \psi$. Para $n=2$, se $\langle a, b\rangle \equiv_{R}\langle c, d\rangle$, pela Proposição $\left.3.4 i v\right)$ segue que $a b=c d$ e $a \in D_{R}^{t}(b, c)$. Logo $a \in D_{R^{\times}}(b, c)$ e $\langle a, b\rangle \equiv_{R^{\times}}\langle c, d\rangle$. Supondo $n \geq 3$, sejam $\varphi=\langle a\rangle \oplus \varphi^{\prime}$ e $\psi=\langle b\rangle \oplus \psi^{\prime}$ formas sobre $R^{\times}$de dimensão $n \operatorname{com} \varphi \equiv_{R} \psi$. Por hipótese, devem existir $\alpha, \beta, z_{3}, \ldots, z_{n} \in R^{\times}$tais que

$$
\begin{aligned}
\langle a, \alpha\rangle & \equiv_{R}\langle b, \beta\rangle \\
\varphi^{\prime} & \equiv_{R}\left\langle a, z_{3}, \ldots, z_{n}\right\rangle \\
\psi^{\prime} & \equiv_{R}\left\langle b, z_{3}, \ldots, z_{n}\right\rangle .
\end{aligned}
$$


Deste modo, por hipótese de indução, segue que

$$
\begin{aligned}
\langle a, \alpha\rangle & \equiv_{R^{\times}}\langle b, \beta\rangle \\
\varphi^{\prime} & \equiv_{R^{\times}}\left\langle a, z_{3}, \ldots, z_{n}\right\rangle \\
\psi^{\prime} & \equiv_{R^{\times}}\left\langle b, z_{3}, \ldots, z_{n}\right\rangle
\end{aligned}
$$

e portanto $\varphi=\langle a\rangle \oplus \varphi^{\prime} \equiv_{R^{\times}}\langle a, \alpha\rangle \oplus\left\langle z_{3}, \ldots, z_{n}\right\rangle \equiv_{R^{\times}}\langle b, \beta\rangle \oplus\left\langle z_{3}, \ldots, z_{n}\right\rangle \equiv_{R^{\times}}\langle b\rangle \oplus \psi^{\prime}=\psi$.

Provemos agora que $X_{R} \rightarrow X_{R^{\times}}$é sobrejetor usando a Proposição $5.23 i v$ ). Seja $\varphi$ forma de Pfister sobre $R^{\times}$de grau $n \geq 1 \mathrm{com}-1 \in D_{R}^{t}(\varphi)$. Pelo Corolário 3.15 iii), vale $-\varphi \equiv_{R} \varphi$ e portanto $\varphi \equiv_{R} 2^{n}\langle 1,-1\rangle$. Logo, pelo provado acima, $\varphi \equiv_{R^{\times}} 2^{n}\langle 1,-1\rangle$ e portanto $-1 \in D_{R^{\times}}(\varphi)$.

Sejam $R$ RS e $a \in R$ não-nulo. Dado $x \in R \operatorname{com} \bar{x} \in R_{a}^{\times}$, podemos assumir $x^{2}=a^{2}$. De fato, como $\bar{x}^{2}=1$ em $R_{a}, x^{2} a^{2}=a^{2}$. Tomando $x^{\prime}=x a^{2}$, temos $\overline{x^{\prime}}=\bar{x}$ em $R_{a}$ e $x^{\prime 2}=a^{2}$. Deste modo, se $\varphi$ é forma sobre $R$ tal que $\bar{\varphi}$ tem entradas inversíveis em $R_{a}$ e $\bar{x} \in D_{R_{a}}^{t}(\bar{\varphi}) \cap R_{a}^{\times}$, então podemos supor sem perda de generalidade que $x \in D_{R}^{t}(\varphi)$.

Teorema 5.25. Seja R RS. São equivalentes:

i) Para todo $a \in R$ não-nulo, $R_{a}$ tem suficientes unidades.

ii) Para todas $k$-formas $\varphi=\left\langle a_{1}, \ldots, a_{k}\right\rangle$ e $\psi=\left\langle b_{1}, \ldots, b_{k}\right\rangle, k \geq 3$, satisfazendo $\varphi \equiv_{R} \psi$, existem $\alpha, \beta \in R$ e $k-2$-forma $\theta$ tais que

$$
\begin{aligned}
\left\langle a_{1}, \alpha\right\rangle & \equiv_{R}\left\langle b_{1}, \beta\right\rangle \\
\left\langle a_{2}, \ldots, a_{k}\right\rangle & \equiv_{R}\langle\alpha\rangle \oplus \theta \\
\left\langle b_{2}, \ldots, b_{k}\right\rangle & \equiv_{R}\langle\beta\rangle \oplus \theta .
\end{aligned}
$$

iii) a) Para todos a, $, c, x, l \in R$ quadrado-constantes (Definição 4.55) com $x \in D_{R}^{t}(a, l), l \in$ $D_{R}^{t}(b, c)$, existe $l^{\prime} \in D_{R}^{t}(a, b)$ com $l^{\prime 2}=l^{2}$ e $x \in D_{R}^{t}\left(l^{\prime}, c\right)$.

b) $\operatorname{Sejam} \varphi=\left\langle a_{1}^{2}, a_{1}\right\rangle \otimes \cdots \otimes\left\langle a_{n}^{2}, a_{n}\right\rangle$ e $d=\Pi_{i=1}^{n} a_{i}^{2}$. Dado $a \in D_{R}^{t}(\varphi)$ com $a^{2}=d^{2}$, existem $x, y \in D_{R}^{t}\left(\left\langle a_{2}^{2}, a_{2}\right\rangle \otimes \cdots \otimes\left\langle a_{n}^{2}, a_{n}\right\rangle\right)$ com $x^{2}=y^{2}=d^{2}$ tais que $a \in D_{R}^{t}\left(x, a_{1} y\right)$.

Demonstração. $i) \Rightarrow i i)$ : Sejam $\varphi=\left\langle a_{1}, \ldots, a_{k}\right\rangle$ e $\psi=\left\langle b_{1}, \ldots, b_{k}\right\rangle, k \geq 3$, satisfazendo $\varphi \equiv_{R} \psi$. Seja $a \in \operatorname{Id}(R) \operatorname{com} D(a)=\operatorname{supp}(\varphi)=\operatorname{supp}(\psi)$. Então em $R_{a} \bar{\varphi} \equiv_{R_{a}} \bar{\psi}$ são formas com entradas invertíveis e isomorfas. Como $R_{a}$ tem suficientes unidades, existem $\alpha, \beta \in R$ e $\theta$ forma sobre $R$ com $\bar{\varphi} k-2$-forma tais que

$$
\begin{aligned}
\left\langle\overline{a_{1}}, \bar{\alpha}\right\rangle & \equiv_{R_{a}}\left\langle\overline{b_{1}}, \bar{\beta}\right\rangle \\
\left\langle\overline{a_{2}}, \ldots, \overline{a_{k}}\right\rangle & \equiv_{R_{a}}\langle\bar{\alpha}\rangle \oplus \bar{\theta} \\
\left\langle\overline{b_{2}}, \ldots, \overline{b_{k}}\right\rangle & \equiv_{R_{a}}\langle\bar{\beta}\rangle \oplus \bar{\theta} .
\end{aligned}
$$

Pelo Comentário 3.22, $\alpha^{2}=\beta^{2}=a$ e existem $z_{3}, \ldots, z_{k} \in R$ tais que $z_{i}^{2}=a$ para todo $i=3, \ldots, k$ e $\bar{\theta} \equiv_{R_{a}}\left\langle\overline{z_{3}}, \ldots, \overline{z_{k}}\right\rangle$. Como o $X_{R_{a}} \cong\left\{\sigma \in X_{R}: \sigma(a)=1\right\}$, segue que

$$
\begin{aligned}
\left\langle a_{1}, \alpha\right\rangle & \equiv_{R}\left\langle b_{1}, \beta\right\rangle \\
\left\langle a_{2}, \ldots, a_{k}\right\rangle & \equiv_{R}\langle\alpha\rangle \oplus \theta \\
\left\langle b_{2}, \ldots, b_{k}\right\rangle & \equiv_{R}\langle\beta\rangle \oplus \theta .
\end{aligned}
$$

ii) $\Rightarrow$ i): Como $R_{a} \cong R_{a^{2}}$, podemos supor $a \in \operatorname{Id}(R)$ não-nulo. Assim, pelo Teorema 5.24, o item $i$ ) diz que em $R_{a}$ tem suficientes unidades.

iii) $\Rightarrow$ i) Seja $h \in R$ não-nulo. Como $R_{h} \cong R_{h^{2}}$, podemos assumir $h$ idempotente. Primeiro vamos provar que $R_{h}^{\times}$é RSG. Sejam $\bar{a}, \bar{b}, \bar{c}, \bar{x}, \bar{l} \in R_{h}^{\times}$tais que $\bar{x} \in D_{R_{h}}^{t}(\bar{a}, \bar{l}), \bar{l} \in D_{R_{h}}^{t}(\bar{b}, \bar{c})$. Assim, podemos assumir sem perda de generalidade que $x \in D_{R}^{t}(a, l), l \in D_{R}^{t}(b, c)$. Pelo item $a$ ), existe 
$l^{\prime} \in D_{R}^{t}(a, b) \operatorname{com} l^{\prime 2}=h$ e $x \in D_{R}^{t}\left(l^{\prime}, c\right)$. Logo $\overline{l^{\prime}} \in D_{R_{a}}^{t}(\bar{a}, \bar{b}) \cap R_{a}^{\times}$e $\bar{x} \in D_{R_{a}}^{t}\left(\overline{l^{\prime}}, \bar{c}\right)$. Portanto, $R_{a}^{\times}$é RSG.

Resta mostrar que o mapa $X_{R_{h}} \rightarrow X_{R_{h}^{\times}}$é sobrejetor. Vamos usar a Proposição 5.23 iii $)$. Seja $\bar{\varphi}$ forma de Pfister sobre $R_{h}^{\times}$. Podemos supor sem perda de generalidade que $\varphi=\left\langle a_{1}^{2}, a_{1}\right\rangle \otimes \cdots \otimes\left\langle a_{n}^{2}, a_{n}\right\rangle$ com $a_{1}, \ldots, a_{n}$ quadrado-constantes, $a_{1}^{2}=h$. Seja $a \in R$ com $a^{2}=h$ e $\bar{a} \in D_{R_{g}}^{t}(\bar{\varphi})$. Assim, $a \in D_{R}^{t}(\varphi)$ e por hipótese existem $x, y \in D_{R}^{t}\left(\left\langle a_{2}^{2}, a_{2}\right\rangle \otimes \cdots \otimes\left\langle a_{n}^{2}, a_{n}\right\rangle\right)$ tais que $x^{2}=y^{2}=h$ e $a \in D_{R}^{t}\left(x, a_{1} y\right)$. Logo $\bar{a} \in D_{R_{a}}^{t}\left(\bar{x}, \overline{a_{1} y}\right) \operatorname{com} \bar{x}, \bar{y} \in D_{R_{a}}^{t}\left(\left\langle 1, \overline{a_{2}}\right\rangle \otimes \cdots \otimes\left\langle 1, \overline{a_{n}}\right\rangle\right)$. Portanto, pela Proposição 5.23, segue que $X_{R_{h}} \rightarrow X_{R_{h}^{\times}}$é sobrejetor.

i) $\Rightarrow$ iii): A volta utiliza argumentos e simplificações parecidas com a ida.

Definição 5.26. Seja $R$ RS. $R$ tem suficientes qc-formas se satisfaz qualquer uma das condições acima.

Teorema 5.27. A classe dos RSs com suficientes qc-formas e classe dos RSs com suficientes unidades são Horn-Geométrica axiomatizável na linguagem $\mathcal{L}_{R S}=\left\{\cdot,-, D^{t}, 0,1\right\}$. Em particular, essa classe é fechada por produtos reduzidos e colimits indutivos.

Demonstração. Segue direto do item $i i i)$ do Teorema 5.25 pois a fórmula $v \in D^{t}\left(v_{1}, \ldots, v_{n}\right)$ é uma pp-fórmula.

Comentário 5.28. Se $R$ tem suficientes qc-formas, então a isometria de soma ortogonais de qcformas é de primeira ordem. Mais especificamente, dados $n \geq k \geq 1$, a pp-fórmula $\theta_{n, k}(\bar{x}, \bar{y})$ na linguagem $\mathcal{L}_{R S}$ descrita na demonstração do Teorema 3.23 satisfaz o seguinte: dadas formas $\varphi=\left\langle a_{1}, \ldots, a_{n}\right\rangle, \psi=\left\langle b_{1}, \ldots, b_{k}\right\rangle$ que são somas ortogonais de qc-formas, vale

$$
\varphi \equiv_{R} \psi \text { se, e somente se, } R \models \theta_{n, k}\left(a_{1}, \ldots, a_{n}, b_{1}, \ldots, b_{k}\right) .
$$

Definição 5.29 ([Mar7]). Seja $R$ RS. Diz-se que $R$ tem muitas unidades se para todo $a, c \in R$ com $[[c \geq 0]] \subseteq[[a \neq 0]]$ existir $a^{\prime} \in R^{\times}$tal que para todo $\sigma \in[[c \geq 0]]$ vale $\sigma\left(a^{\prime}\right)=\sigma(a)$.

Exemplo 5.30. - Seja $R$ RS satisfazendo $R=R^{\times} \operatorname{Id}(R)$. Então $R$ tem muitas unidades. De fato, sejam $a, c \in R$ com $[[c \geq 0]] \subseteq[[a \neq 0]]$. Tomando $r \in R^{\times}$tal que $a=r a^{2}$, temos $a=r$ em $[[c \geq 0]]$. Em particular, todo RS Booleano e von Neumann tem muitas unidades.

- Se $A$ é um anel semi-real com muitas unidades, então $G_{A}$ é RS com muitas unidades (Proposição 8.1.4 de [Mar7]).

Teorema 5.31. Seja $R R S$ com muitas unidades. Então

i) $R^{\times}$é $R S G$.

ii) Para todos $a_{1}, \ldots, a_{n} \in R^{\times}$, vale $D_{R^{\times}}\left(a_{1}, \ldots, a_{n}\right)=D_{R}^{t}\left(a_{1}, \ldots, a_{n}\right) \cap R^{\times}$.

Em particular, $R$ tem suficientes unidades.

Demonstração. Pela Proposição 2.7 de [DP1], dada forma $\varphi$ sobre qualquer $R S$, vale que $D_{R}^{t}(\varphi) \cap$ $R^{\times}=D_{R}(\varphi) \cap R^{\times}$. Com isso, os itens $i$ ) e ii) seguem do Lema 8.1.6 de [Mar7] e, portanto, pela Proposição 5.23 todo RS com muitas unidades tem suficientes unidades.

Corolário 5.32. Seja $R$ RS Booleano. Então $R$ tem suficientes qc-formas.

Demonstração. Basta notar que, para todo $a \in \operatorname{Id}(R)$ não-nulo, $R_{a}$ também é RS Booleano (é imagem de Booleano) e portanto tem muitas unidades. 
Comentário 5.33. Para um RS $R$ qualquer, as condições $i$ ) e $i i$ ) do Teorema acima são equivalentes a: dados $a, a_{1}, \ldots, a_{n} \in R^{\times}$

$$
a \in D_{R}^{t}\left(a_{1}, \ldots, a_{n}\right) \Rightarrow \text { existe } b \in D_{R}^{t}\left(a_{2}, \ldots, a_{n}\right) \cap R^{\times} \text {tal que } a \in D_{R}^{t}\left(a_{1}, b\right) .
$$

Mais geralmente, as condições $i$ e $i i$ ) valem para $R_{a}$ para todo $a \in R$ não-nulo se, e somente se, dados $a, a_{1}, \ldots, a_{n} \in R$ quadrado-constantes

$$
a \in D_{R}^{t}\left(a_{1}, \ldots, a_{n}\right) \Rightarrow \text { existe } b \in D_{R}^{t}\left(a_{2}, \ldots, a_{n}\right) \text { tal que } b^{2}=a^{2}, a \in D^{t}\left(a_{1}, b\right) .
$$

Definição 5.34. Seja $R$ RS com suficientes unidades. Definimos o conjunto $\operatorname{Id}_{c}(R):=\{x \in$ $\operatorname{Id}(R)$ : Existe $y \in \operatorname{Id}(R)$ tal que $x \vee y=0$ e $x \wedge y=1\}$. Note que, pelas leis de $\operatorname{Morgan}, \operatorname{Id}_{c}(R) \subseteq$ $\operatorname{Id}(R)$ é álgebra Booleana com as operações de reticulado induzidas.

Seja $R$ com suficientes unidades. Dado $x \in \operatorname{Id}_{c}(R)$, vamos denotar por $x^{c} \in \operatorname{Id}_{c}(R)$ o único elemento satisfazendo $x \vee x^{c}=0, x \wedge x^{c}=1$. Além disso, o elemento $y \in D^{t}\left(x,-x^{c}\right)$ satisfaz para $\sigma \in X_{R}$

$$
\begin{gathered}
\text { se } \sigma(x)=0, \text { então } \sigma(y)=-1 \\
\text { se } \sigma(x)=1, \text { então } \sigma(y)=1 .
\end{gathered}
$$

Assim $\nabla(x)=y \in R^{\times}$(e $D^{t}\left(x,-x^{c}\right)$ tem único elemento). De maneira análoga, dado qualquer $a \in R, \nabla(a x) \in D^{t}\left(a x,-x^{c}\right)$.

Proposição 5.35. Seja $R$ RS com suficientes unidades. Então o conjunto $\nabla_{R}:=\nabla\left(\operatorname{Id}_{c}(R)\right) \subseteq R^{\times}$ vN-conjunto.

Demonstração. Sejam $e, f \in \operatorname{Id}_{c}(R), e^{\prime} \in D^{t}(e, f)$ e $g \in R^{\times}$. De forma inteiramente análoga ao que foi feito no Lema 5.8, prova-se, usando as operações de reticulado induzidas da álgebra de Post de $R$, que as seguintes expressões valem:

- $\nabla(e) \vee \nabla(f)=\nabla(e \cdot f)$.

- $\nabla(e) \wedge \nabla(f)=\nabla\left(e^{\prime}\right)$.

- $\nabla(e) \vee g=\nabla(e g)$.

- $\nabla(e) \wedge g=\nabla(e) \cdot g \cdot \nabla(e g)$.

- $-\nabla(e)=\nabla\left(e^{c}\right)$.

Mas como o mapa $X_{R} \rightarrow X_{R^{\times}}$é sobrejetor, segue que as expressões acima também valem em $R^{\times}$ utilizando as operações induzidas de sua envoltória Booleana. Logo $\nabla_{R} \subseteq R^{\times}$é vN-conjunto.

Seja $R$ RS com suficientes unidades. Dado $a=\nabla(e) \in \nabla_{R}$, vamos denotar por $\nabla^{-1}(a)$ o idempotente $e$. Se $f: R \rightarrow G$ é morfismo de RSs com suficientes unidades, note que $f\left(\operatorname{Id}_{c}(R)\right) \subseteq$ $\operatorname{Id}_{c}(G)$ e portanto $f\left(\nabla_{R}\right) \subseteq \nabla(G)$.

Teorema 5.36. Seja $R R S$ com suficientes unidades. Então existe morfismo $I_{R}: R S\left(R^{\times}, \nabla_{R}\right) \rightarrow R$ tal que dado morfismo $f: V \rightarrow R, V R S$ von Neumann, existe único morfismo $\bar{f}: V \rightarrow R S\left(R^{\times}, \nabla_{R}\right)$ tal que o diagrama

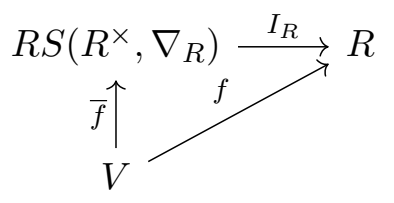

comuta. 
Demonstração. Pela Proposição 5.35, $\left(R^{\times}, \nabla_{R}\right)$ é vN-par. Considere então $R^{\prime}=R S\left(R^{\times}, \nabla_{R}\right)$ e o mapa $I_{R}: R S\left(R^{\times}, \nabla_{R}\right) \rightarrow R$ dado por $I_{R}(\gamma(g, a))=(g \vee a) \cdot \nabla^{-1}(a)$ (comparar com a definição do mapa na demonstração do Teorema $5.14 \mathrm{ii})$ ). Vamos verificar que $I_{R}$ satisfaz as hipótese da Proposição 5.12. Considere o isomorfismo RSG $I v: R^{\times} \rightarrow R^{\prime \times}$ dado pelo Teorema 5.14. Sejam $g \in R^{\times}, a \in \nabla_{R}$. Para simplificar a exposição, vamos escrever $I$ no lugar de $I_{R}$.

i) $I(\gamma(g, 1))=(g \vee 1) \nabla^{-1}(1)=g$. Logo $I\left(R^{\prime \times}\right) \subseteq R^{\times}$e $\left.I\right|_{R^{\prime \times}}: R^{\prime \times} \rightarrow R^{\times}$é morfismo de RSG pois $\left.I\right|_{R^{\prime \times}}=I v^{-1}$.

ii) $I(\gamma(1, a))=(1 \vee a) \nabla^{-1}(a)=a \nabla^{-1}(a)$. Mas como vale $y \nabla(y)=y$ para todo $y \in \operatorname{Id}_{c}(R)$, temos $a \nabla^{-1}(a)=\nabla^{-1}(a)$ e $\operatorname{assim} I\left(I d\left(R^{\prime}\right)\right) \subseteq I d_{c}(R) \subseteq I d_{\nabla}(R)$.

iii) Pelo Lema 5.13, temos que $\gamma(g, a)^{2}=\gamma(1, a)$ e $\nabla(\gamma(g, a))=\gamma(g \vee a, 1)$. Deste modo,

$$
I\left(\gamma(g, a)^{2}\right) I(\nabla(\gamma(g, a)))=I(\gamma(1, a)) I(\gamma(g \vee a, 1))=\nabla^{-1}(a)(g \vee a)=I(\gamma(g, a)) .
$$

iv) Também pelo Lema 5.13, temos $\nabla(I(\gamma(1, a)))=\nabla\left(\nabla^{-1}(a)\right)=a=I(\gamma(a, 1))=I(\nabla(\gamma(1, a)))$.

Assim, pela Proposição 5.12, $I_{R}: R S\left(R^{\times}, \nabla_{R}\right) \rightarrow R$ é morfismo de RS. Agora seja $f: V \rightarrow R$ morfismo de RS com $V$ von Neumann. Então $f\left(V^{\times}\right) \subseteq R^{\times}$e $f\left(\nabla_{V}\right) \subseteq \nabla_{R}$. Pelo Teorema 5.14, existe morfismo $\bar{f}^{\prime}: R S\left(V^{\times}, \nabla_{V}\right) \rightarrow R S\left(R^{\times}, \nabla_{R}\right)$ tal que o diagrama

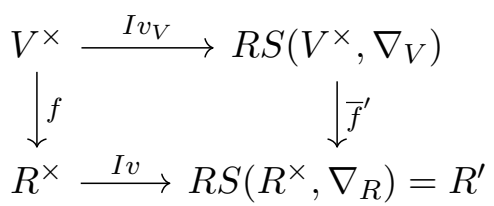

é comutativo. Por outro lado, pelo Lema 5.15, existe isomorfismo canônico $g: V \rightarrow R S\left(V^{\times}, \nabla_{V}\right)$. Considere então $\bar{f}=\bar{f}^{\prime} \circ g$. Deste modo, dado $v \in V^{\times}$, temos

$$
I_{R}(\bar{f})=I_{R}(\gamma(f(v), 1))=f(v) .
$$

Pela Proposição $2.55 v$ ), segue que $f=\bar{f} \circ I$. A unicidade de tal $\bar{f}$ também segue pela Proposição $2.55 v)$ pois $\left.I\right|_{R^{\prime \times}}: R^{\prime \times} \rightarrow R^{\times}$é isomorfismo de RSG.

Comentário 5.37. No teorema acima, a imagem de $I_{R}$ é constituída dos $x \in R$ tal que $x^{2} \in \operatorname{Id}_{c}(R)$ e $x=g x^{2}$ para algum $g \in R^{\times}$.

Definição 5.38. Sejam $D$ categoria e $C$ subcategoria fielmente plena de $D$. A subcategoria $C$ é dita birreflexiva se o funtor de inclusão admite adjunto à esquerda e à direita.

Corolário 5.39. Sejam RS e vNRS as categorias dos RSs e RSs von Neumann, respectivamente. Se todo RS tem suficientes unidades, então a subcategoria vNRS de RS é birreflexiva.

Demonstração. O Teorema 4.14 fornece o adjunto à esquerda e o Teorema 5.36 o adjunto à direita do funtor de inclusão vNRS $\hookrightarrow$ RS.

Lema 5.40. Sejam $G$ RSG, $H$ um pré-RSG e $f: G \rightarrow H$ um morfismo de pré-RSG. Suponha que para todo morfismo $g: F \rightarrow H$ de pré-RSG com $F$ RSG, exista único morfismo $\bar{g}: F \rightarrow G$ tal que $g=\bar{g} \circ f$. Então $f$ é isomorfismo e $H$ é RSG.

Demonstração. Sejam $x, a \in H$ tais que $x \in D_{H}(1, a)$. Vamos mostrar que existe RSG $F$, morfismo $g: F \rightarrow H$ e $x^{\prime}, a^{\prime} \in F$ tais que $x^{\prime} \in D_{F}\left(1, a^{\prime}\right)$ e $g\left(x^{\prime}\right)=x, g\left(a^{\prime}\right)=a$. Considere $F=\mathbb{Z}_{2}^{3}$ álgebra Booleana de 8 elementos vista como RSG. Sejam $x^{\prime}=(-1,1,1)$ e $a^{\prime}=(-1,-1,1)$. Então $F=$ 
$\left\{1,-1, x^{\prime},-x^{\prime}, a^{\prime},-a^{\prime}, x^{\prime} a^{\prime},-x^{\prime} a^{\prime}\right\}$ é um $\mathbb{F}_{2}$-espaço vetorial com base $\left\{-1, x^{\prime}, a^{\prime}\right\}$ e valem as seguintes relações:

$$
\begin{array}{r}
D_{F}(1,1)=\{1\}, D_{F}(1,-1)=F, D_{F}\left(1, x^{\prime}\right)=\left\{1, x^{\prime}\right\} \\
D_{F}\left(1,-x^{\prime}\right)=\left\{1,-x^{\prime},-a, x^{\prime} a^{\prime}\right\}, D_{F}\left(1, a^{\prime}\right)=\left\{1, a^{\prime}, x^{\prime}, x^{\prime} a^{\prime}\right\} \\
D_{F}\left(1,-a^{\prime}\right)=\left\{1,-a^{\prime}\right\}, D_{F}\left(1, x^{\prime} a^{\prime}\right)=\left\{1, x^{\prime} a^{\prime}\right\} \\
D_{F}\left(1,-x^{\prime} a^{\prime}\right)=\left\{1, x^{\prime},-a^{\prime},-x^{\prime} a^{\prime}\right\} .
\end{array}
$$

Defina $g: F \rightarrow H$ morfismo de $\mathbb{F}_{2}$-espaços vetoriais por $g(-1)=-1, g\left(x^{\prime}\right)=x$ e $g\left(a^{\prime}\right)=a$. Assim, $g$ é morfismo de grupos de expoente 2 com elemento distinguido e uma rápida verificação permite concluir que dados $p, q \in F$ com $p \in D_{F}(1, q)$ vale $g(p) \in D_{H}(1, g(q))$. Logo, $g$ é morfismo de pré-RSG.

Assim, usando a propriedade universal descrita no lema, existem $x^{\prime}, a^{\prime} \in G$ tais que $x^{\prime} \in D_{G}(1, a)$ e $f\left(x^{\prime}\right)=x, f\left(a^{\prime}\right)=a$. Isto já nos dá que $f$ é sobrejetora e regular. Para concluir que é isomorfismo, resta a injetividade. Sejam $a, b \in G$ tal que $f(a)=f(b)$. Considere o fan $F=\{1,-1, x,-x\}$ de rank 1. Defina $g_{1}$ e $g_{2}$ de $F$ para $G$ enviando $x$ em $a$ e $b$, respectivamente. Então $g_{1}$ e $g_{2}$ são morfismos de RSG e $f \circ g_{1}=f \circ g_{2}$. Pela unicidade da propriedade universal, segue que $g_{1}=g_{2}$. Portanto $a=b$.

Teorema 5.41. Sejam $\boldsymbol{R S}$ e $\boldsymbol{v} \boldsymbol{N R S}$ as categorias dos RSs e RSs von Neumann, respectivamente. Se a subcategoria $\boldsymbol{v} \boldsymbol{N R} \boldsymbol{S}$ de $\boldsymbol{R} \boldsymbol{S}$ é birreflexiva, então os inversiveis de todo RS é $R S G$.

Demonstração. Suponha que a subcategoria dos RSs von Neumann é birreflexiva. Deste modo, fixado RS $R$, existe RS von Neumann $V$ e morfismo $I: V \rightarrow R$ final com relação aos morfismo saindo de um RS von Neumann e chegando em $R$. Em particular, a restrição $\left.I\right|_{V^{\times}}: V^{\times} \rightarrow R^{\times}$ satisfaz as hipóteses do Lema 5.40. Logo $\left.I\right|_{V^{\times}}: V^{\times} \rightarrow R^{\times}$é isomorfismo e $R^{\times}$é RSG.

\subsubsection{Envoltória RS-Booleana}

Na última subseção, é definido a envoltória RS-Booleana de um RS qualquer e monomorfismos, epimorfismos e imerções completas de RSs em geral são classificadas em termos das envoltórias von Neumann e RS-Booleana e dos aneis de Witt. Os principais resultados são:

- Dado RS $R$, o candidato a envoltória RS-Booleana $B(R)$, definida como um subconjunto apropriado da envoltória von Neumann, é, de fato, RS e o morfismo de inclusão $B(R) \hookrightarrow V(R)$ é final com relação a morfismos saindo de $B(R)$ para RS von Neumann (Teorema 5.48).

- Dado $R$ RS, o Teorema 5.50 descreve algumas propriedades algébricas de $B(R)$ como seus espectro primo e real, seu corpos residuais e seu anel de Witt em função das respectivas noções em $R$.

- O Teorema 5.52 apresenta o funtor $B: \mathbf{R S} \rightarrow$ BoolRS e prova que $B$ é adjunto à esquerda do funtor de inclusão BoolRS $\hookrightarrow$ RS.

- Caracterizações de epimorfismos de RSs utilizando aneis de Witt e as envoltórias von Neumann e RS-Booleana (Teorema 5.63). É uma versão do Teorema 10.2 de [DM1] expandida para os RSs.

- Caracterizações de imersões completas de RSs também utilizando aneis de Witt e as envoltórias von Neumann e RS-Booleana (Teorema 5.64). É uma generalização do Teorema V.4.5 de [DP2].

Definição 5.42. Uma álgebra de Post é uma estrutura $(L, \vee, \wedge,-, \nabla, \top, \underline{c})$ satisfazendo as seguintes \{postDef\} condições

i) $(L, \vee, \wedge, \top)$ é um reticulado distributivo com maior elemento $\top$. 
ii) O operador unário - (negação) satisfaz:

- $-(-x)=x$.

- $-(x \wedge y)=-x \vee-y$.

iii) O operador unário $\nabla$ satisfaz:

- $-x \vee \nabla(x)=\top$.

- $x \wedge-x=-x \wedge \nabla(x)$.

- $\nabla(x \wedge y)=\nabla(x) \wedge \nabla(y)$.

iv) $-\underline{c}=\underline{c}$.

Exemplo 5.43. Seja $3=\{1,0,-1\}$ ordem linear com $1<0<-1$. Então 3 é álgebra de Post com $\nabla(x)=x$ para $x \in\{1,-1\}, \nabla(0)=-1, \top=-1, \underline{c}=0$.

Seja $(L, \vee, \wedge,-, \nabla, \top, \underline{c})$ uma álgebra de Post. Considere as seguintes definições:

- Dados $x, y \in L$, a diferença simétrica $\triangle$ é definida por $x \triangle y=(-x \wedge y) \vee(x \wedge-y)$.

- Dados $x, y, z \in L$

$$
x \in D_{L}(y, z) \Leftrightarrow y \wedge z \wedge \underline{c} \leq x \leq y \vee z \vee \underline{c} .
$$

Considere também as constantes $-1=\top, 1=-\top$ e $0=\underline{c}$.

Exemplo 5.44. Seja $X$ espaço topológico booleano e considere $C(X, 3)=\{1,0,-1\})$ o espaço das funções contínuas onde 3 é discreto. Considere a ordem $1<0<-1$ em $\mathbf{3}$ e o produto usual. Dados $f, g, h \in C(X, \mathbf{3})$,

- $f \wedge g(x)=f(x) \wedge g(x)$ para todo $x \in X$

- $f \vee g(x)=f(x) \vee g(x)$ para todo $x \in X$.

- $(-f)(x)=-1 \cdot f(x)$ para todo $x \in X$.

\{postRepr\}

$$
\nabla(f)(x)= \begin{cases}f(x) & \text { se } f(x) \neq 0 \\ -1 & \text { se } f(x)=0\end{cases}
$$

Então $(C(X, \mathbf{3}), \wedge, \vee,-, \nabla,-1,0)$ é álgebra de Post. Além disso, a diferença simétrica em $C(X, \mathbf{3})$ é o produto ponto a ponto.

Teorema 5.45. Seja $(L, \vee, \wedge,-, \nabla, \top, \underline{c})$ álgebra de Post. Seja $X_{L}$ o espaço dos morfismos de álgebra de Post de $L$ em 3 com a topologia induzida de $3^{L}$ (3 discreto). Então $X_{L}$ é espaço Booleano e o mapa da valoração ev: $L \rightarrow C\left(X_{L}, 3\right)$ é isomorfismo de álgebras de Post.

Demonstração. Teorema X.4.5 de [BD].

Proposição 5.46. Seja $(L, \vee, \wedge,-, \nabla, \top, \underline{c})$ uma álgebra de Post. Então $(L, \triangle, 1,0,-1)$ é um semigrupo ternário e $\left(L, \triangle, 0,1,-1, D_{L}\right)$ é um semigrupo real von Neumann. Além disso, dados $x, y, z \in L$,

$$
x \in D_{L}^{t}(y, z) \Leftrightarrow(y \wedge \nabla(z)) \vee(z \wedge \nabla(y)) \leq x \leq(y \vee \nabla(z)) \wedge(z \vee \nabla(y)) .
$$

Demonstração. A proposição segue de vários resultados de [DP2]. A Proposição IV.2.1 mostra que $(L, \triangle, 1,0,-1)$ é um semigrupo ternário e o Teorema I.V.2.7 que $\left(L, \triangle, 0,1,-1, D_{L}\right)$ é semigrupo real. Por último, o Teorema IV.3.2 item (4).(i) afirma que em $L$ vale a propriedade $i i i)$ do Teorema 2.58 (todo elemento de $L$ tem complemento) e portanto $L$ é von Neumann. 
Seja $R$ RS. Em [DM2], $P(R):=C\left(X_{R}, 3\right)$ é definida, juntamente com o morfismo $\varepsilon_{R}: R \rightarrow$ $P(R)$ dado pela avaliação, como a envoltória de Post do semigrupo real $R$. O que segue resume suas propriedades:

Proposição 5.47. Seja $R$ RS e considere o morfismo $\varepsilon_{R}: R \rightarrow P(R)$. Então

i) $\varepsilon_{R}$ é imersão completa.

ii) $\operatorname{Im}\left(\varepsilon_{R}\right)$ gera $P(R)$ como álgebra de Post.

iii) A associação $R \mapsto P(R)$ é funtorial.

iv) Dado morfismo $f: R \rightarrow P$ de RS com $P$ álgebra de Post, existe único $\bar{f}: P(R) \rightarrow P$ tal que $f=\bar{f} \circ \varepsilon_{R}$.

Demonstração. Seção IV.4 de [DP2].

Sejam $R$ RS e $v: R \rightarrow V(R)$ o morfismo canônico para a envoltória von Neumann. Considere o conjunto $B(R)=\left\{x \in V(R): x^{2} \in v(\operatorname{Id}(R))\right\}$. Note que $B(R)$ é fechado por produto e $0,1,-1 \in$ $B(R)$. Assim, $B(R)$ é semigrupo ternário e podemos considerar a seguinte relação em $B(R)$ : dados $x, y \in B(R), D_{B(R)}^{t}(x, y)=D_{V(R)}^{t}(x, y) \cap B(R)$.

Teorema 5.48. Seja $R$ RS. Então $\left(B(R), D_{B(R)}^{t}, \cdot, 0,1,-1\right)$ é $R S$ Booleano e o mapa de inclusão $i_{R}: B(R) \rightarrow V(R)$ é inicial com relação a todos os morfismos saindo de $B(R)$ para um $R S$ von Neumann. Em particular, $i_{R}: B(R) \rightarrow V(R)$ é imersão completa e $V(B(R)) \cong V(R)$.

Demonstração. Vamos mostrar que existe mapa bijetor de $B(R)$ para um semigrupo real Booleano que preserva e reflete sua representação transversal. Seja $v: R \rightarrow V(R)$ o morfismo canônico para envoltória von Neumann e $\nabla: V(R) \rightarrow V(R)^{\times}$o operador nabla. Considere o subconjunto $\nabla^{\prime}:=\nabla(v(\operatorname{Id}(\mathrm{R}))) \subseteq \nabla_{V(R)} \subseteq V(R)^{\times}$. Então todo elemento de $\nabla^{\prime}$ é particionador e pelo Lema 5.8 segue que $\nabla^{\prime} \subseteq V(R)^{\times}$é reticulado distributivo. Logo $\left(V(R)^{\times}, \nabla^{\prime}\right)$ é B-par e podemos considerar $B^{\prime}=\mathfrak{R}\left(V(R)^{\times}, \nabla^{\prime}\right)$. Pela inclusão $\left(V(R)^{\times}, \nabla^{\prime}\right) \rightarrow\left(V(R)^{\times}, \nabla_{V(R)}\right)$ e pelo isomorfismo $\mathfrak{R}\left(V(R)^{\times}, \nabla_{V(R)}\right) \cong V(R)$ do Lema 5.15 , o Teorema 5.14 ii) fornece morfismo induzido $f: B^{\prime} \rightarrow V(R)$. Ele é dado por $f(\gamma(g, a))=g \nabla^{-1}(a)$.

Note que $\left.f\right|_{B^{\prime x}}: B^{\prime \times} \rightarrow V(R)^{\times}$é injetor (isomorfismo na verdade) e pela demonstração do Lema 5.15 segue que $f$ é injetor. Temos ainda que $\operatorname{Im}(f)=\left\{g \nabla^{-1}(a) \in V(R): a \in \nabla^{\prime}\right\}=\{g v(x) \in$ $V(R): x \in \operatorname{Id}(R)\}=B(R)$ e assim $f$ é uma bijeção entre $B^{\prime}$ e $B(R)$ que preserva produto. Por outro lado, como $\left.f\right|_{B^{\prime \times}}: B^{\prime \times} \rightarrow V(R)^{\times}$é isomorfismo, segue que $f^{*}: X_{V(R)} \rightarrow X_{R}$ é homeomorfismo; $\operatorname{logo} f: B^{\prime} \rightarrow V(R)$ é imersão completa. Deste modo, temos morfismo bijetor $B^{\prime} \rightarrow B(R)$ entre semigrupos ternários que preserva e reflete representação transversal. Logo, $B(R)$ é semigrupo real Booleano.

Para concluir a propriedade universal do mapa de inclusão $B(R) \rightarrow V(R)$, pela Proposição 5.17 é suficiente provarmos que a subálgebra booleana gerado por $\nabla^{\prime}$ é $\nabla_{V(R)}$. Seja $x=\nabla(e) \in \nabla_{V(R)}$, $e \in \operatorname{Id}(V(R))$. Pelo Teorema $4.10 i)$, existem $a_{1}, \ldots, a_{n}, b_{1}, \ldots, b_{n} \in \operatorname{Id}(R)$ tais que

$$
s p(v)(D(e))=\bigcup_{i=1}^{n} D\left(a_{i}\right) \cap D\left(b_{i}\right)^{c} .
$$

Deste modo, em $\operatorname{Id}(V(R))$ vale $e=\bigwedge_{i=1}^{n}\left(v\left(a_{i}\right) \vee v\left(b_{i}\right)^{c}\right)$. Logo $x=\nabla(e)=\bigcap_{i=1}^{n}\left(\nabla\left(v\left(a_{i}\right)\right) \cup\right.$ $\left.-\nabla\left(v\left(b_{i}\right)\right)\right)$, onde $\nabla\left(a_{i}\right), \nabla\left(b_{i}\right) \in \nabla^{\prime}$. Portanto, a subálgebra Booleana gerado por $\nabla^{\prime}$ é $\nabla_{V(R)}$.

Definição 5.49. Seja $R$ RS. Denotamos por $b_{R}: R \rightarrow B(R)$ o morfismo canônico de $R$ para $B(R)$. Se não houver confusão, o denotaremos apenas por $b$.

Teorema 5.50. Seja $R R S$. Considere o morfismo natural $b: R \rightarrow B(R)$. Então 
i) Os mapas induzidos por $b s_{b}: \operatorname{spec}(B(R)) \rightarrow \operatorname{spec}(R)$ e $r s_{b}: X_{B(R)} \rightarrow\left(X_{R}\right)_{\text {cons }}$ são homeomorfismos. Em particular, $b$ é imersão completa.

ii) Para cada $p \in \operatorname{spec}(B(R))$, seja $q=s_{b}(p) \in \operatorname{spec}(R)$. Então o morfismo $b_{p}: K_{q}(R) \rightarrow K_{p}(B(R))$ induzido é isomorfismo.

iii) O mapa induzido $W(b): W(R) \rightarrow W(B(R))$ é isomorfismo de aneis de Witt que preserva o ideal fundamental.

iv) Sejam $a \in R$ e $\varphi$ forma sobre $R$. Entãa $a \in D_{R}^{t}(\varphi)$ se, e somente se, $b(a) \in D_{B(R)}^{t}(b \star \varphi)$.

Demonstração. Seja $v: R \rightarrow V(R)$ o morfismo canônico para a envoltória von Neumann e considere $i: B(R) \rightarrow V(R)$ o morfismo de inclusão que é universal pelo Teorema 5.48. Note que $i \circ b=v$.

i) Note que vale $s_{b} \circ s_{i}=s_{v}$ e $r s_{b} \circ r s_{i}=r s_{v}$. Pelo Teorema 4.10 segue que $s_{i}, s_{v}, r s_{i}, r s_{v}$ são bijetoras; $\log _{0} s_{b}$ e $r s_{b}$ são bijetoras. Ainda, como $B(R)$ é RS Booleano, segue que $X_{B(R)}$ é espaço topológico Booleano e portanto $r s_{b}: X_{B(R)} \rightarrow\left(X_{R}\right)_{\text {const }}$ é homeomorfismo. Resta mostrar que $s_{b}: \operatorname{spec}(B(R)) \rightarrow \operatorname{spec}(R)$ é aberta. Mas dado $x \in \operatorname{Id}(\mathrm{B}(\mathrm{R}))$, por definição existe $y \in \operatorname{Id}(R) \operatorname{com} v(y)=x$ e portanto $s_{b}(D(x))=D(y)$.

ii) Como $s_{v}: \operatorname{spec}(V(R)) \rightarrow \operatorname{spec}(R)$ é bijetor, existe $t \in \operatorname{spec}(V(R))$ tal que $s_{v}(t)=q$. Pelo Teorema 4.10 segue que $v_{t}: K_{q}(R) \rightarrow K_{t}(V(R))$ e $i_{t}: K_{p}(B(R)) \rightarrow K_{t}(V(R))$ são isomorfismo de RSs. Como $i_{t} \circ b_{p}=v_{t}$, segue que $b_{p}$ é isomorfismo.

iii) Pelo Teorema 4.10, segue que $W(v): W(R) \rightarrow W(V(R))$ e $W(i): W(B(R)) \rightarrow W(V(R))$ são isomorfismo que preservam os respectivos ideais fundamentais. Assim, como $W(i) \circ W(b)=$ $W(v)$, o mesmo vale para o morfismo $W(b)$.

iv) Pelo Teorema 4.12, temos

$$
a \in D_{R}^{t}(\varphi) \Leftrightarrow v(a) \in D_{V(R)}^{t}(v \star \varphi) \Leftrightarrow b(a) \in D_{B(R)}^{t}(b \star \varphi) .
$$

Lema 5.51. Sejam $R, G$ RSs Booleanos e $v_{R}: R \rightarrow V(R), v_{G}: G \rightarrow V(G)$ os morfismos canônicos para a envoltória von Neumann. Seja $f: V(R) \rightarrow V(G)$ morfismo de RS. Suponha que para todo $x \in \operatorname{Id}(R), f\left(v_{R}(x)\right) \in v_{G}(\operatorname{Id}(G))$. Então existe único $\bar{f}: R \rightarrow G$ tal que $f \circ v_{R}=v_{G} \circ \bar{f}$.

Demonstração. Pela Proposição 5.17, segue que $\left.v_{G}\right|_{G^{\times}}: G^{\times} \rightarrow V(G)^{\times}$é isomorfismo de RSGs. Assim, dado $x \in R$, temos $f\left(v_{R}(x)\right)=f\left(v_{R}\left(x^{2}\right)\right) f\left(v_{R}(\nabla(x))\right) \in \operatorname{Im}\left(v_{G}\right)$. Como $v_{G}$ é injetora, podemos definir $\bar{f}: R \rightarrow G$ como $\bar{f}(x)=v_{G}^{-1}\left(f\left(v_{R}(x)\right)\right)$. Deste modo, sendo $v_{G}$ imersão completa pelo Teorema 4.10, segue que $\bar{f}$ é morfismo de RS. A unicidade é imediata.

Teorema 5.52. Seja $R$ RS.

i) A associação

$$
R \mapsto B(R) \quad(R \stackrel{f}{\rightarrow} G) \mapsto(B(R) \stackrel{V(f) \mid}{\longrightarrow} B(G))
$$

define funtor da categoria dos RSs para a categoria dos RS Booleanos.

ii) Seja $f: R \rightarrow B$ morfismo de RSs com B Booleano. Então existe único $\bar{f}: B(R) \rightarrow B$ tal que

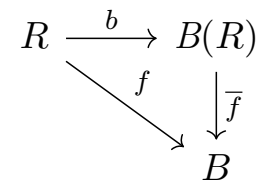

é diagrama comutativo. Em outras palavras, o funtor de inclusão da categoria dos $R S$ Booleanos para a categoria dos RSs tem adjunto á esquerda. 
iii) Com a notação do item acima, se f é imersão completa, então $\bar{f}$ também é.

Demonstração. i) Sejam $f: R \rightarrow G$ morfismos de RSs e $v(f): V(R) \rightarrow V(G)$ o morfismo induzido. Considere ainda os demais morfismos

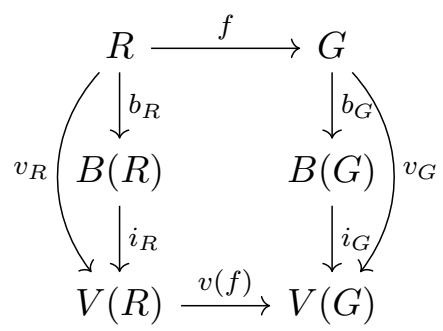

Vamos verificar que o morfismo $v(f)$ satisfaz a condição do Lema 5.51. Dado $x \in \operatorname{Id}(B(R))$, existe $y \in \operatorname{Id}(R)$ tal que $x=b_{R}(y) ; \operatorname{logo}$

$$
v(f)\left(i_{R}(x)\right)=v(f)\left(v_{R}(y)\right)=v_{G}(f(y)) \in i_{G}(\operatorname{Id}(B(G))) .
$$

Logo, como os morfismos $i_{R}$ e $i_{G}$ são universais pelo Teorema 5.48, o Lema 5.51 garante a existência de único morfismo $b(f): B(R) \rightarrow B(G)$ fazendo o diagrama acima comutar. A unicidade da construção de $b(f)$ garante que dados morfismos $g: R \rightarrow G, h: G \rightarrow P$ de RSs, então $b(h \circ g)=b(h) \circ b(g)$, provando que a associação é funtorial.

ii) Considere o diagrama

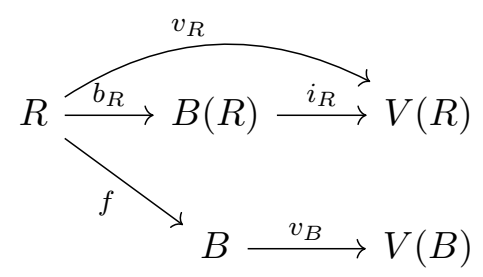

Pela propriedade universal de $v_{R}$, existe único $f^{\prime}: V(R) \rightarrow V(B)$ tal que $f^{\prime} \circ v_{R}=v_{B} \circ f(*)$. Por outro lado, $f^{\prime}$ satisfaz as condições do Lema 5.51: dado $x \in \operatorname{Id}(B(R))$, existe $y \in \operatorname{Id}(R)$ $\operatorname{com} x=b_{R}(y) ; \operatorname{logo} f^{\prime}\left(i_{R}(x)\right)=f^{\prime}\left(v_{R}(y)\right)=v_{B}(f(y)) \in v_{B}(\operatorname{Id}(B))$. Logo, como $i_{R}$ é universal pelo Teorema 5.48, segue do Lema 5.51 que existe único $\bar{f}: B(R) \rightarrow B(* *)$ que faz o diagrama

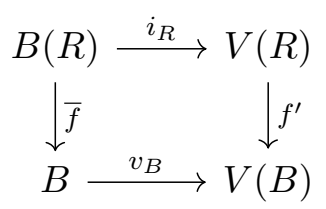

comutar. Pela unicidade da propriedade universal de $v_{B}$ segue que $\bar{f} \circ b_{R}=f(* * *)$. Se houvesse outro morfismo $\bar{f}^{\prime}$ satisfazendo $(* * *)$, a unicidade em $(*)$ implica $v\left(\bar{f}^{\prime}\right)=f^{\prime}$ e unicidade em (**) mostra que $\bar{f}^{\prime}=\bar{f}$.

iii) Suponha $f: R \rightarrow B$ imersão completa. Seguinda a notação da demonstração do item $i i)$ acima, note que $v_{b}: B \rightarrow V(B)$ é imersão completa e portanto $v_{b} \circ f$ também é. Pelo Teorema 4.14.(iii) segue que $f^{\prime}: V(R) \rightarrow V(B)$. Por outro lado, no diagrama comutativo (†) o morfismo $i_{R}: B(R) \rightarrow V(R)$ também é imersão completa. Logo $v_{b} \circ \bar{f}=f^{\prime} \circ i_{R}$ é imersão completa; mas como $v_{b}$ é também, o mesmo vale para $\bar{f}$.

Definição 5.53. Seja $R$ RS. O semigrupo real $B(R)$ é chamado de envoltória RS-Booleana de R.

A seguir é apresentada uma representação da envoltória RS-Booleana na envoltória de Post (análogo ao trabalho feito com a envoltória von Neumann na Proposição 4.19). Depois são discutidas 
as propriedades da envoltória RS-Booleana de RSs-fan ${ }^{3}$ e RSs espectrais ${ }^{4}$.

Lema 5.54. Seja $R$ RS. Seja $\varphi$ forma satisfazendo

i) $\varphi^{2} \cong \varphi$.

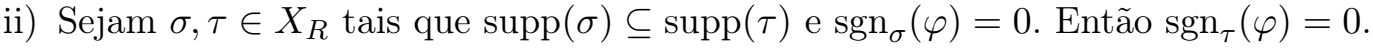

Então existe $a \in \operatorname{Id}(R)$ tal que $\varphi \cong\langle a\rangle$.

Demonstração. Primeiro observe que o item $i$ ) implica que para todo $\sigma \in X_{R}$ vale $\operatorname{sgn}_{\sigma}(\varphi) \in\{0,1\}$. Agora considere o subconjunto $A=\left\{p \in \operatorname{spec}(R): \operatorname{dim}_{p}(\varphi) \equiv 1 \bmod 2\right\} \subseteq \operatorname{spec}(R)$. Como o morfismo $\operatorname{dim}(\varphi):(\operatorname{spec}(R))_{\text {const }} \rightarrow \mathbb{Z}$ é contínuo pela Proposição 3.4. $(i) .(g)$, segue que

$$
A \subseteq(\operatorname{spec}(R))_{\text {const }} \text { é aberto-fechado. }
$$

Por outro lado, as propriedades $i$ ) e $i i$ ) do enunciado implicam que

$$
\text { dado } p \in A \text { e } q \subseteq p \text {, segue que } q \in A \text {. }
$$

De fato, sejam $\sigma, \tau \in X_{R} \operatorname{com} \operatorname{supp}(\sigma)=q$ e $\operatorname{supp}(\tau)=p$. Como $p \in A$, segue que $\operatorname{sgn}_{\tau}(\varphi) \equiv$ $\operatorname{dim}_{\tau}(\varphi)=1 \bmod 2(\star)$. Deste modo, se $\operatorname{sgn}_{\sigma}(\varphi)=0$, teríamos pela propriedade $\left.i i\right)$ que $\operatorname{sgn}_{\tau}(\varphi)=0$, contrariando $(\star)$. Logo, pelo item $i$ ), segue que $\operatorname{sgn}_{\sigma}(\varphi)=1$ e portanto $q \in A$.

Assim, na terminologia de [DST], $A$ é construtível ( $\dagger$ ) e fechado por generalizações $(\ddagger)$. Logo, pelo Teorema 1.5.4 de [DST] (pág. 31), segue que $A \subseteq \operatorname{spec}(R)$ é aberto-compacto. Neste caso existem $a_{1}, \ldots, a_{n} \in \operatorname{Id}(R)$ tais que $A=D\left(a_{1}\right) \cup \cdots \cup D\left(a_{n}\right)$. Tomando $a \in D^{t}\left(a_{1}, \ldots, a_{n}\right)$, temos $A=D(a)$. Assim, pelo item $i)$, segue que para todo $\sigma \in X_{R}$ vale $\operatorname{sgn}_{\sigma}(\varphi)=\sigma(a)$.

Seja $R$ RS. Considere o morfismo $\varepsilon: R \rightarrow P_{R}=C\left(\left(X_{R}\right)_{c o n s t}, 3\right)$ para a envoltória de Post de $R$. Pela Proposição 5.46, o RS $P_{R}$ é von Neumann e em particular Booleano. Logo, pelo Teorema 5.52 , existe único morfismo $\widetilde{\varepsilon}: B(R) \rightarrow P_{R}$ satisfazendo $\varepsilon=\widetilde{\varepsilon} \circ b$.

Proposição 5.55. Seguindo a notação acima, vale:

i) $\widetilde{\varepsilon}: B(R) \rightarrow P_{R}$ é imersão completa. Em particular, $\widetilde{\varepsilon}$ é injetora e dados $a, b, c \in B(R)$ temos que

$$
a \in D_{B(R)}^{t}(b, c) \Leftrightarrow \widetilde{\varepsilon}(a) \in D_{P_{R}}^{t}(\widetilde{\varepsilon}(b), \widetilde{\varepsilon}(c)) .
$$

ii) Dada forma $\varphi \in B(R)$ e $\sigma \in X_{R}$, vale que

$$
\widetilde{\varepsilon}(\varphi)(\sigma)=\operatorname{sgn}_{\sigma}(\varphi) .
$$

iii) As funções $f \in \operatorname{Im}(\widetilde{\varepsilon})$ são caracterizadas pelas seguintes propriedades (comparar com Proposição 4.19.(iii)) :

a) $f:\left(X_{R}\right)_{\text {const }} \rightarrow 3$ são contínuas e dados $\sigma, \tau \in X_{R}$, se $\operatorname{supp}(\sigma) \subseteq \operatorname{supp}(\tau)$ e $f(\sigma)=0$, então $f(\tau)=0$

b) Para todo $p \in \operatorname{spec}(R)$, existe $x \in R$ tal que $\left.f\right|_{X_{p}}=\left.\varepsilon(x)\right|_{X_{p}}$.

Demonstração. Considere os morfismos canônicos $v: R \rightarrow V(R), b: R \rightarrow B(R)$ e a inclusão $i: B(R) \hookrightarrow V(R)$. Lembre-se que $v=i \circ b$. Além disso, seja $\bar{\varepsilon}: V(R) \rightarrow P_{R}$ o morfismo induzido na envoltória von Neumann.

\footnotetext{
${ }^{3}$ Definição VI.2.6 de [DP2]

${ }^{4}$ Definição V.1.8 de [DP2]
} 
i) Pela Proposição 5.47, o morfismo $\varepsilon: R \rightarrow P_{R}$ é imersão completa. Logo pelo Teorema 5.48.(iii) o morfismo $\widetilde{\varepsilon}: V(R) \rightarrow P_{R}$ também é imersão completa.

ii) Por definição, temos

$$
\bar{\varepsilon} \circ i \circ b=\bar{\varepsilon} \circ v=\varepsilon=\widetilde{\varepsilon} \circ b,
$$

pela proporiedade universal de $b$ (Teorema 5.48.(ii)) segue que $\bar{\varepsilon} \circ i=\widetilde{\varepsilon}$. Deste modo, o resultado segue pela Proposição 4.19.(ii).

iii) Seja $\varphi \in B(R)$ forma. Por definição, existe $x \in \operatorname{Id}(R)$ tal que $\varphi \cong\langle x\rangle$. Seja $f=\widetilde{\varepsilon}(\varphi)$. Pela Proposição 4.19.(iii), segue que $f$ satisfaz a propriedade $b$ ). Para $a$ ), sejam $\sigma, \tau \in X_{R}$ tais que $\operatorname{supp}(\sigma) \subseteq \operatorname{supp}(\tau)$ e $f(\sigma)=0$. Neste caso, segue do item $i i)$ que $\sigma(x)=0$. Logo $\tau(x)=0$ e $\operatorname{assim} f(\tau)=0$.

Reciprocamente, seja $f \in P_{R}$ satisfazendo as propriedades a) e b). Pela Proposição 4.19.(iii), existe forma $\varphi \in V(R)$ tal que $f=\bar{\varepsilon}(\varphi)$. Por outro lado, como $f^{2}$ também satisfaz a proporiedade $a$ ), segue que $\varphi^{2}$ satisfaz as hipóteses do Lema 5.54. Logo existe $a \in \operatorname{Id}(R)$ tal que $\varphi^{2} \cong\langle a\rangle$. Considere então a forma $\psi=a \nabla(\varphi)$, onde $\nabla: V(R) \rightarrow V(R)^{\times}$é o operador nabla. Então $\psi \in B(R)$ e

$$
f=\bar{\varepsilon}(\varphi)=\bar{\varepsilon}\left(\nabla(\varphi) \varphi^{2}\right)=\bar{\varepsilon}(\nabla(\varphi) a)=\widetilde{\varepsilon}(\psi) .
$$

Comentário 5.56. A Proposição 5.55 fornece uma forma equivalente de definir a envoltória von Neumann como o subconjunto das funções $f \in P_{R}$ satisfazendo as propriedades $a$ ) e $b$ ) de 5.55.(iii) munido das noções de produto e de representação transveral induzidas da envoltória de Post $P_{R}$.

O próximo resultado apresenta uma caracterização útil de RS-fan. Seja $R$ RS. Pela Proposição VI.1.2 de [DP2], a condição $a$ ) diz os ideais primos de $R$ são linearmente ordenados. Já a condição b) diz que o mapa supp: $X_{R} \rightarrow \operatorname{spec}(R)$ satisfaz a propriedade going-up (Definição 5.3.1 de [DST]).

Teorema 5.57. Seja R RS. São equivalentes:

i) $R$ é $R S$-fan;

ii) $R$ satisfaz as seguintes condições:

a) $\forall a, b \in R\left(a^{2} b^{2}=a^{2}\right.$ ou $\left.a^{2} b^{2}=b^{2}\right)$.

b) $\operatorname{Sejam} \sigma, \tau \in X_{R} \operatorname{com} \operatorname{supp}(\sigma) \subseteq \operatorname{supp}(\tau)$. Então existe $\bar{\tau} \in X_{R}$ tal que $\operatorname{supp}(\bar{\tau})=\operatorname{supp}(\tau)$ e $\sigma^{-1}(0,1) \subseteq \bar{\tau}^{-1}(0,1)$.

c) Dado $p \in \operatorname{spec}(R), K_{R}(p)^{\times}$é $R S G$-fan.

Demonstração. Teorema VI.4.2 de [DP2] (pág. 226).

Lema 5.58. Seja $R$ RS. Suponha que $R$ seja Booleano e satisfaça a condição $b$ ) do Teorema 5.57. Então $R$ é RS von Neumann.

Demonstração. Vamos mostrar que os ideias primos de $R$ são dois a dois incompatíveis. Sejam $p, q \in \operatorname{spec}(R)$ e suponha que $p \subseteq q$. Tome $\sigma, \tau \in X_{R}$ tais que $p=\operatorname{supp}(\sigma)$ e $q=\operatorname{supp}(\tau)$. Pela condição $b)$ do Teorema 5.57 , existe $\bar{\tau} \in X_{R}$ tal que

$$
\operatorname{supp}(\bar{\tau})=\operatorname{supp}(\tau) \text { e } \sigma^{-1}(0,1) \subseteq \bar{\tau}^{-1}(0,1)
$$

Afirmação. Nas condições acima, $\sigma=\bar{\tau}$.

Demonstração. Seja $x \in R$. Vamos mostrar que $\sigma(x)=\bar{\tau}(x)$ em alguns casos. 
- Caso 1: $\sigma(x)=0$.

Neste caso, $\sigma(\nabla(x))=-1$. Assim, por $(\dagger)$ segue que $\tau(x) \in\{0,1\}$ e $\tau(\nabla(x))=-1$. Logo $\tau(x)=0$.

- Caso 2: $\sigma(x)=1$.

Neste caso, $\sigma(\nabla(x))=1$. Assim, por $(\dagger)$ segue que $\tau(\nabla(x))=1$ e portanto $\tau(x)=1$.

- Caso 3: $\sigma(x)=-1$.

Segue do caso acima aplicado a $-x$.

Pela afirmação acima, segue que $p=\operatorname{supp}(\sigma)=\operatorname{supp}(\bar{\tau})=\operatorname{supp}(\tau)=q$.

Proposição 5.59. Seja $R$ RS-fan. Então $B(R)$ satisfaz as condições $a$ ) e $c$ ) do Teorema 5.57. Além disso, $B(R)$ é RS-fan somente no caso em que $R$ é 0 -RSG.

Demonstração. Primeiro note que, para um semigrupo real $G$, a Proposição VI.1.2 de [DP2] (pág 207) diz que a condição $a$ ) do Teorema 5.57 é equivalente aos ideias primos de $G$ serem linearmente ordenados. Deste modo, pelo Teorema 5.50.(i) segue que

$$
\operatorname{spec}(B(R)) \cong \operatorname{spec}(R)
$$

e portando os ideias primos de $B(R)$ são linearmente ordenados. Logo, $B(R)$ satisfaz $a$ ). Ainda pelo Teorema 5.50.(ii) temos que $B(R)$ satisfaz a condição $c$ ).

Agora suponha que $B(R)$ seja RS-fan, ou seja, satisfaça a condição $b$ ). Então pelo Lema 5.58 $B(R)$ é RS von Neumann. Mas como os ideias primos de $B(R)$ são linearmente ordenados, o conjunto $\operatorname{spec}(B(R))$ é unitário. Assim pela bijeção $(\dagger)$ segue que $\operatorname{spec}(R)$ é unitário e portanto $R$ é 0-RSG.

Em seguida, as propriedades da envoltória RS-Booleana de um RS espectral são exploradas.

Proposição 5.60. Seja $R$ semigrupo real espectral ${ }^{5}$ e considere o morfismo induzido $\bar{\varepsilon}: B(R) \rightarrow P_{R}$ na envoltória de Post.

i) $\operatorname{Im}(\bar{\varepsilon})$ é o conjunto das funções $f \in P_{R}$ satisfazendo

$$
\operatorname{Dados} \sigma, \tau \in X_{R}, \text { se } \operatorname{supp}(\sigma) \subseteq \operatorname{supp}(\tau) \text { e } f(\sigma)=0 \text {, então } f(\tau)=0 \text {. }
$$

ii) O mapa $\operatorname{supp}_{B(R)}: X_{B(R)} \rightarrow \operatorname{spec}(B(R))$ é bijetor

Em particular, $B(R)$ é espectral somente no caso em que $R$ é álgebra de Post.

Demonstração. Note que pelo Teorema V.9.1 de [DP2] (pág. 199), o mapa espectral

$$
\operatorname{supp}_{R}: X_{R} \rightarrow \operatorname{spec}(R)
$$

é homeomorfismo. Em particular, pelo Teorema 5.50.(i) segue o item ii). Além disso, para todo $p \in \operatorname{spec}(R), X_{p}$ é unitário. Assim, a condição b) da Proposição 5.55.(iii) é satisfeita para qualquer $f \in P_{R}$, de onde segue o item $i$ ).

Deste modo, se $B(R)$ é espectral, então novamente pelo Teorema V.9.1 de [DP2] temos que o

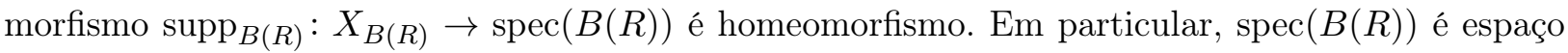
Booleano. Logo pelo Teorema 5.50. $(i)$ o espaço spec $(R)$ é Booleano. Assim pelo homeomorfismo ( $\dagger)$ o espaço $X_{R}$ é Booleano também.

Para concluir, considere $\operatorname{Sp}(R)=C\left(X_{R}, 3_{s p}\right) \subseteq P_{R}$ a envoltória espectral de $R$ (Definição V.4.1 de [DP2]). Como $X_{R}$ é Booleano, segue que $\operatorname{Sp}(R)=P_{R}$. Por outro lado, pelo Corolário V.4.6 de [DP2] (pág. 81), vale $R \cong \mathrm{Sp}(R)$. Logo $R$ é álgebra de Post.

\footnotetext{
${ }^{5}$ Confira Definição V.1.8 de [DP2] (pág. 173) ou caracterização de primeira ordem no Teorema V.7.4 de [DP2] (pág. 194)
} 
Nesta parte, é mostrado que a envoltória RS-Booleana preserva quocientes. Porém, diferentemente do resultádo análogo para envolótira von Neumann (Proposição 4.22), não sabemos que a envoltória RS-Booleana preserva morfismo forte nem se preserva morfismo transversalmente 2regular.

Proposição 5.61. Seja $f: R \rightarrow G$ morfismo quociente de RSs. Então $b(f): B(R) \rightarrow B(G)$ é quociente.

Demonstração. Vamos provar que $b(f): B(R) \rightarrow B(G)$ é quociente na categoria dos RSs Booleanos, isto é, satisfaz a propriedade universal da Definição 1.9. Seja $g: B(R) \rightarrow B$ morfismo para RS Booleano $B$ satisfazendo $\operatorname{ker}(B(f)) \subseteq \operatorname{ker}(g)$. Então pela Proposição 1.10

$$
\operatorname{ker}(f) \subseteq \operatorname{ker}\left(v_{G} \circ f\right)=\operatorname{ker}\left(b(f) \circ v_{R}\right) \subseteq \operatorname{ker}\left(g \circ b_{R}\right)
$$

Assim, como $f$ é quociente, existe $\widetilde{g}: G \rightarrow B$ tal que $g \circ b_{R}=\widetilde{g} \circ f$.

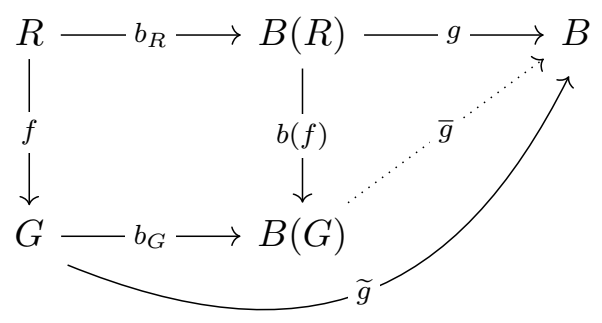

Por outro lado, pela propriedade universal da envoltória RS-Booleana $b_{G}$, existe $\bar{g}: B(G) \rightarrow B$ tal que $\widetilde{g}=\bar{g} \circ b_{G}$. Deste modo,

$$
g \circ b_{R}=\bar{g} \circ b_{G} \circ f=\bar{g} \circ b(f) \circ b_{R} .
$$

Pela unicidade da propriedade universal da envoltória RS-Booleana $b_{R}$, segue que $g=\bar{g}$ 。 $b(f)$. A unicidade de $\bar{g}$ segue pela unicidade das propriedades universais de $f$ e $b_{G}$. Assim, $b(f)$ é quociente.

Proposição 5.62. i) Todo monomorfismo de semigrupos reais na categoria RS é injetor.

ii) Todo monomorfismo de semigrupos reais von Neumann na categoria vNRS é injetor.

Demonstração. i) Seja $f: R \rightarrow G$ monomorfismo na categoria dos semigrupos reais. Sejam $a, b \in$ $R$ com $f(a)=f(b)$. Considere o RS-fan $F=\left\{1,0,-1, x,-x, x^{2},-x^{2}\right\}$ gerado por um elemento (exemplo VI.3.3 de [DP2]) e defina dois morfismos de semigrupos ternários $g_{1}: F \rightarrow$ $R, g_{2}: F \rightarrow R$ dados por $g_{1}(x)=a, g_{2}(x)=b$. Note que $f \circ g_{1}=f \circ g_{2}$. Como $F$ é RS-fan, segue que $g_{1}$ e $g_{2}$ são morfismos de RSs. Deste modo, sendo $f$ monomorfismo, temos $g_{1}=g_{2}$ e portando $a=b$.

ii) Seja $f: V \rightarrow G$ monomorfismo na categoria dos RSs von Neumann. Sejam $a, b \in V \operatorname{com} f(a)=$ $f(b)$. Pela demonstração do item $i$ ) acima, existem morfismos de RSs $g_{1}: F \rightarrow V, g_{2}: F \rightarrow V$ e $x \in R$ tais que $g_{1}(x)=a, g_{2}(x)=b$ e $f \circ g_{1}=f \circ g_{2}$. Seja $v: F \rightarrow V(F)$ o morfismo para envoltória von Neumann. Então $V\left(g_{1}\right): V(F) \rightarrow V, V\left(g_{2}\right): V(F) \rightarrow V$ satisfazem $f \circ V\left(g_{1}\right) \circ v=$ $f \circ g_{1}=f \circ g_{2}=f \circ V\left(g_{2}\right) \circ v$ mas como $v$ é epimorfismo, segue que $f \circ V\left(g_{1}\right)=f \circ V\left(g_{2}\right)$. Portanto $V\left(g_{1}\right)=V\left(g_{2}\right)$ e assim $f(a)=\left(f \circ V\left(g_{1}\right) \circ v\right)(x)=\left(f \circ V\left(g_{2}\right) \circ v\right)(x)=f(b)$.

Teorema 5.63. Seja $f: R \rightarrow G$ morfismo de RS. Então as seguintes afirmações são equivalentes:

i) $f: R \rightarrow G$ é epimorfismo. 
ii) $P(f): P(R) \rightarrow P(G)$ é sobrejetora.

iii) $V(f): V(R) \rightarrow V(G)$ é epimorfismo.

iv) $B(f): B(R) \rightarrow B(G)$ é epimorfismo.

v) $W(f): W(R) \rightarrow W(G)$ é epimorfismo.

vi) Dado $\varphi \in W(G)$, existe $k \geq 1$ tal que $2^{k} \varphi \in \operatorname{Im}(W(f))$.

vii) $\operatorname{sper}(f): X_{G} \rightarrow X_{R}$ é injetora.

Demonstração. Primeiro vamos provar as equivalências $i i) \Leftrightarrow v i i)$ e iii) $\Leftrightarrow v$ ).

ii $\Rightarrow$ vii): Sejam $\sigma, \tau \in X_{G}$ com $\sigma \circ f=\tau \circ f$. Se $\sigma \neq \tau$, então como $X_{G}$ espaço Booleano existe $g: X_{G} \rightarrow \mathbf{3}$ contínuas com $g(\sigma) \neq g(\tau)$. Por outro lado, como $P(f)$ é sobrejetora, existe $h: X_{R} \rightarrow \mathbf{3}$ tal que $g(\omega)=h(\omega \circ f)$ para todo $\omega \in X_{G}$. Logo $g(\sigma)=h(\sigma \circ f)=h(\tau \circ \sigma)=g(\tau)$, um absurdo. $\log \sigma=\tau$.

vii) $\Rightarrow i i):$ Se $\operatorname{sper}(f): X_{G} \rightarrow X_{R}$ é injetora, então pela Proposição 4.9 de [DM1] segue que o morfismo induzido $C\left(\left(X_{R}\right)_{\text {const }},\{1,-1\}\right) \rightarrow C\left(\left(X_{G}\right)_{\text {const }},\{1,-1\}\right)$ entre as álgebra Booleanas é sobrejetor e portanto $\left.P(f)\right|_{P(R)^{\times}}: P(R)^{\times} \rightarrow P(G)^{\times}$é sobrejetor. Assim, dado $g \in P(G)$, existem $h_{1}, h_{2} \in P(R)^{\times}$tais que $P(f)\left(h_{1}\right)=\nabla(g)$ e $P(f)\left(h_{2}\right)=\nabla\left(g^{2}\right)$. Defina $e:\left(X_{R}\right)_{\text {const }} \rightarrow\{0,1\}$ por

$$
e(\sigma)= \begin{cases}1 & \text { se } \sigma\left(h_{2}\right)=1 \\ 0 & \text { se } \sigma\left(h_{2}\right)=-1\end{cases}
$$

Então e é contínua, $e \in \operatorname{Id}(P(R))$ e $\nabla(e)=h_{2}$. Seja $h=h_{1} e$. Então como $\nabla(P(f)(e))=P(f)\left(h_{2}\right)=$ $\nabla\left(g^{2}\right)$, segue que pela Proposição 2.55 que $P(f)(e)=g^{2}$. Logo $P(f)(h)=\nabla(g) g^{2}=g$.

iii) $\Leftrightarrow v$ ): Segue do Teorema 4.63 .

Agora prosseguimos com o restante das equivalências:

$i) \Rightarrow i v)$ : Pelo Teorema 5.52, o funtor que associa a envoltória RS-Booleana é adjunto à esquerda. Logo, preserva epimorfismo.

$i v) \Rightarrow$ iii) : Pelo Teorema 5.48, $V(R)$ é a envoltória von Neumann de $B(R)$. Logo, sendo a envoltória von Neumann adjunto à esquerda (Teorema 4.14), ela preserva epimorfismo.

iii $) \Rightarrow$ vii) : Como $V(f): V(R): V(G)$ é epimorfismo, o mapa induzido $\operatorname{sper}(f):\left(X_{G}\right)_{\text {const }} \rightarrow$ $\left(X_{R}\right)_{\text {const }}$ entre espaços Booleanos é injetor.

$i i) \Rightarrow v i):$ Considere $i_{R}: W(R) \rightarrow C\left(\left(X_{R}\right)_{\text {const }}, \mathbb{Z}\right), i_{G}: W(G) \rightarrow C\left(\left(X_{G}\right)_{\text {const }}, \mathbb{Z}\right)$ os morfismos de inclusão. Seja $g \in G$. Como $P(f)$ é sobrejetora, existe $h \in P(R)$ tal que $P(f)(h)=h \circ \operatorname{sper}(f)=$ $i_{G}(\langle g\rangle)$. Pelo Teorema 8.3.1 de [Mar7], existe $k \geq 1$ e forma $\varphi$ sobre $R$ tais que $2^{k} h=i_{R}(\varphi)$. Assim, dado $\sigma \in X_{G}$, temos $2^{k} \sigma(g)=2^{k} h(\sigma \circ f)=\operatorname{sgn}_{\sigma \circ f}(\varphi)=\operatorname{sgn}_{\sigma}(f \star \varphi)$. Logo $W(f)(\varphi)=2^{k}\langle g\rangle$. Como as formas com apenas uma entrada gera $W(G)$, temos o resultado.

$v i) \Rightarrow v i i)$ : Sejam $\sigma, \tau \in X_{G}$ tais que $\sigma \circ f=\tau \circ f$. Seja $g \in G$. Por hipótese, existe forma $\varphi$ sobre $R$ e $k \geq 1$ tais que $2^{k}\langle g\rangle=f \star \varphi$ em $W(G)$. Logo $2^{k} \sigma(g)=\operatorname{sgn}_{\sigma}(f \star \varphi)=\operatorname{sgn}_{\sigma \circ f}(\varphi)=$ $\operatorname{sgn}_{\tau \circ f}(\varphi)=\operatorname{sgn}_{\tau}(f \star \varphi)=2^{k} \tau(g)$. Logo $\sigma(g)=\tau(g)$. Como $g \in G$ foi arbitrário, segue que $\sigma=\tau$.

vii) $\Rightarrow i$ ): Sejam $g_{1}, g_{2}: G \rightarrow H$ dois morfismos de RSs tais que $g_{1} \circ f=g_{2} \circ f$. Seja $g \in G$.

Dado $\sigma \in X_{H}$, temos $\sigma \circ g_{1} \circ f=\sigma \circ g_{2} \circ f$. Como $\operatorname{sper}(f)$ é injetor, segue que $\sigma \circ g_{1}=\sigma \circ g_{2}$ e portanto $\sigma\left(g_{1}(g)\right)=\sigma\left(g_{2}(g)\right)$. Como $g \in G$ foi arbitrário, segue que $g_{1}=g_{2}$.

Teorema 5.64. Seja $f: R \rightarrow G$ morfismo de semigrupos reais. Então as seguintes afirmações são equivalentes:

i) $f: R \rightarrow G$ é imersão completa.

ii) Dada forma de Pfister $\varphi$ sobre $R$ e $x \in R$, se $f(x) \in D_{G}^{t}(f \star \varphi)$, então $x \in D_{R}^{t}(\varphi)$.

iii) sper $(f): X_{G} \rightarrow X_{R}$ é sobrejetor. 
iv) $W(f): W(R) \rightarrow W(G)$ é injetor.

v) $P(f): P(R) \rightarrow P(G)$ é injetor.

vi) Dadas formas $\varphi, \psi$ sobre $R$, se $f \star \varphi \equiv_{G} f \star \psi$, então $\varphi \equiv_{R} \psi$.

vii) $V(f): V(R) \rightarrow V(G)$ é imersão completa.

viii) $B(f): B(R) \rightarrow B(G)$ é imersão completa.

Demonstração. Primeiro vamos provar $i) \Rightarrow i i) \Rightarrow i i i) \Rightarrow i$ ).

i) $\Rightarrow$ ii): Seja $\varphi=\bigotimes_{i=1}^{n}\left\langle a_{i}^{2}, a_{i}\right\rangle$ forma de Pfister e considere $a=\Pi_{i=1}^{n} a_{i}^{2}$. Seja $x \in R$ com $f(x) \in D_{G}^{t}(f \star \varphi)$. Pela Proposição 3.14, segue que $f(x)=f(a x)$ e $f \star x \varphi \cong_{G} f \star \varphi$. Como $f$ é imersão completa, segue que $x=a x$ e $x \varphi \cong_{R} \varphi$. Novamente pela Proposição 3.14 temos $x \in D_{R}^{t}(\varphi)$.

$i i) \Rightarrow i i i)$ : Primeiro observe que $\operatorname{sper}(f):\left(X_{G}\right)_{\text {const }} \rightarrow\left(X_{R}\right)_{\text {const }}(*)$ é contínua. Como $\left(X_{G}\right)_{\text {const }}$ é compacto e $\left(X_{R}\right)_{\text {const }}$ Hausdorff, para mostrarmos que sper $(f)$ é sobrejetora basta provarmos que $(*)$ tem imagem densa. Sejam $a_{1}, \ldots, a_{n}, x \in R, x$ idempotente, tais que $\bigcap_{i=1}^{n}\left[\left[a_{i}=1\right]\right] \cap[[x=0]] \neq \emptyset$ em $X_{R}$. Sejam $\varphi=\bigotimes_{i=1}^{n}\left\langle a_{i}^{2}, a_{i}\right\rangle$ e $a=\prod_{i=1}^{n} a_{i}^{2}$. Como $\bigcap_{i=1}^{n}\left[\left[a_{i}=1\right]\right] \cap[[x=0]]=\bigcap_{i=1}^{n}\left[\left[a_{i}=\right.\right.$ $1]] \cap[[a x=0]]$, podemos supor sem perda de generalidade que $x=a x$. Pelo Comentário 3.13 segue que $x \varphi \neq_{R} \varphi$. Assim, pela Proposição 3.14 vale $x \notin D_{R}^{t}(\varphi)$ e portanto por hipótese $f(x) \notin D_{G}^{t}(f \star \varphi)$. Novamente pela Proposição 3.14 segue que $f \star x \varphi \approx_{G} f \star \varphi$ e portanto existe $\sigma \in X_{G}$ tal que $\sigma(f(x))=0, \sigma\left(f\left(a_{i}\right)\right)=1$ para todo $i=1, \ldots, n$.

iii) $\Rightarrow$ i): Imediato.

Agora vamos prosseguir com as seguintes equivalências $i) \Leftrightarrow i v), i i i) \Leftrightarrow v), v i) \Rightarrow i i)$ e $i i i) \Rightarrow v i)$.

$i) \Leftrightarrow i v)$ : Direto da definição.

iii $\Rightarrow v$ ): Sejam $g_{1}, g_{2} \in P(R)$ tais que $g_{1} \circ \operatorname{sper}(f)=g_{2} \circ \operatorname{sper}(f)(*)$. Se $g_{1} \neq g_{2}$, existe $\sigma \in X_{R}$ tal que $g_{1}(\sigma) \neq g_{2}(\sigma)$. Porém, como $\operatorname{sper}(f)$ é sobrejetor, existe $\tau \in X_{G}$ tal que $\tau \circ f=\sigma$. Assim, por $(*)$, segue que $g_{1}(\sigma)=g_{1}(\tau \circ f)=g_{2}(\tau \circ f)=g_{2}(\sigma)$, um absurdo. Logo $g_{1}=g_{2}$ e $P(f)$ é injetor.

$v) \Rightarrow$ iii): Se $P(f)$ é injetor, então em particular

$$
\left.P(f)\right|_{P(R)^{\times}}: P(R)^{\times}=C\left(\left(X_{R}\right)_{\text {const }},\{1,-1\}\right) \rightarrow C\left(\left(X_{G}\right)_{\text {const }},\{1,-1\}\right)=P(G)^{\times}
$$

é injetor. Então pela Proposição 4.9 de [DM1] temos $\operatorname{sper}(f): X_{G} \rightarrow X_{R}$ injetor.

$v i) \Rightarrow$ ii): Sejam $\varphi=\bigotimes_{i=1}^{n}\left\langle a_{i}^{2}, a_{i}\right\rangle$ forma de Pfister, $a=\prod_{i=1}^{n} a_{i}^{2}$ e $x \in R$. Suponha que $f(x) \in D_{G}^{t}(f \star \varphi)$. Pelo Corolário 3.15, temos

$$
f \star\left(x \varphi \oplus a\langle 1,-1\rangle^{n}\right) \equiv_{G} f \star\left(\varphi \oplus x^{2}\langle 1,-1\rangle^{n}\right) .
$$

Portanto, por hipótese temos $x \varphi \oplus a\langle 1,-1\rangle^{n} \equiv_{R} \varphi \oplus x^{2}\langle 1,-1\rangle^{n}$. Novamente pelo Corolário 3.15 segue que $x \in D_{R}^{t}(\varphi)$.

$i i i) \Rightarrow v i)$ : Direto da definição.

Por último, vamos provar que $i i i) \Rightarrow v i i) \Rightarrow v i i i) \Rightarrow i i i)$.

iii $) \Rightarrow$ vii): Pelo Teorema 4.10, os morfismos canônicos $v_{R}: R \rightarrow V(R)$ e $v_{G}: G \rightarrow V(G)$ induzem homeomorfismos $X_{V(R)} \cong\left(X_{R}\right)_{\text {const }}$ e $X_{V(G)} \cong\left(X_{G}\right)_{\text {const }}$. Assim, se $\operatorname{sper}(f)$ é sobrejetor, $\operatorname{sper}(V(f))$ também o é.

vii $\Rightarrow$ viii): Sejam $\varphi, \psi$ formas sobre $B(R)$ tais que $B(f) \star \varphi \cong_{B(G)} B(f) \star \psi$. Aplicando o morfismo de inclusão $B(G) \rightarrow V(G)$ e tendo em mente que $B(f)=\left.V(f)\right|_{B(G)}$, temos $V(f) \star \varphi \cong_{V(G)}$ $V(f) \star \psi$. Como $V(f)$ é imersão completa, segue que $\varphi \equiv_{V(R)} \psi$. Por outro lado, pelo Teorema 5.48, o morfismo de inclusão $B(R) \rightarrow V(R)$ satisfaz a propriedade universal da envoltória von Neumann. Logo, pelo Teorema 4.10, o mapa $B(R) \rightarrow V(R)$ é imersão completa e portanto $\varphi \cong_{B(R)} \psi$. 
viii $\Rightarrow$ iii): Pelo Teorema 5.50, os morfismos canônicos $b_{R}: R \rightarrow B(R)$ e $b_{G}: G \rightarrow B(G)$ induzem homeomorfismos $X_{B(R)} \cong X_{R}$ e $X_{B(G)} \cong X_{G}$. Assim se $\operatorname{sper}(B(f)): X_{B(G)} \rightarrow X_{B(R)}$ é sobrejetor, então $\operatorname{sper}(f): X_{G} \rightarrow X_{R}$ também é.

Proposição 5.65. Seja $f: R \rightarrow G$ morfismo de RSs von Neumann. Então

i) $\operatorname{spec}(f): \operatorname{spec}(G) \rightarrow \operatorname{spec}(R)$ é sobrejetor $\left.\Leftrightarrow f\right|_{I d(R)}: I d(R) \rightarrow I d(G)$ é injetor.

ii) $\operatorname{spec}(f): \operatorname{spec}(G) \rightarrow \operatorname{spec}(R)$ é injetor $\left.\Leftrightarrow f\right|_{I d(R)}: \operatorname{Id}(R) \rightarrow I d(G)$ é sobrejetor.

Demonstração. Dado um semigrupo real $S$, pelo Corolário I.6.19 de [DP2] o espectro $\operatorname{spec}(S)$ é homeomorfo ao espectro de ideals primos do reticulado $\operatorname{Id}(\mathrm{S})$. Assim, no caso von Neumann, os itens $i$ ) e $i$ i) são reformulações das correspondências de injetividade e sobrejetividade na dualidade de Stone (conteúdo da Proposição 4.9 de [DM1]). 


\section{Capítulo 6}

\section{Conclusões}

\subsection{Resultados parciais}

i) Na seção 3.4 introduzimos o conceito de índice de Witt para semigrupos reais como uma função avaliada no espectro primo. Também foi visto que a continuidade o índice de Witt se relaciona com a validade do Teorema da Decomposição de Witt. O Teorema 3.34 fornece alguns exemplos de RS em $W I(R S)$ bem como construções nesta classe. Por outro lado, seguindo a demonstração do Lema 2.5 de [Ken], é possível provar que

Teorema 6.1. Todo RS von Neumann é limite de um diagrama de produtos e ultraprodutos de $0-R S G s$.

Deste modo, pelo Teorema 3.34 se conseguirmos provar que a classe $W I(R S)$ é fechada por limites, então a classe $W I(R S)$ consiste de todos os RS von Neumann.

ii) No terceiro capítulo, apresentamos uma possível generalização de isotropia. Seu grande problema é que não sabemos se vale o Teorema da Decomposição de Witt em todo RS von Neumann. Uma alternativa é a seguinte definição:

Definição 6.2. Sejam $R$ RS von Neumann e $\varphi$ forma.

- Suponha $\varphi$ qc-forma $\operatorname{com} \operatorname{supp}(\varphi)=D(e), e \in \operatorname{Id}(R)$. A forma $\varphi$ é dita isotrópica* se $e,-e \in D^{t}(\varphi)$. Por outro lado, $\varphi$ é dito anisotrópica* se não for isotrópica*. A forma nula $\langle 0\rangle$ é por definição anisotrópica*.

- Suponha $\left(\varphi_{1}, \ldots, \varphi_{k}\right)$ sistema ortogonal para $\varphi$. Então $\varphi$ é isotrópica* se existe $1 \leq i \leq k$ tal que $\varphi_{i}$ é isotrópica* e $\varphi$ é anisotrópica* se não for isotrópica*.

Comentários 6.3. - Sejam $R$ RS von Neumann e $\varphi$ uma forma com coeficientes inversíveis. Então $\varphi$ é anisotrópica* se, e somente se, $\varphi$ é anisotrópica no RSG $R^{\times}$(comparar com $3.26)$.

- Se uma forma é anisotrópica, então ela é anisotrópica* mas a volta não vale em geral (3.26).

- Seja $\left(\varphi_{1}, \ldots, \varphi_{k}\right)$ sistema ortogonal com $\operatorname{supp}\left(\varphi_{i}\right)=D\left(e_{i}\right)$. Então $\varphi=\varphi_{1} \oplus \cdots \oplus \varphi_{k}$ é isotrópica* se, e somente se, existe $1 \leq i \leq k$ tal que $e_{i},-e_{i} \in D^{t}(\varphi)$.

Proposição 6.4. Sejam $R$ RS von Neumann e $\varphi$ uma qc-forma $\operatorname{com} \operatorname{supp}(\varphi)=D(e)$. São equivalentes:

i) $\varphi$ é isotrópica*

ii) $0 \in D^{t}(\varphi)$.

iii) Existe forma $\theta$ tal que $\varphi \equiv e\langle 1,-1\rangle \oplus \theta$. 
Demonstração. $i) \Rightarrow i i)$ : Se $\varphi$ é isotrópica*, então $0 \in D^{t}(e,-e) \subseteq D^{t}(\varphi)$.

ii) $\Rightarrow$ iii): Lema 3.36 .

iii $\Rightarrow$ ) : Primeiro note que por $e\langle 1,-1\rangle \oplus \theta \equiv \varphi=e \varphi \equiv e\langle 1,-1\rangle \oplus e \theta$ temos $e \theta \equiv \theta$. Seja $l \in D^{t}(\theta)$. Então $e,-e \in D^{t}(e,-e, l e) \subseteq D^{t}(\varphi)$.

Comentários 6.5. - Na linguagem dos RSGs, a isotropia de formas é descrita por uma fórmula Horn-geométrica e o mesmo acontece pela proposição anterior com a noção de isotropia* para qc-formas na linguagem dos RSs von Neumann. No entanto, a situação se inverte com a noção de isotropia adotada ao longo da tese. Pela demonstração do Teorema 3.34 ii), a noção de anisotropia em RSs von Neumann é capturada por uma fórmula Horn-geométrica (o Corolário 3.38 sugere fórmula Horn-geométrica alternativa).

- A anisotropia* é descrita por uma fórmula Horn-geométrica. De fato, seja $\varphi$ uma $k$-forma, $k \geq 0$. Então $\varphi=\left\langle a_{1}, \ldots, a_{n}\right\rangle$ é anisotrópica* se, e somente se,

$$
0 \in D^{t}(\varphi) \Rightarrow a_{1}=\cdots=a_{n}=0 .
$$

Portanto, a anisotropia* para qc-formas é descrita por uma fórmula Horn-geométrica. Juntando essa fórmula com aquelas construídas no Teorema 3.23, é possível descrever a anisotropia* por uma fórmula Horn-geométrica. Por outro lado, pelo item anterior segue que a isotropia* é descrita por uma fórmula Horn.

Uma possível grande diferença entre as noções isotropia e isotropia* é a validade da decomposição de Witt (comparar com o Teorema 3.29, iii)):

Teorema 6.6 (Teorema da decomposição de Witt). Sejam $R R S$ von Neumann e $\varphi$ forma. Então existe forma $\psi$ anisotrópica* ${ }^{*}$ forma $\theta$ sobre $\operatorname{Id}(R)$ tal que $\varphi \equiv \theta\langle 1,-1\rangle \oplus \psi$.

Também é possível obter uma versão mais forte do Teorema da Subforma de Pfister do que em 3.43 .

Teorema 6.7 (Teorema da subforma de Pfister revisitado). Sejam $R$ RS von Neumann e $\varphi, \psi$ formas. Suponha que $\psi$ seja anisotrópica* ${ }^{*}$ e que para todo morfismo de multi-aneis $f: R \rightarrow G$ com $G$ hipercorpo especial valha $D_{G}^{t}(f \star \varphi) \subseteq D_{G}^{t}(f \star \psi)$. Então existe forma $\theta$ sobre $R$ tal que $\psi \equiv_{R} \varphi \oplus \theta$.

Comentários 6.8. Pela Proposição 3.39, se $\varphi, \psi$ são formas com $\psi$ anisotrópica e existe forma $\theta \operatorname{com} \psi \equiv \varphi \oplus \theta$, então $D^{t}(\varphi) \subseteq D^{t}(\psi)$. No entanto, se trocarmos anisotrópica por anisotrópica* não temos o mesmo resultado. De fato, sejam $R$ von Neumann e $x \in \operatorname{Id}(R) \operatorname{com} x \neq 1$. Então $\left\langle-1,-x^{c}\right\rangle \equiv\langle x\rangle \oplus\langle-1,-1\rangle$ e $\left\langle-1,-x^{c}\right\rangle$ é anisotrópica* mas $D^{t}(\langle x\rangle)=\{x\} \nsubseteq D^{t}\left(-1,-x^{c}\right)$.

A facilidade em encontrar a parte anisotrópica* garantida pelo Teorema 6.6 acima se estende para formas de Pfister. Seja $\varphi$ uma forma de Pfister num RS von Neumann. Pelo Corolário $3.15, \varphi$ é hiperbólica $(\varphi \cong\langle 0\rangle)$ se, e somente se, é isotrópica*. Provado o teorema da subforma de Pfister, podemos obter uma caracterização das formas de Pfister.

Definição 6.9. Sejam $R$ RS von Neumann e $\varphi=\left\langle a_{1}, \ldots, a_{n}\right\rangle$ forma $\operatorname{com} \operatorname{supp}(\varphi)=D(e)$, $e \in \operatorname{Id}(R)$. A forma $\varphi$ é dita multiplicativa se $e \in D^{t}(\varphi)$ e para todo morfismo de multi-aneis $f: R \rightarrow G$ com $G$ hipercorpo especial tem-se $f\left(a_{1}\right)+\cdots+f\left(a_{n}\right) \subseteq G$ subconjunto fechado por multiplicação.

Teorema 6.10. Sejam $R R S$ von Neumann e $\varphi$ uma forma multiplicativa anisotrópica* Então $\varphi$ é uma soma ortogonal de formas de Pfister. Em particular, toda qc-forma multiplicativa $e$ anisotrópica* é uma forma de Pfister. 


\subsection{Representações de grupos especiais e de semigrupos reais}

Posta uma abstração de um conceito matemático, é questão natural determinar a extensão da abstração realizada. Assim, o problema (original) da representação em teoria abstratas de formas quadráticas remonta à década de 1980: inicialmente posto por M. Marshall no contexto da categoria dos espaços de ordem abstratos, uma formulação dual da noção de grupo especial reduzido, questiona se todo objeto nesta categoria é isomorfo a algum espaço de ordens de um corpo formalmente real e pitagórico.

Resultados parciais, do final dos anos 1970 ([Cra], [Kul]), garantem que os grupos especiais reduzidos de comprimento de cadeia finito (isto inclui os RSGs finitos) são representáveis na acepção acima: por conta de que estes são contruídos recursivamente a partir de $\mathbb{Z}_{2}=\{-1,1\}$ tomando produtos finitos e extensões finitas. Outro resultado bem conhecido é que os RSGs provenientes de álgebras booleanas são representáveis (por corpos SAP). Aparentemente estes foram os últimos resultados neste tema por três décadas até ter-se mostrado em [AMa] que os grupos especiais reduzidos profinitos também são representáveis. Deve-se mencionar que há um sentimento dominante na comunidade de teorias abstratas de formas quadráticas de que a resposta ao problema assim posto é negativa, mas alguns pesquisadores até mesmo suspeitam de que esta possa ser independente dos axiomas da teoria dos conjuntos.

O problema da representação admite uma quantidade considerável de variantes fracas, algumas delas com implicações interessantes, geralmente descritas por ampliações em duas frentes:

(a) Extensão da classe dos RSGs considerados "representados": retira-se a limitação de utilizarse apenas os corpos formalmente reais e pitagóricos (e com a pré-ordem dada pelos quadrados) para considerar os RSGs obtidos, de alguma maneira, a partir de anéis pré-ordenados.

(b) Extensão da classe de morfismos de um RSG com alvo em algum RSG "representado": retira-se a limitação de utilizar apenas $S G$-isomorfismos para considerar também algum tipo de mergulho em representados (mergulho elementar, mergulho puro, ...).

Sobre o caso (a):

Em [DM8] mostra-se que um RSG qualquer é isomorfo ao RSG associado a algum grupo multiplicativo de invertíveis do anel de funções contínuas a valores reais e definidas em um dado espaço booleano (mais precisamente, no próprio espaço de ordens do RSG).

Sobre o caso (b):

Esta versão se conecta com os estudo dos princípios locais-globais, como o princípio local-global de Pfister (sobre reflexão de isometria) e o princípio de reflexão de isotropia do Marshall, através das flechas canônicas associadas, respectivamente, com a envoltória Booleana e envoltória profinita ([MM2]). Segue de resultados em [KMS] que para todo RSG $G$ existe um corpo formalmente real e pitagórico $F$ e $j: G \longmapsto \mathcal{G}(F)$ um mergulho que reflete isotropia. Em [MM1], utilizando informações sobre envoltória especial profinita, foi possível incrementar ligeiramente este resultado obtendo um mergulho $G \longmapsto \mathcal{G}(F)$ que reflete subformas: isto já permitiu extrair, como consequência direta, o "Teorema de Pfister para subformas", no contexto geral dos RSGs. A idéia geral aqui é que a Teoria dos Grupos Especiais sendo uma teoria matemática de primeira-ordem, permite o uso de instrumentos da Lógica -mais precisamente, da Teoria dos Modelos-, para obter, por iteração com informações específicas da matemática em questão, resultados por meio de "transferência": o estabelecimento de métodos gerais que garantam a existência de uma flecha, que preserva e reflete certas noções lógicas, partindo de um grupo especial e a valores em um outro grupo especial "bem conhecido"(= representado) permite inferir a validade geral de algum resultado a partir da validade deste resultado em uma subclasse específica de objetos representados, estes obtidos através da disponibilidade de recursos adicionais deste contexto (veja [DM4], [Mar9], [AT], [Mrn2], [MM3]). 
Outro problema de representação importante na teoria algébrica de formas quadráticas é descobrir qual a classe de semigrupos reais é representável por p-aneis via o funtor do Teorema 2.26. Na página 177 de [Mar7] é mostrado, na linguagem dual dos ARS, que existe um semigrupo real spectral que não realizável por nenhum p-anel. Concretamente, está em aberto determinar se todo RS von Neumann é realizável por p-anel. No caso afirmativo, o Teorema 4.33 implicaria que todo RS von Neumann é representável por um p-anel von Neumann regular. Deste modo, teríamos um resultado na direção do item (a) acima: todo RSG seria representável por um quociente de todos os inversíveis de anel von Neumann regular pelos inversíveis de uma pré-ordem.

Ainda sobre semigrupos reais, um problema em aberto é determinar se todo RS tem suficientes unidades (ou equivalentemente se todo RS tem suficientes qc-formas). Se isso for verdade, hveria implicações para a teoria dos aneis fielmente quadráticos: dado p-anel $(A, T)$ satisfazendo bounded inversion $\left(1+T \subseteq A^{\times}\right)$, valeria que o pré-RSG $G_{T}(A)$ seria RSG e a $T$-isometria coincidiria com a isometria em $G_{T}(A)$. Assim, para $A$ ser $T$-fielmente quadrático bastaria verificar que vale $[T-F Q 2]$ da Definição 3.1, p. 27, de [DM10].

Para o futuro, há algumas estratégias para atacar o problema da representação:

- Para o Prof. Francisco Miraglia Neto, técnicas de forcing podem ser úteis para mostrar a consistência da representação de todo RSG por corpos;

- O Prof. Peter utilizou métodos da teoria de topos para delinear uma demonstração de que se um grupo especial é elementarmente equivalente a um SG de um corpo, então eles são isomorfos.

- Questões sobre representação em outras formulações abstratas de formas quadráticas (abstract real spectra, real semigroups, multirings) também são postas e têm sido motivação para nosso estudo preliminar iniciado em [RRM2].

\subsection{Outros tópicos para pesquisas futuras}

- Na construção apresentada da envoltória von Neumann de um RS $R, V(R)$, utilizamos o anel de Witt de $R, W(R)$. Notamos que também é possível construir a envoltória von Neumann de $R$ a partir do anel graduado de Witt $W_{g}(R)$ e, como consequência do isomorfismo natural $W(V(R)) \cong W(R)$, temos que o anel graduado de Witt $W_{g}(R)$ codidifica (e é codificado) pelo anel de Witt $W(R)$.

De fato, para mostrar isso, pela Proposição 4.23 podemos supor $R$ von Neumann. Vamos utilizar a notação do início da subseção 4.2.1. Com a soma de formas, $\bar{I}_{n}(R)=$ $I^{n}(R) / I^{n+1}(R)$ é grupo de expoente 2 com elemento distinguido $\top_{n}=2^{n}=\otimes_{i=1}^{n}\langle 1,1\rangle$. Pelo Teorema 4.36, o morfismo $\beta: R^{\times} \rightarrow \bar{I}_{1}(R)$ dado por $\beta(g)=\langle 1,-g\rangle$ é isomorfismo de grupos $\operatorname{com} \beta(-1)=\top_{1}$.

Por outro lado, considerando a multiplicação $*: \bar{I}_{1}(R) \times \bar{I}_{1}(R) \rightarrow \bar{I}_{2}(R)$, sabe-se que dados $a, b, c, d \in R^{\times}$vale

$$
\begin{aligned}
\langle a, b\rangle \equiv_{R^{\times}}\langle c, d\rangle & \Leftrightarrow a b=c d \text { e }\langle 1,-a\rangle \otimes\langle 1,-b\rangle \equiv_{R^{\times}}\langle 1,-c\rangle \otimes\langle 1,-d\rangle \\
& \Leftrightarrow \beta(a)+\beta(b)=\beta(c)+\beta(d) \text { e } \beta(a) * \beta(b)=\beta(c) * \beta(d) .
\end{aligned}
$$

Portanto, as equivalências acima sugerem como definir estrutura de grupo especial reduzido em $\bar{I}_{1}(R)$ usando apenas a estrutura de anel graduado de modo que $\beta: R^{\times} \rightarrow \bar{I}_{1}(R)$ seja isomorfismo de RSG.

Pelo Teorema 4.36, temos $\beta\left(\nabla_{R}\right)=\bar{I}_{0}(R) \otimes 2$. Logo $\beta:\left(R^{\times}, \nabla_{R}\right) \rightarrow\left(\bar{I}_{1}(R), \bar{I}_{0}(R) \otimes\right.$ 2 ) é isomorfismo de $B$-pares. Assim, pelo Lema 5.15 temos que $\mathfrak{R}\left(\bar{I}_{1}(R), \bar{I}_{0}(R) \otimes 2\right) \cong$ 
$\mathfrak{R}\left(R^{\times}, \nabla_{R}\right) \cong R$. De forma mais explícita,

$$
\begin{aligned}
R & \rightarrow \mathfrak{R}\left(\bar{I}(R), \bar{I}_{0}(R) \otimes 2\right) \\
a & \mapsto \gamma\left(\langle 1,-\nabla(a)\rangle,\left\langle 1,-\nabla\left(a^{2}\right)\right\rangle\right)=\gamma\left(\left\langle 1,1,-a,-a^{2}\right\rangle,\langle 1,-a\rangle \otimes 2\right)
\end{aligned}
$$

é isomorfismo de RSs von Neumann.

O próximo passo seria desenvolver uma $K$-teoria para semigrupos reais (ou von Neumann RS) e há um indicativo de que os multi-aneis podem oferecer caráter unificador uma vez que o Kaique M. Andrade, utlizando o conceito de multi-corpo, obeteve uma $K$-teoria que estende simultaneamente a $K$-teoria de Milnor para corpos e a $K$-teoria de grupos especiais.

- Os métodos Booleanos na teoria dos grupos especiais reduzidos se mostraram importantes na resolução das conjecturas de Lam e Marshall para corpos. Neste contexto, dois invariantes Booleanos da isometria se destacam: os de Horn-Tarski e os de Stiefel- Whitney. Eles moram na envoltória Booleana de um RSG e classificam a isometria de formas. Seria interessante obtê-los para RSs utilizando a envoltória de Post. A principal dificuldade é a presença do zero nas entradas das formas de modo que o desenvolvimento de invariantes completos passa primeiro por "uniformizar"os zeros de uma forma ou modificar os invariantes para que não sejam sensíveis aos zeros. Uma primeira estratégia seguindo a uniformização dos zeros para RS von Neumann é considerar formas cujo número de entradas coincida com sua dimensão máxima (isso não impõe restrição real pela Proposição 3.31) e a partir delas desenvolver os invariantes completos.

- Um dos objetivos iniciais desse trabalho era entender até onde os semigrupos reais poderiam fornecer uma teoria de formas quadráticas razoável (como uma isometria de formas de primeira ordem que classifique a representação transversal) e o desenvolvimento da tese mostrou que noções simples de isometria e Witt-equivalência num RS geral leva de maneira natural a noções bem comportadas num RS von Neumann. Outra questão, porém mais secundária, é sobre a possibilidade da definição e desenvolvimento de uma teoria (de primeira-ordem) não-reduzida, como uma expansão simultânea da teoria dos grupos especiais e a teoria dos semigrupos reais, o que levou a formulação e obtenção de vários resultados desse trabalho através do uso do espectro primo de um RS e não do seu espectro real (Exemplos: Teorema 3.8 e Proposição 5.10). Uma potencialmente natural linha de pesquisa seria considerar certos multi-aneis para desenvolver uma teoria não necessariamente reduzida que conseguisse reunir os bons ingredientes da isometria em RS von Neumann. Nesse sentido, a seção 1.4 fornece o embrião dessa teoria: um hiper-anel von Neumann geométrico cujas fibras sejam hipercorpos especiais. Por ser geométrico, a ideia é ao invés de usar o teorema de separação dos RSs, utilizar o feixe estrutural para colar informações das fibras que são RSGs. Suspeito que seja relativamente tranquilo obter axiomas Horn-geométricos para esses objetos e dado anel von Neumann $A$, é possível provar que $A /{ }_{m} A^{\times 2}$ é um primeiro exemplo prototípico dessa teoria. Em tópicos, seria interessante obter futuramente:

- axiomas Horn-geométricos simples para os hiper-aneis von Neumann geométricos com fibras sendo hipercorpos especiais;

- algum princípio local-global focado no espectro primo para os objetos do item acima;

- funtor canônico da categoria dos aneis (ou de certos pares de subconjuntos de um anel) para essa nova categoria que, embora possa não ser sempre representável pelo quociente de Marshall, possa ser útil por razões teóricas;

- exemplos utilizando o quociente de Marshall. 


\section{Apêndice A}

\section{Quocientes em Categorias Quase-Concretas}

Definição A.1. Uma categoria quase-concreta é um par $(C, T)$ onde $C$ é uma categoria e $T: C \rightarrow$ Set é funtor.

Definição A.2. Seja $(C, T)$ uma categoria quase-concreta. Seja $f: X \rightarrow Y$ morfismo em $C$.

$\{$ defqc\}

$\{\operatorname{ker} U\}$

- O núcleo de $f$ é $\operatorname{ker}_{T}(f)=\{(a, b) \in T(X) \times T(X): T(f)(a)=T(f)(b)$ em $T(Y)\}$.

- O morfismo $f$ é dito injetor (respectivamente: sobrejetor, bijetor) se $T(f): T(X) \rightarrow T(Y)$ é injetor (respectivamente: sobrejetor, bijetor).

Note que $f$ é injetor se, e somente se, $\operatorname{ker}_{T}(f)=\operatorname{ker}_{T}\left(I d_{X}\right)$.

Comentário A.3. É um resultado bem conhecido (e de simples verificação) que se $(C, T)$ é uma categoria concreta, i.e. $T: C \rightarrow$ Set é um funtor fiel, se $T(f)$ é injetor (respectivamente: sobrejetor), então $f$ é monomorfismo em $C$ (respectivamente: epimorfismo). Além disso, se $T$ é pleno e fiel e $T(f)$ é bijetor, então $f$ é um isomorfismo em $C$.

Definição A.4. Seja $(C, T)$ uma categoria quase-concreta. Um morfismo $f: X \rightarrow Y$ em $C$ é dito $T$ quociente se para todo morfismo $h: X \rightarrow Z$ satisfazendo $\operatorname{ker}_{T}(f) \subseteq \operatorname{ker}_{T}(h)$, existe único morfismo $\bar{h}: Y \rightarrow Z$ tal que $h=\bar{h} \circ f$.

Note que todo isomorfismo em $C$ é trivialmente um $T$-quociente bijetor.

Proposição A.5. Seja $(C, T)$ uma categoria quase-concreta. Sejam $f: A \rightarrow B, g: B \rightarrow C$ morfismos em $C$. Então

i) $\operatorname{ker}_{T}(f) \subseteq \operatorname{ker}_{T}(g \circ f)$.

ii) Todo morfismo $T$-quociente é epimorfismo.

iii) Suponha que $f, g \circ f$ sejam morfismos $T$-quocientes. Então $g: B \rightarrow C$ é morfismo $T$-quociente.

iv) Suponha $f, g$ sejam morfismos $T$-quocientes. Se $f$ é sobrejetora, então $g \circ f: A \rightarrow C$ é morfismo $T$-quociente.

v) Suponha que $f$ seja $T$-quociente. Se $f$ é injetora, então $f$ é isomorfismo.

vi) Suponha $f$ morfismo $T$-quociente e $f^{\prime}: A \rightarrow B^{\prime}$ outro morfismo $T$-quociente com $\operatorname{ker}_{T}(f)=$ $\operatorname{ker}_{T}\left(f^{\prime}\right)$. Então existe um único isomorfismo $h: B \rightarrow B^{\prime}$ tal que $f^{\prime}=h \circ f$.

Demonstração. i) Imediato da definição.

ii) Segue do item anterior. 
iii) Seja $h: B \rightarrow Z$ morfismo com $\operatorname{ker}_{T}(g) \subseteq \operatorname{ker}_{T}(h)$. Assim $\operatorname{ker}_{T}(g \circ f) \subseteq \operatorname{ker}_{T}(h \circ f)$. Como $g \circ f$ é morfismo $T$-quociente, existe único morfismo $\bar{h}: C \rightarrow Z \operatorname{com} h \circ f=\bar{h} \circ(g \circ f)$.

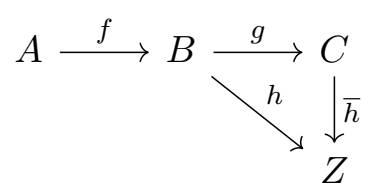

Mas como $f$ é epimorfismo - isto segue do item ii) pois $f$ é um $T$-quociente (por hipótese) temos $h=\bar{h} \circ g$. Novamente, como $f$ é epimorfismo também temos a unicidade de $\bar{h}$. Assim $g$ é morfismo $T$-quociente.

iv) Seja $h: A \rightarrow V$ morfismo com $\operatorname{ker}_{T}(g \circ f) \subseteq \operatorname{ker}_{T}(h)$. Como $f$ é mapa quociente, pelo item $\left.i\right)$ existe $\tilde{h}: B \rightarrow V$ tal que $h=\tilde{h} \circ f$. Por outro lado, dados $\left(b, b^{\prime}\right) \in \operatorname{ker}_{T}(g)$, sejam $a, a^{\prime} \in T(A)$ satisfazendo $T(f)(a)=b, T(f)\left(a^{\prime}\right)=b^{\prime}$. Então $T(g \circ f)(a)=T(g \circ f)\left(a^{\prime}\right)$ e assim $T(h)(a)=$ $T(h)\left(a^{\prime}\right)$. Logo $T(\tilde{h})(b)=T(\tilde{h})(T(f)(a))=T(h)(a)=T(h)\left(a^{\prime}\right)=T(\tilde{h})\left(T(f)\left(a^{\prime}\right)\right)=T(\tilde{h})\left(b^{\prime}\right)$ e assim $\operatorname{ker}_{T}(g) \subseteq \operatorname{ker}_{T}(\tilde{h})$. Deste modo, sendo $g$ mapa quociente, existe $\bar{h}: C \rightarrow V$ tal que $\tilde{h}=\bar{h} \circ g$.

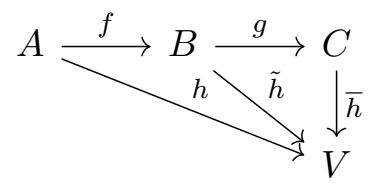

Assim $h=\bar{h} \circ(g \circ f)$. Pelo item $i i)$ temos que $g \circ f$ é epimorfismo e portanto $\bar{h}$ é o único mapa que faz o diagrama acima comutar. Logo, $g \circ f$ é mapa $T$-quociente.

v) Se $f: A \rightarrow B$ é injetor, então $\operatorname{ker}_{T}(f)=\operatorname{ker}_{T}\left(I_{A}\right)$. Assim, pela propriedade universal de $f$, existe $g: B \rightarrow A$ tal que o diagrama

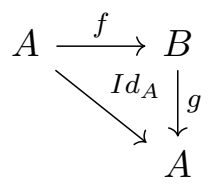

comuta. Deste modo $f \circ g \circ f=I d_{B} \circ f$ mas como $f$ é epimorfismo temos $f \circ g=I d_{B}$. Logo $f$ é isomorfismo.

vi) Como $f$ e $f^{\prime}$ são quocientes, existem e são únicos $h_{1}: B \rightarrow B^{\prime}$ e $h_{2}: B^{\prime} \rightarrow B$ tais que $f=h_{2} \circ f^{\prime}$ e $f^{\prime}=h_{1} \circ f$. Então $f=h_{2} \circ f^{\prime}=\left(h_{2} \circ h_{1}\right) \circ f$ e $f^{\prime}=\left(h_{1} \circ h_{2}\right) \circ f^{\prime}$. Como $f, f^{\prime}$ são epimorfismos, segue que $h_{2} \circ h_{1}=I d_{B}$ e $h_{1} \circ h_{2}=I d_{B^{\prime}}$.

$\{q u o I n j\}$

Definição A.6. Seja $(C, T)$ categoria quase-concreta. Uma subclasse $Q$ de morfismos de $C$ é dita quociente-injetiva se:

- todo morfismo de $Q$ é $T$-quociente e sobrejetor;

- dado morfismo $f: X \rightarrow Y$ em $C$, existe morfismo $\pi: X \rightarrow \bar{X}$ em $Q \operatorname{com} \operatorname{ker}_{T}(\pi)=\operatorname{ker}_{T}(f)$.

Proposição A.7. Sejam $(C, T)$ categoria quase-concreta e $Q$ subclasse quociente-injetiva. Sejam $f: X \rightarrow Y$ morfismo e $\pi: X \rightarrow \bar{X}$ em $Q \operatorname{com}^{\operatorname{ker}_{T}}(\pi)=\operatorname{ker}_{T}(f)$.

i) Como $\pi$ é $T$-quociente, seja $\bar{f}: \bar{X} \rightarrow Y$ o único morfismo satisfazendo $f=\bar{f} \circ \pi$. Então $\bar{f}$ é injetor. 
ii) Se $\pi^{\prime}: X \rightarrow X^{\prime}$ é outro morfismo em $Q \operatorname{com} \operatorname{ker}_{T}\left(\pi^{\prime}\right)=\operatorname{ker}_{T}(f)$, então existe um único isomorfismo $h: \bar{X} \rightarrow X^{\prime}$ tal que $\pi^{\prime}=h \circ \pi$.

Demonstração. $\quad$ i) Dado $(a, b) \in \operatorname{ker}_{T}(\bar{f})$, pela sobrejetividade de $\pi: X \rightarrow \bar{X}$ existem $a^{\prime}, b^{\prime} \in T(\bar{X})$ tais que $T(\pi)\left(a^{\prime}\right)=a, T(\pi)\left(b^{\prime}\right)=b . \operatorname{Logo}\left(a^{\prime}, b^{\prime}\right) \in \operatorname{ker}_{T}(f)=\operatorname{ker}_{T}(\pi)$ e assim $a=b . \operatorname{Logo} \bar{f}$ é injetora.

ii) Consequência direta do item vi) da Proposição A.5.

Definição A.8. Sejam $(C, T)$ categoria quase-concreta e $Q$ subclasse quociente-injetiva. Dado morfismo $f: X \rightarrow Y$, denotamos por $\pi_{f}: X \rightarrow \bar{X}$ o único morfismo em $Q$ a menos de isomorfismo (pela Proposição A.7) satisfazendo $\operatorname{ker}_{T}\left(\pi_{f}\right)=\operatorname{ker}_{T}(f)$ e por $\bar{f}: \bar{X} \rightarrow Y$ o único mapa satisfazendo $f=\bar{f} \circ \pi_{f}$.

Teorema A.9. Sejam $(C, T)$ categoria quase-concreta e $Q$ subclasse quociente-injetiva. Então \{quocientQC os morfismos de $Q$ classificam todos morfismos T-quocientes de $C$. Mais especificamente, sejam $f: X \rightarrow Y$ morfismo T-quociente e $\pi_{f}: X \rightarrow \bar{X}$ o morfismo T-quociente em $Q$ associado a $f$. Então $\bar{f}: \bar{X} \rightarrow Y$ é isomorfismo. Em particular, todo morfismo T-quociente é sobrejetor.

Demonstração. Pelo item i) da Proposição A.7, o morfismo $\bar{f}$ é injetor. Como, por hipótese, $f$ é $T$-quociente, então pelo item iii) da Proposicão $A .5$, temos que $\bar{f}$ é um $T$-quociente e, como este é injetor, segue do item v) da Proposição $A .5$ que $\bar{f}: \bar{X} \rightarrow Y$ é um isomorfismo. Além disso, como $\pi_{f}$ é sobrejetor, segue $f=\bar{f} \circ \pi_{f}$ também é sobrejetor. 


\section{Índice Remissivo}

0-RSG, 29

anel

de Witt, 80

abstrato, 117

regular, 101

graduado de Witt, 100

real, 116

B-conjunto, 124

B-par, 124

cone primo, 33

conjectura de Marshall, 102

elemento particionador, 123

envoltória

RS-Booleana, 141

von Neumann, 90

espectro

primo, 7

real, 33

feixe, 17

forma, 55

anisotrópica, 71

assinatura de, 56

de Pfister, 64

dimensão de, 56

isotrópica, 71

qc-, 59

regular, 83

suporte de, 59

fracamente inversível, 6

fórmula

geométrica, 15

Horn-geométrica, 6

hiper-anel, 5

von Neumann, 19

hipercorpo, 6

de frações, 11

de Krasner, 6

de sinais, 6 especial, 37

real reduzido, 36

ideal

fundamental, 87

gerado, 7

maximal, 7

primo, 7

T-convexo, 38

real, 34

imersão completa, 93

isometria, 56

limite indutivo, 14

localização, 10

mono-prefeixe, 17

morfismo

de multi-aneis, 6

de RS transversalmente 2-regular, 53

forte, 6

p-, 34

quociente, 8

multi-anel, 5

geométrico, 17

p-, 34

real reduzido, 36

semi-real, 34

multicorpo, 6

multidomínio, 5

núcleo, 8

ordem, 33

partição da unidade, 20

propriedade $a$-inversível, 16

pré-feixe estrutural, 17

pré-ordem, 34

quadrado-constante, 117

quociente

de Marshall, 12

por ideal, 9 
representação, 27

transversal, 27

reticulado, 122

semigrupo

real, 28

Booleano, 46

com muitas unidades, 136

com suficientes qc-formas, 136

com suficientes unidades, 133

von Neumann, 46

ternário, 27

sistema ortogonal, 60

subcategoria birreflexiva, 138

subgrupo

von Neumann, 22

saturado, 52

teorema

da decomposição de Witt, 72

da separação, 30

da subforma de Pfister, 79

Haupsatz de Arason-Pfister, 101

vN-conjunto, 124

vN-par, 124

Witt-equivalência, 56

álgebra de Post, 139

índice de Witt, 71 


\section{Referências Bibliográficas}

[AM] P.Arndt, H.L. Mariano, The von Neumann-regular Hull of (preordered) rings and quadratic forms, South American Journal of Logic 2 (2016), 201-244. 89

[AMa] V. Astier and H. L. Mariano, Realizing profinite reduced special groups, Pacific Journal of Mathematics, 250(2):257.285, 2011. 153

[AS] V. Astier, Pfister's subform theorem for reduced special groups, Journal of Pure and Applied Algebra, 189 (2004). 79, 80

[AT] V. Astier and M. Tressl, Axiomatization of local-global principles for pp-formulas in spaces of orderings, Archive of Mathematical Logic, 44:77.95, 2005. 153

[BD] R. Balbes, P. Dwinger, Distributive Lattices, Univ. of Missouri Press, 1974. 140

[CK] C.C. Chang, H.J. Kiesler, Model Theory, Studies in Logic and the Foundations of Mathematics, vol. $73,1990.6,19$

[Cra] T. C. Craven, Characterizing Reduced Witt Rings of Fields, Journal of Algebra 53 (1978), 68-77. 153

[DM1] M. Dickmann, F. Miraglia, Special Groups: Boolean-Theoretic Methods in the Theory of Quadratic Forms, Memoirs of the AMS 689, Providence, USA, 2000. 1, 3, 6, 29, 50, $89,100,101,108,122,124,132,139,148,149,150$

[DM2] M. Dickmann, F. Miraglia, On Quadratic Forms whose total signature is zero mod $2^{n}$. Solution to a problem of M. Marshall, Inventiones Mathematicae 133 (1998), 243-278. 1, 3, $89,110,141$

[DM3] M. Dickmann, F. Miraglia, Lam's Conjecture, Algebra Colloquium 10 (2003), 149-176.

[DM4] M. Dickmann, F. Miraglia, Elementary Properties of the Boolean Hull Functor and Reduced Quotient Functors, The Journal of Symbolic Logic 68 (2003), 946-971. 153

[DM5] M. Dickmann, F. Miraglia, Bounds for the representation of quadratic forms, Journal of Algebra 268 (2003), 209-251.

[DM6] M. Dickmann, F. Miraglia, Commutative Rings and Special Groups, preprint (2004).

[DM7] M. Dickmann, F. Miraglia, Algebraic K-theory of Special Groups, Journal of Pure and Applied Algebra 204 (2006), 195-234.

[DM8] M. Dickmann, F. Miraglia, Representation of reduced special groups in algebras of continuous functions, Quadratic forms - algebra, arithmetic and geometry 493, Contemp. Math., Amer. Math. Soc., Providence, RI, 2009. 153

[DM9] M. Dickmann, F. Miraglia, Quadratic form theory over preordered von Neumann-regular rings, J. Algebra 319(4) (2008), 1696-1732. 51, 103 
[DM10] M. Dickmann, F. Miraglia, Faithfully Quadratic Rings, Memoirs of the AMS 1128(4), Providence, USA, 2015. 43, 103, 154

[DMP] M. Dickmann, F. Miraglia, A. Petrovich, Constructions in the category of real semigroups, Ordered algebraic structures and related topics. Providence: AMS 2017. 64, 74

[DP1] M. Dickmann, A. Petrovich, Real Semigroups and Abstract Real Spectra, Contemporary Mathematics AMS 344 (2004), 99-119. 2, 27, 29, 30, 35, 136

[DP2] M. Dickmann, A.Petrovich, Real Semigroups, Real Spectra and Quadratic Forms over Rings, in preparation. $1,2,3,15,27,29,30,32,35,37,46,48,52,53,55,64,65,83,89,98,110,139$, $140,141,144,145,146,147,150$

[DST] M. Dickmann, N. Schwartz, M. Tressl, Spectral Spaces, Cambridge University Press, 2019. $30,35,144,145$

[Jun] J. Jun, Algebraic Geometry over Hyperrings, arXiv:1512.04837v1, 2015, 37pp. 2, 15

[Ken] J.F. Kennison, Integral Domain Type Representations in Sheaves and Other Topoi, Math. Z., 151, 35-36, 2006. 151

[Kul] M. Kula, Fields with prescribed quadratic form schemes, Mathematische Zeitschrift 167(3) (1979), 201-212. 153

[KMS] M. Kula, M. A. Marshall and A. Sladek, Direct limits of finites spaces of orderings, Pacific Journal of Mathematics, 112:391.406, 1984. 153

[Lam1] T. Y. Lam, The algebraic theory of quadratic forms, W. A. Benjamin, Massachusetts, USA, 1973.

[Lam2] T. Y. Lam, Orderings, valuations and quadratic forms, Regional Conference Series in Mathematics 52, American Mathematical Society, Providence, USA, 1983.

[Lam3] T. Y. Lam, Ten Lectures on Quadratic Forms over Fields, Queen's Papers in Pure and Applied Mathematics 46 (1977), 1-102.

[Lam4] T. Y. Lam, An introduction to real algebra, Rocky Mountain Journal of Mathematics 14 (1984), 767-814.

[Lam5] T. Y. Lam, Introduction to quadratic forms over fields, Graduate Studies in Mathematics, vol. 67, American Mathematical Society, Providence, RI, 2005.

[Lir] A. L. de Lima, Les groupes spéciaux. Aspects Algébriques et Combinatoires de la Théorie des Espaces d'Ordres Abstraits, Thèse de doctorat, Université Paris VII, Paris, France, 1996.

[Mar1] M. A. Marshall, A reduced theory of quadratic forms, Queen's Papers in Pure and Applied Mathematics 46 (1977), 569-579.

[Mar2] M. A. Marshall, Classification of Finite Spaces of Orderings, Canadian Journal of Mathematics 31 (1979), 320-330.

[Mar3] M. A. Marshall, Quotients and Inverse Limits of Spaces of Orderings, Canadian Journal of Mathematics 31 (1979), 604-616.

[Mar4] M. A. Marshall, Spaces of Orderings IV, Canadian Journal of Mathematics 32 (1980), 603-627.

[Mar5] M. A. Marshall, The Witt Ring of a Space of Orderings, Transaction of the AMS 258 (1980), 505-521. 
[Mar6] M. Marshall, Abstract Witt Rings, Queen's Papers in Pure and Applied Mathematics 57, Queen's University, Canada, 1980. 110

[Mar7] M. A. Marshall, Spaces of Orderings and Abstract Real Spectra, Lecture Notes in Mathematics 1636, Springer-Verlag, Berlin, Germany, 1996. 1, 27, 30, 46, 51, 79, 86, 96, 116, $122,136,148,154$

[Mar8] M. A. Marshall, Open questions in the theory of spaces of orderings, Journal of Symbolic Logic 67 (2001), 341-352.

[Mar9] M. A. Marshall, Local-global properties of positive primitive formulas in the theory of spaces of orderings, Journal of Symbolic Logic 71 (2006), 1097-1107. 153

[Mar10] M. Marshall, Real reduced multirings and multifields, Journal of Pure and Applied Algebra 205 (2006), 452-468. 2, 5, 6, 7, 9, 12, 27, 33, 35, 36

[Mir] F. Miraglia, Introduction to Partially Ordered Structures and Sheaves, Polimetrica Scientific Publishers, Contemporary Logic Series 1, Milan, 2007. 15

[MM1] Mariano, H.L., Miraglia, F., The Profinite Hull of Special Groups and Local-Global Principles, Stud Logica 97 (2011), 127-160. 3, 89, 153

[MM2] H. L. Mariano and F. Miraglia, The Boolean and profinite hulls of reduced special groups, Logic Journal of IGPL, 21(2):160.182, 2013. 153

[MM3] H. L. Mariano and F. Miraglia, Model-theoretic applications of the profinite hull functor of special groups, South American Journal of Logic, 1(1): 111.133, 2015. 153

[Mrn1] H. L. Mariano, Contribuições à teoria dos Grupos Especiais, Tese de Doutoramento, Universidade de São Paulo, São Paulo, Brasil, 2003.

[Mrn2] H. L. Mariano, The Von Neumann-regular Hull of (preordered) rings and quadratic forms, em preparação. 153

[DMR] M. Dickmann, F. Miraglia, H.R.O. Ribeiro, Special Groups and Quadratic Forms over Rings with non Zero-Divisor Coefficients, submitted (2021).

[MR] F. Miraglia, H.R.O. Ribeiro, Boolean Real Semigroups, To appear in Tributes, College Publications (2020). 2, 27, 46, 47, 52, 121, 123

[RM1] H.R.O. Ribeiro, H.L. Mariano, Witt rings for Real Semigroups, in preparation.

[RM2] H.R.O. Ribeiro, H.L. Mariano, Hulls for Real Semigroups and applications, in preparation.

[RM3] H.R.O. Ribeiro, H.L. Mariano, von Neumann regular Hyperrings and applications to Real Reduced Multirings. Disponível em https://arxiv.org/abs/2101.06527. 46, 97, 99, 102

[RM4] H.R.O. Ribeiro, H.L. Mariano, Horn-Geometric Axioms to Faithfully Quadratic Rings, Submetido para publicação no proceedings do EBL 2019.

[RRM1] H.R.O. Ribeiro, K.M.A Roberto, H.L. Mariano, Functorial relationship between multirings and the various abstract theories of quadratic forms, to appear in São Paulo Journal of Mathematical Sciences, preliminary version available at https://arxiv.org/pdf/1610.00816.pdf, 2016, 27pp. 2, 5, 9, 29, 31, 37

[RRM2] H.R. Ribeiro, K.M.A. Roberto, H.L. Mariano, Promiscuously Quadratic Rings, arXiv preprint (2016), https://arxiv.org/abs/1611.09270. 154 